\title{
Rotation compatibility approach to moment redistribution for design and rating of steel I-girders
}

Jennifer E. Righman

West Virginia University

Follow this and additional works at: https://researchrepository.wvu.edu/etd

\section{Recommended Citation}

Righman, Jennifer E., "Rotation compatibility approach to moment redistribution for design and rating of steel I -girders" (2005). Graduate Theses, Dissertations, and Problem Reports. 2670.

https://researchrepository.wvu.edu/etd/2670

This Dissertation is protected by copyright and/or related rights. It has been brought to you by the The Research Repository @ WVU with permission from the rights-holder(s). You are free to use this Dissertation in any way that is permitted by the copyright and related rights legislation that applies to your use. For other uses you must obtain permission from the rights-holder(s) directly, unless additional rights are indicated by a Creative Commons license in the record and/ or on the work itself. This Dissertation has been accepted for inclusion in WVU Graduate Theses, Dissertations, and Problem Reports collection by an authorized administrator of The Research Repository @ WVU.

For more information, please contact researchrepository@mail.wvu.edu. 


\title{
Rotation Compatibility Approach to Moment Redistribution for Design and Rating of Steel I-Girders
}

\author{
Jennifer E. Righman
}

Dissertation submitted to the College of Engineering and Mineral Resources

at

West Virginia University in partial fulfillment of the requirements

for the degree of

\author{
Doctor of Philosophy \\ in \\ Civil Engineering
}

\author{
Karl E. Barth, Ph.D., Chair \\ Michael G. Barker, Ph.D. \\ Julio F. Davalos, Ph.D. \\ Jacky C. Prucz, Ph.D. \\ John P. Zaniewski, Ph.D.
}

Department of Civil and Environmental Engineering

Morgantown, WV

2005

Keywords: Steel, Bridges, Design, Rating, Inelastic, Experimental Data, Finite Elements

(C) 2005 Jennifer E. Righman 


\title{
ABSRACT \\ Rotation Compatibility Approach to Moment Redistribution for Design and Rating of Steel I-Girders
}

\author{
Jennifer E. Righman
}

Moment redistribution refers to the design practice where the inherent ductility of continuous-span steel bridge structures is acknowledged and consideration for the redistribution of the large negative bending moments at interior supports to the less heavily stressed positive bending regions is provided. One of the key assumptions made in moment redistribution procedures is that members have sufficient ductility to sustain a given moment capacity throughout the level of rotation required for redistribution moments to develop. However, there is presently no explicit verification that this assumption is satisfied. Instead, it is assumed that sufficient ductility can be obtained by: (1) limiting the range of girders for which moment redistribution is permissible to relatively compact members and (2) limiting the amount of moment that may be redistributed. Furthermore, the restriction of these procedures to relatively compact members has limited the potential for application of these procedures to the rating and permitting processes for existing bridge structures.

The research presented herein is aimed at overcoming this limitation through a rotation compatibility approach. This method consists of direct comparison of rotation requirements for moment redistribution to available rotation (ductility) of composite or non-composite steel I-girders. An analysis procedure for determining rotation requirements is discussed along with research results illustrating the relationship between intended level of redistribution moment and required rotation for typical bridge designs. An investigation into the available rotation of typical steel I-girders is also described. It is shown that this available rotation can also be related to the intended level of redistribution moment. Thus, relating both the available and required rotations to a common parameter facilitates a direct comparison between these two quantities.

The advantages of this approach compared to current design practices are also discussed. The rotation compatibility approach offers increased design economy by providing a rational basis for determining the class of sections for which moment redistribution is valid and thereby extending the applicability of the specifications. In addition to the benefits offered by this approach in new designs, there is significant potential for economic savings through application of these procedures to bridge rating. 


\section{ACKNOWLEDGEMENTS}

Numerous individuals and organizations are deserving of recognition for their contributions towards the successful completion of this dissertation. I would first like to thank my advisor, Dr. Karl Barth, for his integral and dedicated role in helping me to achieve my professional goals.

The experimental work performed in this project was facilitated by the assistance of Messrs. Bill Comstock, Nate Bowe, Brandon Enochs, Laco Corder, and Alex Stinard. In particular, the dedicated effort of Mr. Bill Comstock is sincerely appreciated.

The contributions of Ms. Lili Yang, who was instrumental in the development of many of the tools used in the finite element analysis conducted herein, are gratefully acknowledged. I would also like to thank Mr. Brandon Enochs for his assistance with the final formatting of the appendices of this report.

The members of the doctoral committee for this work are Drs. Julio Davalos, Michael Barker, Jacky Prucz, and John Zaneiwski and they are deserving of thanks for their respective contributions to this project. I am also appreciative of Dr. David Martinelli, Chair of the WVU Civil and Environmental Engineering Department, for his support of this work.

Financial support for this project was provided by the West Virginia Department of Transportation Division of Highways and is gratefully acknowledged. Fabrication of the experimental girders included in this work was generously donated by American Bridge Manufacturing in Coraopolis, PA. The assistance of Mr. Darko Jurkovic at American Bridge Manufacturing is especially appreciated. 
I lovingly acknowledge my greatest teachers and parents, Mr. and Mrs. Jerry and Nancy Righman, for their continuous support. And lastly, I would like to express my deep appreciation of the countless sacrifices made by my future husband, Mr. Jesse McConnell, who has done everything in his ability to make this task as easy as possible. 


\section{TABLE OF CONTENTS}

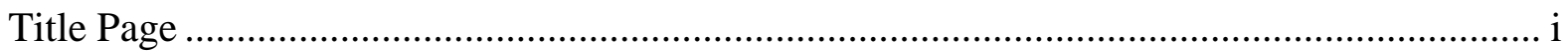

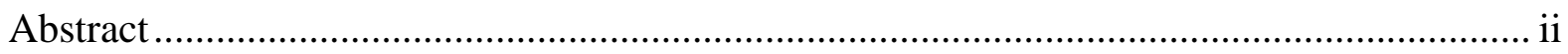

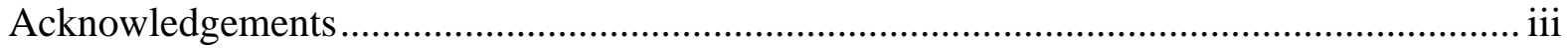

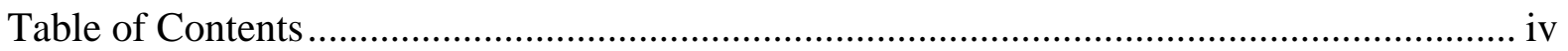

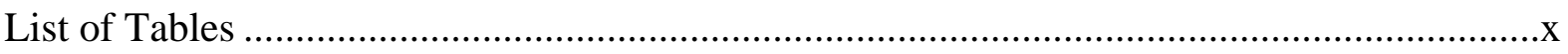

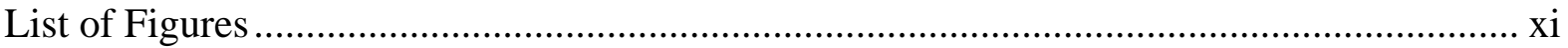

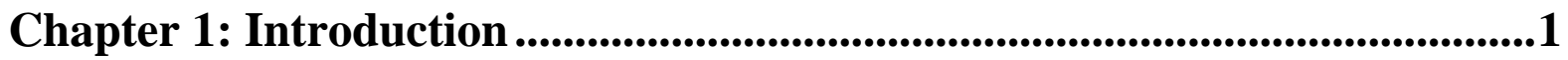

1.1 General Theory of Moment Redistribution...................................................................5

1.2 General Theory of Rotation Compatibility ............................................................4

1.3 Need for Current Research ............................................................................4

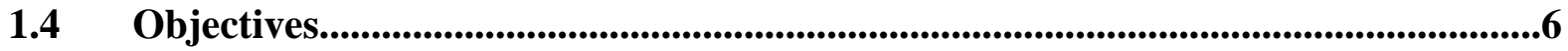

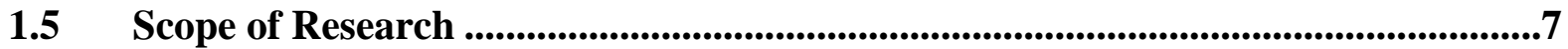

1.6 Dissertation Organization .................................................................................8

Chapter 2: Literature Review...............................................................................11

2.1 Moment Redistribution Design...............................................................................11

2.1.1 Initial Inelastic Provisions (AAHSTO 1973) ..................................................11

2.1.2 Alternate Load Factor Design (AASHTO 1986)...............................................12

2.1.3 Unified Autostress Method (Schilling 1989) .....................................................15

2.1.4 AASHTO LRFD Specifications (AASHTO 1994 and 1998)...............................15

2.1.5 AASHTO $3^{\text {rd }}$ Edition LRFD Specifications (2004) .........................................16

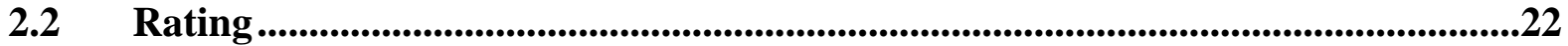

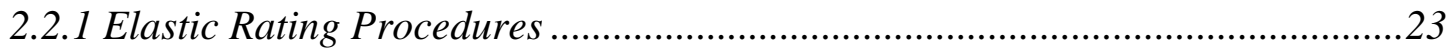

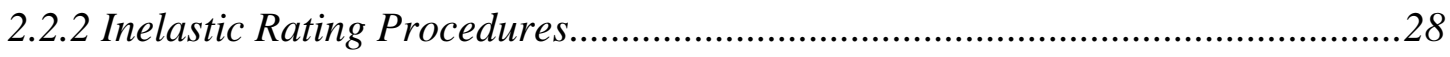

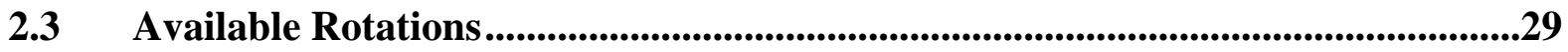

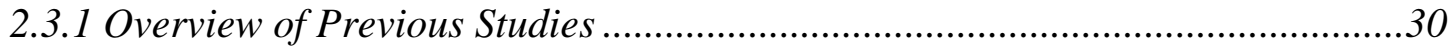

2.3.1.1 Experimental Studies.............................................................................................................. 


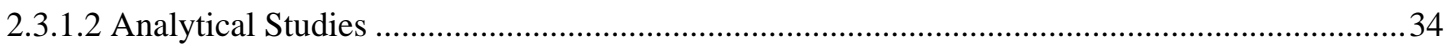

2.3.2 Factors Influencing Available Rotations ......................................................38

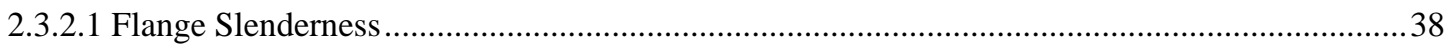

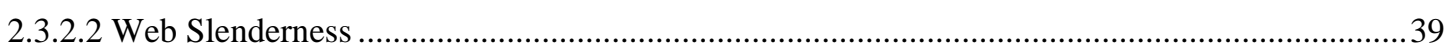

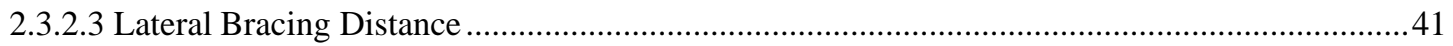

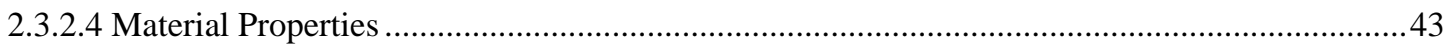

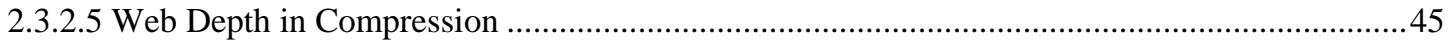

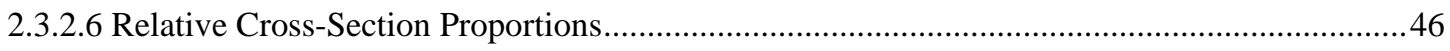

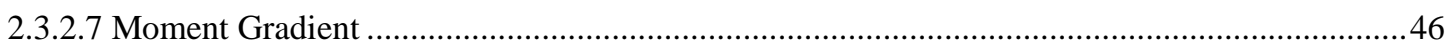

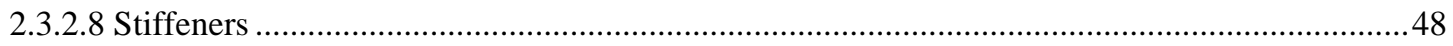

2.3.2.9 Shear Force

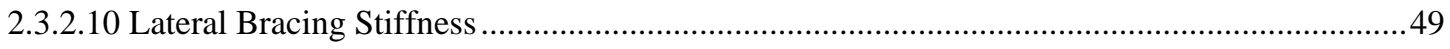

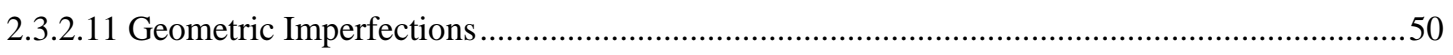

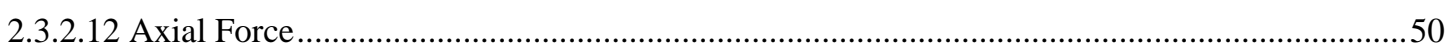

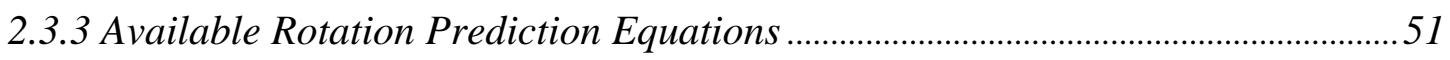

$2.4 \quad$ Rotation Requirements......................................................................................................56

Chapter 3: Experimental Testing......................................................................62

3.1 General Information ...................................................................................................62

3.2 Parametric Values.........................................................................................................66

3.2.1 Web Slenderness ..........................................................................................68

3.2.2 Compression Flange Slenderness ......................................................................69

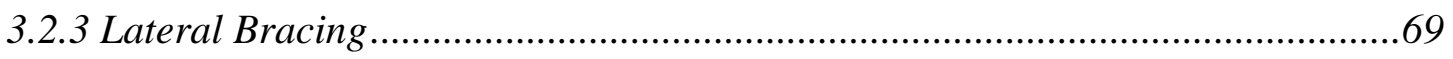

3.2.4 Cross-Section Aspect Ratio.............................................................................

3.2.5 Material Specifications ......................................................................................70

3.2.6 Percentage of Web Depth in Compression .........................................................70

3.2.7 Comparison of Parametric Values to Previous Experimental Girders ...............71

$3.3 \quad$ Individual Girder Properties ...............................................................................................71

3.4 Testing Procedure ...............................................................................................................78

3.4.1 General Considerations .................................................................................78

3.4.2 Data Collection ...........................................................................................

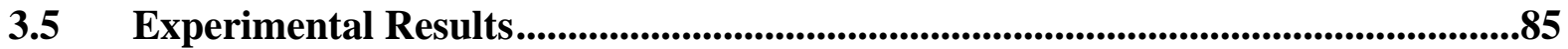

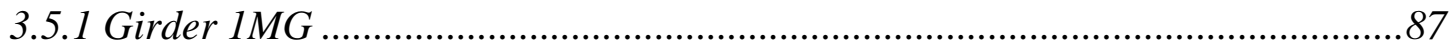

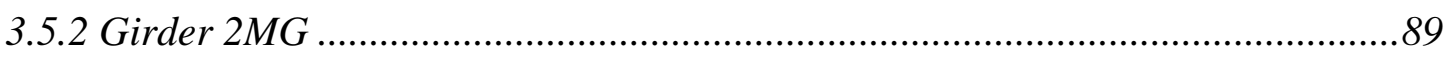




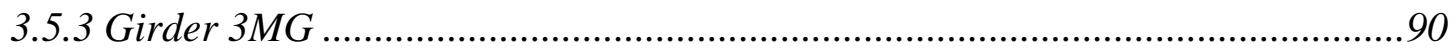

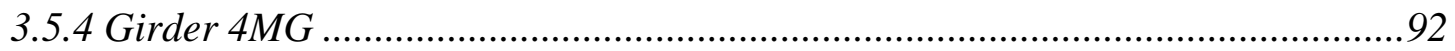

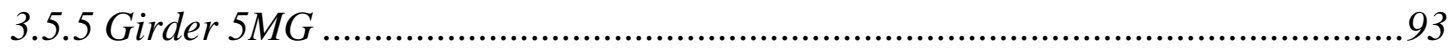

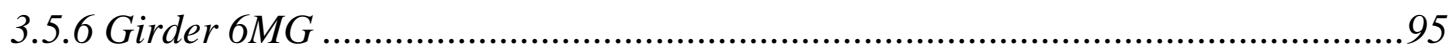

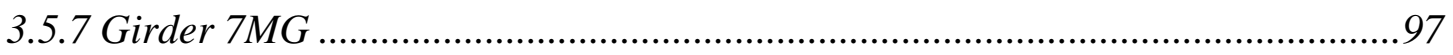

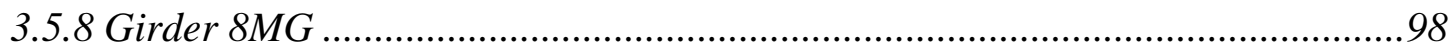

3.5.9 Girder 9MG …………………………………................................101

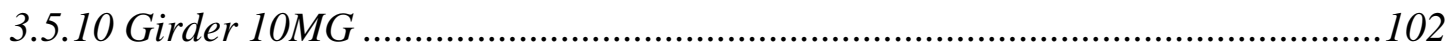

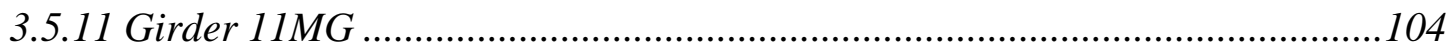

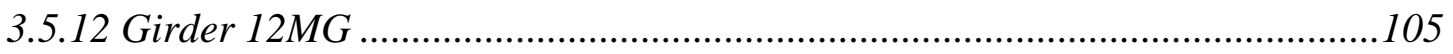

3.5.13 Summary of Experimental Results ...............................................................107

3.5.13.1 Moment Capacity …………………………………………………………………....107

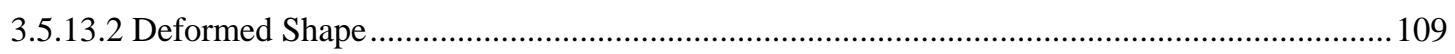

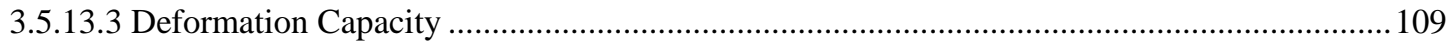

\section{Chapter 4: Nonlinear FEA Modeling Procedures .............................................111}

4.1 Mesh Density .....................................................................................................................111

4.2 Element Selection .................................................................................................112

4.2.1 Element Naming Convention .....................................................................112

4.2.2 General Purpose Shell Elements ...................................................................112

4.3 Material Modeling ...............................................................................................................115

4.3.1 Von Mises Yield Criterion.............................................................................116

4.3.2 Associated Flow Rule..................................................................................117

4.3.3 Isotropic Hardening ...................................................................................117

4.3.4 Stress-Strain Relationship..........................................................................118

4.4 Geometric Imperfections................................................................................120

4.5 Modeling of Residual Stresses.....................................................................................124

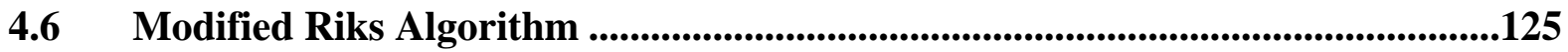

$4.7 \quad$ Summary...................................................................................................................130

\section{Chapter 5: FEA of Steel I-Girders for the Determination of Moment-} Rotation Characteristics..................................................................131

5.1 FEA of Experimental Girders..................................................................................132 
5.2 FEA Parametric Study .....................................................................................147

5.3 FEA of Design Study Girders ....................................................................................152

5.4 FEA of Extended Design Studies.........................................................................156

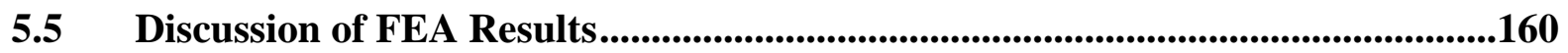

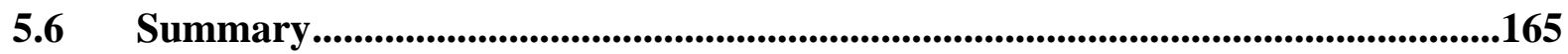

Chapter 6: Rotation Requirements for Steel Bridge I-Girders .................167

6.1 Background .........................................................................................................................168

6.2 Computation of Rotation Requirements...................................................................169

6.2.1 Required Plastic Rotation ...........................................................................169

6.2.2 Required Rotation Capacity.............................................................................175

6.3 Representative Designs ..................................................................................................176

6.4 Discussion of Rotation Requirements .........................................................................181

6.4.1 Moment of Inertia Considerations .................................................................182

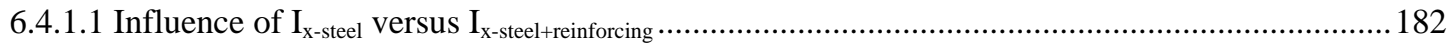

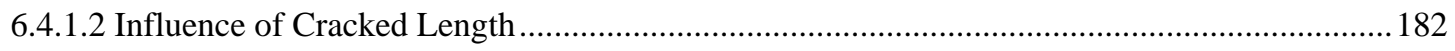

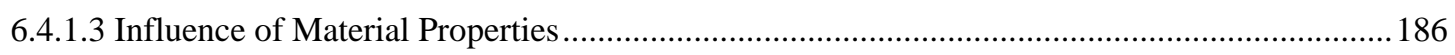

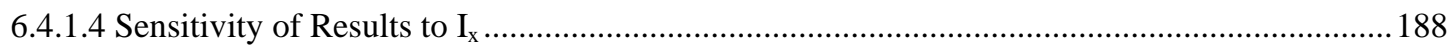

6.4.2 Influence of Span Length ................................................................................191

6.4.3 Influence of Redistribution Moment....................................................................193

6.4.4 Influence of Number of Spans ..........................................................................197

6.4.5 Required Rotation Capacity...........................................................................198

6.4.6 Development of Required Rotation Prediction Equations..................................203

\section{Chapter 7: Rotation Compatibility -}

Design and Rating Specifications .....................................................................207

7.1 Comparison of Available and Required Rotations ....................................................208

7.1.1 Summary of Available Rotations....................................................................208

7.1.2 Summary of Required Rotations ...................................................................209

7.1.3 Comparison of Available and Required Rotations ..........................................210

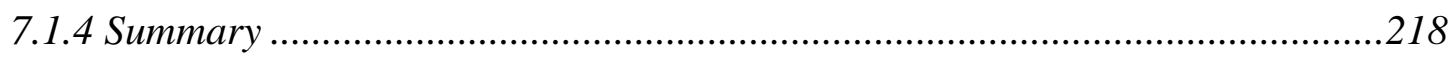

7.2 Rotation Compatibility Design Specifications...............................................................219 


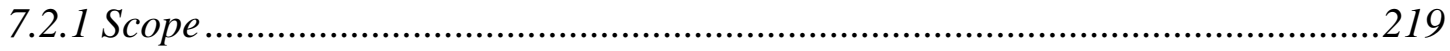

7.2.2 Overview of Design Procedure...................................................................219

7.2.3 Rotation Compatibility Design Specification Equations ....................................220

7.2.4 Comparison to AASHTO 2004 Moment Redistribution Procedures ..................221

7.3 Rotation Compatibility Rating Specifications..............................................................224

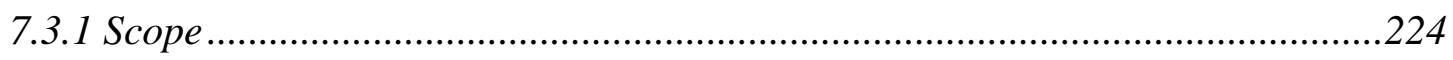

7.3.2 Overview of Rating Procedure......................................................................224

7.3.3 Rotation Compatibility Rating Specification Equations .....................................225

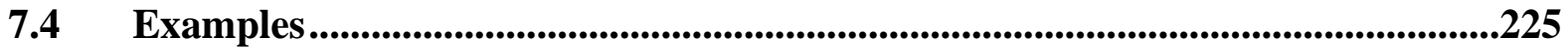

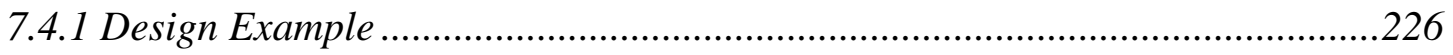

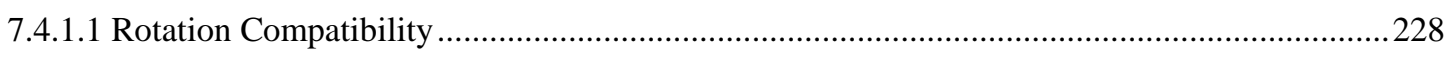

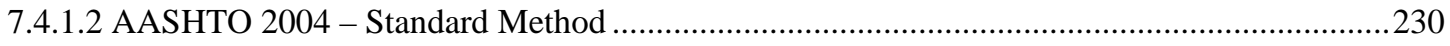

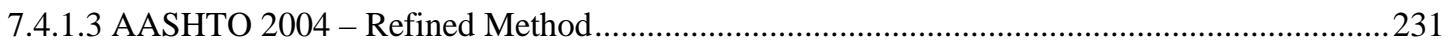

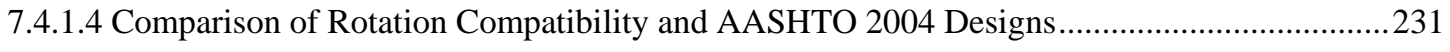

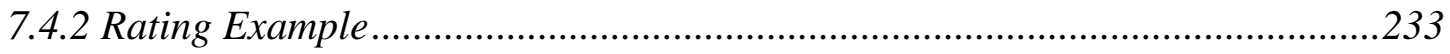

$7.5 \quad$ Summary .......................................................................................................................236

Chapter 8: Summary, Conclusions, and Recommendations........................2237

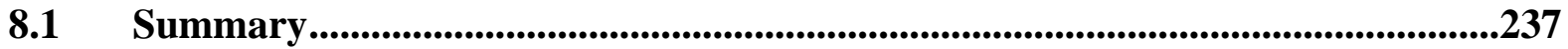

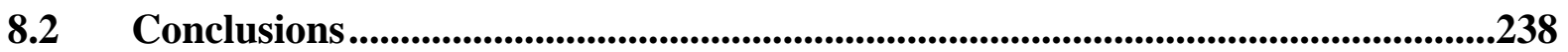

8.3 Recommendations for Future Research ................................................................242

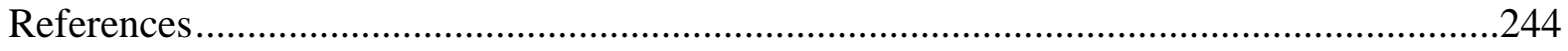

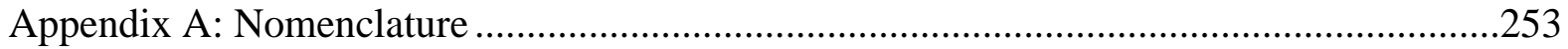

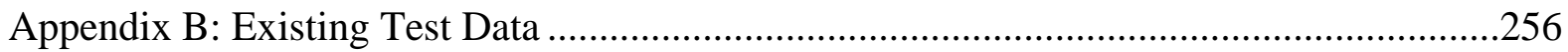

Appendix C: Material Testing Results...........................................................................296

Appendix D: FEA Moment-Rotation Curves .....................................................................302

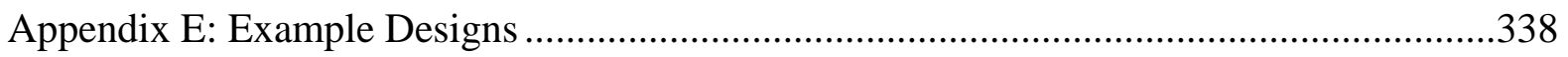




\section{LIST OF TABLES}

Table 3-1. Stiffener Thicknesses used in Experimental Girders (in.) .........................68

Table 3-2. Parametric Values of Experimental Girders...............................................72

Table 3-3. Nominal Dimensions and Yield Strengths for Experimental Girders ......73

Table 3-4. Actual Dimensions and Yield Strengths for Experimental Girders...........74

Table 3-5. Target and Actual Parametric Values in Experimental Girders ..............75

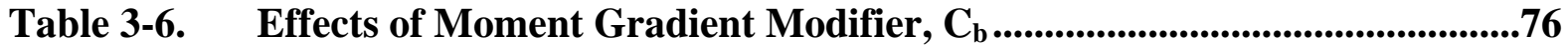

Table 3-7. Average Initial Imperfections in Experimental Girders ............................78

Table 3-8. Comparison of Experimental and AASHTO (2004) Capacities

(ft-kips) ........................................................................................................85

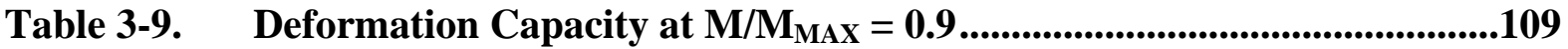

Table 5-1. Comparison of Experimental and FEA Maximum Capacities................139

Table 5-2. $\quad \theta_{\text {RL }}$ Values in $\operatorname{mrad}($ part 1) ........................................................................147

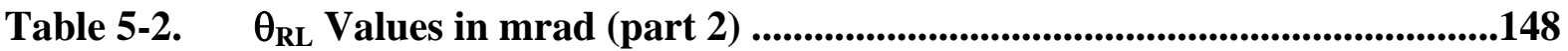

Table 5-3. Scope of Parametric Study ...........................................................................149

Table 6-1. Comparison of Rotation Requirements for Hybrid versus Homogeneous Girders .........................................................................187

Table 6-2. Comparison of Rotation Requirements for Un-stiffened versus Stiffened Girder

Table 6-3. Comparison of Rotation Requirements for Sub-Optimal Girders..........190

Table 6-4. Rotation Requirements Resulting From Analysis of Experimental

Data

Table 7-1. Comparison of Performance Ratios from Rotation Compatibility and Moment Redistribution Designs 


\section{LIST OF FIGURES}

Figure 1-1. Illustration of Moment Redistribution Concept ...............................................2

Figure 2-1. Illustration of Effective Plastic Moment Concept .....................................13

Figure 2-2. Barth and White Moment-Rotation Model ..............................................17

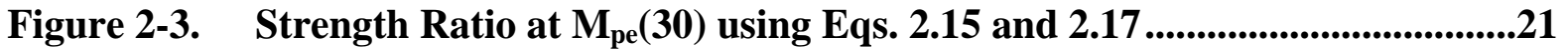

Figure 2-4. Strength Ratio at $\mathrm{M}_{\mathrm{pe}}(9)$ using Eqs. 2.16 and 2.18 ......................................21

Figure 2-5. Strength Ratio at $\mathbf{M}_{\text {pe }}(30)$ using $A L F D ~ M_{p e}$ Equations ...............................22

Figure 2-6. Strength Ratio at $M_{p e}(9)$ using ALFD $M_{p e}$ Equations ..................................22

Figure 2-7. Flowchart for LRFR ...............................................................................25

Figure 2-8. AASHTO Legal Loads..................................................................................27

Figure 2-9. Illustration of Variable Definitions used by Kemp (1986) .........................35

Figure 2-10. Schematic Diagram of FEA Techniques used by Lääne and Lebet (2005)

Figure 2-11. Available Rotation versus Lateral Slenderness Ratio by Kemp and Dekker (1991) ...................................................................................42

Figure 2-12. Diagonal Stiffener Proposed by Kemp (1986) ..............................................49

Figure 2-13. Equivalent Section used by Kato (1989) ...............................................52

Figure 2-14. Available Rotation Equation Suggested by Lääne and Lebet (2005) Compared to Experimental Data ..............................................................56

Figure 2-15. Definition of Rotation Capacity ..................................................................57

Figure 2-16. Rotation Requirements - Kemp and Dekker (1991) ................................59

Figure 3-1. Typical Continuous-Span Girder and Test Specimen..................................62

Figure 3-2. Photograph of Experimental Setup, Viewed from Above...........................63

Figure 3-3. Photograph of Experimental Setup, Viewed from End of Girder.............64

Figure 3-4. General Test Girder Configuration, Elevation View................................64

Figure 3-5. Lateral Bracing System used in Experimental Testing.............................66

Figure 3-6. Lateral Frames Providing Longitudinal Restraint in Latter Stages of Experiment

Figure 3-7. Initial Geometric Imperfections Considered in Experimental Girders....77

Figure 3-8. Schematic Diagram of Instrumentation Used in Experimental Testing...80

Figure 3-9. $\quad$ Photograph of Instrumentation at Ends of Girder 
Figure 3-10. Photograph of Instrumentation at Girder Centerline ...................................82

Figure 3-11. Photograph of Instrumentation at SL and SR .......................................83

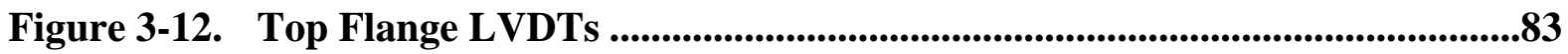

Figure 3-13. Bottom Flange LVDTs.............................................................................84

Figure 3-14. Web LVDTs.........................................................................................8

Figure 3-15. Deformed Shape of Girder 1MG .......................................................88

Figure 3-16. Load versus Displacement Data for Girder 1MG.....................................88

Figure 3-17. Deformed Shape of Girder 2MG ..............................................................89

Figure 3-18. Load versus Displacement Data for Girder 2MG....................................90

Figure 3-19. Deformed Shape of Girder 3MG ................................................................91

Figure 3-20. Load versus Displacement Data for Girder 3MG.........................................92

Figure 3-21. Deformed Shape of Girder 4MG .......................................................................93

Figure 3-22. Load versus Displacement Data for Girder 4MG......................................94

Figure 3-23. Deformed Shape of Girder 5MG ..............................................................94

Figure 3-24. Load versus Displacement Data for Girder 5MG....................................95

Figure 3-25. Deformed Shape of Girder 6MG .................................................................96

Figure 3-26. Load versus Displacement Data for Girder 6MG.........................................97

Figure 3-27. Deformed Shape of Girder 7MG ........................................................................98

Figure 3-28. Load versus Displacement Data for Girder 7MG ...................................99

Figure 3-29. Deformed Shape of Girder 8MG ........................................................100

Figure 3-30. Load versus Displacement Data for Girder 8MG.......................................100

Figure 3-31. Deformed Shape of Girder 9MG .......................................................101

Figure 3-32. Load versus Displacement Data for Girder 9MG...................................102

Figure 3-33. Deformed Shape of Girder 10MG ................................................................103

Figure 3-34. Load versus Displacement Data for Girder 10MG................................104

Figure 3-35. Deformed Shape of Girder 11MG ...................................................................105

Figure 3-36. Load versus Displacement Data for Girder 11MG....................................106

Figure 3-37. Deformed Shape of Girder 12MG .....................................................107

Figure 3-38. Load versus Displacement Data for Girder 12MG.....................................108

Figure 4-1. $\quad$ Element Natural Coordinate System ............................................................114

Figure 4-2. Von Mises Yield Criterion...................................................................................116

Figure 4-3. Stress-Strain Relationship for Grade 50 Steel...........................................118

Figure 4-4. Stress-Strain Relationship for Grade 70 Steel............................................119 
Figure 4-5. Initial Geometric Imperfections ......................................................................121

Figure 4-6. Residual Stress Pattern...............................................................................125

Figure 4-7. Modified Riks Algorithm ............................................................127

Figure 5-1. FEA and Experimental Load versus Displacement Data -

Girder 1MG

Figure 5-2. FEA and Experimental Load versus Displacement Data -

Girder 2MG

Figure 5-3. FEA and Experimental Load versus Displacement Data -

Girder 3MG.

Figure 5-4. FEA and Experimental Load versus Displacement Data -

Girder 4MG.

Figure 5-5. FEA and Experimental Load versus Displacement Data -

Girder 5MG

Figure 5-6. FEA and Experimental Load versus Displacement Data -

Girder 6MG.

Figure 5-7. FEA and Experimental Load versus Displacement Data -

Girder 7MG.

Figure 5-8. FEA and Experimental Load versus Displacement Data -

Girder 8MG .136

Figure 5-9. FEA and Experimental Load versus Displacement Data -

Girder 9MG.

Figure 5-10. FEA and Experimental Load versus Displacement Data -

Girder 10MG

Figure 5-11. FEA and Experimental Load versus Displacement Data -

Girder 11MG

Figure 5-12. FEA and Experimental Load versus Displacement Data -

Girder 12MG

Figure 5-13. FEA and Experimental Deformed Shapes - Girder 1MG

Figure 5-14. FEA and Experimental Deformed Shapes - Girder 2MG.

Figure 5-15. FEA and Experimental Deformed Shapes - Girder 3MG.

Figure 5-16. FEA and Experimental Deformed Shapes - Girder 4MG.

Figure 5-17. FEA and Experimental Deformed Shapes - Girder 5MG.

Figure 5-18. FEA and Experimental Deformed Shapes - Girder 6MG 
Figure 5-19. FEA and Experimental Deformed Shapes - Girder 7MG ........................143

Figure 5-20. FEA and Experimental Deformed Shapes - Girder 8MG .......................144

Figure 5-21. FEA and Experimental Deformed Shapes - Girder 9MG .....................144

Figure 5-22. FEA and Experimental Deformed Shapes - Girder 10MG .....................145

Figure 5-23. FEA and Experimental Deformed Shapes - Girder 11MG .....................145

Figure 5-24. FEA and Experimental Deformed Shapes - Girder 12MG ....................146

Figure 5-25. Moment versus Rotation - Girder CF-1-65-4-50 ....................................149

Figure 5-26. Moment versus Rotation - Girder SF-30-65-3-H ...................................149

Figure 5-27. Moment versus Rotation for Design Study Girders - Group 1 ..............154

Figure 5-28. Moment versus Rotation for Design Study Girders - Group 2 ...............155

Figure 5-29. Moment versus Rotation for Design Study Girders - Group 3 ................155

Figure 5-30. Moment versus Rotation for Design Study Girders - Group 4 ................156

Figure 5-31. Moment versus Rotation for Permutations of

Girder 120-2-E-50-20-A...............................................................................157

Figure 5-32. Moment versus Rotation for Permutations of

Girder 120-2-E-H-10-B-(S) 158

Figure 5-33. Moment versus Rotation for Permutations of

Girder 180-2-E-50-20-B.

Figure 5-34. Moment versus Rotation for Permutations of

Girder 210-2-E-50-10-E.

Figure 5-35. $\quad \theta_{\mathrm{RL}}$ versus Unbraced Length for Girders Violating Current Moment

Redistribution Lateral Bracing Limits ...............................................163

Figure 5-36. Moment Rotation Model for Compact Girders (AASHTO 2004)...........164

Figure 5-37. Moment Rotation Model for Girders Violating Eq. 5.3 ..........................165

Figure 5-38. Moment versus Rotation - Girder CF-1-65-4-50 .......................................165

Figure 5-39. Moment versus Rotation - Girder SF-30-65-3-H ..................................166

Figure 6-1. Computation of Required Rotations in a Two-Span Continuous

Beam with Equal Span Lengths

Figure 6-2. Computation of Required Rotations in a Four-Span Continuous

Beam .........................................................................................................171

Figure 6-3. Definition of Variables used in Equation 6.2...........................................172

Figure 6-4. Definition of Rotation Capacity (Galambos 1968)...................................175 
Figure 6-5. Representation of Negative Bending Section of Continuous-Span Girder as Simply-Supported Beam ............................................................176

Figure 6-6. Bridge Cross-Section for Two-Span Designs..........................................177

Figure 6-7. Bridge Cross-Section for Three- and Four-Span Designs.........................178

Figure 6-8. Deck Casting Sequence for Two-Span Designs .........................................179

Figure 6-9. Deck Casting Sequence for Three-Span Designs ...................................180

Figure 6-10. Deck Casting Sequence for Four-Span Designs .......................................180

Figure 6-11. Required Rotation versus Cracked Length (ft), $M_{r d}=20 \% M_{e}$, Transition from Cracked to Composite occurs in Pier Cross-Section....184

Figure 6-12. Required Rotation versus Cracked Length (ft), $M_{r d}=20 \% M_{e}$, Transition from Cracked to Composite occurs in Negative Bending Region. .185

Figure 6-13. Required Rotation versus Cracked Length (as a percentage of total span length), $M_{r d}=20 \% M_{e}$,Transition from Cracked to Composite occurs in Negative Bending Region

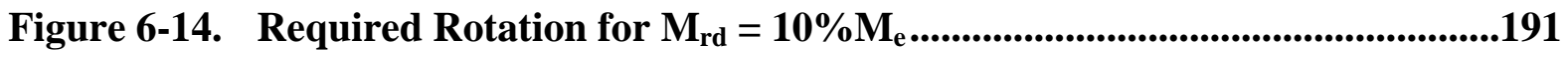

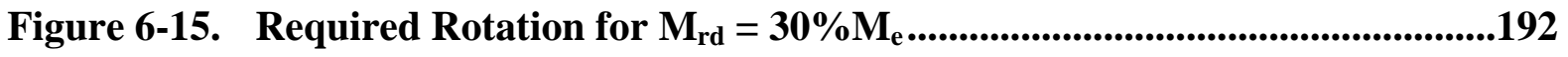

Figure 6-16. Required Rotation for $M_{r d}=40 \% M_{e}$

Figure 6-17. Normalized Rotation Requirements (by percentage of redistribution moment) for $M_{r d}=10 \% M_{e}$

Figure 6-18. Normalized Rotation Requirements (by magnitude of redistribution moment) for $M_{r d}=10 \% M_{e}$

Figure 6-19. Relationship Between Percentage of Redistribution Moment and Required Plastic Rotation

Figure 6-20. Relationship Between Magnitude of Redistribution Moment and Required Plastic Rotation 196

Figure 6-21. End Rotations in Prismatic Simply-Supported Beam .197

Figure 6-22. Relationship Between Percentage of Moment Redistributed and Required Rotation Capacity .

Figure 6-23. Illustration of Procedure to Determine Rotation Requirements from Experimental Moment Rotation Curves. 
Figure 6-24. Relationship Between Percentage of Moment Redistributed and Required Rotation Capacity as Determined From Experimental Results

Figure 6-25. Relationship Between Required Plastic Rotation and Percentage of Redistribution Moment for Two-Span Grade 50 Designs ........................205

Figure 7-1. Moment-Rotation Models ...............................................................209

Figure 7-2. Example Girder..........................................................................211

Figure 7-3. Comparison of Available and Required Rotations for Example Girder with $L_{b}$ satisfying Eq. 7.1

Figure 7-4. Comparison of Available and Required Rotations for Example

Girder with $L_{b}$ violating Eq. 7.1 ...................................................................217

Figure 7-5. Design Example Bridge Cross-Section.............................................................226

Figure 7-6. Casting Sequence for Design Example....................................................227

Figure 7-7. Rotation Compatibility and Moment Redistribution Designs ..................232

Figure 7-8. Girder Elevation Rating Example..............................................................233

Figure 8-1. Moment Rotation Model ..........................................................................239 


\section{Chapter 1: INTRODUCTION}

This dissertation is focused on inelastic design and rating procedures for continuous-span steel I-girder bridges. Design procedures incorporating inelastic methods are currently incorporated in AASHTO Load and Resistance Factor Design (LRFD) Specifications (2004) as optional provisions, termed "moment redistribution" procedures. These methods assume that the ductility necessary for moment redistribution can be attained by implementing two restrictions. These restrictions limit (1) the types of girders that may be used in conjunction with moment redistribution procedures and (2) the magnitude of moment that may be redistributed. Consequently, these requirements decrease the potential economic benefits of inelastic design procedures. Furthermore, at the present time, moment redistribution procedures are included in the design specifications only; these procedures are not included in the AASHTO Load and Resistance Factor Rating (LRFR) Specifications (2003). One primary reason for this is that the majority of existing bridge girders do not satisfy the current requirements for use of moment redistribution provisions. Therefore, by extending the range of girders for which moment redistribution is applicable, there is the potential for significant economic benefits to both the design and rating processes.

To develop improved moment redistribution procedures that are valid for more slender sections than permitted by current specifications is the primary objective of this work. This is facilitated through a new approach to moment redistribution, termed "rotation compatibility”. This method is founded on an explicit evaluation of the rotations required for moment redistribution and comparison of these values to the ductility of individual girders. The ultimate result of these procedures is design and rating specifications that do not contain the previous restrictions on girder geometry and maximum permissible levels of redistribution. Instead, the rotation compatibility procedures developed herein: are valid for any girder cross-section that satisfies the cross-section proportion limits specified by AASHTO (2004) for general steel I-girders, explicitly compute maximum levels of redistribution, and are in a format such that the suggested procedures may be conveniently adopted into both the AASHTO LRFD and LRFR Specifications. 


\subsection{General Theory of Moment Redistribution}

In a continuous-span structure, maximum negative bending moments (at supports) typically exceed peak positive bending moments (near midspan). Thus, as the girder is subjected to increasing loads, yielding first occurs at the locations of peak negative moment. This yielding (which may occur at stress levels below the yield stress of the girder due to the presence of initial stresses resulting from the girder fabrication processes) results in permanent inelastic rotations and residual stresses in the girder. In statically indeterminate structures, these residual stresses are balanced by the development of residual reactions. The moment caused by the residual reactions is termed the redistribution moment $\left(\mathrm{M}_{\mathrm{rd}}\right)$ and is necessarily zero at abutments and varies linearly between supports. This redistribution moment is illustrated by the light gray line in Fig. 1-1 for a two-span continuous girder.

Figure 1-1 also illustrates the conventional elastic moment envelope for a two-span continuous girder by the heavy black lines. Summing this elastic moment and the redistribution moment gives the moment diagram represented by the dark gray lines in Fig. $1-1$, which is the design moment used in moment redistribution procedures. Thus, in comparison to the elastic design moments, the moment redistribution design moments are lower in negative bending and higher in positive bending. These lower negative bending moments are the reason that moment redistribution procedures are particularly favorable for

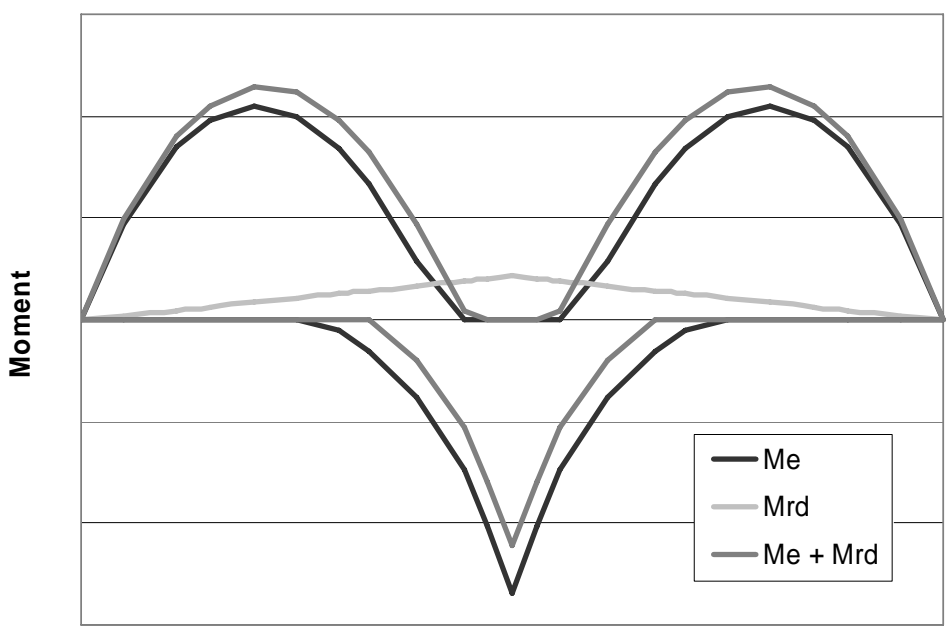

Distance Along Span

Figure 1-1. Illustration of Moment Redistribution Concept 
use with composite I-girder systems, which for a given cross-section have lower negative bending capacities than positive bending capacities due to the ineffectiveness of the concrete slab in tension. In other words, for a continuous-span composite beam of uniform crosssection, the negative bending stresses are higher while the negative bending capacity is lower compared to the positive bending region. Thus, moment redistribution procedures effectively lead to a more uniform distribution between peak negative bending and positive bending moments and a more efficient design. Because the design of the pier region of continuousspan girders is typically controlled by bending strength requirements, designing for this reduced level of moment has significant economic benefits. Not only does this allow for the use of smaller cross-sections at locations of peak negative moments, but the number of flange transitions can be reduced (which leads to both material and fabrication cost savings) and the use of cover plates (which have unfavorable fatigue characteristics) can be eliminated. The increased moments resulting in positive bending typically have no influence on economy as the design of sections experiencing the highest positive bending stresses are usually not controlled by strength requirements; instead, fatigue or serviceability considerations most commonly govern.

In order for the assumed moment redistribution to occur, the pier section must be sufficiently ductile such that the girder is capable of maintaining the needed moment capacity throughout the range of rotation necessary for moment redistribution to occur. In other words, moment redistribution will not occur without the development of inelastic rotations at the pier. The girder must have sufficient ductility to develop the levels of inelastic rotation corresponding to the assumed magnitude of the redistribution moments while maintaining a moment capacity greater than or equal to the moment redistribution moments in negative bending. Present moment redistribution specifications assume that this ductility requirement can be satisfied by (1) limiting the range of members for which moment redistribution is permitted to relatively compact members and (2) limiting the maximum amount of moment that may be redistributed to $20 \%$ of the elastic moment at the piers. However, these restrictions are conservative assumptions that limit the applicability of the moment redistribution specifications and do not guarantee adequate ductility. Instead, adequate ductility is only guaranteed in the present moment redistribution specifications (AASHTO 2004) by the 
optional "refined method" for moment redistribution. Alternatively, when moment redistribution design is performed using the more simplistic moment redistribution methods, there is no guarantee of adequate ductility.

\subsection{General Theory of Rotation Compatibility}

The primary objective of the rotation compatibility procedures developed herein is to develop a procedure to explicitly evaluate girder ductility requirements. This allows for an accurate assessment of negative bending strength and also provides a rational means for investigating the incorporation of more slender members in moment redistribution specifications. Additionally, the maximum permissible level of redistribution moment can be directly computed using this method.

The rotation compatibility approach is based on direct comparison of the ductility available at a given level of moment for a particular girder to the required ductility for the corresponding level of moment redistribution. This available ductility is assessed through moment versus rotation relationships. Specifically, the moment versus rotation relationships are obtained for a wide range of experimental and analytical girders and then compared to the rotation required to redistribute moment from negative bending sections to positive bending sections. In other words if the available rotation of a member is greater than that required for moment redistribution, the intended level of moment redistribution is acceptable. Otherwise, the intended level of moment redistribution must be reduced in accordance with the available rotation capacity of the member.

\subsection{Need for Current Research}

As suggested by the above discussion, there is a need for improving the existing moment redistribution specifications for steel I-girder bridges. The primary limitation of the existing procedures is that the conservative assumptions made in an attempt to provide adequate ductility restrict the range of girders for which moment redistribution is permissible and the maximum levels of moment that may be redistributed. Not only does this decrease the economy that may result from implementing these procedures in new designs, but also renders the procedures too restrictive to be used in rating, as many existing bridges have 
geometries that do not satisfy the current limits. Furthermore, as there is no explicit evaluation of girder ductility in the basic moment redistribution provisions, there is the potential to assume that higher levels of moment may be redistributed than that which actually occurs, ultimately leading to failure in the pier regions of these girders. Thus a more rational assessment of girder ductility is needed.

The current range of girders that may be used in conjunction with moment redistribution provisions excludes many typical bridges from use unless they are specifically designed to target these limits. These limitations are most significant in terms of required lateral bracing distance and compression flange slenderness. The current lateral bracing limit is particularly restrictive such that (1) many existing bridges would not satisfy this requirement, which has implications for the rating process, and (2) the number of cross-frames necessary in new bridge designs incorporating moment redistribution procedures is typically increased compared to the requirements for a conventional elastic design. Thus, there is a specific need to increase the range of applicability of the moment redistribution specification with respect to these two limits. Research addressing extending the range of flange slenderness and lateral bracing values applicable for moment redistribution is anticipated to have positive economic impacts.

Additionally, there is a need to better assess the maximum levels of redistribution that are permissible. While the current procedures assume that the same level of redistribution (20\% of the maximum negative moment) may be used for all designs, it is more rational to relate the amount of moment that may be redistributed to the girder and bridge properties. This is especially true if the range of girders for which moment redistribution is applicable is increased, as a lower level of redistribution may be required for more slender members.

There are cases where the current moment redistribution procedures result in required crosssections in negative bending regions that are larger than those required using elastic procedures. This occurs because, while the moment redistribution procedures allow for a decrease in applied moment, the moment capacity is also reduced in some cases, irrespective of the amount of moment being redistributed. Thus, because the sum of the moment capacity 
of the girder and the redistribution moment must equal a quantity greater than the elastic moment, in cases where the allowable moment capacity that results from the use of the moment redistribution procedures is substantially lower than the conventional (elastic) moment capacity and the amount of moment being redistributed is relatively small (typically $10 \%$ or less of the elastic moment), the sum of these two quantities, and therefore the elastic moment which the girder can support, is less than the elastic moment capacity that results from use of the elastic strength prediction equations. Consequently, moment redistribution is of no benefit in these cases. Research is needed to address this inconsistency between the elastic and moment redistribution procedures.

Current AASHTO (Load and Resistance Factor Rating, LRFR, 2003 and LRFD 2004) provisions assume a consistent level of strength in both the design and rating specifications. Therefore, it should by extension also be possible to utilize the optional moment redistribution specifications contained in the LRFD specifications for load rating. However, no method exists at the present time for incorporating these methods into the rating procedures. The creation of moment redistribution specifications for bridge rating is anticipated to have positive economic impacts as the reserve capacity of continuous-span bridges can be accounted for during the rating process and the bridges most in need of corrective action can then be identified.

\subsection{Objectives}

The primary objectives of this work are as follows.

- Explicitly evaluate the ductility requirements for moment redistribution resulting in increased safety.

- Investigate the use of moment redistribution procedures for a broader range of girder geometries, particularly with respect to longer unbraced lateral distances and increased compression flange slenderness. This may also allow for the procedures to be utilized with in-service bridges that were not necessarily designed to meet specific compactness requirements.

- Evaluate the maximum permissible levels of moment that may be safely redistributed. 
- Develop moment redistribution specifications for steel I-girder rating. This will result in a more uniform level of safety throughout the bridge inventory, as suggested by Barker and Zacher (1997).

\subsection{Scope of Research}

This research focuses on the inelastic behavior of steel bridge I-girders and the scope of this project consists of four primary components: experimental testing; finite element analysis; study of rotation requirements; and development of design and rating specifications.

Experimental testing of twelve large-scale plate girders is conducted to obtain the momentrotation characteristics of members that are typical of those used in the negative bending sections of continuous-span I-girders. These girders are unique in that the slenderness properties (i.e., flange slenderness, web slenderness, and lateral slenderness) of these girders are higher than the values of these parameters that have been incorporated in previous experimental programs. Because one of the primary reasons for presently limiting the range of applicability of moment redistribution designs to relatively compact members is a lack of experimental evidence suggesting that such procedures are appropriate for more slender girders, such testing is necessary in order to investigate the applicability of moment redistribution procedures to girders that are more slender than currently permitted by these procedures.

The results of this testing are further used to validate the finite element analysis (FEA) procedures implemented in this work. A comprehensive finite element study is conducted where the two primary focuses are a parametric study and FEA of girders resulting from a moment redistribution design study. From these efforts data regarding the available rotations of a large number of typical girders is obtained.

In order to investigate rotations requirements for moment redistribution, consideration is first given to determining an appropriate analysis procedure. This analysis procedure is then used to calculate rotation requirements for typical moment redistribution designs and these 
resulting rotation requirements are used to determine empirical rotation requirement expressions.

The final component of this work is the development of the rotation compatibility procedure, which facilitates direct comparison of the available and required rotations. The conditions necessary to assure that the available rotations are greater than the required rotations are investigated and this information is then synthesized into a format appropriate for incorporation into AASHTO design and rating specifications.

\subsection{Dissertation Organization}

The body of this report consists of eight chapters. This first chapter, Introduction, provides general background information of this project, discusses the need for this research, and highlights the main objectives of this work.

The second chapter, Literature Review, discusses the previous research that has been conducted related to the present work. Specifically, this chapter is organized into four sections. These are: (1) an overview of inelastic bridge design methods that have previously been proposed and/or implemented into AASHTO Specifications; (2) a description of AASHTO rating procedures and previous studies on inelastic rating procedures; (3) a presentation of previous research on the moment-rotation characteristics, i.e., ductility, of steel and steel-concrete composite I-girders; and (4) a review of studies focused on the rotation requirements for inelastic design.

The experimental testing program is described in Chapter 3, Experimental Testing. Here information on the testing configuration and procedures is given along with a detailed description of each of the test girders and the experimental results. The experimental results presented include the maximum moment capacities, deformed shapes, and rotation characteristics of each girder. 
Chapter 4, Nonlinear FEA Modeling Procedures, discusses the finite element procedures used in this work. Mesh densities, element types, material modeling procedures, geometric imperfections, residual stresses, and solution algorithms are considered in this chapter.

The FEA parametric study is presented in Chapter 5, Moment - Rotation Characteristics of Steel I-Girders. The first section of this chapter validates the FEA methods described in Chapter 4 by presenting the results from FEA of each of the experimental girders discussed in Chapter 3 and comparing this data to the experimental results. The ductility of negative bending sections of continuous-span I-girders is then explored through additional FEA studies. Specifically, this work consists of two components. First, a parametric study is presented where the ductility of girders with high slenderness ratios are investigated. Additionally, a series of typical bridge designs is created and FEA results of these girders provide further insight into the moment-rotation characteristics of negative bending sections. This chapter concludes with the development of moment-rotation models that may be used to describe steel I-girder behavior.

Chapter 6, Rotation Requirements for Steel Bridge I-Girders, details the investigation into the rotation requirements for moment redistribution. While there have been no previous studies specifically focused on rotation requirements for moment redistribution, previous studies have researched rotation requirements for other purposes; the first section of this chapter discusses the relevance of these studies, which are presented in Chapter 2, to the present work. The second section of this chapter then presents the analysis method used to determine rotation requirements. Because rotation requirements for a given member are a function of its section properties, determining representative section properties was also necessary. Thus a series of design studies was conducted to obtain representative section properties; a discussion of this design study is presented in the third section of Chapter 6. The final section of this chapter discusses the affects of the various parameters investigated on rotation requirements and concludes with the presentation of mathematical expressions to predict the rotation requirements in continuous-span I-girders. 
The development of the rotation compatibility procedure is the focus of Chapter 7, Rotation Compatibility - Design and Rating Specifications. First, the expressions for available and required rotations are used to establish the conditions necessary to assure that the available rotation is greater than that required for moment redistribution. This information is then used to develop the rotation compatibility specifications for design and rating. This section concludes with the presentation of design and rating examples illustrating the use of the suggested procedures.

Chapter 8, Summary, Conclusions, and Recommendations, summarizes the results of this work. Recommendations for future research in this area are also given.

The body of this dissertation is followed by a list of references cited and five Appendices. Appendix A defines the nomenclature used in this dissertation. The moment-rotation behavior of girders tested in the present and previous experimental studies is presented in Appendix B. Appendix C contains data resulting from tension testing performed on coupons taken from the steel used to fabricate the experimental girders tested in this work. The moment-rotation behavior of the girders analyzed in the FEA study is included in Appendix D. Lastly, Appendix E contains the designs resulting from the moment redistribution design study described in Chapter 6 . 


\section{Chapter 2: LITERATURE REVIEW}

As discussed in Chapter 1, the objective of this work is to develop moment redistribution specifications for design and rating of steel I-girder bridges that assure the available rotation of a cross-section is greater than the rotation required to redistribute moment. Consequently, the literature review for this work consists of four components: a review of previous moment redistribution design provisions; an overview of rating procedures with a specific focus on inelastic rating methods; and a presentation of previous research related to the available and required rotations of steel members. The available literature on each of these three subjects is summarized in this chapter. Specifically, Section 2.1, Moment Redistribution Design, presents a historical overview of the various inelastic design procedures that have been incorporated into past editions of AASHTO Specifications. Section 2.2, Inelastic Rating Methods, provides an overview of bridge rating procedures and summarizes previous research on inelastic rating methods. In order for moment redistribution procedures to be based on a rotation compatibility approach, both the required rotation and available rotation must be known. Studies on the behavior of steel I-girders in negative bending, from which conclusions can be made regarding the available rotation of these members are presented in Section 2.3, Available Rotation. A discussion of previous research on rotation requirements for these types of members is given in Section 2.4, Rotation Requirements.

\subsection{Moment Redistribution Design}

\subsubsection{Initial Inelastic Provisions (AAHSTO 1973)}

Past editions of the American Association of State Highway and Transportation Officials (AASHTO) specifications have accounted for the increase in strength due to inelastic behavior using various methods. A simple method for approximating the effects of inelastic behavior was first incorporated in the $11^{\text {th }}$ edition of the specifications (AASHTO, 1973). Specifically, these specifications accounted for the inelastic behavior of continuous span girders in two ways. First, the maximum moment capacity of the girder was increased from the yield moment to the plastic moment for girders satisfying certain compactness criteria. 
Secondly, $10 \%$ of the peak negative moment was permitted to be redistributed to the positive bending section prior to evaluating the capacity at the maximum load level.

\subsubsection{Alternate Load Factor Design (AASHTO 1986)}

Comprehensive inelastic procedures were first given in the Guide Specifications for Alternate Load Factor Design (ALFD) (AASHTO 1986), which were applicable to compact sections. Similar to the AASHTO Load Factor Design (LFD) procedures, ALFD procedures required a service load check, overload check, and maximum load check. The service load checks were identical for both design philosophies but alternative methods were used for the evaluation of the overload and maximum load checks in ALFD.

In the overload check in ALFD, which is analogous to the Service II limit state in the current AASHTO LRFD procedures, the moment capacity at the pier was obtained using the beamline method. This method made use of a continuity relationship (which was a function of the applied loading) and a moment-rotation relationship (which was based on experimental results). A simultaneous solution of these two expressions yielded the moment capacity and corresponding rotation at that load level.

The maximum load check in ALFD (Strength I in AASHTO LRFD) was based on a mechanism (plastic collapse) approach. The design requirements at this load level were simplified by using the concept of an effective plastic moment as suggested by Grubb and Carskaddan (1981), discussed below, in that if the applied moment was less than the effective plastic moment, then the maximum load check was assumed to be satisfied.

The concept of an effective plastic moment was first introduced by Haaijer et al. (1980). This idea was initiated out of a desire to extend the applicability of plastic design procedures to a broader range of cross sections and thereby increase the economical benefits that could be achieved by using these methods. At the time, plastic design procedures for buildings were specified by the American Institute of Steel Construction (AISC 1973), but were limited to sections satisfying rather stringent web and compression flange slenderness ratios; 
specifically, a cross section was required to have flange and web slenderness ratios satisfying (adapted from Schilling et al., 1997)

$$
\begin{gathered}
b_{f c} / 2 t_{f c} \leq 0.291 \sqrt{E / F_{y f}} \text { and } \\
2 D_{c p} / t_{w} \leq 2.3 \sqrt{E / F_{y w}},
\end{gathered}
$$

where variable definitions for all equations are presented in Appendix A. These requirements were based on the premise that sections need to be rather compact in order to have sufficient rotation capacity.

However, Haaijer et al. (1980) observed that some sections that do not satisfy the requirements of Eqs. 2.1 and 2.2 may also exhibit significant rotation capacity, although at a reduced level of moment as shown in Fig. 2-1 where the moment-rotation behavior of compact and non-compact girders is illustrated. Furthermore, through experimental testing of a plate girder satisfying the above slenderness limits (Eqns. 2.1 and 2.2), Grubb and Carskaddan (1981) found that compact sections demonstrated a minimum rotation capacity of 63.3 mrad. Therefore, it was reasoned that any section that achieved this value of rotation capacity at a particular level of moment would be suitable for plastic design at that moment capacity; this level of moment was termed the effective plastic moment $\left(\mathrm{M}_{\mathrm{pe}}\right)$ of the girder. Therefore, Haaijer et al. (1980) concluded that a section is valid for inelastic design if its rotation capacity at $\mathrm{M}_{\mathrm{pe}}$ is greater than $63.3 \mathrm{mrad}$.

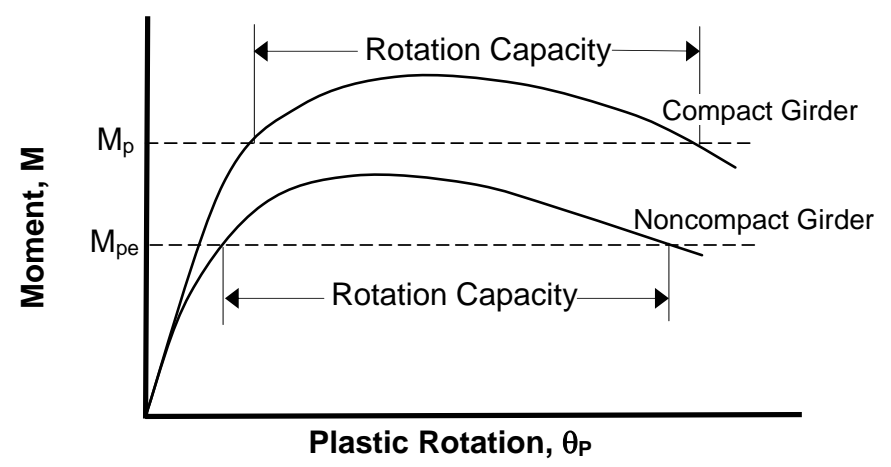

Figure 2-1. Illustration of Effective Plastic Moment Concept 
To calculate $\mathrm{M}_{\mathrm{pe}}$ for a section that does not meet the requirements for plastic design (Eqns. 2.1 and 2.2), Haaijer et al. (1980) proposed that effective yield strengths first be calculated for the flange $\left(\mathrm{F}_{\mathrm{yfe}}\right)$ and web ( $\left.\mathrm{F}_{\mathrm{ywe}}\right)$ using the following equations.

$$
\begin{gathered}
F_{y f e}=9800\left(t_{f c} / b_{f c}\right)^{2} \leq F_{y f} \\
F_{y w e}=38,300\left(t_{w} / D_{c p}\right)^{2} \leq F_{y f}
\end{gathered}
$$

Equations 2.3 and 2.4 are obtained by simple algebraic manipulation of the slenderness limits in Eqs. 2.1 and 2.2, substituting $\mathrm{F}_{\mathrm{yfe}}$ and $\mathrm{F}_{\mathrm{ywe}}$ for $\mathrm{F}_{\mathrm{yf}}$ and $\mathrm{F}_{\mathrm{yw}}$, respectively, and assuming that the modulus of elasticity (E) is equal to 29,000 ksi. These effective yield strengths are then normalized by the actual yield strength of the compression flange to obtain the following reduction factors.

$$
\begin{aligned}
& R_{f}=F_{y f e} / F_{y f} \\
& R_{w}=F_{y w e} / F_{y f}
\end{aligned}
$$

The effective plastic moment is then given by the following.

$$
\begin{gathered}
M_{p e}=R_{f} M_{p f}+R_{w} M_{p w} \\
M_{p w}=\frac{F_{y w} t_{w}}{2}\left[\left(D-D_{c p}\right)^{2}+D_{c p}{ }^{2}\right] \\
M_{p f}=M_{p}-M_{p w}
\end{gathered}
$$

Grubb and Carskaddan (1981) confirmed the validity of using this method to calculate the effective plastic moment by evaluating the rotation capacity obtained at $\mathrm{M}_{\mathrm{pe}}$ (calculated using Eqn. 2.7) in previous experimental results. The results from 49 previous tests conducted by various researchers were analyzed. These tests included specimens having flange slendernesses ranging from 5.0 to 14.7 and web slendernesses ranging from 29.9 to 138 . It was found that the rotation capacity at $\mathrm{M}_{\mathrm{pe}}$ was greater than that of a compact shape (assumed to be 63.3 mrads) in all cases, thus assuring that the above equations allow a section to have sufficient rotation capacity at $\mathrm{M}_{\mathrm{pe}}$. As a result, the Guide Specifications for ALFD (1986) prescribe that $\mathrm{M}_{\mathrm{pe}}$ be calculated in this manner, although the procedure is somewhat simplified by making direct use of the effective yield stresses computed in Eqns. 
2.3 and 2.4; these effective yield stresses are used to compute the effective plastic moment in the same manner as done with the actual yield stresses when computing the plastic moment.

\subsubsection{Unified Autostress Method (Schilling 1989)}

Work by Schilling (1989) aimed to extend the applicability of moment redistribution specifications to girders with slender webs. As a result of this effort, Schilling proposed an alternative method to ALFD that was termed the Unified Autostress Method (UAM) due to the fact that in contrast to ALFD the same method is used in both the overload and maximum load checks. Specifically, this method utilizes moment-rotation curves to determine the girder capacity at both load levels.

\subsubsection{AASHTO LRFD Specifications (AASHTO 1994 and 1998)}

Comprehensive inelastic design procedures were first implemented in the primary specifications with the adoption of the AASHTO Load and Resistance Factor Design Specifications in 1994. The ALFD procedures described above serve as a foundation for the inelastic design methods in the $1^{\text {st }}$ and $2^{\text {nd }}$ Editions of the AASHTO LRFD Specifications (AASHTO 1994 and 1998). In these specifications, the Service II limit state may be evaluated using either the beam-line (as described above for ALFD procedures) or the unified autostress methods, both of which require the use of moment-rotation curves. AASHTO (1994 and 1998) specifies that the ascending portion (which, as explained by Schilling et al. (1997), is the only portion necessary for service limit states) of this response may be expressed as:

$$
\mathrm{M} / \mathrm{M}_{\mathrm{n}}=0.7+60 \theta_{\mathrm{P}}
$$

where $M / M_{n}$ is the moment normalized by $M_{n}$, the nominal moment capacity computed per AASHTO Specifications, and $\theta_{\mathrm{P}}$ is the plastic rotation in radians.

The procedures described above for the maximum load check in ALFD are incorporated into the $1^{\text {st }}$ and $2^{\text {nd }}$ editions of the AASHTO LRFD Specifications (1994 and 1998) for Strength I design with only minor modifications and additions. Alternatively, Strength I requirements may be evaluated using the unified autostress method. 


\subsubsection{AASHTO ${ }^{\text {rd }}{ }^{\text {Edition LRFD Specifications (2004) }}$}

Because the ALFD based procedures required significant additional effort for engineers (i.e., the need to iteratively use both the girder continuity relationship and the moment-rotation relationship) and applied only to a limited range of girder geometries, significant improvements were made to the inelastic design specifications in the $3^{\text {rd }}$ Edition of the LRFD Specifications. These changes are based on a collaborative effort (Barth et al. 2004), where a design approach suggested by Schilling et a. (1997) and effective plastic moment equations developed by Barth and White (1998) are synthesized. These procedures are applicable for straight girders satisfying the following requirements:

- $\quad$ Skew is no more than 10 degrees;

- $\quad$ Nominal yield strength of the steel does not exceed $70 \mathrm{ksi}$;

- There are no staggered crossframes in the bridge system;

- There are no holes in the tension flange within a distance equal to twice the web depth from each side of the pier where moments are redistributed from;

- The cross-section is prismatic within the unbraced length from which moment is redistributed;

- The shear force is less than the nominal shear capacity of the web neglecting tension field action and post-buckling shear resistance;

- $\quad D / t_{w} \leq 150$;

- $\quad 2 D_{c} / t_{w} \leq 6.8 \sqrt{E / F_{y c}}$;

- $\quad D_{c p} \leq 0.75 D$;

- $\quad D / b_{f c} \leq 4.25$;

- $\quad b_{f c} / 2 t_{f c} \leq 0.38 \sqrt{E / F_{y c}}$; and

- $\quad L_{b} \leq\left[0.1-0.06\left(M_{1} / M_{2}\right)\right] r_{t} E / F_{y c}$.

These procedures are much easier to apply than past inelastic design procedures and are valid for both compact and noncompact sections. Using these procedures the girder is evaluated using shakedown (where loads less than the maximum applied load are resisted elastically and deformations stabilize) rather than a mechanism approach. This leads to a 
straightforward procedure where the conventional elastic moment diagram is used in conjunction with an assumed pattern of redistribution moments.

This procedure is implemented in the evaluation of both the strength and service limit states. It is assumed that shakedown occurs if the sum of the effective plastic moment and the redistribution moment is less than the elastic moment (determined from conventional moment envelopes) at the pier. Thus the redistribution moment redistribution at interior supports is equal to:

$$
M_{r d}=M_{e}-M_{p e} \geq 0
$$

The redistribution moment at other locations along the span is determined by connecting the redistribution moments computed at each interior support using Eq. 2.11 with straight lines and extending these lines to a value of zero moment at each abutment. The direction of these redistribution moments is determined to be positive as the inelastic rotation that occurs at the piers during moment redistribution is only possible under equivalent positive bending moments. If the sum of the redistribution moment and the elastic moment envelope is less than the moment capacity of the section along the entire length of the beam, then the strength limit check is satisfied. This condition may be expressed algebraically as follows.

$$
M_{e}+M_{r d} \leq M_{n}
$$

In Barth and White (1998) and White and Barth (1998) the authors propose the momentplastic rotation model shown in Fig. 2-2. This model consists of a linear pre-peak region,

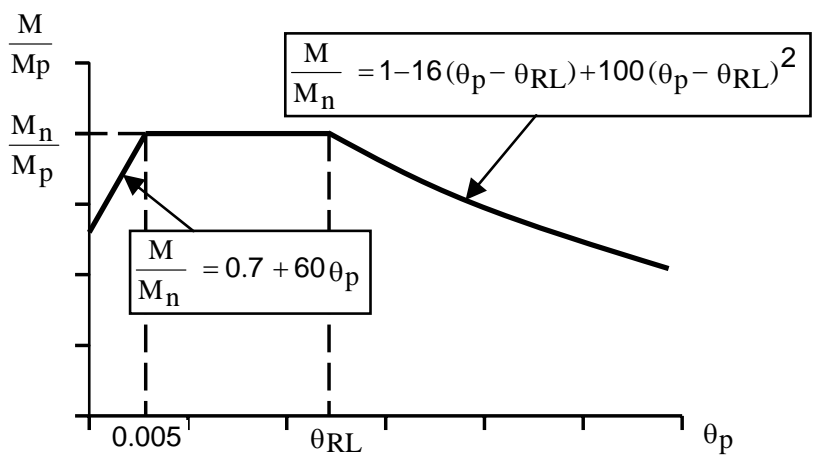

Figure 2-2. Barth and White Moment-Rotation Model 
followed by a plateau at $\mathrm{M}_{\mathrm{n}} / \mathrm{M}_{\mathrm{p}}$, and then a quadratic unloading portion. This suggested moment rotation model was developed using the data obtained from a reasonably comprehensive set of finite element parametric studies.

In this model the pre-peak region is the same as the loading portion of the moment-rotation curve specified in the current AASHTO LRFD Specifications $(1994,1998)$ given in Eqn. 2.10, which is an approximate fit to experimental test data. Results from finite element analyses in which representative girder dimensions and residual stresses have been used are also well represented by this relationship.

The moment capacity predicted by the model is equal to the nominal moment capacity of the girder between a plastic rotation of $0.005 \mathrm{rad}$ and $\theta_{\mathrm{RL}}$, where $\theta_{\mathrm{RL}}$ can be as small as 0.005 in which case there is no plateau. For general sections $\theta_{\mathrm{RL}}$ is expressed as

$$
\theta_{R L}=0.128-0.0119 \frac{b_{f c}}{2 t_{f c}}-0.0216 \frac{D}{b_{f c}}+0.002 \frac{b_{f c}}{2 t_{f c}} \frac{D}{b_{f c}} \geq 0.005 .
$$

For sections having either a transverse stiffener spaced at D/2 or less on each side of the interior support or ultracompact webs (defined as $2 D_{c p} / t_{w} \leq 2.3 \sqrt{E / F_{y c}}$ ), the first coefficient in Eqn. 2.13 may be increased from 0.128 to 0.137 (Barth et al., 2004).

The unloading portion of the curve is expressed as:

$$
\frac{M}{M_{n}}=1-16\left(\theta_{p}-\theta_{R L}\right)+100\left(\theta_{p}-\theta_{R L}\right)^{2}
$$

In White and Barth (1998), the authors compare this moment-rotation model to the results from nearly twenty experimental tests conducted by previous researchers (Carskaddan, 1980; Grubb and Carskaddan, 1981; Schilling, 1985; Schilling and Morcos, 1988; Tansil, 1991; and FHWA, 1992). The general shape of the moment rotation response of the experimental girders is well captured by the proposed model. In addition, the model accurately predicts the flexural capacity for all of the experimental data with the exception of two girders. There is evidence to suggest that for one of these, the experimental results may be erroneous. For 
the other the maximum allowable brace spacing incorporated in the development of the model is exceeded by $16 \%$.

The use of this moment-rotation model is implemented into the AASHTO (2004) Specifications through a simplification where a constant value of rotation is assumed for all girders. This idea of utilizing a constant value of rotation in design procedures was first suggested by Schilling (1985). Schilling (1986) later conducted a series of fifty design studies that showed the plastic rotation ranged from 0 to 29 mrads at the maximum (Strength I) load level in these designs. Therefore, a plastic rotation of 30 mrads is assumed to be an upper-bound value of rotation for all girders under this loading. Also, as a result of trial designs conducted by Schilling (1989) the maximum plastic rotation under overload (Service II) conditions for composite girders was found to be 8.9 mrads and the maximum plastic rotation when considering only the steel girder alone was 9.6 mrads; consequently, a plastic rotation of 9 mrads is assumed to be an upper-bound value for the Service II limit state.

These rotation values are employed to determine the effective plastic moment $\left(\mathrm{M}_{\mathrm{pe}}\right)$ for the girder under consideration. Effective plastic moment equations for the Strength I and Service II limit states can be derived from Eq. 2.13 by using the assumed values of plastic rotation discussed above. Specifically, the quadratic term of $\left(\theta_{P}-\theta_{R L}\right)$ is approximated by $(0.030$ $0.005=0.025)$ or $(0.009-0.005=0.004)$ and either 0.030 or 0.009 is substituted for $\theta_{\mathrm{P}}$ in the linear term depending on the limit state under consideration. For general I-girders this procedure yields:

$$
\begin{aligned}
& \frac{M_{p e}{ }^{(30)}}{M_{n}}=2.63-2.3 \frac{b_{f_{c}}}{t_{f c}} \sqrt{\frac{F_{y c}}{E}}-0.35 \frac{D}{b_{f_{c}}}+0.39 \frac{{ }^{f_{c}}}{t_{f_{c}}} \sqrt{\frac{F_{y c}}{E}} \frac{D}{b_{f_{c}}} \leq 1 \\
& \frac{M_{p e^{(9)}}}{M_{n}}=2.90-2.3 \frac{b_{f c}}{t_{f c}} \sqrt{\frac{F_{y c}}{E}}-0.35 \frac{D}{b_{f c}}+0.39 \frac{{ }^{b} f_{c}}{t_{f c}} \sqrt{\frac{F_{y c}}{E}} \frac{D}{b_{f c}} \leq 1
\end{aligned}
$$

For I-girders having either a transverse stiffener spaced at D/2 or less on each side of the interior support (over a length greater than or equal to $\mathrm{D} / 2$ ) or ultracompact webs, such that the first coefficient in Eqn. 2.13 may be increased to 0.137, the effective plastic moments are: 


$$
\begin{gathered}
\frac{M_{p e}(30)}{M_{n}}=2.78-2.3 \frac{{ }_{f c}}{t_{f c}} \sqrt{\frac{F_{y c}}{E}}-0.35 \frac{D}{b_{f c}}+0.39 \frac{b_{f c}}{{ }_{t}} \sqrt{\frac{F_{y c}}{E}} \frac{D}{b_{f c}} \leq 1 \\
\frac{M_{p e}(9)}{M_{n}}=1
\end{gathered}
$$

Barth et al. (2004) have evaluated the accuracy of Eqns. 2.15-2.18 by comparing the effective plastic moments predicted by these equation to the measured values of moment at rotations of 9 and 30 mrads from 68 experimental tests (Basler, 1959; Schilling, 1985; Schilling and Morcos, 1988; Barth, 1996; Grubb and Carskaddan, 1979; Grubb and Carskaddan, 1981; Carskaddan, 1980; Holtz and Kulak, 1973; Adams et al., 1964; Lukey et al., 1969; Hartnagel, 1997; Climenhaga and Johnson, 1972; Yakel et al., 1999; Tansil, 1991). These experimental tests represent various loading conditions, girder geometries, and material properties. The experiments also include steel and composite girders. Specimens from the referenced studies in which the effective cross frame spacing exceeded the maximum allowed for inelastic design by more than $5 \%$ were not included in the comparisons.

A strength ratio equal to the ratio of the effective plastic moment predicted by Eqns. 2.152.18 to the moment measured at the corresponding rotation in the experimental work is computed for each of the 68 girders considered. Histograms of these strength ratios at $M_{p e}(30)$ and $M_{p e}(9)$ are shown in Figs. 2-3 and 2-4, respectively. These figures show that the proposed equations are appropriately conservative for most of the girders considered. For $\mathrm{M}_{\mathrm{pe}}$ (30) a maximum strength ratio of 1.09 , a minimum strength ratio of 0.70 , and an average strength ratio of 0.92 are obtained; for $\mathrm{M}_{\mathrm{pe}}(9)$ a maximum strength ratio of 1.09, a minimum strength ratio of 0.83 , and an average strength ratio of 0.97 are computed.

The girders that give the lowest strength ratios typically have stockier cross sections and are subjected to a moment gradient. This leads to moment capacities significantly exceeding $M_{p}$ for several cases. In these situations, the performance of the suggested effective plastic moment expressions cannot be improved unless girder capacities in excess of $M_{p}$ are permitted, which would consequently complicate design specifications. 


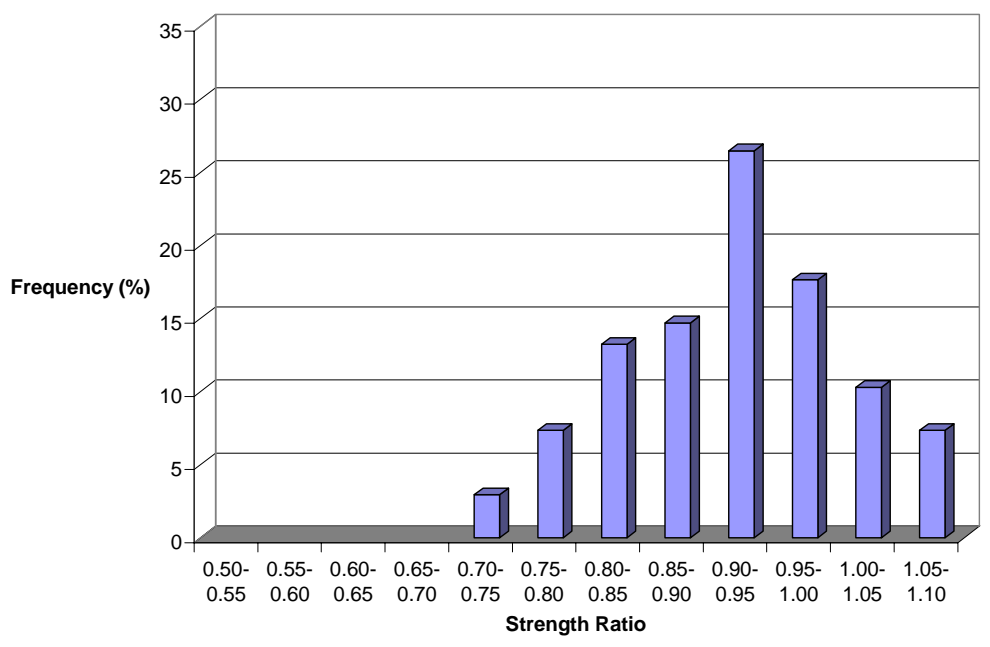

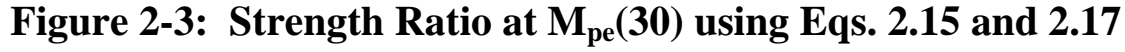

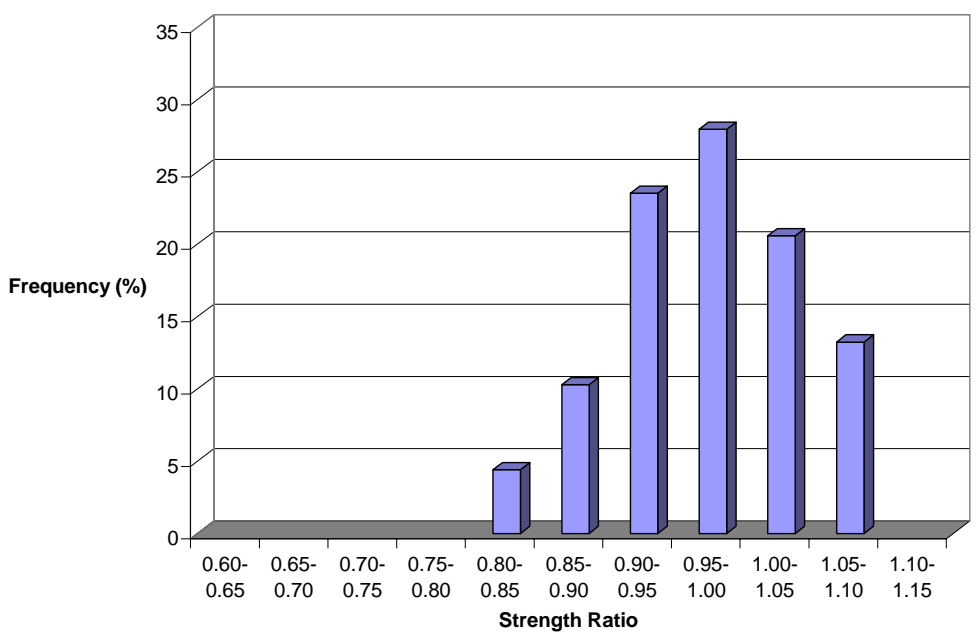

Figure 2-4: Strength Ratio at $M_{\text {pe }}(9)$ using Eqns. 2.16 and 2.18

For comparison purposes, Barth et al. (2004) also compute the strength ratio for each of the same experimental girders using the effective plastic moments implemented in the $1^{\text {st }}$ and $2^{\text {nd }}$ Editions of the LRFD Specifications. Histograms of these strength ratios are shown in Figs. 2-5 and 2-6. For $\mathrm{M}_{\mathrm{pe}}(30)$ these effective plastic moment expressions give a maximum strength ratio of 1.06, minimum strength ratio of 0.53 , and an average strength ratio of 0.79 . At $\mathrm{M}_{\mathrm{pe}}(9)$ a maximum strength ratio of 1.12 , a minimum ratio of 0.61 , and an average strength ratio of 0.93 result. Evaluating the two methods and comparing Fig. 2-3 to Fig. 2-5 


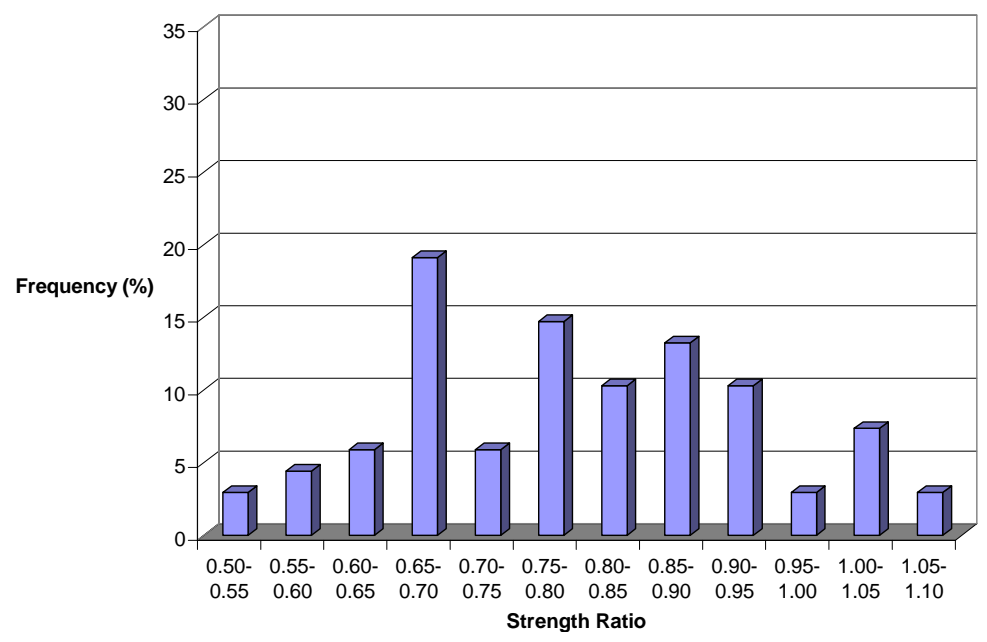

Figure 2-5: Strength Ratio at $\mathbf{M}_{\mathrm{pe}}(30)$ using ALFD $\mathbf{M}_{\mathrm{pe}}$ Equations

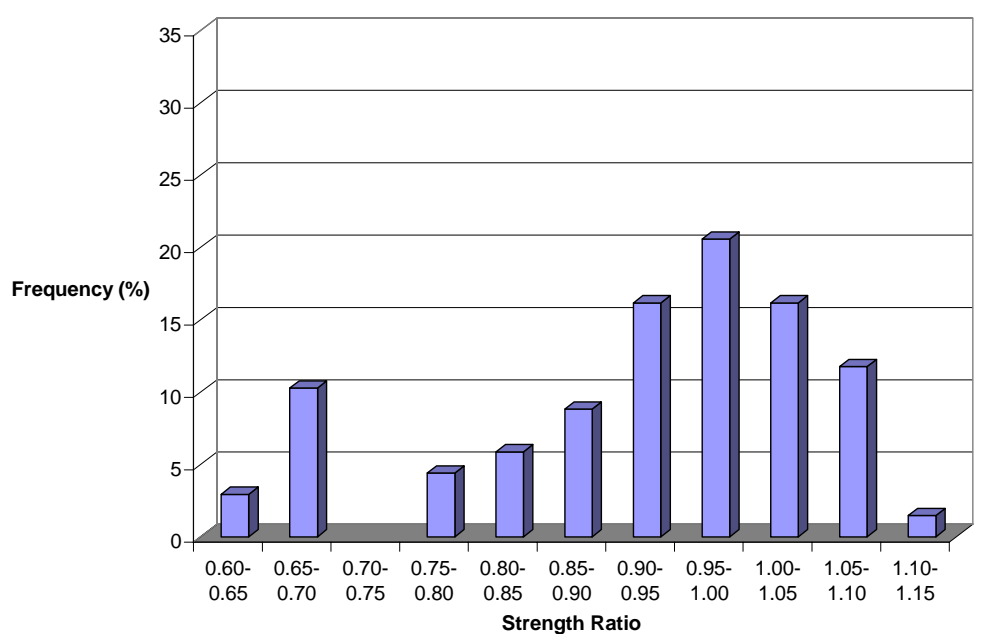

Figure 2-6: Strength Ratio at $M_{p e}(9)$ using ALFD $M_{p e}$ Equations

and Fig. 2-4 to Fig. 2-6, it is evident that the AASHTO (2004) effective plastic moment equations more accurately and consistently predict the behavior of the actual test girders.

\subsection{Rating}

In addition to the benefits inelastic design offers for new bridge designs, inelastic rating procedures more accurately predict the strength of existing bridges, thereby increasing the predicted capacity of bridges that may have otherwise been rated as structurally deficient. However, rating provisions such as these have yet to be incorporated into AASHTO 
specifications. This section will describe the current elastic AASHTO rating procedures and present previous research on inelastic rating methods.

\subsubsection{Elastic Rating Procedures}

In 2003, AASHTO adopted Load and Resistance Factor Rating (LRFR) Specifications that are consistent with the current Load and Resistance Factor Design Specifications (AASHTO 2003 and 2004, respectively). These procedures allow for a seamless transition between design and rating using load and resistance factor procedures. The differences between the design and rating specifications reflect the alternative philosophies associated with these

procedures; the LRFD Specifications are intended to predict the maximum load effects to which a structure may be subjected, while the LRFR Specifications are focused on a balance between safety and economy. These alternative approaches to design and rating are quantitatively represented through use of a lower reliability index, generally equal to 2.5 , in LRFR compared to a reliability index of 3.5 in LRFD. The lower reliability index used in rating is also justified by considering that during the design process there are a greater number of uncertainties regarding geometric and material issues that must be conservatively accounted for. However, the rating procedure is based on the actual condition of the structure, and therefore, many of these factors can be more precisely determined leading to a reduced need for conservatism.

The ultimate result of the LRFR procedures is obtaining one or more rating factors. These rating factors represent the multiple of the loading being considered that can be safely resisted by the structure or structural component under evaluation such that a rating factor greater than or equal to one indicates adequate capacity for the considered loading, while a rating factor less than one indicates insufficient capacity. Rating factors are computed as

$$
R F=\frac{C-\left(\gamma_{D C}\right)(D C)-\left(\gamma_{D W}\right)(D W) \pm\left(\gamma_{P}\right)(P)}{\left(\lambda_{L}\right)(L L+I M)}
$$

where:

$$
\begin{aligned}
\mathrm{RF}= & \text { rating factor } \\
\mathrm{C}= & \varphi_{\mathrm{c}} \varphi_{\mathrm{s}} \varphi \mathrm{R}_{\mathrm{n}}>0.85 \varphi \mathrm{R}_{\mathrm{n}} \text { for Strength Limit States and } \mathrm{f}_{\mathrm{r}} \text { for Service Limit States } \\
& \varphi_{\mathrm{c}}=\text { condition factor (discussed below) }
\end{aligned}
$$


$\varphi_{\mathrm{s}}=$ system factor (reflecting the redundancy of a given bridge)

$\varphi=$ LRFD resistance factor

$\mathrm{R}_{\mathrm{n}}=$ nominal LRFD computed member resistance (using as-inspected dimensions and material properties)

$\gamma_{\mathrm{DC}}=$ load factor for dead loads due to structural components and attachments equal to 1.25

for the Strength Limit State and 1.00 for the Service Limit State of steel structures

$\gamma_{\mathrm{DW}}=$ load factor for wearing surface and utility loads equal to 1.50 unless the wearing surface thickness is measured, in which case the load factor equals 1.25

$\gamma_{\mathrm{p}}=$ load factor for permanent loads other than dead loads (e.g., prestressing forces) equal to 1.00

$\gamma_{\mathrm{L}}=$ live-load factor (discussed below)

$\mathrm{DC}=$ dead load effect due to structural components and attachments

DW = dead load effect due to wearing surface and utilities

$\mathrm{P} \quad=$ permanent loads other than dead loads

$\mathrm{LL}=$ live-load effect

$\mathrm{IM}=$ dynamic load allowance accounting for impact

Equation 2.19 may be used to determine rating factors at three primary rating levels: designload rating, legal-load rating, and permit-load rating. A flowchart depicting the relationship of these three rating levels is given in Fig. 2-7. The design-load rating process is considered to be a screening process for determining if subsequent legal-load rating is necessary. The design-load rating is carried out at the design level of reliability and consists of two alternative rating levels, the operating rating and the inventory rating. The inventory rating level generally utilizes load factors equivalent to those used during design and results in a loading that the bridge can sustain for an indefinite life-span. The operating load level uses lower load factors, resulting in a higher rating factor; this rating factor represents the maximum load level that the bridge may sustain, although unlimited cycles of this load may reduce the life-span of the bridge. Furthermore, an inventory rating factor greater than or equal to one indicates that there is adequate capacity for all AASHTO and State legal loads. An operating rating factor greater than or equal to one indicates that there is adequate 


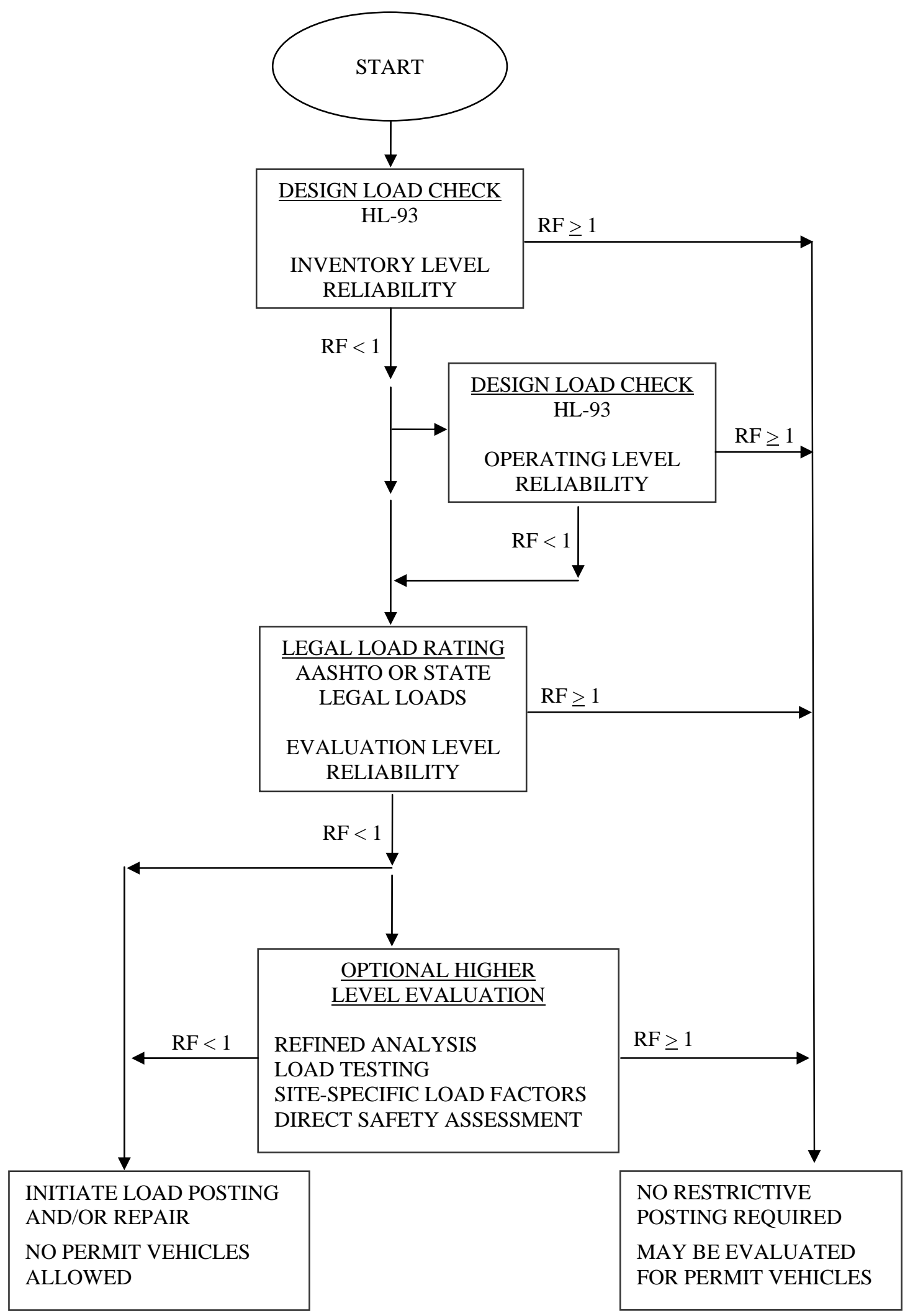

Figure 2-7. Flowchart for LRFR 
capacity for all AASHTO legal loads, but adequate capacity to resist other loadings that are considered legal by individual states is not guaranteed.

If the design-load rating results in RF less than one, legal-load rating is then performed to assess the need for posting or strengthening of the bridge. This process is carried out using the various AASHTO legal loads (Type 3, Type 3S3, and Type 3-3 Trucks, as well as associated lane loads as depicted in Fig. 2-8) and other state specific legal loads as applicable. The live-load factors for legal-load rating range between 1.4 and 1.8 as a function of the ADTT of the subject bridge and are based on achieving a reliability index of 2.5. If the legal-load rating factor is less than one, posting or strengthening the bridge should be considered. If the option for posting is selected, the safe posting load is computed using Eqn 2.20,

$$
\text { Safe Posting Load }=\frac{\mathrm{W}}{0.7}(\mathrm{RF}-0.3)
$$

where $\mathrm{W}$ is the weight of the controlling rating vehicle and $\mathrm{RF}$ is the rating factor. If the controlling rating factor is based on lane loading, $\mathrm{W}$ is taken as equal to 40 tons.

If the legal-load rating is greater than or equal to one the bridge may be suitable for carrying permit vehicles. Load rating for permit vehicles is performed using the actual axle weights and configuration of the specific permit vehicle. Live-load factors ranging between 1.1 and 1.85 are used depending on the permit type (routine or annual permits as opposed to special or limited crossing permits) and the ADTT of the bridge.

While all members of the bridge must be periodically rated for capacity, the members applicable to the present study on moment redistribution rating procedures are the steel girders. The primary reasons for a change in rating factors for these members are a change in the loads applied to the bridge or a change in cross-sectional properties due to corrosion. In the case of section losses due to corrosion, the LRFR Specifications suggest several methods for accounting for this change. One suggested procedure, suggested when there is uniform corrosion throughout a member, is to decrease the original nominal capacity of the member by the ratio of the corroded section modulus to the original section modulus to determine the 


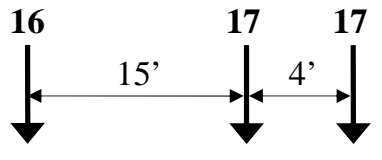

(a) Type 3 Truck

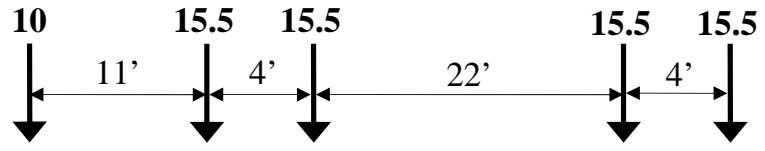

(b) Type 3S2 Truck

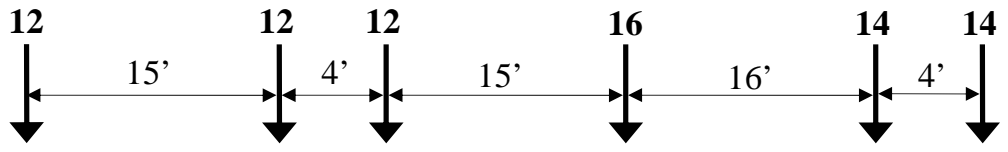

(c) Type 3-3 Truck

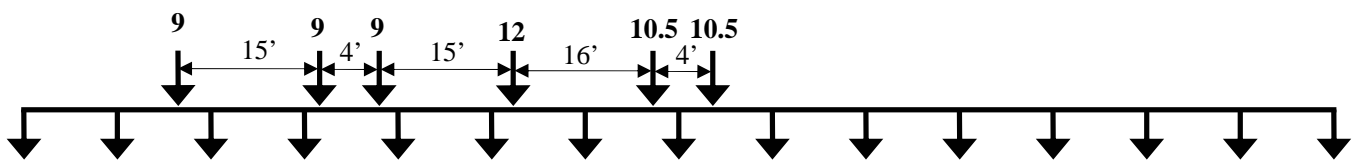

(d) Lane Load for spans greater than $200 \mathrm{ft}$. and all load effects

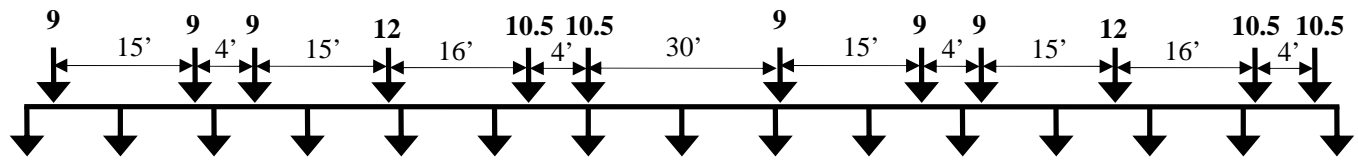

(e) Lane Load for negative moment and interior pier reaction

Figure 2-8. AASHTO Legal Loads 
corroded member capacity. Alternatively, it is suggested that in areas of localized corrosion, the capacity of tension and compression flanges can be conservatively approximated by analyzing the flange as an independent member loaded in tension or compression. Furthermore, condition factors, $\varphi_{c}$, may also be used to account for the corrosion characteristics of members at the engineer's discretion. For members in good or satisfactory condition $\varphi_{\mathrm{c}}$ is taken as 1.0 , for members in fair or poor condition $\varphi_{\mathrm{c}}$ is equal to 0.95 or 0.85 , respectively. Not only do these factors account for the reduced certainty in predicting the strength of corroded members, but they also account for the increased likelihood of future corrosion between rating cycles that may further reduce the capacity of the members.

\subsubsection{Inelastic Rating Procedures}

Previous research on inelastic rating methods is summarized in NCHRP Report 352 (Galambos et. al. 1993). The primary conclusions of this work are that elastic rating procedures are very conservative for continuous-span structures and that the use of inelastic rating procedures is justified. Two alternative inelastic procedures are thus suggested, which are based on the same philosophy as moment redistribution. The first is a grid type of analysis applicable for complete bridges with compact beams; the second is termed residual damage analysis (RDA), which considers individual girders and is applicable for compact or noncompact sections. The result from the grid analysis procedure is a rating factor at the shakedown limit state, while the RDA provides the residual deformation of a structure at a given rating factor.

The grid analysis method suggested by Galambos et al. (1993) for inelastic bridge rating consists of a series of computer programs. The first of these programs acts to compute the girder capacities and discretize the bridge into the grillage model. The second program then performs an elastic analysis of the grillage model. The last program performs an inelastic analysis of the grillage model and determines the redistribution moments. The inelastic rating factor is then computed as

$$
\mathrm{RF}\left(\mathrm{M}_{\mathrm{LL}}\right)+\mathrm{M}_{\mathrm{DL}}+\mathrm{M}_{\mathrm{RD}}=\mathrm{M}_{\mathrm{P}}
$$

where $R F$ is the rating factor, $\mathrm{M}_{\mathrm{LL}}$ is the elastic live load moment, $\mathrm{M}_{\mathrm{DL}}$ is the elastic dead load moment, $\mathrm{M}_{\mathrm{RD}}$ is the redistribution moment, and $\mathrm{M}_{\mathrm{p}}$ is the moment capacity of the beam, 
which is equal to the full plastic moment because compact members are assumed in the analysis. Thus the inelastic rating equation given by Eq. 2.21 is of similar format to the elastic rating equation given by 2.19. However, a grid analysis must be performed to obtain the redistribution moments, which adds significant complexity to the rating process.

The residual deformation method (Galambos et al. 1993, Dishongh and Galambos 1992) may be used to determine the permanent deformations resulting from moment redistribution. Although the method is suggested for use with rating applications, the procedure is equally suited to design applications where it is possible to use the method to compute initial camber recommendations. The residual deformation method is based on the conjugate beam method of analysis; the unknowns in the analysis are the elastic rotation, inelastic rotation, moment at each interior support, the inelastic rotation near the midspan of each span, and elastic rotation at each end support, while the two equilibrium equations for each span and the momentrotation relationships at each hinge give the number of equations needed to solve for each of the unknowns. For example, for a three-span continuous bridge there are eleven unknowns and a system of eleven simultaneous equations. The applied loading consists of a uniformly distributed dead load and any appropriate live-load vehicle or multiple thereof corresponding to a particular rating factor. The loading vehicle is positioned in various positions to produce the maximum rotation at each of the hinge locations. By using this method to compute the inelastic rotations at each pier, the corresponding permanent deflections can also be computed and these values can provide a rational, though rigorous, means for evaluating permanent deformations due to the selected magnitude of live-load vehicle.

\subsection{Available Rotations}

There has been a substantial amount of previous research providing information on the available rotation capacity of steel I-shaped members. These previous works are introduced below in Section 2.3.1, Overview of Previous Studies, which is subdivided into descriptions of experimental and analytical studies. Subsequently the results of these studies are

presented in Section 2.3.2, Factors Influencing Available Rotations, and Section 2.3.3, Available Rotation Prediction Equations. 


\subsubsection{Overview of Previous Studies}

\subsubsection{Experimental Studies}

Several investigators have previously analyzed the moment-rotation behavior of the negative bending region of continuous-span I-girders through experimental testing. This section will serve to introduce these works. In some cases, these studies resulted in conclusions regarding the influence of various parameters on available rotations. These results are subsequently presented in Section 2.3.2. In other situations, only a small number of specimens were tested. The most significant information resulting from these tests is the experimental moment versus rotation relationships obtained. Where available, relevant moment versus rotation plots resulting from these works are presented in Appendix B.

Prasad and Galambos (1963) present the experimental testing of four rolled beams. These beams contained five lateral bracing panels and were loaded such that the center three panels were in uniform bending. The purpose of this testing was to compare the performance of these girders to earlier, similar testing that subjected only one lateral bracing panel to uniform moment.

Adams et al. (1964) experimentally tested beams in both uniform bending and under moment gradient for the purpose of determining the rotation capacity of members fabricated from ASTM A441 steel. The uniform bending tests were conducted using W10x25 rolled beams and the moment gradient tests were conducted on 8B13 weak beam sections. Thus, within a given series, all the beams are of uniform cross-section. The varied parameter in these tests is the lateral bracing distance, which ranged from 25 to 45 times $\mathrm{r}_{\mathrm{y}}$ in the uniform bending tests and 23 to 73 times $\mathrm{r}_{\mathrm{y}}$ in the moment grading tests.

An experimental study primarily aimed at investigating the relationship between flange slenderness and rotation capacity was conducted by Lukey et al. in 1969. The secondary influences of web slenderness, brace spacing, and moment gradient were also studied. Tests were conducted on five-series of rolled beams with a nominal yield strength of $44 \mathrm{ksi}$. Series A, B, and C are characterized by differing web slenderness values (of 32.7, 45.0, and 54.5, respectively) with the flange slenderness varying between 7.0 and 9.7 for each specimen in a 
given series. In the remaining two series a constant web slenderness of 43.6 is used and a constant flange slenderness of 7 is used for Series D, while the flange slenderness is fixed at 9 for Series E. The varied parameters in the series D and E tests are the unbraced length (where the $\mathrm{L}_{\mathrm{b}} / \mathrm{r}_{\mathrm{y}}$ ratio varies between 38 and 147) and the moment gradient within the unbraced length.

Climenhaga and Johnson (1972) report the experimental testing of composite beams conducted to assess the effects of local buckling on available rotations. The primary variables considered in this study were flange and web slenderness, where the flange slenderness ranged between 5.0 and 8.5 and the web slenderness ranged between 23 and 62 . The specimens were fabricated from Grade 43A structural steel; the actual yield strength of the material ranged between 42 and $56 \mathrm{ksi}$. The use of concrete decks versus the use of an increased tension flange producing equivalent sections properties was also investigated in as was the use of longitudinal web stiffeners. Other variables were the force ratio, which the authors define as the ratio between the tensile strength of the concrete reinforcement and the tensile strength of the steel beam, and the shear ratio, which is the ratio between the applied shear and the shear capacity. Lateral torsional buckling was prevented by providing adequate bracing.

Two studies by Holtz and Kulak (1973 and 1975) investigate the effects of web slenderness on available rotations. The first study (Holtz and Kulak 1973) was aimed at determining the appropriate level of web slenderness that would assure that beams are able to reach a moment capacity equal to $M_{p}$. The web slenderness of these specimens ranged between $2.95 \sqrt{E / F_{y}}$ and $5.35 \sqrt{\mathrm{E} / \mathrm{F}_{\mathrm{y}}}$, while the flange slenderness and lateral bracing distances satisfied the compact limits. The beams tested by Holtz and Kulak in 1975 were used to determine the web slenderness necessary to obtain a minimum moment capacity of $\mathrm{M}_{\mathrm{y}}$. The flange slenderness of these specimens was equal to 15 or $0.59 \sqrt{\mathrm{E} / \mathrm{F}_{\mathrm{y}}}$, which is the non-compact flange slenderness limit given in the Canadian Specifications (CSA 1969). In both cases the beams were fabricated from 44 ksi nominal strength steel and were tested in uniform bending. 
Grubb and Carskaddan (1979) tested three beams with uniform cross-sections to determine the moment-rotation characteristics. Specifically, a flange slenderness of 8 and a web slenderness of 60 were used for all three beams. The length of the beams was variable in order to investigate the influences of moment gradient. Lateral bracing was provided to restrain lateral buckling modes of failure. The girders were doubly-symmetric, but a cover plate was used to shift the elastic neutral axis such that $57 \%$ of the web was in compression. All steel used in the specimens was nominal Grade 50.

Similar testing by Grubb and Carskaddan was reported in 1981 with a focus on evaluating the influences of web and flange slenderness on moment-rotation behavior. The effective flange slenderness (normalized for nominal yield strength of 50 ksi compared to the actual flange yield strength of 53.6) of these specimens was varied between 6.00 and 7.24. The effective web slenderness ranged between 47.8 and 63.3. All of these specimens were proportioned to have a $\mathrm{D}_{\mathrm{cp}} / \mathrm{D}$ ratio equal to 0.67 .

Carskaddan (1980) performed experimental testing of a two-girder system with a composite concrete deck. The girders used were identical to those used in the testing conducted by Grubb and Carskaddan (1979) except that the cover plate was replaced with the steel reinforcement in the concrete deck.

In 1985, Schilling conducted moment gradient testing on three girders that were more slender than previous experimental girders. The flange and web slenderness values of these girders were proportioned to target the maximum allowable web slenderness for girders without longitudinal stiffeners and the maximum allowable flange slenderness for non-compact sections, which were 163 and 9.8, respectively, for Grade 50 steel. The varied parameters were the span length and the percentage of the web depth in compression, with two of the three specimens having a larger tension flange than compression flange, such that $73 \%$ of the web was in compression at $\mathrm{M}_{\mathrm{p}}$.

Schilling and Morcos (1988) experimentally tested three plate girders in three-point bending with varying web slenderness values. Specifically, the specimens had web slenderness 
values of 81, 117, and 154. All of the girders were fabricated from Grade 50 steel, had a flange slenderness of 6, were doubly-symmetric, had compact lateral bracing, and possessed one-sided transverse stiffeners located a distance of one-half the web depth from midspan.

In a 1991 study, Tansil reports the experimental testing of a composite plate girder in negative bending. The girder is designed to represent the negative bending section of a halfscale model of a two-span continuous girder and consists of a 85 in. deep plate girder and a 9 in. deep by 156 in. wide concrete slab. The nominal yield stress of the steel is $50 \mathrm{ksi}$ and the concrete has a nominal compressive strength of $4 \mathrm{ksi}$. The girder had a compact compression flange, web, and lateral bracing.

Six Grade 50 plate girders were tested by Barth (1996) for the purpose of studying the effects of flange slenderness, web slenderness, lateral bracing distance, and moment gradient. These girders were tested in three-point bending and contained single-sided transverse stiffeners placed at one-third of the web depth on each side of the midspan location. Additional stiffeners were placed such that the maximum shear in each panel was less than $60 \%$ of the maximum shear capacity to avoid shear interaction effects. Furthermore, four lateral bracing segments were used in all but one test to accurately simulate the boundary conditions of the critical (center) lateral bracing panels. The flanges of these girders were proportioned to have slenderness ratios equal to 7.1 and 9.0, while the web slenderness ratios were equal to 125 or 160 . The lateral bracing distance was compact for all girders.

Hartnagel (1997) reports the testing of four simply-supported, compact girders, which were ultimately used to validate inelastic design procedures suggested by the author. Three of these girders were subjected to a monotonically increasing load, with two of these being composite with varying reinforcement areas and the third being a non-composite girder. The fourth girder was also a composite girder and was loaded with loads of varying magnitudes placed at varying positions in order to simulate cyclic truck traffic.

Experimental testing of two girders in three-point bending was conducted by Yakel et al (1999) to investigate the performance of girders fabricated from HPS-70W. Both of these 
girders had a flange slenderness of 5.4, or $70 \%$ of the compact limit for Grade 70 steel. One of the specimens had a web slenderness exactly equal to the compact limit using the specifications in effect at the time of the testing, while the second girder had a web slenderness that exceeded this limit by $18 \%$. Compact bracing distances were used in both specimens.

Green et al. (2001, 2002) conducted an experimental study of I-girders fabricated from HSLA-80 steel. The experimental program consisted of beams loaded in moment gradient, cyclic moment gradient, and uniform moment. The test specimens were designed so that flange local buckling, web local buckling, or tension flange yielding were the predicted failure modes. Variable flange and web slenderness ratios were investigated, with all of the specimens having a web slenderness between $27 \%$ and $76 \%$ of the compact limit and a flange slenderness between $41 \%$ and $128 \%$ of the compact limit. All of the specimens were compact with respect lateral slenderness and lateral torsional buckling did not occur during any of the experiments.

\subsubsection{Analytical Studies}

Kemp (1986) presents theoretical predictions for the available rotation at maximum moment in beams subjected to moment gradient by computing the length of the beam in the plastic region. For example, considering Fig. 2-9, the length of the plastic region, $\mathrm{L}_{\mathrm{p}}$, is the distance over which the moment exceeds $M_{p}$. Consideration is given to the distance of $L_{p}$ for the three conventional modes of failure: flange local buckling, web local buckling, and lateral torsional buckling, and it is assumed that the failure mode corresponding to the minimum distance of $\mathrm{L}_{\mathrm{p}}$ is the dominant failure mode. These three theoretical equations identify several key factors influencing $L_{p}$, and therefore $M_{\max }$ and the available rotation capacity, as will be discussed in Section 2.3.2. Kemp also compares these theoretical predictions to experimental results.

Kato (1989 and 1990) derives closed-form solutions for the rotation capacity of simplified structural models as a function of the material yield ratio, which is the ratio between the yield strength and ultimate strength of the material. This analysis is conducted for tension 


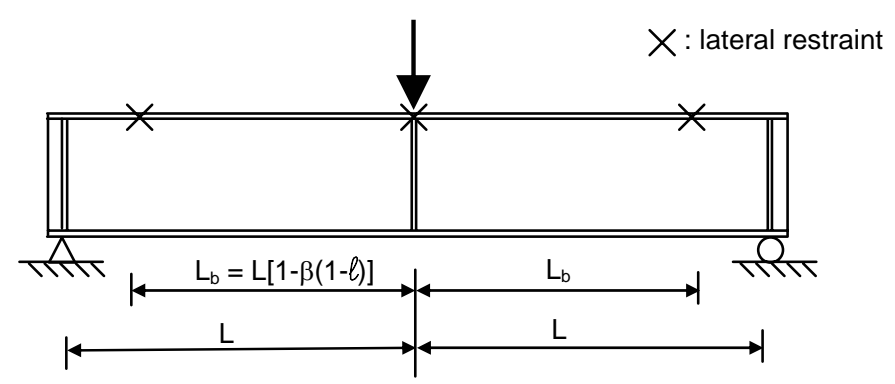

(a) Layout of Beam

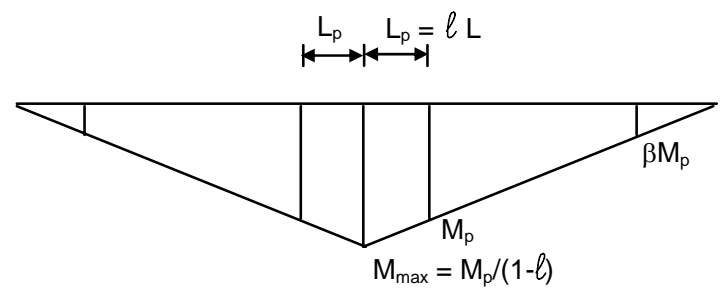

(b) Bending Moment Diagram

Figure 2-9. Illustration of Variable Definitions used by Kemp (1986)

members, columns, beams, and beam-columns although the present discussion is focused on the analysis of beams as this is most relevant to the current research. The rotation capacity of beams is studied using a cantilevered I-beam where contributions of the web are neglected.

Kemp and Dekker (1991) evaluate the available rotations resulting from previous experimental testing (conducted by Lukey and Adams 1969, Kemp 1985, Kemp 1986, and Roik and Kuhlmann 1987) for the purpose of developing mathematical expressions to predict available rotations. This work is based on predicting the length of the plastic section in a steel member as previously considered by Kemp (1986). The expressions resulting from this work are discussed subsequently in Section 2.3.3.

A FEA evaluation of I-girder moment-rotation behavior was conducted by Barth (1996). Symmetry about the center of the negative bending region is employed, thus the FEA models represent one-half of the actual negative bending section with appropriate boundary conditions. The girders are modeled using 9-node shell elements and the steel is represented as a J2 material with kinematic strain hardening. Residual stresses due to flame cutting and welding are incorporated in the analyses and geometric imperfections based on American 
Welding Society tolerances are also included. The varied parameters in this work include: normalized laterally unbraced length, cross-section aspect ratio, transverse stiffener spacing, span to depth ratio, flange slenderness, web slenderness, and percentage of the web depth in compression. The parametric values used for each of these variables are as follows. The alternative compact lateral bracing distances specified in the ASCE (1971) Plastic Design Code and the longer bracing distance permitted by the original AASHTO LRFD Specifications (1994) are the two lateral bracing distances evaluated. Cross-section aspect ratios of $\mathrm{d} / \mathrm{b}_{\mathrm{fc}}$ equal to 3.00 and 4.25 were selected. Stiffener configurations where the first stiffener from midspan is located at $\mathrm{d} / 3$ and where the first stiffener is placed to obtain sufficient shear capacity such that the applied shear is not greater than $60 \%$ of the shear capacity were studied. The ratio between girder length and depth is used as a measure of the moment gradient within the girder; L/d ratios of 20 and 30 were evaluated. Flange slenderness values equal to the compact limit 9.2 and 7.0 were included in the study. Low, intermediate, and high values of web slenderness, equal to 86, 125, and 163, were incorporated. Lastly, $\mathrm{D}_{\mathrm{cp}} / \mathrm{d}$ ratios equal to 0.625 and 0.75 were used in this study.

Gioncu and Petcu (1997a, 1997b) present a study focused on the available rotation capacity of steel wide-flange shapes. Specifically this work is focused on the theoretical development, evaluation of, and parametric results obtained from a computer program (DUCTROT 96) that calculates available rotation capacities for beams and beam-columns. This program functions by computing the moment-rotation behavior due to the development of in-plane and out-of-plane local buckling (Gioncu and Petcu 1997a). In a companion paper (Gioncu and Petcu 1997b), the accuracy of the DUCTROT 96 program is evaluated against experimental and FEA results. Compared to the results of 75 experimental tests by independent researchers (Lukey and Adams 1969; Kemp 1985; Kuhlmann 1986 and 1989; Spangemacher 1991; Kemp and Dekker 1991; Boeraeve et al. 1993; and Suzuki et al. 1994), the average ratio of computed to experimental available rotation was 1.065 and the coefficient of variation was 0.379 . Thus, the average is relatively favorable; however the authors propose a safety factor of 1.5 be applied to the results to counter the large coefficient of variation. The DUCTROT 96 results are also compared against FEA results by 
Spangemacher (1991), where the authors conclude that the program performs adequately in comparison to these results.

Dinno and Birkmoe (2001) conducted an FEA study using the commercial software ANSYS to investigate the influence of flange slenderness and web slenderness values exceeding the compact limits. Variable material properties are also evaluated. The material properties investigated were yield strength, ratio between elastic modulus and strain hardening modulus, and ratio between strain hardening strain and yield strain. These girders simulated four-point bending conditions and were modeled using four-node shell elements. All girders had compact lateral bracing. Residual stresses and geometric imperfections are both included in the analyses and the influences of various magnitudes and patterns of geometric imperfections is considered.

Thomas and Earls (2003) present the results of a finite element evaluation of the available rotation capacity of HPS70W girders. The analysis is carried out using ABAQUS software and four-node reduced integration shell elements (ABAQUS S4R). Lateral constraints at the midspan and support regions are assumed to be fully fixed, while the lateral constraints at intermediate bracing locations is provided by "SPRING1" elements. Four parameters are investigated in this study: flange slenderness, web slenderness, lateral bracing distance between midspan and the first lateral brace, and bracing stiffness.

Lääne and Lebet (2005) present the results of a parametric study investigating the influential parameters affecting available rotations and consequently propose an equation to calculate the available rotation of steel plate girders. This research is conducted using ABAQUS software to conduct FEA of analytical models of the region in the immediate vicinity of the pier. Details of the model including loading and boundary conditions are presented in Fig. 210. Here it is shown that the boundary conditions used assume that the cross-section is fully fixed at the pier location and that rotation in the vertical (y) direction is restrained at the opposite end of the model, where vertical and horizontal forces are applied. The length of the girder modeled is represented by $a$ in the figure and is equal to the height of the web in most cases. The beam is modeled using ABAQUS S9R5 elements. 

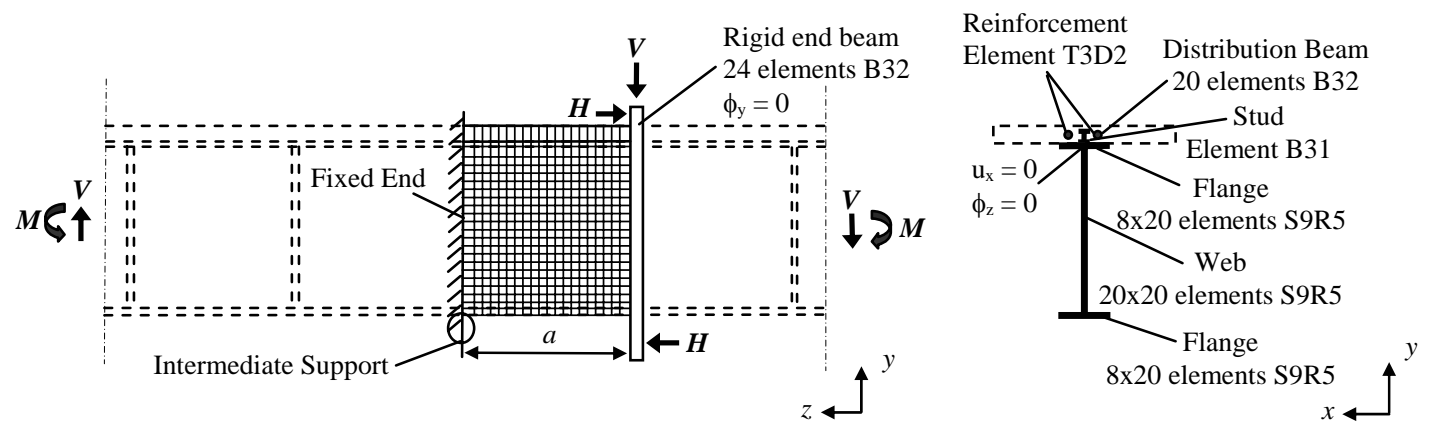

Figure 2-10. Schematic Diagram of FEA Techniques used by Lääne and Lebet (2005)

\subsubsection{Factors Influencing Available Rotations}

\subsubsection{Flange Slenderness}

It is unanimous among previous researchers that flange slenderness decidedly influences the available rotation of steel I-girders. Furthermore, nearly all researchers to investigate this topic (Lukey et al 1969, Kemp 1986, Barth 1996, Gioncu and Percu 1997b; Dinno and Birkemoe 2001, Thomas and Earls 2003) agree that there is a decrease in available rotations with increasing flange slenderness, particularly for compact flanges and when failure is controlled by flange local buckling.

The experimental testing used to determine the current AASHTO and AISC flange compactness limit is presented by Lukey et al. (1969) and Lukey and Adams (1969). Lukey et al. suggest that when failure is by local buckling, the flange slenderness ratio has a significant influence on the available rotations. However, when lateral buckling controls, they conclude that the flange slenderness ratio is of little importance. Similarly, Dinno and Birkemoe (2001) suggest that the interaction between flange and web slenderness has an influence on rotation capacity when flange local buckling occurs, but that flange slenderness has little influence on rotation capacity when web local buckling is the cause of failure.

Additionally, Lukey and Adams present data that suggests a relatively linear relationship between flange slenderness and available rotation for flange slenderness values between 7 and 9. However, once a flange slenderness of 9 is exceeded, the influences of flange slenderness become much less apparent. 
As discussed above, Kemp (1986) presents the results of a study focused on the available rotation capacity of members as a function of the length of the member that is in the plastic region, $L_{p}$. For the limit state of flange local buckling, the following iterative equation is proposed for computing $\mathrm{L}_{\mathrm{p}}$.

$$
\frac{b}{t_{f}}=\sqrt{\frac{2}{1.5 \varepsilon_{b}-C_{f}\left(\pi t_{f} / l_{f} L\right)^{2}}}
$$

where $b$ is the width of the compression flange, $t_{f}$ is flange thickness, $\varepsilon_{b}=\varepsilon_{y f}\left(s+0.5 e \frac{l_{f}}{1-l_{f}}\right), C_{\mathrm{f}}$ is a boundary condition coefficient equal to 0.5 for no web or lateral constraint or 1.0 for web and lateral restraint, $l_{p f}$ is $L_{p f} / L, L$ is the half span length, $L_{p f}$ is $L_{p}$ for the flange local buckling limit state, $\varepsilon_{y f}$ is yield strain of the flange. This equation is based on the assumption that the flange local buckling occurs when the length of the plastic region is greater than the full wavelength of the flange buckle as proposed by Lay and Galambos $(1965,1967)$ and is derived by rearranging the expressions and modifying the assumptions used by Lay and Galambos, Southward (1969), and Stowell (1950).

Lääne and Lebet (2005) investigated three values of flange slenderness in their parametric study: 8.1 (the Eurocode Class 2 limit for Grade 50 steel), 7.3 (the Eurocode Class 1 limit), and 6 (a common value for composite bridges in Switzerland according to the authors). Results indicate that, counter to intuition, the most compact flanges possessed the least rotation capacity and the flanges with a slenderness of 7.3 exhibited the most rotation capacity. Because of the significant body of evidence contradicting these conclusions, this may be taken as a sign that the solution procedures (e.g., boundary and loading conditions) used by Lääne and Lebet may be flawed.

\subsubsection{Web Slenderness}

Several previous researchers report increasing available rotations with decreasing web slenderness values. For example, experimental testing of three girders with varying web slenderness ratios ranging between 81 and 154 by Schilling and Morcos (1988) illustrates that for these girders there is a clear decrease in both moment capacity and available rotations with increasing web slenderness values. Thomas and Earls (2003) investigated web 
slendernesses between 18 and 72 of HPS-70W beams, where 72 is approximately equal to the compact limit in AASHTO (1998) of $3.76 \sqrt{E / F_{y}}$ for $F_{y}$ of $70 \mathrm{ksi}$. A decreasing relationship between rotation capacity and increasing web slenderness is evident based on these FEA results. Lääne and Lebet (2005) investigated five different web slenderness values ranging between 100 and 200 in increments of 25 where it was shown that increasing web slenderness contributes to decreased rotation capacity.

Kemp and Dekker (1991) and Gioncu and Petcu (1997b) report that there is a inversely proportional relationship between web slenderness and available rotations when the web slenderness is above a certain limit, however, below this limit increasing rotations are reported with increasing web slenderness. Specifically, Kemp and Dekker (1991) report that an increase in web slenderness from 24.5 to 30 resulted in an increase in available rotation capacity; a decrease in rotation capacity is then exhibited for beams with increasing web slendernesses between 33 and 40. A similar trend was reported by Gioncu and Petcu (1997b), who evaluated web slenderness values between 23 and 67. The results of this study showed that the minimum available rotation was obtained for the beam with a web slenderness of 38. Gioncu and Petcu attribute this behavior to the fact that beams with a higher web slenderness will fail by elastic-plastic web buckling and beams with a lower web slenderness will fail by plastic flange buckling.

Dinno and Birkemoe (2001) present the influences of web slenderness for 28 FEA girders. Here it is shown that, while for low web slenderness ratio $(d / t w<30)$ there is a significant increase in available rotations with decreasing web slenderness, there is typically an upper limit of web slenderness where further increases no longer influence available rotations. This value of web slenderness may be as low as 50 or may be greater than 70 depending on other parameters of the girder, e.g. low flange slenderness values increase the web slenderness value at which the moment-rotation curves converge.

These findings by Dinno and Birkemoe are consistent with the FEA results presented by Barth (1996). Barth investigated girders with a higher web slenderness than considered by previous researchers where the minimum web slenderness was equal to 86 . These results 
indicated even though decreased moment capacity exists for girders with higher web slenderness values, the influence on available rotations is small as the moment-rotation curves for girders with varying web slenderness values eventually converge.

Similar to Eq. 2.22 above, Kemp (1986) gives the following iterative equation to predict $\mathrm{L}_{\mathrm{p}}$ (and consequently rotation capacity) as a function of web slenderness.

$$
\left(\frac{h_{c p}}{t_{w}}\right)=\frac{1}{C_{w}} \sqrt{\frac{0.97}{\sqrt{e} \varepsilon_{y w}}}\left(\frac{2 C_{w} h_{f c}}{l_{w} L}+\frac{l_{w} L}{2 C_{w} h_{c p}}\right)
$$

where $h_{c p}$ is the distance between the plastic neutral axis and the center of the compression flange, $t_{w}$ is the web thickness, $C_{w}=B-0.25 n w, B$ is 0.75 if the flange is buckled and 1 if the flange is not buckled, nw is the ratio of applied axial force to force required to fully yield the full web depth, $\varepsilon=E / E_{s t}, \varepsilon_{y w}$ is yield strain of the web, $l_{w}=L_{p w} / L$ where $L_{p w}$ is $L_{p}$ corresponding to the limit state of web local buckling. This equation is developed from classical buckling theory assuming that the web depth in compression may be satisfactorily represented by an equivalent depth subject to uniform stress equal to the yield stress. $\mathrm{L}_{\mathrm{pw}}$ represents the full wavelength of the web buckle using these assumptions.

\subsubsection{Lateral Bracing Distance}

It was suggested by Prasad and Galambos (1963) that lateral torsional buckling is a failure mode with little rotation capacity available. This conclusion was based on the results of experimental testing of beams with five lateral bracing panels loaded such that the center three panels were in uniform bending. This is an extreme situation compared to the actual moment conditions at the pier region of continuous beams, which approximates a linear moment gradient. However, current researchers often cite this work and suggest that failure by lateral torsional buckling should be prevented.

The majority of previous investigations into the influences of lateral bracing distance on available rotations have been conducted on simply-supported beams with two lateral bracing segments. Thus there is no torsional restraint to the critical lateral bracing panels as would exist in actual continuous-span beams and the results from these investigations may be unduly conservative. One such study is that of Adams et al. (1964) where it is shown that 
there is a distinct relationship between increased lateral bracing distance and decreasing available rotations. Similarly, Lukey et al. (1969) show that there is a significant decrease in available rotations at $M_{p}$ as the normalized unbraced length $\left(\mathrm{L}_{\mathrm{b}} / \mathrm{r}_{\mathrm{y}}\right)$ is increased from 70 to 105.

Kemp and Dekker (1991) plot the observed available rotations in 44 experimental tests (Lukey and Adams 1969; Kemp 1985; Kemp 1986; and Roik and Kuhlmann 1987) versus the authors' definition of lateral slenderness ratio, $L_{i} / i_{z c} \varepsilon$, where $L_{i}$ is the distance between locations of maximum and zero moment (which is also equal to the lateral bracing distance), $i_{z c}$ is the radius of gyration of the compression flange and portion of the web in compression, and $\varepsilon$ is $\sqrt{235 / F_{y}}$. These experimental girders depicted in the graph reproduced in Fig. 2-11 represent relatively compact sections with lateral bracing provided at the midspan and end supports only. Kemp and Dekker conclude that failure of beams with a lateral slenderness greater than 60 will be dominated by lateral torsional buckling, which they term "Mode 1" failure; conversely failure of beams with a lateral slenderness less than 60 will be dominated by local buckling, termed “Mode 2" failure. From the data presented in Fig. 2-11, the authors conclude that there is a quadratic relationship between lateral slenderness ratio and available rotations. This influence appears clearer, with less scatter, for the girders with a lateral slenderness exceeding 60.

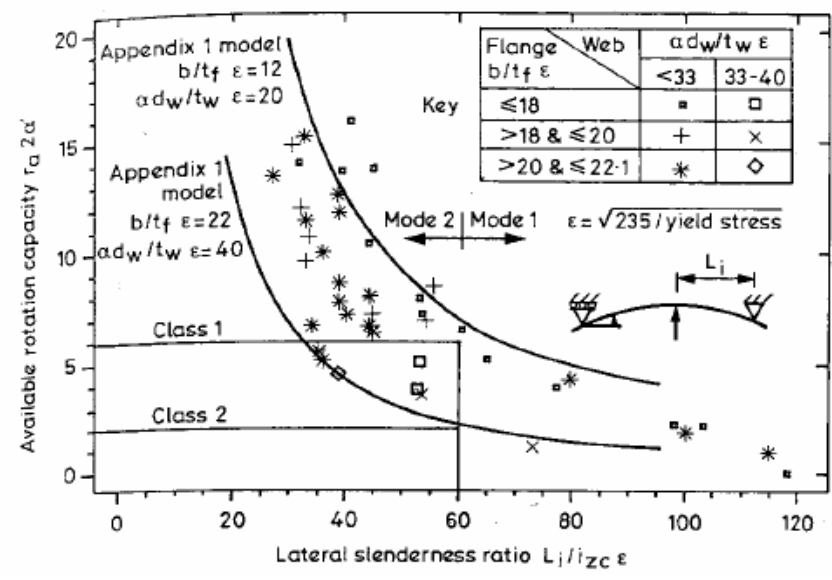

Figure 2-11. Available Rotation versus Lateral Slenderness Ratio by Kemp and Dekker (1991) 
Only the studies by Barth (1996) and Thomas and Earls (2003) consider multiple lateral bracing segments such that representative boundary conditions are applied to the critical panels. Barth illustrates through FEA and experimental testing that lateral bracing distances less than the compact limit provide little benefit in terms of increasing available rotations. Thomas and Earls (2003) varied the lateral bracing distance between $0.5 \mathrm{~d}$ and $2 \mathrm{~d}$ ( $\mathrm{d}$ is the depth of the cross-section) at midspan. However, it appears that a significantly larger lateral bracing distance was used away from midspan of the girders such that the panel where failure occurred may have been the second panel from midspan in many cases. It is hypothesized that failure occurred in these "second panels" in all of the girders where the lateral bracing distance at midspan is $0.5 \mathrm{~d}$, as all of these girders exhibited rotation capacities that were significantly less than the rotation of corresponding girders with lateral bracing distances of 1.0d.

Kemp (1986) considers lateral torsional buckling of a compressive strut comprised of the compression flange and the portion of the web in compression. Based on buckling theory, Kemp gives the following iterative equation for predicting the plastic length of the beam (and consequently rotation capacity) as a function of the lateral slenderness.

$$
\frac{L_{b}}{r}=\sqrt{\frac{\pi^{2}}{\varepsilon_{y f}}} \frac{(1.08-0.04 \beta)(m-\beta)}{(1-\beta) \sqrt{0.6+0.4 \beta}+(m-1) / C_{t} \sqrt{e(0.6 m+0.4)}}
$$

where $r$ is the radius of gyration of the portion of the section in compression, $\beta$ is the ratio of moment at the lateral constraint to the plastic moment, $\mathrm{m}$ is $1 /(1-l t)$, and $C_{t}$ is 1 if the flange is not buckled and 0.667 if the flange is buckled.

\subsubsection{Material Properties}

Based on previous research, it is widely accepted that increased steel yield strength corresponds to a reduction in available rotation capacity. For example, Dinno and Birkemoe (2001) have reported members with higher yield strengths exhibit lower rotation capacity compared to lower grade steels based on analytical results of beams subjected to four-point bending with compact lateral bracing. Furthermore, they suggest that this trend is most significant for cases where failure is caused by flange local buckling. Dinno and Birkemoe also investigated the influences of varying ratios of elastic modulus to strain hardening 
modulus, which was found to be insignificant, and ratios of strain hardening strain to yield strain, which was insignificant for values less than or equal to 10 . At ratios of strain hardening strain to yield strain greater than 10 the available rotation was shown to decrease in one instance.

Gioncu and Petcu (1997b) have researched the available rotation capacity of girders comprised of steel having three different yield strengths: 33 ksi, 39 ksi, and 50 ksi. Girders having flanges and webs with each of these materials are considered, resulting in a total of nine girders. Comparing the rotation capacities of each of these girders shows that the yield strength of the web has a negligible influence on the rotation capacity, but that decreased rotation capacity results with increasing flange yield strength. A change in flange yield strength from 33 to 50 ksi results in a $63 \%$ decrease in rotation capacity.

Green et al (2001) have investigated the available rotation capacity of high performance steel (HSLA-80) members compared to the rotation capacity of members fabricated from A36 steel. This evaluation indicated that members fabricated from high performance steel may have significantly less rotation capacity than A36 members with the same cross-sectional geometry, i.e., the slenderness values are not normalized to account for the difference in yield stress. Specifically, there is a 70 to $83 \%$ decrease in the available rotation of the high performance steel members compared to the corresponding A36 members.

Lääne and Lebet (2005) considered two grades of steel in their parametric study: S355 and S460, which approximately correspond to Grades 50 and 65, respectively. Results of the study show that the higher grade steel has less ductility even though the compression flange slenderness was decreased such that girders from both materials have the same normalized flange slenderness (although web dimensions are equal). The authors suggest that because a higher buckling stress develops in girders with higher yield strengths, the web is also loaded with a higher stress and cannot support the flange as efficiently as with the lower grade steel.

Kato (1990) suggests that the widely recognized characteristic that an increase in yield strength corresponds to a decrease in rotation capacity is actually due to the increase in yield 
ratio that typically accompanies increased yield strengths. It is a fairly intuitive concept that a smaller yield ratio will permit a larger extension of the plastic region and therefore increased rotation capacity. This hypothesis is explored via a theoretical investigation utilizing the moment-curvature relationship where steel with a yield stress of $35 \mathrm{ksi}$, YR of $0.6, \mathrm{E} / \mathrm{E}_{\mathrm{st}}=50$, and $\varepsilon_{\mathrm{st}} / \varepsilon_{\mathrm{y}}=5$ is taken as the reference material. This evaluation shows that when $F_{y}$ is altered and the other three parameters are left unchanged, there is not a significant influence on rotation capacity. However, when $\mathrm{YR}$ is varied and $\mathrm{F}_{\mathrm{y}}, \mathrm{E} / \mathrm{E}_{\mathrm{st}}$, and $\varepsilon_{\mathrm{st}} / \varepsilon_{\mathrm{y}}$ are constant, the change in rotation capacity is rather dramatic. The influences of varying $E / E_{s t}$ and $\mathrm{e}_{\mathrm{st}} / \mathrm{e}_{\mathrm{y}}$ are also presented, where it is shown that these parameters are also influential, but to a lesser extent than the yield ratio. Thus it is proposed that the influence of material properties on rotation capacity is best attributed to the complementary energy of the steel, which conveniently takes into account all applicable parameters: the yield stress, modulus of elasticity, ultimate stress, strain hardening strain, and strain hardening modulus, but that the yield ratio is the single parameter most influential to available rotations.

\subsubsection{Web Depth in Compression}

Grubb and Carskaddan (1979) first reported the significant influences that the percentage of the web depth in compression may have on available rotations. This observation resulted from available rotations that were lower than expected compared to previous testing. Because the previous tests were on doubly-symmetric sections and the tests by Grubb and Carskaddan were singly-symmetric with $57 \%$ of the web in compression in the elastic range, it was reasoned that the lower available rotations were due to this influence.

Subsequent research has supported that there is a relationship between increasing percentage of depth of web in compression and available rotations, but it is suggested that this influence is of little significance for girders with more than half of the web depth in compression and that this behavior is better attributed to the decreased moment capacity that results with increasing web depth in compression. For example, Barth (1996) investigated the use of $\mathrm{D}_{\mathrm{cp}} / \mathrm{d}$ ratios equal to 0.625 and 0.75 and found that the primary influence of the higher ratio was to decrease the peak moment capacity; no clear relationship exists between $D_{c p} / d$ ratio and available rotations based on the results presented in this work. Similarly, Gioncu and 
Petcu (1997b) investigated the influence of variable tension flange area on the available rotation capacity. The tension flange area was varied between 0 and $1.8 \mathrm{~A}_{\mathrm{fc}}$, which corresponds to a $\mathrm{D}_{\mathrm{cp}} / \mathrm{D}$ ratio varying between 0.2 and 0.74 for the given beam geometry. Results indicated that there is decreasing rotation available with increasing $\mathrm{D}_{\mathrm{cp}} / \mathrm{D}$ ratio, however, for beams with a $\mathrm{D}_{\mathrm{cp}} / \mathrm{D}$ ratio greater than 0.5 the reduction in rotation capacity becomes less severe. Lääne and Lebet (2005) investigated six different percentages of elastic web depth in compression ranging between 42.8 and $63.2 \%$, with three of these less than 0.5 and three with $\mathrm{D}_{\mathrm{c}} / \mathrm{D}$ greater than 0.5. They suggest that when more than half of the web is in compression there is a negligible influence on rotation capacity, however, if the elastic neutral axis is less than $0.5 \mathrm{D}$, there is a considerable increase in ductility with decreasing percentage of web in compression.

\subsubsection{Relative Cross-Section Proportions}

Barth (1996) studies the influence of the cross-section aspect ratio, which is defined as the web depth divided by the compression flange width. Here it is reported that increasing $d / b_{\mathrm{fc}}$ ratios from 3 to 4.25 result in an average decrease in moment capacity of $6 \%$. This decrease in moment capacity correspondingly influences the available rotations at a given level of moment.

Lääne and Lebet (2005) investigated seven combinations of relative compression flange, tension flange, and web areas. The area of the tension flange was varied between 20 and $30 \%$ of the total area; the area of the compression flange represented between 35 and $45 \%$ of the total girder area; and the area of the web ranged between 30 and $40 \%$ of the total area. Results of this analysis showed that the minimum ductility results when the compression flange area is maximized and the web area is minimized, while the maximum ductility results when the web area is maximized and the compression flange area is minimized.

\subsubsection{Moment Gradient}

There are variable opinions regarding the influence of moment gradient on available

rotations. Grubb and Carskaddan (1979) aimed to investigate the influences of moment gradient through experimental testing of three I-girders that were identical except for changes 
in the span length of the specimens. At $\mathrm{M}_{\mathrm{p}}$ the available rotations were $11 \mathrm{mrad}$ for the shortest beam, 7 mrads for the beam of intermediate length, and 0 mrads for the longest beam. Grubb and Carskaddan also plot the available rotations from the experimental testing conducted by Adams et al. (1964) and Lukey and Adams (1969) as a function of the L/d ratio of the specimens, where $L$ is half the span length and $d$ is the total section depth. Data from similar tests are grouped into series and it is shown that there is an increasing relationship between available rotation and L/d ratio. The depth of all sections considered is relatively constant, therefore it could be stated that there is an increasing relationship between span length and available rotation. Grubb and Carskaddan hypothesize that this phenomenon occurs because the yielding is less localized with a longer negative bending length. They attribute the contrast between this behavior and their own experimental results to the higher web and flange slenderness values of their own specimens and suggest that the flatter moment gradient that exists with the longer span lengths increases the length of the web subjected to a high compressive stress rendering the web more susceptible to buckling.

Barth (1996) examines the influence of moment gradient for L/d ratios equal to 20 and 30, which are typical L/d ratios for continuous-span bridges. Here it is also concluded that there is a decrease in available rotations due to lower moment gradient. However, this data suggests that this influence is sufficiently small such that it is reasonable to neglect these effects.

Kemp (1986) reports the results of two experimental tests that are nearly identical except that one beam had twice the span length of the other. These results are opposite to those reported by Grubb and Carskadan and Barth in that the increase in span length led to a drop in available rotation capacity at the maximum moment from 7.4 to 1.4. It is suggested that this influence is due to the fact that as the span length is increased, the yielded length remains constant and consequently the proportion of the span that is plastic decreases resulting in a lower rotation capacity. Similarly, Gioncu and Petcu (1997b) investigated the influence of span length on beams ranging from 2 to $6 \mathrm{~m}$ in length. Consequently, it was concluded that beams with a span length of $4 \mathrm{~m}$ had approximately half the available rotation capacity of 
beams with a span length of $2 \mathrm{~m}$. Beams with a span length of $6 \mathrm{~m}$ had approximately $75 \%$ of the available rotation of the beams with a span length of $4 \mathrm{~m}$.

\subsubsection{Stiffeners}

It was concluded by Huang (1995) that stiffeners located closer to the peak moment location, termed inelastic restraint stiffeners, may result in greater available rotations. This behavior occurs because these stiffeners act to restrain buckling at peak moment locations, thus delaying the decrease in moment capacity. It is suggested that stiffeners placed at one-third the web depth away from the pier location will result in optimal moment-rotation characteristics.

Similarly, Lääne and Lebet (2005) observed that when the web panel length was less than the height of the web, a substantial increase in rotation capacity was obtained. They suggest that this may be caused by a stiffener being located where compression flange buckling would normally occur, thus delaying or suppressing this mode of failure.

Barth (1996) subsequently investigated the influence on moment-rotation behavior due to the inelastic restraint stiffeners suggested. Barth observed minimal benefits to the use of these additional stiffeners and thus does not recommend their use due to the associated increased fabrication costs.

Kemp (1986) proposes a diagonal stiffener having the configuration shown in Fig. 2-12 be placed at sections of maximum negative moment. Test results on five pairs of experimental girders with and without this diagonal stiffener show that the stiffened girders exhibit approximately twice the rotation capacity of their unstiffened counterparts for cases where failure was caused by local buckling. This stiffener arrangement is designed to stiffen the compression flange and portion of the web in compression in order to delay buckling in the section, however strengthening the portion of the member in tension is avoided to increase the extent of tensile yielding in the beam, which further increases the rotation capacity. Therefore the stiffener height, $h_{s}$, should be approximately equal to the web depth in 

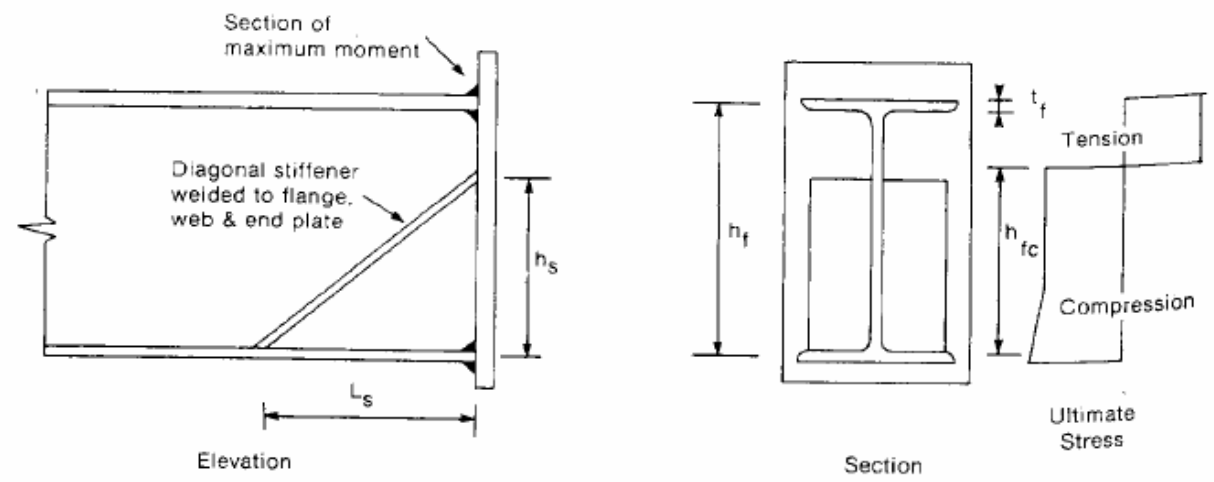

Figure 2-12. Diagonal Stiffener Proposed by Kemp (1986)

compression. Based on the research by Kemp, 5\% of the negative bending length is suggested to be an optimal value for the length of the stiffener, $L_{s}$.

\subsubsection{Shear Force}

The experimental girders tested by Holtz and Kulak (1973) included three girders with relatively high levels of shear. Specifically, the shear was approximately equal to $90 \%$ of the shear capacity of these beams. The experimental results indicated that this had little or no effect on the moment capacity of the beams.

Grubb and Carskaddan (1979) also conclude that the influence of shear force on available rotation is negligible. They reason that increasing shear theoretically reduces the moment capacity of the beam. Thus, if the available rotation is evaluated at a lower moment capacity, higher available rotation results. Because both of these influences are small and offset one another, Grubb and Carskaddan recommend that the effects of shear may be neglected.

\subsubsection{Lateral Bracing Stiffness}

Thomas and Earls (2003) investigated the influence of lateral bracing stiffness in their parametric study focusing on the available rotation capacity of HPS70W girders. Here lateral bracing was applied in the FEA in two alternative methods: rigid lateral constraints at all lateral bracing locations or rigid lateral constraints at midspan and bearing locations and flexible (spring element) constraints at all other lateral bracing locations. These results 
demonstrated that the varying stiffness assigned to the spring elements contributes a negligible influence to rotation capacity when the lateral bracing distance is between 4 and 10 times the minimum required stiffness specified in the 1999 AISC Specifications. Only one girder is discussed where the stiffness was outside of this range and this girder had a lateral bracing stiffness equal to the AISC specified stiffness, $\beta$. For this single girder, the rotation capacity was approximately half the rotation capacity of similar girders with stiffness of $4 \beta$ to $10 \beta$. However, the rigid constraints (which may be thought of as having infinite stiffness) provided a stiffness only slightly higher than the beam with stiffness equal to $\beta$.

\subsubsection{Geometric Imperfections:}

Holtz and Kulak (1973) specifically address the influence of initial out-of-flatness of the webs of the girders tested in their experiments. They found that the six girders (out of a total of 10 girders) that did not reach the plastic moment capacity were also the girders with the highest values of initial web imperfections although all but one of these girders satisfied the imperfection tolerances given by CSA Standard W29.1. It was observed that webs with the higher imperfection values began laterally deflecting at lower levels of load, leading to earlier buckling and reduced moment capacity.

In the FEA work conducted by Dinno and Birkmoe (1991) the influences of five different patterns of imperfections on the available rotations were investigated. This included imperfections that were: a function of the sine function, a function of cosine function, a function of a combination of sine and cosine, based on buckling analysis results for the first buckling mode, and the real imperfections mapped from similar experimental testing. It was demonstrated that the choice of imperfection pattern is influential and should be selected to represent the actual imperfections to the extent possible.

\subsubsection{Axial Force}

Kemp (1986) suggests that the available rotation should be a function of the parameter $h / 2 h_{c p}$, where $h$ is the distance between the center of the flanges and $h_{c p}$ is the distance between the plastic neutral axis and the center of the compression flange. This parameter is said to capture the decrease in rotation capacity corresponding to axial compressive force. 


\subsubsection{Available Rotation Prediction Equations}

Galambos (1968) reports the following equation for available rotation capacity of beams with the lateral bracing distance equal to $\frac{\pi r_{y}}{K \sqrt{\varepsilon_{y}(1+0.56 h)}}$ under uniform moment, which was determined theoretically assuming lateral and local buckling occur simultaneously:

$$
\left[\frac{s-1}{0.7 h}\right]\left[\frac{\pi^{2}}{\varepsilon_{y}\left(\frac{K L}{r_{y}}\right)}-1\right]
$$

where $s$ is the ratio between the strain hardening strain and the yield strain, $h$ is the ratio between the strain hardening modulus and the elastic modulus, $\varepsilon_{\mathrm{y}}$ is the yield strain, $\mathrm{K}$ is the

effective length ratio, $\mathrm{L}$ is the unbraced length, and $\mathrm{r}_{\mathrm{y}}$ is the radius of gyration about the $\mathrm{y}$ (vertical) axis. This equation was shown by Galambos (1968) to be a satisfactory representation of the available rotation at $95 \%$ of $\mathrm{M}_{\mathrm{p}}$ in several experimental girders. It is noted that this equation is conservative for beams with shorter lateral bracing distances. Similarly, the following equation was proposed to predict the available rotation (in radians) of beams under moment gradient:

$$
2.84 \varepsilon_{y}(s-1) \frac{b t}{d w}\left(\frac{A_{w}}{A_{f}}\right)^{1 / 4}\left(1+\frac{V_{1}}{V_{2}}\right)
$$

where $V_{1}$ and $V_{2}$ represent the shear immediately to the left and right of the hinge locations (Galambos 1968). The accuracy of this equation was evaluated compared to experimental results where the test rotation was measured as the difference between the elastic rotation at $\mathrm{M}_{\mathrm{p}}$ and the rotation when unloading began since few tests were carried out to the point where the girder unloaded back down to $\mathrm{M}_{\mathrm{p}}$. The equation was shown to be conservative for 11 out of 13 experiments in which compact beams were used.

Kemp (1986) gives the following equation for predicting the rotation capacity at the maximum moment based on moment curvature relationships

$$
R_{m}=\frac{h}{2 h_{c p}} l\left(2 s-1+e \frac{l}{1-l}\right)
$$


where $h$ is the depth between the centers of the two flanges, $h_{\mathrm{cp}}$ is the distance between the plastic neutral axis and the center of the compression flange, $l$ is $\mathrm{L}_{\mathrm{p}} / \mathrm{L}$ as shown in Fig. 2-9, $\mathrm{s}$ is the ratio of the strain hardening strain to yield strain, and e is the ratio of elastic modulus to strain hardening modulus. $\mathrm{L}_{\mathrm{p}}$ is taken as the minimum $\mathrm{L}_{\mathrm{p}}$ from the limit states of flange local buckling, web local buckling and lateral torsional buckling as computed using Eqs. 2.22, 2.23, and 2.24, respectively. The theoretical and experimental values of $\mathrm{R}_{\mathrm{m}}$ are compared for 11 experimental girders, where the correlation between the two is relatively good considering the complexity of the problem. It should be pointed out that Eq. 2.27 is intended to predict the rotation capacity at the maximum moment. Thus, the available rotation capacity after the girder decreases to a given moment capacity will be greater than that predicted by this equation. A general guideline is that the available rotation at $\mathrm{M}_{\mathrm{p}}$ is equal to twice the rotation at the maximum moment. However the accuracy of this rule of thumb varies significantly for different beams, and may result in conservative or unconservative predictions.

Kato (1989) derives the available rotation of I-shaped steel beams by representing the Isection as an equivalent two flange model as shown in Fig. 2-13, where the plastic moment capacity and cross-sectional area are the same in both the original section and the equivalent section and a web thickness of $0.6 \mathrm{t}_{\mathrm{f}}$ is further assumed. Based on the moment-curvature relationship of this equivalent section, Eqn 2.28 is proposed to predict the available rotation capacity of I-shaped steel beams as a function of geometric and material characteristics,

$$
R=\frac{1}{s}\left[\frac{E}{E_{s t}} \frac{I}{I_{c}}(s-1)^{2}+\frac{h}{h_{c}} \frac{\varepsilon}{\varepsilon_{s t}}(s-1)\right]
$$

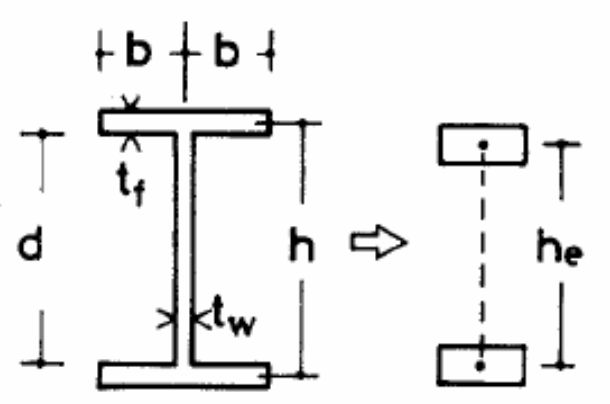

Figure 2-13. Equivalent Section used by Kato (1989) 
where $\mathrm{s}$ is the normalized critical stress $\sigma_{\mathrm{cr}} / \mathrm{F}_{\mathrm{y}}$ and $\sigma_{\mathrm{cr}}$ is the critical stress governed by local buckling.

To format the above equation as an expression of terms more frequently used in design, the following equation is proposed to compute the critical stress as a function of the flange and web slenderness

$$
\frac{1}{s}=0.6003+\frac{1.600}{\alpha_{f}}+\frac{0.1535}{\alpha_{w}}
$$

where $\alpha_{f}=E / F_{y f}\left(t_{f} / b\right)^{2}$ and $\alpha_{w}=E / F_{y w}\left(t_{w} / d\right)^{2}$. This equation is based on a linear regression of experimental data from 68 stub-column tests. By solving Eqn. 2.28 for 1/s, equating this with Eqn. 2.29, and incorporating a correction factor to account for the uniform stress distribution in the experimental stub-columns as opposed to the stress gradient that exists in beams, the following equation is obtained.

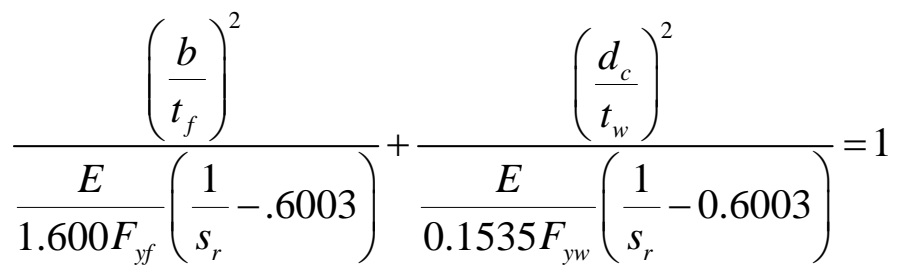

Then, by computing the value of $1 / s_{r}$ corresponding to different levels of required rotation, the permissible flange and web slenderness can be determined. For example, by assuming $\mathrm{E} / \mathrm{E}_{\mathrm{st}}=70, \varepsilon_{\mathrm{st}} / \varepsilon_{\mathrm{y}}=10$, and $\mathrm{F}_{\mathrm{yw}}=\mathrm{F}_{\mathrm{yf}}=\mathrm{F}_{\mathrm{y}}$, the following is obtained for a required rotation capacity of four,

$$
\frac{\left(\frac{b_{f}}{2 t_{f}}\right)^{2}}{\left(\frac{181}{\sqrt{F_{y}}}\right)^{2}}+\frac{\left(\frac{D}{t_{w}}\right)^{2}}{\left(\frac{1170}{\sqrt{F_{y}}}\right)}=1 .
$$

Similarly, Equation 2.32 is obtained for a required rotation capacity of two, 


$$
\frac{\left(\frac{b_{f}}{2 t_{f}}\right)^{2}}{\left(\frac{200}{\sqrt{F_{y}}}\right)^{2}}+\frac{\left(\frac{D}{t_{w}}\right)^{2}}{\left(\frac{1279}{\sqrt{F_{y}}}\right)^{2}}=1 .
$$

Thus, it is assumed in these equations that the required rotation capacity of a section is a function of the interaction between flange and web slenderness and the material properties. It has been assumed in the development of these equations that lateral torsional buckling of the girders is prevented.

Based on his findings that the rotation capacity of beams could best be represented as a function of the complementary energy of the steel and using the same procedure as in previous work (Kato 1989), Kato (1990) suggested the following equation to predict the available rotation capacity at the maximum moment

$$
R=Y R\left[\frac{C_{2}+C_{3}}{C_{1}}-2\left(\frac{1}{Y R}-1\right)\right] .
$$

where $C_{1}, C_{2}$, and $C_{3}$ refer to components of the complementary energy of the material, and YR is yield ratio. The constants $C_{1}, C_{2}$, and $C_{3}$ are expressed by the following equations:

$$
\begin{gathered}
C_{1}=\sigma_{y}{ }^{2} / 2 E=\sigma_{y} \varepsilon_{y} / 2, \\
C_{2}=\left(\frac{1}{Y R}-1\right) \sigma_{y} \varepsilon_{s t}, \text { and } \\
C_{3}=\frac{(1 / Y R-1)^{2} \sigma_{y}{ }^{2}}{2 E_{s t}} .
\end{gathered}
$$

Eq. 2.33 represents the rotation capacity assuming that local buckling is prevented such that the ultimate stress of the steel can be achieved. The possibility that the maximum stress $\sigma_{\text {cr }}$ may be less than $\sigma_{\mathrm{u}}$ is accounted for and the equations are simplified for design by utilizing regression analysis to obtain typical values of $\sigma_{\mathrm{cr}}$ resulting in the following equation for predicting the available rotation capacity

$$
R=\frac{R_{b}-1}{R_{b}}\left[\frac{E}{E_{s t}}\left(R_{b}-1\right)+2 \frac{\varepsilon_{p}}{\varepsilon_{y}}\right]
$$


where $\mathrm{R}_{\mathrm{b}}$ is $\sigma_{\mathrm{cr}} / \sigma_{\mathrm{y}}$ and $\sigma_{\mathrm{cr}}$ is determined using empirical equations as a function of flange slenderness, web slenderness, and steel type and $\varepsilon_{\mathrm{p}}=\varepsilon_{\mathrm{st}}-\varepsilon_{\mathrm{y}}$.

Kemp and Dekker (1991) expand on previous theoretical methods (Kemp 1985 and Kemp 1986) to develop the following equation for the prediction of the available rotation capacity of steel members classified as Class 1 or Class 2 sections according to Eurocode Specifications

$$
R_{a}=3\left(60 / \lambda_{e}\right)^{1.5} / 2 \alpha^{\prime}
$$

where

$\lambda_{e}=K_{f} K_{w}\left(L_{i} / i_{z c} \varepsilon\right), \quad K_{f}=\left(b_{f} / 2 t_{f} \varepsilon\right) / 20, \quad K_{w}=K_{w 2}=\alpha d_{w} / 33 t_{w} \varepsilon$ for $33<\alpha d_{w} / t_{w} \varepsilon \leq 40$

and $K_{w}=K_{w 1}=\left[460-\left(L_{i} / i_{z c} \varepsilon\right)\right] \sqrt{K_{w 2}} / 400$ for $\alpha d_{w} / t_{w} \varepsilon \leq 33, \alpha$ is the percentage of the depth of section in compression between the centers of the two flanges. This equation is based on conservative inelastic material properties of $\mathrm{E} / \mathrm{E}_{\text {st }}$ equal to 50 and $\varepsilon_{\mathrm{st}} / \varepsilon y$ equal to 10 .

Lääne and Lebet (2005) evaluated the available rotation capacity of 30 finite element models at a level of moment equal to $90 \%$ of $\mathrm{M}_{\mathrm{y}}$. The models included in this evaluation consisted of girders with the following attributes: flange slenderness of either the Eurocode Class 1 limit or 6; web slenderness values of 100, 125, 150, 175, or 200; Grade S355 and Grade 460 steel; and cross-sections proportioned such that the tension flange represented $25 \%$ of the total area of the girder, the compression flange area was 35 or $45 \%$ of the total area, and the web was 30 or $40 \%$ of the total area. The available rotation of each of these models is then expressed as a function of the modified reference slenderness for web buckling,

$$
\lambda_{p}{ }^{\prime}=\frac{2 D_{c p}}{t_{w}} \frac{1.05}{\sqrt{k}} \sqrt{\frac{F_{y}}{E}}
$$

where $\mathrm{k}$ is the plate buckling coefficient of the web. Based on this data, Lääne and Lebet proposed the Equation 2.40 to predict the available cross-section of I-girders in negative bending,

$$
\theta_{a v}=\frac{15.75}{\left(\lambda_{p}\right)^{2}}
$$


The relationship between Eq. 2.40 and experimental data for girders exceeding the Eurocode Class 1 compactness limit is shown below in Fig 2-14. For girders with a modified reference slenderness for web buckling less than the Class 1 compactness limit, the available rotation is assumed to be 63 mrads based on the previous results of Grubb and Carskaddan (1981) who found that compact sections demonstrated a minimum rotation capacity of 63 mrad. Figure 2-14 illustrates that this prediction equation is conservative in all cases and inappropriately conservative in several cases.

\subsection{Rotation Requirements}

In the U. S., the majority of research on plastic design and behavior has been focused on building-type applications. Consequently, general guidelines for rotation requirements were suggested for this purpose, as summarized in this section, which also serve as the basis for the AASHTO compactness limits. A more appropriate representation of AASHTO rotation requirements would be based on an analysis of rotation requirements for continuous span beams. A more accurate representation of rotation requirements for bridge members would result based on the analysis of continuous-span beams. Several researchers have investigated

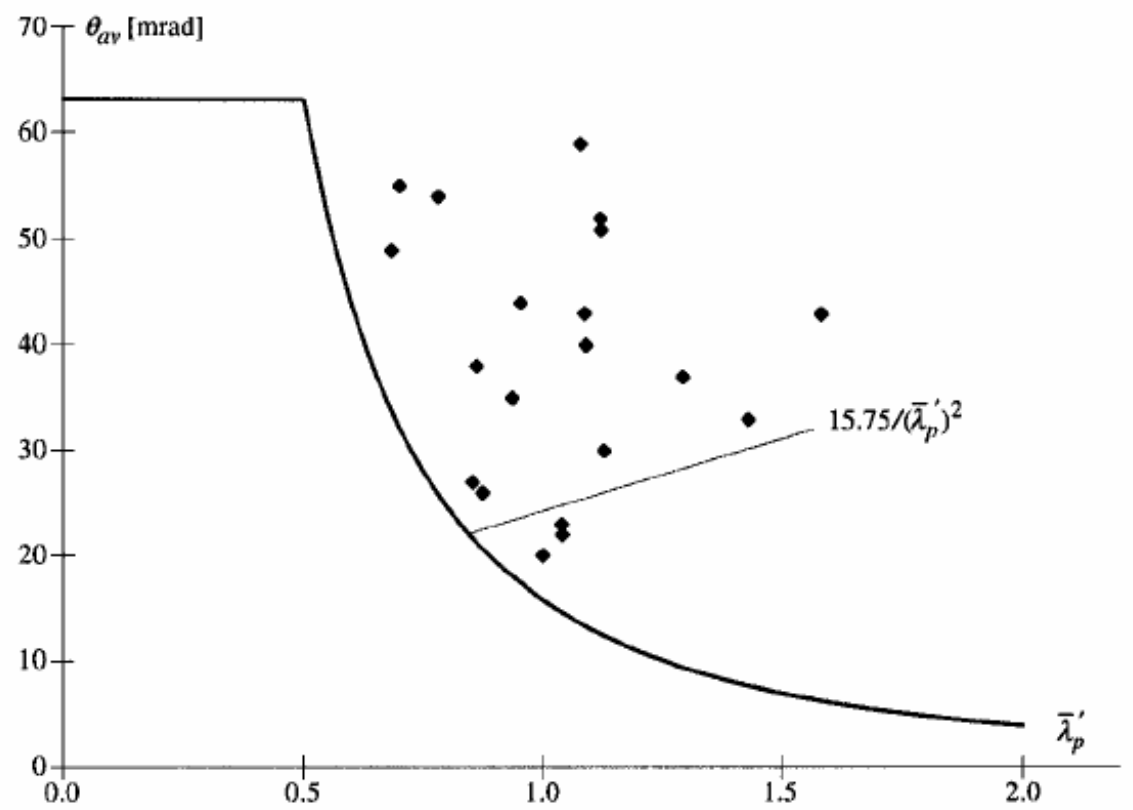

Figure 2-14. Available Rotation Equation Suggested by Lääne and Lebet (2005) Compared to Experimental Data 
this issue and one particularly important aspect of this research is investigations relating rotation requirements to intended degree of moment redistribution. These as well as other relevant findings and theoretical considerations from these studies are summarized below.

Early work in plastic design for building applications sought to determine the approximate degree of rotation required in order for plastic design to be valid. This requirement was typically expressed in the non-dimensional quantity of rotation capacity equal to $\theta_{2} / \theta_{1}-1$, where $\theta_{2}$ and $\theta_{1}$ are as shown in Fig. 2-15. Through the years, various rotation capacity requirements have been suggested. For example, in 1963, Prasad and Galambos suggested a rotation capacity of 10 as a rough estimate of required rotation and utilized this rotation requirement in evaluating lateral bracing limits for plastically designed beams.

In 1974, the AISC ASD Specifications were revised and local and lateral buckling limits were based on rotation capacity requirements of 3. Yura et al. (1978) suggested that this level of rotation was sufficient for most civil engineering applications and this conclusion seems to be based on research by Galambos (1968) and Neal (1963) suggesting that a rotation capacity of 2 was generally conservative, although higher rotation requirements may be necessary for some cases. Specifically, in a summary of rotation requirements for some general structures, Galambos (1968) revealed that for practical structures the required rotation capacity is less than 2 , although rotation requirements may be higher for single story

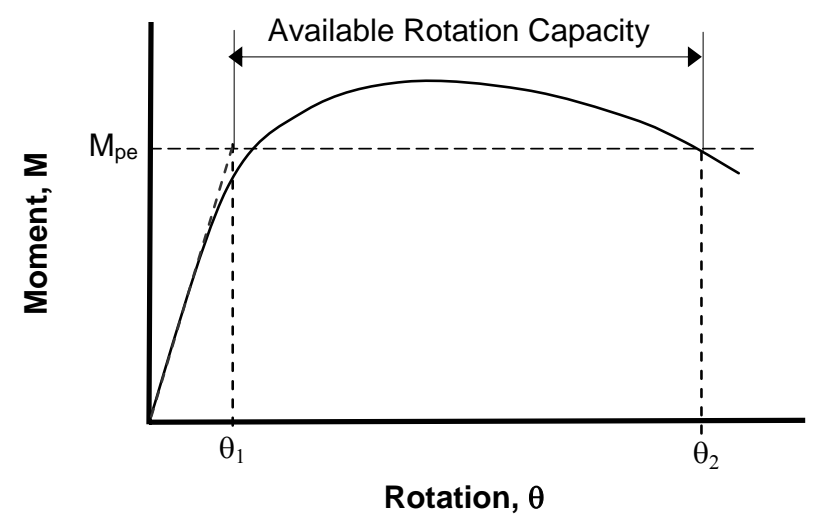

Figure 2-15. Definition of Rotation Capacity 
frames with very steep gables and in areas of high moment gradient. Similar findings have been reported by Neal (1963) where it was shown that rotation capacities of 2 are adequate for continuous beams with concentrated loads.

The rotation requirement of 3 was later adopted as the criteria for determining compact section requirements in the AISC LRFD Specifications (Yura et al. 1978). Similarly, the compact section criteria adopted in the current AASHTO LFRD elastic provisions are also based on a rotation requirement of 3 .

Kemp (1990) calculated required rotations by superimposing the rotations due to the applied loads and end (redistribution) moment assuming that members are simply-supported, i.e., each span of continuous-span beams and beams of portal frames are analyzed as simply supported. However, this approach results in an upper bound of the required rotation because it assumes that a hinge is present in the structure before load has been applied and thus neglects the increased stiffness of the structure due to continuity prior to yielding at the hinge locations. Results of these efforts were presented in the form of graphs giving required rotation capacity for typical three and five span bridges as a function of the ratio between positive and negative bending moment resistance and ratio of distance to the inflection point to the span length. Previous research by Kemp (1987) also suggested that the distance to the inflection point was of importance as larger rotations were required for beams where the distance to the inflection point was reduced due to low ratios between the stiffness of the negative bending section to the stiffness of the positive bending section.

Kemp and Dekker (1991) present the required rotation of symmetrical two-span continuous beams as shown in Fig. 2-16. As shown in the figure, the required rotation is plotted as a function of both percentage of moment redistributed and the ratio between negative and positive bending strength of the cross-section. It is assumed in this work that the beam is of a uniform cross-section with uniform flexural-rigidity, but other analysis details and assumptions are not provided. From the data presented in Fig. 2-16, it is suggested that for an intended degree of redistribution less than $20 \%$, a rotation capacity less than 1.0 is 


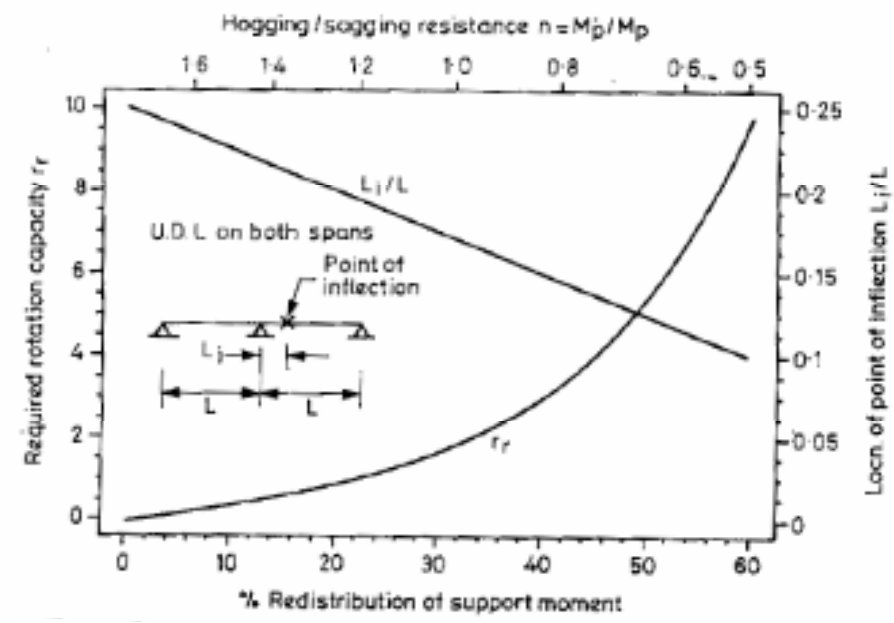

Figure 2-16. Rotation Requirements - Kemp and Dekker (1991)

required and for moment redistribution of less than 30\%, rotation capacities less than 2.0 are required.

One of the most comprehensive studies on rotation requirements to date is presented by $\mathrm{Li}$, Choo, and Nethercot (1995). The primary purpose of this research was to determine the rotations necessary to redistribute moment from pier sections to the midspan cross-section, where it was assumed that the required rotation occurs at connections rather than in members. In this work, the required rotation is quantified as the rotation necessary to achieve moments in excess of $\mathrm{M}_{\mathrm{y}}$ yet below $\mathrm{M}_{\mathrm{p}}$ in the mid-span cross-section.

Li et al. (1995) conducted their analysis of required rotations by decomposing the rotation into elastic and plastic components. Rotation requirements were calculated by first representing a continuous beam as equivalent simple span beams. Thus the procedure was similar to that used by Kemp (1990) in that the continuity at the pier prior to hinge formation is neglected. The elastic component of rotations is that resulting in the simply-supported beam with end moments equal to the elastic moment at each end of the span in the continuous beam and applied loads of either a uniformly distributed load, central concentrated load, or two third-point loads. This elastic component of rotation is calculated as a function of: ratios between end moment(s) to the maximum span moment, the ratio of 
the relative stiffness along the girder (cracked vs. uncracked stiffness as only beams of uniform cross-section are considered), the maximum span moment, span length, and stiffness of the positive moment region. The plastic rotation is considered to be that caused by plastic deformation at midspan. Thus, by this definition of plastic rotation, if the positive bending moment is less than $\mathrm{M}_{\mathrm{y}}$, no plastic rotation will occur regardless of the moment conditions at the supports. The required plastic rotation is determined by the area of the curvature diagram, where the curvature is determined using empirical formulas developed by the authors.

Later work by the same authors (Nethercot, Li, and Choo 1995) focused on identifying the most influential parameters affecting required rotations. Through regression analyses, empirical equations were developed relating required rotations to percentage of moment redistribution. Empirical equations are developed for cases of equal end moments and one end moment equaling zero. These equations are a function of six variables: span length, moment of inertia, modulus of elasticity, yield moment of the cross-section, plastic moment of the cross section, and the nominal moment capacity of the beam in positive bending. The empirical equations give results that are generally with $15 \%$ of the theoretical results for 1360 designs.

Using these empirical equations, plots are constructed of required rotation versus the ratio between negative and positive moments $\left(\mathrm{M}^{\prime} / \mathrm{M}_{\mathrm{d}}\right)$ and required rotation versus degree of moment redistribution. This information allowed for the determination of the maximum degree of moment redistribution acceptable, which revealed that the maximum degree of redistribution is typically $30 \%$ or more for semi-rigid composite frames. It is also concluded that for a fixed amount of available rotation, span length is the most significant factor affecting allowable degree of redistribution. The required rotations for several representative beams to achieve their maximum moment capacity at midspan are calculated. This shows that the required rotations are typically in the range of 36-50 mrad to achieve this purpose.

Earlier research by Hope-Gill (1979) also investigated the topic of the maximum permissible amount of redistribution. Details of the analysis methods are not presented, but results of 
computer analyses indicate $40 \%$ may be considered a maximum percentage of permissible redistribution for compact sections for the case where moment is redistributed from the pier to positive bending regions. For slender sections, a maximum allowable moment capacity equal to $75 \% \mathrm{M}_{\mathrm{p}}$ is used in the analysis, which results in a maximum degree of redistribution of 10 to $20 \%$.

One important factor that must be considered in computing required rotations is the appropriate value to use for the moment of inertia $\left(\mathrm{I}_{\mathrm{x}}\right)$ of the pier section. The previously mentioned studies have used various $I_{x}$ quantities to represent the effectiveness of the concrete deck in negative bending. For example, Nethercot et al (1995) assumed the moment of inertia of negative bending sections to be equal to the average of the cracked and uncracked moments of inertia. However, it is noted that both elastic and plastic rotations are computed in this work, so both the cracked and uncracked sections are applicable. In work by Hope-Gill (1979) two alternative $I_{x}$ values are implemented: uncracked and cracked over $15 \%$ of the span length. It was concluded that assuming the beam to be cracked a predetermined amount does not accurately predict actual behavior. Kemp (1990) includes the effect of concrete cracking in negative moment regions as an additional component of available rotation capacity equal to I/I' - 1, where I and I' are the uncracked and cracked section moments of inertia, respectively.

Based on the above referenced research on rotation requirements, the following conclusions are proposed. Approximate suggestions were developed for rotation requirements of building members, and it was determined that these members, which are typically compact, had more available rotation than required. Thus, American specifications have not previously investigated rotation requirements in detail, and consequently, restrict the use of moment redistribution procedures to relatively compact members. The findings of $\mathrm{Li}$ et al. (1995), Nethercot et al. (1995), and Kemp (1990) provide information pertinent to addressing the issue of rotation requirements for continuous-span beams. General analysis methods and considerations of appropriate representation of the cracked pier section are of particular interest. However, these works compute rotation requirements assuming a hinge exists at each pier before load is placed on the girder, leading to overly conservative results. 


\section{Chapter 3: EXPERIMENTAL TESTING}

\subsection{General Information}

Twelve large-scale steel I-girders were tested in three-point bending for the purpose of obtaining moment-rotation characteristics of girders having slender geometries, which was necessary in order to extend the applicability of moment redistribution specifications to more slender girders. As will be detailed subsequently, these experimental girders represent the only known series of experiments to have such geometries. The girders were designed to be representative of the negative bending sections of continuous-span girders where the inflection points in the continuous span girder were represented as simple supports in the test specimen and the pier reaction is represented by an applied load in the test specimen as shown in Fig. 3-1. This loading configuration is further suited to testing of negative bending sections as the moment gradient produced is similar to the negative moment envelope that exists in the negative moment regions of continuous span girders.

This chapter discusses the attributes, testing procedures, and experimental results of these girders. Information that is consistent for all specimens is discussed in Section 3.1, General Information. The parametric values considered in the experimental testing are given in Section 3.2, Parametric Values. Section 3.3, Individual Girder Properties, discusses the

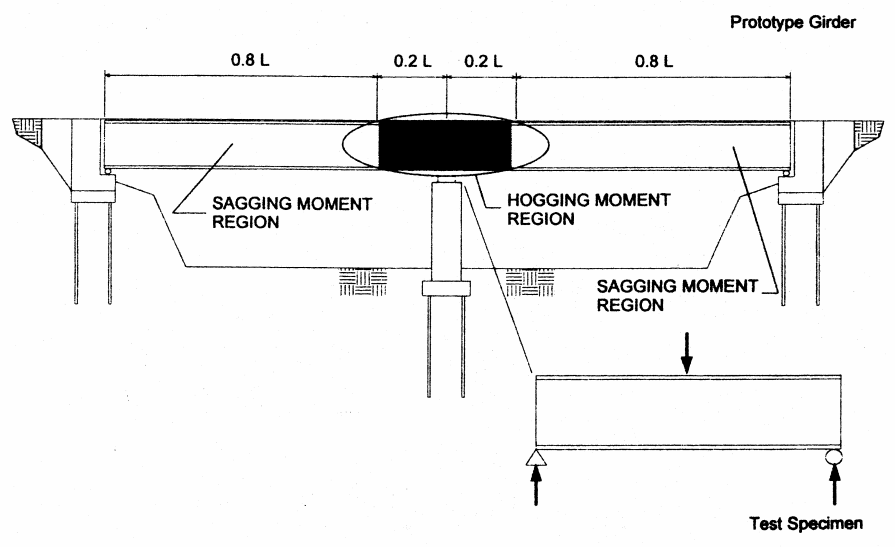

Figure 3-1. Typical Continuous Span Girder and Test Specimen 
parametric values assigned to and general properties of each girder. The testing procedure and data collection details are presented in Section 3.4, Testing Procedure. Results of these tests are discussed in Section 3.5, where particular emphasis is placed on ultimate capacities, moment-rotation characteristics, and failure modes.

The general experimental setup may be viewed in the photographs presented in Figs. 3-2 and $3-3$, where it may be observed that the girders included in this work were of relatively large scale. Specifically, the bearing length of the girders varied between 20 and $39 \mathrm{ft}$; the web depth ranged between 25 and 43 in.; and the flange widths varied between 7 and 17 in. The girder webs and flanges were fabricated from plate thicknesses of $5 / 16,3 / 8,1 / 2,5 / 8,3 / 4$, and $7 / 8$ in.

All girders were symmetric about the centerline of the girder. Lateral bracing was also symmetric about the centerline of the girder and was provided at the midspan and bearing locations of all girders in addition to two or four other locations along the span, as shown in

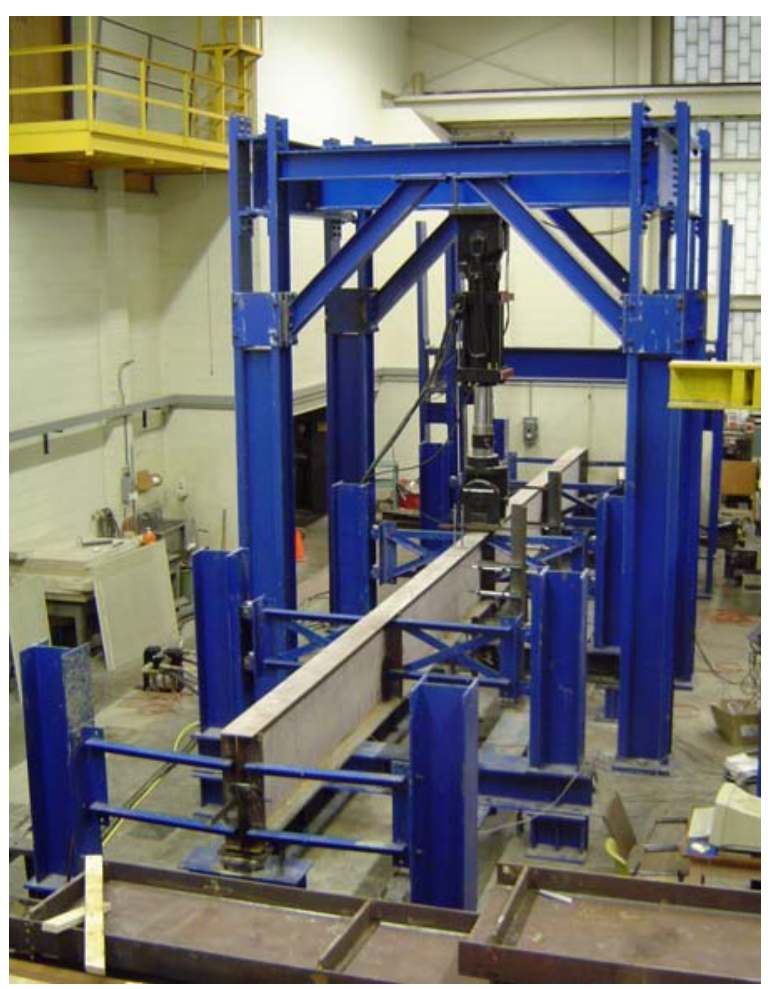

Figure 3-2. Photograph of Experimental Setup, Viewed from Above 


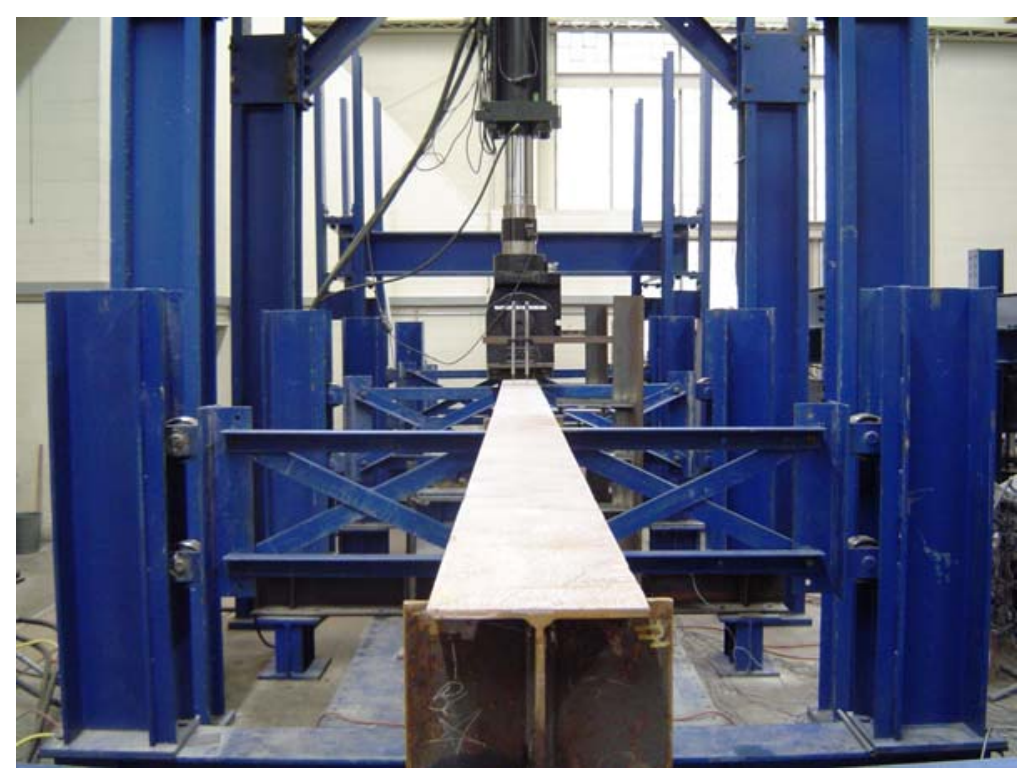

Figure 3-3. Photograph of Experimental Setup, Viewed from End of Girder

Fig. 3-4, creating a total of either 4 ( $\mathrm{L}_{\mathrm{b} 3}$ equals zero in Fig. 3-4) or 6 lateral bracing segments. A minimum of 4 lateral bracing segments was necessary to provide torsional restraint to the critical (center) lateral bracing panels, while six lateral bracing panels were used in some cases in order to increase the magnitude of moment that could be generated by providing a longer girder length.

A constant lateral bracing distance was used when possible; however, there were two situations where a constant lateral bracing distance was not provided. The first was the case where using a constant lateral bracing distance would result in a total girder length greater than $39 \mathrm{ft}$, which was the maximum bearing length of girder possible given the length of steel

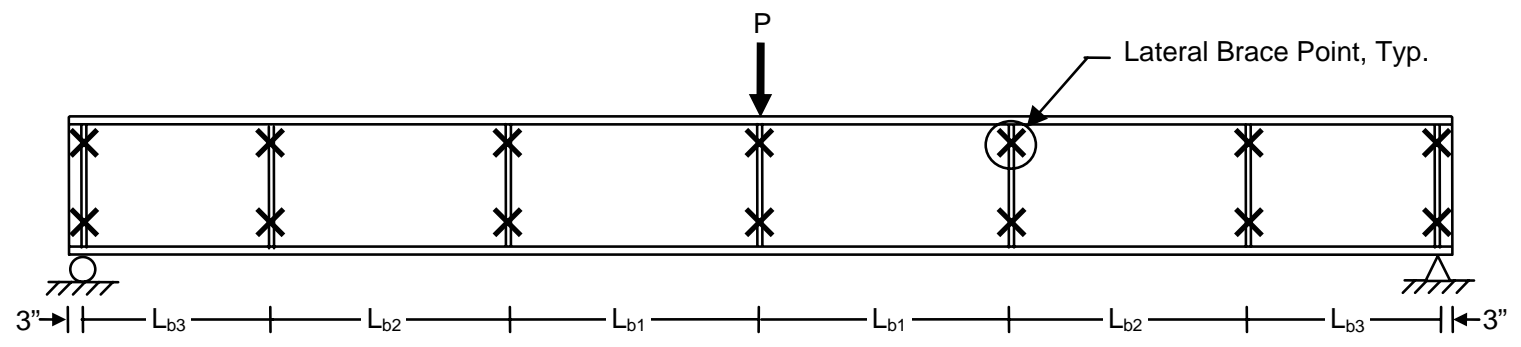

Figure 3-4. General Test Girder Configuration, Elevation View 
plate available ( $40 \mathrm{ft}$ ) and the necessity to eliminate full penetration groove welds due to the associated increased fabrication cost. Thus, in this situation the target bracing distance was used at the lateral bracing segments closest to midspan ( $\mathrm{L}_{b 1}$ and, if possible, $\mathrm{L}_{\mathrm{b} 2}$ ) and the bracing distances in the end segments of the girder $\left(\mathrm{L}_{\mathrm{b} 3}\right.$ and, if necessary, $\mathrm{L}_{\mathrm{b} 2}$ ) were reduced. The second situation in which a constant lateral bracing distance was not provided is if 6 panels with a length equal to the target lateral bracing distance result in a total girder length that was not sufficient to produce a maximum applied moment in the girder $10 \%$ higher than the predicted capacity of the girder when the full capacity of the actuator (330 kips) was applied. In this scenario, the length of the lateral bracing segments closest to the end of the girder were increased in order to be able to generate a larger amount of moment in the girder. When this approach was used, the capacity of each lateral bracing segment was evaluated to assure that the segments closest to the midspan of the girder remain the critical segment where failure will occur.

Lateral bracing for the girders was provided by a system of lateral frames having the configuration shown in Fig. 3-5. This framing system consisted of steel cross-frames comprised of L3x3x3/8 standard shapes, which were bolted to stiffeners welded to the girder at each lateral bracing location. The opposite end of the cross-frames was bolted to the web of steel WT6x29s. Each WT section had two plates, which support a 1.5 in. steel rod and high-capacity forged steel wheels, welded onto its flange near each end of the WT, as shown in Fig. 3-5. Lateral movement of this system was restrained by W12x72s with C10x20s welded onto the inside face of the W sections. These vertical W12x72s were in turn bolted to a horizontal W12x72, which was ultimately bolted to the strong floor. This same lateral bracing system was used at the bearing locations of the girder, except that (1) the wheels were omitted and the WT sections were bolted directly onto the vertical W12x72 members, since there was no vertical displacement at these locations; and (2) the diagonal members of the cross-frames were omitted as it was anticipated that the lateral forces in the ends of the member would be significantly less than along the span of the girder.

This lateral framing system prevented lateral movement of the girder while allowing for the vertical deflection of the beam through the presence of the steel wheels. Furthermore, 


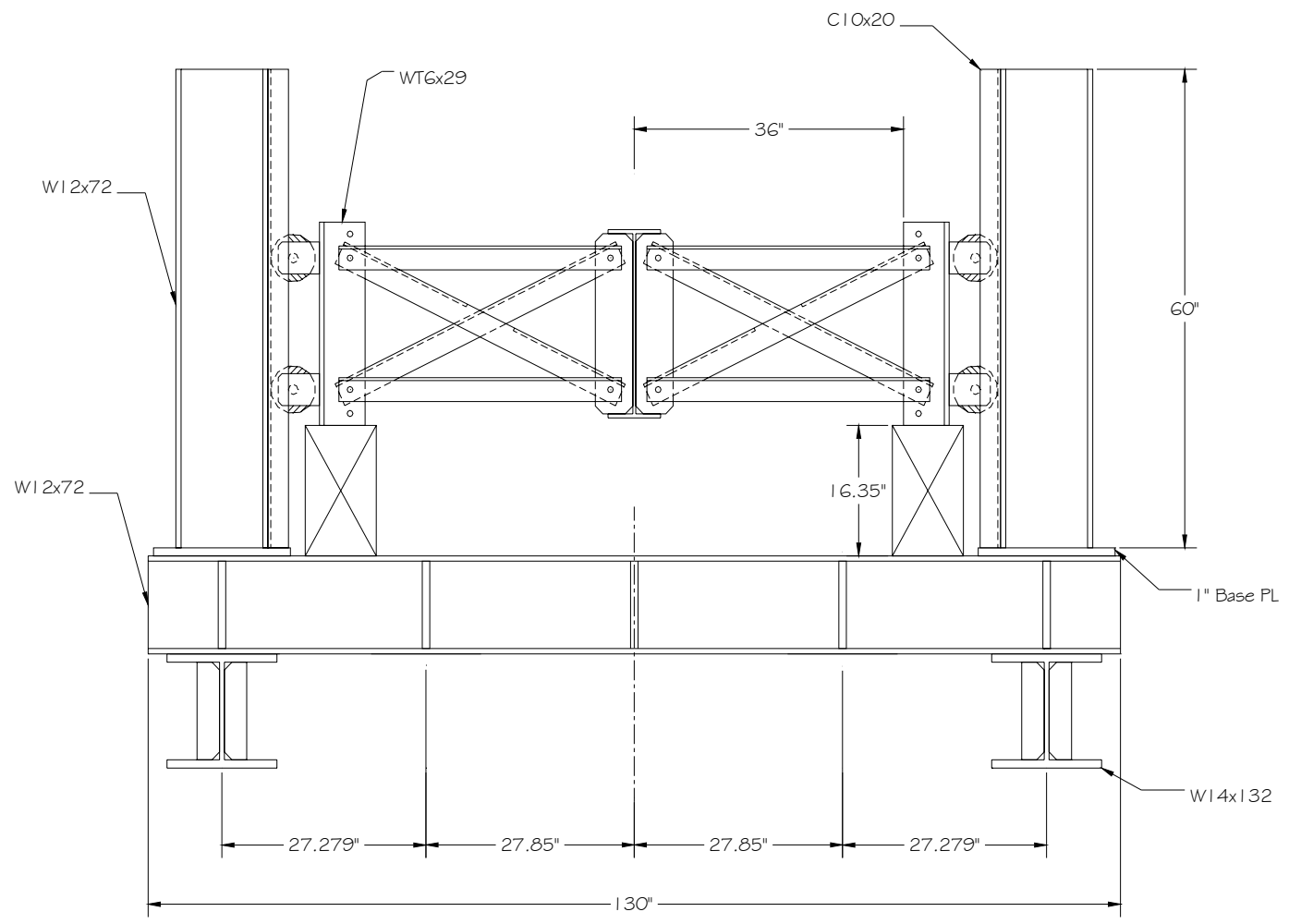

Figure 3-5. Lateral Bracing System Used in Experimental Testing

longitudinal movement of the girders was unrestrained up to the point where the plates supporting the steel wheels come into contact with the flanges of the channel due to significant rotation of the girder about the vertical axis. This situation occurred in the latter stages of several of the tests when significant lateral distortions of the girder were present and is illustrated in Fig. 3-6. However, as will be discussed subsequently, it is possible to calculate the amount of force (if any) being resisted by the lateral frames so that this influence can be quantified and it is demonstrated that there is no influence on the test data from this effect.

All girder stiffeners were designed to resist an applied load corresponding to a moment of $110 \%$ of $\mathrm{M}_{\mathrm{p}}$ (the plastic moment capacity of the girder) based on stiffener capacities predicted by Section 6.10 .8 of the $2^{\text {nd }}$ Edition of the AASHTO LRFD Bridge Design Specifications (AASHTO 1998). There were three different types of stiffeners on each girder: end bearing stiffeners, center bearing stiffeners, and double-sided transverse 


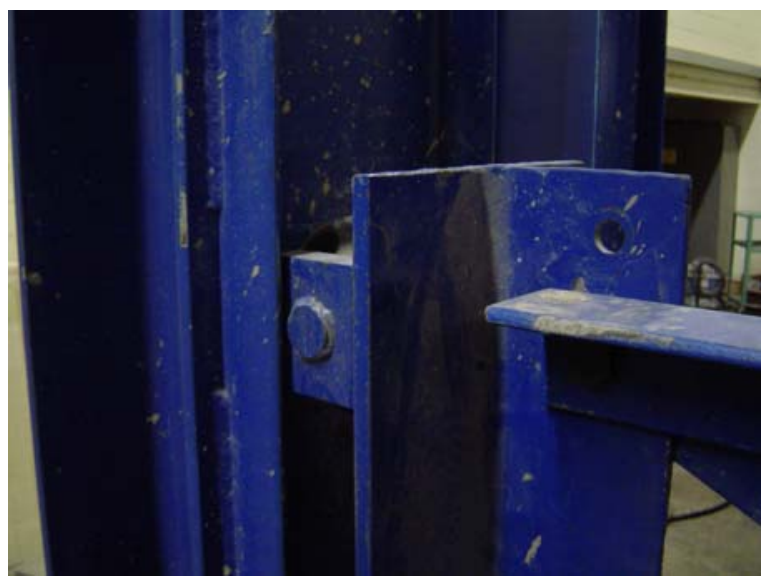

(a) Plates Supporting Wheels in Contact With Channel Flanges

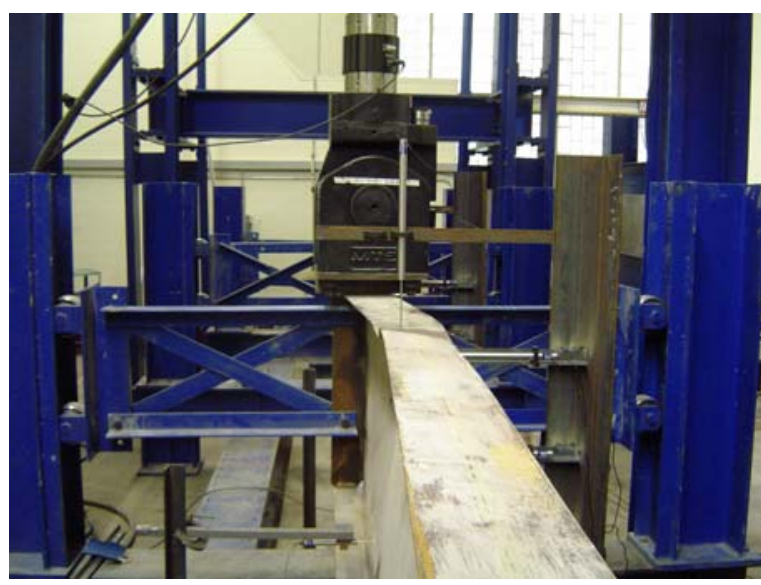

(b) Corresponding Girder Deformation Causing Cross-frames to Rotate About Vertical Axis

Figure 3-6. Lateral Frames Providing Longitudinal Restraint in Latter Stages of Experiment

stiffeners. The primary purpose of the double-sided transverse stiffeners was to simply provide a connection to the lateral bracing frames and as such one set of these stiffeners was located at each lateral bracing location. All transverse stiffeners were 5 in. wide by $1 / 2$ in. thick and were tight-fit against both the top and bottom flanges. Both the end and center bearing stiffeners were milled-to-bear against the top and bottom flanges. These stiffeners were also 5 in. wide in all cases; however increased thicknesses of 0.875 in., 1.125 , or 1.25 in. were used in some cases in order to resist the higher concentrated loads anticipated at these locations. Table 3-1 lists the specific stiffener thicknesses for each girder.

One-quarter inch fillet welds were used to weld all stiffeners to both the webs and flanges of the girders. Additionally, the same size fillet welds were used at the web-flange junctions in all girders.

\subsection{Parametric Values}

The primary objective of these girders was to investigate the behavior of girders designed at several key slenderness limits, particularly slenderness limits adopted in the $3^{\text {rd }}$ edition of the AASHTO LRFD Specifications (AASHTO 2004). Specifically, two alternative web 
Table 3-1. Stiffener Thicknesses used in Experimental Girders (in.)

\begin{tabular}{|cccc|}
\hline Specimen & $\begin{array}{c}\text { Transverse } \\
\text { Stiffener }\end{array}$ & $\begin{array}{c}\text { End Bearing } \\
\text { Stiffener }\end{array}$ & $\begin{array}{c}\text { Center } \\
\text { Bearing } \\
\text { Stiffener }\end{array}$ \\
\hline \hline 1MG & 0.500 & 0.500 & 1.125 \\
2MG & 0.500 & 0.875 & 1.125 \\
3MG & 0.500 & 0.500 & 0.875 \\
4MG & 0.500 & 0.500 & 0.875 \\
5MG & 0.500 & 0.875 & 1.250 \\
6MG & 0.500 & 0.500 & 0.500 \\
$7 M G$ & 0.500 & 0.500 & 0.875 \\
8MG & 0.500 & 0.500 & 0.875 \\
9MG & 0.500 & 0.500 & 0.500 \\
$10 M G$ & 0.500 & 0.500 & 0.500 \\
$11 M G$ & 0.500 & 0.500 & 0.875 \\
$12 M G$ & 0.500 & 0.500 & 0.875 \\
\hline
\end{tabular}

slenderness limits, two alternative flange slenderness limits, and three lateral bracing limits were evaluated as discussed below. Variations in cross-section aspect ratio and material configuration were also considered as described below based on the results of the literature review suggesting that these are the two additional parameters most influential to available rotations. Additionally, consideration is also given to the percentage of the web depth in compression in the plastic range, $\mathrm{D}_{\mathrm{cp}} / \mathrm{D}$.

\subsubsection{Web Slenderness}

The web slenderness is quantified in this work as the ratio of twice the web depth in compression to the web thickness. The two web slenderness ratios considered were the compact and noncompact web limits from AASHTO (2004). The web is classified as compact (meaning that the maximum moment capacity of the girder may equal $\mathrm{M}_{\mathrm{p}}$ provided other compactness criteria are also satisfied) if the following equation is satisfied,

$$
\frac{2 D_{c p}}{t_{w}} \leq \lambda_{p w}=\frac{\sqrt{E / F_{y c}}}{\left(0.54 M_{p} / R_{h} M_{y}-0.1\right)^{2}}
$$

where the web depth in compression $\left(D_{\mathrm{cp}}\right)$ is determined based on a fully yielded crosssection. Alternatively, the web is considered noncompact (and the maximum moment capacity of the girder is equal to $\mathrm{M}_{\mathrm{y}}$ ) if Eqn. 3.1 is violated, but Eqn. 3.2 is satisfied 


$$
2 D_{c} / t_{w} \leq 5.7 \sqrt{E / F_{y c}}
$$

where the web depth in compression $\left(D_{c}\right)$ is based on elastic section properties.

\subsubsection{Compression Flange Slenderness}

The flange slenderness ratio is defined as the ratio between half the flange width to the flange thickness, $\mathrm{b}_{\mathrm{fc}} / 2 \mathrm{t}_{\mathrm{fc}}$. The two flange slenderness ratios considered were: (1) the compact flange slenderness ratio equal to $0.382 \sqrt{E / F_{y c}}$, where AASHTO (2004) specifies girders satisfying this flange slenderness equation may have a maximum moment capacity of $\mathrm{M}_{\mathrm{p}}$ and (2) the AASHTO (2004) maximum allowable flange slenderness ratio, which is equal to 12 and is regarded as a practical upper limit to ensure the flange will not distort excessively when welded to the web.

\subsubsection{Lateral Bracing}

Three alternative lateral bracing distances were targeted in this research; these were the compact limit, the noncompact limit, and the midpoint between these two limits. The compact limit, $\mathrm{L}_{\mathrm{p}}$, is the limiting unbraced length to achieve a maximum moment capacity of $\mathrm{M}_{\mathrm{p}}$, which is equal to $r_{t} \sqrt{E / F_{y c}}$. The noncompact limit, $\mathrm{L}_{\mathrm{r}}$, is considered the limiting unbraced length to achieve the onset of yielding in the flange under uniform bending and is equal to $\pi r_{t} \sqrt{E / F_{y c}}$. The third lateral bracing limit investigated is an intermediate bracing distance, which is equal to the average between the compact and noncompact limits, or $2.07 r_{t} \sqrt{E / F_{y c}}$.

An additional consideration with respect to lateral bracing distances is the moment gradient effect. The moment capacity prediction equations given in the AASHTO (2004) Specifications are based on beams in uniform bending. Therefore, when moment gradient conditions exist, as in these experimental girders, the computed moment capacities are increased by the "moment gradient modifier", which is represented by the variable $\mathrm{C}_{\mathrm{b}}$; however, the maximum moment capacity is limited to a maximum of that which would result from the use of compact brace spacing. This influence was also considered in the design of 
the experimental girders with the goal of having several girders with moment capacities less than the maximum allowable for lateral torsional buckling.

\subsubsection{Cross-Section Aspect Ratio}

The cross-sectional aspect ratio is defined as the ratio between the web depth and compression flange width, $\mathrm{D} / \mathrm{b}_{\mathrm{fc}}$. This parameter was included in the experimental program based on preliminary analyses suggesting that higher $\mathrm{D} / \mathrm{b}_{\mathrm{fc}}$ ratios result in lower moment capacities. It is thought that this behavior may be attributed to the decreased restraint provided to the flange by the web, and vice versa, that occurs as the $\mathrm{D} / \mathrm{b}_{\mathrm{fc}}$ ratio increases. The majority of the experimental girders had a $\mathrm{D} / \mathrm{b}_{\mathrm{fc}}$ ratio equal to 4 , however one girder is proportioned to have a $\mathrm{D} / \mathrm{b}_{\mathrm{fc}}$ value equal to 3 and one girder with a $\mathrm{D} / \mathrm{b}_{\mathrm{fc}}$ ratio of 5 is included to investigate the influence of this parameter. These $\mathrm{D} / \mathrm{b}_{\mathrm{fc}}$ values are representative of typical bridge girder designs, with most efficient designs typically having $\mathrm{D} / \mathrm{b}_{\mathrm{fc}}$ values in the range between three and four.

\subsubsection{Material Specifications}

Both Grade 50 and HPS 70W steel were used to fabricate the girders tested in this research and these alternative grades of steel were incorporated for two primary reasons. First, as discussed in Chapter 2, previous studies have suggested that there is decreased rotation capacity available for girders fabricated from higher yield strengths. Second, because HPS $70 \mathrm{~W}$ is a relatively new material, it was of interest to include girders fabricated from this steel. Thus, the experimental program included homogeneous girders of both grades and hybrid girders comprised of grade 70 flanges and grade 50 webs.

\subsubsection{Percentage of Web Depth in Compression}

Because the percentage of the web in compression can influence girder behavior, this parameter was also considered. Since higher $\mathrm{D}_{\mathrm{cp}} / \mathrm{D}$ ratios typically result in reduced ductility, a constant value of $\mathrm{D}_{\mathrm{cp}} / \mathrm{D}$ equal to 0.65 was targeted for all girders. This value is a relatively high value for the negative bending region of composite steel-concrete bridge girders. 


\subsubsection{Comparison of Parametric Values to Previous Experimental Girders}

Considering the parametric values discussed above, it is demonstrated that the focus of this work is on girders with relatively slender geometries. These girders are unique in this respect as few of the girders tested in previous studies represent geometries typical of the most slender bridge girders. This is shown by comparison of the parametric values discussed above to those of the girders contained in Appendix B, which were introduced in Chapter 2.

A review of the girders presented in Appendix B shows that the majority of these girders are doubly-symmetric girders. In other words, the $\mathrm{D}_{\mathrm{cp}} / \mathrm{D}$ ratio of these girders is equal to 0.5 and thus higher ductility is expected from these girders compared to girders with higher $\mathrm{D}_{\mathrm{cp}} / \mathrm{D}$ ratios, which frequently occur in practice. The only singly-symmetric girders shown in Appendix B are those tested by Grubb and Carskaddan (1979 and 1981), Carskaddan (1980), Schilling (1985), Climenhaga and Johnson (1972), and Tansil (1991). Of these tests, those by Carskaddan and Tansil are composite beam tests and insufficient information exists to determine the slenderness properties of these girders. Similarly, the geometry of the girders tested by Climenhaga and Johnson is not available.

The seven girders tested by Grubb and Carskaddan all have compact flanges, compact lateral bracing distances, and $\mathrm{D} / \mathrm{b}_{\mathrm{fc}}$ ratios less than three. Four of these girders also have compact webs. Thus, determining the behavior of slender girders based on these results is not possible.

The two girders tested by Schilling that are mono-symmetric have non-compact webs and non-compact lateral bracing distance. Thus, only these two girders of the sixty-eight girders contained in Appendix B are relevant to the current focus on slender girder behavior. This discussion serves to emphasize the need for and importance of the experimental testing conducted herein.

\subsection{Individual Girder Properties}

The test girders were designed to target as many combinations of the above parametric values as possible while varying a minimum number of parameters in each girder in order to 
ascertain the influence of individual variables. The target values of web slenderness, flange slenderness, $\mathrm{D} / \mathrm{b}_{\mathrm{fc}}$ ratio, lateral bracing distance, nominal yield strength, and $\mathrm{D}_{\mathrm{cp}} / \mathrm{D}$ ratio assigned to each girder are listed in Table 3-2. The specific purpose of each girder was as follows.

- Specimen $1 \mathrm{MG}$ was designed to be a baseline girder. It was a compact $50 \mathrm{ksi}$ girder with a $\mathrm{D} / \mathrm{b}_{\mathrm{fc}}$ ratio of 3 .

- Specimen 2MG was a baseline, compact, Grade 50 girder with a $\mathrm{D} / \mathrm{b}_{\mathrm{fc}}$ ratio of 4 .

- Specimen 3MG was a variation of 2MG with a noncompact web.

- Specimen $4 \mathrm{MG}$ was a variation of 3MG with intermediate bracing distance.

- Specimen $5 \mathrm{MG}$ was a variation of $4 \mathrm{MG}$ with a $\mathrm{D} / \mathrm{b}_{\mathrm{fc}}$ ratio of 5 .

- Specimen $6 \mathrm{MG}$ was a Grade 50 girder with a $\mathrm{D} / \mathrm{b}_{\mathrm{fc}}$ ratio of 4 and maximum slenderness ratios for web, flange, and lateral slenderness. This was also a variation of Specimen 4MG with increased flange and lateral slenderness.

- Specimen 7MG was a variation of 4MG with a hybrid material configuration.

- Specimen 8MG was a variation of 7MG with a slender flange.

- Specimen 9MG was a variation of 7MG with noncompact bracing distance.

- Specimen 10MG was a variation of 8MG with noncompact bracing distance, a variation of 9MG with a slender flange, and a variation of 6MG with a hybrid material configuration.

Table 3-2. Parametric Values of Experimental Girders

\begin{tabular}{|ccccccc|}
\hline Specimen & $\begin{array}{c}\text { Web } \\
\text { Slenderness }\end{array}$ & $\begin{array}{c}\text { Flange } \\
\text { Slenderness }\end{array}$ & D/b $_{\mathrm{fc}}$ & Bracing Distance & $\mathbf{F}_{\mathrm{y}}$ & $\mathbf{D}_{\mathrm{cp}} / \mathbf{D}$ \\
\hline \hline $1 \mathrm{MG}$ & $\lambda \mathrm{pw}$ & $0.382(\mathrm{E} / \mathrm{Fy})^{1 / 2}$ & 3 & $\mathrm{Lb}=\mathrm{Lp}$ & 50 & 0.65 \\
$2 \mathrm{MG}$ & $\lambda \mathrm{pw}$ & $0.382(\mathrm{E} / \mathrm{Fy})^{1 / 2}$ & 4 & $\mathrm{Lb}=\mathrm{Lp}$ & 50 & 0.65 \\
$3 \mathrm{MG}$ & $5.7(\mathrm{E} / \mathrm{Fy})^{1 / 2}$ & $0.382(\mathrm{E} / \mathrm{Fy})^{1 / 2}$ & 4 & $\mathrm{Lb}=\mathrm{Lp}$ & 50 & 0.65 \\
$4 \mathrm{MG}$ & $5.7(\mathrm{E} / \mathrm{Fy})^{1 / 2}$ & $0.382(\mathrm{E} / \mathrm{Fy})^{1 / 2}$ & 4 & $\mathrm{Lb}=\mathrm{Lp}+0.5(\mathrm{Lr}-\mathrm{Lp})$ & 50 & 0.65 \\
$5 \mathrm{MG}$ & $5.7(\mathrm{E} / \mathrm{Fy})^{1 / 2}$ & $0.382(\mathrm{E} / \mathrm{Fy})^{1 / 2}$ & 5 & $\mathrm{Lb}=\mathrm{Lp}+0.5(\mathrm{Lr}-\mathrm{Lp})$ & 50 & 0.65 \\
$6 \mathrm{MG}$ & $5.7(\mathrm{E} / \mathrm{Fy})^{1 / 2}$ & 12 & 4 & $\mathrm{Lb}=\mathrm{Lr}$ & 50 & 0.65 \\
$7 \mathrm{MG}$ & $5.7(\mathrm{E} / \mathrm{Fy})^{1 / 2}$ & $0.382(\mathrm{E} / \mathrm{Fy})^{1 / 2}$ & 4 & $\mathrm{Lb}=\mathrm{Lp}+0.5(\mathrm{Lr}-\mathrm{Lp})$ & Hybrid $(50 / 70)$ & 0.65 \\
$8 \mathrm{MG}$ & $5.7(\mathrm{E} / \mathrm{Fy})^{1 / 2}$ & 12 & 4 & $\mathrm{Lb}=\mathrm{Lp}+0.5(\mathrm{Lr}-\mathrm{Lp})$ & Hybrid $(50 / 70)$ & 0.65 \\
$9 \mathrm{MG}$ & $5.7(\mathrm{E} / \mathrm{Fy})^{1 / 2}$ & $0.382(\mathrm{E} / \mathrm{Fy})^{1 / 2}$ & 4 & $\mathrm{Lb}=\mathrm{Lr}$ & Hybrid $(50 / 70)$ & 0.65 \\
$10 \mathrm{MG}$ & $5.7(\mathrm{E} / \mathrm{Fy})^{1 / 2}$ & 12 & 4 & $\mathrm{Lb}=\mathrm{Lr}$ & Hybrid $(50 / 70)$ & 0.65 \\
$11 \mathrm{MG}$ & $5.7(\mathrm{E} / \mathrm{Fy})^{1 / 2}$ & $0.382(\mathrm{E} / \mathrm{Fy})^{1 / 2}$ & 4 & $\mathrm{Lb}=\mathrm{Lr}$ & 70 & 0.65 \\
$12 \mathrm{MG}$ & $5.7(\mathrm{E} / \mathrm{Fy})^{1 / 2}$ & 12 & 4 & $\mathrm{Lb}=\mathrm{Lr}$ & 70 & 0.65 \\
\hline
\end{tabular}


- $\quad$ Specimen 11MG was a variation of 9MG with a homogeneous (HPS 70W) material configuration.

- Specimen $12 \mathrm{MG}$ was a variation of $11 \mathrm{MG}$ with a slender flange and a variation of 6MG and 10MG with a homogeneous, HPS 70W material configuration.

Once this matrix of parameters was determined, the test girders were designed to satisfy these target slenderness parameters based on the nominal material properties (yield strength and modulus of elasticity) of the steel. The resulting dimensions were then used to determine quantities of plate needed. Once the plate that would be used to fabricate the girders was received, three tension coupons were taken from each plate and the actual yield strengths of each plate were determined. The average yield strength from all of the coupons taken from the same heat (where in some cases there were multiple plates from the same heat of steel) were used to finalize the dimensions of the girders; however, the nominal value of elastic modulus, 29,000 ksi, was still used in the final girder designs as there was little variation in this parameter (the average variation in elastic modulus is only $4.5 \%$ compared to an average variation in yield strength of 26\%). The resulting dimensions specified for each girder are as presented in Table 3-3. The reader is referred to Appendix C for additional data and information relating to the tension testing.

Table 3-3. Nominal Dimensions and Yield Strengths for Experimental Girders

\begin{tabular}{|c|c|c|c|c|c|c|c|c|c|c|c|c|}
\hline Specimen & $\begin{array}{l}\mathrm{L}_{\mathrm{b} 1} \\
\text { (in) }\end{array}$ & $\begin{array}{l}\mathrm{L}_{\mathrm{b} 2} \\
\text { (in) }\end{array}$ & $\begin{array}{l}\mathrm{L}_{\mathrm{b} 3} \\
\text { (in) }\end{array}$ & $\begin{array}{c}\text { D } \\
\text { (in) }\end{array}$ & $\begin{array}{c}t_{w} \\
\text { (in) }\end{array}$ & $\begin{array}{l}b_{\mathrm{fc}} \\
\text { (in) }\end{array}$ & $\begin{array}{l}t_{\mathrm{fc}} \\
\text { (in) }\end{array}$ & $\begin{array}{l}\mathbf{b}_{\mathrm{ft}} \\
\text { (in) }\end{array}$ & $\begin{array}{l}t_{f t} \\
\text { (in) }\end{array}$ & $\begin{array}{c}F_{y c} \\
(k s i)\end{array}$ & $\begin{array}{c}F_{y t} \\
(k s i)\end{array}$ & $\begin{array}{l}F_{y w} \\
\text { (ksi) }\end{array}$ \\
\hline $1 \mathrm{MG}$ & 36 & 48 & 60 & 24.750 & 0.750 & 8.125 & 0.500 & 14.000 & 0.875 & 50.00 & 50.00 & 50.00 \\
\hline $2 \mathrm{MG}$ & 30 & 48 & 66 & 27.000 & 0.750 & 7.250 & 0.500 & 17.000 & 0.875 & 50.00 & 50.00 & 50.00 \\
\hline $3 \mathrm{MG}$ & 42 & 42 & 36 & 31.000 & 0.313 & 7.875 & 0.500 & 12.125 & 0.500 & 50.00 & 50.00 & 50.00 \\
\hline $4 \mathrm{MG}$ & 96 & 96 & 0 & 30.000 & 0.313 & 7.875 & 0.500 & 12.125 & 0.500 & 50.00 & 50.00 & 50.00 \\
\hline $5 \mathrm{MG}$ & 84 & 84 & 66 & 43.000 & 0.500 & 8.250 & 0.500 & 13.000 & 0.750 & 50.00 & 50.00 & 50.00 \\
\hline $6 \mathrm{MG}$ & 132 & 102 & 0 & 30.000 & 0.313 & 7.500 & 0.313 & 9.500 & 0.500 & 50.00 & 50.00 & 50.00 \\
\hline $7 \mathrm{MG}$ & 78 & 78 & 0 & 29.000 & 0.313 & 7.000 & 0.500 & 10.750 & 0.500 & 70.00 & 70.00 & 50.00 \\
\hline $8 \mathrm{MG}$ & 84 & 84 & 0 & 28.000 & 0.313 & 8.000 & 0.375 & 9.750 & 0.500 & 70.00 & 70.00 & 50.00 \\
\hline $9 \mathrm{MG}$ & 114 & 114 & 0 & 29.000 & 0.313 & 7.000 & 0.500 & 10.750 & 0.500 & 70.00 & 70.00 & 50.00 \\
\hline $10 \mathrm{MG}$ & 126 & 108 & 0 & 28.000 & 0.313 & 8.000 & 0.375 & 9.750 & 0.500 & 70.00 & 70.00 & 50.00 \\
\hline $11 \mathrm{MG}$ & 114 & 114 & 0 & 32.000 & 0.375 & 7.125 & 0.500 & 15.500 & 0.500 & 70.00 & 70.00 & 70.00 \\
\hline $12 \mathrm{MG}$ & 114 & 114 & 0 & 32.000 & 0.375 & 8.250 & 0.375 & 14.000 & 0.500 & 70.00 & 70.00 & 70.00 \\
\hline
\end{tabular}


Once the girders were received from the fabricator each girder was carefully measured to determine the actual dimensions of the girder. Measurements were taken at a minimum of four cross sections along the length of the girder and then averaged. The measurements of plate thicknesses and flange widths less than 12 in. were taken using calipers with an accuracy of 0.001 in. Flanges with a width greater than 12 in., web depths, and lateral distances were taken using a tape measure and these measurements were rounded to the nearest 0.0625 in. Table 3-4 reports these actual dimensions of the test girders along with the actual yield strengths obtained from the respective tension coupons.

As discussed above, each girder was designed based on five different geometric requirements: web slenderness, flange slenderness, lateral slenderness, $D / b_{\mathrm{fc}}$ ratio, and $\mathrm{D}_{\mathrm{cp}} / \mathrm{D}$ ratio. As it was not possible to satisfy all five of these requirements simultaneously as changing the dimensions of the girder to better satisfy one limit (such as web slenderness) often results in the target value of another limit (e.g., percentage of web in compression) deviating more from the desired value. However, given this difficulty and the limitation of the six available plate thicknesses, it is believed that the intended parametric values are well satisfied. This is demonstrated in Table 3-5, which summarizes the target parametric values assigned to each girder and the resulting values based on the nominal and actual dimensions for each girder. Note that the designation of "nominal value” in Table 3-5 refers to the value

Table 3-4. Actual Dimensions and Yield Strengths for Experimental Girders

\begin{tabular}{|c|c|c|c|c|c|c|c|c|c|c|c|c|}
\hline Specimen & $\begin{array}{l}\mathrm{L}_{\mathrm{b} 1} \\
\text { (in) }\end{array}$ & $\begin{array}{l}\mathrm{L}_{\mathrm{b} 2} \\
\text { (in) }\end{array}$ & $\begin{array}{l}L_{b 3} \\
\text { (in) }\end{array}$ & $\begin{array}{c}\text { D } \\
\text { (in) }\end{array}$ & $\begin{array}{c}t_{w} \\
\text { (in) }\end{array}$ & $\begin{array}{l}b_{\mathrm{fc}} \\
\text { (in) }\end{array}$ & $\begin{array}{l}t_{f c} \\
\text { (in) }\end{array}$ & $\begin{array}{l}b_{\mathrm{ft}} \\
\text { (in) }\end{array}$ & $\begin{array}{c}t_{f t} \\
\text { (in) }\end{array}$ & $\begin{array}{c}F_{y c} \\
\text { (ksi) }\end{array}$ & $\begin{array}{c}F_{y t} \\
\text { (ksi) }\end{array}$ & $\begin{array}{l}F_{y w} \\
\text { (ksi) }\end{array}$ \\
\hline $1 \mathrm{MG}$ & 36 & 48 & 60 & 24.750 & 0.750 & 8.262 & 0.505 & 14.000 & 0.878 & 64.6 & 56.0 & 72.6 \\
\hline $2 \mathrm{MG}$ & 30 & 48 & 66 & 27.000 & 0.750 & 7.318 & 0.515 & 17.000 & 0.875 & 64.6 & 44.3 & 72.6 \\
\hline $3 \mathrm{MG}$ & 42 & 42 & 36 & 30.958 & 0.316 & 8.038 & 0.499 & 12.250 & 0.499 & 64.6 & 64.6 & 62.6 \\
\hline $4 \mathrm{MG}$ & 96 & 96 & 0 & 29.688 & 0.315 & 7.995 & 0.520 & 12.375 & 0.521 & 66.3 & 66.3 & 58.5 \\
\hline $5 \mathrm{MG}$ & 84 & 84 & 66 & 42.980 & 0.503 & 8.401 & 0.502 & 13.175 & 0.764 & 64.6 & 72.6 & 64.6 \\
\hline $6 \mathrm{MG}$ & 132 & 102 & 0 & 29.828 & 0.319 & 7.647 & 0.321 & 9.584 & 0.502 & 62.6 & 64.6 & 58.5 \\
\hline $7 \mathrm{MG}$ & 78 & 78 & 0 & 28.908 & 0.320 & 7.129 & 0.526 & 10.835 & 0.531 & 88.2 & 88.2 & 58.5 \\
\hline $8 \mathrm{MG}$ & 84 & 84 & 0 & 27.859 & 0.317 & 8.135 & 0.383 & 9.940 & 0.532 & 94.8 & 88.2 & 58.5 \\
\hline $9 \mathrm{MG}$ & 114 & 114 & 0 & 28.750 & 0.322 & 7.047 & 0.526 & 10.750 & 0.526 & 88.2 & 88.2 & 62.6 \\
\hline $10 \mathrm{MG}$ & 126 & 108 & 0 & 27.875 & 0.327 & 8.086 & 0.386 & 9.954 & 0.524 & 94.8 & 88.2 & 58.5 \\
\hline $11 \mathrm{MG}$ & 114 & 114 & 0 & 31.844 & 0.375 & 7.133 & 0.528 & 15.594 & 0.531 & 88.2 & 88.2 & 94.8 \\
\hline $12 \mathrm{MG}$ & 114 & 114 & 0 & 32.750 & 0.390 & 8.257 & 0.524 & 14.188 & 0.524 & 88.2 & 88.2 & 94.8 \\
\hline
\end{tabular}




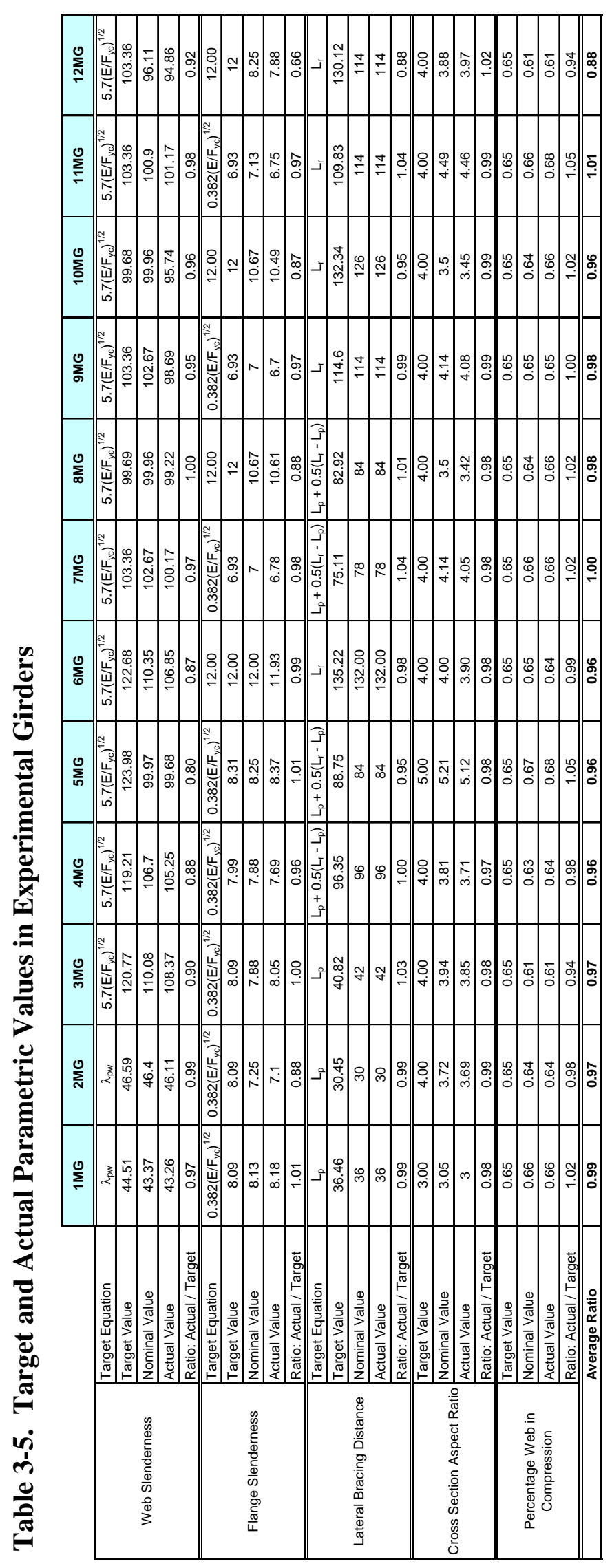


based on the nominal dimensions of the girder but the actual material properties of the girder as these were known prior to designing the girders. This shows that each parametric value assigned to the girders was typically satisfied within $5 \%$, i.e., the ratio between the actual and target parametric values was between 0.95 and 1.05 in most cases. Furthermore, the average ratio is 0.97 with a standard deviation of 0.06 . It will be noticed that the intended flange slenderness ratio for Girder $12 \mathrm{MG}$ is not well satisfied. This is due to a fabrication error where a flange width much narrower than the intended flange width was used. This also affects the other performance ratios of this girder to a smaller degree. However, the objective of Girder 12MG, to investigate a more slender flange compared to Girder 11MG, can still be evaluated.

As discussed above, it was desired to have several girders where the lateral bracing distance was of sufficient length so that the moment capacity computed from the lateral torsional buckling equations would be less than the maximum allowable capacity for this limit state. Table 3-6 demonstrates the girder characteristics with respect to moment gradient effects. Here it is shown that 4 (6MG, 10MG, $11 \mathrm{MG}$, and 12MG) of the 10 girders with lateral bracing distances greater than the compact limit will give moment capacities less than the maximum allowable for the lateral torsional buckling.

Table 3-6. Effects of Moment Gradient Modifier, $C_{b}$

\begin{tabular}{|ccc|}
\hline $\begin{array}{c}\text { Girder } \\
\text { Number }\end{array}$ & $\mathbf{C b}$ & $\mathbf{M}=\mathbf{M}_{\text {MAX-LTB }}$ \\
\hline \hline $1 \mathrm{MG}$ & 1.13 & yes \\
$2 \mathrm{MG}$ & 1.10 & yes \\
$3 \mathrm{MG}$ & 1.19 & yes \\
$4 \mathrm{MG}$ & 1.30 & yes \\
$5 \mathrm{MG}$ & 1.20 & yes \\
$6 \mathrm{MG}$ & 1.34 & no \\
$7 \mathrm{MG}$ & 1.31 & yes \\
$8 \mathrm{MG}$ & 1.30 & yes \\
$9 \mathrm{MG}$ & 1.30 & yes \\
$10 \mathrm{MG}$ & 1.32 & no \\
$11 \mathrm{MG}$ & 1.30 & no \\
$12 \mathrm{MG}$ & 1.30 & no \\
\hline
\end{tabular}


In addition to the measurements taken of the girder dimensions, the geometric imperfections of the girders were also recorded. These initial imperfections were considered to be comprised of three components as shown in Fig. 3-7: an out-of-flatness of the web panels $\left(\delta_{\text {ow }}\right)$, a tilt of the compression flange $\left(\delta_{\text {of }}\right)$, and a lateral sweep of the compression flanges $\left(\delta_{\mathrm{oL}}\right)$. The initial out-of-flatness of the web panel, illustrated in Fig. 3-7(a), was measured by holding a straightedge with a 24 in. length flush against the surface of the web. The maximum gap between the straightedge and the surface of the web, which typically occurs near the midheight of the web, was then recorded as $\delta_{\text {ow }}$. The initial tilt of the compression flange, as shown in Fig. 3-7(b), was assumed to consist of uniform rotation of the flange about the web. This type of imperfection was observed in several of the girders; however, an equally typical situation was that the flange was not straight across its width with the flange deformed such that both edges of the flange were rotated towards the web. In cases where the flange exhibited uniform rotation, $\delta_{\text {of }}$ was measured by holding a level at one edge of the flange and measuring the difference between level and the opposite edge of the flange and then dividing this value by two in order to obtain the average difference in height between the web centerline and the flange edge. If both edges of the flange were rotated towards the web, $\delta_{\text {of }}$ was measured by holding a level at the center of the flange and measuring the distance between level and each edge of the flange then averaging these two values. The sweep of the compression flange, shown in Fig. 3-7(c) was measured by holding a level in

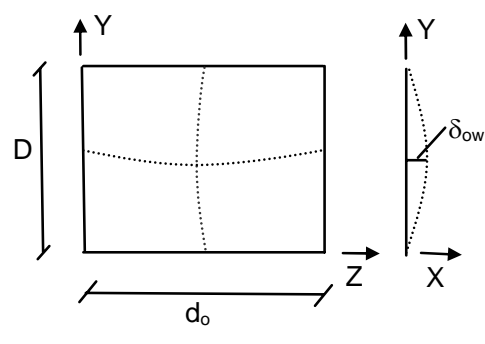

(a) Initial out-of-flatness of

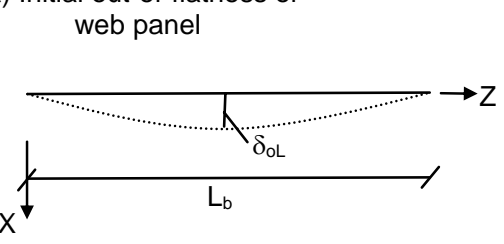

(c) Initial sweep of the compression flange

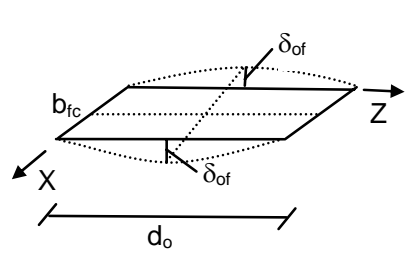

(b) Initial tilt of compression flange

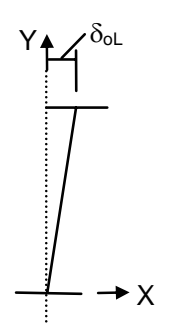

Figure 3-7. Initial Geometric Imperfections Considered in Experimental Girders 
the vertical position against the bottom of the web and then measuring the difference between the top of the level and the surface of the web. Each of these imperfection measurements is taken at the center of each lateral bracing panel of each girder. For several girders, additional measurements were also taken at other points along the girders, especially when it was observed that more severe imperfections occurred at an alternate location. In this case, the reported imperfection value is based on a weighted average, where a higher weight is given to imperfection values closer to midspan as these values are assumed to have a more significant influence of girder behavior. The accuracy of this weighted average procedure is shown to be accurate through comparisons to FEA results. The average of the recorded initial imperfections is given for each girder in Table 3-7.

\subsection{Testing Procedure}

\subsubsection{General Considerations}

Prior to conducting each experiment, the girders were coated with a mixture of lime and water so that yielding in the girders could be visually detected with ease. This method worked well to indicate the progress of yielding in the beams. Small cracks began to appear in the lime coating as yielding initiated and as yielding in the girder progressed, so did the size and number of cracks in the lime coating.

Table 3-7. Average Initial Imperfections in Experimental Girders

\begin{tabular}{|cccc|}
\hline Specimen & $\delta_{\text {ow }}$ & $\delta_{\text {of }}$ & $\delta_{\text {oL }}$ \\
\hline \hline 1MG & 0.00 & 0.08 & 0.21 \\
2MG & 0.00 & 0.10 & 0.09 \\
3MG & 0.24 & 0.08 & 0.22 \\
$4 \mathrm{MG}$ & 0.11 & 0.09 & 0.13 \\
$5 \mathrm{MG}$ & 0.12 & 0.09 & 0.10 \\
$6 \mathrm{MG}$ & 0.06 & 0.06 & 0.19 \\
$7 \mathrm{MG}$ & 0.13 & 0.10 & 0.31 \\
$8 \mathrm{MG}$ & 0.20 & 0.19 & 0.20 \\
$9 \mathrm{MG}$ & 0.05 & 0.09 & 0.18 \\
$10 \mathrm{MG}$ & 0.03 & 0.22 & 0.31 \\
$11 \mathrm{MG}$ & 0.09 & 0.11 & 0.20 \\
$12 \mathrm{MG}$ & 0.03 & 0.09 & 0.15 \\
\hline
\end{tabular}


Load was applied to the girders using a 330-kip actuator, which was mounted on a steel tension frame with equal capacity as viewed in Fig. 3-2. The tests were conducted in a displacement control mode using small displacement increments and allowing for loads to stabilize before applying the subsequent displacement increment. Specifically, displacement increments ranging between $0.10 \mathrm{in}$. and $0.20 \mathrm{in}$. were used during the portions of the test where a linear-elastic response is expected; smaller increments of $0.05,0.075$, or $0.1 \mathrm{in}$. were used during portions of the test when inelastic behavior was expected. For displacement intervals less than or equal to $0.1 \mathrm{in}$. the displacement was applied over a time interval of one minute. A duration of up to 2 minutes was used for larger displacement increments.

At the completion of each displacement interval, the force output from the actuator was monitored to ascertain when a stable load was achieved. In the elastic range of behavior, a constant force reading was typically obtained after ten minutes or less. However, once yielding of the girder began, the work input by the actuator caused plastic deformations of the girder to occur and hence less force is subsequently required from the actuator to maintain the prescribed amount of vertical deflection. Because time is required for these plastic deformations to develop, approximately 20 minutes was required for the loading to become stable in the inelastic range of the testing. Allowing for the load to become stable before proceeding to the subsequent load step assures that the static load-deflection curve is obtained.

\subsubsection{Data Collection}

A significant amount of data was recorded at each load step during the girder tests. This data not only included load and vertical displacement measurements required to obtain momentrotation relationships, but also vertical and horizontal displacements at two cross-sections in proximity to and symmetric about midspan of the girder to obtain quantitative measurements to aid in the categorization of failure modes, i.e., flange local buckling, web local buckling, lateral distortional buckling, or a combination of these modes. Figure 3-8 schematically illustrates the instrumentation layout for all tests, while Figs. 3-9 through 3-14 show photographs of these sensors. 


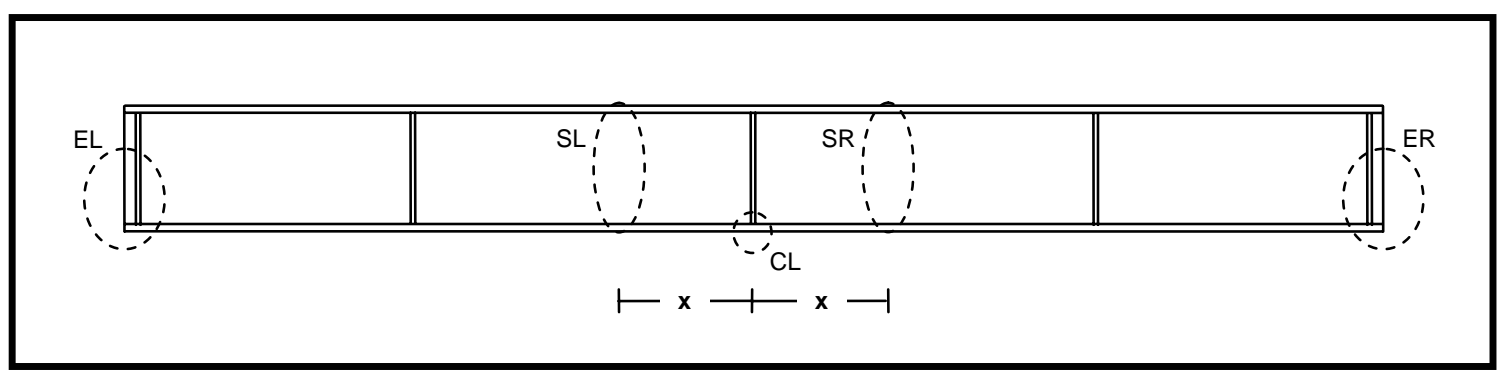

(a) Instrumentation Locations

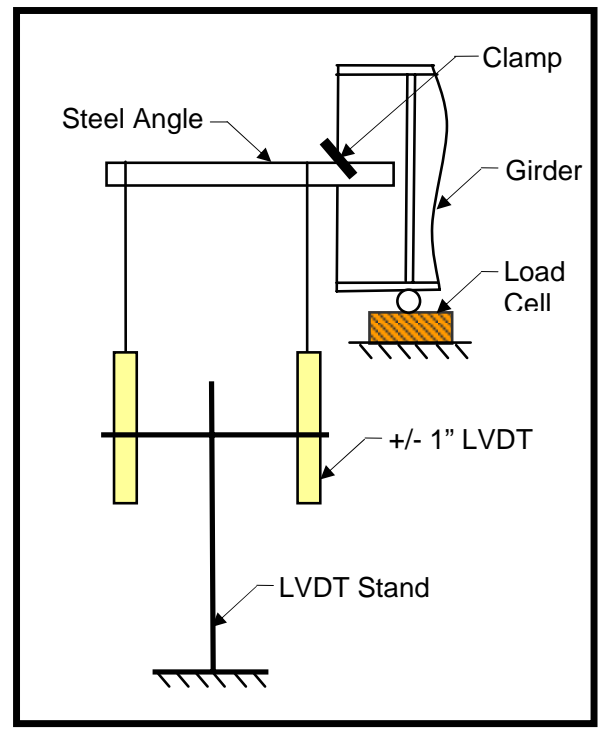

(b) Instrumentation at EL and ER

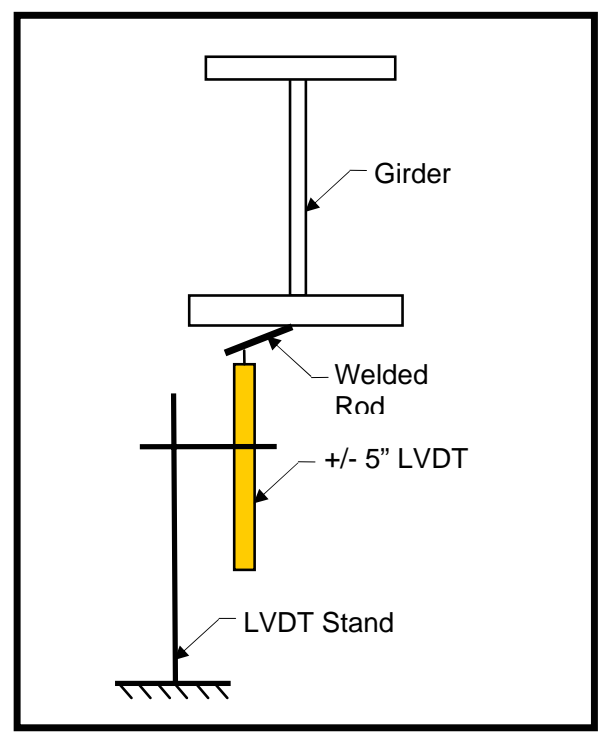

(c) Instrumentation at CL

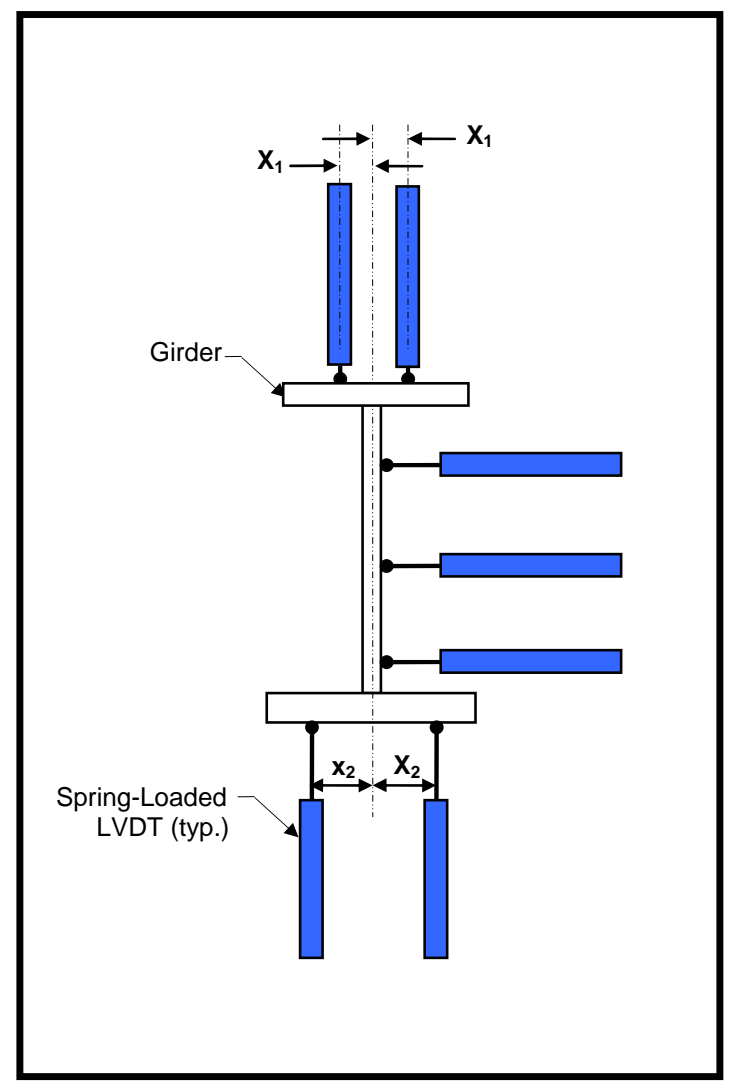

(d) Instrumentation at SL and SR

Figure 3-8. Schematic Diagram of Instrumentation Used in Experimental Testing 
As shown in Fig. 3-8(a) instrumentation was placed at five cross-sections along the length of the beam (labeled EL, SL, ER, SR, and CL, where "E" designates end locations, "S" designates locations along the span, "L" refers to the left side of the beam, "R" refers to the right side of the beam, and "CL" designates the centerline of the bean). Figures 3-8(b, c, and d) show schematic diagrams of the instrumentation placed at each of these locations as discussed below. Additionally, at each displacement increment the force and displacement readings output from the actuator are also recorded.

Figures 3-8(b) and 3-9 depict the instrumentation installed at the left and right ends of the beam, labeled as sections EL and ER, respectively. Specifically, at each end of the beam, there were two linear variable differential transducers (LVDTs) for the purposes of obtaining end rotations of the girder as well as low profile load cells for determining end reactions. The force readings from the load cells also served as a comparison to the actuator force for verification that the reported forces are accurate and assessment of the amount of force (if any) resisted by the lateral frames.

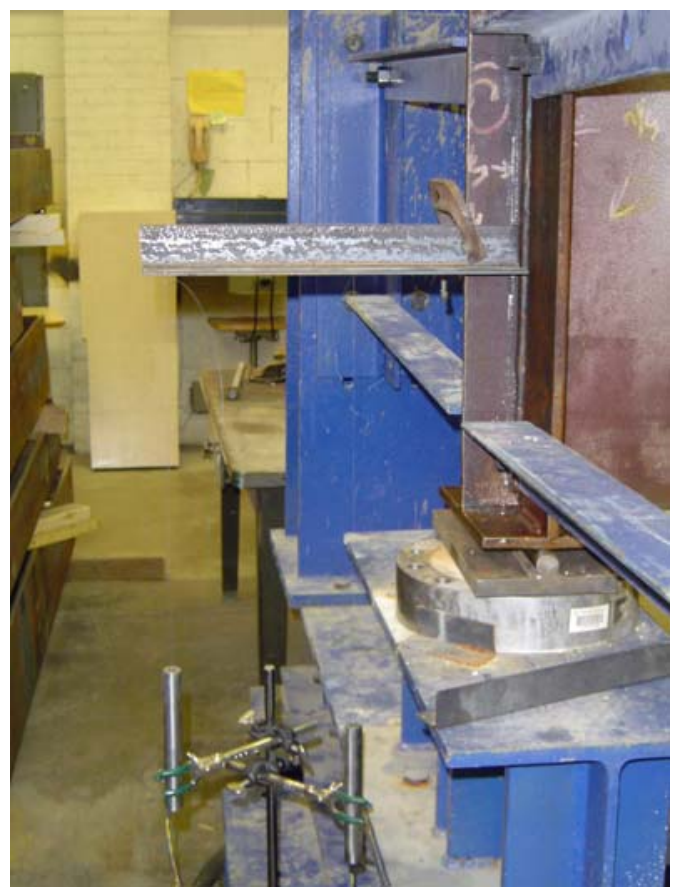

Figure 3-9. Photograph of Instrumentation at Ends of Girder 
Figure 3-8(c) illustrates the instrumentation provided at the center of the beam, labeled as cross-section CL. At this location a small-diameter rod was welded on the bottom surface of the girder at the longitudinal and transverse center of the beam from which a LVDT was suspended in order to measure vertical displacement of the beam at this location. This welded rod was necessary due to limited clearance between the bottom surface of the girder and the testing frame (as shown in Fig. 3-10), which prohibited installation of the LVDT directly under the centerline of the girder.

An arrangement of spring-loaded LVDTs as depicted in Figs. 3-8(d) was placed at crosssections SL and SR in order to measure the deformations of the cross-section. Specifically, at each of these sections there are a total of seven LVDTs as shown in Fig. 3-11: two measuring vertical deformation of the top flange (see Fig. 3-12), two measuring vertical deformation of the bottom flange (see Fig. 3-13), and three measuring lateral deformation of the web (see Fig. 3-14). Based on the readings from these sensors, the rotation of both the top and bottom flanges can be determined in addition to the deformed shape of the web. The use of three LVDTs on the web allowed for assessing if the web deformation was linear (as would be expected if lateral-torsional buckling occurred) or quadratic (as would occur during web local buckling).

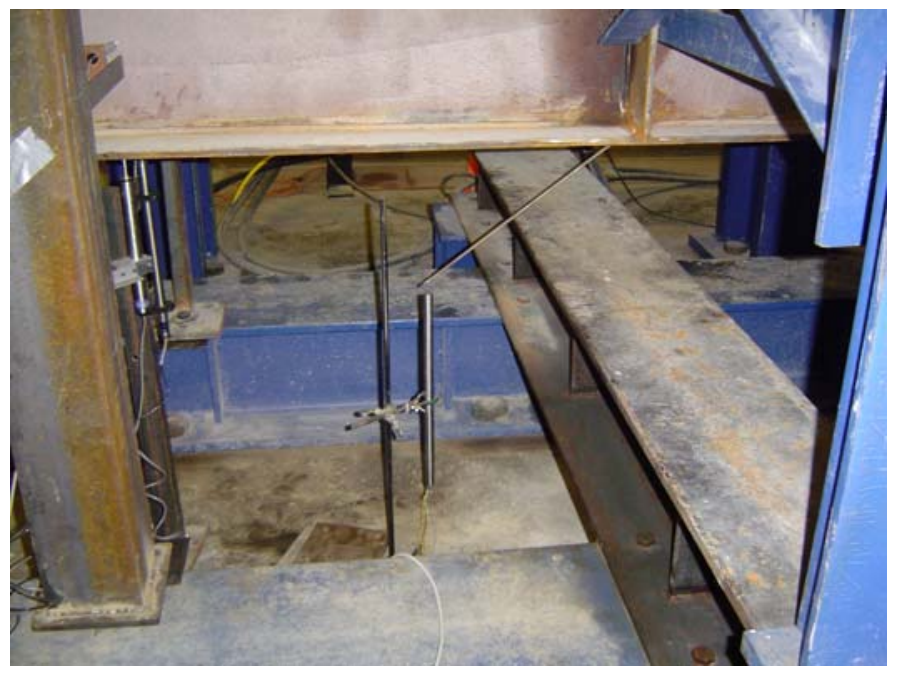

Figure 3-10. Photograph of Instrumentation at Girder Centerline 


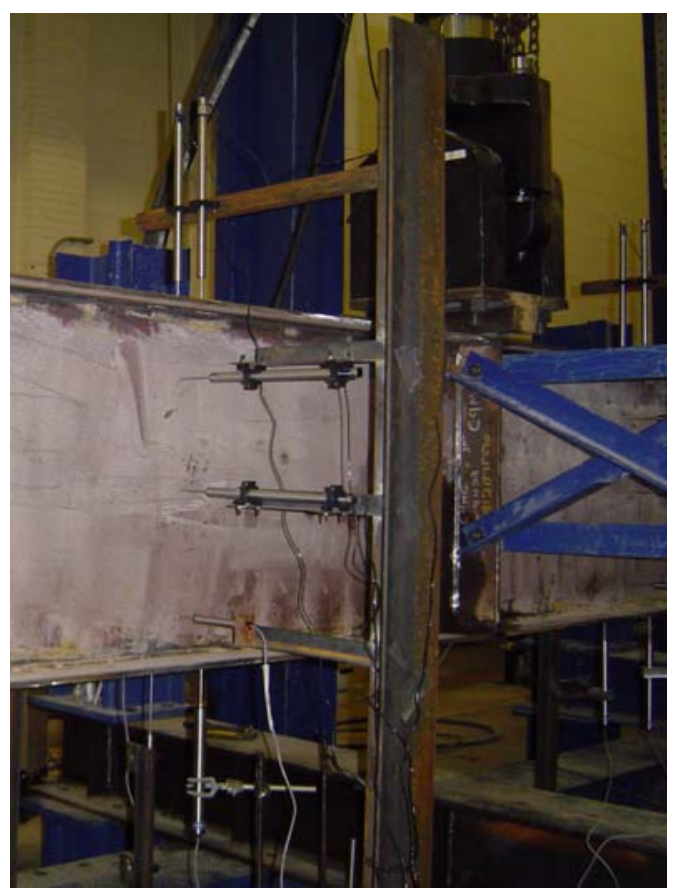

Figure 3-11. Photograph of Instrumentation at SL and SR

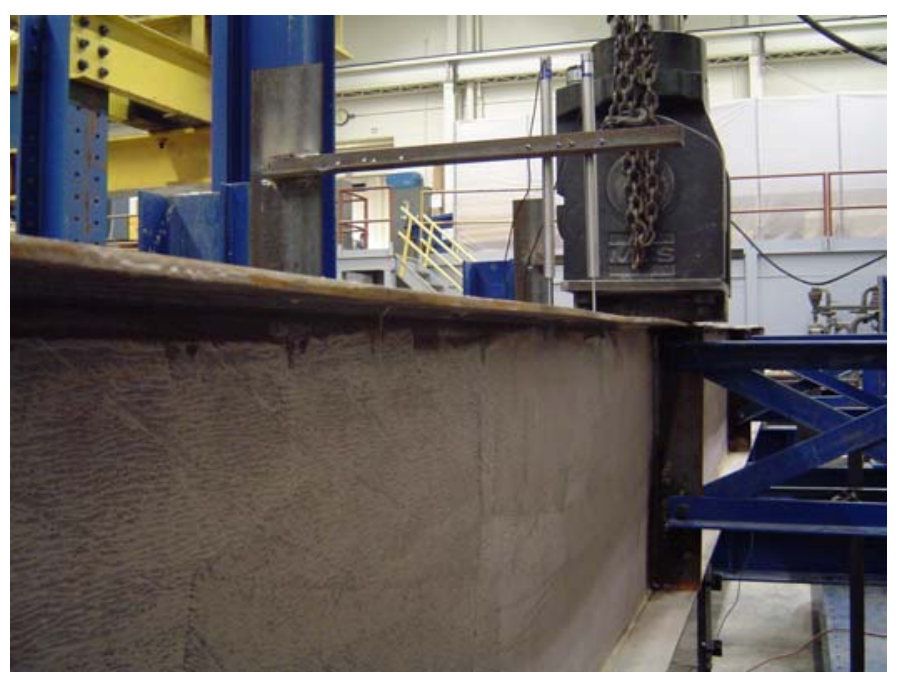

Figure 3-12. Top Flange LVDTs

The pair of LVDTs on the bottom flange was centered about the web of the girder with a typical spacing of 6 in. between the two sensors, but varied based on the width of the bottom flange. The LVDT pair on the top flange was also centered about the web, but with a smaller distance between these two gages of 3 in. to attempt to capture the displacement of the top 


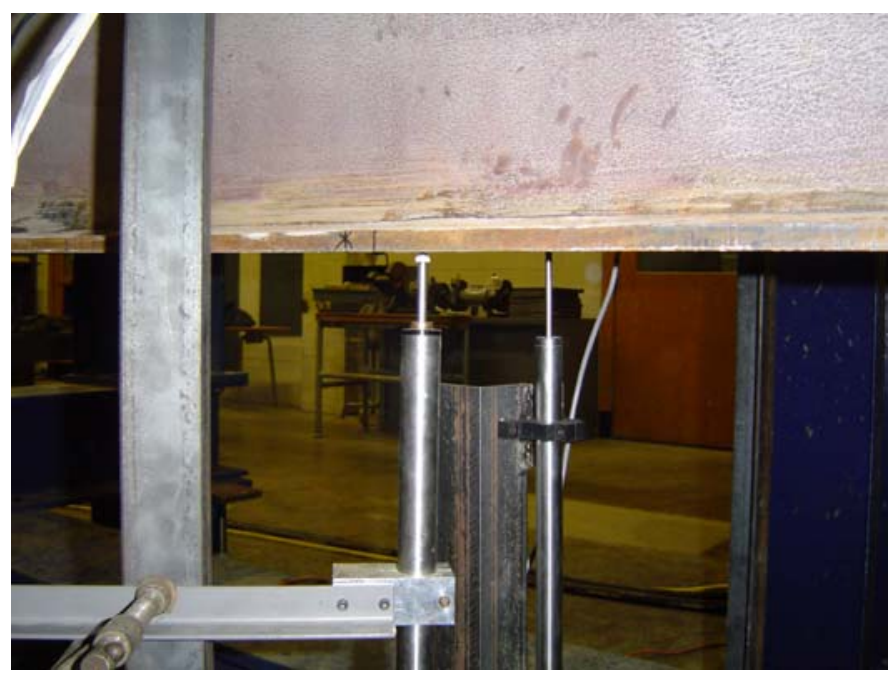

Figure 3-13. Bottom Flange LVDTs

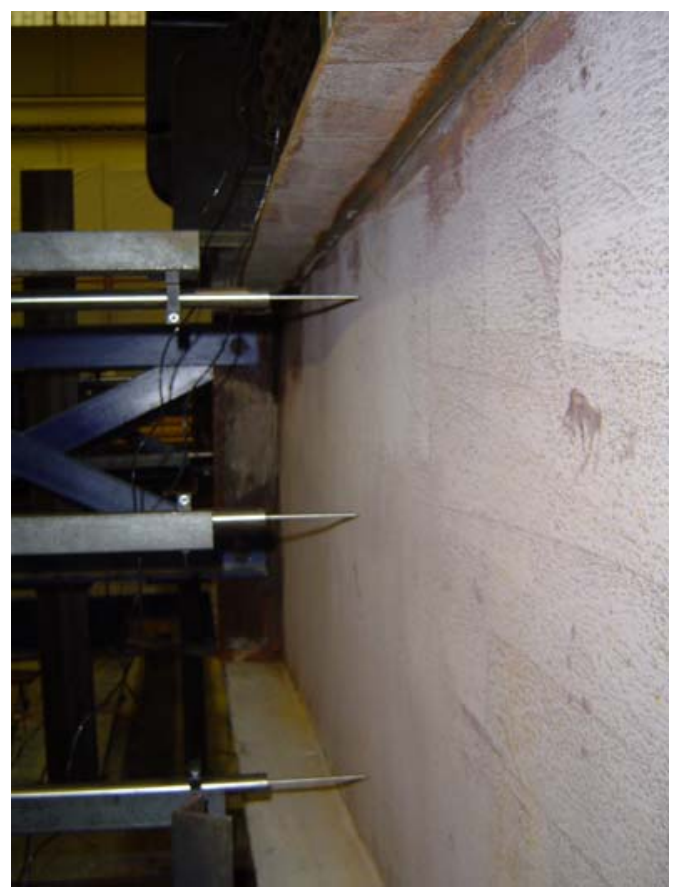

Figure 3-14. Web LVDTs

flange even if significant lateral movement of the top flange occurred, i.e., the LVDTs are mounted independently of the girder such that the displacements captured by the LVDTs represent the displacement at a fixed point in space as opposed to the displacement of a fixed point on the girder. The three web LVDTs were placed such that they were located 
approximately equal distances apart to the extent permitted by the LVDT stands. The web LVDT closest to the top flange is located at least 5 in. from the top flange to allow for a minimum of this amount of deflection. The longitudinal location of these sensors, labeled as $\mathrm{x}$ in Fig. 3-8(a), varied for each girder and was determined by conducting a finite element analysis of the girders prior to each experiment in order to predict the location where the most critical buckling was expected.

\subsection{Experimental Results}

There are three primary points of interest regarding the experimental results. These are (1) maximum moment capacities and comparison of these strengths to those predicted by AASHTO specifications, (2) the failure modes as determined from visual assessment of the girder and quantitative measurements resulting from the LVDT readings, and (3) the load versus deflection response of the girders. These three attributes of each girder are discussed in the following sections, followed by a discussion of general conclusions formed on the basis of these experimental results and will subsequently be used to verify the FEA techniques implemented in later chapters.

The maximum moment capacities of each girder and the respective moment capacities predicted by AASHTO (2004) Specifications are given below in Table 3-8. A discussion of this data is given in the subsequent sections detailing the results of each girder individually.

Table 3-8. Comparison of Experimental and AASHTO (2004) Capacities (ft-kips)

\begin{tabular}{|cccc|}
\hline Specimen & $\mathbf{M}_{\text {EXP }}$ & $\mathbf{M}_{\text {AASHTO }}$ & $\begin{array}{c}\text { Percent } \\
\text { Difference }\end{array}$ \\
\hline \hline 1MG & 1635 & 1640 & 0.3 \\
2MG & 1757 & 1805 & 2.8 \\
3MG & 985 & 991 & 0.6 \\
4MG & 978 & 971 & 0.8 \\
5MG & 1803 & 2026 & 12.4 \\
6MG & 519 & 582 & 12.2 \\
$7 M G$ & 951 & 1043 & 9.7 \\
8MG & 809 & 777 & 4.0 \\
$9 M G$ & 897 & 1060 & 18.2 \\
$10 M G$ & 834 & 796 & 4.5 \\
11MG & 1125 & 1317 & 17.1 \\
12MG & 1445 & 1584 & 9.6 \\
\hline
\end{tabular}


It is noted that the optional provisions given in Appendix A of Article 6.10 of the specifications (AASHTO 2004), which allow for the prediction of moment capacities in excess of $\mathrm{M}_{\mathrm{y}}$, are applicable for these girders and have been used to compute predicted moment capacities. Furthermore, it is noted that in the current (2004) specifications the controlling failure modes are either computed to be local buckling (which is based on the slenderness properties of the flange, i.e., flange local buckling), lateral-torsional buckling, or tension flange yielding.

The LVDT readings from the sensors located along the span of the girder are used to generate a plot of the deformed shape of each girder, which are presented for each girder in the following sections. The presented data is the data from the panel of the girder to the right or left of midspan (i.e, SR or SL in Fig. 3-8(a)) experiencing the most severe distortions and, in cases where such data is available, is the data after removing the load from the girder at the end of the test, thus representing the final deformed shape of the girder. Where this data is not available due to the need for removing sensors in order to avoid damage (as was done in the testing of girders $2 \mathrm{MG}, 9 \mathrm{MG}$, and $12 \mathrm{MG}$ ), the data presented is the data from the last available load step. Unless noted otherwise, the deformed shape presented is qualitatively representative of the deformed shape of the girder throughout the unloading range although the magnitude of the deformations increases as the vertical displacement applied to the girder increases. In each plot the original undeformed shape of the girder is represented by square data points and the deformed shape is represented by a series of diamonds. Furthermore, filled data points represent actual test data and shaded data points represent approximate data in cases where readings from top flange LVDTs were not available due to significant lateral movement of the flange such that the LVDT is no longer in contact with the flange.

Load versus deflection plots are subsequently presented for each of the twelve experimental girders. It is noted that there is a linear relationship between load and moment as well as between deflection and rotation, so the shape of the moment versus rotation curves is the same as the shape of the load versus deflection relationships presented below. For many of the girders, the girder was loaded multiple times and in these cases the load versus deflection relationship of each loading cycle is presented. In most cases, the deflection data presented 
in the load versus deflection plots represents an average of the readings obtained from the internal LVDT contained within the actuator and the readings obtained from the external LVDT located beneath the centerline of the girder. In this situation, the data in the load versus deflection plots is represented by a series of squares. It is noted that the actuator displacement data may over predict the actual displacements in the girder as the actuator has the ability to move longitudinally and laterally in addition to vertically. Conversely, the external LVDT may under predict the actual displacements due to lateral or longitudinal movement of the point on the bottom flange of the girder to which the LVDT is affixed. Thus, averaging these two values gives a realistic representation of the actual girder deflection in most cases. However, there are instances when the external LVDT data is deemed unreliable due to substantial movement of the bottom flange of the girder at midspan, resulting in LVDT readings that are significantly less than the actual vertical deflection of the girder. In these instances, the data presented in the load versus deflection

plots is the actuator data only. This is indicated by using a series of triangles to represent the data in the plots.

\subsubsection{Girder $1 M G$}

Table 3-8 indicates that the maximum moment capacity of girder $1 \mathrm{MG}$ is well predicted by the AASHTO (2004) specifications. Specifically, the predicted moment capacity of $1640 \mathrm{ft}-$ kips matches the experimental capacity of the girder (1635 ft-kips) within $0.3 \%$ and is controlled by flange local buckling.

The deformed shape of the girder is shown in Fig. 3-15, where it is shown that the predominant deformations are in the compression flange and portion of the web in compression. It is also observed that the top flange and the top of the web remain approximately perpendicular to one another throughout the testing range.

As shown in Fig. 3-16, girder 1MG exhibits relatively ductile behavior as anticipated for this compact girder. The ductility of the girder may be quantified by the deformation capacity of the girder at a particular level of load, where the deformation capacity is computed as the ratio between the plastic displacement (total displacement minus elastic displacement) and 


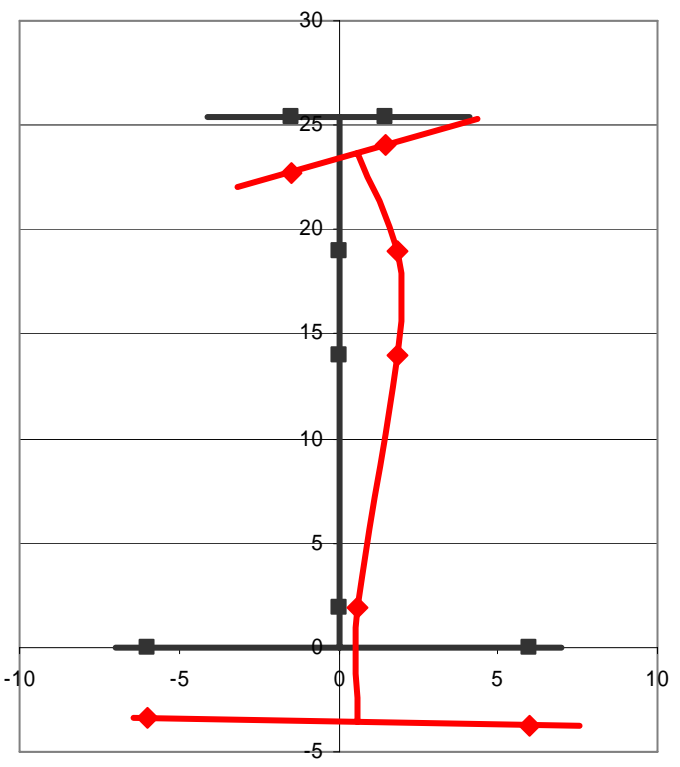

Figure 3-15. Deformed Shape of Girder 1MG

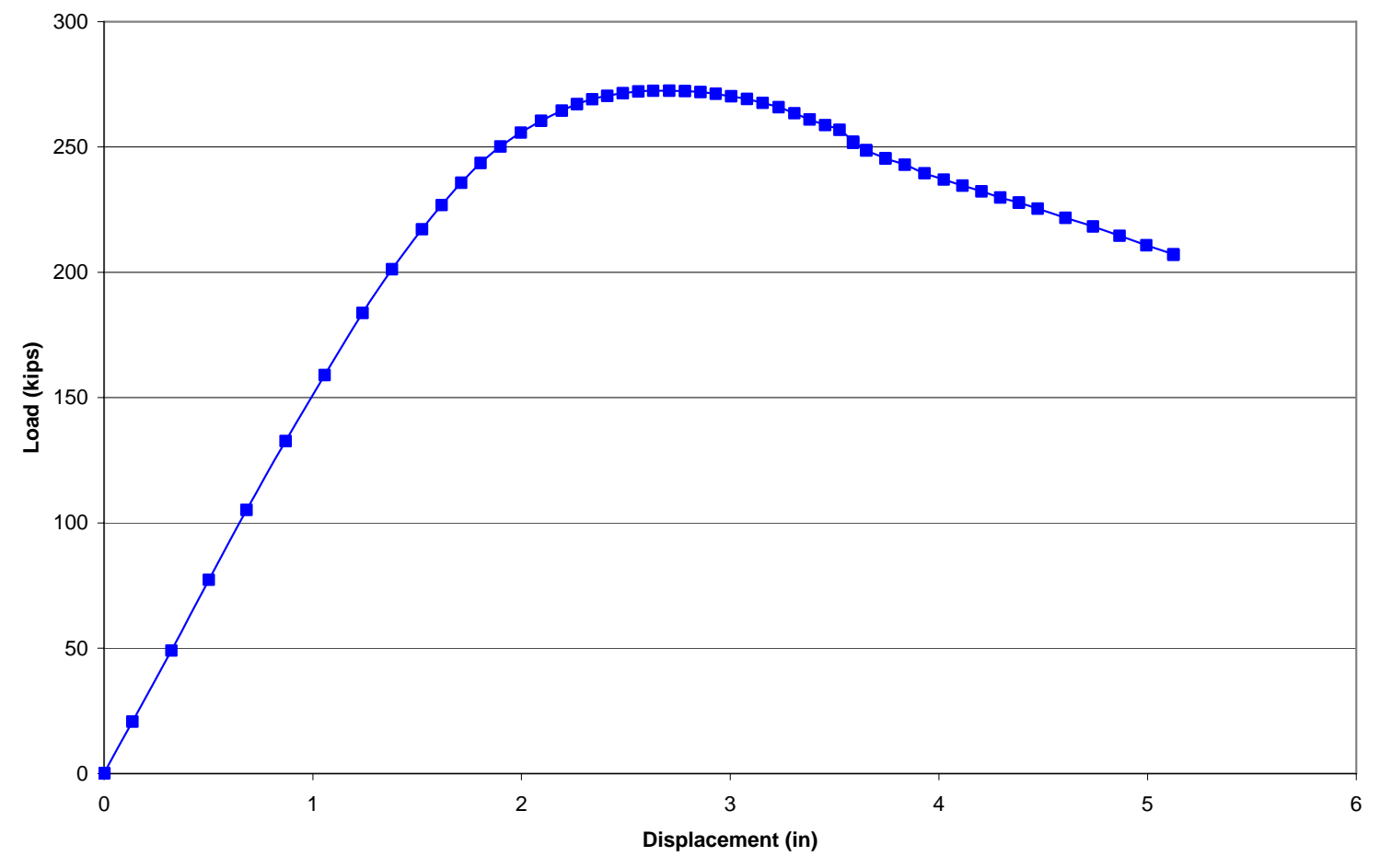

Figure 3-16. Load versus Displacement Data for Girder 1MG 
the elastic displacement. At a load equal to 246 kips (which is equal to $90 \%$ of the maximum load), the deformation capacity of this girder is 2.0 .

\subsubsection{Girder $2 M G$}

The AASHTO (2004) specifications accurately predict the moment capacity of Girder 2MG by predicting a moment capacity of 1805 ft-kips, which differs from the experimental capacity of 1757 ft-kips by only 2.7\%. Furthermore, the specifications predict the same moment capacity for all three strength calculations: flange local buckling, lateral torsional buckling, and tension flange yielding.

The deformed shape of Girder 2MG is shown below in Fig. 3-17. Here it is shown that the most significant deformations are lateral movement of the girder. However, it is important to note that testing of this girder was terminated prior to achieving significant deformations in the girder due to failure of one of the lateral braces, which contributed to the lateral movement of the beam depicted in the figure. Additionally, rotation of the compression flange about the web and web buckling are also captured by the LVDT readings presented in Fig. 3-17.

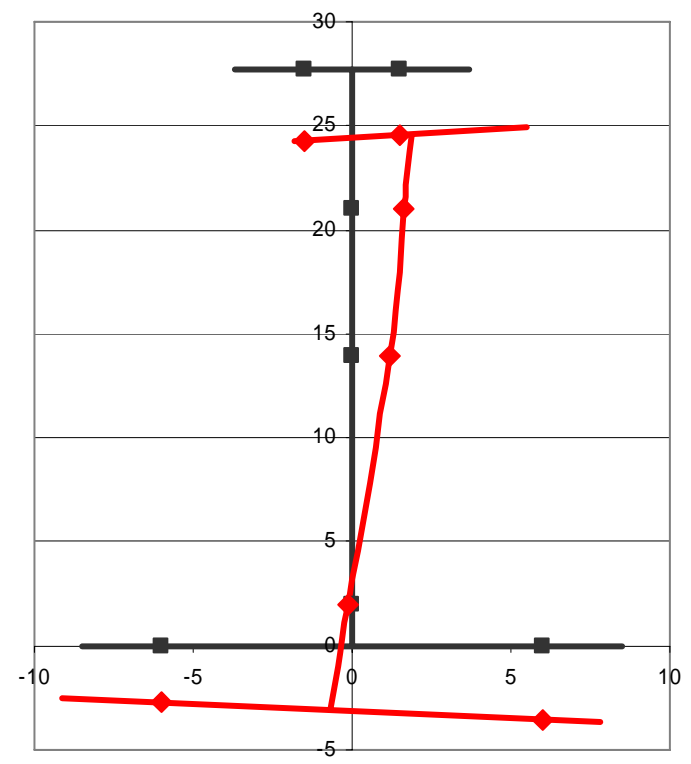

Figure 3-17. Deformed Shape of Girder 2MG 
Girder 2MG also displays a ductile load versus displacement relationship typical of a compact beam, which is shown in Fig. 3-18. At a load of 264 kips, equal to 90\% of the maximum load, the deformation capacity of girder 2MG is 2.3. Thus, comparing the deformation capacities Girder $1 \mathrm{MG}$ with a $\mathrm{D} / \mathrm{b}_{\mathrm{fc}}$ ratio of 3 (and a deformation capacity equal to 2.0) and Girder $2 \mathrm{MG}$ with a $\mathrm{D} / \mathrm{b}_{\mathrm{fc}}$ ratio of 4 (and a deformation capacity equal to 2.3) illustrates that there is not a significant influence on girder ductility with varying $\mathrm{D} / \mathrm{b}_{\mathrm{fc}}$ ratio for these compact girders.

\subsubsection{Girder 3MG}

As shown in Table 3-8, the AASHTO (2004) Specifications accurately predict the capacity of girder 3MG. The difference between the experimental capacity of $985 \mathrm{ft}$-kips and the predicted capacity of $991 \mathrm{ft}$-kips is only $0.6 \%$. The predicted capacity is computed using Appendix A and is controlled by the computations based on flange local buckling or lateral torsional buckling.

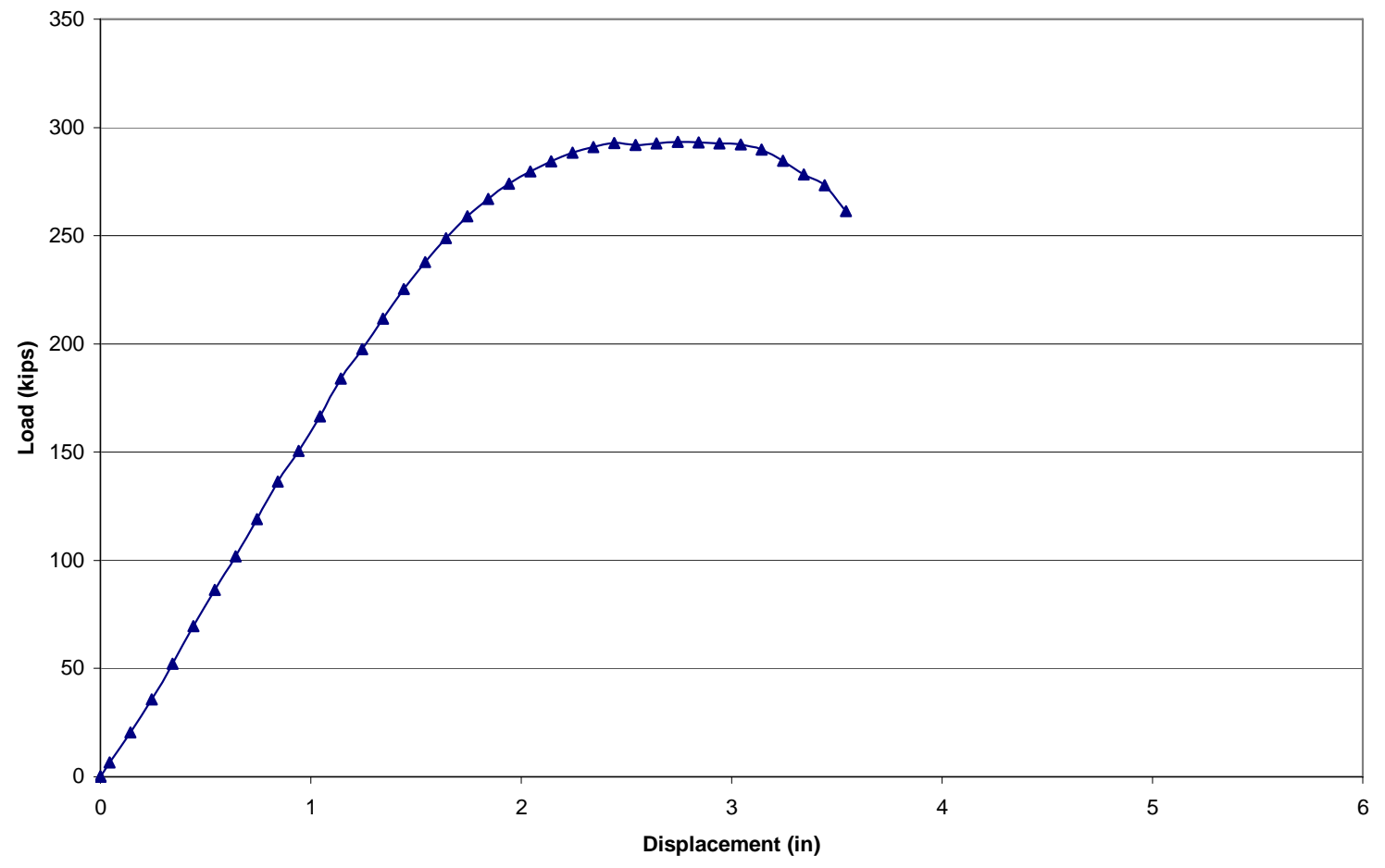

Figure 3-18. Load versus Displacement Data for Girder 2MG 
Two plots of the deformed shape of Girder 3MG are given below in Fig. 3-19. The plot on the left represents the deformed shape of the girder at the maximum load where it is clear that web buckling is the only deformation present. The plot on the right in Fig. 3-19 shows the deformed shape of the girder after the testing has been completed. This plot indicates that, as web buckling progresses, flange buckling also begins to occur due to the decreased restraint to the flange as the web deformations increase. These experimental results support the anticipated failure mode of web local buckling, which was assumed based on the noncompact web, compact flanges, and compact brace spacing of this girder. Furthermore, comparing Figs. 3-17 and 3-19, the influence of the more slender flange in Girder 3MG is clearly observed.

Figure 3-20 illustrates the load versus deflection data for Girder 3MG. This graph shows that there is a nearly linear relationship between load and deflection in both the loading and unloading phases. However, the girder is still relatively ductile, with a deformation capacity of 1.9 at $90 \%$ of the maximum load. Thus, the use of a non-compact web appears to result in only slightly less deformation compared to the compact girders (1MG and 2MG) discussed above.
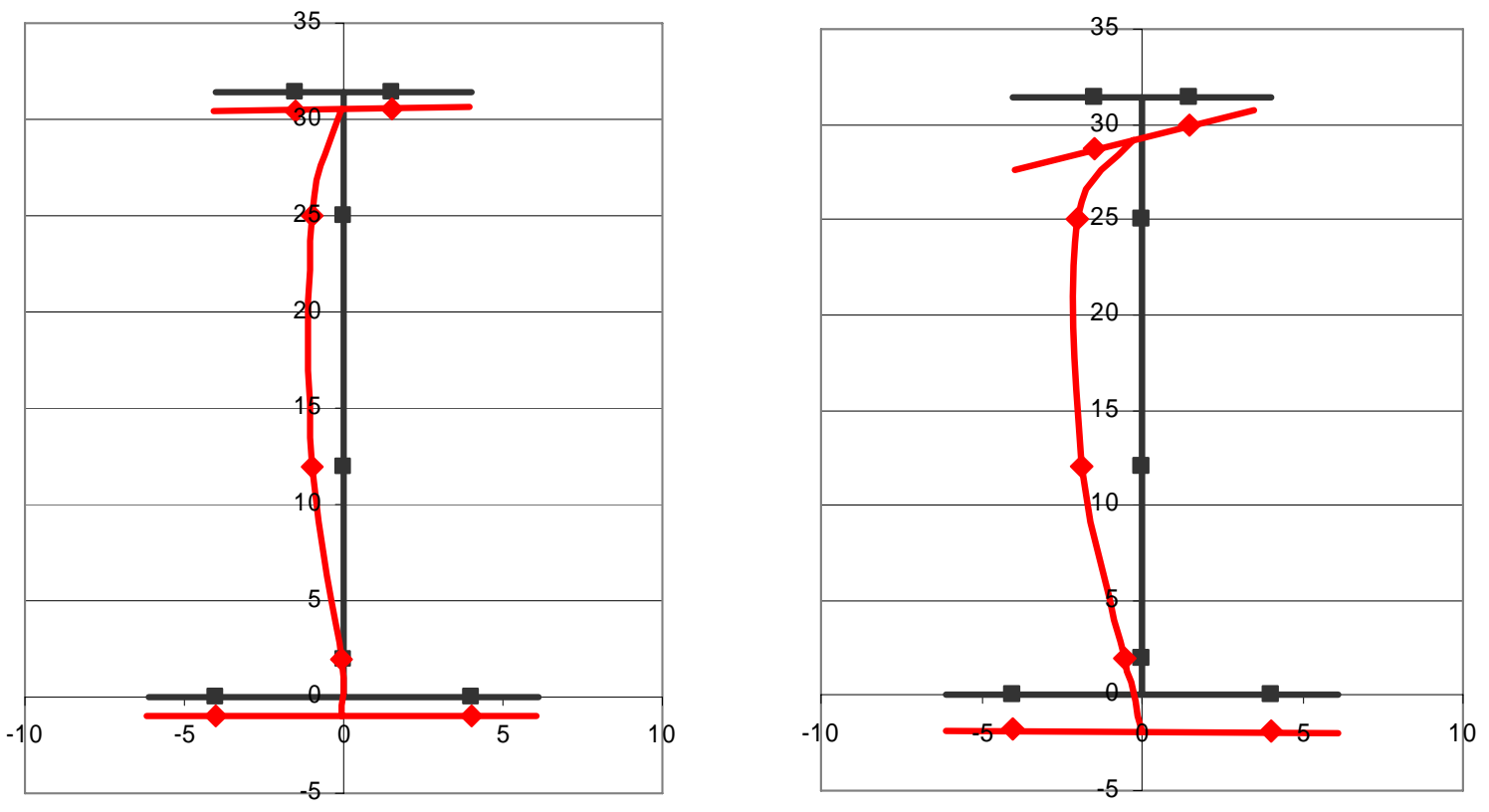

Figure 3-19. Deformed Shapes of Girder 3MG 


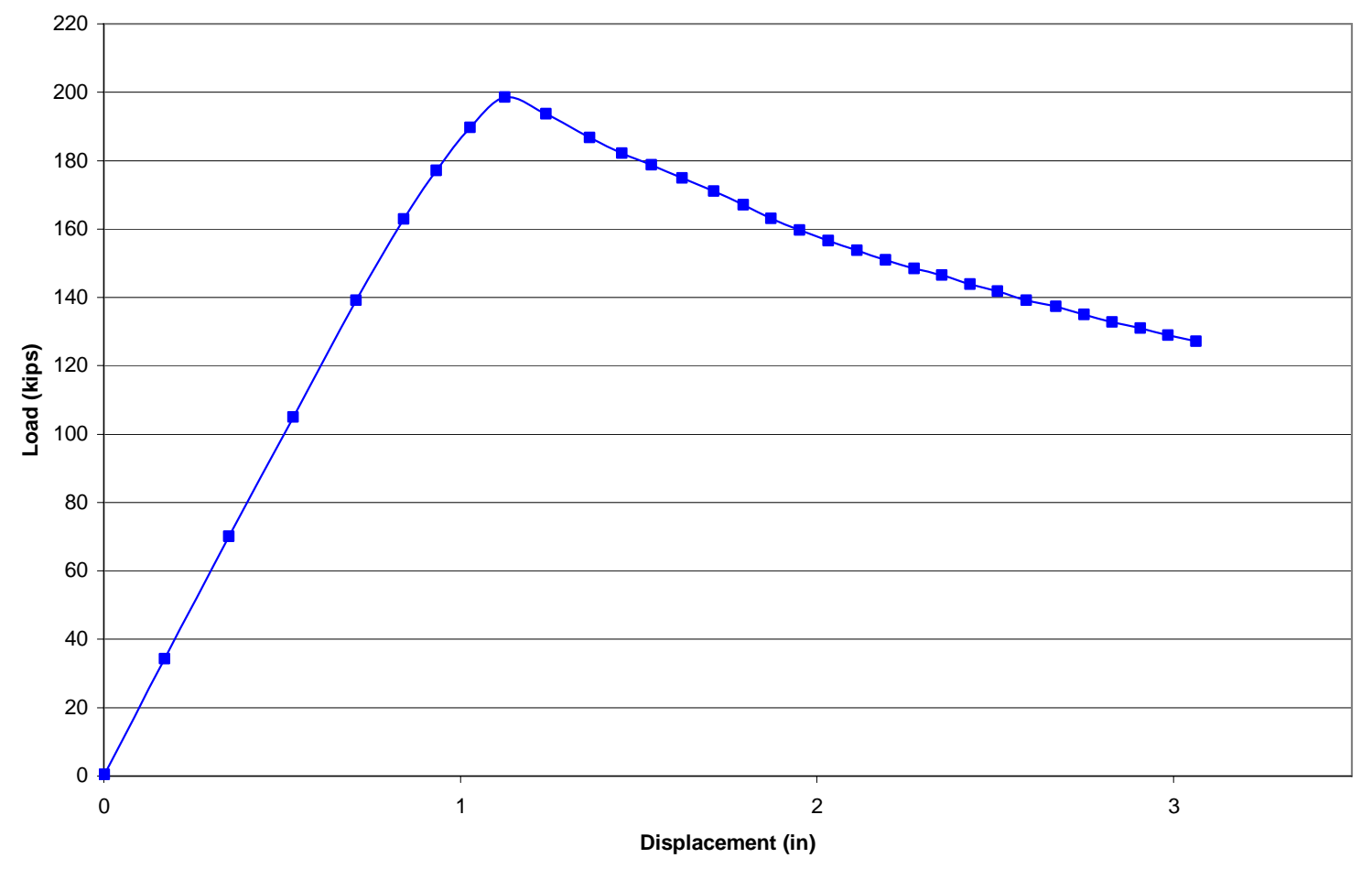

Figure 3-20. Load versus Displacement Data for Girder 3MG

\subsubsection{Girder $4 M G$}

The AASHTO (2004) Specifications result in a predicted moment capacity of $971 \mathrm{ft}$-kips for Girder 4MG, which is based on flange local buckling or lateral torsional buckling. This capacity differs from the experimental moment capacity of $978 \mathrm{ft}-\mathrm{kips}$ by only $0.7 \%$.

Figure 3-21 shows the deformed shape of Girder 4MG at the sensor locations, which are located approximately $4 \mathrm{in}$. from the point where the most severe girder deformations occurred. From the figure, lateral movement of the compression flange and deformation of the web are the most prominent deformations; however significant flange local buckling also occurred in the girder, but due to the small amplitude of this buckle, this deformation was not well captured by the LVDTs. Comparing Figs. 3-19 and 3-21, the influence of the longer bracing distance in Girder 4MG is shown to be increased lateral movement of the compression flange and portion of the web in compression. 


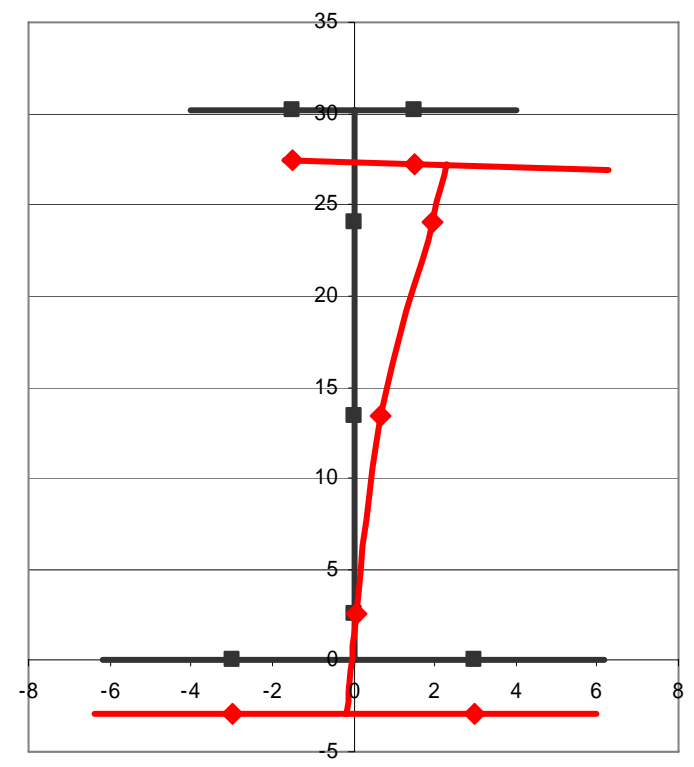

Figure 3-21. Deformed Shape of Girder 4MG

The influences of longer lateral bracing distance can also be seen by analyzing the load versus displacement graphs presented in Figs. 3-20 and 3-22. From Fig. 3-22, it can be determined that Girder 4MG has significantly less ductility than the girders previously discussed; at a load of 108 kips ( $90 \%$ of the maximum load), the deformation capacity is only 1.1. In other words, the deformation capacity decreases by $42 \%$ for Girder $4 \mathrm{MG}$ with an intermediate lateral bracing distance compared to Girder 3MG with a compact lateral bracing distance.

\subsubsection{Girder 5MG}

As shown by Table 3-8, the AASHTO (2004) Specifications over predict the capacity of Girder 5MG by $12.4 \%$. This predicted capacity is controlled by flange local buckling and lateral torsional buckling, where both failure modes predict equal capacity.

Figure 3-23 illustrates the deformed shape of girder 5MG, where the primary deformation is lateral movement of the compression flange accompanied by a small amount of rotation between the compression flange and web. However, a local buckle was also present in the compression flange of the girder, but was not well captured by the instrumentation because 


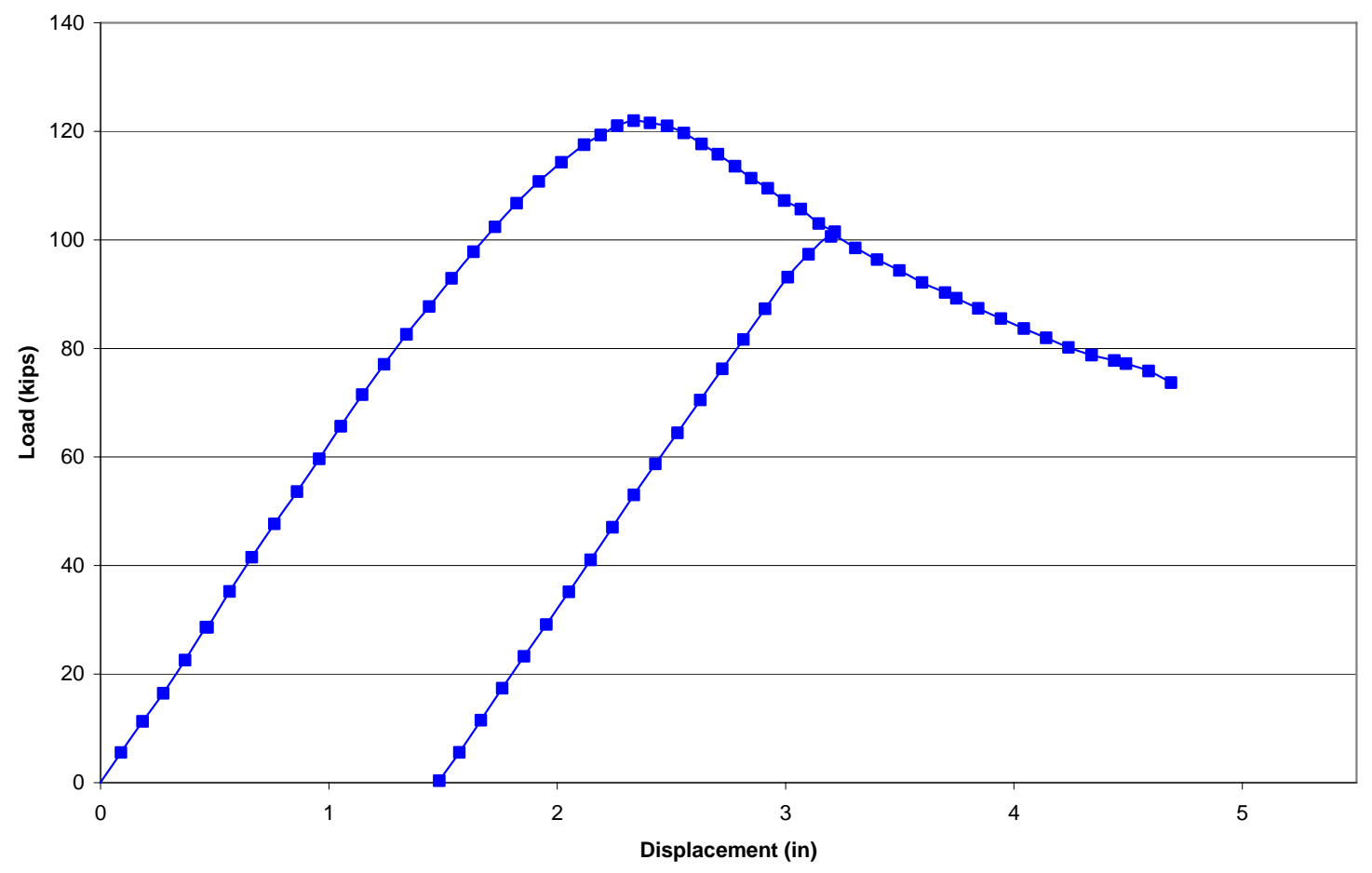

Figure 3-22. Load versus Displacement Data for Girder 4MG

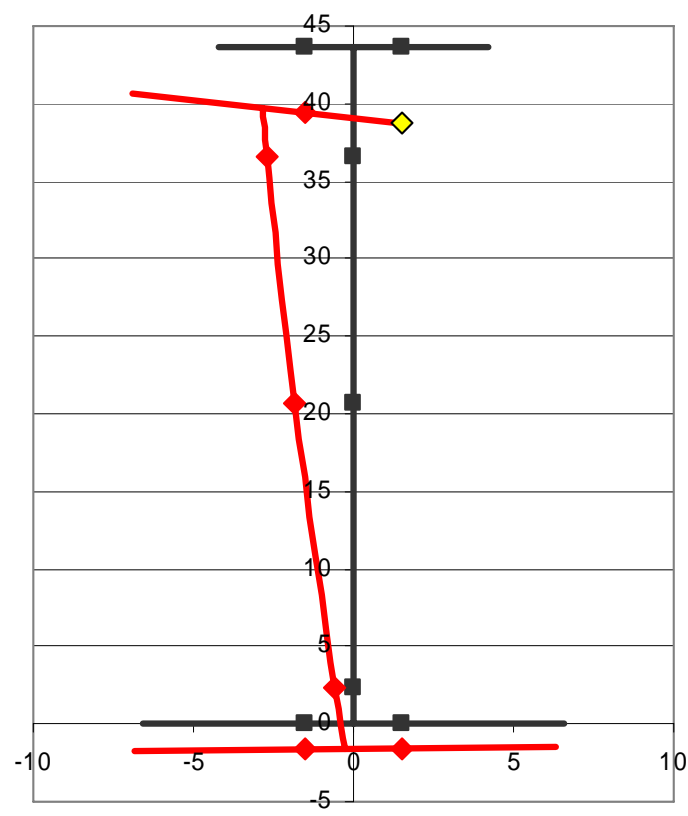

Figure 3-23. Deformed Shape of Girder 5MG 
the LVDTs were located 3 in. from the center of the flange buckle. Comparing Fig. 3-23 to Fig. 3-21, shows that the higher $\mathrm{D} / \mathrm{b}_{\mathrm{fc}}$ ratio used in Girder 5MG does not have an influence of the deformed shape.

The load versus displacement curve for Girder 5MG is shown below in Fig. 3-24. From this data it is determined that at a load of 163 kips ( $90 \%$ of the maximum load) the deformation capacity of the girder is 1.0. Comparing this data to the corresponding data for Girder 4MG (deformation capacity of 1.1), it is shown that the deformation capacity of the two girders are approximately equal. Thus it is concluded that the increased $\mathrm{D} / \mathrm{b}_{\mathrm{fc}}$ ratio of Girder $5 \mathrm{MG}$ does not have a significant influence on girder behavior.

\subsubsection{Girder 6MG}

The AASHTO (2004) equations also over-predict the capacity of Girder 6MG, as shown in Table 3-8. Specifically, the predicted capacity of $582 \mathrm{ft}$-kips is $12.2 \%$ higher than the

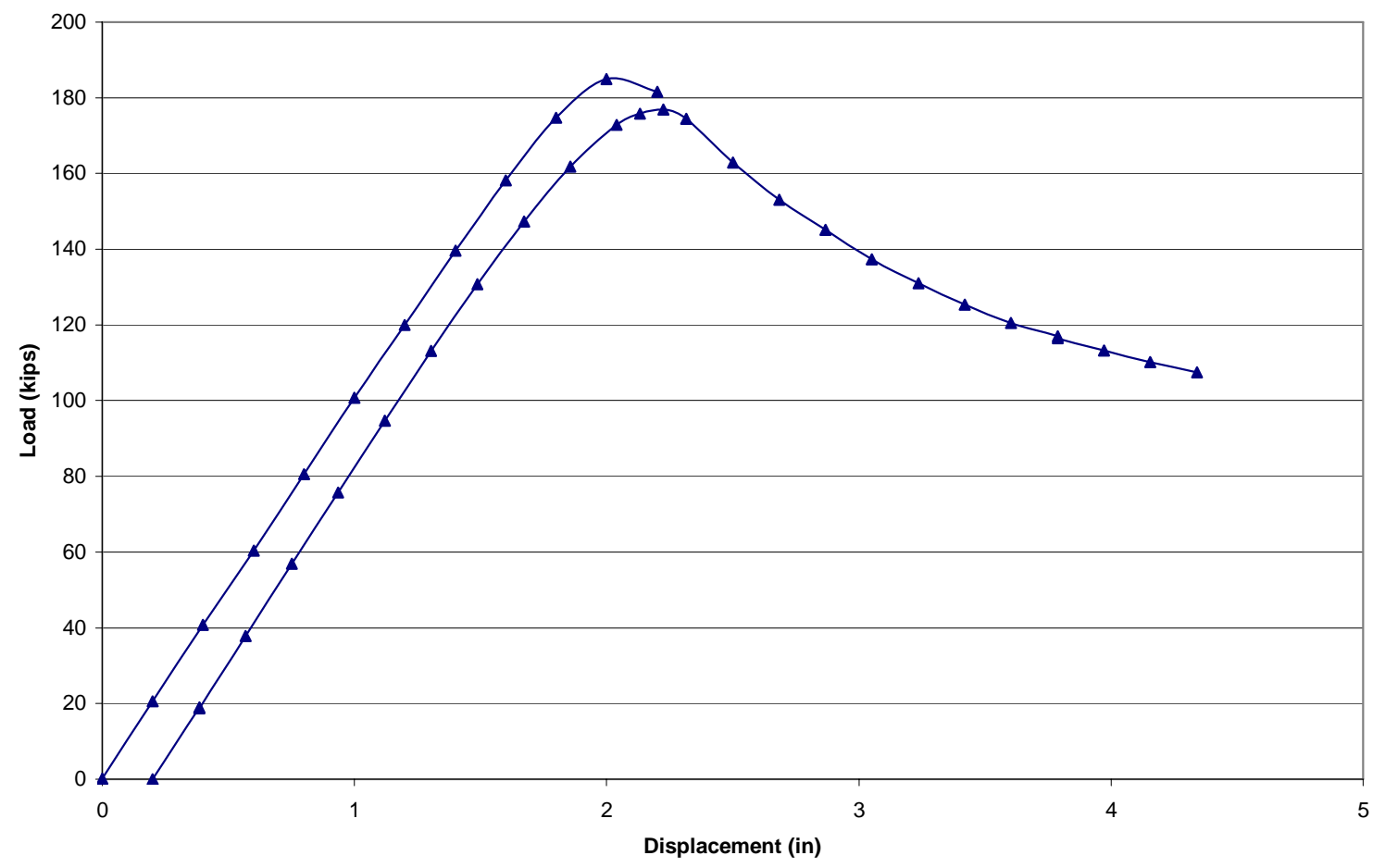

Figure 3-24. Load versus Displacement Data for Girder 5MG 
experimentally observed capacity of 519 ft-kips. The predicted capacity is controlled by flange local buckling.

Figure 3-25 shows the deformed shape of Girder 6MG. This deformed shape plot seems to depict a lateral torsional buckling failure mode, however the LVDTs were located approximately 6 in. from the location of the girder where the maximum deformations occurred. At this location, significant flange and web local buckling was also observed. Thus the deformed shape of the girder is as anticipated considering that all three slenderness ratios were maximized in this girder.

The load versus displacement data for Girder 6MG shown below in Fig. 3-26 indicates that this girder has the least ductility of all of the girders examined thus far, with the load dropping relatively quickly after the maximum load is attained. Computation of the deformation capacity of Girder 6MG supports this observation where at a load equal to 90\% of the maximum load (48 kips), the deformation capacity is determined to be 0.7 .

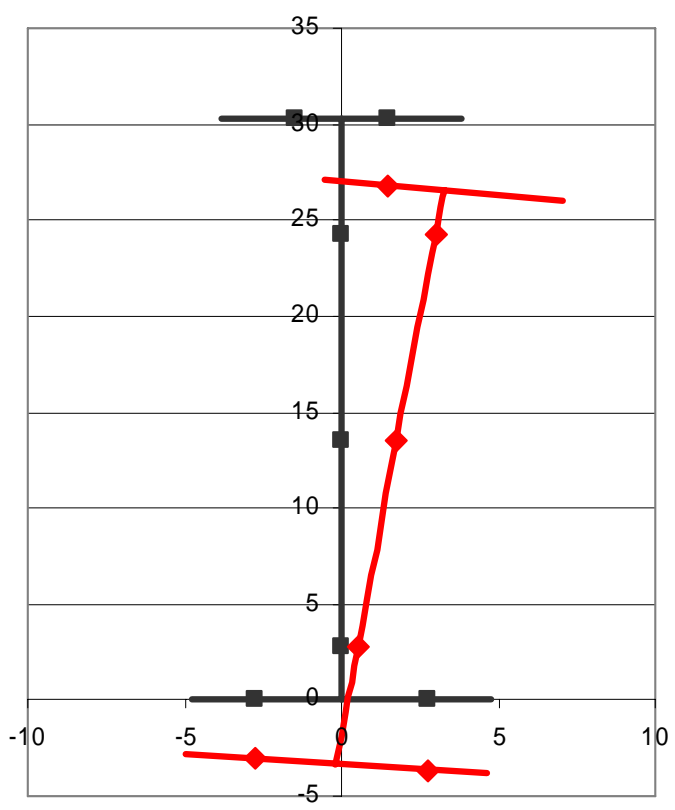

Figure 3-25. Deformed Shape of Girder 6MG 


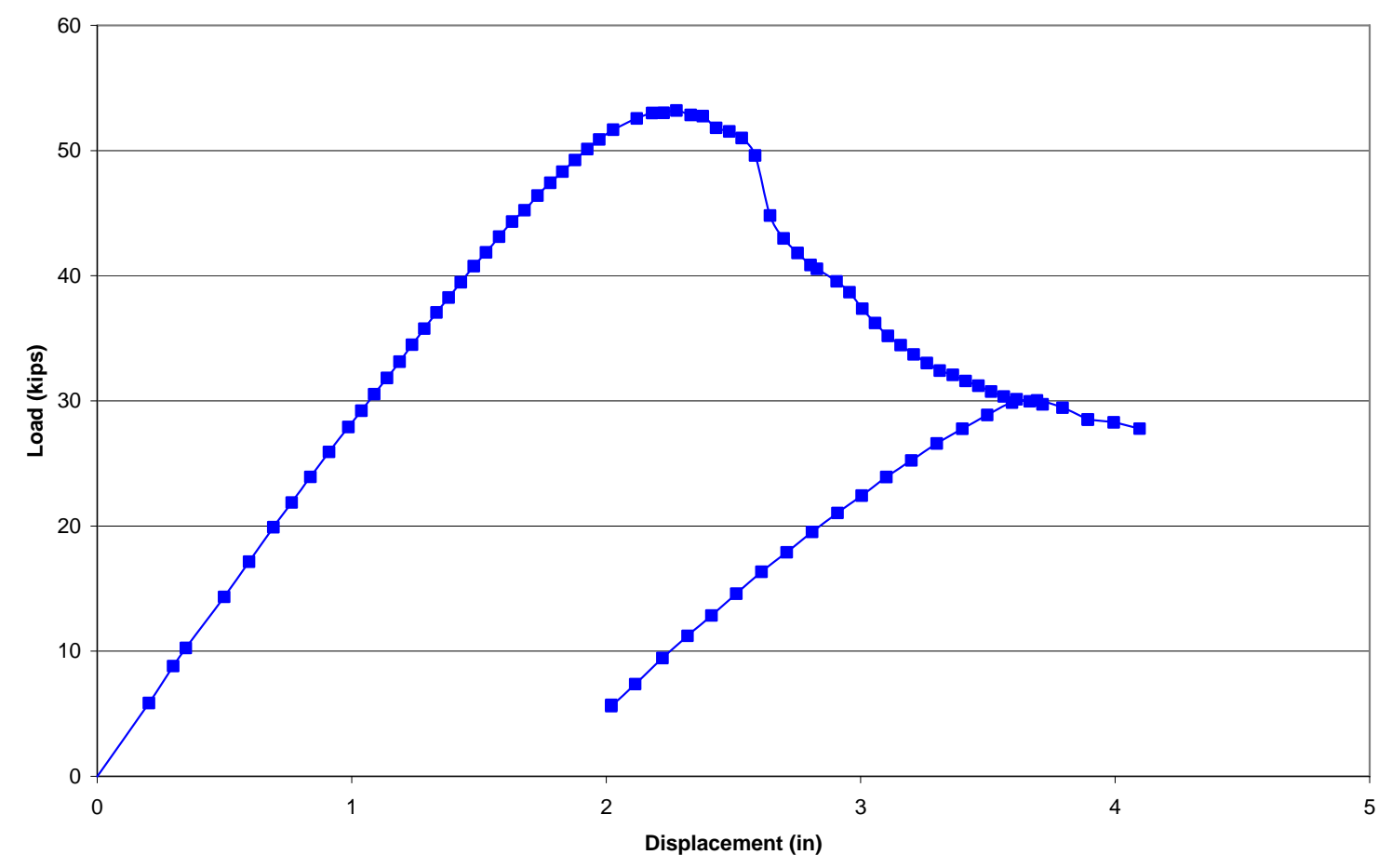

Figure 3-26. Load versus Displacement Data for Girder 6MG

\subsubsection{Girder 7MG}

The experimental and predicted moment capacities shown in Table 3-8 indicate that the AASHTO (2004) specifications over predict the moment capacity of Girder 7MG. Specifically, the capacity predicted from the specifications is equal to $1043 \mathrm{ft}$-kips, which is 9.7\% higher than the experimental capacity of $951 \mathrm{ft}-\mathrm{kips}$. Furthermore, the predicted capacity is based on both flange local buckling and lateral torsional buckling.

The graph on the left of Fig. 3-27 shows the girder displacements at the maximum load, where web buckling is the only deformation observed. Additionally, the graph on the right of Fig. 3-27 shows the displacement at the end of loading. Here it is shown that flange buckling also develops in the girder. However, this flange buckling is attributed to a consequence of the web buckling causing decreased restraint to the compression flange. This behavior is as anticipated for a girder with a compact flange and non-compact web. Girder 7MG also has an intermediate lateral bracing distance, however this distance results in predicted moment capacities that are the same as the predicted capacity for a compact 

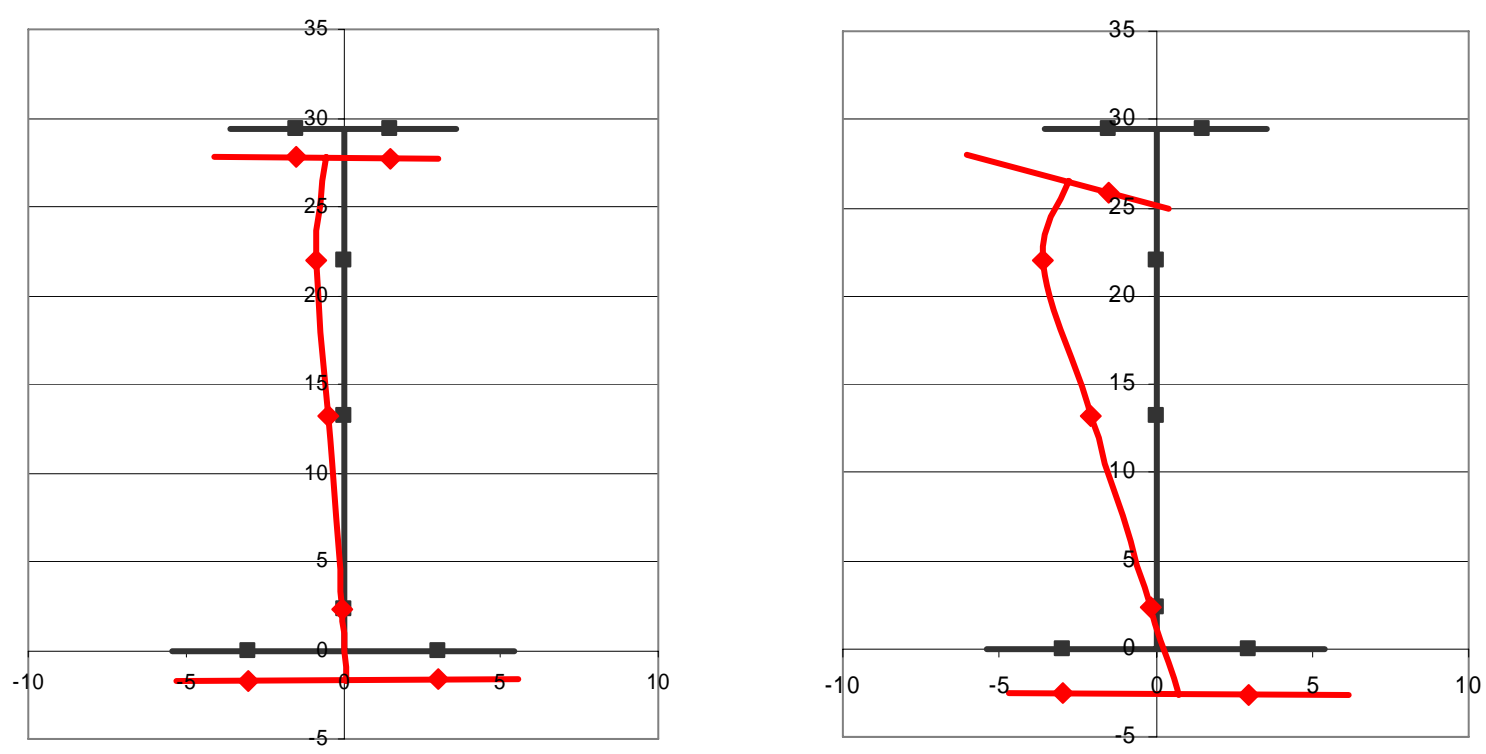

Figure 3-27. Deformed Shape of Girder 7MG

bracing distance due to moment gradient $\left(\mathrm{C}_{\mathrm{b}}\right)$ effects. It is noted that these deformed shapes differ from the deformed shape of Girder 4MG, where all three buckling modes were observed, although the two girders only differ in that Girder 7MG is a hybrid girder while Girder 4MG is a homogeneous Grade 50 girder.

Figure 3-28 indicates that Girder 7MG has a moderate amount of ductility. Evaluating the deformation capacity at 131 kips (90\% of the maximum load) gives a deformation capacity of 1.2. Comparing these results to the deformation capacity of Girder 4MG (1.1), it is determined that the results for the two girders are nearly equal, although slightly higher deformation capacity results for the hybrid girder.

\section{$\underline{\text { 3.5.8 Girder } 8 M G}$}

As shown in Table 3-8, the specifications predict the moment capacity of girder 8MG within an acceptable level of accuracy. Specifically, the predicted capacity of $777 \mathrm{ft}-\mathrm{kip}$, which is based on the flange local buckling equations, is $4.0 \%$ conservative compared to the actual girder capacity of 809 ft-kips. 


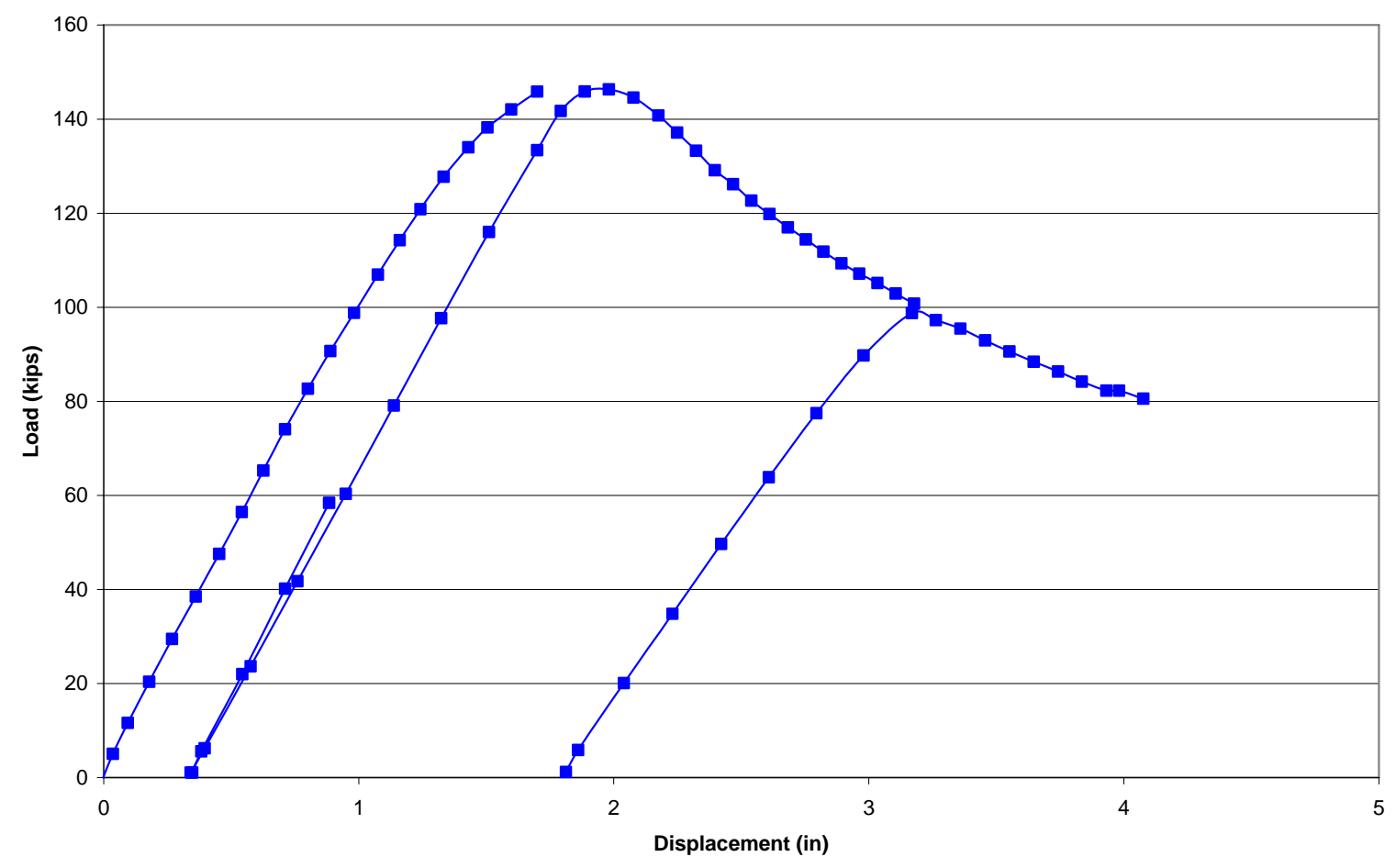

Figure 3-28. Load versus Displacement Data for Girder 7MG

The deformed shape of Girder 8MG is shown below in Fig. 3-29. This figure indicates that the primary girder deformations are web deformations and lateral movement of the compression flange. However, in actuality, the most significant deformations were a flange buckle with amplitude of 3 in. that developed in the girder 15 in. from the LVDT locations. Comparing the deformed shape of Girder 8MG to Girder 7MG, flange buckling only occurs in Girder 7MG as a consequence to web buckling, while flange local buckling is the predominant failure mode in Girder 8MG. Thus, the deformed shapes of these two girders are as expected given that the two girders are similar except that Girder 8MG has a slender flange, while Girder 7MG has a compact flange.

Figure 3-30 shows the load versus displacement relationship for Girder 8MG, where it is observed that the load rapidly decreases once the maximum moment capacity is attained. 


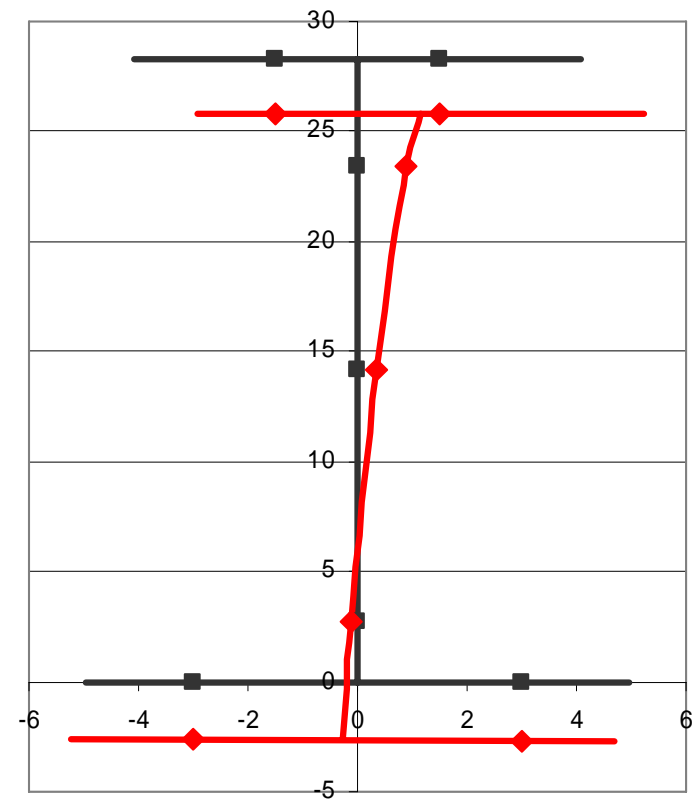

Figure 3-29. Deformed Shape of Girder 8MG

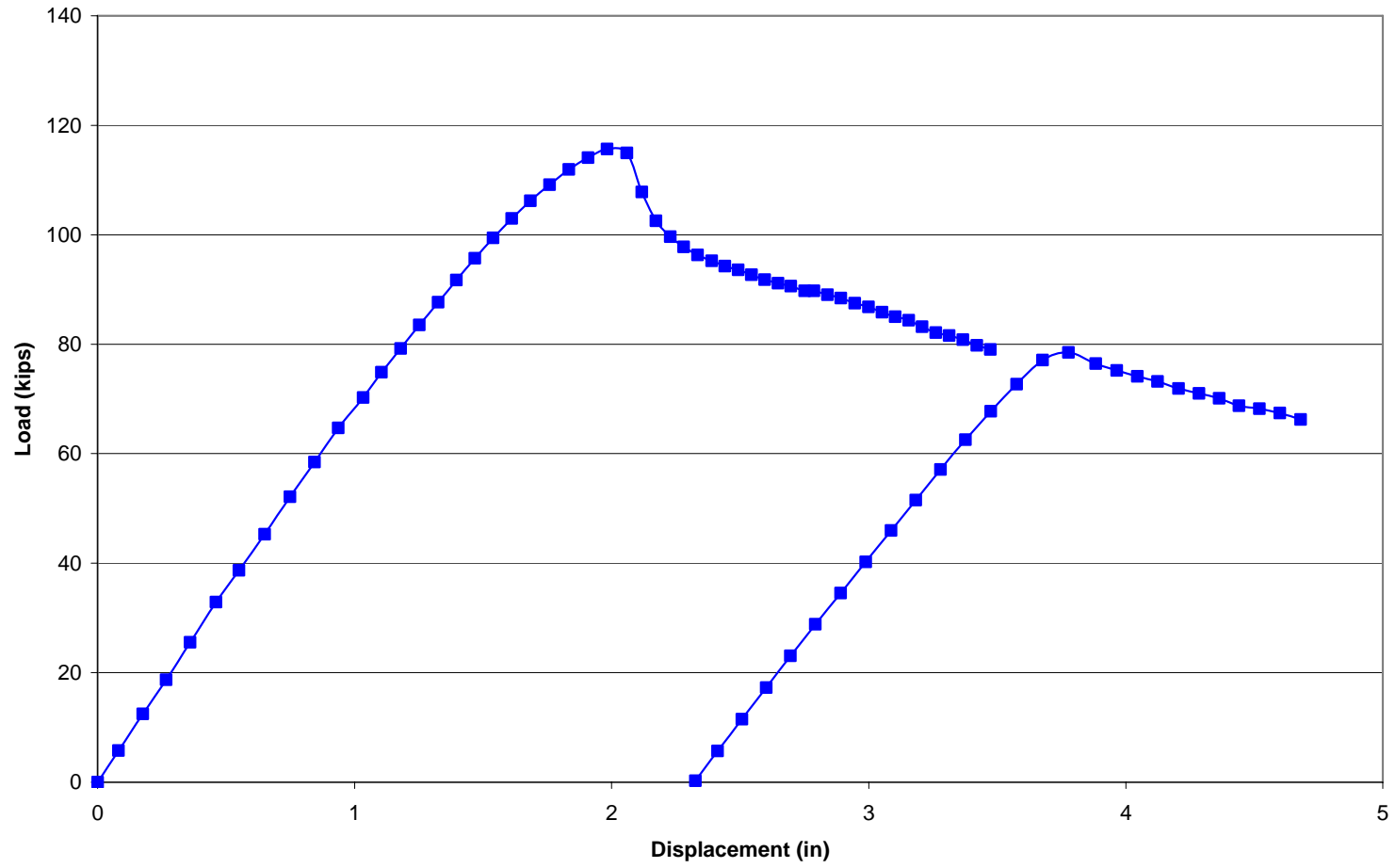

Figure 3-30. Load versus Displacement Data for Girder 8MG 
Computation of the deformation capacity at a load of 104 kips ( $90 \%$ of the maximum load) gives a result of 0.7 . Thus, compared to Girder $7 \mathrm{MG}$ with a deformation capacity of 1.2, the increased flange slenderness of Girder 8MG results in a significant decrease in deformation capacity.

\subsubsection{Girder $9 M G$}

The specifications predict a moment capacity that is highly unconservative for Girder 9MG. Here the controlling capacity is based on both flange local buckling and lateral torsional buckling and is equal to $1060 \mathrm{ft}$-kips, which is $18.2 \%$ higher than the actual capacity of 897 ft-kips.

Figure 3-31 shows the deformed shape of the girder is dominated by web local buckling and lateral movement of the compression flange. This is also the predicted behavior of the girder as the girder has a non-compact web, non-compact brace spacing and a compact flange. Girders 7MG and 9MG differ only in that a shorter bracing distance is used in Girder 7MG and comparing these deformed shape of Girder 9MG to the final deformed shape of Girder 7MG shown on the right side of Fig.3-27, it is observed that the two deformed shapes are similar, although more flange deformation is observed in Girder 7MG.

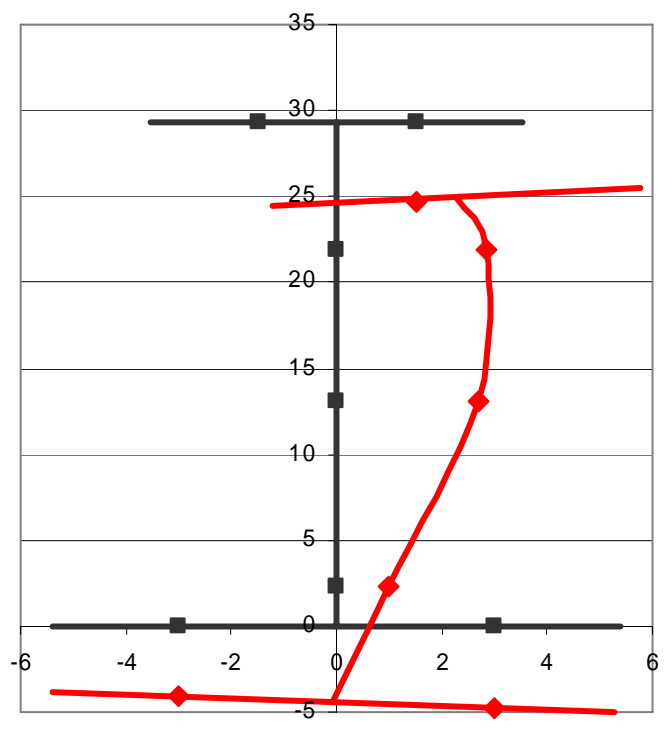

Figure 3-31. Deformed Shape of Girder 9MG 
Figure 3-32 shows the load versus displacement data for girder 9MG, where it is observed that the load quickly reduces after the maximum load is reached and then a more gradual unloading occurs. At $90 \%$ of the maximum load, 85.2 kips, the slope of the unloading curve is still relatively steep, resulting in a deformation capacity of only 0.4. Comparing this deformation capacity to the deformation capacity of Girder 7MG (1.2), it is concluded that the increased lateral bracing distance of Girder 9MG significantly reduces the deformation capacity. Furthermore, in comparison to the deformation capacity of Girder 8MG (0.7), which differs from Girder 7MG in that a slender flange is used, it is concluded that, for the values investigated, increased lateral bracing distance is more detrimental to deformation capacity than increased flange slenderness.

\subsubsection{Girder 10MG}

Table 3-8 shows that the AASHTO (2004) Specifications are 4.5\% conservative for Girder 10MG. The predicted capacity of $796 \mathrm{ft}$-kips is based on flange local buckling compared to the experimental capacity of $834 \mathrm{ft}-\mathrm{kips}$.

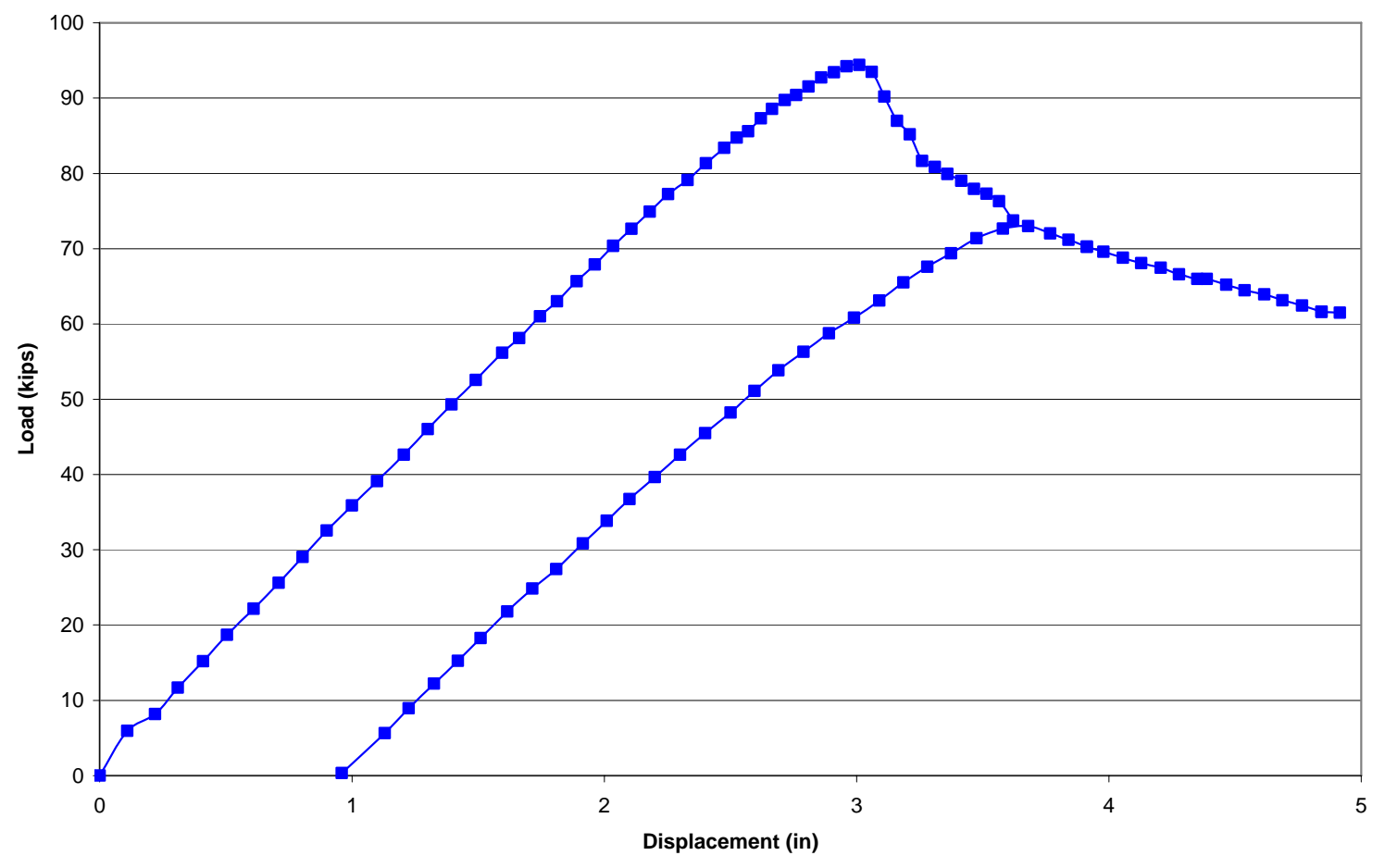

Figure 3-32. Load versus Displacement Data for Girder 9MG 
The deformed shape of Girder 10MG is shown in Fig. 3-33. Here the primary deformations are observed to be lateral movement of the compression flange and web deformation; however, significant flange local buckling and web local buckling also occur in the girder at a location $15 \mathrm{in}$. from the LVDT locations, and thus these deformations are not reflected in Fig. 3-33. Comparing these deformed shapes to those of Girder 8MG (which is similar to Girder 10MG except a longer bracing distance is used in Girder 10MG) it is shown that the deformed shapes of the two girders are very similar. Girder 10MG is also similar to Girder 9MG except that a slender flange is used in Girder 10MG. By comparing Figs. 3-33 and 331 it is shown that with the compact flange and slender web, web buckling predominates; however, for a slender web and slender flange, flange buckling and web buckling occur at more similar magnitudes. Girder 10MG is a hybrid version of Girder 6MG and comparing Figs. 3-33 and 3-25, it is shown that the deformed shapes of these two girders are similar, but increased web deformations result in the hybrid girder.

The load versus displacement data of Girder 10MG is shown in Fig. 3-34. It is shown here that this girder also has relatively low ductility. Computing the deformation capacity of the girder at 77 kips ( $90 \%$ of the maximum load) gives a result of 0.5 . Comparing these results

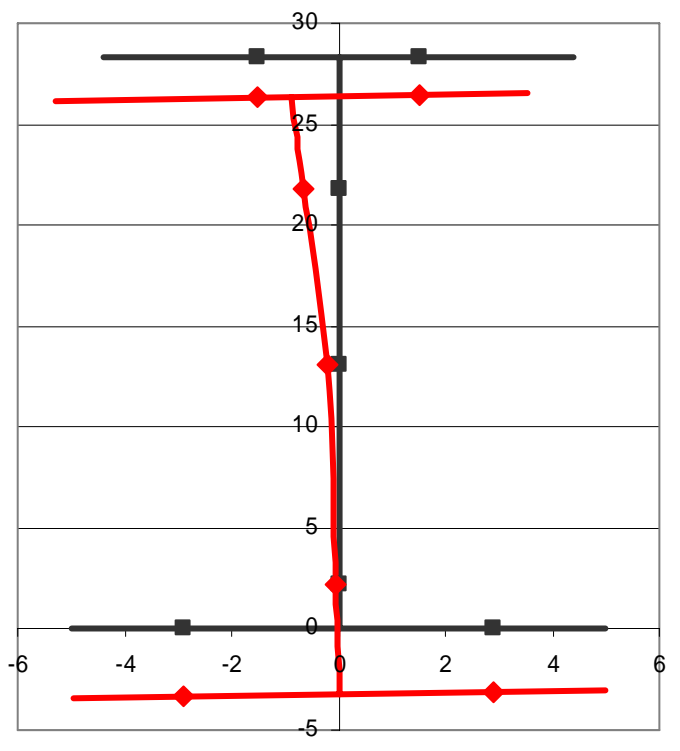

Figure 3-33. Deformed Shape of Girder 10MG 


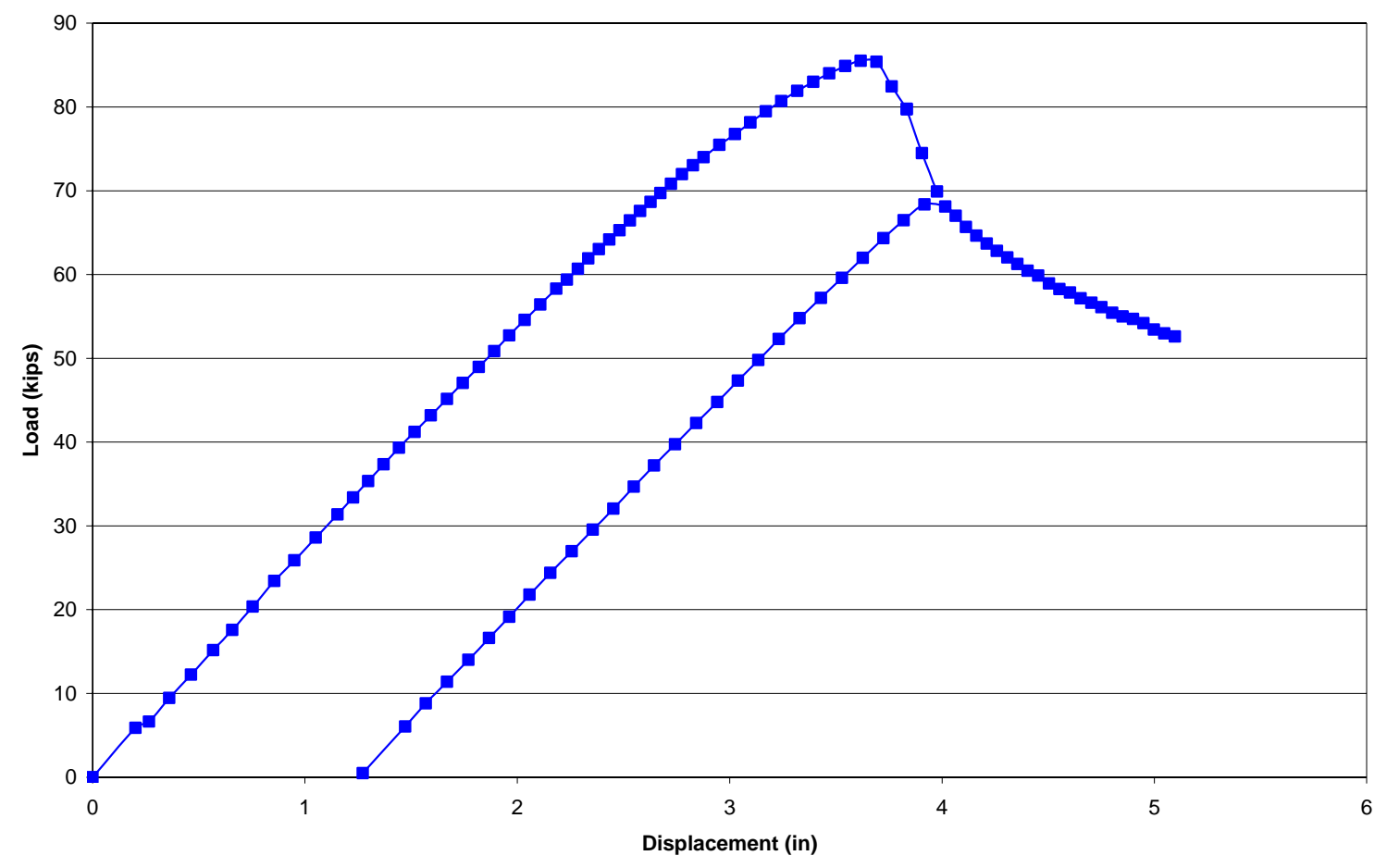

Figure 3-34. Load versus Displacement Data for Girder 10MG

to the deformation capacity of Girder 8MG at the same normalized load (0.7), it is shown that the longer bracing distance slightly reduces the deformation capacity. Similarly, comparison with the deformation capacity of Girder 9MG (0.4), it is shown that the increased flange slenderness of Girder 10MG does not have a detrimental influence on the girder ductility. Lastly, comparing the deformation capacities of Girder 10MG (0.5) and Girder 6MG (0.7), it is seen that the hybrid girder has slightly less deformation capacity.

\subsubsection{Girder 11MG}

The specifications predict a moment capacity of 1317 ft-kips for Girder 11MG. This capacity is $17.1 \%$ higher than the experimental capacity of $1125 \mathrm{ft}$-kips and is based on the lateral torsional buckling equations.

The deformed shape of Girder 11MG consists of a combination of lateral torsional buckling and web buckling, as shown in Fig. 3-35. This deformed shape consisting predominantly of web buckling in combination with lateral buckling is as expected behavior for this girder 


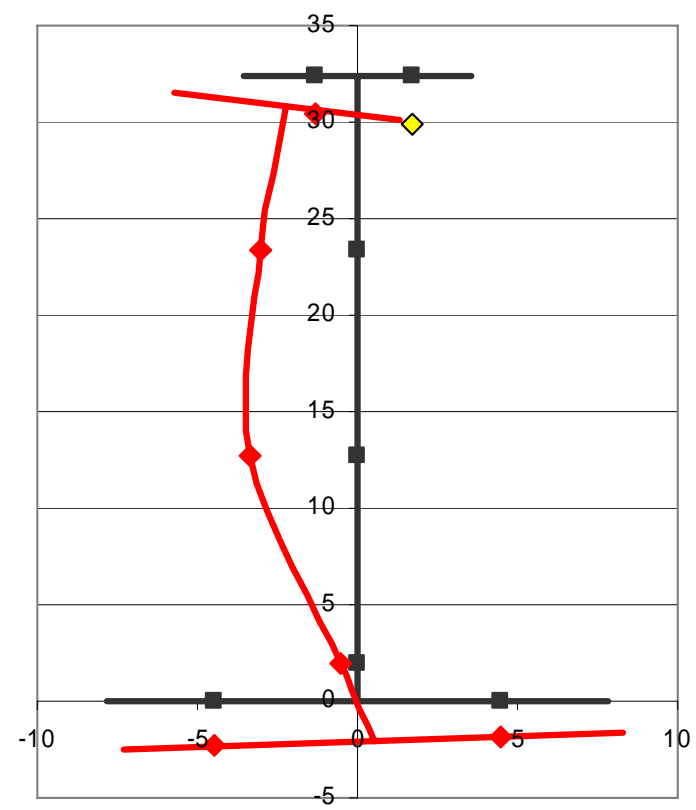

Figure 3-35. Deformed Shape of Girder 11MG

with non-compact web slenderness, non-compact bracing distance, and compact flanges. Girder 11MG is similar to Girder 9MG except that the web of Girder 11MG is comprised of steel having a higher yield strength. Thus, the deformed shapes of the two girders (shown in Figs. 3-35 and 3-31) are similar, but there is less web buckling in Girder 11MG.

Figure 3-36 illustrates the load versus displacement data for Girder 11MG. It is shown here that there is a comparatively small amount of ductility for this girder. Computing the deformation capacity at 107 kips (90\% of the maximum load) gives a deformation capacity of 0.5 , which closely corresponds to the deformation capacity of the similar hybrid girder, Girder 9MG, which had a deformation capacity of 0.4 .

\subsubsection{Girder $12 M G$}

The maximum moment capacity of Girder 12MG is $1445 \mathrm{ft}-\mathrm{kips}$, as shown in Table 3-8. The specifications predict a moment capacity 9.6\% higher than this, $1584 \mathrm{ft}-\mathrm{kips}$, based on flange local buckling. 


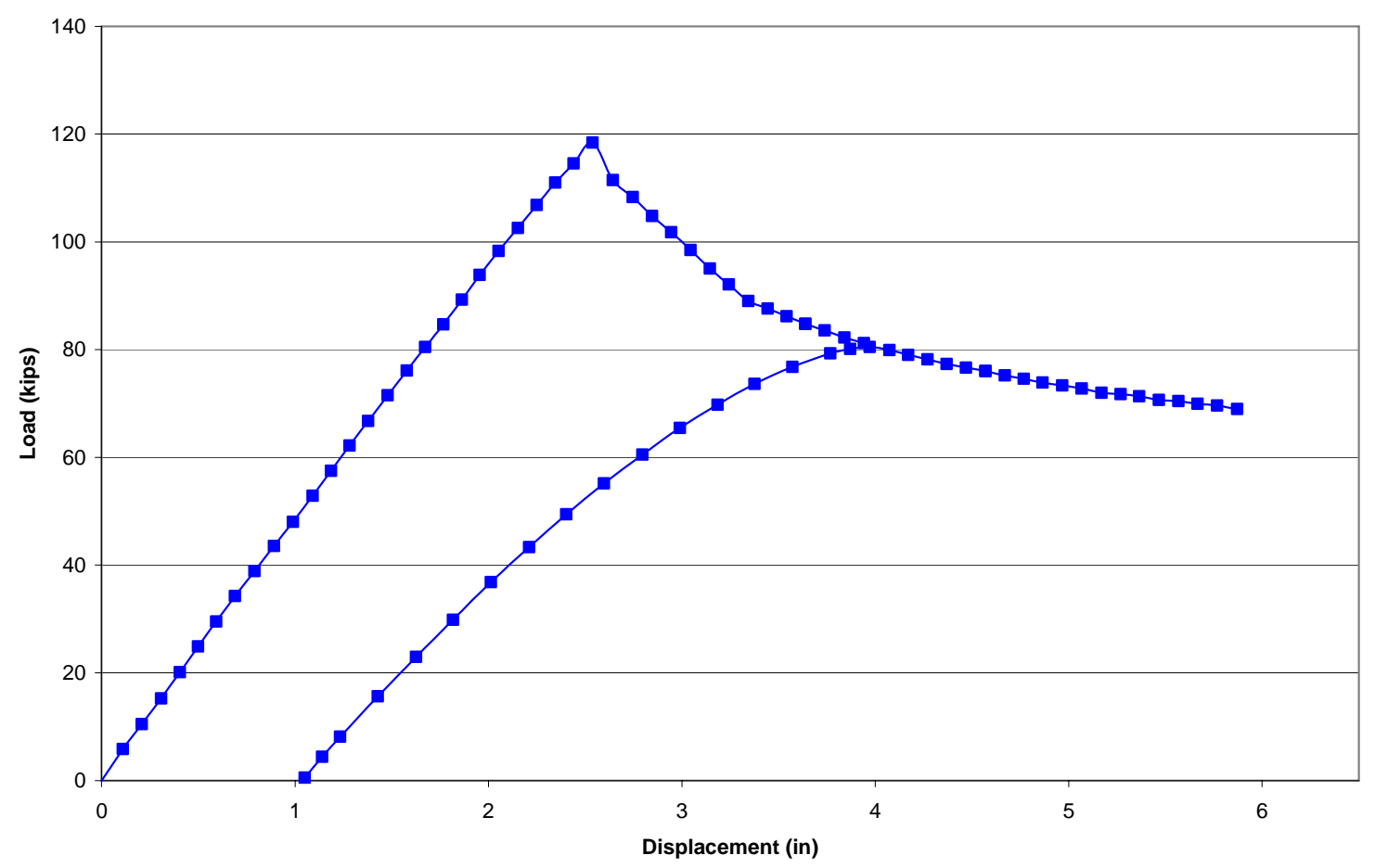

Figure 3-36. Load versus Displacement Data for Girder 11MG

The deformed shape of Girder 12MG is shown in Fig. 3-37. As shown in the figure, the main type of deformation in Girder 12MG consists of lateral movement of the compression flange in combination with rotation of the compression flange about the web. Girder 12MG is similar to Girder 11MG except that Girder 12MG has a slightly more slender compression flange. The influence of the higher compression flange slenderness is shown to be that flange and web buckling occur at approximately the same magnitude when the flange slenderness is increased, compared to Girder 11MG where web buckling is prominent. Girder 12MG is also similar to Girder 9MG except steel of a higher yield stress is used in Girder 12MG. Comparing Fig. 3-37 and 3-31, it is observed that the influence of the higher web strength is less bending in the compression region of the web. Girder 12MG is also similar to Girder 6MG, but with two differences, which are a higher yield strength of $70 \mathrm{ksi}$ used in Girder 12MG compared to a yield strength of $50 \mathrm{ksi}$ in Girder 6MG and a lower flange slenderness is used in Girder 12MG. Comparing the deformed shapes of these two girders shows that the deformed shapes of the two girders are very similar, especially when it 


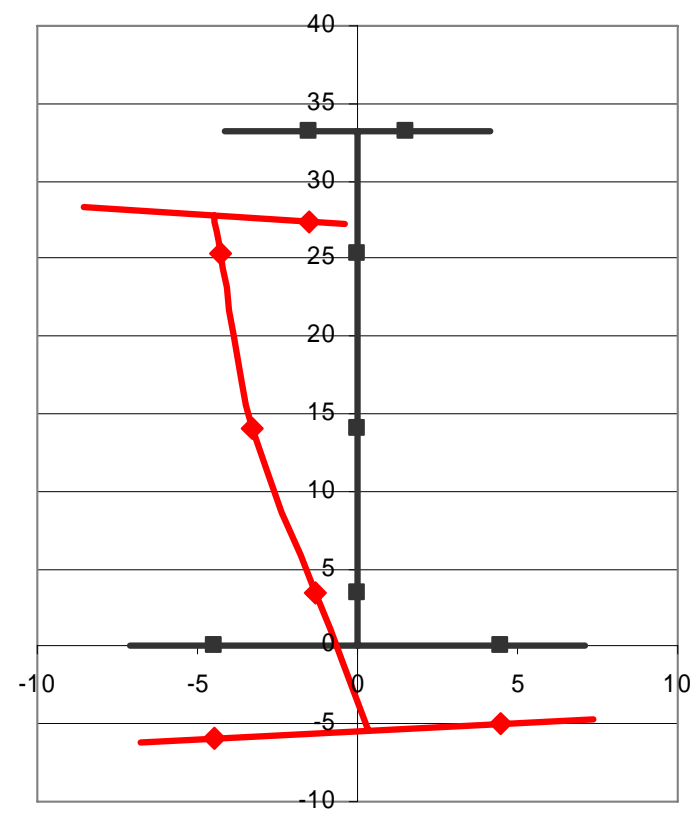

Figure 3-37. Deformed Shape of Girder 12MG

is considered that the deformed shape of Girder 6MG shown in Fig. 3-25 does not capture the web and flange deformations that occurred away from the LVDT locations in this girder.

Figure 3-38 shows the load versus displacement data for Girder 12MG. The ductility of this girder is also shown to be relatively limited, as the load rapidly descends after reaching the maximum load. The deformation capacity of this girder is computed to be 0.6 at a load of 137 kips (90\% of the maximum load). This closely corresponds to the deformation capacity of similar girders such as Girder 11MG (0.5), Girder 9MG (0.4), and Girder 6MG (0.7).

\subsubsection{Summary of Experimental Results}

\subsubsection{Moment Capacity}

The above comparisons of the experimental moment capacity to that predicted by the AASHTO (2004) Specifications reveal several trends. First, for the compact girders included in this study (Girders 1MG and 2MG) the specifications give accurate predictions of the moment capacities (within 3\% of the experimental capacities). 


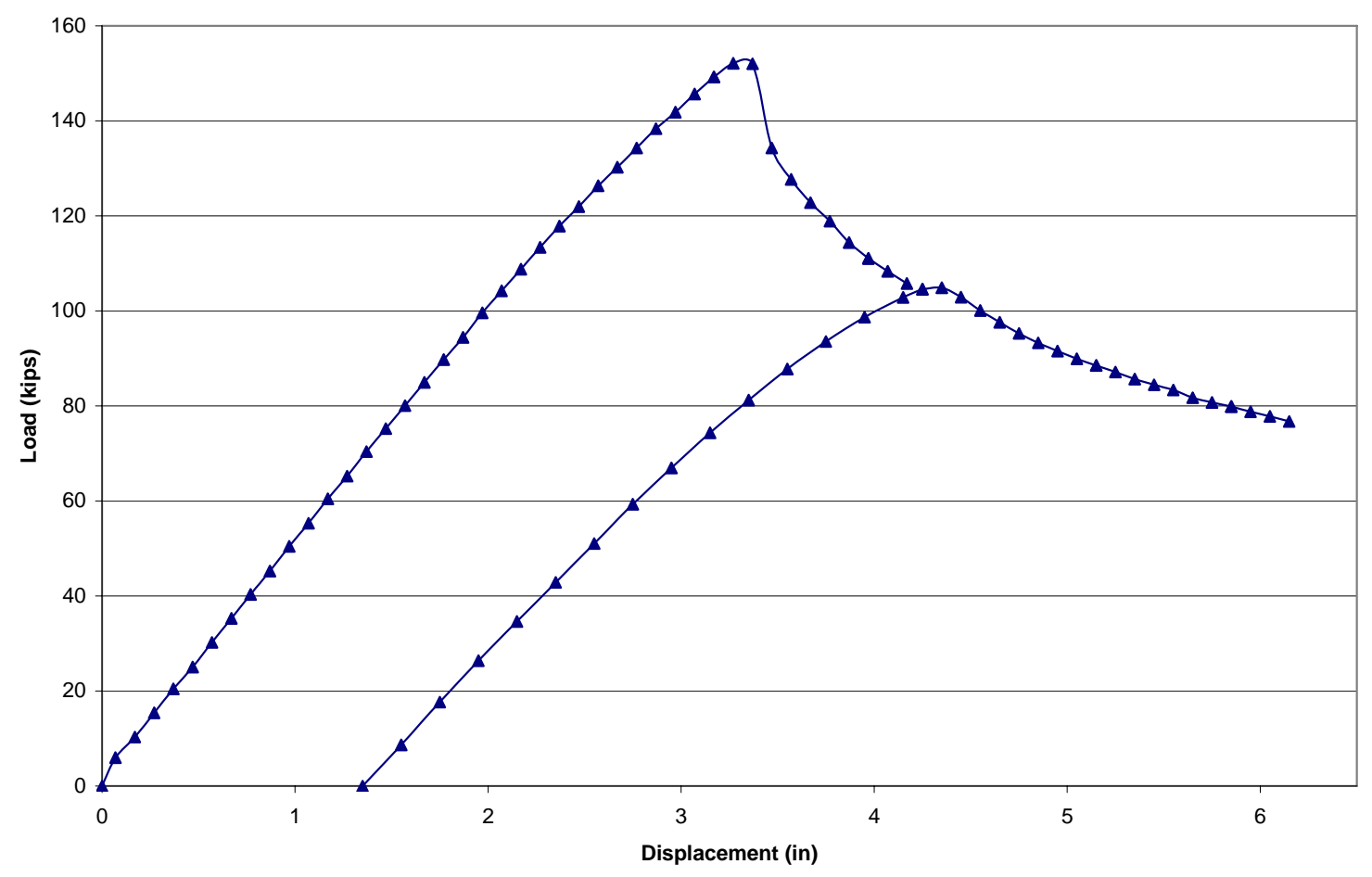

Figure 3-38. Load versus Displacement Data for Girder 12MG

Three of the girders in this work (Girders 3MG, 4MG, and 5MG) were nominal Grade 50 girders with non-compact webs and compact flanges. For two of these, the AASHTO Specifications give accurate predictions of the experimental capacity. For the third Grade 50 girder with a non-compact web and compact flange, the predicted capacities are unconservative by $12.4 \%$. For the Grade 50 girder with a non-compact web, non-compact compression flange, and non-compact lateral bracing distance (Girder 6MG), the specifications are unconservative.

The predicted moment capacities are also unconservative for the three girders with compact flanges incorporating Grade 70 steel (Girders 7MG, 9MG, and 11MG), but conversely, are conservative for both girders with slender flanges incorporating Grade 70 steel (Girders 8MG and 10MG). 


\subsubsection{Deformed Shape}

The deformed shape of each girder was presented above and comparisons were made between these deformed shapes and those of similar girders. In general, it may be concluded that the deformed shapes observed in the tests were as anticipated based on the slenderness properties of each girder.

\subsubsection{Deformation Capacity}

The previously reported deformation capacities of each girder are summarized below in Table 3-9. Analyzing these results with respect to the individual properties of each girder reveals that the most significant trend in these results is that the deformation capacity decreases as the lateral bracing distance of the girder increases. Girders with a compact lateral bracing distance have a deformation capacity between 1.9 and 2.3; girders with an intermediate lateral bracing distance have a deformation capacity between 0.8 and 1.2 and; girders with a non-compact lateral bracing distance have a deformation capacity between 0.4 and 0.7. Thus, once the compact bracing limit is exceeded, significantly lower deformation capacities result.

The experimental data also suggest that for girders with intermediate bracing distances (Girders 7MG and 8MG), the increase from a compact to a slender flange results in decrease in deformation capacity from 1.2 to 0.7. However for girders with maximum bracing

Table 3-9. Deformation Capacity at $\mathrm{M} / \mathrm{M}_{\mathrm{MAX}}=0.9$

\begin{tabular}{|cc|}
\hline Specimen & $\begin{array}{c}\text { Deformation } \\
\text { Capacity }\end{array}$ \\
\hline \hline $1 \mathrm{MG}$ & 2.0 \\
$2 \mathrm{MG}$ & 2.3 \\
$3 \mathrm{MG}$ & 1.9 \\
$4 \mathrm{MG}$ & 1.1 \\
$5 \mathrm{MG}$ & 1.0 \\
$6 \mathrm{MG}$ & 0.7 \\
$7 \mathrm{MG}$ & 1.2 \\
$8 \mathrm{MG}$ & 0.8 \\
$9 \mathrm{MG}$ & 0.4 \\
$10 \mathrm{MG}$ & 0.5 \\
$11 \mathrm{MG}$ & 0.5 \\
$12 \mathrm{MG}$ & 0.6 \\
\hline
\end{tabular}


distances (Girders 9MG through 12MG), variable flange slenderness does not have an appreciable influence of deformation capacity.

Based on the experimental data, a clear trend can not be determined between deformation capacity and the other variables investigated (web slenderness, material properties, $\mathrm{D} / \mathrm{b}_{\mathrm{fc}}$ ratio). The influences of these variables will be further explored through the FEA presented in Chapter 5. 


\section{Chapter 4: NONLINEAR FEA MODELING PROCEDURES}

This chapter will discuss the methods and procedures employed to conduct nonlinear FEA in this research. In summary, FEA was performed using commercially available FEA software, namely ABAQUS version 6.3.1. Initially, FEMAP software (version 8.3) was used to create the geometry (nodes and elements) for each girder being analyzed. FEMAP is then capable of writing this information in the form of an ABAQUS input file. This input file is then modified by the user to include material, load, and analysis technique information. Additionally, the node coordinates in the input file are modified to account for initial geometric imperfections using a FORTRAN program specially created for this purpose. The new input file and a user-defined sub-routine specifying residual stresses are then analyzed by ABAQUS using the modified Riks method. Thorough details of these procedures are presented herein. The reader will notice that throughout this chapter reference is made to specific commands used in ABAQUS; these are given by the notation *command.

\subsection{Mesh Density}

Initial considerations that must be correctly addressed are element size and mesh density. The selection of relatively large elements will result in unrealistically low predicted strengths due to the effects of stress concentrations, while relatively small elements can cause an overestimation of the energy dissipation capacity (Bazant and Cedolin 1980). By selecting the appropriate mesh density, these situations will be avoided, and accurate results can be obtained. Previous research by Yang (2004) has evaluated the ideal mesh density for steel Igirders of the type investigated in this study. This assessment included an evaluation of the accuracy and processing time for models with three different mesh densities: a relatively course mesh with 4 elements across the width of each flange and 6 elements throughout the height of the web, an intermediate mesh density with 6 elements across the width of the flange and 10 elements throughout the height of the web, and a fine mesh density with 10 elements across the flange width and 20 elements through the web height. Yang concluded that the ideal mesh density was the combination of 10 elements across the flange width and 
20 elements throughout the web height, which resulted in less than $1 \%$ error compared to selected experimental results. Thus, this same element size is used in the current research.

Furthermore, the aspect ratio is minimized (made closest to 1) to the extent possible. Using the mesh density discussed above, there are twice as many elements in the web as there are in each flange. However, the web height is typically three to four times the width of the compression flange. Because it is desirable for the web and flange elements to have equal lengths so that these elements will share coincident nodes, it is not possible to choose the element length so that the web and flange will both have an aspect ratio of 1 . Instead, an element length is selected that gives an equal aspect ratio in the compression flange and the web, which typically results in an aspect ratio of approximately 1.4 for all elements.

\subsection{Element Selection}

ABAQUS/Standard provides the user with a large number of available element types. Therefore, it is necessary to initially investigate the suitability of a selected element type for the given problem. As proven by several researchers (White et. al. 1987; Huang 1995; Barth 1996; Earls and Shah 2000), shell elements are both sufficient and necessary for modeling the physical behavior of the type of plate girders analyzed in this study.

\subsubsection{Element Naming Convention}

The element naming convention used in ABAQUS conveys the key attributes of the element. For example, the first letter of the element name refers to the general element type, with "S"

representing a shell element. This is followed by a number indicating the number of nodes in the element. This character may be followed by the letter "R", which indicates that reduced integration is used. Lastly, a " 5 " at the end of an element name indicates that the element only contains five degrees of freedom (three translations and two in-plane rotations), otherwise all six degrees of freedom are considered.

\subsubsection{General-Purpose Shell Elements}

Previous research on the performance of negative bending regions of steel I-girders has shown that the S4R element results in the most accurate prediction of actual girder behavior 
(Yang 2004). S4R is a 4-node general-purpose shell element. These elements are intended to provide robust, accurate solutions for both thin and thick shells, using classical (Kirchhoff) shell theory when appropriate for relatively thin shells and thick (Mindlin) shell theory as the shell thickness increases. These elements allow for finite membrane strains and rotations of the shell. Therefore, they are suitable for large-strain analysis involving inelastic deformation of materials with nonzero effective Poisson's ratio. These elements allow for change in shell thickness as a function of the membrane strain. Transverse shear deformation is also included. These elements are not subject to hourglass effects or transverse shear locking.

Other popular shell elements available in the ABAQUS element library are S4, S4R5, and S9R5. As suggested by the element labels, the difference between the S4 and S4R elements is the number of integration points used in the analysis; "full" integration is used for S4 elements, while "reduced" integration is used for S4R elements. In full integration, a sufficient number of integration points is selected such that integration of the element stiffness matrix is exact, i.e., for first order elements, such as S4, each element has four integration points. However, reduced integration elements use one order less than that required for full integration of the element stiffness matrix. Thus, for a S4R element only one integration point is used to form the element stiffness matrix. However, the mass matrix and force matrix are still integrated exactly. Displacements are then computed at each node. Linear interpolation is then used to determine the displacements at other locations using

$$
\mathrm{u}=\sum \mathrm{f}_{\mathrm{i}} \mathrm{u}_{\mathrm{i}}
$$

where $u_{i}$ are the displacements at each node and $f_{i}$ is the following shape function

$$
f_{i}=\frac{1}{4}\left(1+\xi_{0}\right)\left(1+\eta_{0}\right)
$$

and $\xi_{0}$ and $\eta_{0}$ refer are expressed as

$$
\begin{aligned}
& \xi_{0}=\xi_{i} \xi \\
& \eta_{0}=\eta_{i} \eta
\end{aligned}
$$


where $\xi$ and $\eta$ refer to the natural coordinates of each node, as illustrated in Fig. 4-1. For example, at node 1 , $\xi$ equals -1 and $\eta$ equals -1 . Thus, $\xi_{0}$ equals - $\xi$ and $\eta_{0}$ equals $-\eta$ and Eq. 4.2 becomes

$$
\mathrm{f}_{1}=\frac{1}{4}(1-\xi)(1-\eta)
$$

S4R elements offer several advantages over the fully integrated S4 elements. For example, reduced integration of isoparametric elements computes strains and stresses at the locations known to provide optimal accuracy; thus, reduced integration usually produces more accurate results provided the elements are not disturbed or loaded in in-plane bending. Furthermore, reduced integration typically softens the response of the elements, which also leads to increased accuracy by counteracting the overly stiff response usually encountered in FEA. The use of fewer integration points also benefits the user by resulting in reduced computing time and storage requirements. The primary disadvantage of using reduced integration is that deformation modes that cause no strain at the integration points may develop. This may lead to inaccurate results if these zero-energy modes propagate through the structure in a phenomenon commonly known as hourglassing. However, ABAQUS prevents hourglassing

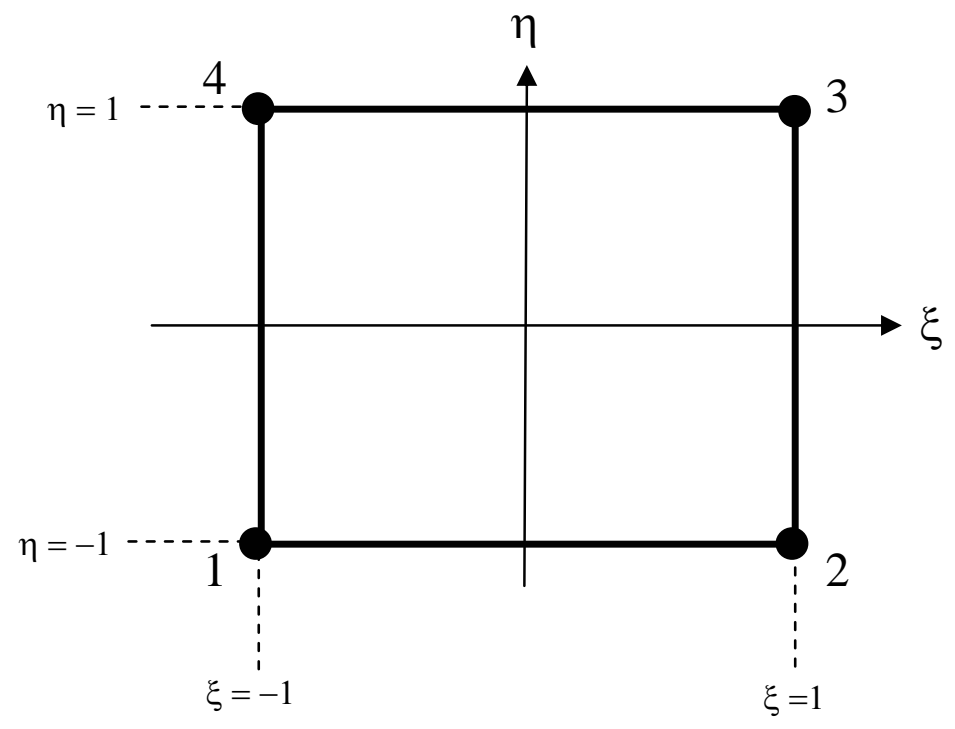

Figure 4-1. Element Natural Coordinate Svstem 
for S4R elements by introducing a small artificial stiffness associated with zero-energy deformation modes.

S4R5 and S9R5 are three-dimensional shell elements whose behavior is governed by classical (Kirchoff) shell theory. Thus, these elements are only appropriate for cases where transverse shear flexibility is negligible and the shell normal remains orthogonal to the shell reference surface, i.e, for relatively thin plates or shells. Because thin shell elements neglect transverse shear, these elements have only five degrees of freedom (three displacement components and two in-surface rotation components) at each node. Consequently, use of these two elements to model thick shells may give inaccurate results. ABAQUS (2002) suggests that thin shell elements are appropriate when the shell thickness is less than approximately $1 / 15$ of the characteristic length (distance between supports) of the shell. Because of the high mesh density used in this work, the characteristic length of the elements is relatively small ( 1 to 2 in. for most cases). Therefore, it is not appropriate to consider these elements as thin shells as this would require element thicknesses less than 2/15 of an inch, which is thinner than the plate thicknesses utilized in this work (5/16 in. or greater).

\subsection{Material Modeling}

An elastic-plastic constitutive law including strain hardening effects is used in this work. From a numerical viewpoint, the implementation of a constitutive model involves the integration of the state of the material at an integration point over a time increment. Therefore, ABAQUS utilizes the incremental plasticity form of the elastic-plastic model, in which the increment of plastic strain is related to the state of stress and the stress increment. Incremental models are formulated in terms of: a yield surface, a flow rule, and a hardening rule. Of the many material modeling options available in ABAQUS, an elastic-plastic constitutive model with standard Mises yield surfaces, associate plastic flow rule, and isotropic work hardening has been found to be suitable to represent rate-independent behavior of a metal subjected to a relatively monotonic loading where creep effects are not important (Earls and Shah 2000). Consequently, this approach is used in this study. 


\subsubsection{Von Mises Yield Criterion}

The yield criterion defines the elastic limit of a material under combined states of stress. In general, the elastic limit, or yield stress, is a function of the state of stress, $\sigma_{i j}$, and one or more material constants and can be expressed as

$$
f\left(\sigma_{i j}, k_{1}, k_{2}, \ldots\right)=0
$$

where $k_{1}, k_{2}, \ldots$ are experimentally determined material constants.

Dating from 1913, the von Mises yield criterion is one of the most commonly used yield criteria for metal materials. It is a pressure-independent yield criterion and states that yielding begins when the strain energy of distortion reaches a critical value $k$,

$$
f\left(J_{2}\right)=J_{2}-k=0
$$

where $J_{2}=\frac{1}{2} S_{i j} S_{i j}$ is the second invariant of the deviatoric stress tensor, $s_{i j}$, and $k=\frac{\sigma_{0}}{\sqrt{3}}$, in which $\sigma_{0}$ is the uniaxial yield stress of the material. Thus, if $f\left(J_{2}\right)<0$, the material will behave elastically; however, if $f\left(J_{2}\right)=0$ yielding will occur. The von Mises criterion represents a circular cylinder in principle stress space, as shown in Fig. 4-2(a).

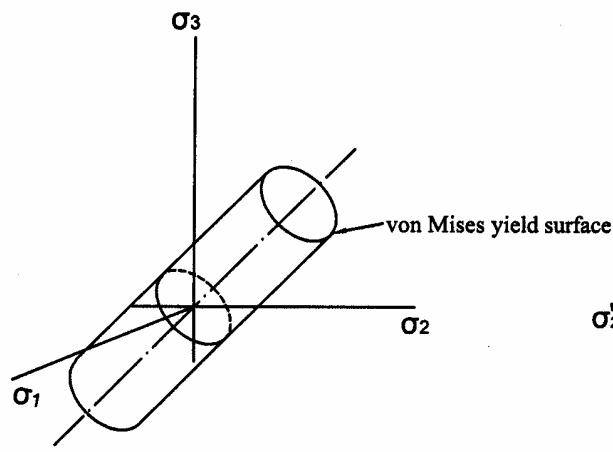

(a) Von Mises yield surface in principal stress space

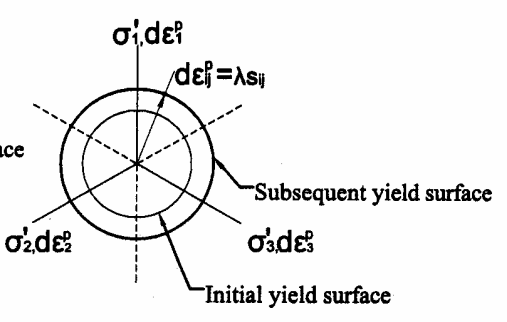

(b) Subsequent von Mises yield surface with isotropic hardening and associate flow rule in deviatoric space

Figure 4-2. Von Mises Yield Criterion 


\subsubsection{Associated Flow Rule}

The flow rule defines both the direction and the relative magnitudes of the plastic strain increment vector $d \varepsilon_{i j}^{p}$ once the elastic limit has been exceeded. In 1928, von Mises proposed the concept of the plastic potential function, which is a scalar function of the stresses, $g\left(\sigma_{i j}\right)$. Then the plastic flow equations can be written in the form of

$$
d \varepsilon_{i j}^{p}=d \lambda \frac{\partial g}{\partial \sigma_{i j}}
$$

where $d \lambda$ is a positive scalar factor of proportionality, which is nonzero only when plastic deformations occur.

The simplest flow rule is termed associated flow rule, which means that the yield function and the plastic potential function coincide, i.e. $f=g$. Thus,

$$
d \varepsilon_{i j}^{p}=d \lambda \frac{\partial f}{\partial \sigma_{i j}}
$$

This implies that the plastic flow develops normal to the yield surface $\frac{\partial f}{\partial \sigma_{i j}}$. For the von Mises criteria, the associated flow rule has the simple form:

$$
d \varepsilon_{i j}^{p}=d \lambda \frac{\partial f}{\partial \sigma_{i j}}=d \lambda s_{i j}
$$

\subsubsection{Isotropic Hardening}

The hardening rule specifies the manner in which the initial yield surface changes during the process of plastic flow. The most widely used flow rules are those of isotropic hardening, kinematic hardening, and mixed hardening, which is a combination isotropic and kinematic hardening. Isotropic hardening is the simplest hardening rule and is based on the assumption that the initial yield surface expands uniformly without distortion or translation as plastic flow occurs. This hardening rule generally gives realistic results except for cases where complex loading paths with stress reversals are considered.

For isotropic hardening, the size of the yield surface is governed by the value of $k^{2}$, which is expressed as a function of effective strain, $\varepsilon^{p}$, where the value of $\varepsilon^{p}$ depends on the loading 
history. The equation for the subsequent yield surface can be written in the general form

$$
F\left(\sigma_{i j}\right)=k^{2}\left(\varepsilon^{p}\right)
$$

or for the von Mises yield function, $F\left(\sigma_{i j}\right)=J_{2}$, Eqn. (4.11) becomes

$$
J_{2}=\frac{1}{2} s_{i j} s_{i j}=k^{2}\left(\varepsilon^{p}\right)
$$

The von Mises criterion with associated plastic flow rule and isotropic work hardening is illustrated in deviatoric space in Fig. 4-2(b).

\subsubsection{Stress-Strain Relationship}

A multi-linear relationship consisting of an elastic region, a yield plateau, a strain-hardening region, and a second plateau are used to represent the stress-strain characteristics used in the material modeling. These relationships for Grade 50 and Grade 70 steel are shown in Figs. 4-3 and 4-4, respectively. For Grade 50 steel, a typical value for the modulus of elasticity (E

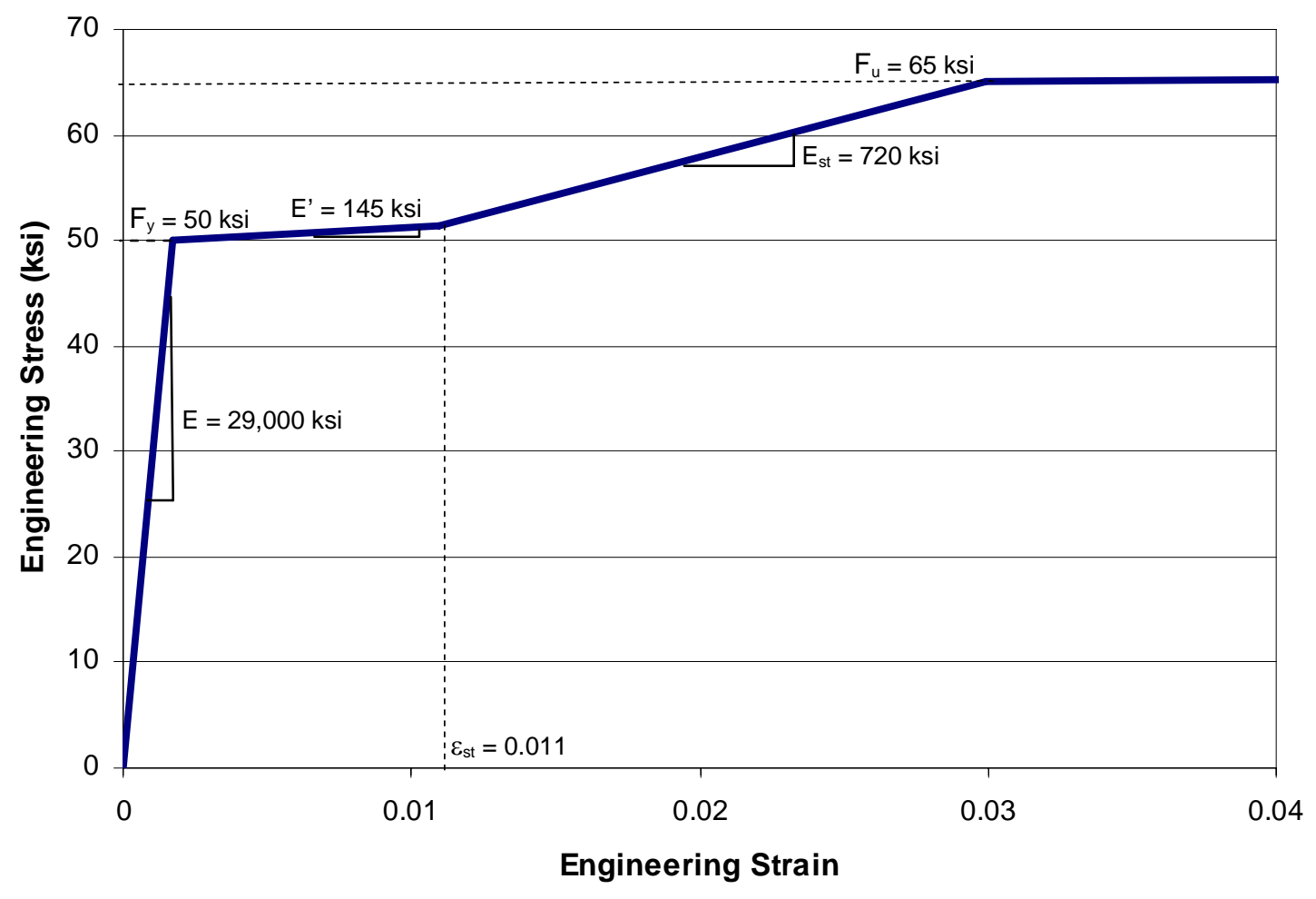

Figure 4-3. Stress-Strain Relationship for Grade 50 Steel 


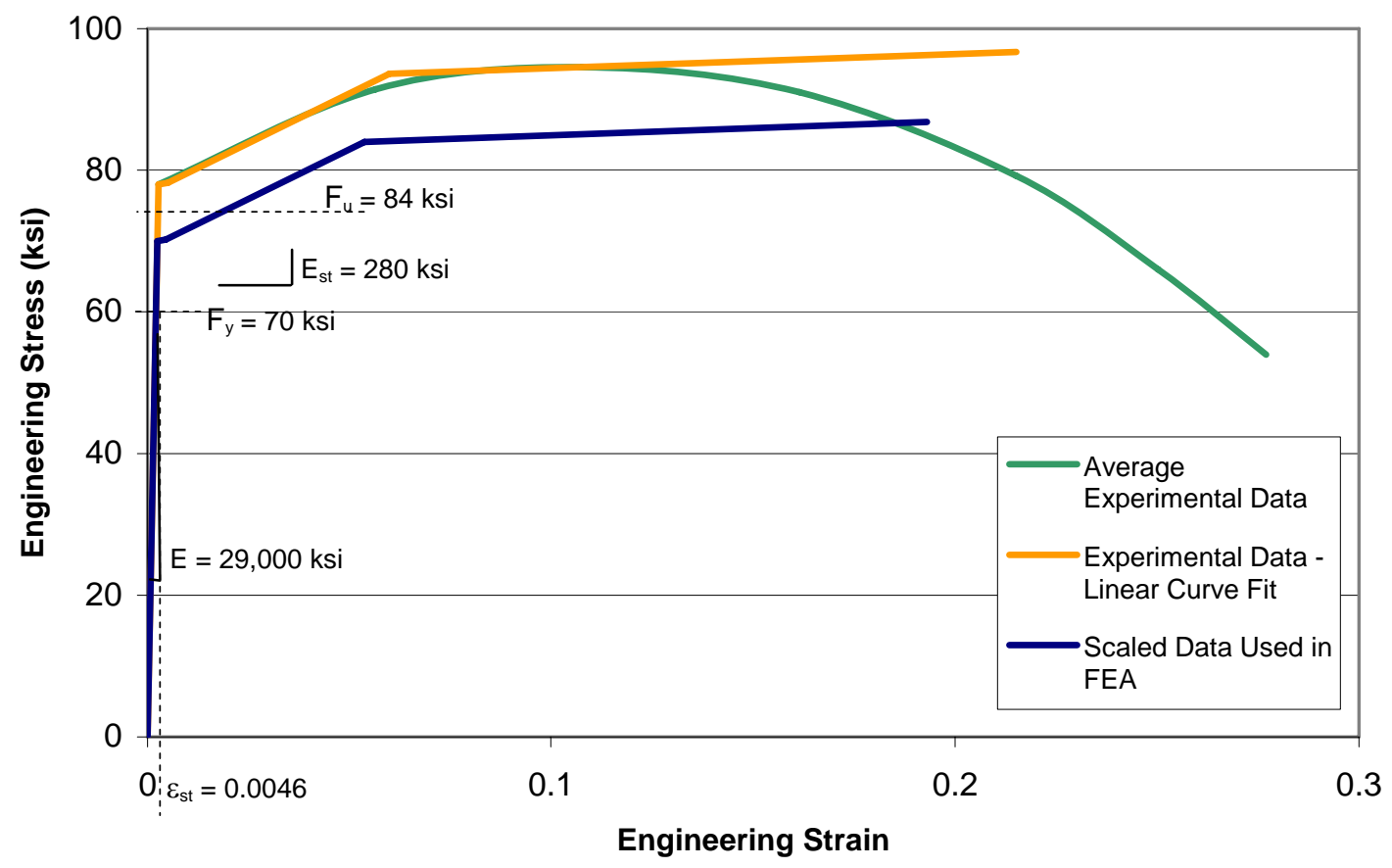

Figure 4-4. Stress-Strain Relationship for Grade 70 Steel

$=29,000 \mathrm{ksi})$ and the nominal yield stress value $\left(\mathrm{F}_{\mathrm{y}}\right)$ of $50 \mathrm{ksi}$ are specified. The values used to define key points in the non-linear region of the curve are largely based on testing conducted by Alpsten (1972). He reported a distinct relationship between plate thickness and resulting values for the strain hardening modulus $\left(E_{\mathrm{st}}\right)$ and strain hardening strain $\left(\varepsilon_{\mathrm{st}}\right)$. Specifically, for $1 / 2$ in. thick plates, average values of $E_{\mathrm{st}}=470 \mathrm{ksi}$ and $\varepsilon_{\mathrm{st}}=0.019$ are reported, while for $1-1 / 2$ in. plate, average values of $E_{\mathrm{st}}=830 \mathrm{ksi}$ and $\varepsilon_{\mathrm{st}}=0.006$ are given. Giving consideration to the range of plate thicknesses typically used in bridge construction and those used in this study, values of $\mathrm{E}_{\mathrm{st}}=720 \mathrm{ksi}$ and $\varepsilon_{\mathrm{st}}=0.011$ are used in this study. The nominal value of ultimate stress $\left(F_{u}\right)$ of $65 \mathrm{ksi}$ is also selected. The slope of the yield plateau region (E') is chosen to be $145 \mathrm{ksi}$ to represent a very small slope (E/200) that is greater than zero in order to avoid numerical difficulties. The slope of the stress-strain curve after strain hardening terminates is selected to be E/2,000, or $14.5 \mathrm{ksi}$. The stress-strain relationship described above is then converted into terms of true stress and true strain, as required for input into ABAQUS. 
The stress-strain model for Grade 70 steel is based on experimental testing conducted by the Federal Highway Administration (Wright 1997). Specifically, Fig. 4-4 shows the average experimental results from six tension tests of grade HPS70W steel, along with a multi-linear fit to this data. Because the test specimens exhibited yield strengths greater than $70 \mathrm{ksi}$, the data is scaled so that $F_{y}$ is equal to $70 \mathrm{ksi}$. As before, the standard value of $\mathrm{E}=29,000 \mathrm{ksi}$ is also assumed. The experimental data indicates that this material has a small yield plateau, with $\varepsilon_{\mathrm{st}}=1.9 \varepsilon_{\mathrm{y}}=0.0046$ (for $\mathrm{F}_{\mathrm{y}}=70 \mathrm{ksi}$ ) and the slope of the yield plateau is assumed to be $100 \mathrm{ksi}$. The experimental data also gives $E_{s t}=280 \mathrm{ksi}$ and the slope of the stress-strain relationship once strain hardening terminates is again approximated as $14.5 \mathrm{ksi}$. Lastly, the test data indicates that the yield stress is $83 \%$ of the ultimate stress; using this information along with $\mathrm{F}_{\mathrm{y}}=70 \mathrm{ksi}$, gives $\mathrm{F}_{\mathrm{u}}=84 \mathrm{ksi}$. Again, this data is converted into terms of true stress and true strain for input into ABAQUS.

\subsection{Geometric Imperfections}

The nonlinearity in response due to the presence of initial imperfections of the girder has a measurable impact on girder ductility, especially on the maximum moment capacity and unloading shape of moment-plastic rotation curves. Furthermore, from the numerical analysis point of view, the modified Riks method of analysis used in this work is a type of post-buckling analysis. Thus, a continuous response is required as opposed to bifurcation. This can be accomplished by introducing a geometric imperfection pattern in the "perfect" geometry so that some degree of buckling occurs before the critical load is reached, as would occur in actual girders. Therefore, introduction of geometric imperfections is a critical step in this type of analysis, and consequently, the application of geometric imperfections is carefully studied in this work.

In welded plate girders, initial geometric imperfections are generally generated during the welding process and/or due to initial out-of-flatness of the long steel plates. Three types of geometric imperfections are considered in this work in order to capture these characteristics:

an out-of-flatness of the web, a tilt of the compression flange, and a lateral sweep of the compression flange. These imperfections were previously illustrated in Fig. 3-7 and are reproduced here in Fig. 4-5. 


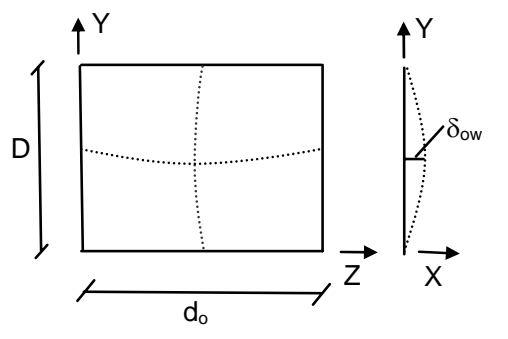

(a) Initial out-of-flatness of

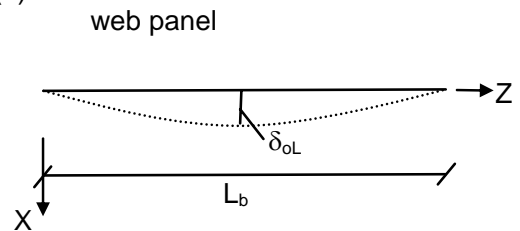

(c) Initial sweep of the compression flange

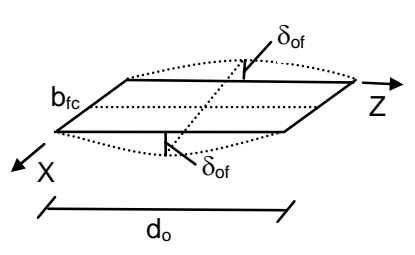

(b) Initial tilt of compression flange

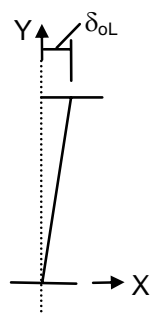

Figure 4-5. Initial geometric imperfections

The values prescribed for these three types of imperfections are based on maximum allowable tolerances specified by the American Welding Society (AWS) and engineering judgment. For example, AWS specifies alternative tolerances for the initial out-of-flatness of the web, depending on if the girder is stiffened. For girders with one-sided transverse stiffeners, the maximum allowable initial out-of-flatness of the web, $\delta_{\text {ow }}$ (see Fig. 4-5), is $d / 67$, where $d$ is the minimum panel dimension, either the web depth (D) or distance between stiffeners $\left(\mathrm{d}_{0}\right)$. Alternatively, the maximum allowable value is $\mathrm{D} / 150$ for unstiffened girders. In this study $\delta_{\text {ow }}$ is prescribed to be equal to $d / 100$, which is chosen to represent a midpoint between the above two requirements. This maximum value of distortion occurs at the center of each web panel and the amount of out-of-flatness at all other locations in the web panel decreases in a half sine wave pattern, in both the $\mathrm{X}$ and $\mathrm{Y}$-directions (see Fig. 4-5). Furthermore, the direction of $\delta_{\text {ow }}$ alternates in adjacent web panels.

The maximum allowable tilt of the flanges, $\delta_{\text {of }}$, specified by AWS is equal to $b_{f} / 100$ or $1 / 4$ in., whichever is greater. However, it is felt that it is unlikely that the distortion of the flange would be this severe in girders with relatively short panel lengths. Therefore, $\delta_{\text {of }}$ is assigned to be the lesser value of $b_{f c} / 150$ or $0.3 d_{o} / 150=d_{o} / 500$. This results in values slightly less than that permitted by AWS for girders with long panel lengths (i.e., $b_{f c}<0.3 d_{0}$ ), while for 
short panels, $\delta_{\text {of }}$ may be significantly less than AWS tolerances. The maximum value of $\delta_{\text {of }}$ occurs at the horizontal center of each web panel along the flange edge. The value of $\delta_{\text {of }}$ decreases in a sine wave pattern along the length of the girder (Z-direction, in Fig. 4-5) and decreases linearly along the width of the flange (X-direction). The direction of $\delta_{\text {of }}$ also alternates in adjacent panels.

AWS limits the variation in straightness of welded girders to $1 / 960^{\text {th }}$ of the girder length. In this work, a lateral sweep of the compression flange $\left(\delta_{\mathrm{oL}}\right)$ is specified to be somewhat less than this limit, with a maximum value equal to $\mathrm{L}_{b} / 1500$, where $\mathrm{L}_{b}$ is the distance between lateral bracing. This value is prescribed at the center of the lateral bracing segment at the web-compression flange junction. The value of $\delta_{\mathrm{oL}}$ varies in a sine wave pattern along the Zdirection of the girder and varies linearly in the Y-direction (see Fig. 4-5). As with the other imperfections, the direction of $\delta_{\mathrm{oL}}$ alternates in adjacent lateral bracing segments. Furthermore, $\delta_{\mathrm{oL}}$ and $\delta_{\text {ow }}$ are prescribed in the same direction within each web panel so that the effects of these two imperfections are cumulative.

Several approaches may be used to implement these imperfections, all of which involve various methods of altering the initial coordinates of each node. One commonly employed approach is to define imperfections as a linear superposition of buckling eigenmodes from a previous buckling or frequency analysis. This approach can be accomplished by first using the *buckle or *frequency commands in ABAQUS to establish probable lower energy collapse buckling modes; then specifying the output file of the buckling analysis in addition to one or more buckling modes multiplied by the scale factor by way of *imperfection command. A similar approach is based on the solution obtained from a previous *static analysis. Alternatively, geometric imperfections can be directly specified in the input file.

In the initial stages of this research, the buckling analysis method was used to apply initial geometric imperfections. However, although the buckling analysis approach is widely used in the nonlinear analysis of plate girders, the resulting buckling mode shapes were generally found to be too localized to represent the initial shape of the girders at a global level. 
Furthermore, the predominant displacements resulting from buckling analyses were associated with buckling of the relatively slender webs. In order to obtain a more suitable buckled shape, it was deemed necessary to increase the web thickness used in the buckling analysis (usually twice the thickness of the actual girder). At the beginning of this work, this approach was adopted and initially worked well for girders with relatively short lateral bracing distances. However, when girders with larger lateral bracing distances were later studied, it was difficult to obtain buckling modes giving a desired geometric imperfection pattern.

Other disadvantages of this method also become evident. First, it is not possible to control the values of the three different types of imperfections simultaneously, even if several different buckling modes are superimposed. For instance, high values are normally obtained for out-of-flatness of the web, but it is difficult for the desired values for the tilt of the compression flange to be obtained at the same time. Furthermore, it is not possible to separate these three types of imperfections to study their effects on the moment-rotation capacity of the girders independently, nor is it possible to have a uniform degree of imperfections along the length of the girder as would be encountered in actual girders. Instead, the imperfections are most severe near midspan of the girder and are minimal near the supports. Lastly, this method is time consuming due to the need to first run a buckling analysis and then carry out the separate nonlinear analysis, and also creates additional files that occupy valuable CPU space.

Because the objective of performing the buckling analysis is to simply modify the nodal coordinates, the same outcome can also be achieved through the use of a FORTRAN program developed to fulfill the same function, which also eliminates the shortcomings of the buckling analysis approach described above. The purpose of the program is to apply initial imperfections to the girder by changing the initial global coordinates of each node to give the desired imperfections outlined above. The program is compiled using FORTRAN2000 language and functions by reading the data from the input file generated by FEMAP and processing user input to create a new input file with modified nodal coordinates. This 
program was successfully implemented in all FEA conducted in this work and the basic structure of the program is as follows.

1. Because different parts of the girder (web, flange, etc.) have different imperfection modes, it must first be determined if a given node is a web node, a compression flange node, or neither. The program accomplishes this by first reading the coordinates of the "reference node", which is defined as the node at the junction of the web and bottom flange at one end of the girder. A given node is then determined to be on the web if the X coordinate of the node is the same as the reference node. The user also specifies the web depth and if the top or bottom flange is in compression flange in order for the compression flange nodes to be identified.

2. The distance between stiffeners is given by the user and then the out-of-flatness of the web is introduced to all nodes previously identified as web nodes according to the value of $\delta_{\text {ow }}$ provided by the user. Furthermore, the user may specify different values of $\delta_{\text {ow }}$ to be used within each panel if warranted.

3. If a node is on the compression flange, the tilt of the flange is added based on user input for the flange width and $\delta_{\text {of }}$. Again, the user may specify the same value of $\delta_{\text {of }}$ for every panel or vary the values within each panel in any manner desired.

4. After completing the previous procedures, the lateral sweep of the compression flange is superimposed within each lateral bracing segment, resulting in additional modification of the coordinates of all web and the compression flange nodes. These imperfections are based on user input regarding the number of lateral bracing segments, the length of each segment, and the value of $\delta_{\mathrm{oL}}$ for each segment.

5. Lastly, the node coordinates that have been changed and all other unchanged parts of the original ABAQUS input file are saved as a new ABAQUS input file. A text file is also generated for the user's convenience that provides information on the applied imperfection modes as a convenient check of user input.

\subsection{Modeling of Residual Stress}

The longitudinal residual stresses in welded I-girders are primarily caused by flame cutting of the plates and longitudinal welding between the flanges and the web. Typically, the tensile residual stresses are essentially equal to the yield stress of the material within a small area, 
termed the heat affected zones, while a smaller, near-constant self-equilibrating compression stress is developed within the other regions of the plates. The residual stress distribution may be idealized by assuming that when the section is free of external forces, the residual stresses over the entire cross-section must satisfy equilibrium and sum to zero.

In this study, residual stress effects are represented by specifying initial stress conditions at the beginning of the analysis through a user-defined sub-routine, which automatically applies a prescribed magnitude of initial (residual) stress to each element depending on the elements location in the girder. When initial stresses are given, the initial stress state may not be in exact equilibrium for the finite element matrix. Therefore, an initial step is included to allow ABAQUS to check for equilibrium and iterate, if necessary, to achieve equilibrium. Specifically, a *static step, where girder dead load is also applied, is implemented before the Riks analysis to insure that equilibrium is satisfied once residual stresses have been included.

The residual stress pattern that is used in this study is shown in Fig. 4-6. This stress distribution is considered a reasonable approximation of the actual residual stresses induced by welding and flame cutting in typical plate girders.

\subsection{Modified Riks Algorithm}

The girders studied in this work are analyzed using the Modified Riks algorithm available in ABAQUS. Because it was of key importance to obtain information on girder behavior once

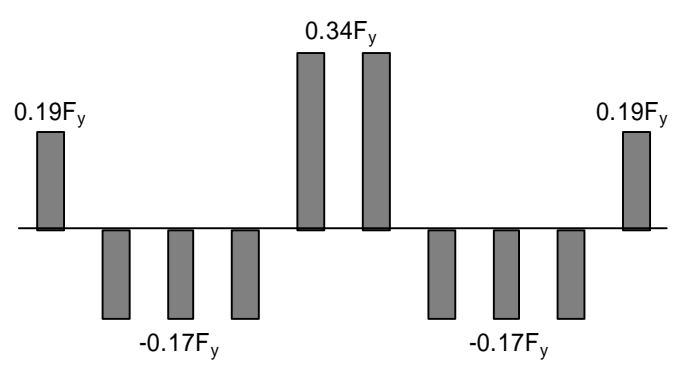

(a) Residual Stress Pattern for Flanges

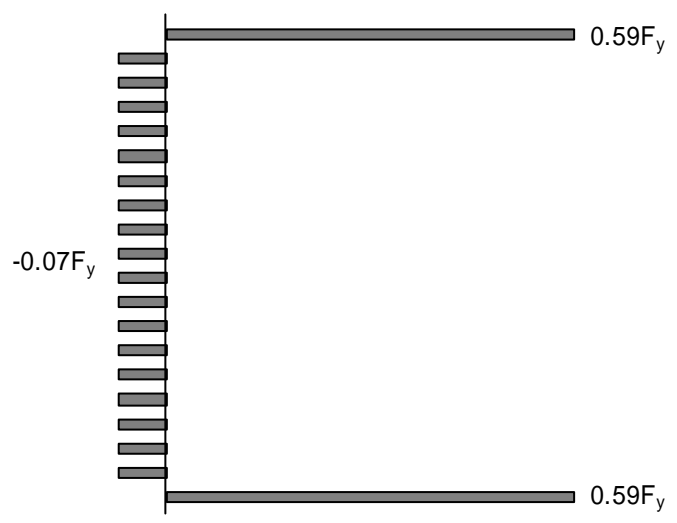

(b) Residual Stress Pattern for Webs

Figure 4-6. Residual Stress Pattern 
maximum loads had been exceeded for this research, it was necessary to employ a solution method capable of capturing this response. The Modified Riks method was selected for use because it has been shown to be one of the most versatile and efficient of such methods (Huang 1994), capable of obtaining a complete nonlinear solution and giving information on girder behavior in both the loading and unloading regions.

The Modified Riks method was initially introduced by Riks (1979), and later improved upon by several researchers (Crisfield 1983, 1981; Ramm 1981; Powell and Simons 1981). In addition to the ability to pass beyond the limit point and trace the unloading portion of the nonlinear equilibrium path, the Riks method also allows solutions to be achieved regardless of whether the response is stable or unstable. This method provides efficient usage of computational resources during the nonlinear solution process. In short, the Modified Riks method may be considered as a combination of the displacement control procedure, the arclength method, and Newton iteration techniques.

One basic assumption used in the Modified Riks method is that the loading is proportional, i.e., all load magnitudes vary with a single scalar parameter. Furthermore, the load magnitude is used as an additional unknown during the solution. Because the progress of the solution is independent of the load increment, ABAQUS uses the "arc length", which is the distance along the static equilibrium path in load-displacement space, to control the increment size. This value is initially provided by the user and is later adjusted by the ABAQUS automatic load increment algorithm based on the convergence rate. The essence of the method is then to find the solution of a single equilibrium path in a space that is defined by the nodal variables and the loading parameter. The solution during each increment is found by moving a given distance along the tangent line to the current solution point and then searching for equilibrium in the plane that not only passes through the point thus obtained, but also is orthogonal to the same tangent line. Once this plane is determined, Newton's method remains the basic algorithm to solve the equilibrium equations. Additionally, it is assumed that the response is reasonably smooth, i.e., sudden bifurcations do not occur. The algorithm of modified Riks method is shown in Fig. 4-7 and is briefly described below. 


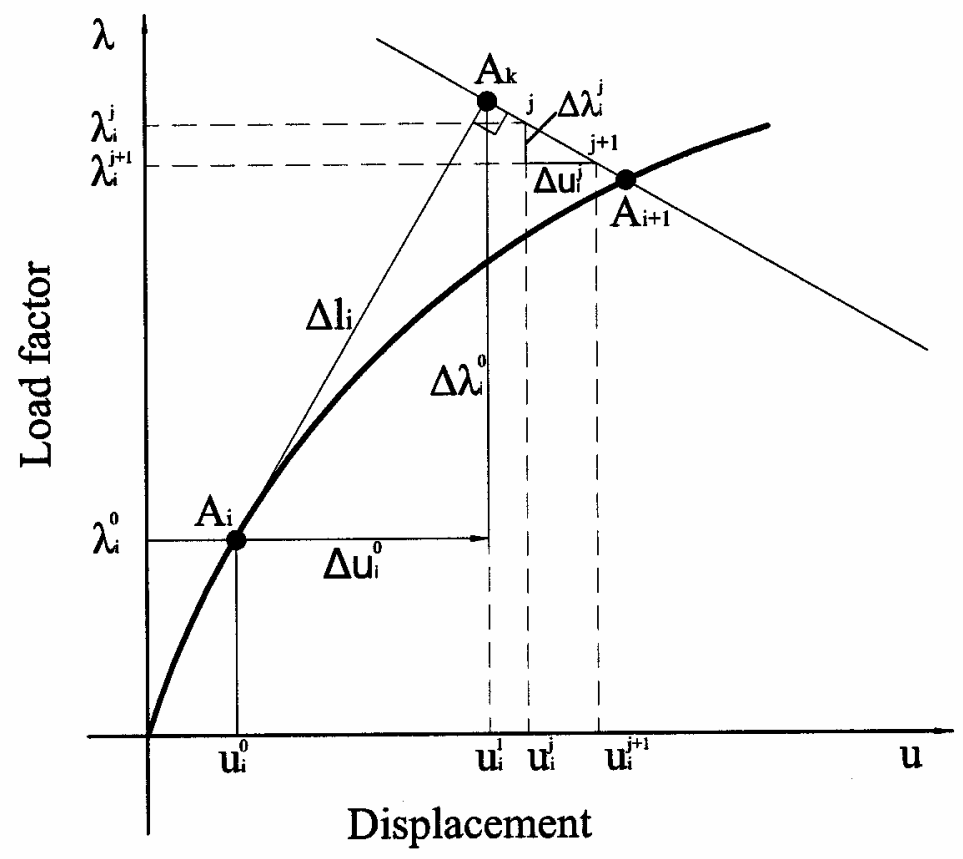

Figure 4-7. Modified Riks Algorithm

The initial arc length increment along the static equilibrium path, $\Delta l_{\text {in }}$, is given in the input file of ABAQUS. The initial load proportional factor increment, $\Delta \lambda_{\text {in }}$, is computed as

$$
\Delta \lambda_{\text {in }}=\frac{\Delta l_{\text {in }}}{l_{\text {period }}}
$$

where $l_{\text {period }}$ is the total arc length scale factor that is also given in the ABAQUS input file. This value of $\Delta \lambda_{\text {in }}$ is only used during the first iteration of the initial increment.

For subsequent increments, suppose the solution has already been obtained at point $A_{i}$ $\left(u_{i}^{0}, \lambda_{i}^{0}\right)$. Next the equilibrium state of the structure at the $\mathrm{i}+1$ load increment is sought as shown in Fig. 4-7. First, the arc length is used to determine $\Delta l_{i}$, the distance traveled along a line tangent to the current solution point. As discussed above, Newton's method is then used to obtain a solution on the plane orthogonal to $\overrightarrow{A_{i} A_{k}}$. The incremental equilibrium equation after the $j^{\text {th }}$ iteration may be expressed as a linear system of equations:

$$
K_{i}^{j} \Delta u_{i}^{j}=\Delta F_{i}^{j}
$$


where $K_{i}^{j}$ is the tangent stiffness matrix for the load increment $\mathrm{i}$ at the end of the $\mathrm{j}^{\text {th }}$ iteration; $\Delta u_{i}^{j}$ is the incremental displacement vector; and $\Delta F_{i}^{j}$ is a load vector which may be described as

$$
\Delta F_{i}^{j}=\Delta P_{i}^{j}+R_{i}^{j}
$$

in which $\Delta P_{i}^{j}$ is the load increment at the $\mathrm{j}^{\text {th }}$ iteration of load increment $\mathrm{i}$ which can be expressed as a fraction of the total applied load $\mathrm{P}$ as:

$$
\Delta P_{i}^{j}=\Delta \lambda_{i}^{j} P
$$

where $\Delta \lambda_{i}^{j}$ is the increment of the load magnitude parameter. $R_{i}^{j}$ is the out-of-balance force vector which equals the difference between the external and internal forces at the beginning of iteration j, i.e.

$$
R_{i}^{j}=P_{i}^{j}-Q_{i}^{j}
$$

where $Q_{i}^{j}$ is the internal force vector. $R_{i}^{j}$ is then used to check equilibrium within the prescribed equilibrium tolerances. If the equilibrium tolerances are satisfied, then the solution is assumed to have converged and this process is repeated for the next load step. Otherwise additional iterations are performed until the out-of-balance force vector (as computed using Eq. 4.17) is within the prescribed tolerances.

The incremental load magnitude parameter can be obtained by first substituting Eqn. 4.14 and 4.16 into the equilibrium equation, Eqn. 4.15, giving

$$
K_{i}^{j} \Delta u_{i}^{j}=\Delta \lambda_{i}^{j} P+R_{i}^{j}
$$

Furthermore, two displacement vectors, $\Delta u_{i}^{a j}$ and $\Delta u_{i}^{b j}$, may be defined corresponding to the two load vectors $P$ and $R_{i}^{j}$,

$$
\begin{aligned}
K_{i}^{j} \Delta u_{i}^{a j} & =P \\
K_{i}^{j} \Delta u_{i}^{b j} & =R_{i}^{j}
\end{aligned}
$$

Substituting $\Delta u_{i}^{a j}$ and $\Delta u_{i}^{b j}$ into Eq. (4.18), gives

$$
\Delta u_{i}^{j}=\Delta \lambda_{i}^{j} \Delta u_{i}^{a j}+\Delta u_{i}^{b j}
$$


For the first iteration $(\mathrm{j}=0)$ at $\mathrm{i}^{\text {th }}$ increment, the structure is in an equilibrium state at the beginning of the increment. Thus, the out-of balance force vanishes at this stage, and consequently $\Delta u_{i}^{b 0}=0$. Substituting this into Eqn. 4.21 gives

$$
\Delta u_{i}^{0}=\Delta \lambda_{i}^{0} \Delta u_{i}^{a 0}
$$

Also, from Fig. 4-7, it is shown that

$$
\Delta l_{i}^{2}=\left(\Delta \lambda_{i}^{0}\right)^{2}+\left\{\Delta u_{i}^{0}\right\}^{T}\left\{\Delta u_{i}^{0}\right\}
$$

The incremental load parameter for the first iteration can thus be obtained by substituting Eqn. 4.22 into Eqn. 4.23

$$
\Delta \lambda_{i}^{0}=\frac{ \pm \Delta l_{i}}{\sqrt{\left\{\Delta u_{i}^{a 0}\right\}^{T}\left\{\Delta u_{i}^{a 0}\right\}+1}}
$$

Because the vector $\overrightarrow{A_{k} A_{i+1}}$ must be orthogonal to $\overrightarrow{A_{i} A_{k}}$, the dot product of these two vectors must equal zero, or symbolically,

$$
\Delta \lambda_{i}^{0} \Delta \lambda_{i}^{j}+\left\{\Delta u_{i}^{0}\right\}^{T}\left\{\Delta u_{i}^{j}\right\}=0
$$

Substituting Eqn. 4.22 for $\Delta u_{i}^{0}$ and Eqn. 4.23 for $\Delta u_{i}^{j}$ into Eqn. 4.25 gives the incremental load parameter for subsequent iterations to be

$$
\Delta \lambda_{i}^{j}=\frac{-\left\{\Delta u_{i}^{a 0}\right\}^{T}\left\{\Delta u_{i}^{b_{j}}\right\}}{\left\{\Delta u_{i}^{a 0}\right\}^{T}\left\{\Delta u_{i}^{a j}\right\}+1}
$$

Finally, the solution point at $\mathrm{j}+1$ can be expressed as

$$
\begin{aligned}
& u_{i}^{j+1}=u_{i}^{0}+\sum_{i=0}^{j} \Delta u_{i}^{j} \\
& \lambda_{i}^{j+1}=\lambda_{i}^{0}+\sum_{i=0}^{j} \Delta \lambda_{i}^{j}
\end{aligned}
$$

Or, by multiplying Eqn. (4.28) by P, we may write

$$
P_{i}^{j+1}=P_{i}^{0}+\sum_{i=0}^{j} P_{i}^{j}
$$


The stiffness matrix is then updated and multiplied by the displacements obtained above (Eqn. 4.27) to find the forces at the current solution point. These forces are in turn used along with the loads obtained above (Eqn. 4.29) to calculate the out-of-balance force vector. This iteration algorithm is repeated until the equilibrium solution is considered to have converged for increment $\mathrm{i}+1$, when all the entries in $R_{i}^{N}$ are sufficiently small. Furthermore, it is also noteworthy to point out that ABAQUS scales the above load-displacement solution space such that the dimensions are approximately the same magnitude on each axis.

\subsection{Summary}

This chapter has discussed the FEA modeling procedures used in this work. Chapter 5 will discuss the use of these modeling procedures to: (1) predict the behavior of the experimental girders discussed in Chapters 3 and (2) obtain inelastic moment-rotation curves of the steel Igirders used in the FEA study. 


\section{Chapter 5: FEA OF STEEL I-GIRDERS FOR THE DETERMINATION OF MOMENT-ROTATION CHARACTERISTICS}

This chapter aims to investigate the available rotation of steel I-girders at various levels of moment, as obtained from experimental and analytical moment versus rotation curves. Previous research has resulted in a reasonably accurate moment-rotation model for compact girders, which is used as the basis for the AASHTO (2004) moment redistribution provisions. This model is applicable for girders with compact flanges, compact lateral bracing distances, and compact or non-compact webs. Thus, the specific objective of this chapter is to determine a similar model that is applicable for more slender classes of girders. Of particular interest is to investigate increased flange slenderness ratios and greater unbraced lengths compared to those permitted by the current AASHTO (2004) moment redistribution procedures contained in Appendix B of the specifications as these are the two areas where the current specifications are most prohibitive.

This chapter consists of five sections. In Section 5.1 FEA of the experimental girders previously introduced in Chapter 3 is presented. Here it is shown that the FEA techniques discussed in Chapter 4 provide an accurate prediction of girder behavior. Section 5.2 then presents the FEA results of a parametric study focused on slender members. Because the available rotations resulting from the analysis of these girders was relatively low, it was not possible to make sound conclusions on the relationship between available rotations and variations in the investigated parameters. Therefore, FEA were conducted for two additional series of girders. The first of these is presented in Section 5.3 where the analysis of a series of representative moment redistribution designs is discussed. Trends in girder behavior are established on the basis of these results and then additional modeling of modified versions of these girders is presented in Section 5.4, where increased lateral bracing distances and flange slenderness ratios are investigated. A synthesis of the experimental and FEA data is presented in Section 5.5. Here the available rotations of steel I-girders is examined with a specific focus on the behavior of slender I-girders and a moment-rotation model for these types of girders is presented. A chapter summary is included in Section 5.6. 


\subsection{FEA of Experimental Girders}

To first validate the FEA procedures introduced in Chapter 4, analyses of each of the experimental girders are presented herein. Girder geometries used in the analyses are the average of the measured dimensions from a minimum of four cross-sections along the length of the girder. Similarly, the material properties of each flange and web are based on the stress-strain data resulting from the tension testing of coupons taken from the same steel plate as the girder component under consideration. The three types of geometric imperfections (see Section 3.3) are measured in each panel of each girder. At a minimum, the geometric imperfections are measured at the center of each panel, and in some cases additional measurements are recorded. Because the imperfection program used in conjunction with these analyses applies a pattern of imperfections based on the magnitude of the imperfections at the center of the panel, when the imperfections are measured at multiple locations, a weighted average is used to obtain the imperfection value input into the program, where higher weights are assigned to imperfection values closer to midspan of the girder. Specifically, imperfection measurements taken within the third of the panel closest to midspan are assigned a weight of 1.0, measurements within the center third of the panel are assigned a weight of 0.8 , and measurements taken from the third of the panel farthest from midspan are assigned a weight of 0.5. To account for the small amount of flexibility and lateral movement of the lateral constraints used in the experimental testing, the lateral constraints used in these analyses are comprised of ABAQUS SPRING1 elements as opposed to fully rigid constraints. The spring elements are applied at the web nodes closest to where the lateral constraints are affixed in the actual girders and are assigned a spring constant of 40 kips/in. All other attributes of the analyses conducted of the experimental girders are the same as those reported in Chapter 4.

The experimental load versus deflection plots previously presented in Figs. 3-16, 3-18, 3-20, 3-22, 3-24, 3-26, 3-28, 3-30, 3-32, 3-34, 3-36, and 3-38 are reproduced below in Figs. 5-1 through 5-12 presenting only the peak data, i.e., the reloading portions of the curves are neglected, and comparing these results to the corresponding FEA results. Figures 5-1 through 5-12 show that the FEA techniques generally produce results that well represent the experimental results. Specifically, these figures show that (1) the shape of the load versus 


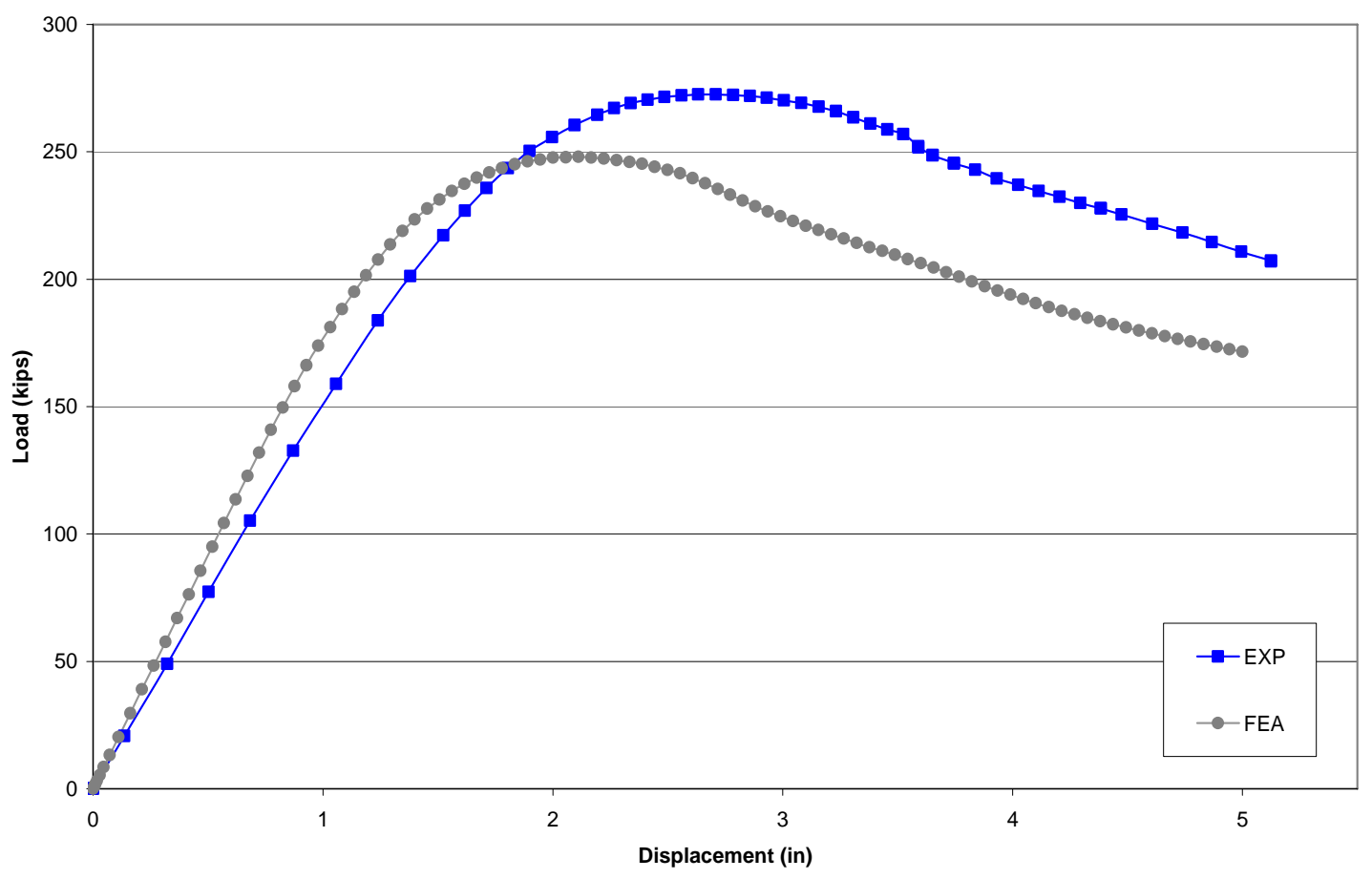

Figure 5-1. FEA and Experimental Load versus Displacement Data Girder 1MG

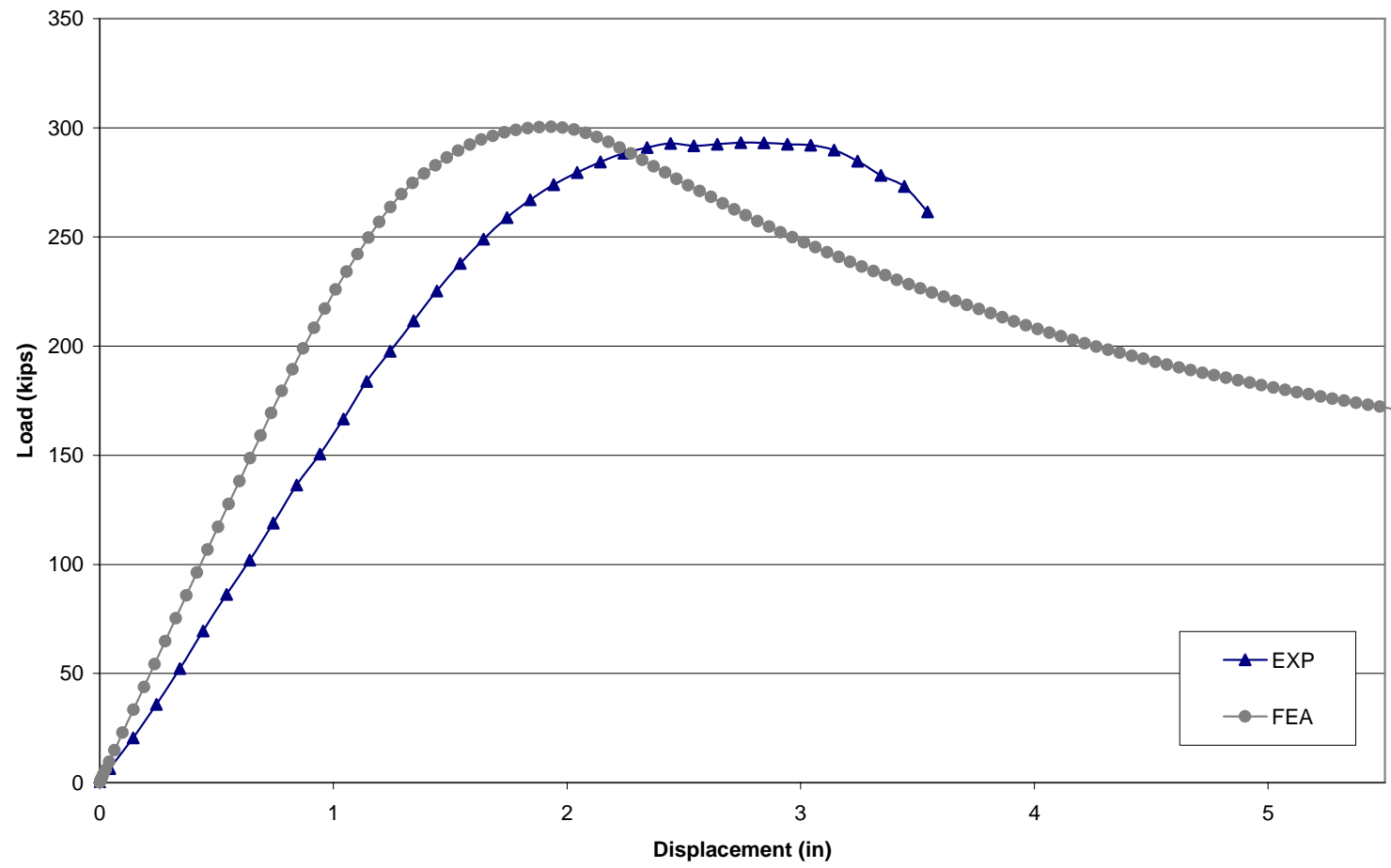

Figure 5-2. FEA and Experimental Load versus Displacement Data Girder 2MG 


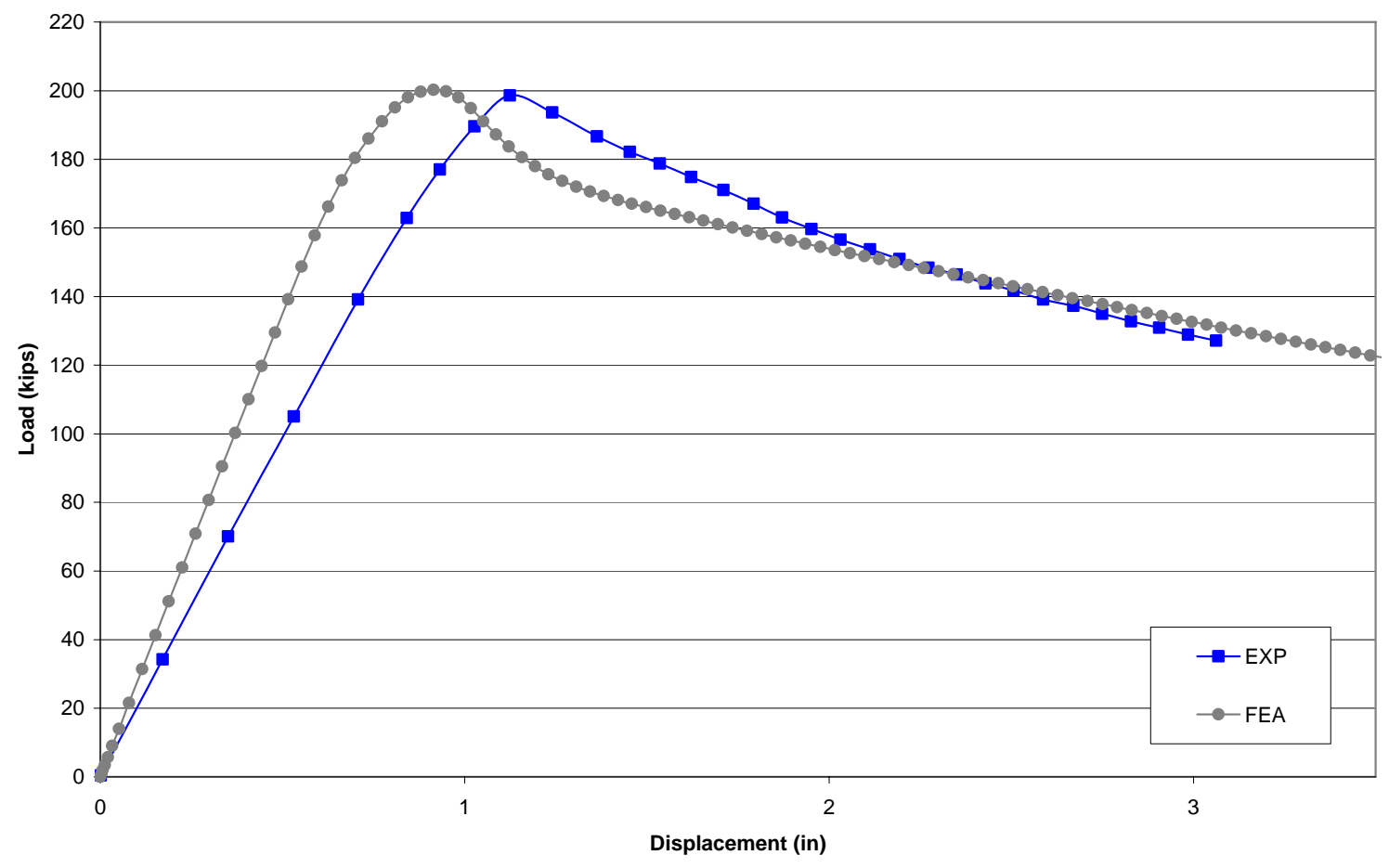

Figure 5-3. FEA and Experimental Load versus Displacement Data Girder 3MG

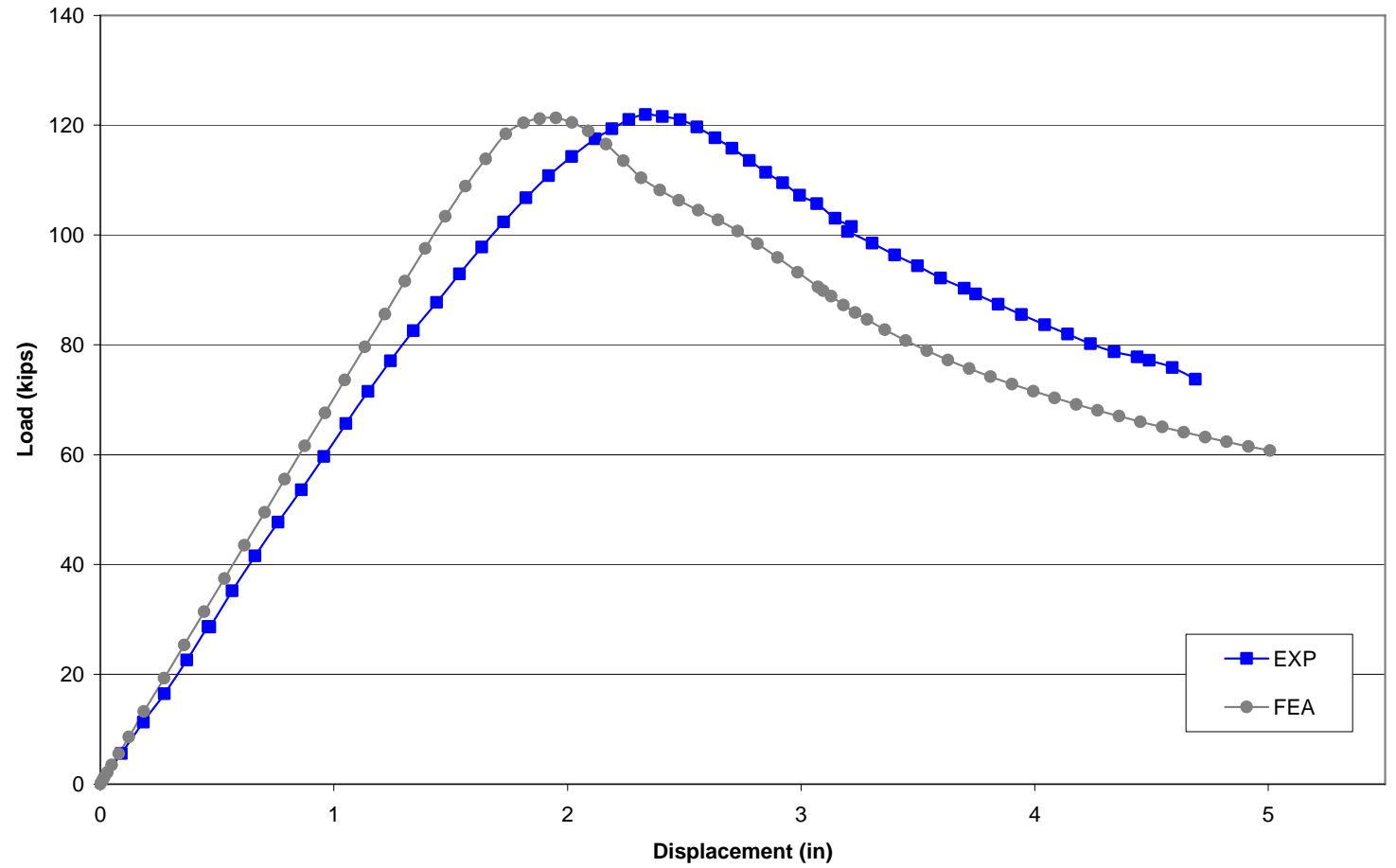

Figure 5-4. FEA and Experimental Load versus Displacement Data Girder 4MG 


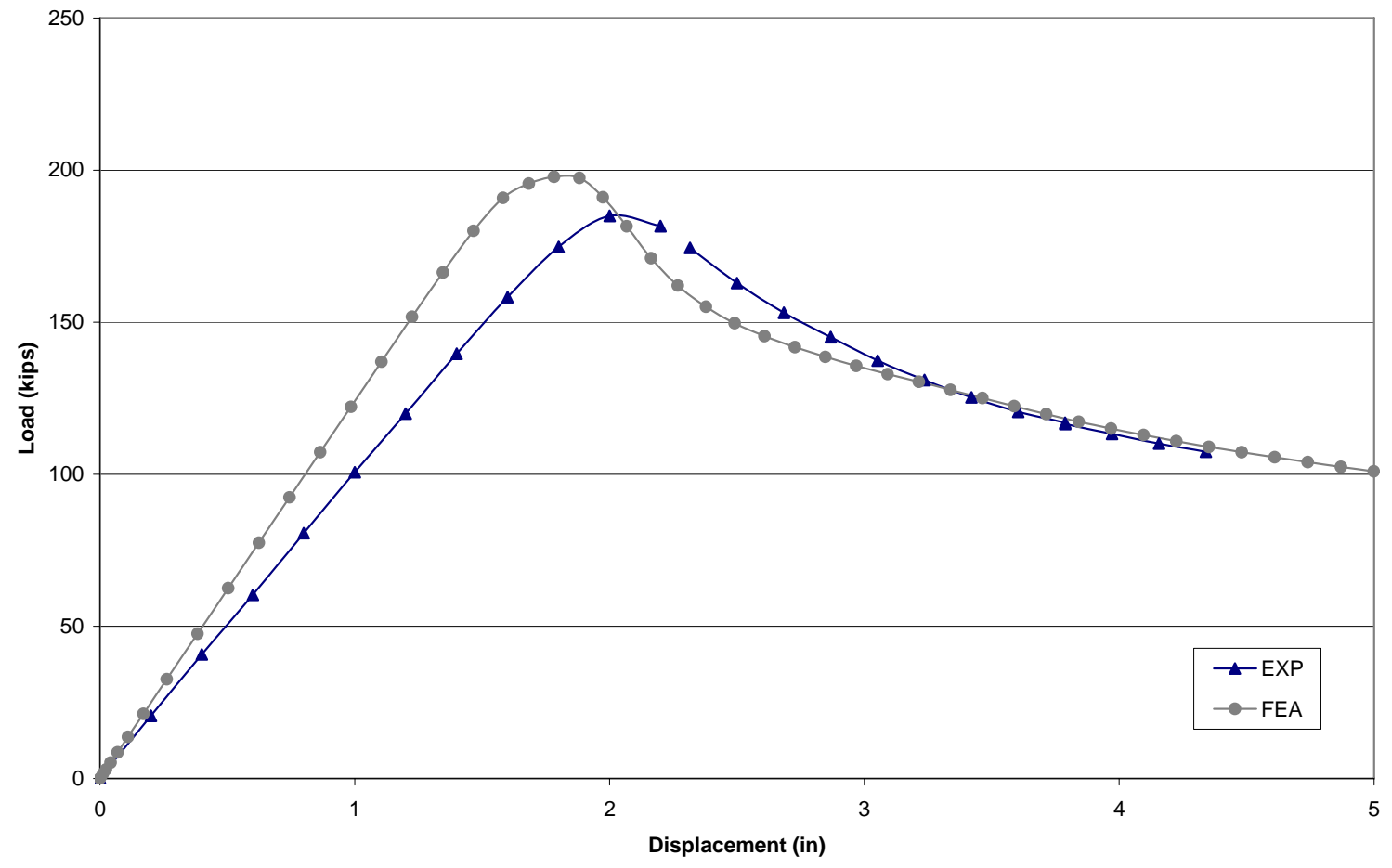

Figure 5-5. FEA and Experimental Load versus Displacement Data Girder 5MG

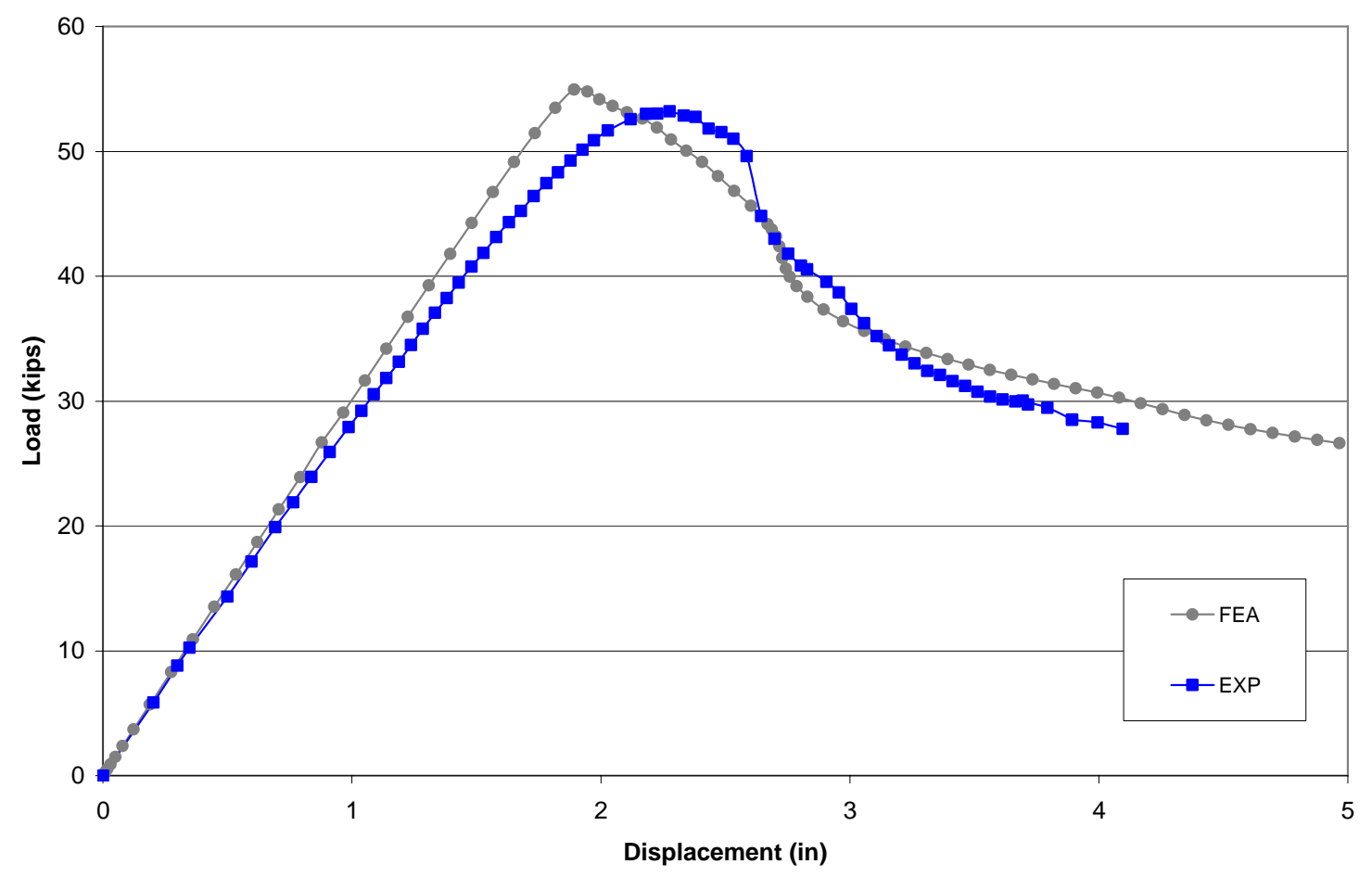

Figure 5-6. FEA and Experimental Load versus Displacement Data Girder 6MG 


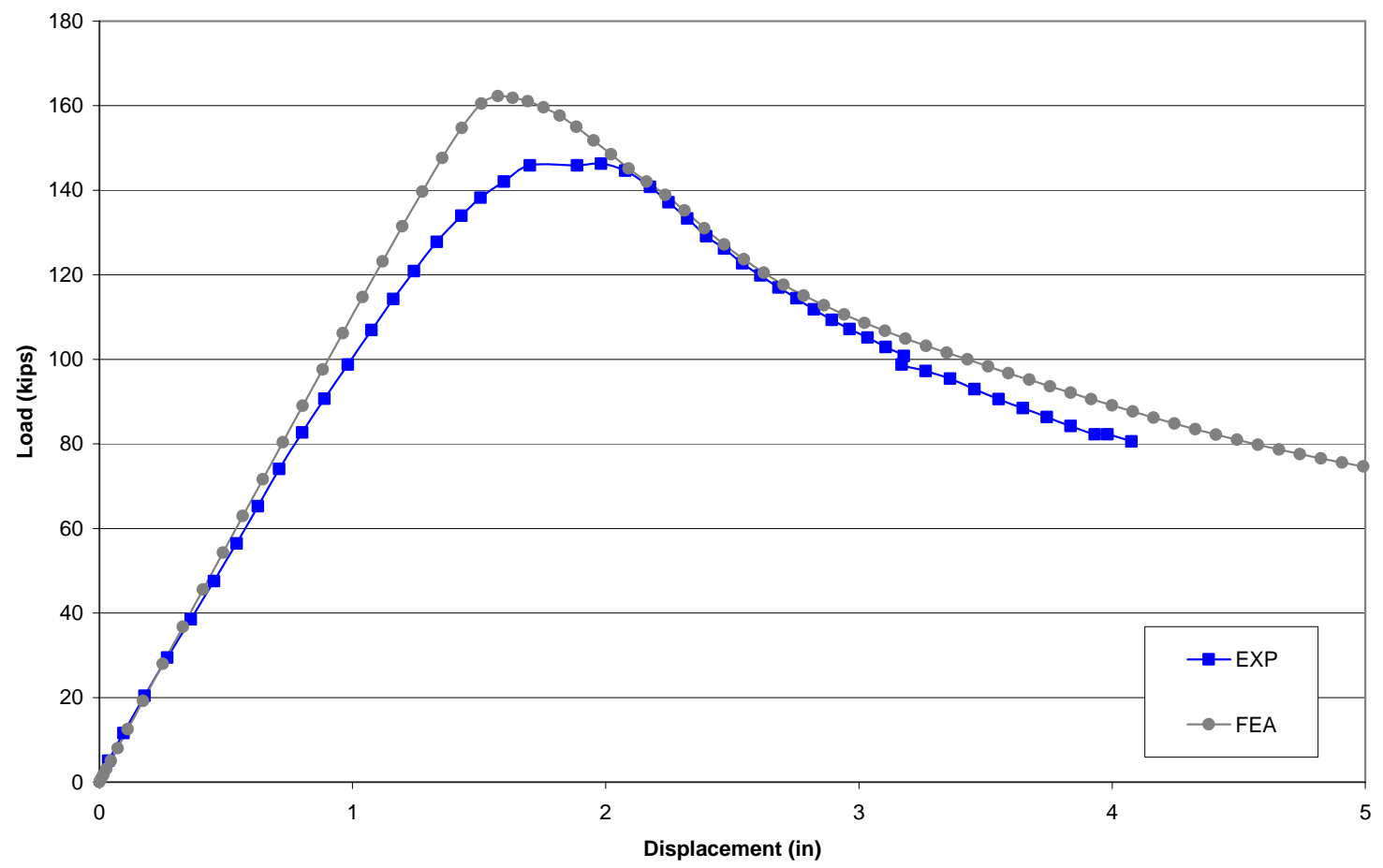

Figure 5-7. FEA and Experimental Load versus Displacement Data Girder 7MG

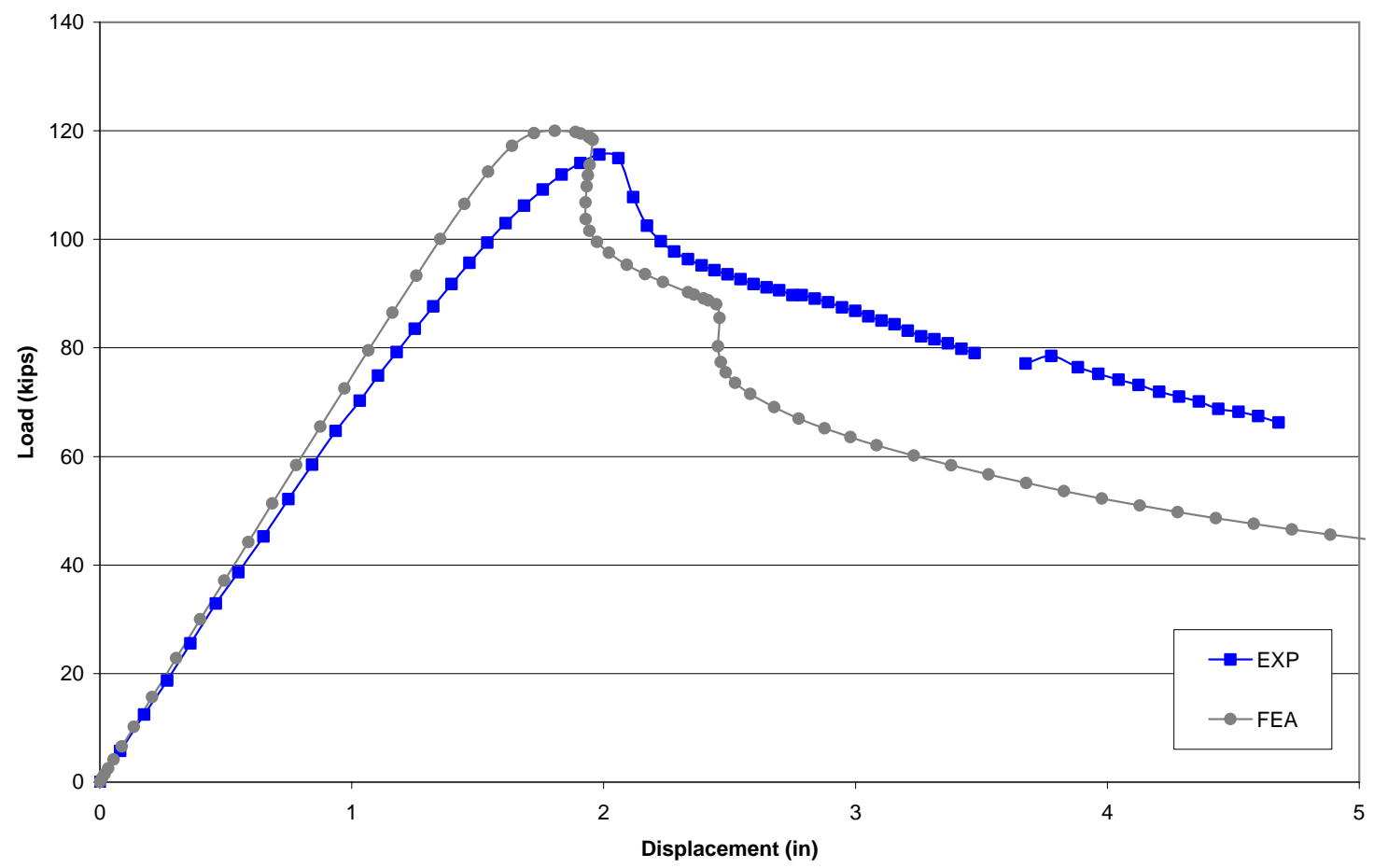

Figure 5-8. FEA and Experimental Load versus Displacement Data Girder 8MG 


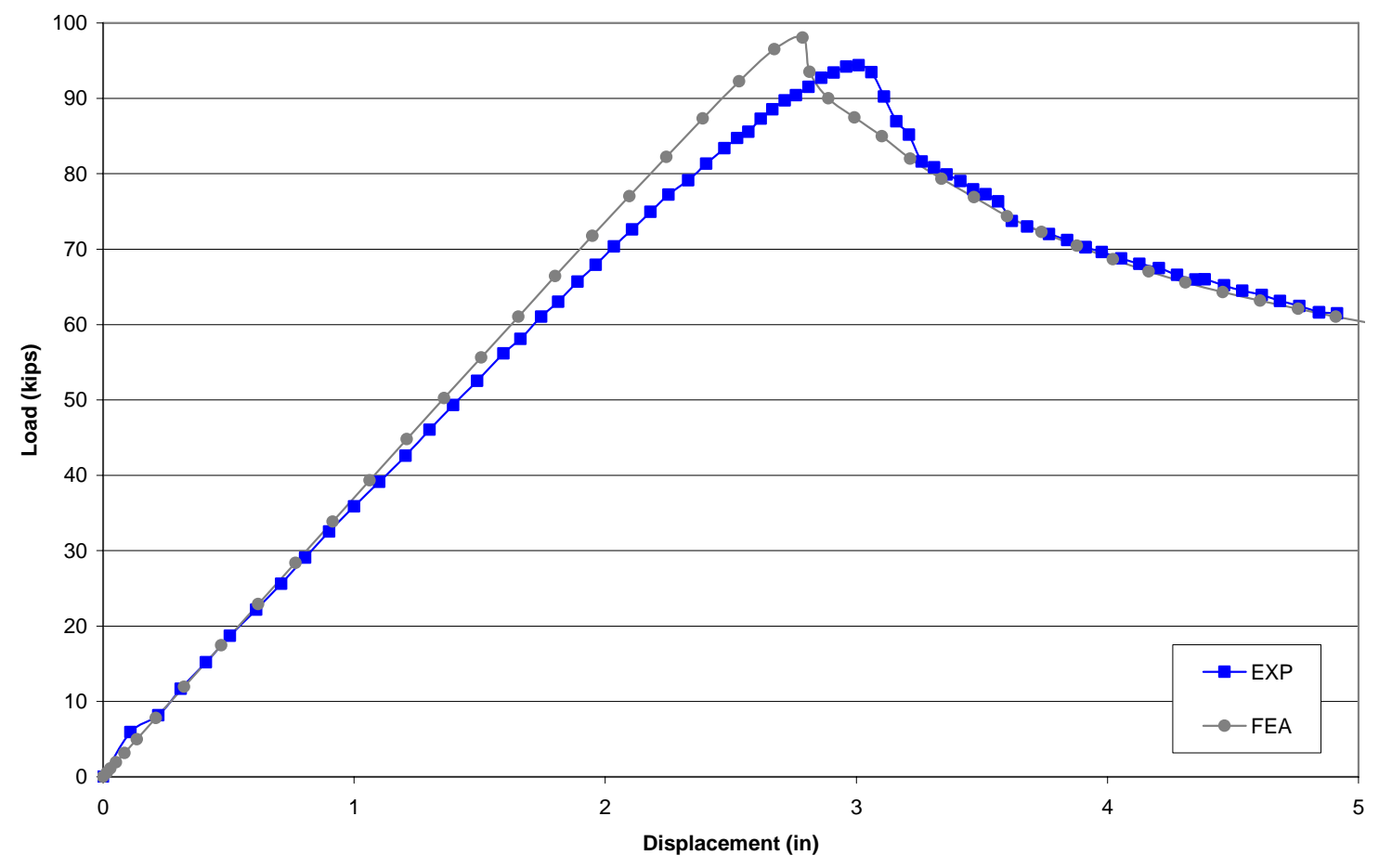

Figure 5-9. FEA and Experimental Load versus Displacement Data Girder 9MG

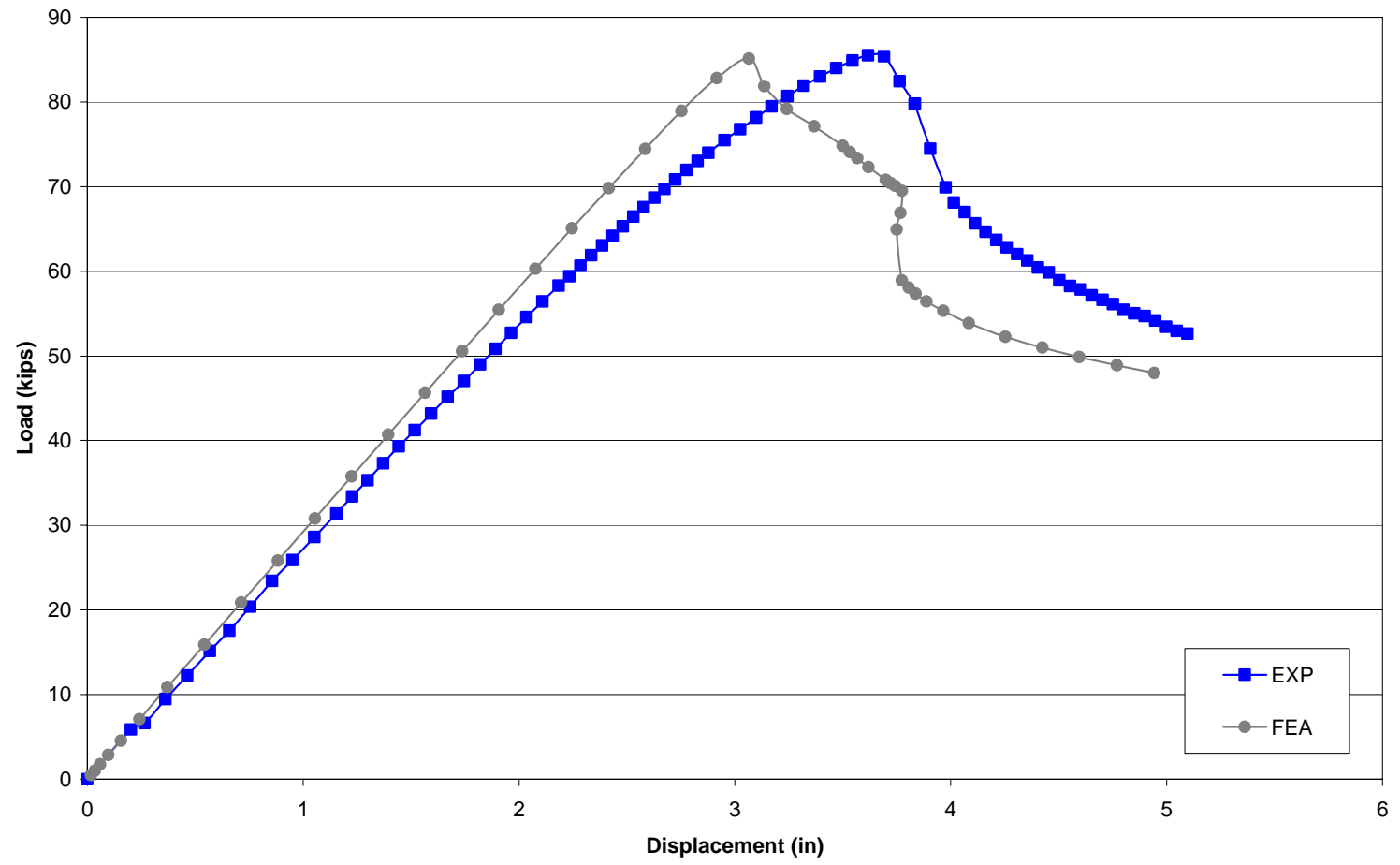

Figure 5-10. FEA and Experimental Load versus Displacement Data Girder 10MG 


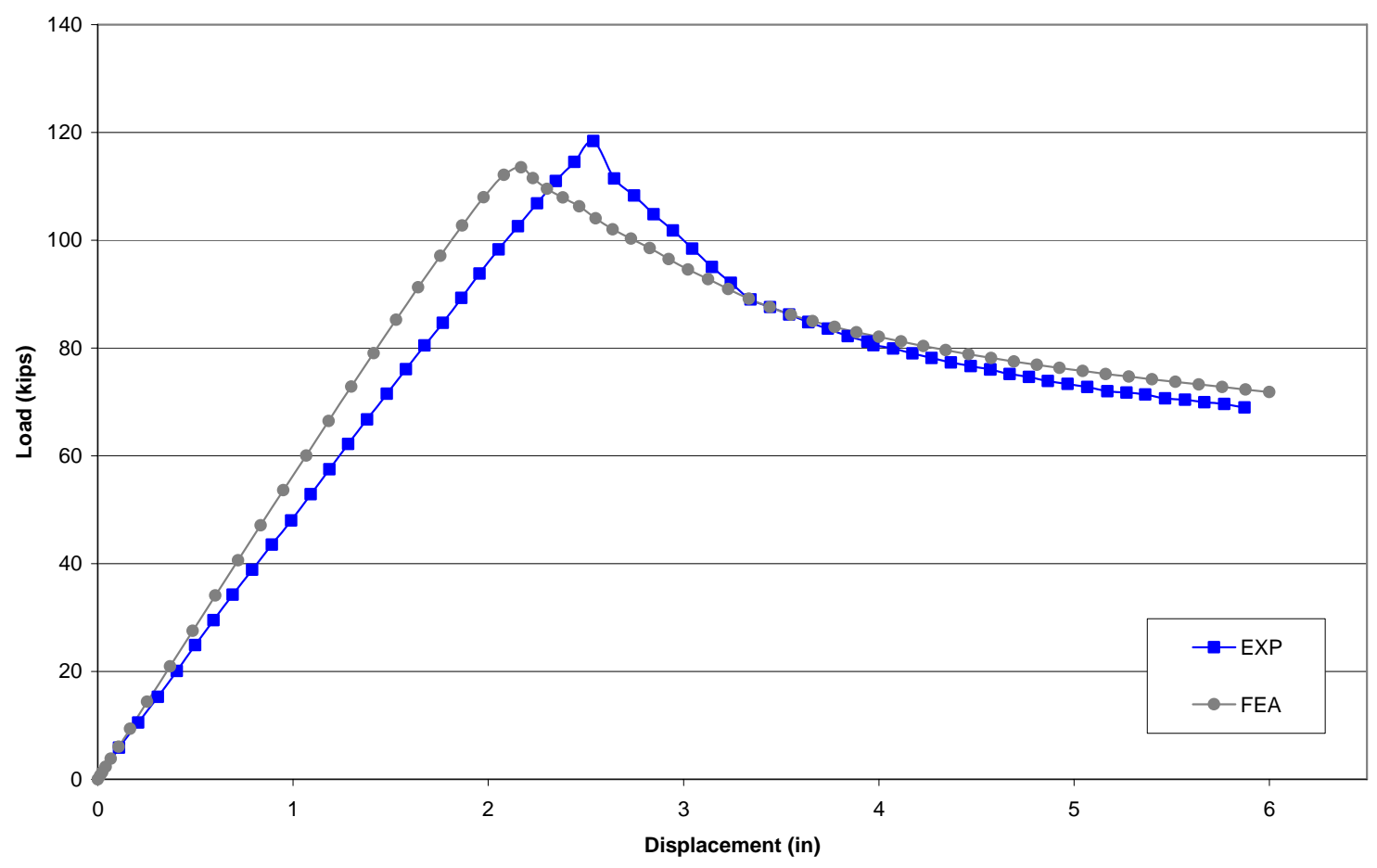

Figure 5-11. FEA and Experimental Load versus Displacement Data Girder 11MG

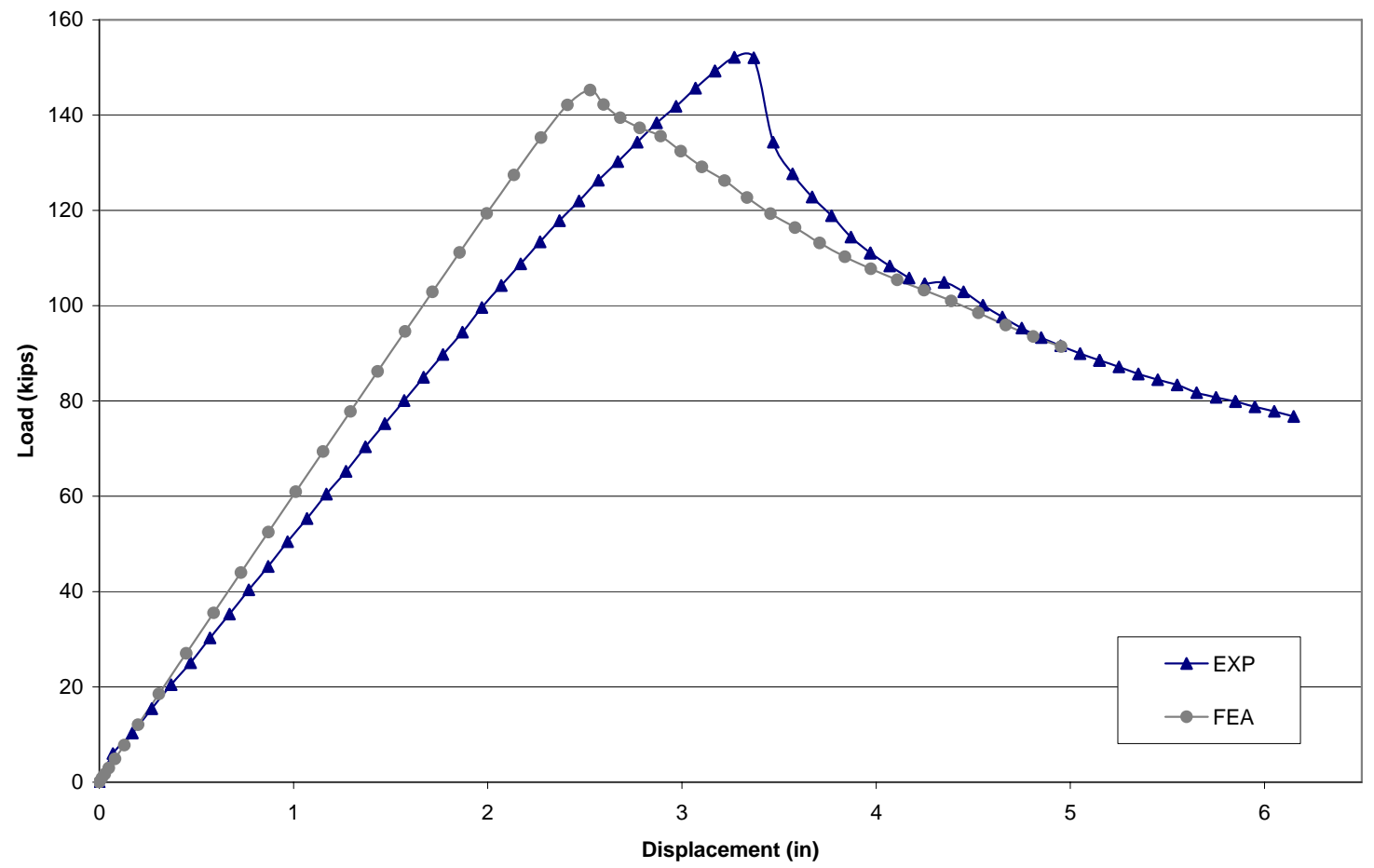

Figure 5-12. FEA and Experimental Load versus Displacement Data Girder 12MG 
deflection response is very similar in both the experimental and analytical results and (2) the maximum moment capacity is well predicted by the FEA. The primary differences between the experimental and FEA results is that, in all cases, the initial stiffness of the girder is greater in the FEA results. In some cases this results in less displacement at a given load throughout the range of loading up to the maximum load (e.g., Girder 2MG) and in other situations the slopes of the two curves are initially equal, but yielding initiates in the actual girder earlier than predicted by the FEA (e.g., Girder 7MG). For some girders, the displacements are also conservative throughout the unloading phase, but in most cases the two curves converge during unloading. Thus, the FEA displacements (and therefore rotations) are either conservative or accurate during the unloading phase, which is most relevant to the present focus of determining available rotations.

Table 5-1 compares the maximum capacities from both the experimental and FEA results. Here a positive percent difference indicates that the FEA produces conservative results. It is shown by Table 5-1 that the FEA and experimental capacities are generally in agreement within 5\%, which is considered to be an accurate strength prediction. Of the three cases where the deviation between the two capacities is greater than $5 \%$, one of these is Girder $7 \mathrm{MG}$; this girder was loaded to $99.7 \%$ of the maximum load, unloaded, reloaded to $39.9 \%$ of the maximum load, and then loaded to the maximum load. Thus, there is the potential that this may have reduced the ultimate capacity that may have otherwise been achieved as it can

Table 5-1. Comparison of Experimental and FEA Maximum Capacities (kips)

\begin{tabular}{|lccc|}
\hline Specimen & $\begin{array}{c}\text { Maximum Load } \\
\text { Experimental }\end{array}$ & $\begin{array}{c}\text { Maximum Load } \\
\text { FEA }\end{array}$ & Percent Difference \\
\hline \hline 1MG & 273 & 248 & 9.0 \\
2MG & 293 & 300 & -2.5 \\
3MG & 199 & 200 & -0.8 \\
$4 M G$ & 122 & 121 & 0.5 \\
$5 M G$ & 185 & 198 & -6.9 \\
$6 M G$ & 53 & 55 & -3.2 \\
$7 M G$ & 146 & 162 & -10.9 \\
8MG & 116 & 120 & -3.8 \\
$9 M G$ & 94 & 98 & -3.9 \\
$10 M G$ & 86 & 85 & 0.4 \\
$11 M G$ & 118 & 114 & 3.7 \\
$12 M G$ & 152 & 145 & 4.7 \\
\hline
\end{tabular}


be seen from Fig. 3-28 that there is a small change in the stiffness of the girder between the $2^{\text {nd }}$ and $3^{\text {rd }}$ loading cycles, indicating that permanent deformations have already been introduced before attaining the maximum load. Girder $1 \mathrm{MG}$ has an actual capacity that is 9.0\% higher than the FEA results. Thus, even though there is a relatively high percent difference the FEA results are conservative for this case. Overall, it is believed that the experimental capacities are well predicted by the FEA results.

Figures 5-13 through 5-24 compare the deformed shapes resulting from the experiments and the FEA. Each of these plots shows the original (undeformed) cross section and LVDT positions with data points, the deformed LVDT positions with data points (as previously presented in Figs. 3-17, 3-19, 3-21, 3-23, 3-25, 3-27, 3-29, 3-31, 3-33, 3-35, 3-37, and 3-39), and the deformed shape from the FEA (without data points). It should be noted that in cases where such data is available the experimental data represents the deformed shape after unloading the girder. However this data was not available for Girders 2MG, 9MG, and $12 \mathrm{MG}$ due to the need for removing the instrumentation prior to unloading the girder in order to avoid damage to this equipment, and consequently, the deformed shapes presented for

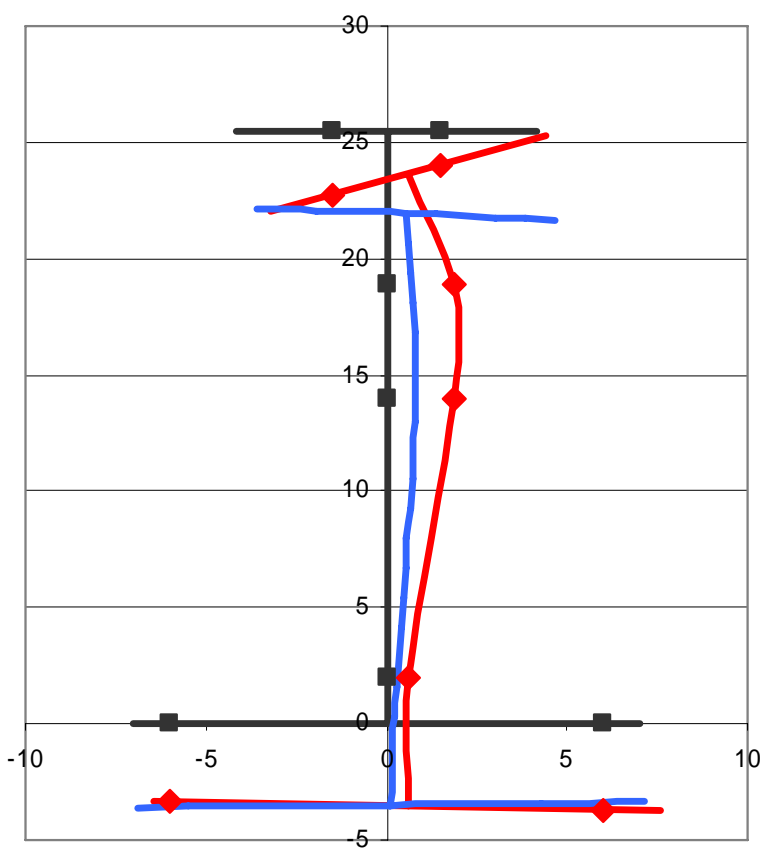

Figure 5-13. FEA and Experimental Deformed Shapes - Girder 1MG 


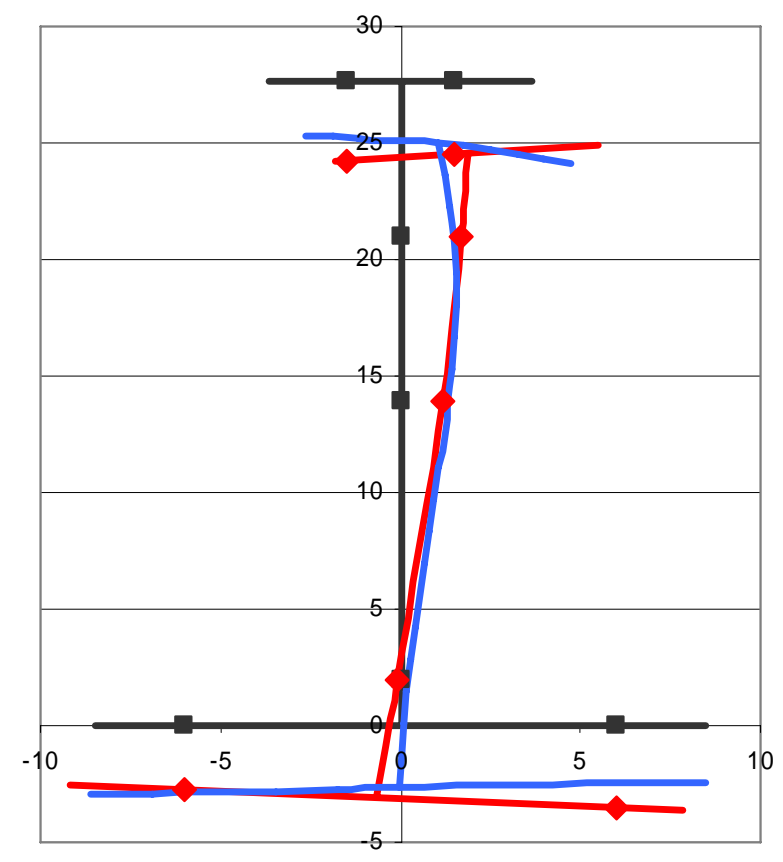

Figure 5-14. FEA and Experimental Deformed Shapes - Girder 2MG

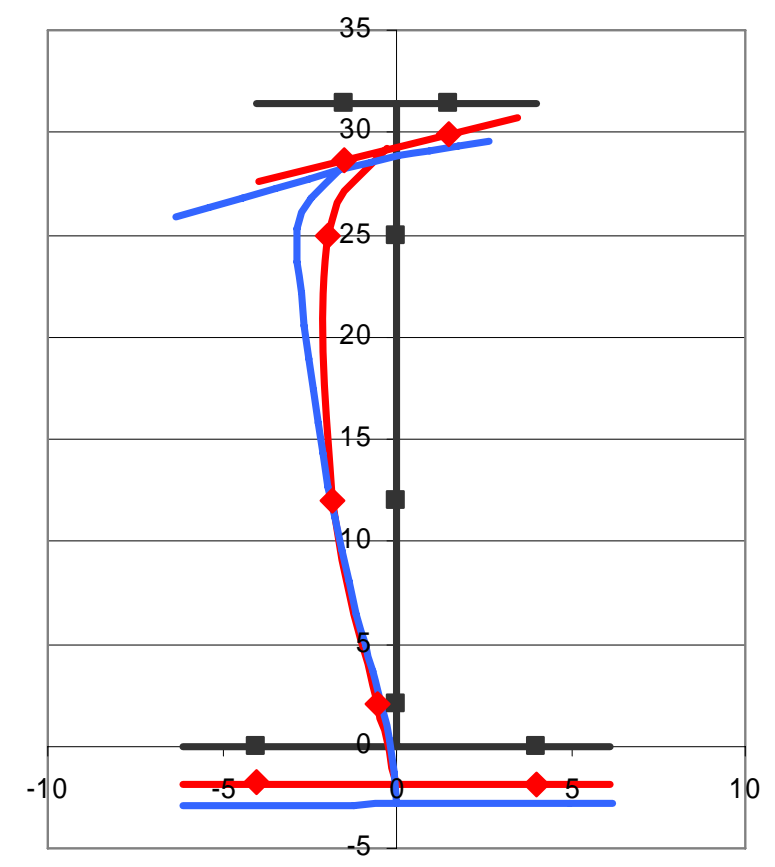

Figure 5-15. FEA and Experimental Deformed Shapes - Girder 3MG 


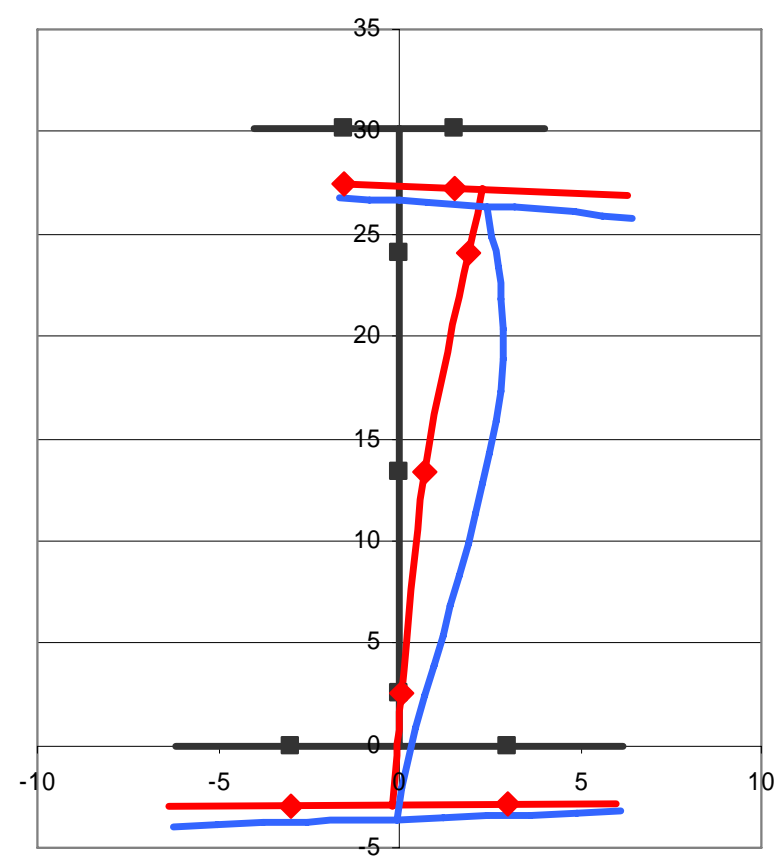

Figure 5-16. FEA and Experimental Deformed Shapes - Girder 4MG

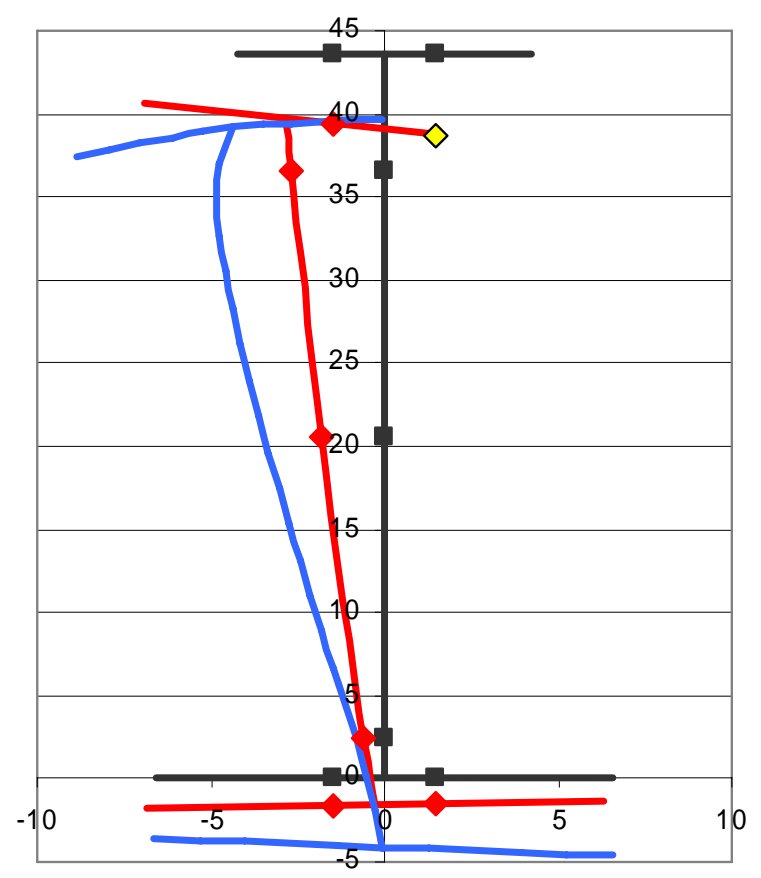

Figure 5-17. FEA and Experimental Deformed Shapes - Girder 5MG 


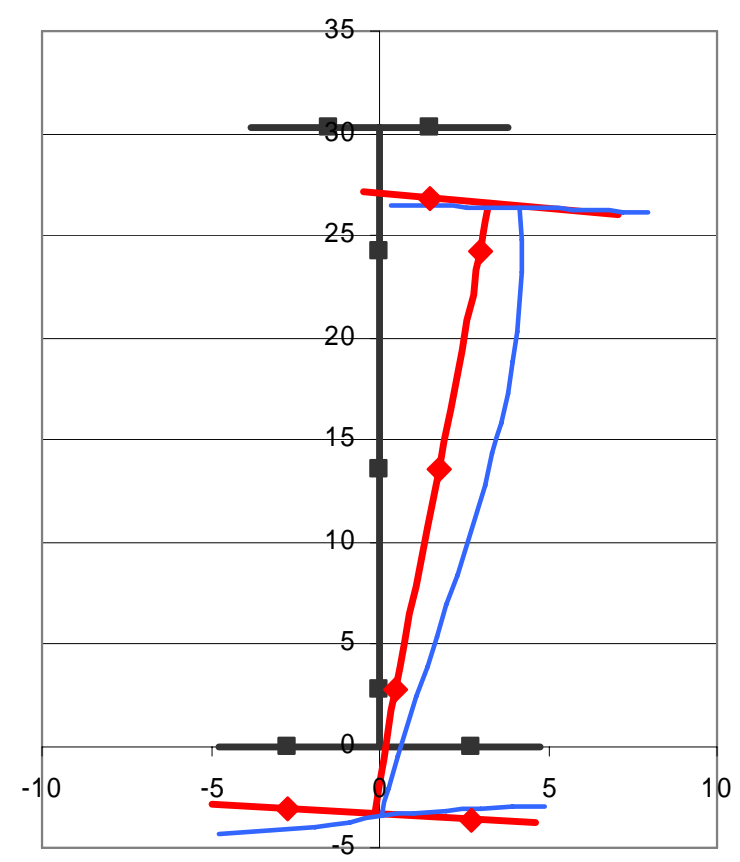

Figure 5-18. FEA and Experimental Deformed Shapes - Girder 6MG

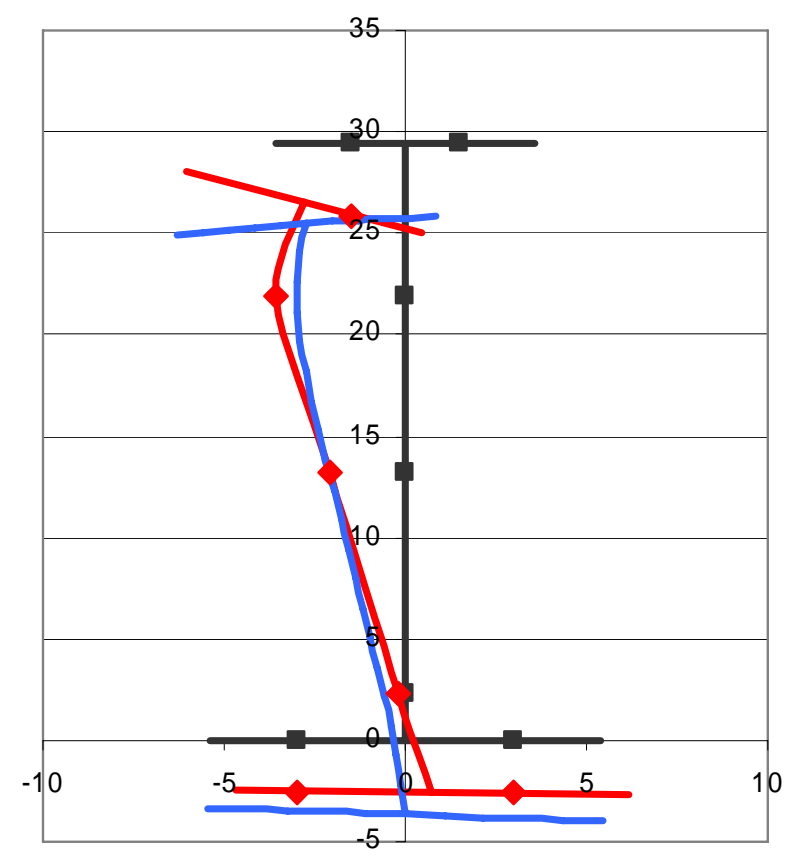

Figure 5-19. FEA and Experimental Deformed Shapes - Girder 7MG 


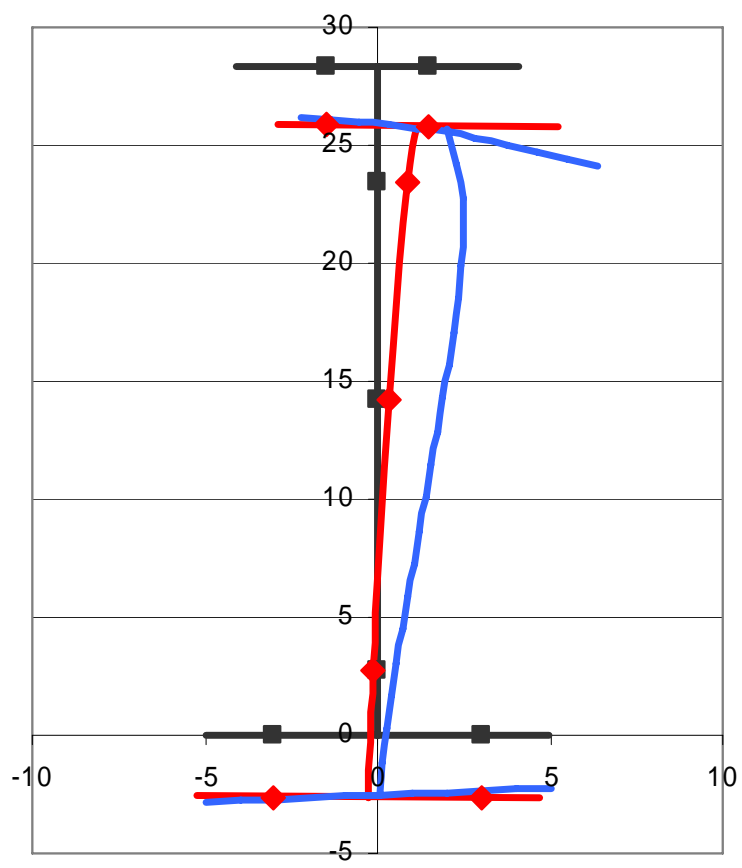

Figure 5-20. FEA and Experimental Deformed Shapes - Girder 8MG

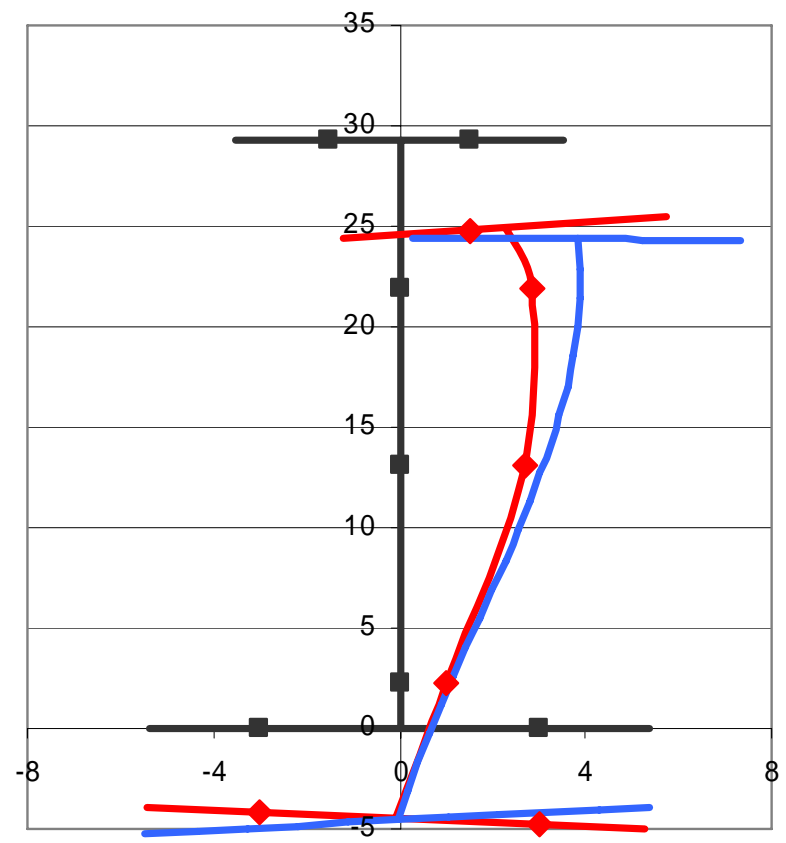

Figure 5-21. FEA and Experimental Deformed Shapes - Girder 9MG 


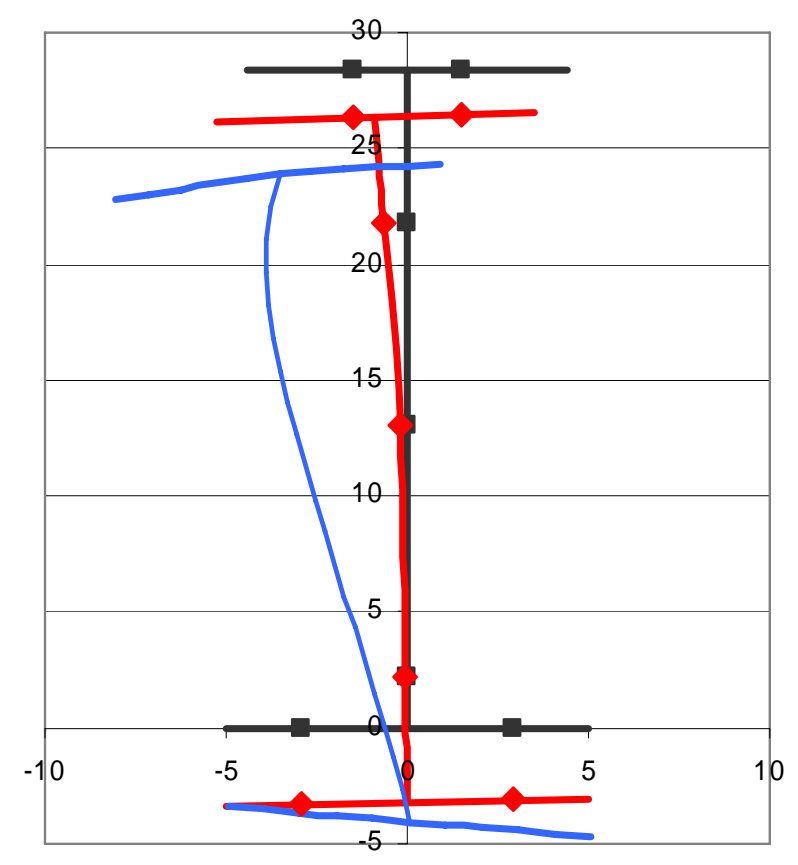

Figure 5-22. FEA and Experimental Deformed Shapes - Girder 10MG

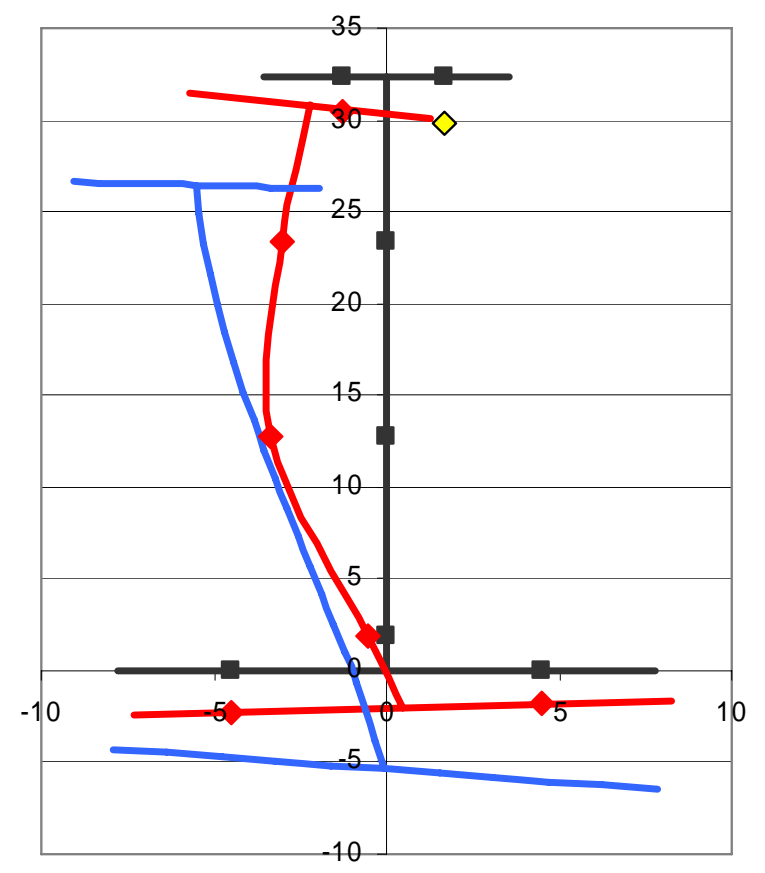

Figure 5-23. FEA and Experimental Deformed Shapes - Girder 11MG 


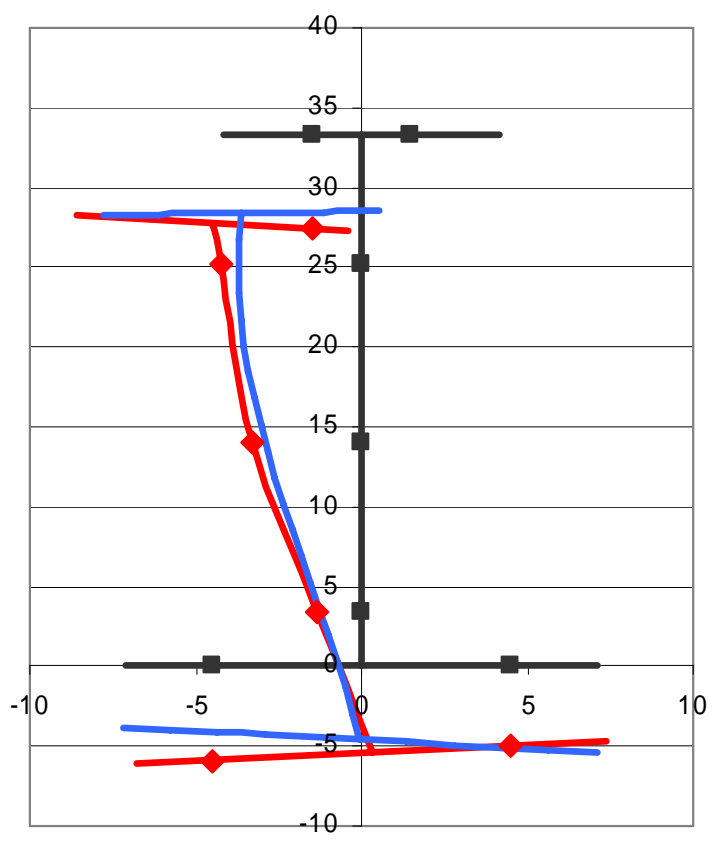

Figure 5-24. FEA and Experimental Deformed Shapes - Girder 12MG

these girders are from the last load step where data is available. The FEA data is the data from the closest corresponding load step at the cross-section closest to the point where the LVDTs are located. The FEA and experimental deformed shapes are shown to be quite similar in most cases, especially within the context of the significant number of parameters involved. For example, in most cases the girders exhibit similar modes of buckling, at the same location along the length of the girder, and with similar magnitudes of deformations. As the experimental deformed shapes, load versus deflection responses, and maximum capacities are all well represented by the FEA it is concluded that the FEA procedures adopted in this work are of reliable accuracy and may be used with confidence to investigate the available rotations of steel I-girders.

The ductility of steel I-girders can be represented by two key parameters. These are the rotation at which the moment level decreases below $\mathrm{M}_{n}$ (termed $\theta_{\mathrm{RL}}$ ) and the equation of the curve representing the unloading behavior below this point. $\theta_{\mathrm{RL}}$ is given below in Table 5-2 for each of the 12 experimental girders and the 71 hypothetical girders to be discussed in this chapter. The $\theta_{\mathrm{RL}}$ values presented in Table 5-2 are determined by analyzing the moment 
Table 5-2. $\theta_{\mathrm{RL}}$ Values in mrad (part 1)

\begin{tabular}{|c|c|c|c|c|c|c|c|c|}
\hline & Girder Label & $\begin{array}{c}\text { Actual } \\
\theta_{\mathrm{RL}}\end{array}$ & $\begin{array}{c}\text { AASHTO } \\
\theta_{\mathrm{RL}}\end{array}$ & $\begin{array}{l}\text { Actual I } \\
\text { AASHTO }\end{array}$ & $\begin{array}{c}2 D_{c p} \\
\theta_{R L}\end{array}$ & $\begin{array}{c}\text { Actual I } \\
2 \mathrm{D}_{\mathrm{cp}}\end{array}$ & $\begin{array}{c}2 \mathrm{D}_{\mathrm{cp}} \text { and } \mathrm{L}_{\mathrm{b}} / \mathrm{r}_{\mathrm{t}} \\
\theta_{\mathrm{RL}}\end{array}$ & $\begin{array}{c}\text { Actual } / \\
2 D_{c p} \text { and } L_{b} / r_{t}\end{array}$ \\
\hline \multirow{12}{*}{ 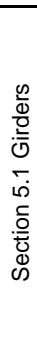 } & $1 \mathrm{MG}$ & 8 & NA & NA & NA & NA & 5.8 & 1.37 \\
\hline & $2 M G$ & 8 & 11.1 & 0.72 & 5.6 & 1.42 & 5.6 & 1.42 \\
\hline & $3 M G$ & 3 & 7.2 & 0.42 & 5.0 & 0.60 & 5.0 & 0.60 \\
\hline & $4 M G$ & 4 & NA & NA & NA & NA & 5.0 & 0.80 \\
\hline & $5 \mathrm{MG}$ & 5 & NA & NA & NA & NA & 5.0 & 1.00 \\
\hline & $6 M G$ & 6 & NA & NA & NA & NA & 5.0 & 1.20 \\
\hline & $7 \mathrm{MG}$ & 5 & NA & NA & NA & NA & 5.0 & 1.00 \\
\hline & $8 M G$ & 3 & NA & NA & NA & NA & 5.0 & 0.60 \\
\hline & 9MG & 2 & NA & NA & NA & NA & 5.0 & 0.40 \\
\hline & $10 \mathrm{MG}$ & 5 & NA & NA & NA & NA & 5.0 & 1.00 \\
\hline & $11 \mathrm{MG}$ & 2 & NA & NA & NA & NA & 5.0 & 0.40 \\
\hline & $12 \mathrm{MG}$ & 3 & NA & NA & NA & NA & 5.0 & 0.60 \\
\hline \multirow{32}{*}{ 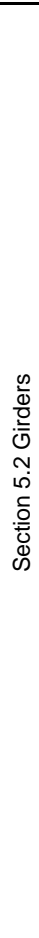 } & CF-1-65-4-50 & 1 & 5.0 & 0.20 & 5.0 & 0.20 & 5.0 & 0.20 \\
\hline & CF-2-65-4-50 & 1 & NA & NA & NA & NA & 5.0 & 0.10 \\
\hline & CF-30-65-4-50 & 1 & NA & NA & NA & NA & 5.0 & 0.20 \\
\hline & SF-1-65-4-50 & 1 & NA & NA & NA & NA & 5.0 & 0.20 \\
\hline & SF-2-65-4-50 & 1 & NA & NA & NA & NA & 5.0 & 0.20 \\
\hline & SF-30-65-4-50 & 0 & NA & NA & NA & NA & 5.0 & 0.04 \\
\hline & SF-1-65-3-50 & 2 & NA & NA & NA & NA & 5.0 & 0.38 \\
\hline & SF-2-65-3-50 & 2 & NA & NA & NA & NA & 5.0 & 0.34 \\
\hline & SF-30-65-3-50 & 1 & NA & NA & NA & NA & 5.0 & 0.26 \\
\hline & CF-1-65-4-H & 2 & 5.0 & 0.44 & 5.0 & 0.44 & 5.0 & 0.44 \\
\hline & CF-2-65-4-H & 0 & NA & NA & NA & NA & 5.0 & 0.06 \\
\hline & CF-30-65-4-H & 2 & NA & NA & NA & NA & 5.0 & 0.40 \\
\hline & SF-1-65-4-H & 1 & NA & NA & NA & NA & 5.0 & 0.20 \\
\hline & SF-2-65-4-H & 1 & NA & NA & NA & NA & 5.0 & 0.18 \\
\hline & SF-30-65-4-H & 2 & NA & NA & NA & NA & 5.0 & 0.44 \\
\hline & CF-0.5-65-3-H & 1 & 8.9 & 0.16 & 5.7 & 0.25 & 5.7 & 0.25 \\
\hline & CF-1-65-3-H & 4 & 8.9 & 0.46 & 5.7 & 0.72 & 5.7 & 0.72 \\
\hline & CF-1.65-65-3-H & 2 & NA & NA & NA & NA & 5.0 & 0.48 \\
\hline & CF-30-65-3-H & 2 & NA & NA & NA & NA & 5.0 & 0.32 \\
\hline & SF-1.75-65-3-H & 2 & NA & NA & NA & NA & 5.0 & 0.34 \\
\hline & SF-30-65-3-H & 1 & NA & NA & NA & NA & 5.0 & 0.28 \\
\hline & CF-1-50-4-50 & 1 & 5.0 & 0.28 & 5.0 & 0.28 & 5.0 & 0.28 \\
\hline & CF-2-50-4-50 & 0 & NA & NA & NA & NA & 5.0 & 0.08 \\
\hline & CF-30-50-4-50 & 0 & NA & NA & NA & NA & 5.0 & 0.02 \\
\hline & SF-1-50-4-50 & 1 & NA & NA & NA & NA & 5.0 & 0.24 \\
\hline & SF-2-50-4-50 & 1 & NA & NA & NA & NA & 5.0 & 0.24 \\
\hline & SF-3.75-50-4-50 & 0 & NA & NA & NA & NA & 5.0 & 0.00 \\
\hline & CF-1-50-4-H & 1 & 5.0 & 0.22 & 5.0 & 0.22 & 5.0 & 0.22 \\
\hline & CF-2-50-4-H & 0 & NA & NA & NA & NA & 5.0 & 0.08 \\
\hline & SF-1-50-4-H & 1 & NA & NA & NA & NA & 5.0 & 0.24 \\
\hline & SF-2-50-4-H & 1 & NA & NA & NA & NA & 5.0 & 0.22 \\
\hline & SF-30-50-4-H & 2 & NA & NA & NA & NA & 5.0 & 0.42 \\
\hline
\end{tabular}

versus rotation plots for each of the experimental and FEA girders, which are presented in Appendices B and D, respectively. It is noted that the maximum capacity of several of the experimental girders was less than $\mathrm{M}_{\mathrm{n}}$; in this case, the reported rotation is the rotation at the maximum moment level, which is conservative in that it is the rotation before the girder experiences any unloading.

\subsection{FEA Parametric Study}

A FEA parametric study was conducted to further investigate the rotation characteristics of slender steel I-girders. The variables included are similar to those included in the experimental testing, although increased combinations of these variables are studied 
Table 5-2, continued. $\theta_{\mathrm{RL}}$ Values in mrad (part 2)

\begin{tabular}{|c|c|c|c|c|c|c|c|c|}
\hline & Girder Label & $\begin{array}{c}\text { Actual } \\
\theta_{\mathrm{RL}}\end{array}$ & $\begin{array}{c}\text { AASHTO } \\
\theta_{\mathrm{RL}} \\
\end{array}$ & $\begin{array}{c}\text { Actual I } \\
\text { AASHTO }\end{array}$ & $\begin{array}{c}2 \mathrm{D}_{\mathrm{cp}} \\
\theta_{\mathrm{RL}}\end{array}$ & $\begin{array}{c}\text { Actual I } \\
2 \mathrm{D}_{\mathrm{cp}}\end{array}$ & $\begin{array}{c}2 D_{c p} \text { and } L_{b} / r_{t} \\
\theta_{R L}\end{array}$ & $\begin{array}{c}\text { Actual } I \\
2 D_{c p} \text { and } L_{b} / r_{t}\end{array}$ \\
\hline \multirow{39}{*}{ 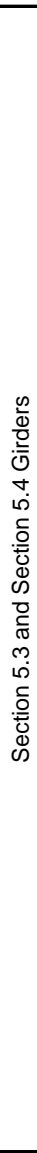 } & 90-2-E-50-10-A & 23 & 27.8 & 0.83 & 18.3 & 1.26 & 18.3 & 1.26 \\
\hline & 90-2-E-50-20-B & 29 & 27.8 & 1.03 & 24.1 & 1.19 & 24.1 & 1.19 \\
\hline & $120-2-E-50-10-A$ & 39 & 36.3 & 1.07 & 28.5 & 1.36 & 28.5 & 1.36 \\
\hline & $120-2-E-50-20-A$ & 27 & 31.4 & 0.87 & 22.0 & 1.24 & 22.0 & 1.24 \\
\hline & $120-2-E-50-20-L b=20$ & 11 & NA & NA & NA & NA & 8.5 & 1.29 \\
\hline & $120-2-E-50-20-L b=30$ & 3 & NA & NA & NA & NA & 5.0 & 0.60 \\
\hline & 120-2-E-50-20-NCF & 16 & NA & NA & NA & NA & 5.0 & 3.20 \\
\hline & 120-2-E-50-20-NCF-Lb=30 & 13 & NA & NA & NA & NA & 5.0 & 2.60 \\
\hline & 120-2-E-50-UF(30)-E & 63 & 31.4 & 1.99 & 35.1 & 1.78 & 35.1 & 1.78 \\
\hline & 120-2-E-50-U(30)-E & 63 & 31.4 & 1.99 & 35.1 & 1.78 & 35.1 & 1.78 \\
\hline & $120-2-\mathrm{H}-10-\mathrm{A}$ & 61 & 25.7 & 2.38 & 36.6 & 1.67 & 36.6 & 1.67 \\
\hline & $120-2-\mathrm{H}-10-\mathrm{B}$ & 60 & NA & NA & NA & NA & 30.3 & 1.98 \\
\hline & $120-2-\mathrm{H}-10-\mathrm{B}(\mathrm{S})$ & 63 & 25.7 & 2.45 & 37.9 & 1.66 & 37.9 & 1.66 \\
\hline & $120-2-H-10-B(S)-16$ & 49 & NA & NA & NA & NA & 33.3 & 1.47 \\
\hline & $120-2-H-10-B(S)-24$ & 36 & NA & NA & NA & NA & 23.5 & 1.53 \\
\hline & $120-2-\mathrm{H}-10-\mathrm{B}(\mathrm{S})-30$ & 23 & NA & NA & NA & NA & 16.1 & 1.43 \\
\hline & $120-2-\mathrm{H}-10-\mathrm{B}(\mathrm{S})-\mathrm{NCF}$ & 25 & NA & NA & NA & NA & 5.0 & 5.00 \\
\hline & 120-2-H-10-B(S)-NCF-30 & 32 & NA & NA & NA & NA & 5.0 & 6.40 \\
\hline & $120-2-\mathrm{H}-20-\mathrm{A}$ & 48 & NA & NA & NA & NA & 35.8 & 1.34 \\
\hline & $120-2-\mathrm{H}-20-\mathrm{A}(\mathrm{S})$ & 27 & NA & NA & NA & NA & 37.0 & 0.73 \\
\hline & $150-2-50-10-A$ & 42 & 27.7 & 1.53 & 28.6 & 1.48 & 27.8 & 1.52 \\
\hline & $150-2-50-20-C$ & 35 & 27.0 & 1.30 & 25.6 & 1.38 & 24.7 & 1.43 \\
\hline & $150-2-50-40-A$ & 37 & 23.7 & 1.56 & 27.5 & 1.35 & 26.5 & 1.40 \\
\hline & 180-2-E-50-10-A & 23 & 27.5 & 0.82 & 22.5 & 1.00 & 22.5 & 1.00 \\
\hline & 180-2-E-50-20-B & 25 & 25.7 & 0.97 & 23.1 & 1.08 & 23.1 & 1.08 \\
\hline & 180-2-E-50-20-B-22.5 & 10 & NA & NA & NA & NA & 12.3 & 0.78 \\
\hline & 180-2-E-50-20-B-30 & 4 & NA & NA & NA & NA & 5.0 & 0.86 \\
\hline & 180-2-E-50-20-NCF & 9 & NA & NA & NA & NA & 5.0 & 1.78 \\
\hline & 180-2-E-50-20-NCF-Lb=30 & 7 & NA & NA & NA & NA & 5.0 & 1.42 \\
\hline & 210-2-E-50-10-E & 10 & 18.8 & 0.54 & 17.8 & 0.57 & 17.8 & 0.57 \\
\hline & 210-2-E-50-10-E-18 & 9 & 18.8 & 0.48 & 17.8 & 0.51 & 17.8 & 0.51 \\
\hline & 210-2-E-50-10-E-26.25 & 3 & NA & NA & NA & NA & 11.1 & 0.28 \\
\hline & 210-2-E-50-10-E-30 & 3 & NA & NA & NA & NA & 8.0 & 0.35 \\
\hline & 210-2-E-50-10-NCF & 19 & NA & NA & NA & NA & 5.0 & 3.80 \\
\hline & 210-2-E-50-10-NCF-30 & 11 & NA & NA & NA & NA & 5.0 & 2.20 \\
\hline & 210-2-E-50-20-F & 14 & 22.1 & 0.62 & 20.2 & 0.68 & 20.2 & 0.68 \\
\hline & 210-2-E-50-30-B & 17 & 19.5 & 0.87 & 21.2 & 0.80 & 21.2 & 0.80 \\
\hline & 210-2-E-50-40-C & 23 & 17.3 & 1.33 & 19.6 & 1.17 & 19.6 & 1.17 \\
\hline & 140-175-140-D & 17 & 27.7 & 0.61 & 35.2 & 0.48 & 35.2 & 0.48 \\
\hline
\end{tabular}

compared to those feasible to include in the experimental testing. The parametric values included in this FEA study are equal to:

- flange slenderness values equal to the compact limit, $0.38 \sqrt{E / F_{y c}}$, and the maximum allowable flange slenderness of 12 ;

- homogeneous Grade 50 girders and hybrid girders comprised of Grade 50 webs and Grade 70 flanges;

- $\mathrm{D} / \mathrm{b}_{\mathrm{fc}}$ ratios of 3 and 4.25 ;

- lateral bracing distances equal to the current AASHTO 2004 Appendix B lateral bracing limit, and various percentages of this limit, up to a maximum lateral bracing distance of $30 \mathrm{ft}$; and

- $\mathrm{D}_{\mathrm{cp}} / \mathrm{D}$ ratios of 0.65 and 0.50 . 
- A constant web slenderness equal to the noncompact limit, $2 D_{c} / t_{w}=5.7 \sqrt{E / F_{y c}}$, was used as the variation in web slenderness results in little influence on moment versus rotation behavior.

In order to minimize the number of analyses required while obtaining complete information on the rotation characteristics of slender I-girders, representative combinations of the above parameters were carefully selected. Table 5-3 summarizes the attributes of each of the 32 analyses comprising this parametric study. This table also introduces the naming convention used for these FEA girders. The first component of the girder label refers to the flange slenderness used; “CF” indicates that the flange is proportioned to have a slenderness equal to the compact limit, while "SF" specifies that the maximum flange slenderness is used. The

Table 5-3. Scope of Parametric Study

\begin{tabular}{|c|c|c|c|c|c|}
\hline Girder Label & $\begin{array}{c}\text { Flange } \\
\text { Slenderness }\end{array}$ & $\begin{array}{l}\text { Lateral Bracing } \\
\text { Distance }\end{array}$ & $\mathrm{D}_{\mathrm{cp}} / \mathrm{D}$ Ratio & $\mathrm{D} / \mathrm{b}_{\mathrm{fc}}$ Ratio & $\begin{array}{c}\text { Material } \\
\text { Configuration }\end{array}$ \\
\hline 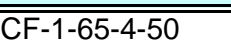 & Compact & $\overline{100 \%}$ & 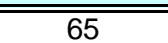 & 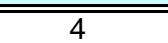 & $\overline{50}$ \\
\hline CF-2-65-4-50 & Compact & $200 \%$ & 65 & 4 & 50 \\
\hline CF-30-65-4-50 & Compact & $30(375 \%)$ & 65 & 4 & 50 \\
\hline SF-1-65-4-50 & Slender & $100 \%$ & 65 & 4 & 50 \\
\hline SF-2-65-4-50 & Slender & $200 \%$ & 65 & 4 & 50 \\
\hline SF-30-65-4-50 & Slender & 30 (375\%) & 65 & 4 & 50 \\
\hline SF-1-65-3-50 & Slender & $100 \%$ & 65 & 3 & 50 \\
\hline SF-2-65-3-50 & Slender & $200 \%$ & 65 & 3 & 50 \\
\hline SF-30-65-3-50 & Slender & 30 (400\%) & 65 & 3 & 50 \\
\hline CF-1-65-4-H & Compact & $100 \%$ & 65 & 4 & $\mathrm{H}$ \\
\hline CF-2-65-4-H & Compact & $200 \%$ & 65 & 4 & $\mathrm{H}$ \\
\hline CF-30-65-4-H & Compact & 30 (429\%) & 65 & 4 & $\mathrm{H}$ \\
\hline SF-1-65-4-H & Slender & $100 \%$ & 65 & 4 & $\mathrm{H}$ \\
\hline SF-2-65-4-H & Slender & $200 \%$ & 65 & 4 & $\mathrm{H}$ \\
\hline SF-30-65-4-H & Slender & 30 (500\%) & 65 & 4 & $\mathrm{H}$ \\
\hline CF-0.5-65-3-H & Compact & $50 \%$ & 65 & 3 & $\mathrm{H}$ \\
\hline CF-1-65-3-H & Compact & $100 \%$ & 65 & 3 & $\mathrm{H}$ \\
\hline CF-1.65-65-3-H & Compact & $165 \%$ & 65 & 3 & $\mathrm{H}$ \\
\hline CF-30-65-3-H & Compact & $30(230 \%)$ & 65 & 3 & $\mathrm{H}$ \\
\hline SF-1.75-65-3-H & Slender & $175 \%$ & 65 & 3 & $\mathrm{H}$ \\
\hline SF-30-65-3-H & Slender & 30 (250\%) & 65 & 3 & $\mathrm{H}$ \\
\hline CF-1-50-4-50 & Compact & $100 \%$ & 50 & 4 & 50 \\
\hline CF-2-50-4-50 & Compact & $200 \%$ & 50 & 4 & 50 \\
\hline CF-30-50-4-50 & Compact & 30 (333\%) & 50 & 4 & 50 \\
\hline SF-1-50-4-50 & Slender & $100 \%$ & 50 & 4 & 50 \\
\hline SF-2-50-4-50 & Slender & $200 \%$ & 50 & 4 & 50 \\
\hline SF-3.75-50-4-50 & Slender & 30 (375\%) & 50 & 4 & 50 \\
\hline CF-1-50-4-H & Compact & $100 \%$ & 50 & 4 & $\mathrm{H}$ \\
\hline CF-2-50-4-H & Compact & $200 \%$ & 50 & 4 & $\mathrm{H}$ \\
\hline SF-1-50-4-H & Slender & $100 \%$ & 50 & 4 & $\mathrm{H}$ \\
\hline SF-2-50-4-H & Slender & $200 \%$ & 50 & 4 & $\mathrm{H}$ \\
\hline SF-30-50-4-H & Slender & 30 (500\%) & 50 & 4 & $\mathrm{H}$ \\
\hline
\end{tabular}


second element of the girder label indicates the multiple used of the Appendix B lateral bracing distance or that the maximum lateral bracing distance of $30 \mathrm{ft}$ is used. The third label refers to the percentage of the web depth in compression. The fourth part indicates the $\mathrm{D} / \mathrm{b}_{\mathrm{fc}}$ ratio. Lastly, the fifth component conveys the material configuration where " 50 " indicates that the girder is a homogeneous Grade 50 girder and " $\mathrm{H}$ " indicates that the girder is a hybrid girder with Grade 70 steel used for the flanges of the negative bending section and Grade 50 steel used elsewhere.

Girder designs satisfying the desired parameters were created for each of the cases presented in Table 5-3. This was accomplished by first selecting a web depth of 60 in. for each girder. The compression flange width was then selected to provide the desired $\mathrm{D} / \mathrm{b}_{\mathrm{fc}}$ ratio. Next, the compression flange thickness was selected to give the desired compression flange slenderness. The web slenderness, tension flange width, and tension flange thickness were then simultaneously selected to give the desired web slenderness and $\mathrm{D}_{\mathrm{cp}} / \mathrm{D}$ ratio. Lastly, the lateral bracing distances were selected.

The selected web depth of 60 in. corresponds to an L/D (span length to web depth) ratio of 30 for a $150 \mathrm{ft}$ span length. It was assumed that $25 \%$ of each span length was in negative bending, resulting in a simply-supported length of $75 \mathrm{ft}$ in each of the models. Thus, the moment gradient induced into each girder is representative of actual loading conditions for typical continuous-span bridges. A minimum of four lateral bracing segments are used to provide warping restraint to the center two critical lateral bracing panels. Thus, for a maximum lateral bracing distance of $30 \mathrm{ft}$, the total girder length is $120 \mathrm{ft}$. Consequently, cantilevered ends of the girder extend beyond the simple supports. All other aspects of the FEA are as discussed in Chapter 4.

Representative moment rotation plots resulting from these analyses are presented in Figs. 525 and 5-26, while this data is presented for each of the parametric study girders in Appendix D. Figures 5-25 and 5-26 show that these girders exhibit a relatively low degree of ductility in that there is little rotation available at high moment levels and the slope of the descending portion of the curve is relatively steep. The ductility resulting from the parametric study 


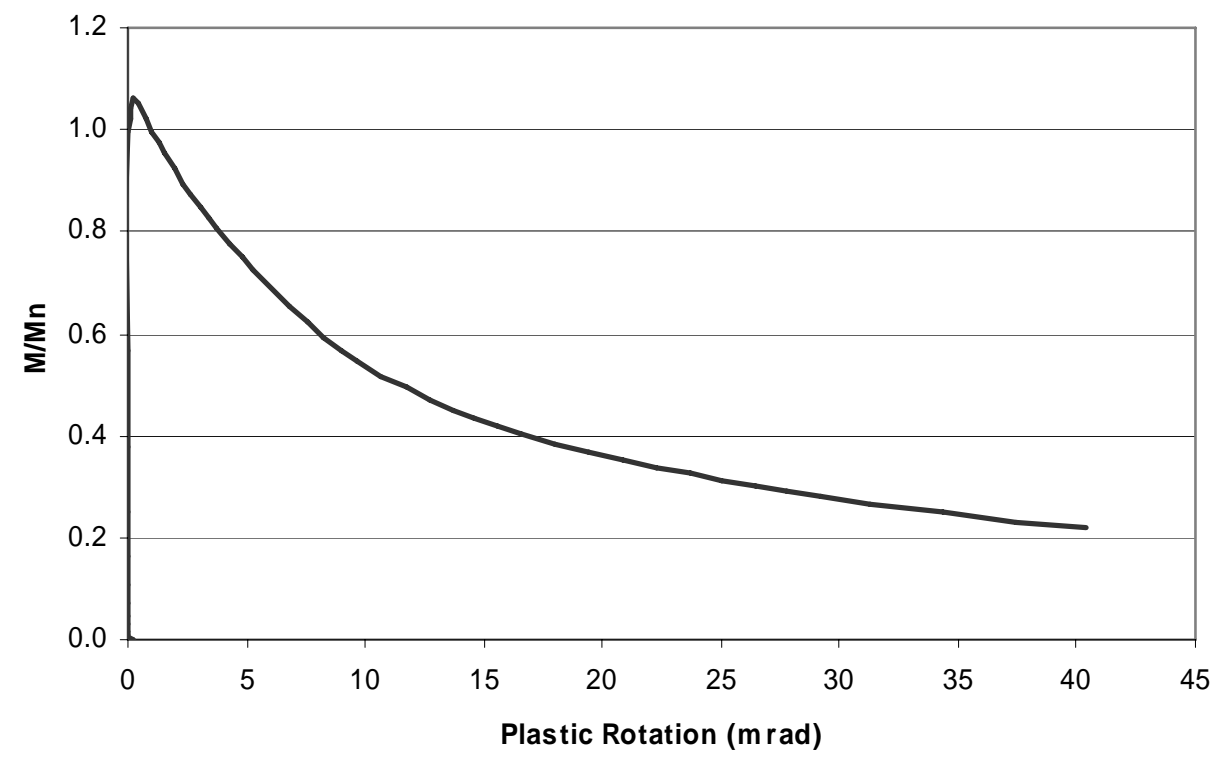

Figure 5-25. Moment versus Rotation - Girder CF-1-65-4-50

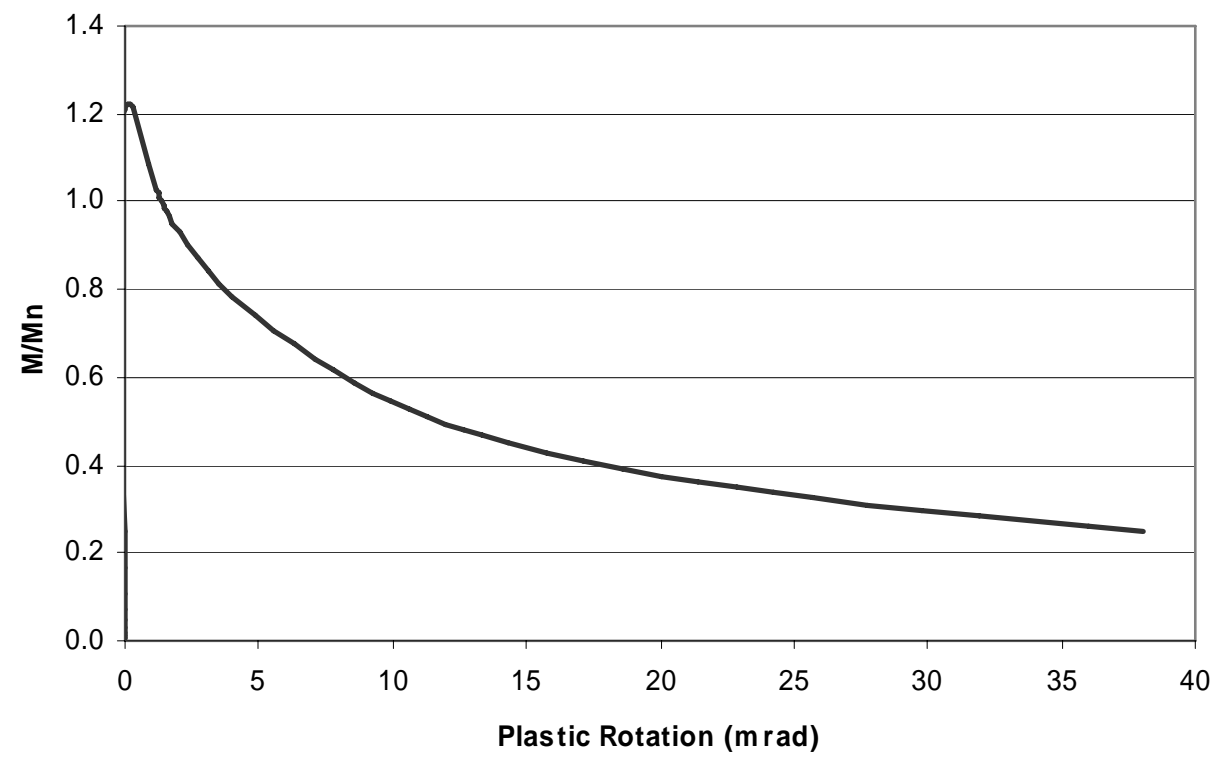

Figure 5-26. Moment versus Rotation - Girder SF-30-65-3-H

designs is further represented by the data presented in Table 5-2. Here $\theta_{\mathrm{RL}}$ is presented for each of the parametric girders, which is determined by analyzing the moment rotation plots (contained in Appendix D) for each of the girders. Here it shown that $\theta_{\mathrm{RL}}$ varies between 0 and 4 for this series of parametric girders. 
It should be noted that because the $\theta_{\mathrm{RL}}$ value for a given girder is directly related to the girder's nominal moment capacity, the value of $\mathrm{M}_{\mathrm{n}}$ of a given girder should also be considered when comparing $\theta_{\mathrm{RL}}$ values for different girders. For example, considering the results presented in Table 5-2 for girders CF-1-65-4-H, CF-2-65-4-H, and CF-30-65-4-H, it is observed that despite the significantly increased lateral bracing distance of CF-30-65-4-H compared to CF-2-65-4-H, a higher $\theta_{\mathrm{RL}}$ value results for the girder with the longer bracing distance. This is somewhat counterintuitive and occurs because the AASHTO (2004) equations predict lower moment capacities for these girders with longer lateral bracing distances (or increasing flange slenderness, etc). Thus, the girders with longer bracing distances exhibit less available rotation at a given magnitude of moment, but when the results are normalized by nominal moment capacity, greater available rotations may be predicted for girders with greater lateral bracing distances.

The relatively low $\theta_{\mathrm{RL}}$ values of the parametric study girders is attributed to the fact that several of the slenderness ratios are simultaneously maximized (to varying degrees) in each of these girders. Because of the narrow range of $\theta_{\mathrm{RL}}$ that exists for these girders, it is necessary to perform additional FEA studies to obtain data and form conclusions on the variation in $\theta_{\mathrm{RL}}$ with the variation in girder geometries. The subsequent series of FEA is performed for girders that are more typical of those that would be encountered in actual bridge designs. Specifically, FEA was conducted of the girders resulting from the design study subsequently presented in Chapter 6. These girders are typical of those that would result from use of the current (AASHTO 2004) moment redistribution procedures. Analyses of these girders are presented in the following section, Section 5.3. To then obtain moment versus rotation behavior of girders that have slenderness properties exceeding the current moment redistribution limits, the slenderness properties of these designs were increased and FEA of these girders were conducted as described in Section 5.4.

\subsection{FEA of Design Study Girders}

Section 6.3 presents the design of a representative series of girders obtained using moment redistribution procedures. These designs were created for the purpose of aiding in the determination of rotation requirements as discussed in the following chapter. FEA of 21 of 
these girders are performed here to obtain the moment versus rotation of typical girders, which are then used to identify trends in this behavior. Girders with similar moment-rotation behavior are subsequently identified and representative girders from each of these groups are selected for investigation of the influences of increased slenderness parameters, which is presented in Section 5.4 .

The design criteria used for these girders are presented in Section 6.3 and Appendix E details each design. To summarize, two-, three-, and four-span continuous homogeneous and hybrid girders were designed targeting specific percentages of redistribution moment. FEA of the negative bending section of representative two- and three-span designs were performed and the moment versus rotation behavior of these girders was obtained. These girders were modeled representing the dead load contraflexure points as the locations of simple supports and cantilevered segments extend beyond these supports as needed to provide additional lateral bracing panels, and consequently, warping restraint to the midspan panels. The models were then loaded with a point load at the center of the simply-supported span, producing the moment gradient typical of negative bending sections. Figures 5-27 through 530 present the resulting data by grouping together the plots for girders with moment versus rotation curves having similar shapes.

These figures refer to specific designs using the following naming convention. For the two span designs, the first number in the label refers to the span length, followed by a label of "2" indicating that the design is a two-span bridge, and the label "E" indicating that both spans are of equal length. For the three-span designs, the first three numbers give the lengths of each span. These span length labels are then followed by a label indicating the material properties of the girder, where " 50 " represents a Grade 50 design and " $\mathrm{H}$ " represents a hybrid girder with Grade 70 flanges in negative bending sections and Grade 50 elsewhere. Next, a label indicating the targeted percentage of redistribution moment is given. In most cases more than one design was performed for each design situation before the optimum design was achieved; the next label in the naming convention simply references the specific design for each situation that was selected as the optimal design. Some of the design labels are then followed by the label "(S)", which indicates that the girder design is a stiffened girder. In 
cases where the design girder consists of a uniform cross-section, this is indicated by the label " $U$ " and in cases where the flange dimensions are uniform but the web thickness varies at field splice locations, this is indicated by the label "UF”.

Figures 5-27 through 5-30 show that these girders exhibit significantly more ductility than the girders previously presented in Section 5.2. This is also demonstrated by the higher qRL values for these girders, presented in Table 5-2. This is expected as the girders in these figures are more compact than those from the parametric study. From each of the plots in Figs. 5-27 through 5-30, the girder with the least ductility is then selected for further study; modified versions of girders 120-2-E-50-20-A, 120-2-E-H-10-B-(S), 180-2-E-50-20-B, and 210-2-E-50-10-E with increased lateral bracing distance and flange slenderness are designed and analyzed as discussed below.

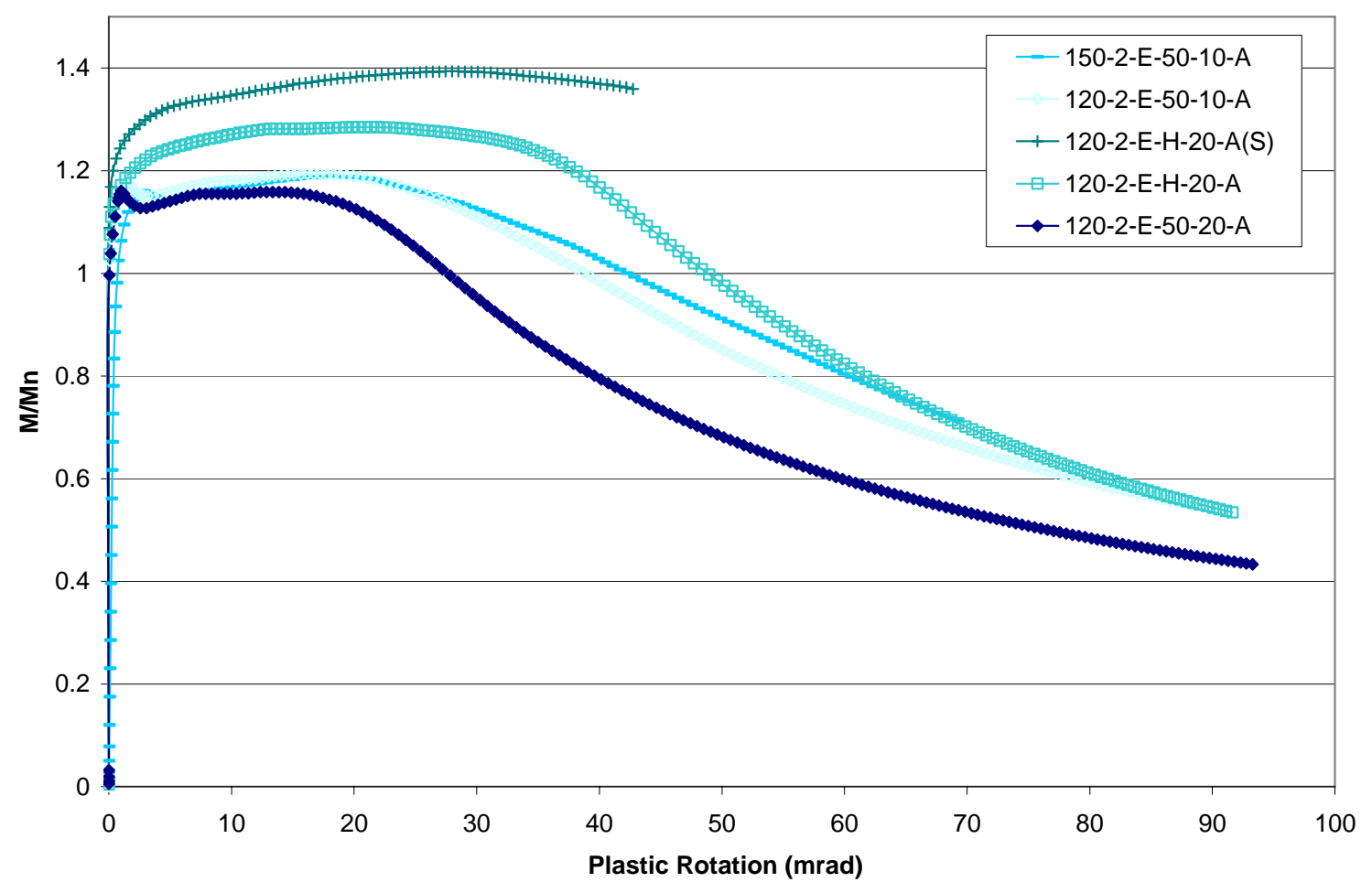

Figure 5-27. Moment versus Rotation for Design Study Girders - Group 1 


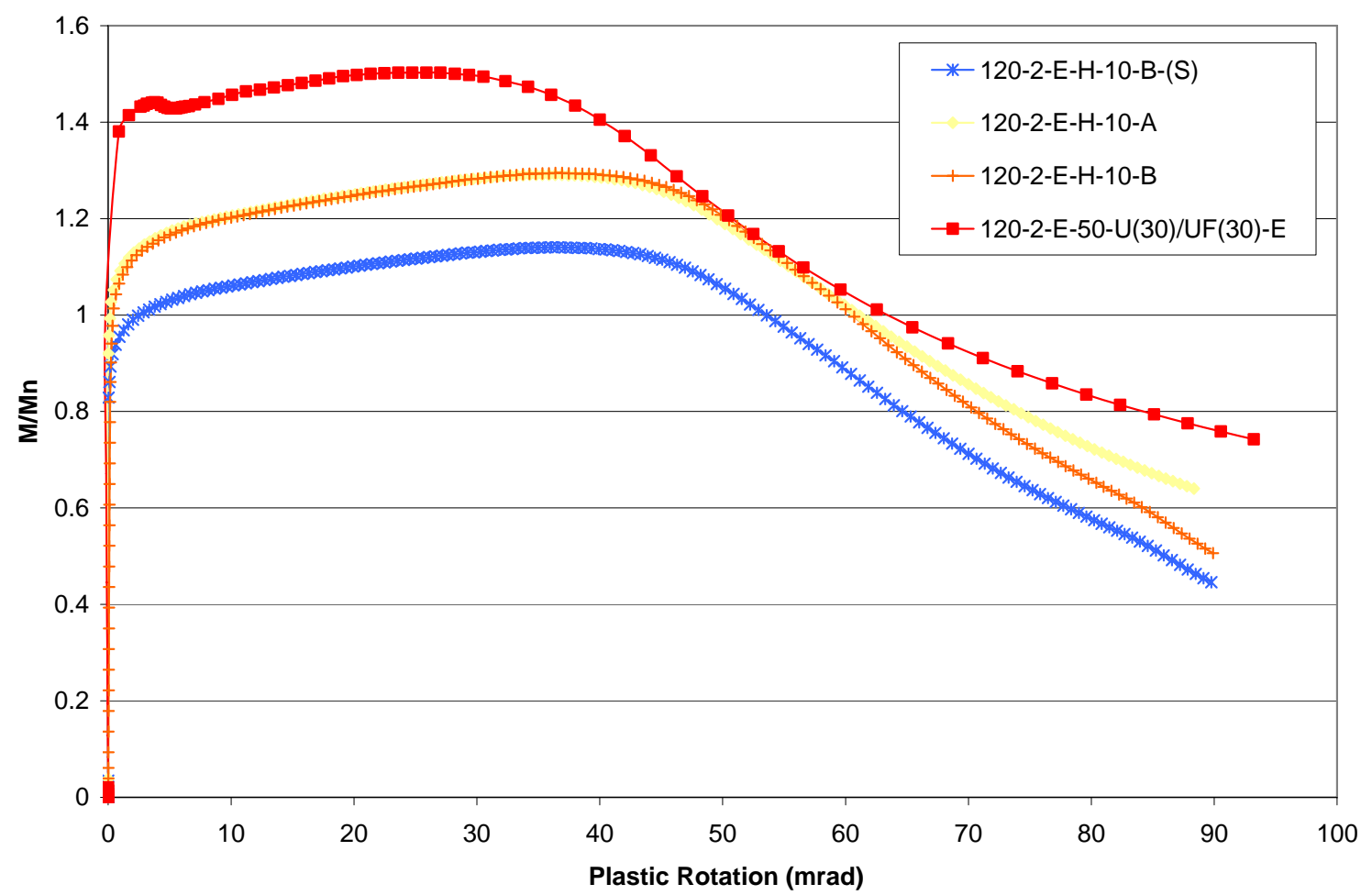

Figure 5-28. Moment versus Rotation for Design Study Girders - Group 2

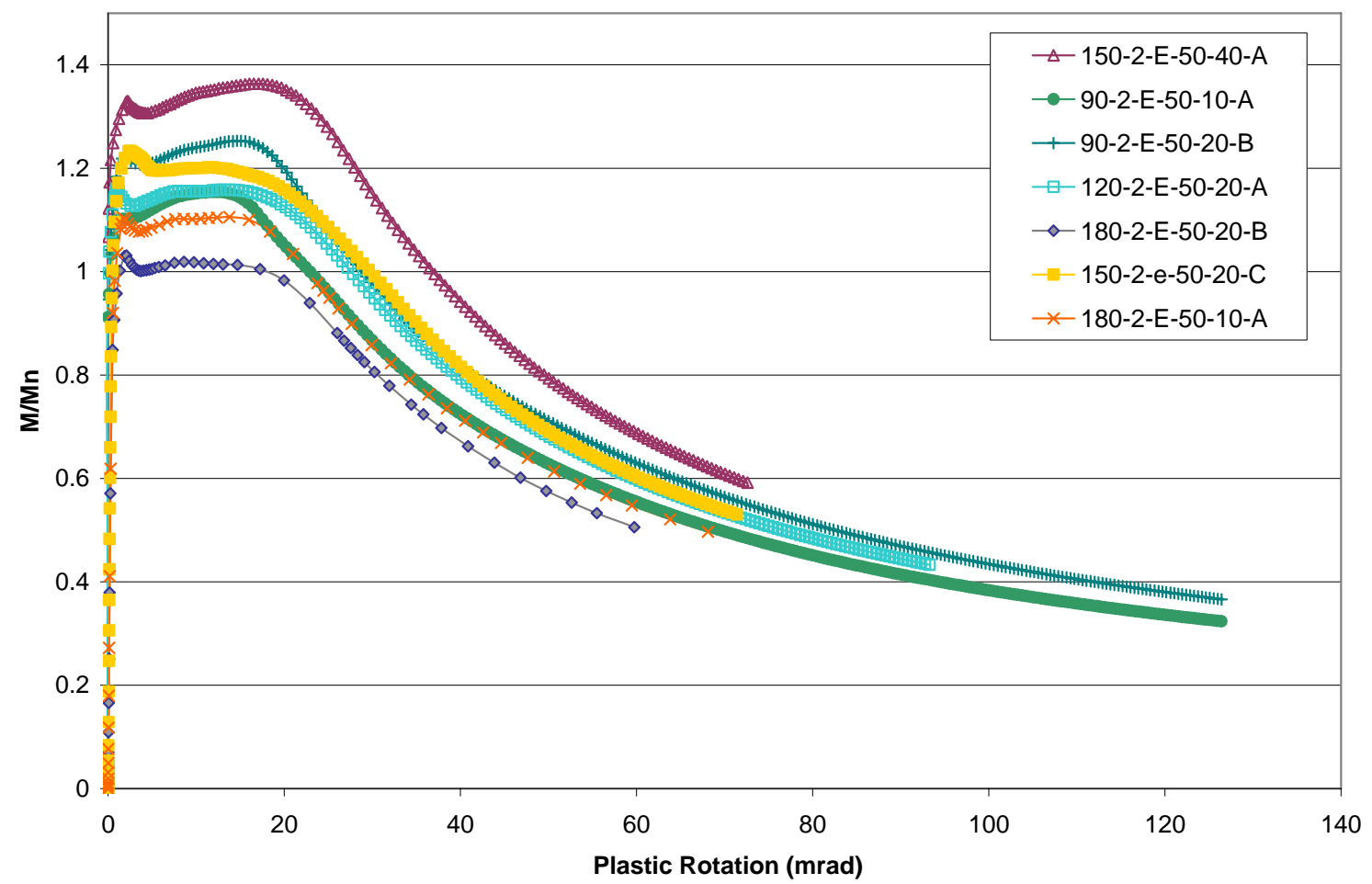

Figure 5-29. Moment versus Rotation for Design Study Girders - Group 3 


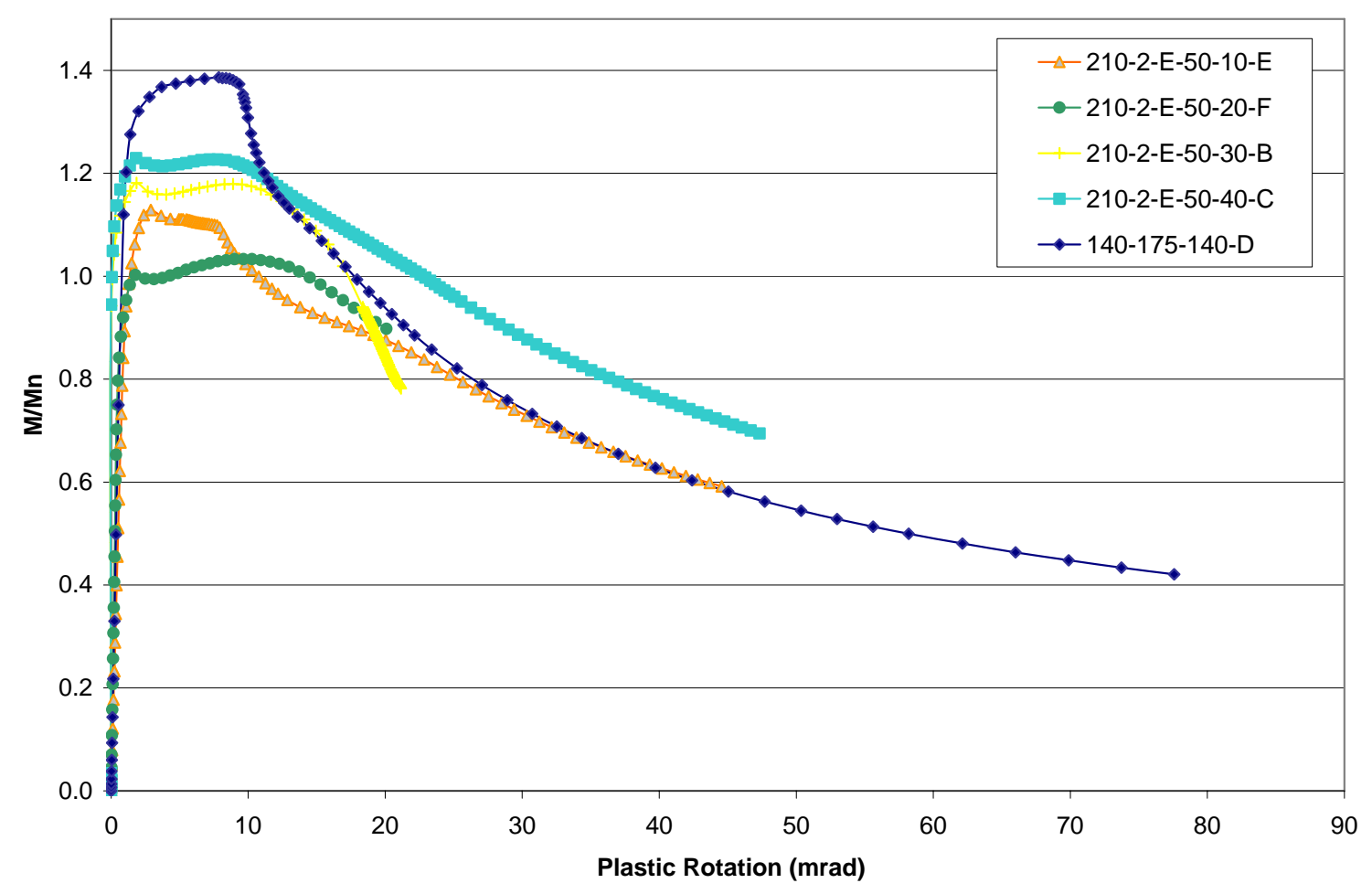

Figure 5-30. Moment versus Rotation for Design Study Girders - Group 4

\subsection{FEA of Extended Design Studies}

After identifying four of the girders from the design studies to be used for an extended study on available rotations, modified versions of these girders were designed with increased flange slenderness and lateral bracing distances. For the designs with increased lateral bracing distances, the same cross-sectional geometry was used as in the original designs and the lateral bracing distances were increased up to a maximum value of $30 \mathrm{ft}$. To produce designs with increased compression flange slenderness, the same web dimensions were used as in the original designs. The flange dimensions were then altered to produce a compression flange slenderness equal to 12 , while producing a moment capacity at least as great as the moment capacity in the original design. Each of the designs with increased compression flange slenderness were designed with two alternative lateral bracing configurations: lateral bracing sucyh that the current moment redistribution requirements are satisfied and a lateral bracing distance of $30 \mathrm{ft}$. FEA of these girders were then performed using the same methods as previously described. 
The results of these efforts are presented in the form of the moment versus rotation plots given in Figs. 5-31 through 5-34. In these figures the girders are labeled using the naming convention described above followed by additional descriptors indicating the alternative lateral bracing distance used (if applicable) and if a non-compact flange (indicated by the label "NCF") is used. Figure 5-31 presents the data for the original and modified versions of girder 120-2-E-50-20-A. Here it is shown that, for the designs with a compact flange, increasing lateral bracing distances correspond to a decrease in the available rotation of the girder. Increased lateral bracing distance also results in reduced rotations for the girders with non-compact flanges, although this influence is much less significant for these girders. In fact the available rotation at $M_{n}$ is nearly equal for the two non-compact flange designs shown in Fig. 5-31 even though the lateral bracing distance varies by 300\%. This difference in behavior between the compact and non-compact flange girders is consistent with the behavior predicted by the AASHTO (2004) moment redistribution lateral bracing limit. For the original, compact flange design for Girder 120-2-E-50-20-A the AASHTO 2004

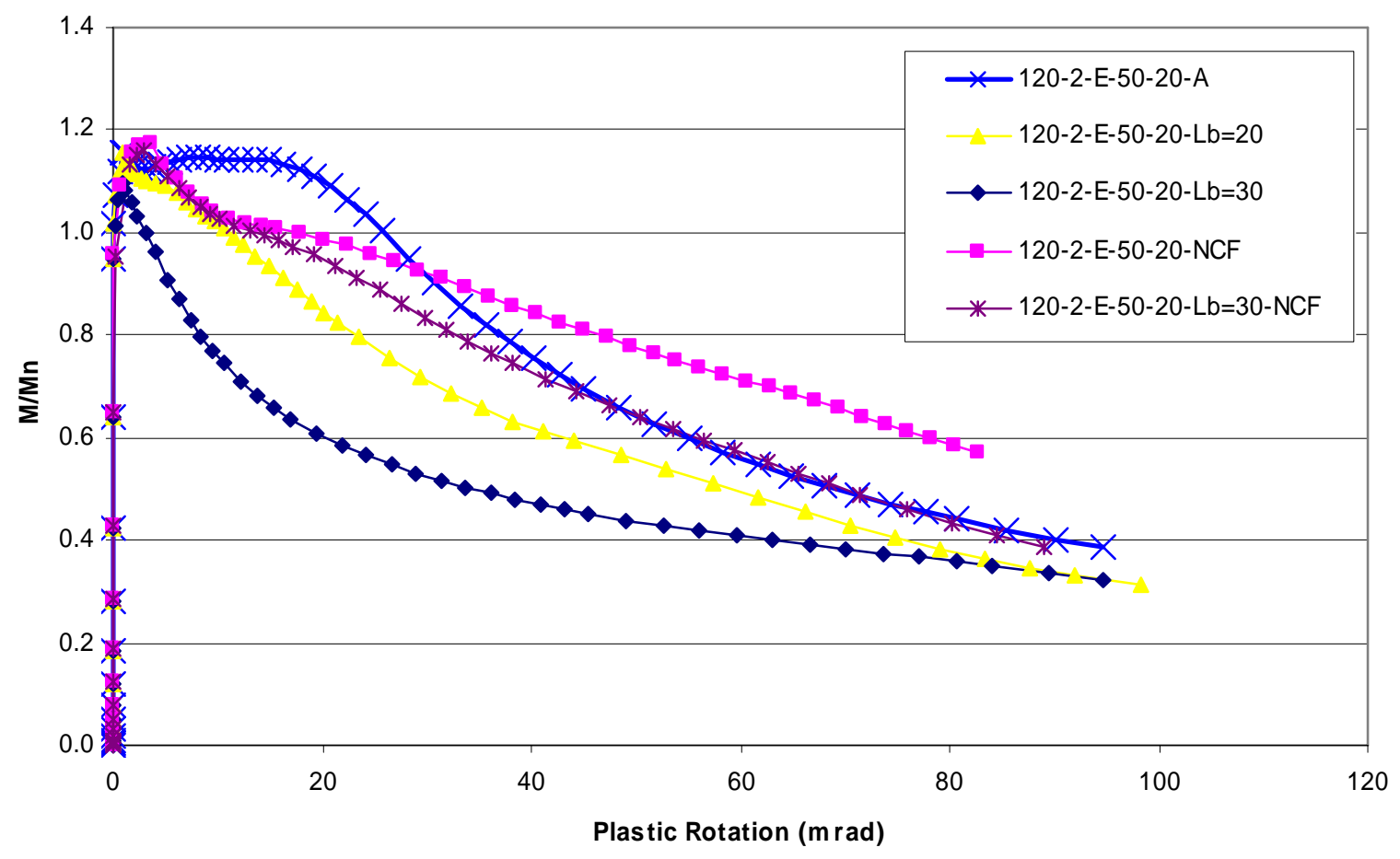

Figure 5-31. Moment versus Rotation for Permutations of Girder 120-2-E-50-20-A 


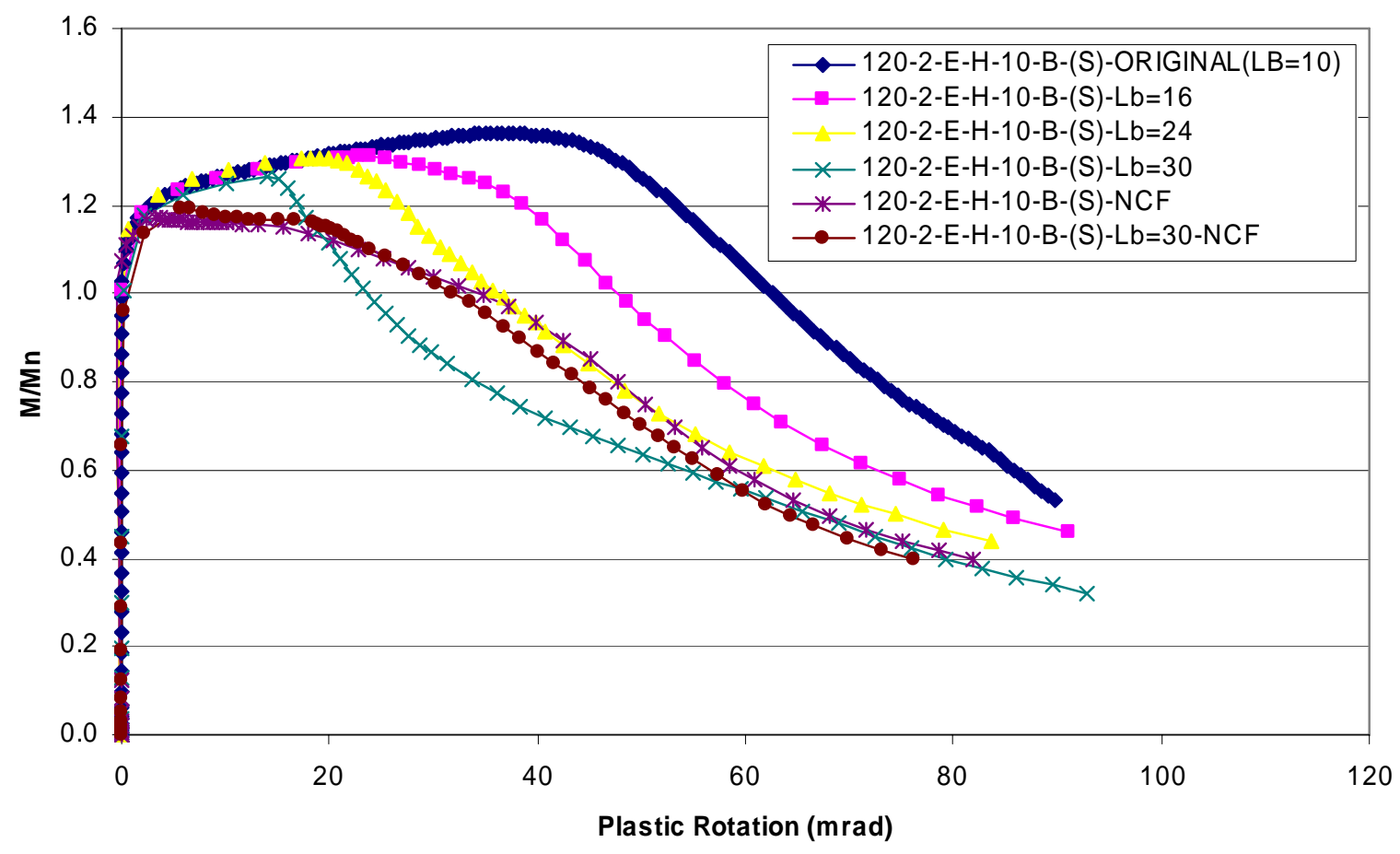

Figure 5-32. Moment versus Rotation for Permutations of Girder 120-2-E-H-10-B-(S)

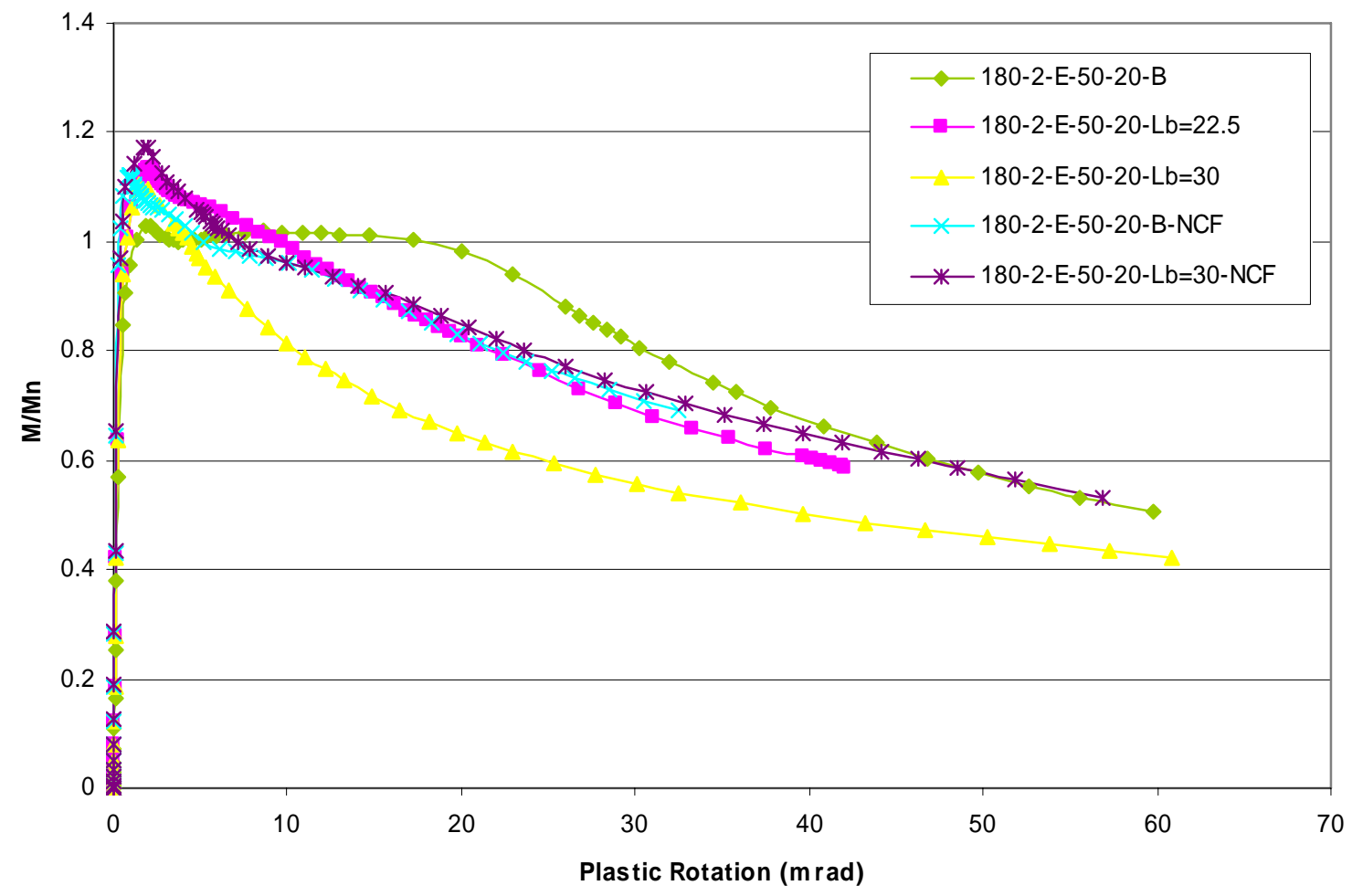

Figure 5-33. Moment versus Rotation for Permutations of Girder 180-2-E-50-20-B 


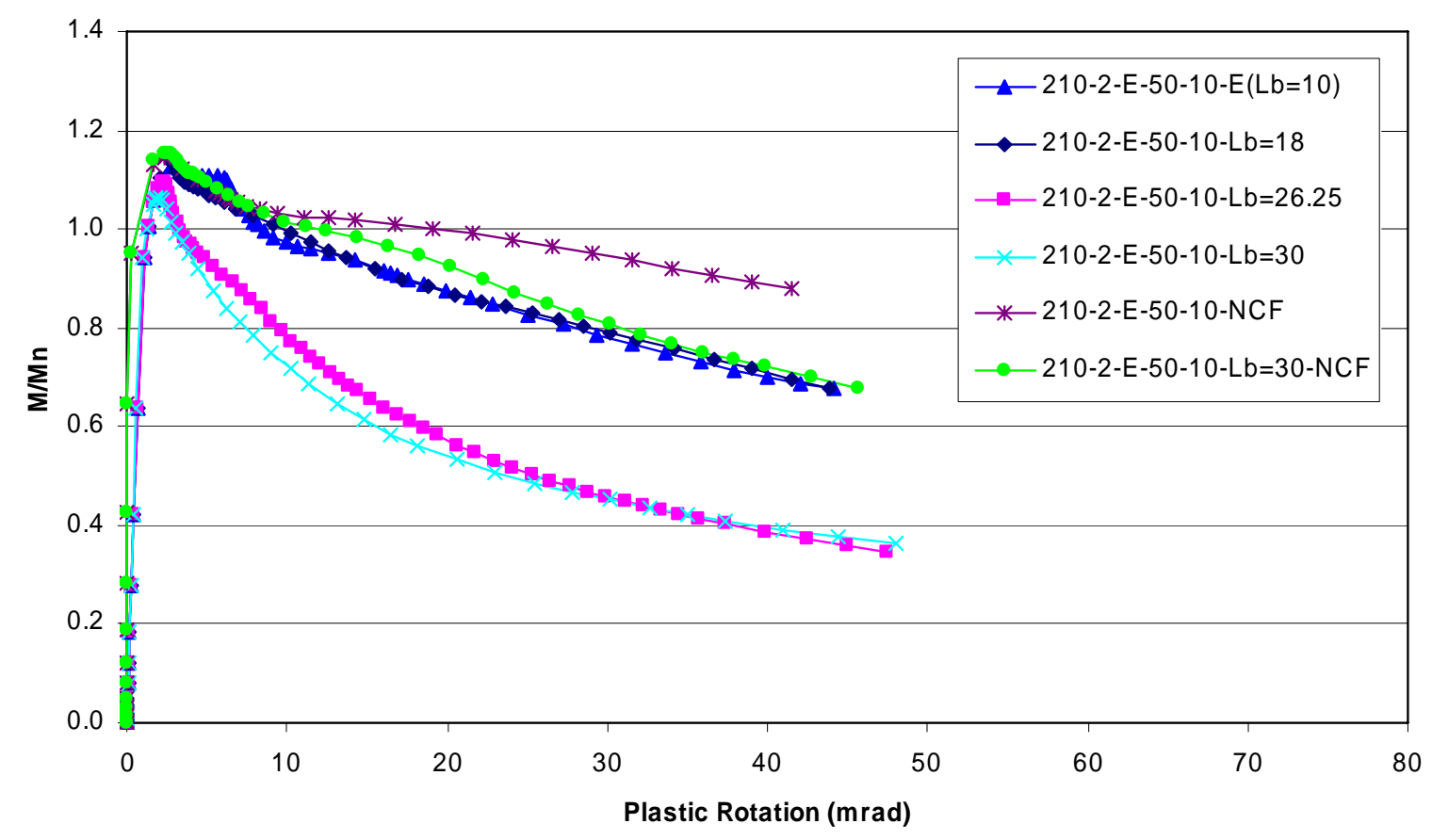

Figure 5-34. Moment versus Rotation for Permutations of Girder 210-2-E-50-10-E

Appendix B limit was $14 \mathrm{ft}$, while the Appendix B limit for the non-compact flange girders is $36 \mathrm{ft}$. Thus, it appears that when the AASHTO Appendix B lateral bracing limit is satisfied, there is little influence of available rotations with varying lateral bracing distances. Comparing the two designs having the original lateral bracing distance of $10 \mathrm{ft}$ in Fig. 5-31 shows that the girder with the compact flange initially exhibits higher rotations than the girder with the non-compact flange, however at a moment capacity of less than $90 \%$ of $\mathrm{M}_{\mathrm{n}}$ on the unloading portion of the curve, the girder with the non-compact flange has the greater ductility. This is attributed to the higher value of $r_{t}$ characteristic of the non-compact flange girder. Similarly, comparing the two designs with a lateral bracing distance of $30 \mathrm{ft}$, the noncompact flange girder is again the more ductile of the two as the higher $r_{t}$ value of this girder provides greater restraint to the lateral torsional buckling mode of failure.

These same trends are evident in Figs. 5-32 through 5-34, which present the data from the modified designs of 120-2-E-H-10-B-(S), 180-2-E-50-20-B, and 210-2-E-50-10-E, respectively. For the designs in Fig. 5-32, the Appendix B lateral bracing requirements are $10 \mathrm{ft}$ for the compact flange girders and $22 \mathrm{ft}$ for the non-compact flange; for the designs in 
Fig. 5-33, these requirements are $15 \mathrm{ft}$ for the compact flange girders and $37 \mathrm{ft}$ for the noncompact flange girders; and for the designs in Fig. 5-34, these requirements are $22 \mathrm{ft}$ for the compact flange girders and $42 \mathrm{ft}$ for the non-compact flange girders. Thus, the data in Fig. 534 further substantiates the claim that when the lateral bracing distance satisfies Appendix B requirements, there is little influence on available rotations. Specifically, the original design (210-2-E-50-10-E) with a lateral bracing distance of $10 \mathrm{ft}$ and the modified design with a lateral bracing distance of $18 \mathrm{ft}$ have nearly identical moment-rotation characteristics as both are below the limit of $22 \mathrm{ft}$.

\subsection{Discussion of FEA Results}

Table 5-2 quantitatively demonstrates that the slender girders have significantly lower levels of available rotation at $\mathrm{M}_{\mathrm{n}}$ than compact girders. For example, examining the $\theta_{\mathrm{RL}}$ values for the experimental girders, it is observed that the compact girders (1MG and 2MG) have available rotations of 8 mrads at $M_{n}$, while the slender girders (3MG through 12MG) have available rotations between 2 and 6 mrads at maximum moment. The available rotations of the parametric study girders presented in Section 5.2, where the slenderness ratios of the girders were maximized, is between 0 and 4 . The design example girders presented in Section 5.3, which have slenderness properties satisfying the current AASHTO moment redistribution provisions, show much higher $\theta_{\mathrm{RL}}$ values, ranging between 14 and 63 mrads. Thus, comparing the results of the girders from Section 5.2 and Section 5.3, it appears that increasing the allowable range of slenderness values results in a significant decrease in available rotations. However, examining the results of the girders presented in Section 5.4 shows that for more realistic girders, where fewer slenderness parameters are simultaneously maximized, the results are more favorable. Specifically, for the Section 5.4 girders, the $\theta_{\mathrm{RL}}$ values range between 3 and 49 mrads.

In addition to the actual $\theta_{\mathrm{RL}}$ values, Table 5-2 also presents the value of $\theta_{\mathrm{RL}}$ predicted by the AASHTO (2004) Specifications, which is given by the equation

$$
\theta_{\mathrm{RL}}=128-143 \frac{\mathrm{b}_{\mathrm{fc}}}{\mathrm{t}_{\mathrm{fc}}} \sqrt{\frac{\mathrm{F}_{\mathrm{yc}}}{\mathrm{E}}}-21.6 \frac{\mathrm{D}}{\mathrm{b}_{\mathrm{fc}}}+24.1 \frac{\mathrm{b}_{\mathrm{fc}}}{\mathrm{t}_{\mathrm{fc}}} \frac{\mathrm{D}}{\mathrm{b}_{\mathrm{fc}}} \sqrt{\frac{\mathrm{F}_{\mathrm{yc}}}{\mathrm{E}}} \geq 5 .
$$


It was observed that the accuracy of this prediction equation could be improved by replacing the quantity $\mathrm{D}$ in the current equation with $2 \mathrm{D}_{\mathrm{cp}}$, resulting in

$$
\theta_{\mathrm{RL}}=128-143 \frac{\mathrm{b}_{\mathrm{fc}}}{\mathrm{t}_{\mathrm{fc}}} \sqrt{\frac{\mathrm{F}_{\mathrm{yc}}}{\mathrm{E}}}-21.6 \frac{2 \mathrm{D}_{\mathrm{cp}}}{\mathrm{b}_{\mathrm{fc}}}+24.1 \frac{\mathrm{b}_{\mathrm{fc}}}{\mathrm{t}_{\mathrm{fc}}} \frac{2 \mathrm{D}_{\mathrm{cp}}}{\mathrm{b}_{\mathrm{fc}}} \sqrt{\frac{\mathrm{F}_{\mathrm{yc}}}{\mathrm{E}}} \geq 5
$$

Comparing the resulting $\theta_{\mathrm{RL}}$ values presented in Table 5-2 using Eqs. 5.1 and 5.2, it is shown that Eq. 5.2 results in either equal or improved predictions in most cases. For the 25 FEA girders from Sections 5.2, 5.3, and 5.4 for which Eq. 5.1 is valid, Eq. 5.2 gives: the same $\theta_{\mathrm{RL}}$ as Eq. 5.1 for 4 of the girders; an improved $\theta_{\mathrm{RL}}$ value for 16 of the girders, and less accurate $\theta_{\mathrm{RL}}$ values in 5 cases. An improved $\theta_{\mathrm{RL}}$ value is defined as either closer to the actual value when both equations are conservative, closer to the actual value when both equations are unconservative, or a conservative prediction resulting from Eq. 5.2 compared to an unconservative prediction resulting from Eq. 5.1. Thus, Eq. 5.2 results in $\theta_{\mathrm{RL}}$ values either equal to or more accurate than those predicted by Eq. 5.1 for $80 \%$ of the FEA girders. For the experimental girders, Eq. 5.2 is more accurate than Eq. 5.1 for both of the girders for which Eq. 5.1 is valid.

Both Eq. 5.1 and 5.2 are limited to a minimum value of 5 mrads. This is believed to be justified, even though several of the girders presented in Table 5-3 have $\theta_{\mathrm{RL}}$ values below this minimum, for the following reasons. It was demonstrated in Section 5.1 that, by comparison to the actual experimental results, the FEA results are conservative with respect to predicting the amount of deflection (and thus rotation) at high load levels. Thus, even though $\theta_{\mathrm{RL}}$ values between 0 and 4 mrads were obtained from the FEA of the girders in Section 5.2, it is believed that the actual rotations of these girders would likely exceed a minimum of 5 mrads. This trend is not unique to the present study as other researchers have also reported that FEA results conservatively predict available rotations. For example, Greschik et al. (1989) also report that FEA results give conservative estimates of rotation capacities compared to experimental results. Specifically, a rotation capacity of 5.75 was obtained in the FEA of a beam that displayed a rotation capacity of 7.10 experimentally. Additionally, Kemp (1990) includes the effect of concrete cracking in negative bending 
regions as an additional component of available rotation, which is conservatively neglected herein.

Neither Eqs. 5.1 nor 5.2 incorporates the effects of unbraced length. Such a consideration was not previously necessary, as it has been shown that for lateral bracing distances less than the current limit, the available rotations are very similar regardless of the value of $\mathrm{L}_{\mathrm{b}}$. However, since it has also been shown that a decrease in available rotations results if the current lateral bracing limit is violated, it is necessary to include this parameter in the $\theta_{\mathrm{RL}}$ prediction equation if the moment redistribution specifications are to be extended to include girders with greater unbraced lengths. Any consideration of the influences of unbraced length should also incorporate the radius of gyration of the compression flange and portion of the web in compression $\left(\mathrm{r}_{\mathrm{t}}\right)$ as lateral torsional buckling is controlled by the value of this parameter. Thus, it is considered appropriate to normalize the unbraced length by $r_{t}$. The variation in $\theta_{\mathrm{RL}}$ with normalized unbraced length is thus shown below in Fig. 5-35 for the designs previously presented in Section 5.4 that violate the current moment redistribution lateral bracing limit. The unbraced lengths used in this figure are equal to the actual unbraced length of the girders for girders that violate the current lateral bracing limit and are equal to the maximum allowable bracing distance for girders that satisfy the limit. This is based on the observation that if the moment redistribution lateral bracing limits are satisfied, nearly constant $\theta_{\mathrm{RL}}$ values result. Figure 5-35 shows that the average slope of the rotation versus unbraced length relationship is 0.5 mrads. Thus the decrease in available rotation with increasing lateral bracing distance is expressed as a function of $0.5 \mathrm{~L}_{\mathrm{b}} / \mathrm{r}_{\mathrm{t}}$. However, it is desirable to incorporate this quantity in such a way as to obtain values of $\theta_{\mathrm{RL}}$ equal to that given by Eq. 5.2 for cases when the current lateral bracing limits are satisfied and then obtain linearly decreasing rotations for lateral bracing distances exceeding this limit. By examining the current lateral bracing limit equation,

$$
\mathrm{L}_{\mathrm{b}} \leq\left[0.10-0.06 \frac{\mathrm{M}_{1}}{\mathrm{M}_{2}}\right] \frac{\mathrm{r}_{\mathrm{t}} \mathrm{E}}{\mathrm{F}_{\mathrm{y}}},
$$

it is revealed that for typical girders this equation results in a maximum normalized lateral bracing distance $\left(\mathrm{L}_{\mathrm{b}} / \mathrm{r}_{\mathrm{t}}\right)$ equal to 30 . Thus, $\theta_{\mathrm{RL}}$ is expressed as 


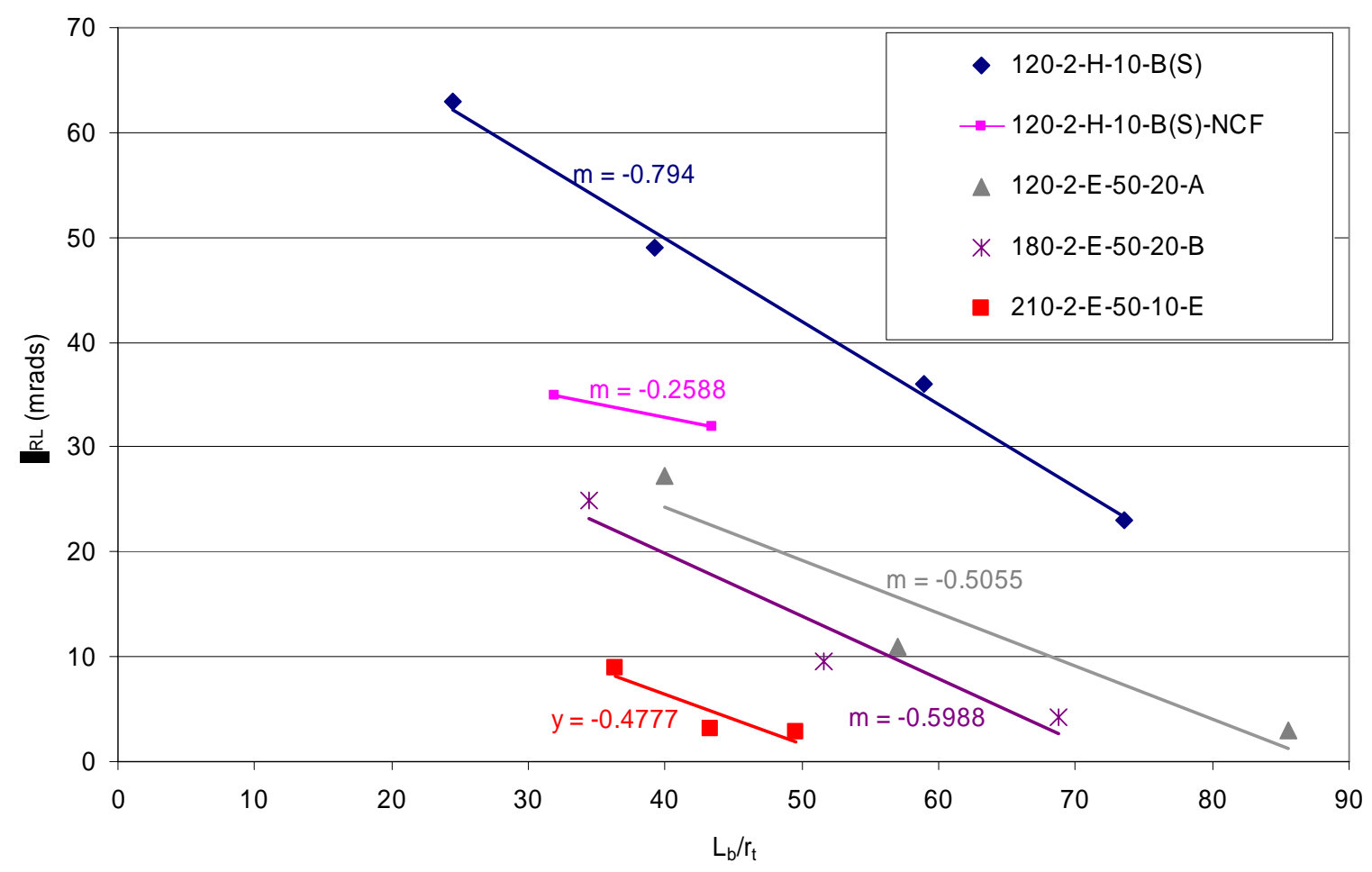

Figure 5-35. $\theta_{\mathrm{RL}}$ versus Unbraced Length for Girders Violating Current Moment Redistribution Lateral Bracing Limits

$$
\theta_{\mathrm{RL}}=128-143 \frac{\mathrm{b}_{\mathrm{fc}}}{\mathrm{t}_{\mathrm{fc}}} \sqrt{\frac{\mathrm{F}_{\mathrm{yc}}}{\mathrm{E}}}-21.6 \frac{2 \mathrm{D}_{\mathrm{cp}}}{\mathrm{b}_{\mathrm{fc}}}+24.1 \frac{\mathrm{b}_{\mathrm{fc}}}{\mathrm{t}_{\mathrm{fc}}} \frac{2 \mathrm{D}_{\mathrm{cp}}}{\mathrm{b}_{\mathrm{fc}}} \sqrt{\frac{\mathrm{F}_{\mathrm{yc}}}{\mathrm{E}}}-\max \left[0,0.5\left(\frac{\mathrm{L}_{\mathrm{b}}}{\mathrm{r}_{\mathrm{t}}}-30\right)\right] \geq 5
$$

It is suggested that Eq. 5.4 can be used to determine $\theta_{\mathrm{RL}}$ for all girders regardless of unbraced length. It is noted that obtaining higher $\theta_{\mathrm{RL}}$ values than would result from Eq. 5.2 is prevented by forcing the last term of the equation to equal zero for cases where Eq. 5.3 is satisfied.

The $\theta_{\mathrm{RL}}$ values resulting from the use of Eq. 5.4 are also shown in Table 5-2. Here the acceptable accuracy of this equation is demonstrated as it is shown that there is a favorable comparison between the accuracy of the current AASHTO (2004) $\theta_{\mathrm{RL}}$ equation, which has an average ratio between actual and predicted $\theta_{\mathrm{RL}}$ values of 0.97 and the accuracy of Eq. 5.4, which has an average ratio between actual and predicted $\theta_{\mathrm{RL}}$ values of 0.96 . 
Although it will be demonstrated in the following chapter that $\theta_{\mathrm{RL}}$ is the only parameter describing the moment-rotation behavior that is necessary in the rotation compatibility procedure, the shape of the unloading curve is also needed to fully represent the momentrotation behavior and will be briefly discussed. It is observed that the unloading curve of the moment-rotation model incorporated in the AASHTO (2004) Specifications, shown below in Fig. 5-36, is accurate in predicting the behavior of compact beams. In fact, it is concluded that the relatively flat unloading curve used in this model is accurate for all cases except where failure is influenced by lateral torsional buckling, i.e., for cases where Eq. 5.3 is violated. When failure is attributed wholly or in part to lateral torsional buckling, the girder unloads more rapidly. The experimental and FEA results previously presented are used to determine a mathematical expression for this relationship. An equation of similar format to that given by the current AASHTO (2004) Specifications is adopted and it is determined that the unloading behavior for girders that violate Eq. 5.3 can be expressed as

$$
\frac{\mathrm{M}_{\mathrm{pe}}}{\mathrm{M}_{\mathrm{n}}}=1-0.060\left(\theta_{\mathrm{p}}-\theta_{\mathrm{RL}}\right)+0.0012\left(\theta_{\mathrm{p}}-\theta_{\mathrm{RL}}\right)^{2},
$$

which is shown graphically in Fig. 5-37. The accuracy of this relationship is shown below for two typical girders in Figs. 5-38 and 5-39. Additional comparisons between the proposed moment-rotation model and actual data are shown for each of the FEA girders in Appendix D.

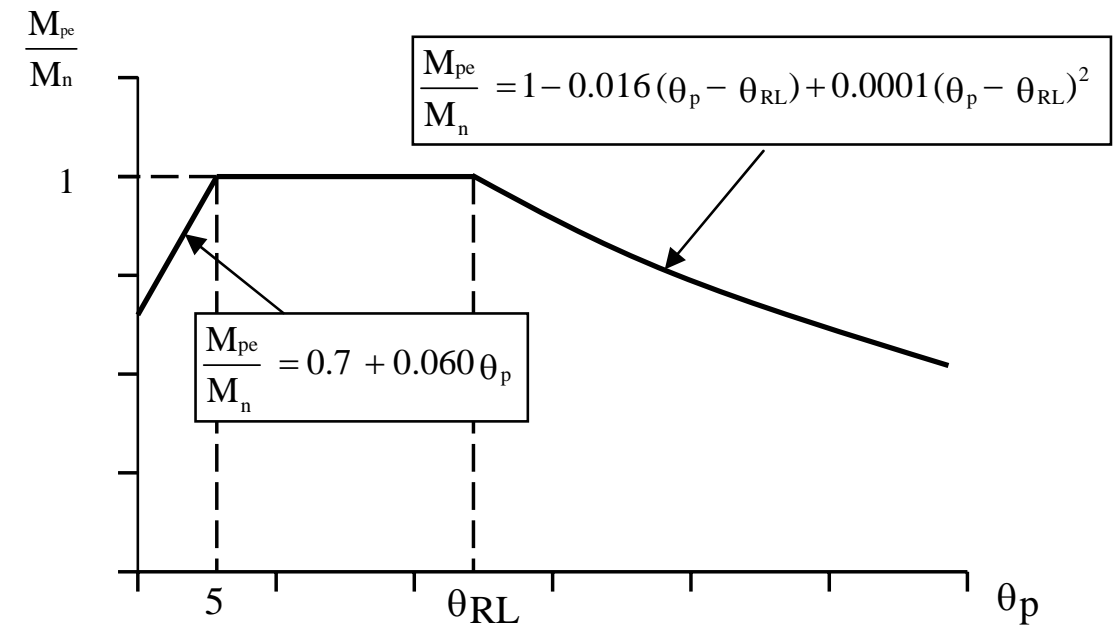

Figure 5-36. Moment Rotation Model for Compact Girders (AASHTO 2004) 


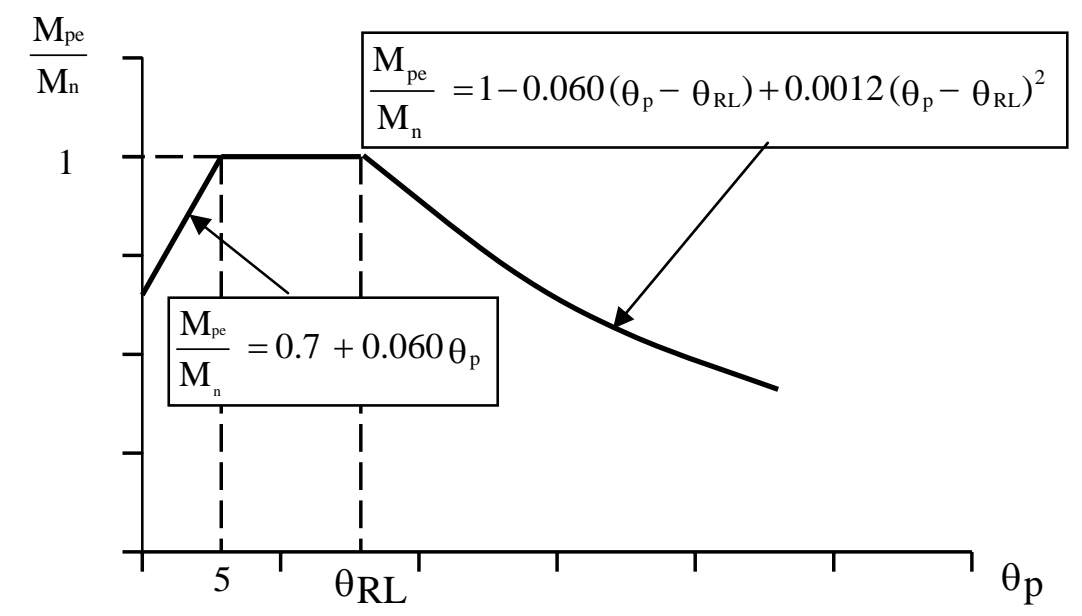

Figure 5-37. Moment Rotation Model for Girders Violating Eq. 5.3

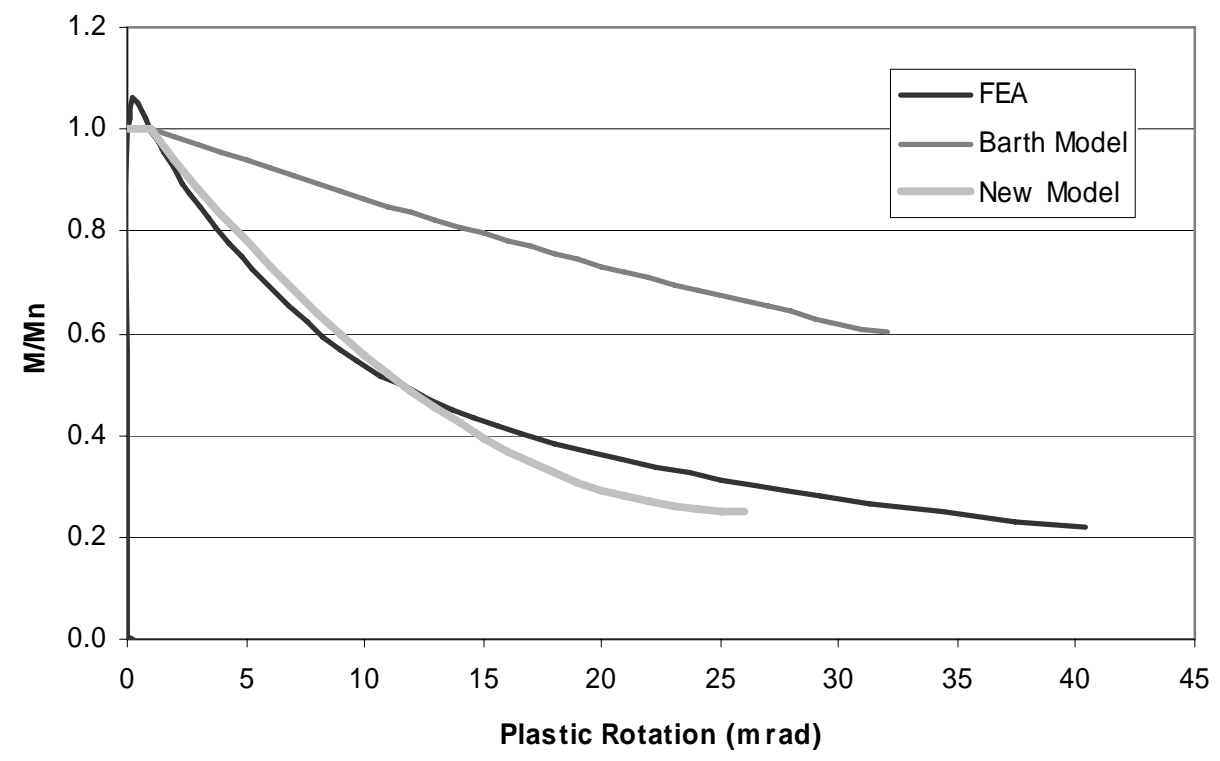

Figure 5-38. Moment versus Rotation - Girder CF-1-65-4-50

\subsection{Summary}

This chapter has investigated the moment-rotation behavior of steel I-girders, with a specific focus on slender I-girder behavior. This study has resulted in an improved $\theta_{\mathrm{RL}}$ prediction equation for girders that satisfy the current AASHTO (2004) requirements for moment redistribution and the development of a $\theta_{\mathrm{RL}}$ equation for girders that are outside of the range 


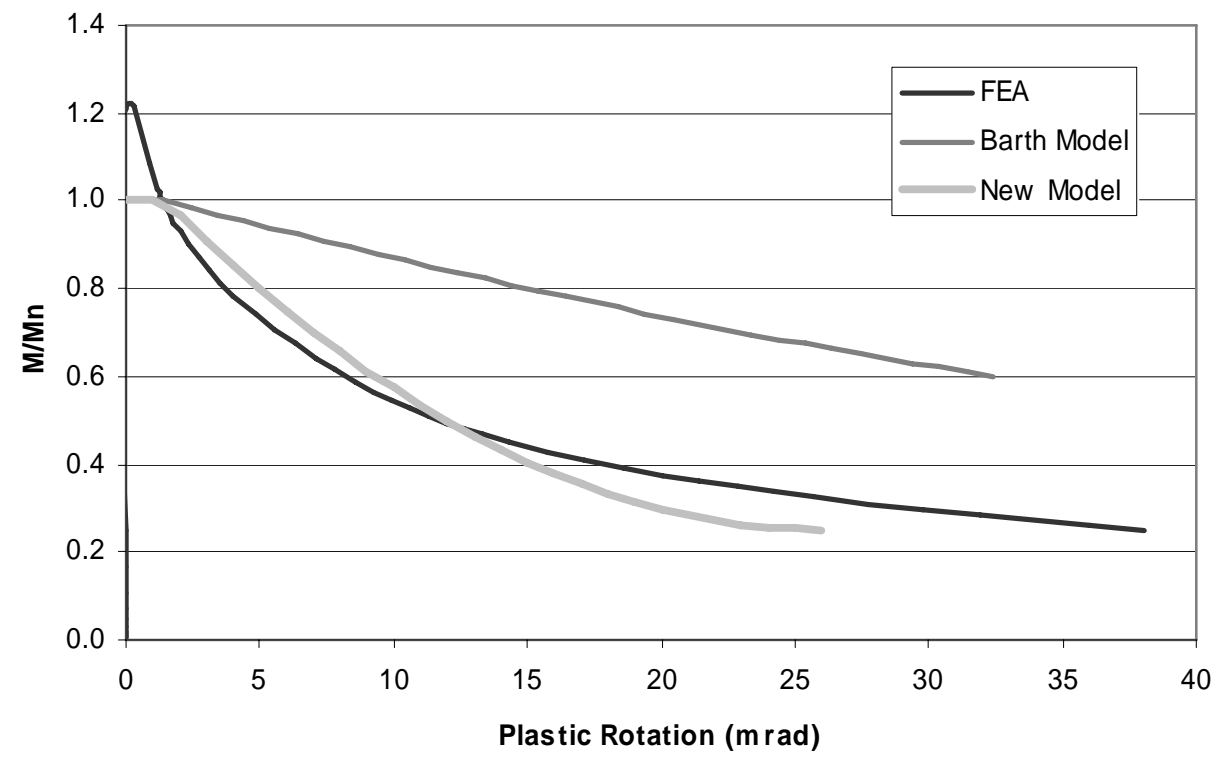

Figure 5-39. Moment versus Rotation - Girder SF-30-65-3-H

of applicability of the current moment redistribution specifications. Additionally, a momentrotation model for girders that are currently not applicable for use with moment redistribution provisions has been presented and will be subsequently used in Chapter 7 to develop moment redistribution specifications for these types of girders. 


\section{Chapter 6: ROTATION REQUIREMENTS FOR STEEL BRIDGE I-GIRDERS}

While the previous chapter focused on the available rotation of steel I-girders, this chapter will determine the rotation requirements for moment redistribution to occur. Chapter 7 will then compare these rotation requirements to the previously determined available rotations, ultimately resulting in the development of rotation compatibility specifications for design and rating of continuous-span steel I-girder bridges.

A literature review of previous rotation requirement studies has been previously presented in Chapter 2. The relevance of these studies to the current work is first presented in the background section of this chapter in Section 6.1.

The rotation requirements suggested in this chapter are determined based on a simple structural analysis procedure, which is discussed in Section 6.2. This method is considered to provide a conservatively accurate representation of the girder behavior that occurs during moment redistribution. In summary, these calculations assume that all loads up to $\mathrm{M}_{\mathrm{pe}}$ are resisted elastically. Once a moment of $\mathrm{M}_{\mathrm{pe}}$ is reached it is assumed that a hinge develops instantaneously. Thus, all moments exceeding $\mathrm{M}_{\mathrm{pe}}$ are redistribution moments. As discussed previously, these redistribution moments then cause positive bending in the girder.

In order to obtain girder properties representative of members designed using moment redistribution procedures it was necessary to create a series of example bridge designs. A discussion of these designs is given in Section 6.3.

Given the analysis procedures discussed in Section 6.2 and the design examples presented in Section 6.3, the resulting rotation requirements are evaluated in Section 6.4. First the relative influence of the parameters affecting the required plastic rotation is explored. Resulting rotation requirements are then presented in terms of both required plastic rotations and required rotation capacity, with particular emphasis on required plastic rotations as these are 
the rotation requirements that will subsequently be used in the development of the rotation compatibility specifications. This section and this chapter conclude with the development of rotation requirement prediction equations.

\subsection{Background}

As discussed previously, rotation requirements specifically applicable to AASHTO moment redistribution procedures have not previously been investigated. Because of this, researchers in this area frequently cite a required rotation capacity of three as the rotation requirement. This limit was originally incorporated into AISC Specifications based on limited data and was by extension later incorporated in AASHTO specifications (the reader is referred to Section 2.3.1 for expanded discussion of this rotation requirement). Assuming that a required rotation capacity of three is appropriate for plastic building design, there are several reasons that suggest rotation requirements for bridge members will likely be significantly less than that required for buildings. First, current inelastic bridge design specifications are based on a shakedown approach instead of mechanism formation as assumed in building design. Because the shakedown loading will necessarily be less than the mechanism loading, the rotation required to achieve shakedown should be less than that required to develop a mechanism. Second, the higher degree of indeterminacy typical of building frames, as compared to bridge girders, dictates that more hinges must be developed in order to redistribute moment. Because the first hinge to develop must have sufficient rotation capacity to allow all subsequent hinges to develop, bridge girders will certainly have lower rotation requirements because there are fewer hinges required. Lastly, experimental studies (e.g., Hartnagel and Tran 2003) have shown that girders with rotation capacities much less than three are still able to satisfy the ductility assumptions inherent in the current AASHTO moment redistribution specifications.

A review of the analysis procedures previously used to determine rotation requirements was also presented in Section 2.3.1. The analysis procedure used in this work is similar to that used in the previous continuous-span rotation studies conducted by Kemp (1990), Li et al. (1995), and Nethercot et al. (1995) with one important modification. This is that in the

previous works it is assumed that a hinge exists at interior support locations prior to the 
application of load on the girder. While this approach is suitable for these previous studies where the applied moment is not decomposed into elastic and plastic components, improved rotation requirement predictions can be obtained when the elastic moments and plastic (redistribution) moments are applied to the girder separately. Specifically, the analysis approach used herein assumes that there is full continuity at piers under elastic levels of moment (i.e., less than or equal to $M_{p e}$ ); plastic hinges are then assumed once $M_{p e}$ is exceeded, thus conservatively predicting the required plastic rotations.

\subsection{Computation of Rotation Requirements}

\subsubsection{Required Plastic Rotation}

The required plastic rotation can be computed using basic structural analysis methods by representing each span of the girder as simply-supported (i.e., hinge at each pier) and then applying end moments (equal to the redistribution moment, $\mathrm{M}_{\mathrm{rd}}$ ) at each pier location. As discussed above, this approach is similar to methods used by Kemp (1990), Nethercot et al (1995), and $\mathrm{Li}$ et al (1995), but differs in the way pier continuity is considered. Consequently, the moments applied to compute required rotations also differ in that the end moments are equal to the redistribution moment as opposed to the total moment. Using the smaller redistribution moment is justified because it is assumed in this work that moments up to $\mathrm{M}_{\mathrm{pe}}$ are resisted elastically. Thus the component of rotation up to $\mathrm{M}_{\mathrm{pe}}$ is elastic rotation at the pier and the rotation corresponding to $\mathrm{M}_{\mathrm{rd}}$ is plastic rotation.

Figure 6.1 illustrates this procedure for a two-span continuous beam with equal span lengths. For a beam with uniform cross-section, the rotation at the end of the beam where moment is applied is computed as

$$
\frac{\theta}{2}=\frac{\mathrm{ML}}{3 \mathrm{EI}} .
$$

Thus the total rotation at the pier is equal to $\theta$ due to equal components of rotation equal to $\theta / 2$ required in each span when equal span lengths exist. For two-span continuous girders with unequal span lengths, the rotations in each span will not necessarily be equal; however, the same approach of summing the required rotation from each span to obtain the total required pier rotation may still be utilized. For continuous-span bridges with three or more 


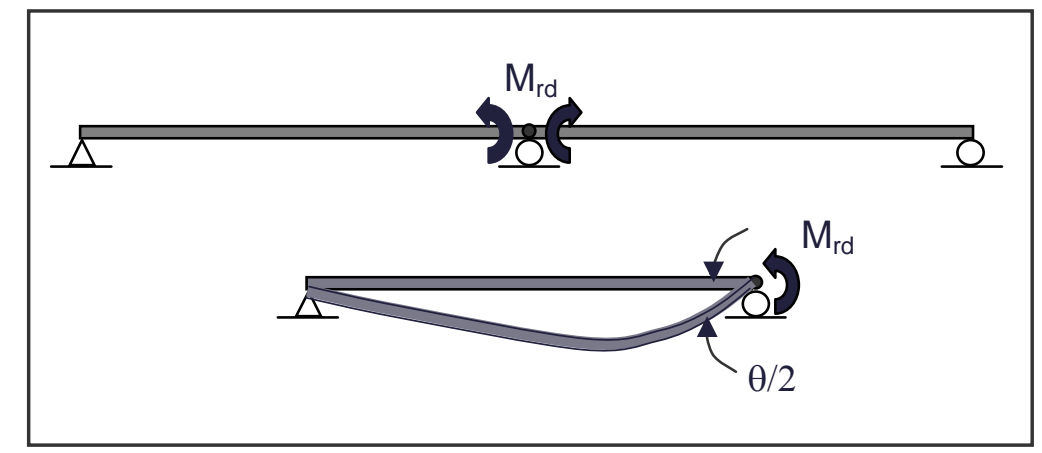

\section{Figure 6-1. Computation of Required Rotations in a Two-Span Continuous Beam with Equal Span Lengths}

spans, it must be recognized that the rotation at a given pier subsequently influences the rotation at all other adjacent pier locations. For example, consider the four-span structure shown in Fig. 6-2. In this case, the required rotation at Pier 1 will be equal to the sum of $\theta_{1}$, $\theta_{2}$, and $\theta_{4}$, while the required rotation at Pier 2 will equal the sum of $\theta_{3}, \theta_{3}, \theta_{6}$, and $\theta_{8}$. This concept can be considered analogous to the formulation of the structure stiffness matrix using matrix structural analysis in that the stiffness of individual elements (which in this case could be represented by each span of the girder) contribute to the global stiffness matrix. Thus, for additional spans, increased stiffness and consequently increased rotation requirements result.

Equation 6.1 shows that the influential parameters affecting required rotation are: the magnitude of the redistribution moment, the length of the span, and the flexural rigidity of the beam. Specifically, there is a linear increasing relationship between the required rotation and the redistribution moment and the same relationship exists between required rotation and girder length. The required rotation is inversely proportional to the quantity EI representing the flexural rigidity of the member. Typically, the flexural rigidity will vary along the length of most bridge girders due to the presence of one or more section transitions. When this occurs the influence of the varying flexural rigidity can be expressed through polynomial expressions. For example, Eqn. 6.2 expresses the required rotation for a span containing $n$ different cross-sections as 


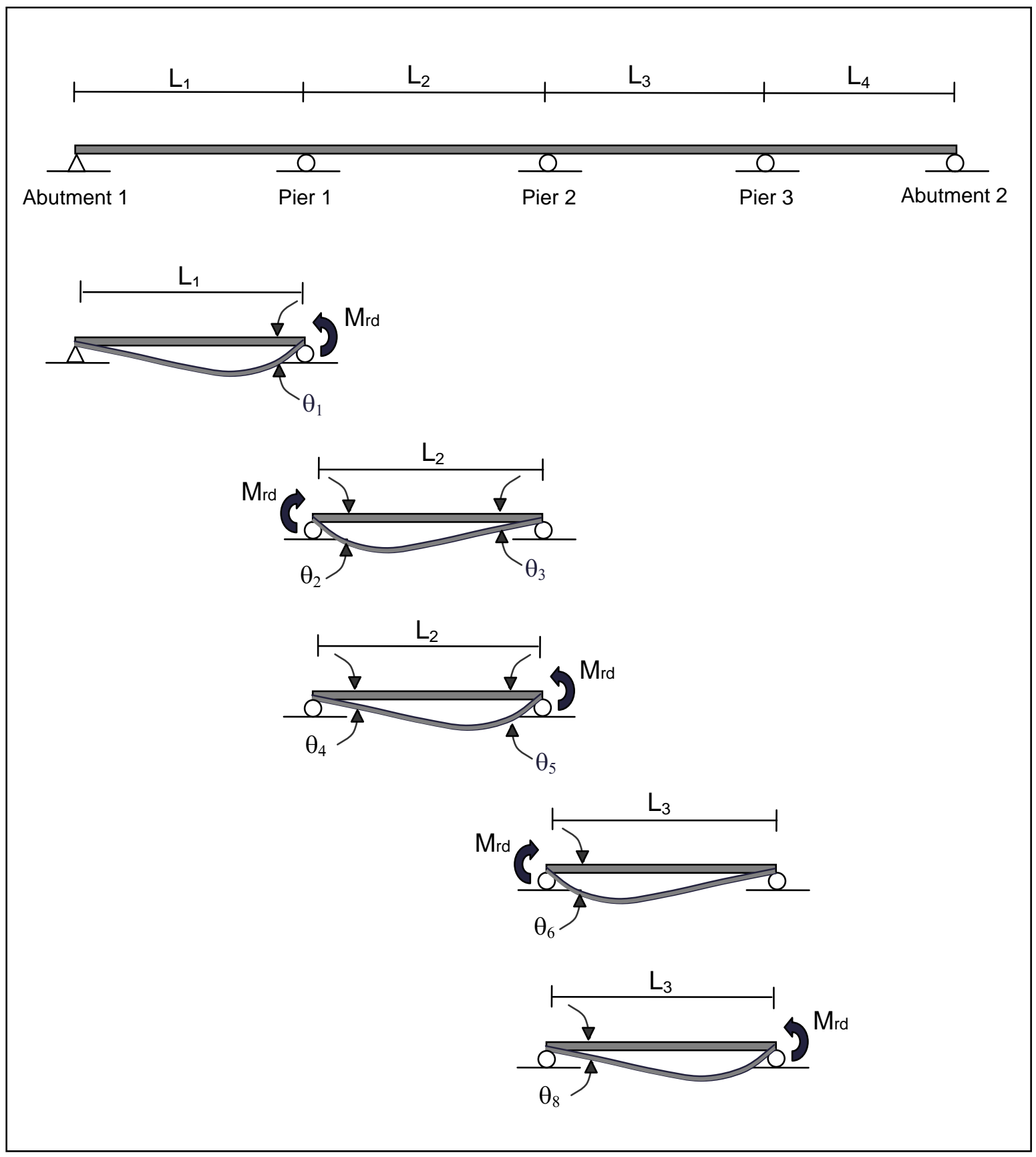

Figure 6-2. Computation of Required Rotations in a Four-Span Continuous Beam

$$
\frac{\theta}{2}=\frac{M_{r d} L}{E I}\left[\frac{\ell_{1}^{3}}{3 x_{1}}+\sum_{j=2}^{n}\left[\left(\ell_{j}\right)\left(\frac{\sum_{i=1}^{j-1} \ell_{i}}{x_{j}}\right)\left(\sum_{i=1}^{j-1} \ell_{i}+\frac{1}{2} \ell_{j}\right)+\left(\frac{\ell_{j}^{2}}{2 x_{j}}\right)\left(\sum_{i=1}^{j-1} \ell_{i}+\frac{2}{3} \ell_{j}\right)\right]\right]
$$

The variables contained in Eq. 6-2 are illustrated in Fig. 6-3 where the variable $\ell$ represents 


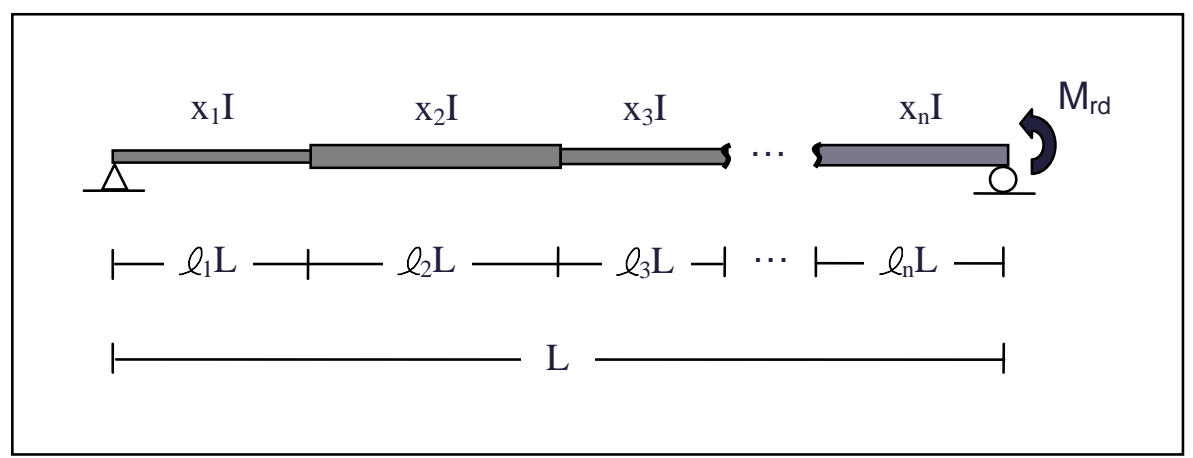

Figure 6-3. Definition of Variables used in Equation 6.2

the ratio of the length of a particular cross-section to the total span length, $\mathrm{x}$ represents the relative ratios between the moment of inertia of various cross-sections, and $\mathrm{x}_{1}$ is set equal to one. Furthermore it is noted that when the span consists of a uniform cross-section, the summation terms in Eqn. 6.2 will equal zero, $\ell_{1}=x_{1}=1$, and thus Eqn. 6.2 reduces to Eqn. 6.1.

Based on Eq. 6.2, it is again shown that the required rotation is a function of span length, magnitude of redistribution moment, modulus of elasticity, and moment(s) of inertia of various cross-sections and the respective length of these cross-sections. For a given design scenario, the span length is known based on the geometry of the subject bridge. The modulus of elasticity is also known from the outset by using the conventional value of modulus of elasticity for steel of $29,000 \mathrm{ksi}$. Thus the parameters $\mathrm{x}_{1}, \ldots, \mathrm{x}_{\mathrm{n}}, \ell_{1}, \ldots, \ell_{\mathrm{n}}$, and $\mathrm{M}_{\mathrm{rd}}$ remain to be determined in order to obtain required rotation values. Because the objective of the present work is to obtain typical required rotations for representative bridges designed using the AASHTO (2004) moment redistribution provisions, a series of design studies was conducted to obtain typical cross-section properties (moments of inertia and cross-section lengths) and redistribution moment values resulting from use of these specifications.

Not only does the moment of inertia typically vary along the length of the beam, but AASHTO specifications also incorporate four different methods of calculating $I_{x}$ (and other section properties) for composite girders. Therefore, some initial consideration should be given to which of these cross-sections is most appropriate for computing rotation 
requirements. The differences in these alternative values are related to the effectiveness of the concrete slab, and furthermore, the assumed level of effectiveness of the concrete slab depends on the loading type under consideration. For example, in the evaluation of positive moment capacity under live loads, it is assumed that the full effective width of the concrete slab is composite with the steel girder. This same cross-section is also used when performing the initial structural analysis of the bridge. A reduced cross-section consisting of one-third of the slab is used for the evaluation of dead loads acting on the composite positive bending section to account for decreased strength due to creep effects in the concrete. Because the concrete slab is in tension in negative bending sections, these sections are assumed to have a fully cracked concrete slab when evaluating the strength of these sections; therefore, the cross-section is represented by the steel girder plus the reinforcing steel in the concrete slab but the slab itself is neglected. Additionally, the cross-section comprised of the girder alone is used to evaluate construction loads prior to deck casting.

The moment of inertia values used to determine rotation requirements are selected to be consistent with the moment of inertia values and typical practices used in the current AASHTO Specifications (2004). Therefore, when computing elastic rotations (needed to compute required rotation capacity, as discussed below) the full composite section is used. The reason for this is that, as illustrated in Fig. 6-1, the redistribution moments producing the plastic rotation are in effect positive bending moments. Thus, by using the full composite section, the same cross-section is used as that used in AASHTO (2004) for the evaluation of positive bending capacity. Not only is this believed to be the most suitable cross-section to determine elastic rotations, this practice is also conservative because the full composite cross-section also results in the highest moment of inertia of the four possible cross-sections. Furthermore, the maximum moment of inertia will result in the minimum elastic rotation and, as will be shown below, the minimum elastic rotation will produce the maximum required rotation capacity.

For the computation of plastic rotations, the full composite section is used over the majority of the span length as the redistribution moments are effectually positive bending moments. However, it is believed that a reduced cross-section should be used in the vicinity of the 
piers. This is due to the likely occurrence of cracking of the concrete slab in these negative bending regions given that moments in excess of $\mathrm{M}_{\mathrm{pe}}$ and relatively large tensile forces in the slab will develop in these regions. Thus, it is believed that the cross-section comprised of the steel girder plus the slab reinforcing would be most appropriate and consistent with current AASHTO provisions as this is the cross-section used to evaluate negative bending capacity. Alternatively, the cross-section comprised of only the steel girder was identified as being a second valid choice for representing the cross-sections in negative bending although, use of the steel girder alone would result in more conservative results due to the smaller moment of inertia values resulting from the use of this cross-section. The influences of using these two alternative $I_{x}$ values on the resulting plastic rotation requirements will be subsequently investigated.

In addition to selecting the appropriate values of moment of inertia to be used, consideration then must also be given to the length over which these moment of inertia values should be used in the computation of required plastic rotations. In other words, the appropriate length over which the slab is considered to be cracked must be determined. This issue has previously been discussed in Chapter 2 where the treatment of this issue by previous researchers was discussed. In summary, Nethercot et al (1995) use a reduced moment of inertia over the entire length of the section that is in negative bending; however, this reduced moment of inertia is equal to the average of the cracked and uncracked moments of inertia, although it is noted that both elastic and plastic rotations are computed in the same calculation, so both the cracked and uncracked sections are applicable. In work by Hope-Gill (1979) two alternative $I_{x}$ values are evaluated: uncracked and cracked over $15 \%$ of the span length. Thus, it was decided in the present work to explore the effects of using a cracked cross-section over: (1) the entire length of the girder between points of dead load contraflexure, (2) varying percentages of the span length, and (3) varying distances. The influences of these cracked distances on rotation requirements will also be investigated subsequently in Section 6.4.1.2. 


\subsubsection{Required Rotation Capacity}

The procedures discussed above detail the method for computing the required plastic rotation used in this work. However, it is also of interest to compute the rotation requirements in terms of required rotation capacity. Figure 6-4 (from Galambos 1968) illustrates the definition of rotation capacity, which is represented by the variable $\mathrm{R}$ in the figure. Figure 64 represents the elastic rotation at $M_{p}$ as $\theta_{p}$ and the total rotation at $M_{p}$ on the unloading portion of the curve is expressed as $\theta_{\max }=\mathrm{R} \theta_{\mathrm{p}}$. Galambos (1968) also gives the following equation for rotation capacity.

$$
\mathrm{R}=\theta_{\max } / \theta_{\mathrm{p}}-1
$$

By simple algebraic manipulation, Eqn. 6.3 could also be expressed by the following.

$$
\mathrm{R}=\left(\theta_{\max }-\theta_{\mathrm{p}}\right) / \theta_{\mathrm{p}}
$$

Since $\theta_{\max }$ is the total rotation and $\theta_{\mathrm{p}}$ is the elastic rotation the difference between the two is the plastic rotation, $\theta_{\text {plastic }}$ and Eqn. 6.4 can be expressed as

$$
\mathrm{R}=\theta_{\text {plastic }} / \theta_{\mathrm{p}}
$$

Realizing that $\theta_{\mathrm{p}}$ is the elastic rotation corresponding to $\mathrm{M}_{\mathrm{p}}$, if the rotation capacity at an alternative level of moment ( $\mathrm{M}_{\mathrm{pe}}$ for example) is desired, the denominator in Eqn. 6.5 should correspond to the elastic rotation at this level of moment, $\theta_{\text {elastic, giving }}$

$$
\mathrm{R}=\theta_{\text {plastic }} / \theta_{\text {elastic }}
$$

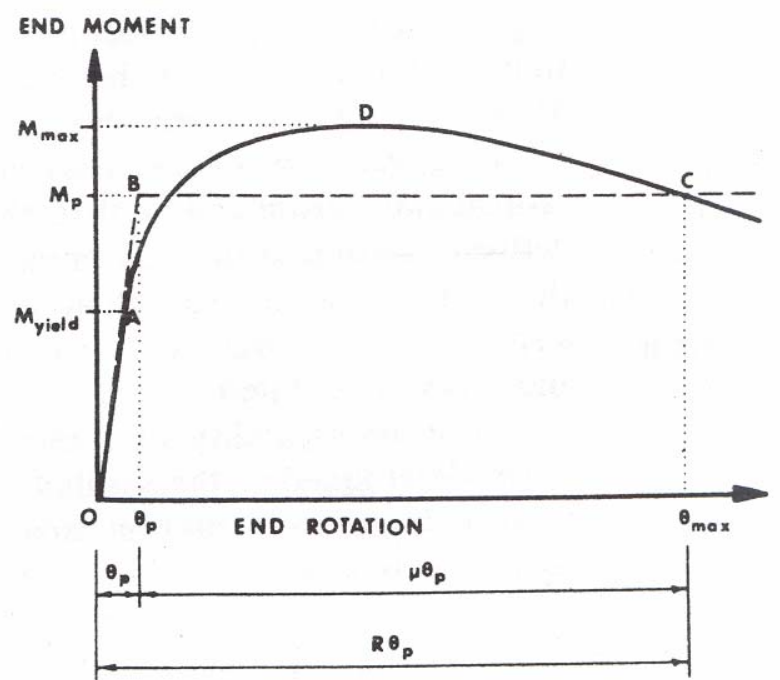

Figure 6-4. Definition of Rotation Capacity (Galambos 1968) 
Equation 6.6 is used to compute the required rotation capacity with the numerator (plastic rotation) computed as outlined above in section 6.2.1 and the denominator (elastic rotation) equal to the elastic rotation at $\mathrm{M}_{\mathrm{pe}}$. This computation of elastic rotation is carried out by representing the portion of the girder between dead load contraflexure points as a simply supported beam with the pier reaction acting as a point load at midspan as shown schematically in Fig. 6-5. The magnitude of the point load is selected such that the moment at midspan induced by the point load is equal to $\mathrm{M}_{\mathrm{p} e}$, and consequently, an equivalent moment gradient exists in both the negative bending region of the continuous span beam and the simply-supported beam representing the negative bending region. The deflection at midspan is then calculated using basic structural analysis procedures, with the moment of inertia computed using the full composite section. The chord between the deflected position at midspan and the end supports is used to compute the rotation at midspan. Thus, rotation at the pier under a moment equivalent to $\mathrm{M}_{\mathrm{pe}}$ is determined, which is the elastic rotation of the girder.

\subsection{Representative Designs}

As discussed above, it was necessary to obtain designs for several representative bridges in order to determine typical moments of inertia, cross-section lengths, and magnitudes of redistribution moments. Therefore, a series of typical designs were generated for a variety of two-span continuous bridges having equal span lengths and a limited number of three- and four-span continuous bridges. It was assumed in these designs that the exterior and interior

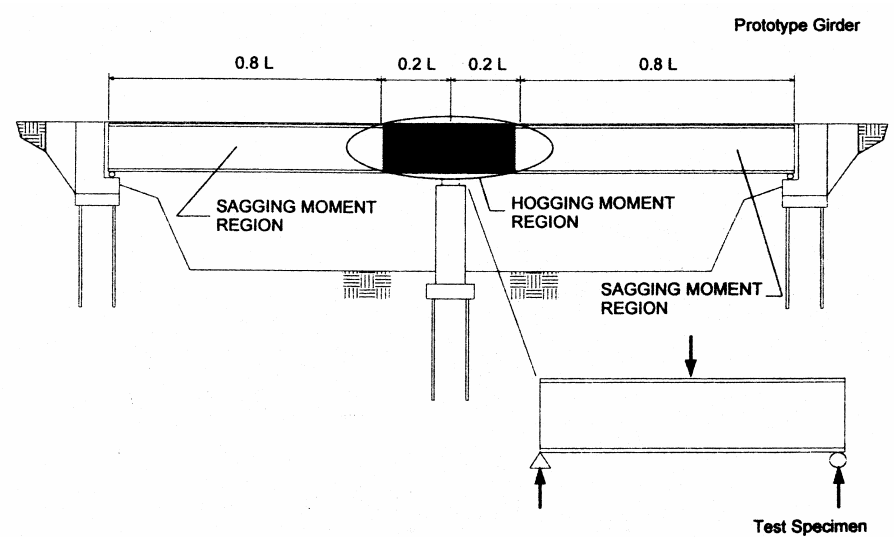

Figure 6-5. Representation of Negative Bending Section of Continuous-Span Girder as Simply-Supported Beam 
girders are identical, and thus, the girders designed satisfy requirements for both. The bridge girders were designed for all applicable limit states, i.e., strength, service, fatigue, and constructibility, according to the AASTHO (2004) Specifications. While it was not attempted to obtain the optimum (minimum weight) design, efficiency of the design was assured by requiring that the performance ratio (applied force divided by section capacity) of each section must be greater than or equal to 0.95 . For the pier sections, the design was typically controlled by strength requirements. Thus, the pier section design was based on achieving the targeted percentage of moment redistribution. Consequently, the performance ratio requirements were not directly applicable; instead a redistribution percentage as close as possible to the targeted redistribution percentage was the goal. Flange transitions were used in the designs when weight savings of at least $1000 \mathrm{lbs}$ of steel could be obtained. Consideration was also given to minimizing the number of plate thicknesses used in a given design.

The cross-section of the two-span bridges is shown in Fig. 6-6 and consisted of four girders spaced at $10 \mathrm{ft}$, which support a two-lane roadway. The roadway width of the two-span bridges was $34 \mathrm{ft}$ and the total bridge width was $37 \mathrm{ft}$. These bridges had a total slab thickness of 8.5 in. where it was assumed that this was comprised of a structural thickness of 8 in. and an integral wearing surface thickness of 0.5 in. A 2 in. haunch (measured from the bottom of the top flange) was also assumed.

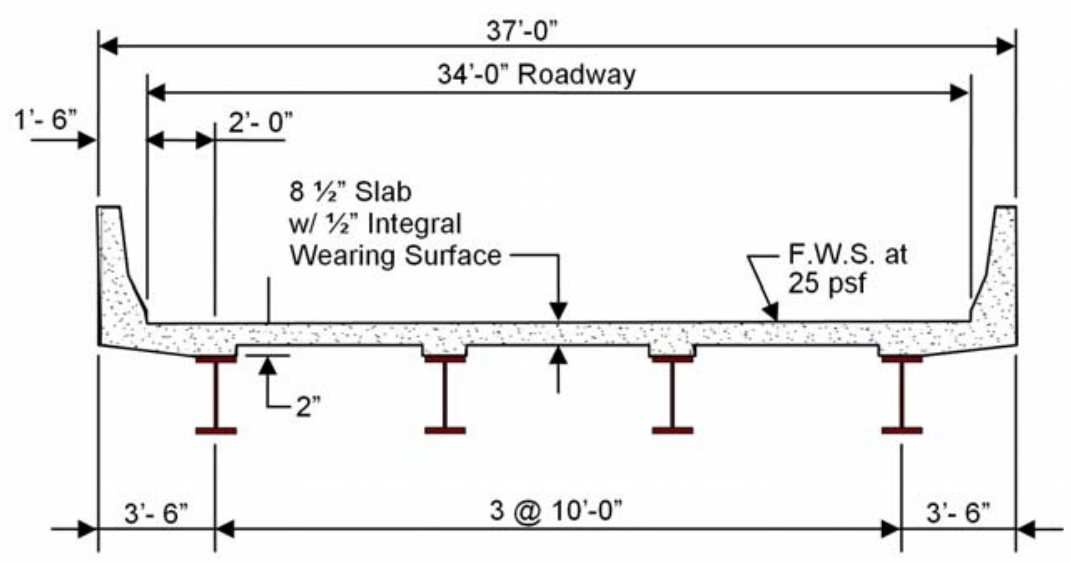

Figure 6-6. Bridge Cross-Section for Two-Span Designs 


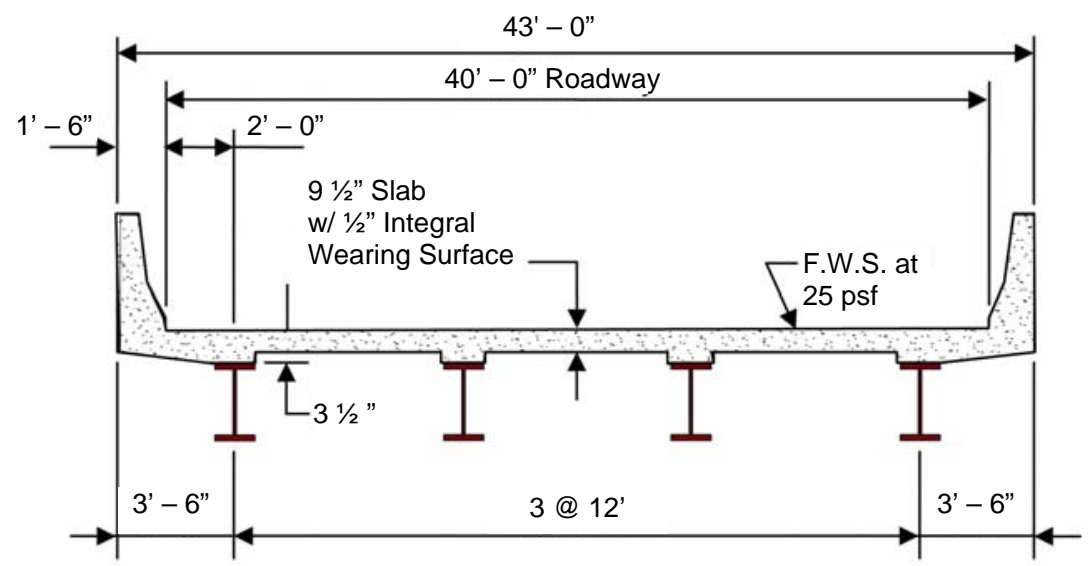

Figure 6-7. Bridge Cross-Section for Three- and Four-Span Designs

Alternatively, the cross-section of the three- and four-span bridges is shown in Fig. 6-7 where the cross-section was slightly altered compared to the two-span designs. The reason for this was to eliminate any influence on rotation requirements that may be related to a specific bridge cross-section (which was found to be negligible) while continuing to use representative bridge cross-sections. Specifically, through the use of variable bridge crosssections, the effective width of the composite section and the slab thickness also vary and thus it is assured that the results presented herein are not related to a specific bridge crosssection. From Fig. 6-7 it may be seen that these bridges consisted of four girders with a wider girder spacing of $12 \mathrm{ft}$, a wider roadway width of $40 \mathrm{ft}$, and a wider total bridge width of $43 \mathrm{ft}$. The three- and four-span bridges also had a larger slab thickness of $9.5 \mathrm{in}$. and a haunch of 3.5 in.; an integral wearing surface thickness of 0.5 in. was again assumed.

In addition to the self-weight of the girders and concrete slab, the girders were also designed to support non-composite dead loads equal to 15 psf for stay-in-place deck forms as well as an additional $5 \%$ of the girder weight, which represented the weight of the cross-frames and other miscellaneous steel details. These non-composite dead loads, designated as DC1 loads in the AASHTO LRFD Specifications (2004), were assumed to be equally distributed to all girders in accordance with recommended design practices. The composite dead load (designated as DC2 in AASHTO LRFD Specifications) consisted of the self-weight of the parapets, which is equal to $520 \mathrm{lbs} / \mathrm{ft}$ per parapet for edge barriers used in this study. The 
girders were also designed for future wearing surface dead loads (designated as DW in AASHTO LRFD Specifications) equal to 25 psf. Both the DC2 and DW loads were also assumed to be equally distributed to all girders.

In evaluating constructibility requirements, lateral bending forces in the exterior girders due to the deck overhang form brackets were considered. It was assumed that these loads may be represented as a uniformly distributed load equal to $275 \mathrm{lbs} / \mathrm{ft}$ comprised of the weight of the deck forms (40 lbs/ft), screed rail (85 lbs/ft), railing (25 lbs/ft), and walkway (125 lbs/ft) in addition to the weight of the finishing machine, which had an assumed weight of 3000 lbs. Additionally, the influence of the deck casting sequence was taken into consideration as mandated by Section 6.10.3.4 of the AASHTO LRFD Specifications (2004). For the twospan bridges, it was assumed that the first slab pours begin at the bearing locations and continue to 50 to $70 \%$ of the span length (which coincides with the location of a section transition in the girder for convenience in the design process) as shown in Fig. 6-8. During the subsequent closure pour over the pier it is assumed that the end sections of each span are composite. Similar deck casting sequences, where positive bending sections are poured prior to negative bending sections, are considered in the three- and four-span designs, which are depicted in Figs. 6-9 and 6-10, respectively.

The varied parameters in the designs include number of spans, span length, percentage of redistribution moment, material yield strength, and stiffened versus unstiffened webs. The purpose of the three- and four-span bridge designs was to investigate the influence of the

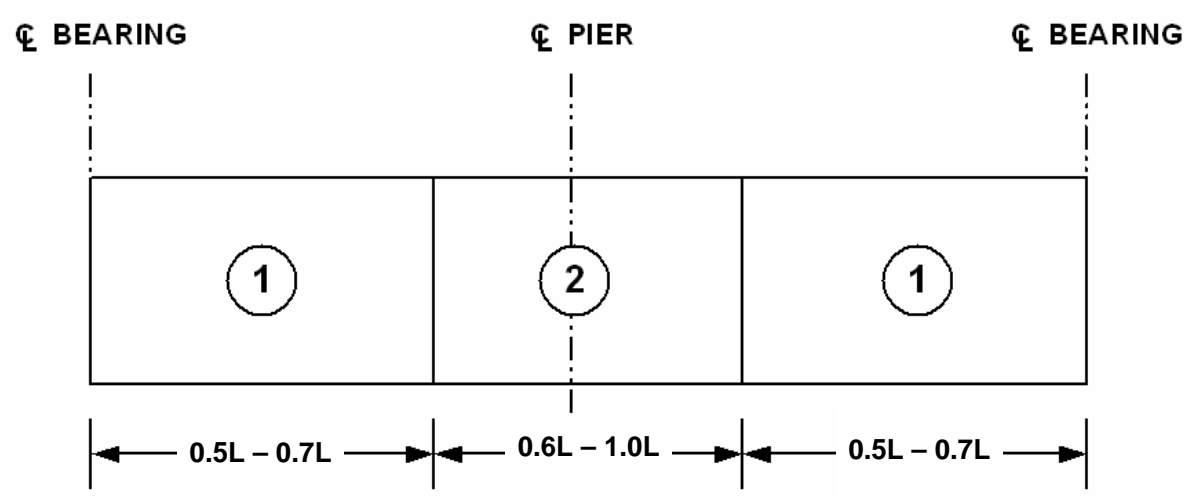

Figure 6-8. Deck Casting Sequence for Two-Span Designs 


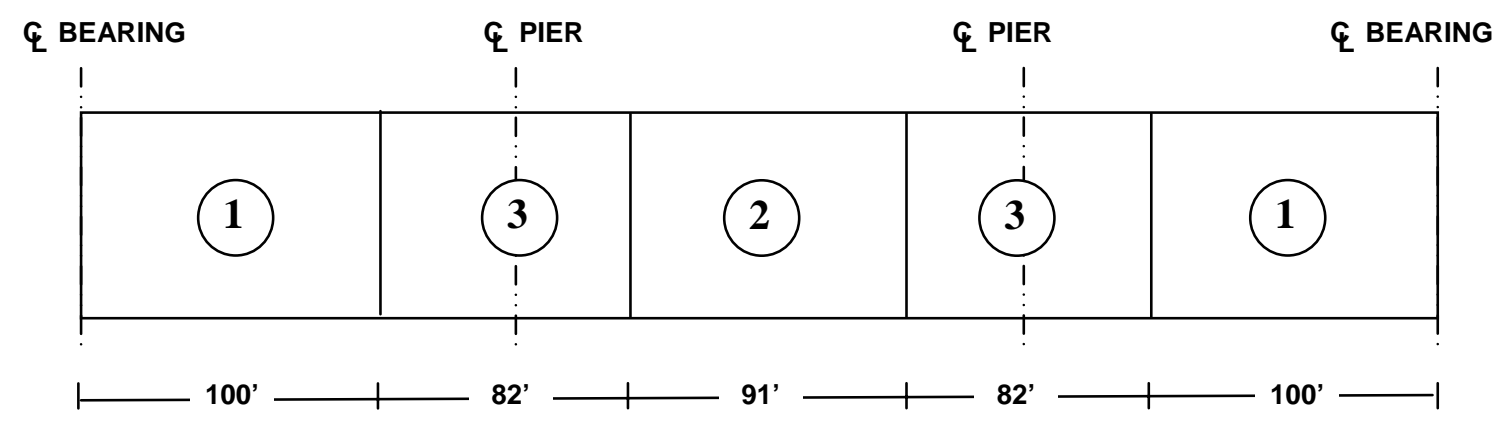

Figure 6-9. Deck Casting Sequence for Three-Span Designs

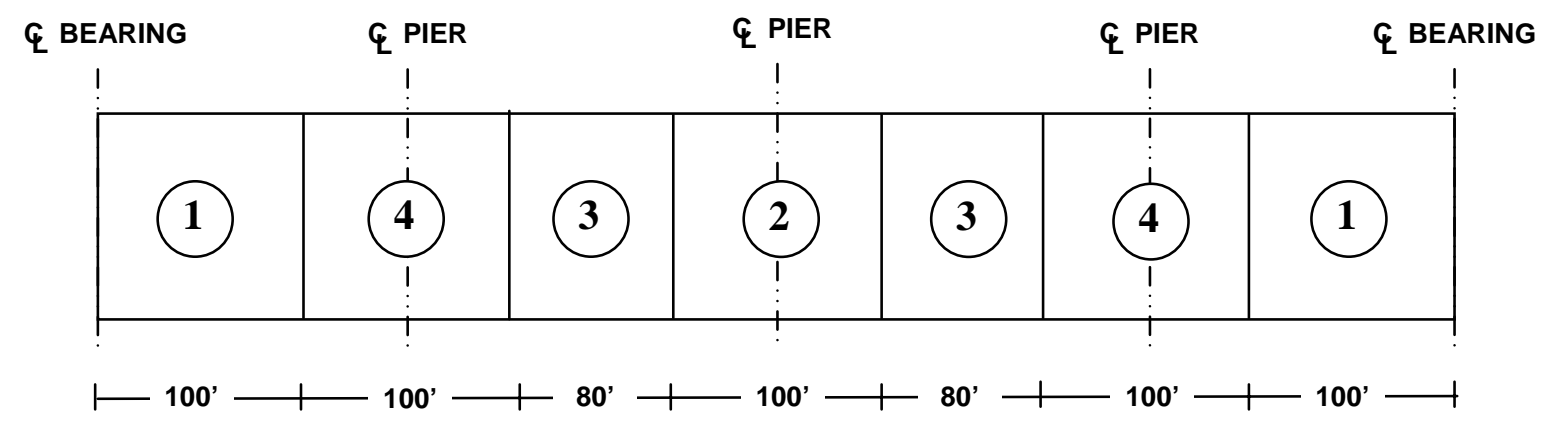

Figure 6-10. Deck Casting Sequence for Four-Span Designs

number of spans only. Consequently, the influence of the other varied parameters was carried out through the two-span designs. For the two-span designs, five different span lengths were used: 90, 120, 150, 180, and $210 \mathrm{ft}$. For each of these span lengths, designs were obtained for targeted levels of moment redistribution of $10 \%$ and $20 \%$ based on use of the AASHTO (2004) moment redistribution procedures and equations. Additionally, higher percentages of redistribution were also investigated in several cases, up to a maximum of $40 \%$ redistribution, which enabled a uniform cross-section to be utilized in some situations. The influences of material properties were evaluated based on alternative homogeneous and hybrid designs with span lengths of $120 \mathrm{ft}$ and $210 \mathrm{ft}$. Similarly, the influences of the use of stiffened versus un-stiffened webs were evaluated by creating alternative designs for the girders with a span length of $120 \mathrm{ft}$. The designs resulting from these efforts are provided in Appendix E. 
In the following sections, specific designs are referenced using the following naming convention. For the two span designs, the first number in the label refers to the span length, where all two span designs consist of spans of equal length, followed by a label of "2" indicating that the design is a two-span bridge. For the three and four-span designs, the first three or four numbers give the lengths of each span. These span length labels are then followed by a label indicating the material properties of the girder, where "50" represents a Grade 50 design and " $H$ " represents a hybrid girder with Grade 70 flanges in negative bending sections and Grade 50 elsewhere. Next, a label indicating the targeted percentage of redistribution moment is given. In most cases more than one design was performed for each design situation before the optimum design was achieved; the next label in the naming convention simply references the specific design for each situation that was selected as the optimal design. Some of the design labels are then followed by the label "(S)", which indicates that the girder design is a stiffened girder. In cases where the design girder consists of a uniform cross-section, this is indicated by the label " $U$ " and in cases where the flange dimensions are uniform but the web thickness varies, this is indicated by the label "UF". For example, Girder 90-2-50-20-B is a two-span continuous, homogeneous Grade 50 girder with $90 \mathrm{ft}$. span lengths and a redistribution moment that is approximately $20 \%$ of the elastic moment. The "B" at the end of this label refers to the specific girder design selected of the several trial designs created for this design situation.

\subsection{Discussion of Rotation Requirements}

Based on the computation procedures outlined in Section 6.2 and the representative designs discussed in Section 6.3, the required rotations for typical continuous-span steel I-girders were obtained. This section will first discuss the influences of variable moment of inertia, span length, redistribution moment, and number of spans on the required plastic rotation. There are also the considerations of the effectiveness of the concrete slab, length of the cracked section, material properties, and the optimization of the design affecting the moment

of inertia and these influences are discussed. Then in order to form comparisons between the rotation requirements suggested in this work and the conventional rotation requirement of three, the required rotation capacities resulting from this work are also presented. This 
section concludes with the presentation of suggested expressions for predicting the required rotations in continuous-span steel I-girders.

\subsubsection{Moment of Inertia Considerations}

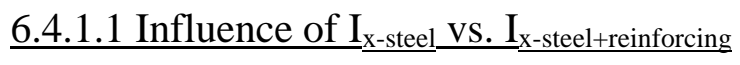

As discussed above, two alternative values may be considered for representing the moment of inertia of cross-sections that are assumed to have a cracked slab: the moment of inertia of the steel girder plus the steel slab reinforcement and the moment of inertia of the steel girder alone. The moment of inertia value for the girder plus reinforcement is considered to be the more appropriate of the two values and is used to form conclusions on rotation requirements in the remaining sections of this work. Furthermore, use of this value of moment of inertia is also supported by Carskaddan (1980). However, investigation of the influence of an alternative, more conservative moment of inertia value was also initially investigated.

Comparisons were made between the required plastic rotations resulting from the use of these two alternative cracked moment of inertia values for each of the design examples created in this work. This showed that the using the moment of inertia of the steel girder alone resulted in required plastic rotations that were 0.3 to 5.0 mrads higher than the plastic rotations required using the larger moment of inertia value of the steel girder plus the steel reinforcement. This represents a 2 to $26 \%$ increase in the required plastic rotations. The girders where the difference in required plastic rotations was largest were generally girders with shorter span lengths and higher levels of redistribution.

\subsubsection{Influence of Cracked Length}

Equation 6.2 may be used not only to represent the variation in moment of inertia due to section transitions, but also the change in moment of inertia due to cracking of the concrete slab. Assuming that the cross-section where the transition from composite cross-section to cracked cross-section occurs may be identified as cross-section $m$ and $\ell_{c}$ represents the

cracked length, Eq. 6.2 may be transformed into Eq. 6.7. Here the summation terms $\sum_{\mathrm{j}=2}^{\mathrm{m}-1}$ and 
$\sum_{\mathrm{j}=\mathrm{m}+1}^{\mathrm{n}}$ are equivalent to the summation term as in Eq. 6.2 although consideration is given to the fact that the cross-section may be composite (designated by the subscript "com" on the moment of inertial terms) or cracked (subscript "cr"). Furthermore, the summation term at $j$ $=m$ is represented by the last four terms of Eqn. 6.7, which account for the variable cracked length.

$$
\begin{aligned}
\theta= & \frac{M_{r d} L}{E I}\left[\frac{\ell_{1}^{3}}{3 x_{1}}+\sum_{j=2}^{m-1}\left[\left(\ell_{j}\right)\left(\frac{\sum_{i=1}^{j} \ell_{i}}{x_{j-c o m}}\right)\left(\sum_{i=1}^{j-1} \ell_{i}+\frac{1}{2} \ell_{j}\right)+\left(\frac{\ell_{j}^{2}}{2 x_{j-c o m}}\right)\left(\sum_{i=1}^{j-1} \ell_{i}+\frac{2}{3} \ell_{j}\right)\right]\right] \\
& +\sum_{j=m+1}^{n}\left[\left(\ell_{j}\right)\left(\frac{\sum_{i=1}^{j-1} \ell_{i}}{x_{j-c r}}\right)\left(\sum_{i=1}^{j-1} \ell_{i}+\frac{1}{2} \ell_{j}\right)+\left(\frac{\ell_{j}^{2}}{2 x_{j-c r}}\right)\left(\sum_{i=1}^{j-1} \ell_{i}+\frac{2}{3} \ell_{j}\right)\right] \\
& +\left(\sum_{i=m}^{n} \ell_{i}-\ell_{c}\right)\left(\frac{\sum_{i=1}^{m-1} \ell_{i}}{x_{m-c o m}}\right)\left(\sum_{i=1}^{m-1} \ell_{i}+\frac{1}{2}\left(\ell_{m}-\ell_{c}\right)\right)+\frac{\left(\sum_{i=m}^{n} \ell_{i}-\ell_{c}\right)^{2}}{2 x_{m-c o m}}\left(\sum_{i=1}^{m-1} \ell_{i}+\frac{2}{3}\left(\ell_{m}-\ell_{c}\right)\right) \\
& +\left(\ell_{c}\right)\left(\frac{\sum_{i=1}^{m} \ell_{i}-\ell_{c}}{x_{m-c r}}\right)\left(\sum_{i=1}^{m} \ell_{i}-\frac{1}{2}\left(\ell_{c}\right)\right)+\frac{\left(\ell_{c}\right)^{2}}{2 x_{m-c r}}\left(\sum_{i=1}^{m} \ell_{i}-\frac{1}{3}\left(\ell_{c}\right)\right)
\end{aligned}
$$

It is noted that Eqn. 6.7 is valid for the case of concrete cracking at one end of the span, i.e., the equation is valid for the end spans of continuous girders. However, the equation is easily modified to account for concrete cracking at both ends of internal spans. This consists of using the first summation term for all cross-sections where a composite cross-section exists, the second summation term for all cross-sections that are fully cracked, and the remaining terms for the two cross-sections where the transition between composite and cracked sections occurs.

Equation 6.7 indicates that for the transition between composite and cracked cross-sections occurring in a particular section transition, the relationship between cracked length and 
required plastic rotation is quadratic. This is shown graphically in Fig. 6-11 for the two-span Grade 50 designs with a targeted redistribution of $20 \%$ of the elastic moment assuming that the transition between composite and cracked sections occurs within the pier cross-section, i.e., prior to the first section transition from the pier. However, Fig. 6-11 also shows that the linear terms of the equation dominate. Specifically, a linear fit to the required rotation versus cracked length curves for each of the five girders results in $\mathrm{R}^{2}$ values ranging between 0.9888 and 0.9994 .

Extending the data shown in Fig. 6-11 to include the full range of cracked lengths considered yields the results presented in Fig. 6-12. Here data is presented illustrating the influence of the assumed cracked length for distances ranging between zero (i.e., composite behavior is assumed throughout the girder) and the point of dead load contraflexure. The maximum variation in required rotation over this range of cracked lengths is 5.1 mrads or $41 \%$ for the data from the $20 \%$ redistribution designs presented in Fig. 6-12. When all the girder designs are considered in this manner, the maximum variation is $10.3 \mathrm{mrad}$ or $42 \%$. When the

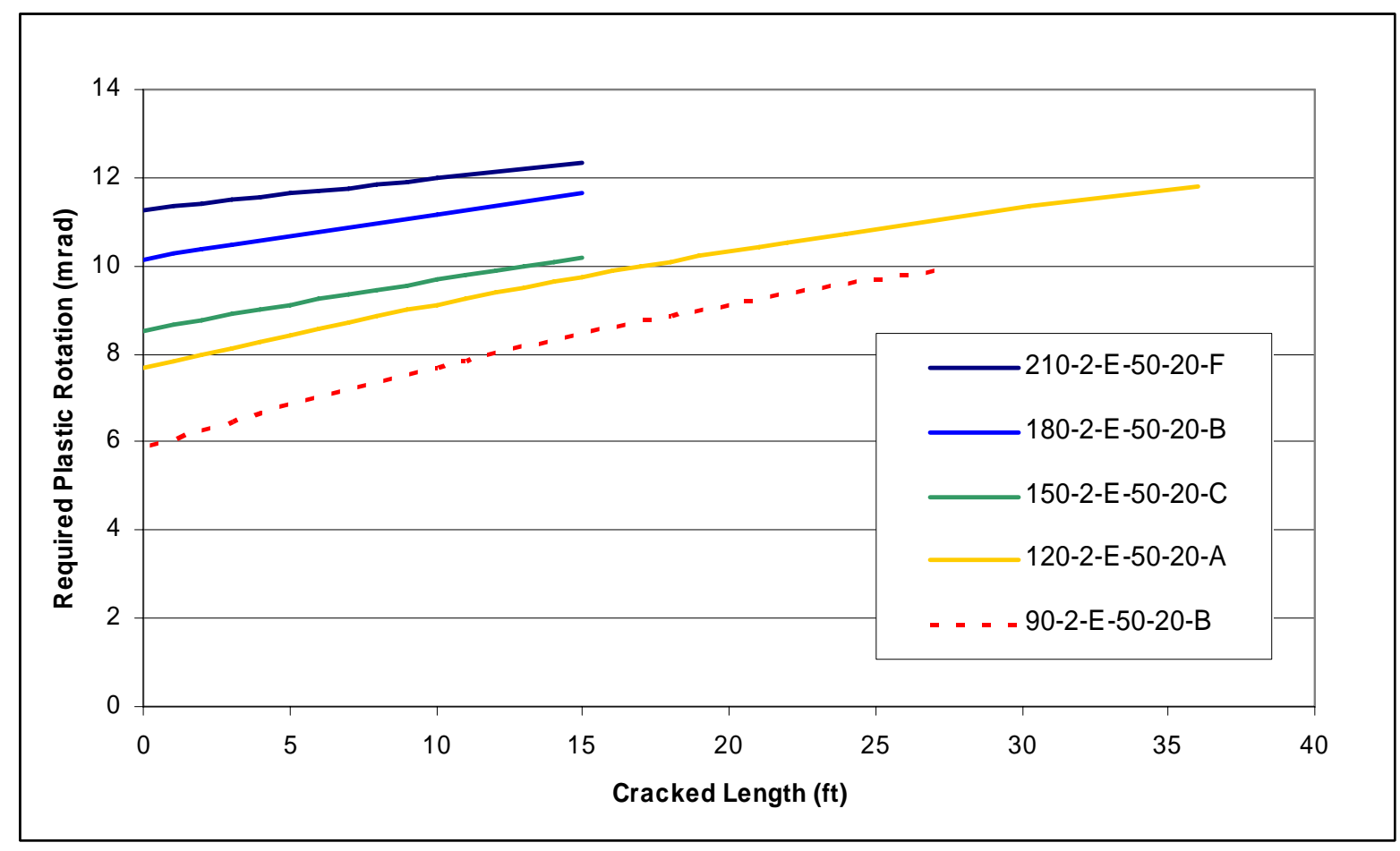

Figure 6-11. Required Rotation versus Cracked Length (ft), $\mathrm{M}_{\mathrm{rd}}=\mathbf{2 0} \% \mathrm{M}_{\mathrm{e}}$, Transition from Cracked to Composite occurs in Pier Cross-Section 


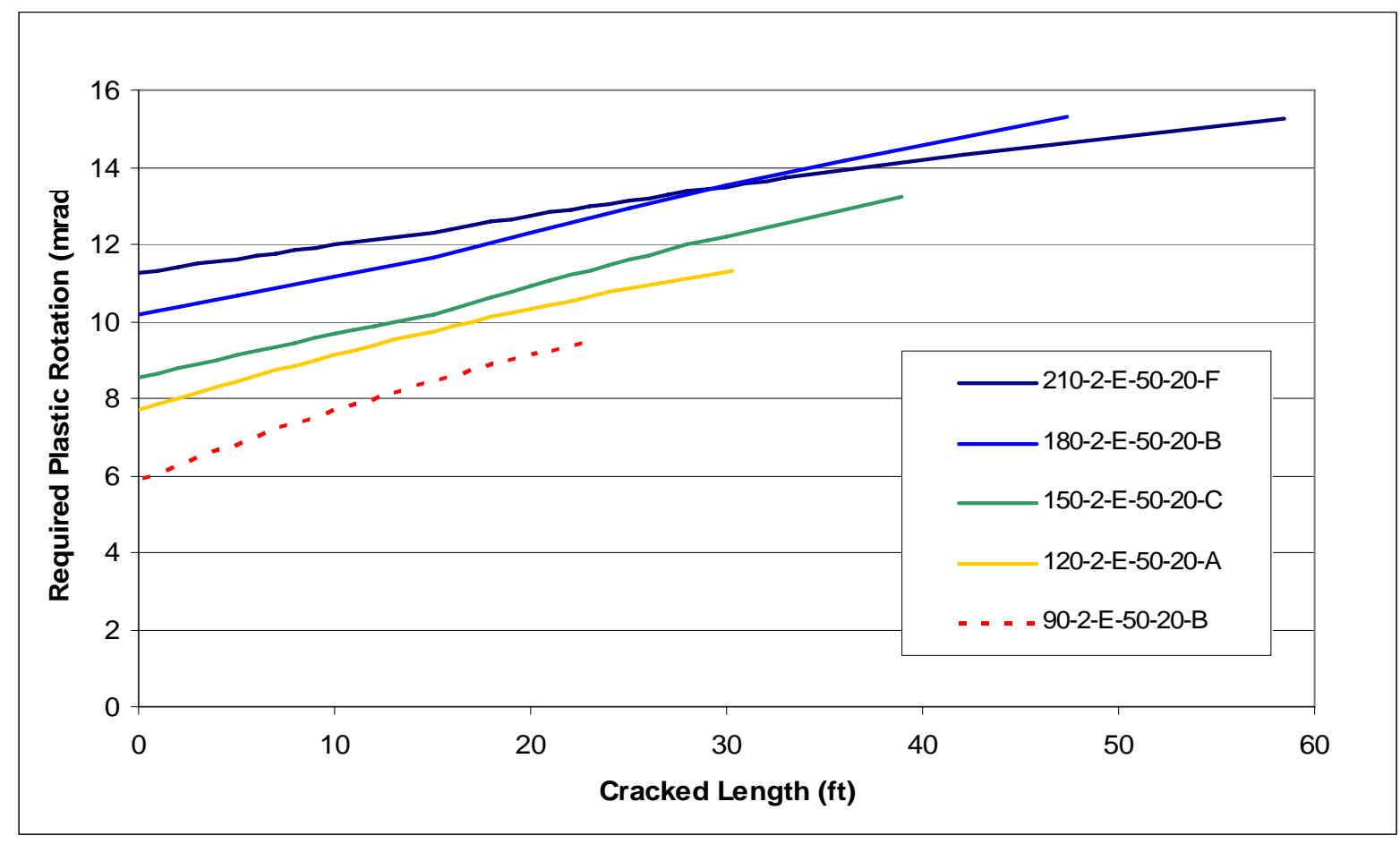

Figure 6-12. Required Rotation versus Cracked Length (ft), $M_{r d}=\mathbf{2 0} \% M_{e}$, Transition from Cracked to Composite occurs in Negative Bending Region

variation is considered in terms of mrads, higher variation results with increasing percentage of redistribution. When the variation is considered in terms of percent difference, the maximum variation results for the shorter span bridges.

The data plotted in Fig. 6-12 is reproduced in Fig. 6-13 plotted versus the cracked length being a percentage of the total span length as opposed to fixed distances. Here it is shown that when the data is plotted in this manner, the increase in required plastic rotation with increasing cracked length is much more uniform, i.e., the slope of each of the lines in Fig. 613 is fairly consistent.

In the absence of experimental evidence on this topic, the amount of concrete cracking that would occur during moment redistribution can not be precisely determined. It seems reasonable to assume that at higher levels of redistribution the extent of the cracked length of the girder would be more significant, but in the absence of data to quantify this assumption, it is necessary to determine conservative approximations for the extent of the cracked cross- 


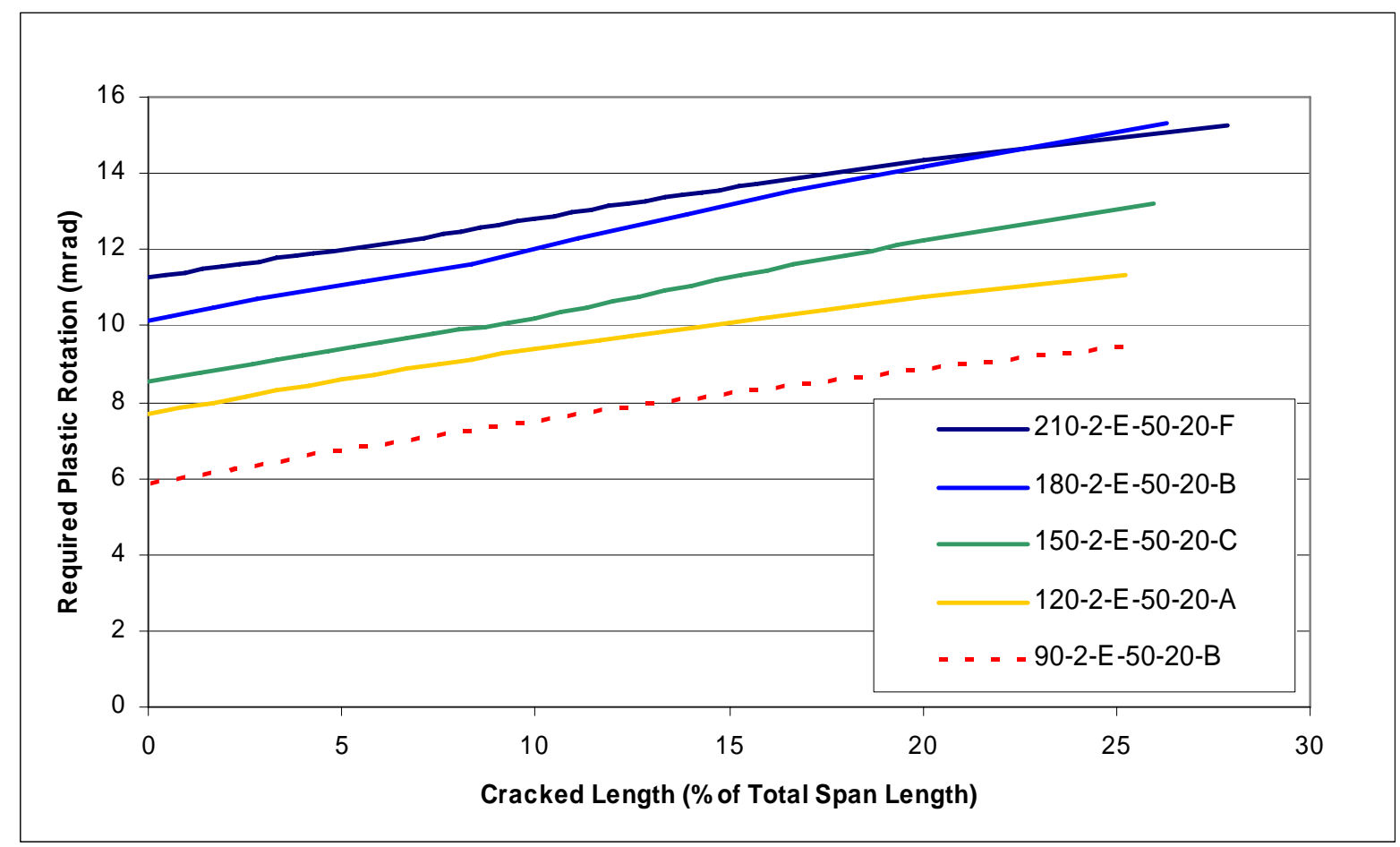

Figure 6-13. Required Rotation versus Cracked Length (as a percentage of total span length), $M_{r d}=20 \% M_{e}$, Transition from Cracked to Composite occurs in

section. Given the data presented above, it is believed that the assumption of a cracked length equal to the length of the girder between dead load contraflexure points is appropriately conservative. For beams of uniform cross-section, the dead load contraflexure point occurs at $30 \%$ of the span length away from the interior pier in a two-span continuous beam with equal span lengths. However, because the pier sections are typically larger than the positive bending sections, the dead load contraflexure point is typically closer to the pier. Specifically, for the designs created in this work the dead load contraflexure point is usually located about $25 \%$ of the span length from the pier (see Fig. 6-13). Consequently, it is believed that to assume that the cracked length of the girder is equal to $25 \%$ of the span length provides uniformity and an appropriate and realistic degree of conservatism.

\subsubsection{Influence of Material Properties}

A second variable that will influence the moment of inertia values is the steel yield strength. This is due to the fact that as higher yield strengths are used, less cross-sectional area is required to meet the same strength requirements. To investigate the influences of the choice 
of material yield strength, designs were created using both of the two most popular material configurations: Grade 50 steel used throughout the girder or Grade 50 steel used for positive bending flanges and all webs in combination with Grade 70 steel used for the flanges of negative bending sections. The results of this evaluation are shown below in Table 6-1 for two-span designs with a span length of $120 \mathrm{ft}$ at targeted redistribution levels of 10 and 20\% and a span length of $210 \mathrm{ft}$ with redistribution levels of 10, 20, 30, and 40\%. It is noted that the use of a hybrid material configuration for higher levels of moment redistribution at the $120 \mathrm{ft}$ span length was not practical because a uniform (Grade 50) cross-section could be used for 30\% redistribution, i.e., there would be no benefit associated with the use of a higher yield strength in negative bending without having a smaller cross-section in negative bending than in positive bending, which is typically regarded as poor design practice. Because, as shown by Eqs .6.1, 6.2, and 6.7, the required rotations are directly proportional to the magnitude of the redistribution moment, it is more appropriate to compare the required rotation values normalized by the magnitude of the redistribution moment or the percentage of redistribution moment. These comparisons are illustrated in Table 6-1 where the required rotation, redistribution moment, percentage of redistribution moment, required rotation normalized by redistribution moment, and required rotation normalized by percentage of redistribution moment are given. The percent difference values refer to the difference in normalized rotation values for each pair of homogeneous and hybrid girders.

The results presented in Table 6-1 show that the influence of the reduced cross-section resulting from the hybrid designs has a significant influence on rotation requirements. In

Table 6-1. Comparison of Rotation Requirements for Hybrid v. Homogeneous Girders

\begin{tabular}{|c|c|c|c|c|c|c|c|}
\hline Design & $\theta_{\mathrm{P}}(\mathrm{mrad})$ & Mrd (ft-kips) & Mrd \% & $\theta_{\mathrm{P}} / \mathrm{Mrd}$ & \% Difference & $\theta_{\mathrm{P}} / \mathrm{Mrd} \%$ & \% Difference \\
\hline 120-2-H-10-B & 6.1 & 825 & 9.5 & 0.00739 & \multirow{2}{*}{24.0} & 0.645 & \multirow{2}{*}{18.2} \\
\hline $120-2-50-10-A$ & 5.8 & 968 & 10.6 & 0.00596 & & 0.546 & \\
\hline $120-2-\mathrm{H}-20-\mathrm{A}$ & 10.2 & 1364 & 15.6 & 0.00750 & \multirow{2}{*}{12.7} & 0.654 & \multirow{2}{*}{12.0} \\
\hline $120-2-50-20-A$ & 11.3 & 1699 & 19.4 & 0.00666 & & 0.584 & \\
\hline $210-2-\mathrm{H}-10-\mathrm{A}$ & 6.9 & 2558 & 9.7 & 0.00268 & \multirow{2}{*}{13.2} & 0.708 & \multirow{2}{*}{8.6} \\
\hline 210-2-50-10-E & 7.1 & 2984 & 10.9 & 0.00237 & & 0.651 & \\
\hline $210-2-\mathrm{H}-20-\mathrm{B}$ & 16.0 & 5141 & 20.0 & 0.00312 & \multirow{2}{*}{9.3} & 0.800 & \multirow{2}{*}{4.5} \\
\hline $210-2-50-20-F$ & 15.0 & 5242 & 19.5 & 0.00285 & & 0.766 & \\
\hline $210-2-\mathrm{H}-30-\mathrm{B}$ & 23.8 & 7516 & 29.9 & 0.00316 & \multirow{2}{*}{15.9} & 0.796 & \multirow{2}{*}{8.8} \\
\hline $210-2-50-30-B$ & 22.0 & 8042 & 30.0 & 0.00273 & & 0.731 & \\
\hline $210-2-\mathrm{H}-40-\mathrm{B}$ & 33.3 & 9936 & 41.1 & 0.00335 & \multirow{2}{*}{14.6} & 0.811 & \multirow{2}{*}{8.5} \\
\hline $210-2-50-40-C$ & 29.9 & 10228 & 40.0 & 0.00293 & & 0.748 & \\
\hline
\end{tabular}


terms of required rotation normalized by the magnitude of redistribution moment the difference is 9 to $24 \%$ and in terms of required rotation normalized by percentage of redistribution moment the difference is 5 to $18 \%$. Thus, the influence of pier section material configuration is significant enough to be considered when developing rotation requirement equations.

\subsubsection{Sensitivity of Results to $\mathrm{I}_{\underline{\mathrm{x}}}$}

A final consideration with respect to the influence of moment of inertia values on required plastic rotation is the sensitivity of the results to these moment of inertia values. It is obvious from Eqs. 6.1, 6.2, and 6.7 that the required rotations are inversely proportional to the moment of inertia values of the girder. Thus, the objective of this section is to investigate the sensitivity of the rotation requirements to the variation in $I_{x}$ values that may occur for a given design situation. In other words, the objective here is not to evaluate changes in rotation requirements across a broad range of moment of inertia values, but instead, to assess the variability in rotation requirements that occurs over the limited range of $I_{x}$ values that will efficiently satisfy the design requirements of a particular design scenario. This is of importance because the goal of this work is to provide definitive conclusions on rotation requirements. Thus, it is necessary to avoid the presentation of results that are linked to specific designs. It should be recognized that for a certain design scenario (i.e., given span lengths and level of intended redistribution) the required moment capacity at various crosssections along the length of the girders will be relatively constant. Consequently, the required cross-section properties (e.g., moment of inertia values) will also be relatively constant. However, various designers may arrive at different cross-section geometries to satisfy the same design requirements. Thus, this influence is examined herein through applying alternative design criteria compared to those used in the development of the initial

designs. Specifically, three of the design criteria that will most significantly affect the resulting moment of inertia values are selected for investigation. These are: the use of “stiffened" versus "un-stiffened" webs, employing different limits for the minimum performance ratio that is acceptable, and implementing alternative criteria for when section transitions are used. 
Un-stiffened webs have a web thickness such that shear requirements are satisfied without the need for transverse stiffeners, while stiffened webs have a reduced web thickness and require the addition of transverse stiffeners. Results of four of the example designs illustrate the influences on rotation requirements from the use of stiffened versus un-stiffened webs. Specifically, the hybrid $120 \mathrm{ft}$ designs with a targeted redistribution of $10 \%$ and $20 \%$ were initially designed as un-stiffened girders and then redesigned with a web thickness 1/16 in. less than the original un-stiffened designs, which is a typical web thickness for stiffened girders. For the case of the designs with $20 \%$ redistribution targeted, the two designs are identical except for the change in web thickness; however, for the designs with $10 \%$ redistribution, it was also necessary to increase the web depth by 1 in. and change the proportions of the top and bottom flanges in order to satisfy design requirements.

Table 6-2 compares the resulting required rotations for the stiffened and un-stiffened designs. As before, the required rotations are normalized by the magnitude of the redistribution moment and the percentage of the redistribution moment, and because the reduction in web thickness also resulted in a significant increase in the redistribution moment, it is not appropriate to directly compare the required rotations of the stiffened versus un-stiffened designs. Table 6-2 shows that the difference in normalized required rotation is less than $2 \%$ in all cases. Also it is shown that when the required rotation is normalized by the magnitude of the redistribution moment, the change from stiffened to un-stiffened girders may result in either an increase or decrease in the normalized required rotation. Thus it is concluded that the influence of stiffened versus un-stiffened webs is negligible.

Different designers may also arrive at varying moment of inertia values for a given design situation due to the use of alternative limits on the minimum allowable performance ratios. As stated previously, it was required that the designs created in this work have a minimum performance ratio of 0.95 for each section transition. Alternatively, three girders were also

Table 6-2. Comparison of Rotation Requirements for Un-stiffened v. Stiffened Girders

\begin{tabular}{|c|c|c|c|c|c|c|c|}
\hline Design & $\theta_{\mathrm{P}}(\mathrm{mrad})$ & Mrd (ft-kips) & Mrd \% & $\theta_{\mathrm{P}} / \mathrm{Mrd}$ & \% Difference & $\theta_{\mathrm{P}} / \mathrm{Mrd} \%$ & \% Difference \\
\hline $120-2-\mathrm{H}-20-\mathrm{A}$ & 10.2 & 1364 & 15.6 & 0.00750 & \multirow{2}{*}{1.8} & 0.654 & \multirow{2}{*}{1.5} \\
\hline $120-2-\mathrm{H}-20-\mathrm{A}-(\mathrm{S})$ & 14.9 & 1953 & 22.5 & 0.00764 & & 0.664 & \\
\hline $120-2-\mathrm{H}-10-\mathrm{B}$ & 6.1 & 825 & 9.5 & 0.00739 & \multirow{2}{*}{0.4} & 0.645 & \multirow{2}{*}{1.4} \\
\hline 120-2-H-10-B-(S) & 11.2 & 1523 & 17.1 & 0.00736 & & 0.654 & \\
\hline
\end{tabular}


designed accepting lower performance ratios and these are compared to the more optimal design for the same situation in Table 6-3. The performance ratios of these alternative designs are as described below.

- In Design 140-175-140-H-B the center cross-section comprising 30\% of the span length has a maximum performance ratio of only 0.91 .

- In Design 150-2-E-30-B one of the three cross-sections is slightly over-designed with a maximum performance ratio equal to 0.94 .

- Design 210-2-E-50-10-A is significantly over-designed, with all of the cross-sections except for the pier section having performance ratios ranging between 0.79 and 0.88 .

Thus it is believed that the first two examples represent alternative reasonable designs and the third example illustrates a more extreme situation where it is unlikely that a designer would consider this to be an appropriate design.

From the results presented in Table 6-3, it can be seen that the percent difference in normalized required rotations is approximately $1 \%$ or less for the two designs that are considered reasonable design alternatives. Additionally, for the significantly over-designed girder the difference is approximately $10 \%$.

An additional cause of variation in the moment of inertia values for a particular design situation is the use of alternative criteria to establish when section transitions are appropriate. This influence is also examined using Design 140-175-140-H-B, which contains a section transition in the end span where the resulting weight savings is only 500 lbs in addition to having a performance ratio of 0.91 along $30 \%$ of the span length. Thus, even though the design criteria used for Design 140-175-140-H-B differs from the criteria used for Design

Table 6-3. Comparison of Rotation Requirements for Alternative Design Criteria Girders

\begin{tabular}{|c|c|c|c|c|c|c|c|}
\hline Design & $\theta_{p}(\mathrm{mrad})$ & Mrd (ft-kips) & Mrd \% & $\theta_{\mathrm{p}} / \mathrm{Mrd}$ & \% Difference & $\theta_{\mathrm{p}} / \mathrm{Mrd} \%$ & \% Difference \\
\hline 140-175-140-H-B & $\overline{17.7}$ & 2635 & $\overline{18.7}$ & 0.00672 & \multirow{2}{*}{0.3} & 0.949 & \multirow{2}{*}{0.9} \\
\hline 140-175-140-H-ORIGINAL & 18.1 & 2696 & 18.9 & 0.00671 & & 0.957 & \\
\hline 150-2-E-50-30-B & 20.8 & 4140 & 31.9 & 0.00502 & \multirow{2}{*}{1.2} & 0.652 & \multirow{2}{*}{1.1} \\
\hline 150-2-E-50-30-C & 21.0 & 4137 & 31.9 & 0.00508 & & 0.659 & \\
\hline 210-2-E-50-10-A & 6.0 & 2786 & 10.0 & 0.00214 & \multirow{2}{*}{9.8} & 0.597 & \multirow{2}{*}{8.4} \\
\hline 210-2-E-50-10-E & 7.1 & 2984 & 10.9 & 0.00237 & & 0.651 & \\
\hline
\end{tabular}


140-175-140-H-ORIGINAL in multiple ways, the resulting rotation requirements differ by less than $1 \%$.

Thus it is concluded from the results presented in Tables 6-2 and 6-3 that, throughout the range of moment of inertia values that are reasonable for a given design situation, the rotation requirements are not sensitive to the range of moment of inertia values that may result due to the use of alternative design criteria. It is consequently suggested that rotation requirements can be accurately based on the results from the initial designs studies.

\subsubsection{Influence of Span Length}

From Figs. 6-11 through 6-13 (presented above) an increasing relationship may be observed between span length and required rotation for designs with $20 \%$ moment redistribution. Figs. 6-14, 6-15, and 6-16 present similar data for designs with $10 \%, 30 \%$, and $40 \%$ redistribution, respectively. Considering the data from all of these figures and keeping in mind that the data of particular interest in these graphs is the required rotation at a cracked length equal to $25 \%$

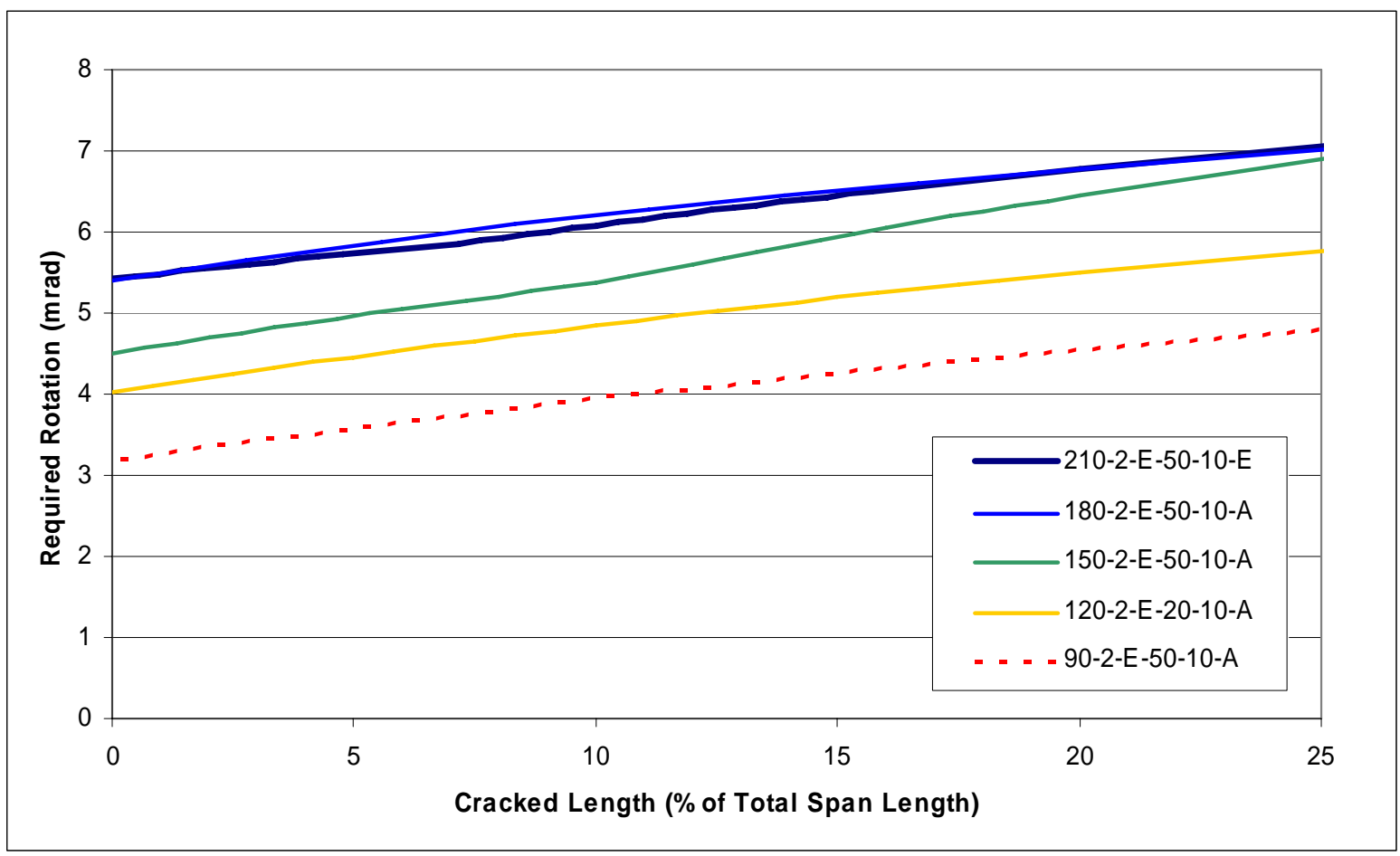

Figure 6-14. Required Rotation for $M_{r d}=10 \% M_{e}$ 


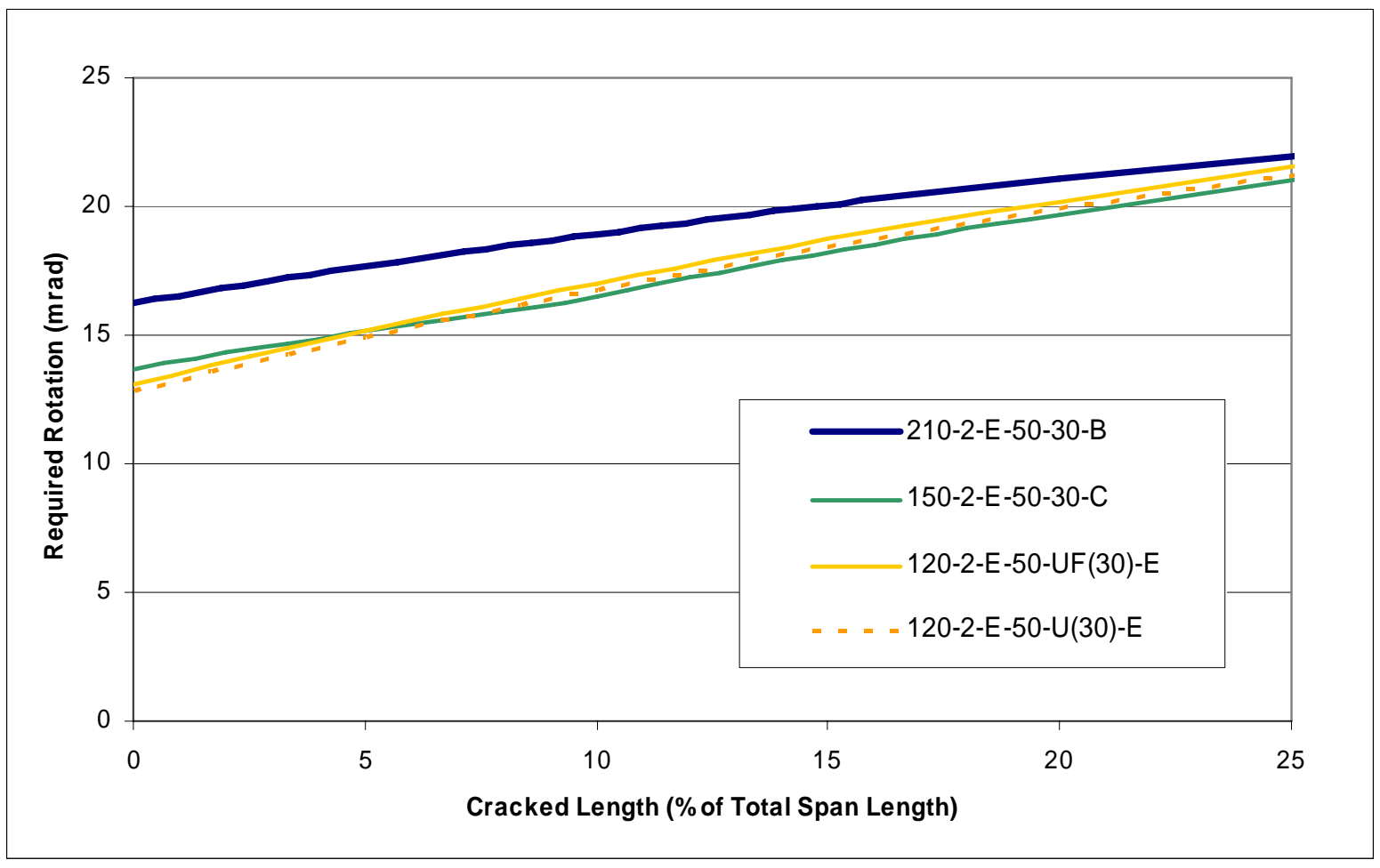

Figure 6-15. Required Rotation for $M_{r d}=30 \% M_{e}$

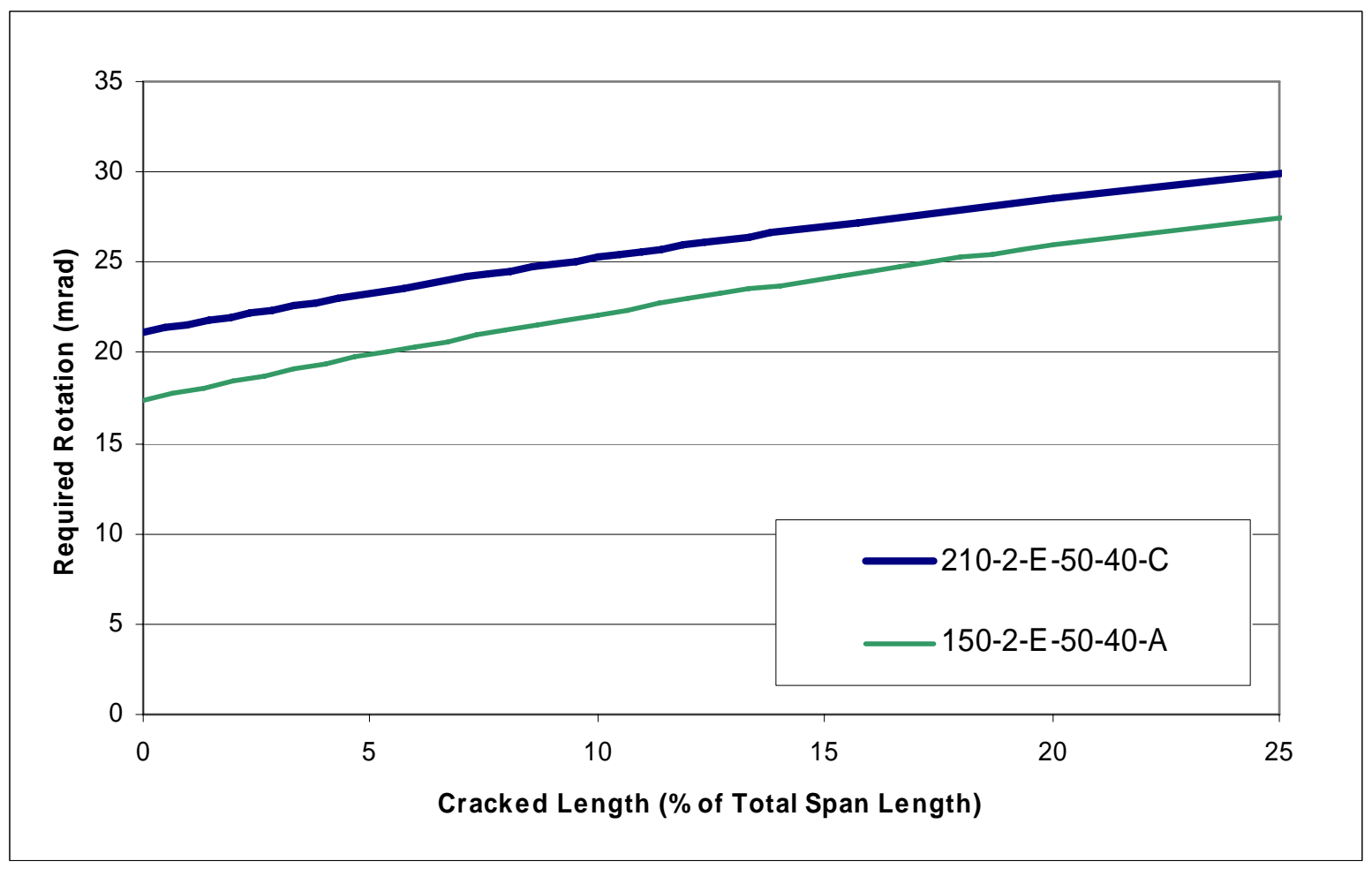

Figure 6-16. Required Rotation for $M_{r d}=40 \% M_{e}$ 
of the total span length (as discussed above), it is shown that in general the highest rotation requirements correspond to the girders with the longest span lengths. However, a longer span length does not necessarily correspond to increased rotation requirements. For example, in Fig. 6-13, a slightly higher rotation is required for the $180 \mathrm{ft}$. girder compared to the $210 \mathrm{ft}$. girder. Also, in Fig. 6-14, approximately the same amount of rotation is required for the

bridges with the three longest span lengths (i.e., $150 \mathrm{ft}$., $180 \mathrm{ft}$., and $210 \mathrm{ft}$.) while approximately the same amount of rotation is required for all of the designs plotted in Fig. 615, where the span length varies from $120 \mathrm{ft}$ to $210 \mathrm{ft}$. Thus it is believed that rotation requirements based on the span lengths producing the greatest rotation requirements are justified.

As with the variables previously discussed, the influence of span length on required plastic rotation is also considered in terms of normalized required rotation. The data is normalized by both the percentage of redistribution moment and the magnitude of the redistribution moment in Figs. 6-17 and 6-18, respectively, for the designs with a targeted redistribution percentage of $10 \%$. The trend in Fig. 6-17 is similar to the results previously presented, which is as anticipated since the previous results were grouped by common percentages of redistribution moment. In Fig. 6-18 it is shown that significantly decreased normalized rotation is required with increasing span length, this same trend exists for the design examples with other levels of redistribution.

\subsubsection{Influence of Redistribution Moment}

The influence of the amount of moment being redistributed has been briefly addressed above as reason for presenting rotation requirements normalized by either the magnitude of the redistribution moment or the redistribution moment percentage (represented as a percentage of the elastic moment). Figure 6-19 examines this influence in greater detail by presenting the required rotation as a function of the percentage of moment being redistributed for the two-span design examples. Here a strong linear relationship is shown between the two variables, which is particularly true when designs of a fixed span length and material 


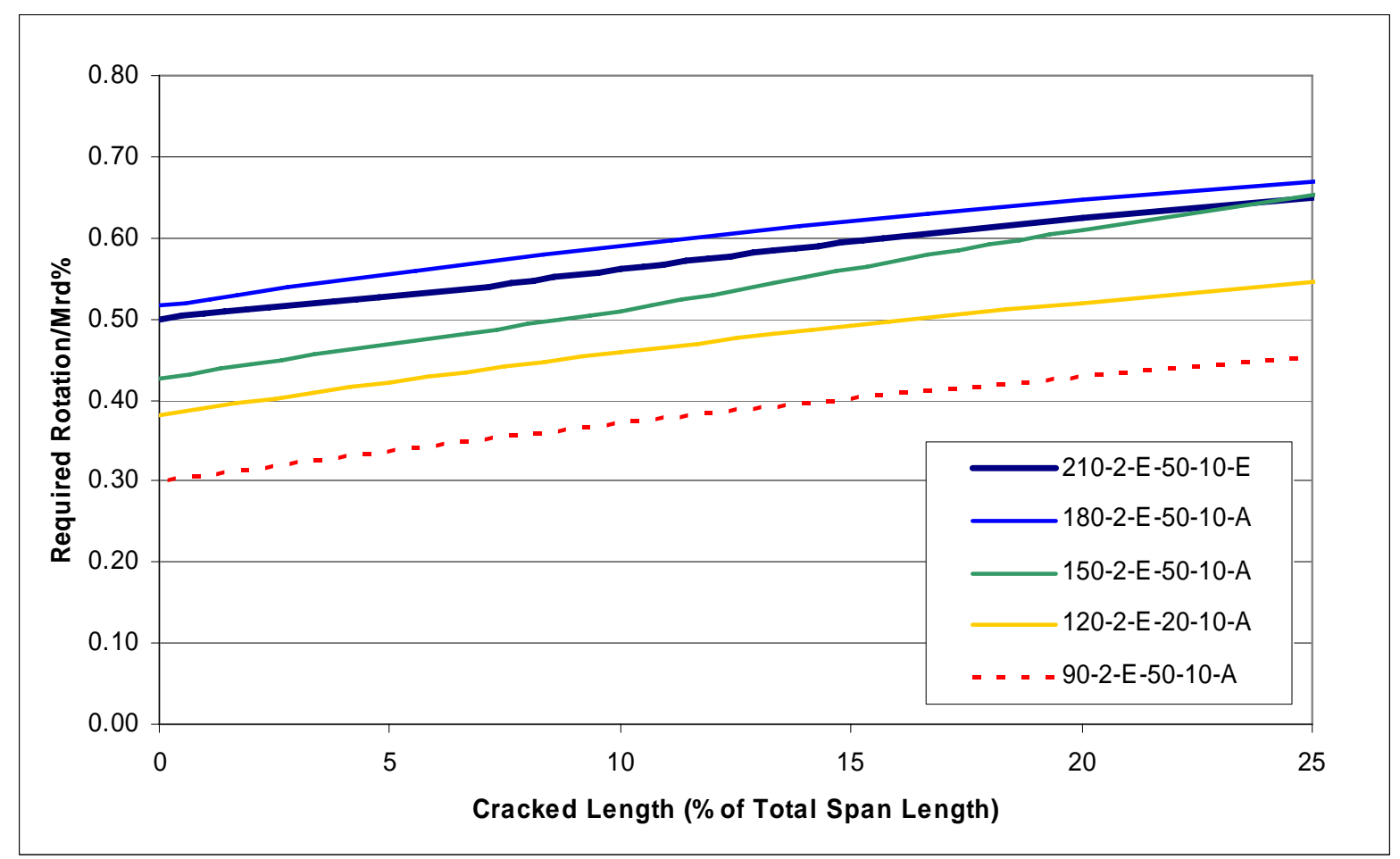

Figure 6-17. Normalized rotation requirements (by percentage of redistribution moment) for $M_{r d}=10 \% M_{e}$

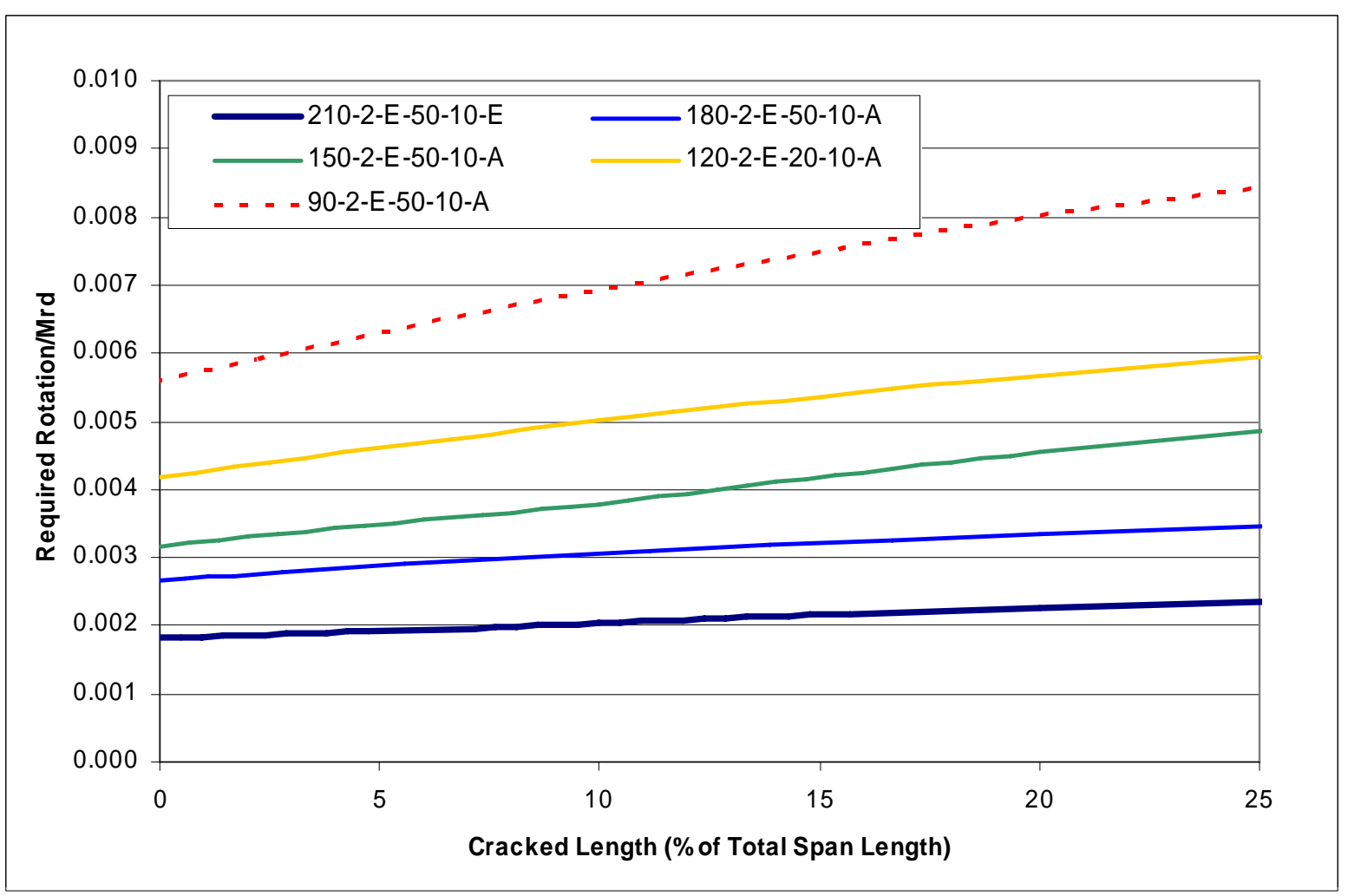

Figure 6-18. Normalized rotation requirements (by magnitude of redistribution moment) for $M_{r d}=10 \% M_{e}$ 


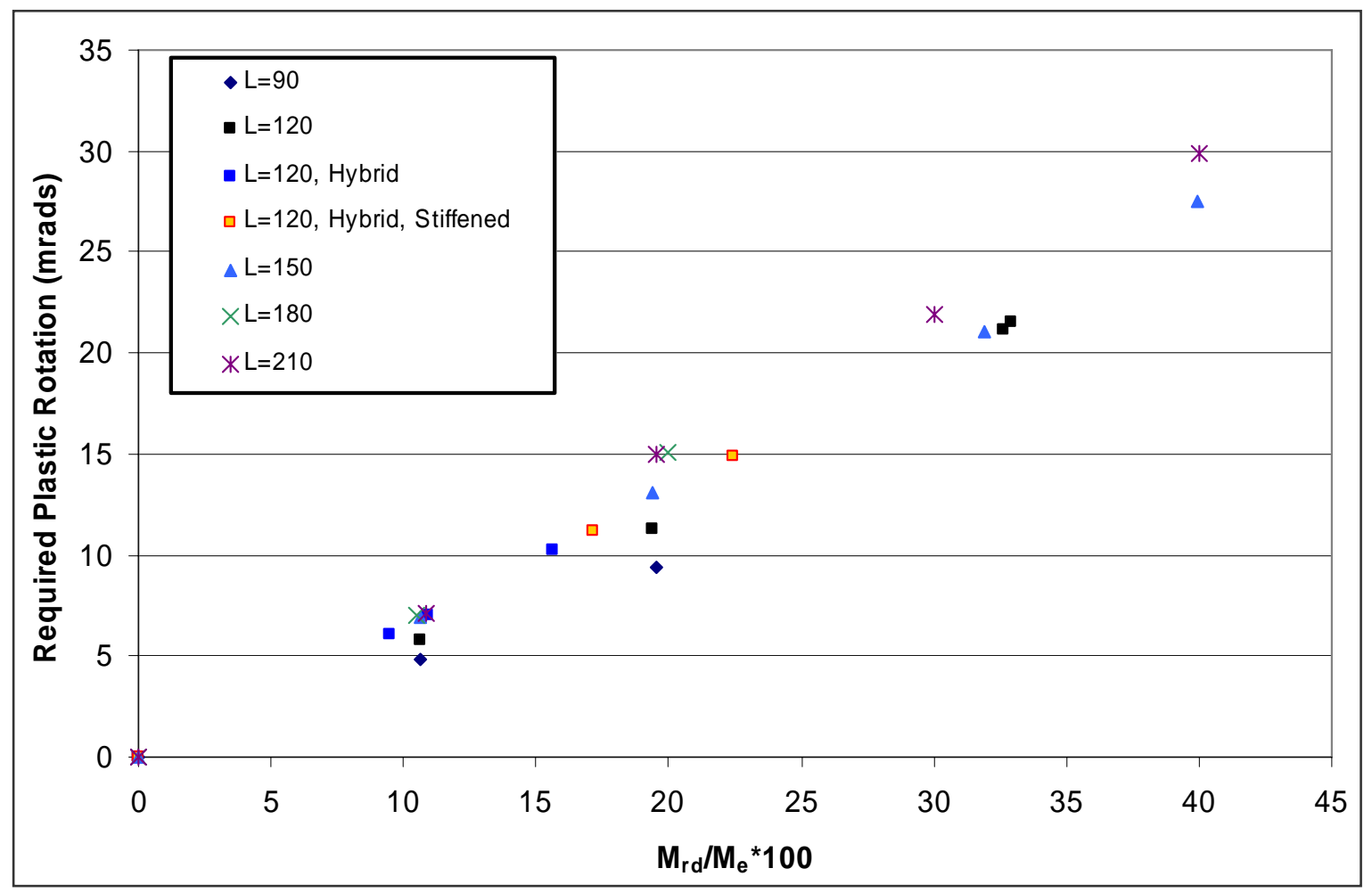

Figure 6-19. Relationship Between Percentage of Redistribution Moment and Required Plastic Rotation

configuration are considered. Figure 6-20 plots the required rotation as a function of the magnitude of the redistribution moment and shows that when the results are plotted in this manner there is still a linear relationship within a particular series, but overall there is considerable scatter within the results. This may be explained by the fact that girders designed for shorter span lengths will have smaller cross-sections; therefore, a redistribution moment of a fixed magnitude will cause greater rotations in the shorter span lengths. When the redistribution moment is expressed as a percentage of the elastic moment, the effect of varying span length is minimized as the larger elastic moments corresponding to increased span length also correspond to larger cross-sections. It is concluded that it is more appropriate to evaluate rotation requirements in terms of the percentage of the moment being redistributed as opposed to the magnitude of this moment.

Once it was determined that the required plastic rotation is best related as a function of the percentage of redistribution moment, the nature of this relationship is sought. As discussed 


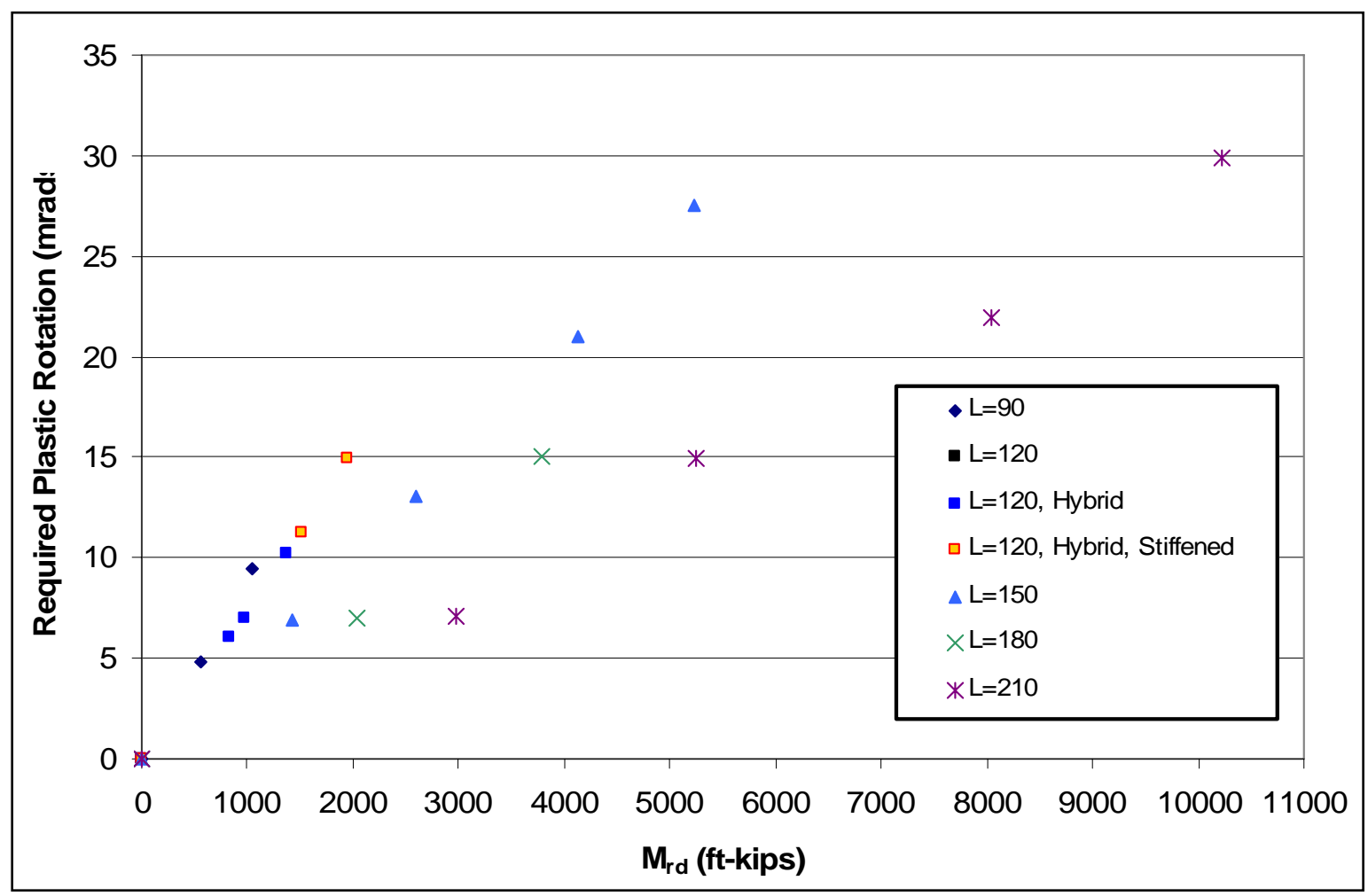

Figure 6-20. Relationship Between Magnitude of Redistribution Moment and Required Plastic Rotation

above and shown by Eqn. 6-1, there is a linear relationship between $\mathrm{M}_{\mathrm{rd}}$ and the required rotations. However, it should be realized that this statement is true assuming that the other variables influencing the required rotations (span length, modulus of elasticity, and moment of inertia) are all constant; although with increasing redistribution moment, smaller pier sections (i.e., reduced moment of inertia values) are required. Therefore, for uniform increments of increasing redistribution, increasing increments of required rotation will result. However, examination of the data in Fig. 6-19 indicates that this issue is negligible; a linear fit to the data for each span length and material configuration yields $\mathrm{R}^{2}$ values between 0.995 and 1.000. As the ultimate goal is to develop rotation requirement relationships suitable for incorporation in design and rating procedures, the simplicity offered by the use of a linear relationship between required plastic rotation and percentage of redistribution moment is favored over the increase in complexity with minimal increase in accuracy that would result by incorporating non-linear equations. 


\subsubsection{Influence of Number of Spans}

The influence of additional spans is first considered in conceptual terms. As was previously discussed in Section 6.2.1 and illustrated in Fig. 6-2, increased rotation requirements are anticipated with increasing numbers of spans. However, it should be noted that this increase is not directly related to the number of spans, but to the pier location. For example, for a two-span continuous girder, there is only one interior pier, which is located between two end spans. In this case, there are is no redistribution moment from adjacent interior piers to consider when determining rotation requirements. However for piers that are located between internal spans, there are components of required rotation that correspond to the redistribution moment at the pier under consideration as well as adjacent piers that must be considered.

A simple span beam of uniform cross-section is first considered to examine the difference in rotation requirements based on pier location (i.e., between two end spans, between one end span and one internal span, or between two internal spans). Basic structural analysis reveals that for such a beam (as shown below in Fig. 6-21) the rotation at the support where the redistribution moment is applied ( $\theta_{1}$ in Fig. 6-21) is equal to twice the rotation at the opposite end of the beam $\left(\theta_{2}\right.$ in Fig. 6-21). Then assuming that the total required rotation at the left pier due to the applied redistribution moment is equal to $2 \theta_{1}$ (due to an additional component of rotation equal to $\theta_{1}$ in the adjacent span, which is true for cases where there is symmetry about the pier), it can be stated that the rotation at the opposite end of the beam is equal to one-fourth the required rotation at the pier where the redistribution moment is applied. Using these assumptions of symmetry about the pier and a prismatic beam while considering the four-span continuous beam shown in Fig. 6-2, it could be stated that the required rotation at

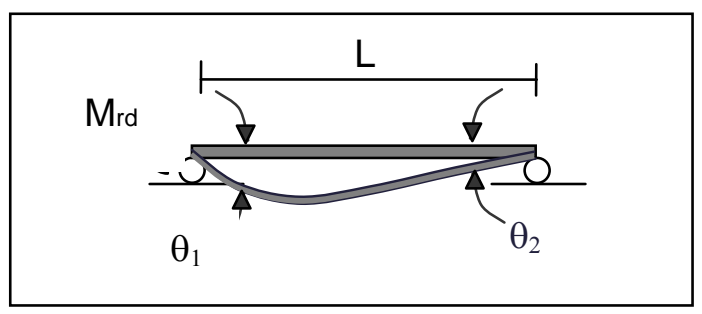

Figure 6-21. End Rotations in Prismatic Simply-Supported Beam 
the center pier would be equal to the sum of: (1) the required rotation at the center pier due to application of a redistribution moment at the center pier, (2) one-fourth the required rotation at the left pier due to application of the redistribution moment at the left pier, and (3) onefourth the required rotation at the right pier due to application of the redistribution moment at the right pier.

It is desirable to investigate the accuracy of assuming that the rotation requirements at piers adjacent to interior spans can be computed using this method of increasing the required rotation due to the redistribution moment applied at the pier under consideration by onefourth of the required rotations at adjacent piers when the redistribution moment is applied at these locations for general girders (with varying cross-sections that are not symmetric about piers) due to the simplicity offered by this approach. This issue is investigated through trial designs of a three-span and a four-span continuous girder. The three-span girder has a span arrangement with lengths of $140 \mathrm{ft}, 175 \mathrm{ft}$, and $140 \mathrm{ft}$ and uses hybrid sections in negative bending locations. The four-span girder has span lengths of $150 \mathrm{ft}, 180 \mathrm{ft}, 180 \mathrm{ft}$, and $150 \mathrm{ft}$ and is a homogeneous Grade 50 girder.

In these design examples the ratio between the component of required rotation that is due to applying the redistribution moment at the adjacent pier and the component of rotation that is due to applying the redistribution moment at adjacent piers is equal to: $24.8 \%$ for the threespan design, $22.3 \%$ for the pier located adjacent to an end span in the four-span girder, and $26.2 \%$ for the pier located between two internal spans in the four-span girder. Thus it is concluded that it is reasonable to compute the required rotation at a pier that is adjacent to one or two internal spans as the sum of the rotation that would be computed assuming the pier was adjacent to two end spans and $25 \%$ of the required rotation that is computed at each adjacent pier assuming these piers are also adjacent to two end spans.

\subsubsection{Required Rotation Capacity}

Required rotation capacities were not of primary interest in this work as the rotation requirements used in the rotation compatibility procedure are in terms of plastic rotation (versus rotation capacity). However, a presentation of the required rotation capacities for 
moment redistribution is presented herein as it is of interest to compare these rotation capacity requirements with the conventional rotation capacity requirement of three (see Section 2.4). Figure 6-22 reproduces the data presented in Fig. 6-19, but plots the rotation requirement in non-dimensional terms of required rotation capacity. Here it is shown that the same linearly increasing relationship exists between percentage of redistribution moment and rotation requirements. Furthermore, for girders with redistribution moments less than the AASHTO (2004) limit (20\% of the elastic moment), it is shown that the required rotation capacity is less than 2. However, as the redistribution moments exceed this limit, increased rotation capacity is required. For a redistribution moment equal to $40 \%$ of the elastic moment, which was the highest percentage of redistribution moment investigated in this study, the required rotation capacity is approximately 4. Comparing these results to the conventional rotation capacity requirement of three, it is shown that this requirement is very conservative for the AASHTO (2004) moment redistribution requirements, however a

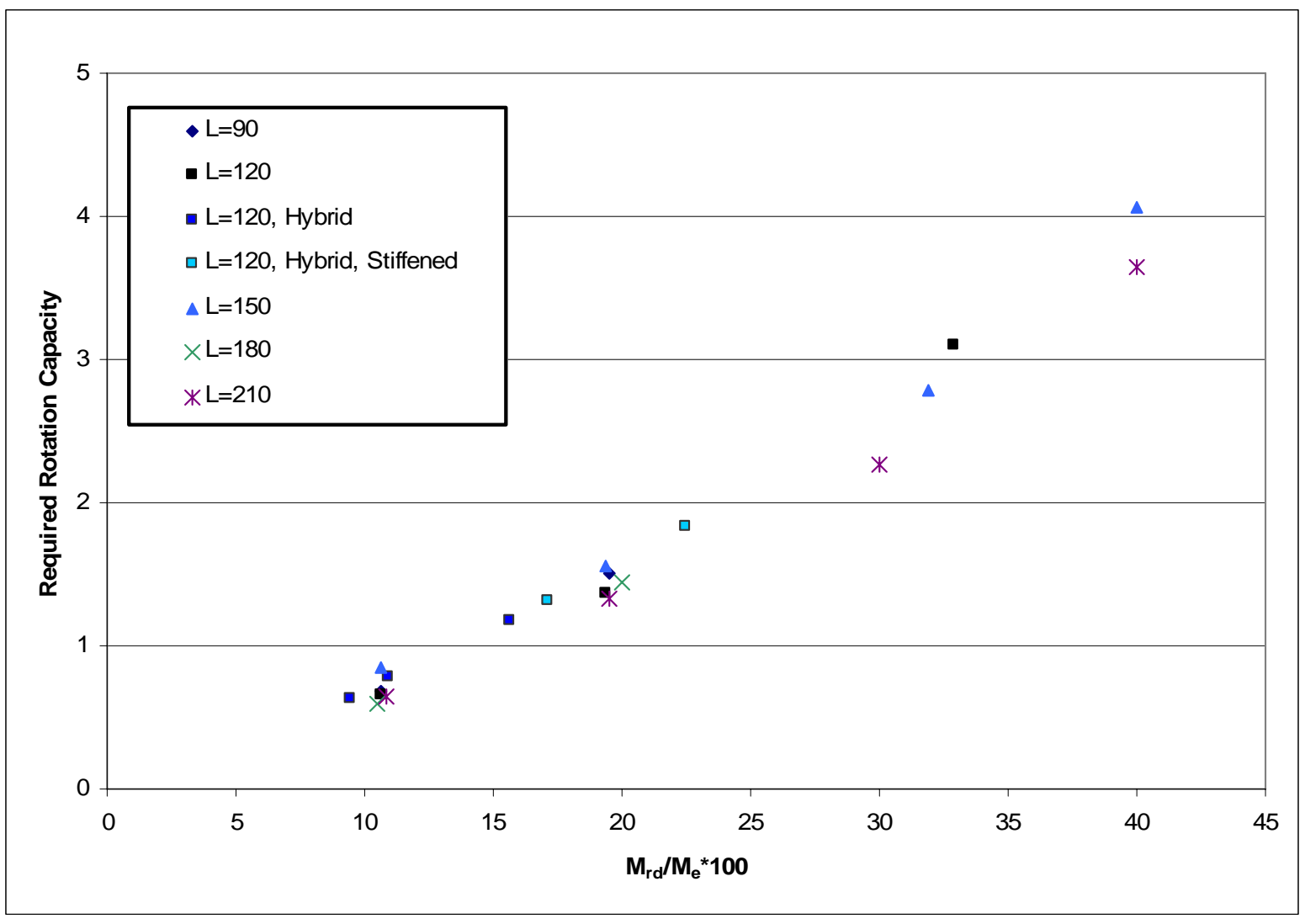

Figure 6-22. Relationship Between Percentage of Moment Redistributed and Required Rotation Capacity 
rotation capacity of three would be unconservative if higher degrees of moment redistribution were permitted.

An alternative approach to evaluating rotation requirements is to utilize moment versus rotation curves from experimental testing. This method is used herein to validate the results presented above. For this evaluation, the experimental data presented in Chapter 3 as well as the data from all three-point bending tests presented in Appendix B is employed. Because the rotation requirements are a function of the global stiffness of the structure and the test specimens represent only the pier region of a continuous span structure, several assumptions must first be made regarding the relationship between the test specimen and the structure of which it is a component. First, it is assumed that the structure consists of a uniform crosssection, i.e, both the positive and negative bending regions have geometry and crosssectional properties equal to that of the test specimen under consideration. Second, as a consequence of assuming that a uniform cross-section is used it can be assumed that the inflection point in the continuous-span structure occurs at a location equal to $30 \%$ of the span length away from the pier. This leads to representing the span length of the continuous-span structure as equal to five-thirds the length of the test specimen.

Once the stiffness of the structure is determined, the required plastic rotation can then be related to the redistribution moment using the linear relationship given by Eq. 6.1,

$$
\frac{\theta}{2}=\frac{\mathrm{ML}}{3 \mathrm{EI}} \text {. }
$$

Furthermore, the redistribution moment can be related to the experimental moment versus rotation curve, which gives $\mathrm{M}_{\mathrm{pe}}$, using Eq. 2.11,

$$
\mathrm{M}_{\mathrm{rd}}=\mathrm{M}_{\mathrm{e}}-\mathrm{M}_{\mathrm{pe}}
$$

and an assumed value of $M_{e}$. It is initially assumed that $M_{e}$ is equal to $120 \% M_{n}$, which represents a redistribution of moment equal to the maximum allowable redistribution currently permitted by AASHTO (2004) specifications. For some of the more slender girders, this assumed level of elastic moment did not give a solution and the elastic moment level was consequently reduced in these cases. 
The procedure used to determine rotation requirements using experimental data is illustrated below in Fig. 6-23, which illustrates the experimental moment versus plastic rotation curve for Girder 4MG, introduced in Chapter 3. Figure 6-23 also shows a required rotation curve. This curve is determined by first assuming that at the elastic moment there is no plastic rotation. Thus, the elastic moment of $1165 \mathrm{ft}$-kips, or $98 \%$ of $\mathrm{M}_{\mathrm{p}}$, determines the $\mathrm{y}$-intercept of the required rotation curve. Secondly, the $x$-intercept of the required rotation curve is determined by realizing that when a hinge exists at the pier, no moment can be resisted at this location and the beam may be analyzed as a simply supported beam with an applied end moment of magnitude equal to the elastic moment. For Girder 4MG, this results in a plastic rotation of 34 mrads. The intersection point where the required rotation curve is less than the available rotation curve is thus the required plastic rotation of the girder. In this case, the required plastic rotation is then 12.40 mrads at a moment of $62 \%$ of $\mathrm{M}_{\mathrm{p}}$. Computing the elastic rotation at this same level of moment using simple structural analysis procedures gives an elastic rotation of 6.85 mrads. The ratio between the plastic and elastic rotations

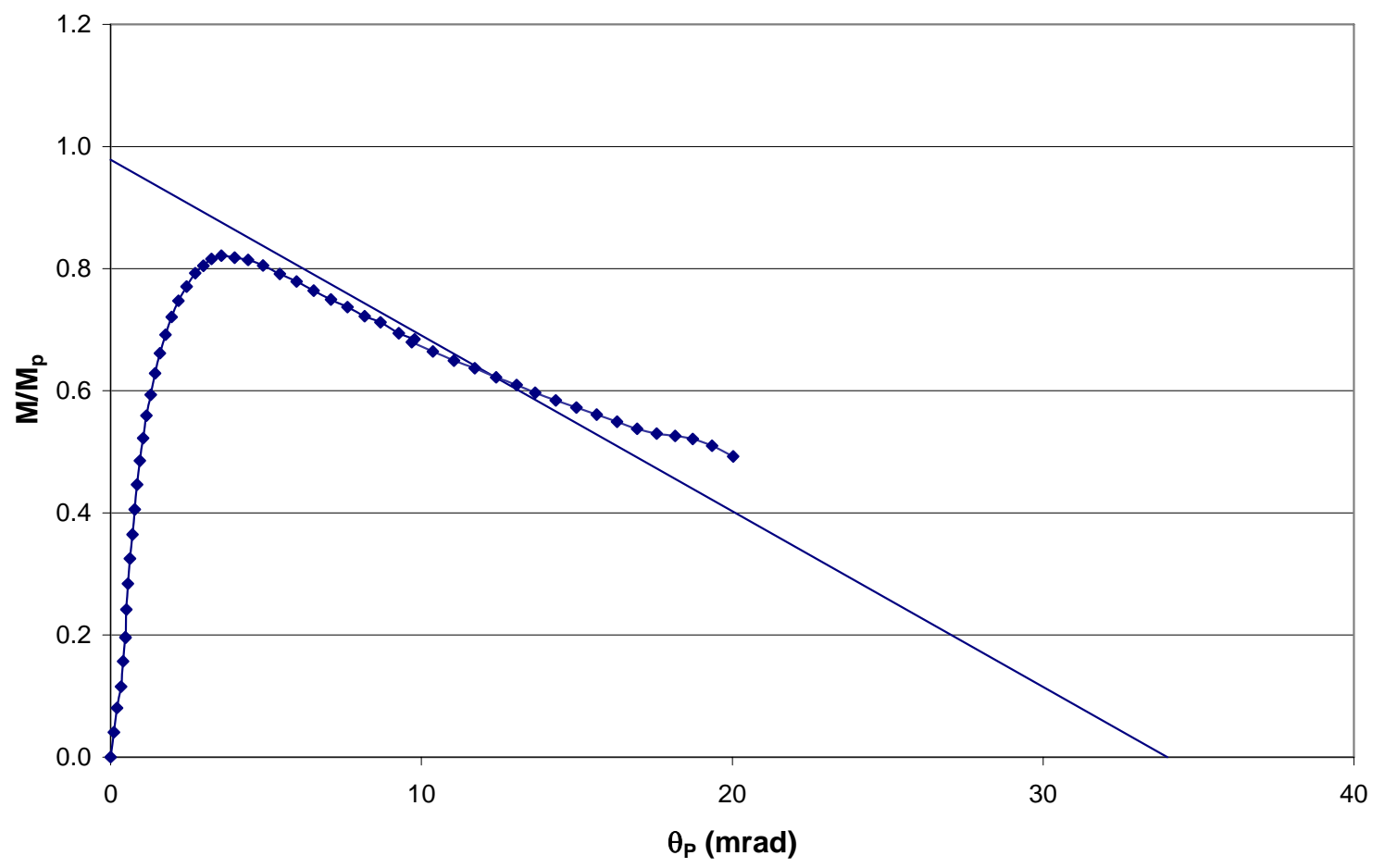

Figure 6-23. Illustration of Procedure to Determine Rotation Requirements from Experimental Moment versus Rotation Curves 
then gives the required rotation capacity, which is equal to 1.81 for Girder 4MG (assuming an elastic moment of $1165 \mathrm{ft}-\mathrm{kips}$ and the global stiffness assumptions outlined above).

This same procedure is used to determine the rotation requirements for each of the 3-point bending tests included in Appendix B and the experimental girders discussed in Chapter 3. Plots illustrating these procedures are also included in Appendix B. This data is summarized in Fig. 6-24, which presents the required rotation capacity for 53 experimental girders as a function of the percentage of redistribution moment. Thus, Fig. 6.24 is analogous to Fig. 622. Comparing Figs. 6-22 and 6-24, it is shown that the rotation requirements illustrated in Fig. 6-22 resulting from the analysis procedure discussed in Section 6.2 and the design studies presented in Section 6.3 are slightly higher than those that result from analysis of the experimental moment versus rotation curves. This is as expected because of the nature of the experimental specimens. Specifically, the experimental girders, except for the Chapter 3 (Righman 2005) girders, are relatively compact. This causes the available and required rotation curves to typically intersect in the pre-peak region of the moment versus rotation response, where the ratio between plastic and elastic rotation is relatively low. With this

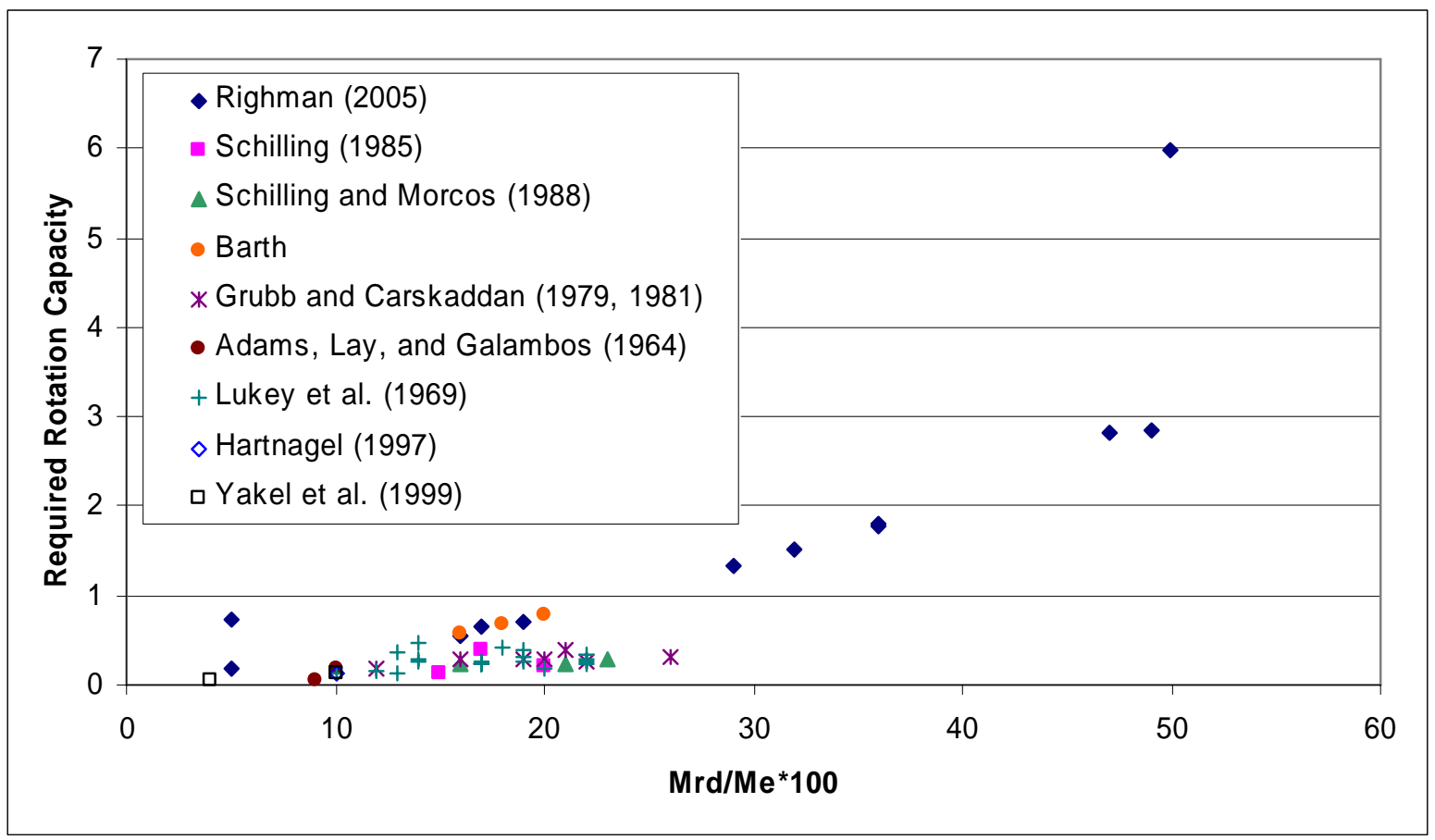

Figure 6-24. Relationship Between Percentage of Moment Redistributed and Required Rotation Capacity as Determined From Experimental Results 
consideration, it is concluded that the comparison between the two methods for computing required rotation capacities is favorable and that the results presented in Fig. 6-22 are appropriately conservative.

The results from determining the rotation requirements from experimental data are also presented in Table 6-4. Here the required plastic rotations, corresponding elastic rotations, required rotation capacities, and the corresponding percentage of the elastic moment that is redistributed are tabulated. Thus, the data in Table 6-4 can be used to compare with the results of Schilling (1986). Schilling reported required plastic rotations between 0 and 29 mrads using the same approach of determining the intersection of the moment versus rotation and required rotation curves for hypothetical girders. From, Table 6-4 it is observed that the required plastic rotation for these experimental girders is between 1 and 17 mrads.

\subsubsection{Development of Required Rotation Prediction Equations}

Based on evaluation of the influential variables affecting rotation requirements identified above, it is concluded that equations to predict the required rotation should be primarily expressed as a linear function of the percentage of redistribution moment. It is also necessary to incorporate the effects of pier location and material properties.

The relationship between percentage of redistribution moment and required rotation is illustrated in Fig. 6-25 for the two-span Grade 50 girders. The relationship

$$
\theta_{\mathrm{pR}}=80 \mathrm{M}_{\mathrm{rd}} / \mathrm{M}_{\mathrm{e}}
$$

is shown by the dark solid line to conservatively represent the available data.

Using Eq. 6.8 and the previously established relationship that the rotation requirements at piers adjacent to internal spans can be computed as the sum of the required rotation at the

pier under consideration assuming the pier is located between end spans and $25 \%$ of the required rotations at adjacent piers assuming these piers are also located between end spans, results in the following rotation requirements: 
Table 6-4. Rotation Requirements Resulting from Analysis of Experimental Data

\begin{tabular}{|c|c|c|c|c|c|}
\hline & Girder & $\theta_{\mathrm{p}}$ & $\theta_{\mathrm{e}}$ & $\mathbf{R}$ & $M_{r d} / M_{e}^{*} 100$ \\
\hline \multirow{12}{*}{ Righman (2005) } & 1MG & 6.77 & 10.49 & 0.65 & 17 \\
\hline & $2 \mathrm{MG}$ & 6.45 & 9.03 & 0.71 & 19 \\
\hline & $3 M G$ & 3.32 & 6.05 & 0.55 & 16 \\
\hline & $4 M G$ & 12.40 & 6.85 & 1.81 & 36 \\
\hline & $5 \mathrm{MG}$ & 11.98 & 4.21 & 2.85 & 49 \\
\hline & $6 \mathrm{MG}$ & 1.23 & 1.67 & 0.74 & 5 \\
\hline & $7 \mathrm{MG}$ & 17.05 & 2.85 & 5.98 & 50 \\
\hline & $8 M G$ & 11.92 & 6.69 & 1.78 & 36 \\
\hline & $9 M G$ & 1.92 & 11.28 & 0.17 & 5 \\
\hline & $10 M G$ & 11.26 & 8.53 & 1.32 & 29 \\
\hline & $11 \mathrm{MG}$ & 16.38 & 5.83 & 2.81 & 47 \\
\hline & $12 \mathrm{MG}$ & 11.47 & 7.61 & 1.51 & 32 \\
\hline \multirow{3}{*}{ Schilling (1985) } & US & 2.22 & 5.53 & 0.40 & 17 \\
\hline & UL & 2.07 & 10.41 & 0.20 & 20 \\
\hline & SL & 1.33 & 10.10 & 0.13 & 15 \\
\hline \multirow{3}{*}{ Schilling and Morcos (1988) } & $\mathrm{S}$ & 4.68 & 16.32 & 0.29 & 23 \\
\hline & M & 2.93 & 12.36 & 0.24 & 21 \\
\hline & $\mathrm{D}$ & 2.98 & 12.12 & 0.25 & 16 \\
\hline \multirow{3}{*}{ Barth (1996) } & $\mathrm{P} 2$ & 6.20 & 9.10 & 0.68 & 18 \\
\hline & P3 & 5.00 & 8.52 & 0.59 & 16 \\
\hline & P6 & 8.70 & 11.20 & 0.78 & 20 \\
\hline \multirow{7}{*}{ Grubb and Carskaddan $(1979,1981)$} & 188-3-1 & 1.85 & 9.58 & 0.19 & 12 \\
\hline & $188-3-2$ & 5.05 & 18.22 & 0.28 & 16 \\
\hline & $188-3-3$ & 10.48 & 32.24 & 0.33 & 26 \\
\hline & $188-3-5$ & 4.59 & 15.41 & 0.30 & 20 \\
\hline & $188-3-6$ & 4.76 & 16.02 & 0.30 & 19 \\
\hline & $188-3-7$ & 3.75 & 14.45 & 0.26 & 22 \\
\hline & $188-3-8$ & 5.60 & 14.51 & 0.39 & 21 \\
\hline \multirow{2}{*}{ Adams, Lay, and Galambos (1964) } & HT-28 & 3.39 & 18.50 & 0.18 & 10 \\
\hline & $\mathrm{HT}-43$ & 0.70 & 12.70 & 0.06 & 9 \\
\hline \multirow{20}{*}{ Lukey et al. (1969) } & A-1 & 3.44 & 23.61 & 0.15 & 12 \\
\hline & $A-2$ & 4.45 & 18.84 & 0.24 & 17 \\
\hline & B-1 & 2.20 & 17.60 & 0.13 & 10 \\
\hline & B-2 & 4.30 & 11.21 & 0.38 & 19 \\
\hline & B-3 & 3.19 & 13.70 & 0.23 & 17 \\
\hline & B-4 & 3.29 & 14.27 & 0.23 & 22 \\
\hline & B-5 & 3.82 & 15.68 & 0.24 & 17 \\
\hline & C-1 & 4.67 & 11.16 & 0.42 & 18 \\
\hline & $\mathrm{C}-2$ & 2.56 & 7.75 & 0.33 & 22 \\
\hline & $\mathrm{C}-3$ & 2.59 & 10.30 & 0.25 & 14 \\
\hline & C-4 & 2.74 & 10.71 & 0.26 & 19 \\
\hline & C-5 & 2.85 & 10.54 & 0.27 & 17 \\
\hline & D-6 & 2.36 & 8.22 & 0.29 & 22 \\
\hline & E-6 & 1.95 & 11.29 & 0.17 & 20 \\
\hline & D-1 & 4.04 & 15.62 & 0.26 & 22 \\
\hline & D-2 & 4.48 & 9.59 & 0.47 & 14 \\
\hline & D-5 & 6.39 & 20.95 & 0.31 & 19 \\
\hline & E-1 & 3.00 & 24.47 & 0.12 & 13 \\
\hline & $E-2$ & 4.80 & 13.30 & 0.36 & 13 \\
\hline & E-5 & 8.42 & 30.32 & 0.28 & 14 \\
\hline Hartnagel (1997) & W14x26 & 2.77 & 19.37 & 0.14 & 10 \\
\hline \multirow{2}{*}{ Yakel et al. (1999) } & $\mathrm{A}$ & 6.34 & 51.85 & 0.12 & 10 \\
\hline & D & 3.32 & 56.79 & 0.06 & 4 \\
\hline
\end{tabular}




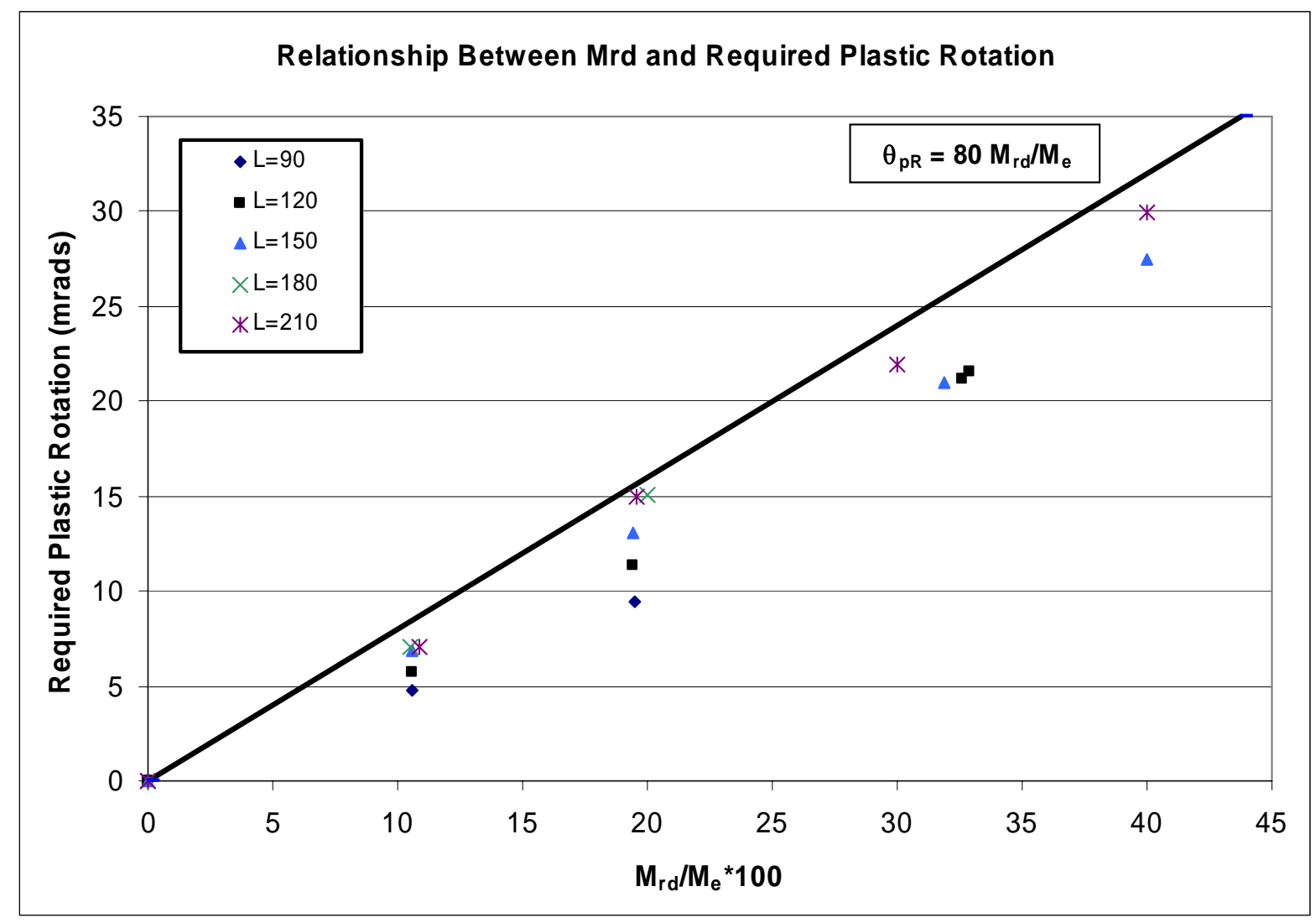

Figure 6-25. Relationship Between Required Plastic Rotation and Percentage of Redistribution Moment for Two-Span Grade 50 Designs

- For homogeneous pier sections between an end span and an internal span

$$
\theta_{\mathrm{pR}}=80 \mathrm{M}_{\mathrm{rd} 1} / \mathrm{M}_{\mathrm{e} 1}+20 \mathrm{M}_{\mathrm{rdA} 1} / \mathrm{M}_{\mathrm{eA} 1} \text {, }
$$

- And, for homogeneous pier sections between two internal spans

$$
\theta_{\mathrm{pR}}=80 \mathrm{M}_{\mathrm{rd}} / \mathrm{M}_{\mathrm{e}}+20 \mathrm{M}_{\mathrm{rdA} 1} / \mathrm{M}_{\mathrm{eA} 1}+20 \mathrm{M}_{\mathrm{rdA} 2} / \mathrm{M}_{\mathrm{eA} 2} \text {, }
$$

where $\mathrm{M}_{\mathrm{rd}} / \mathrm{M}_{\mathrm{e}}$ refers to the redistribution moment at the pier under consideration, while $\mathrm{M}_{\mathrm{rdA} 1} / \mathrm{M}_{\mathrm{eA} 1}$ and $\mathrm{M}_{\mathrm{rdA} 2} / \mathrm{M}_{\mathrm{eA} 2}$ refer to the redistribution moment at adjacent spans.

The relationship between required plastic rotation and percentage of redistribution moment for hybrid girders is evaluated by considering the results previously presented in Table 6-1 where the rotation requirements for homogeneous and hybrid girders are compared. Examining the difference in $\theta_{\mathrm{P}} / \mathrm{M}_{\mathrm{rd}} \%$ ratios for each pair of hybrid and homogeneous girders shows that the maximum difference occurs for the $120 \mathrm{ft}$ designs with $10 \%$ redistribution and 
is equal to 0.099, or approximately 0.1. Because it is shown by Eq. 6.8 that the ratio of $\theta_{\mathrm{P}} / \mathrm{M}_{\mathrm{rd}} \%$ for homogeneous girders is equal to 0.8 , increasing this value by 0.1 gives the resulting relationship for the rotation requirements for two-span hybrid girders,

$$
\theta_{\mathrm{pR}}=90 \mathrm{M}_{\mathrm{rd}} / \mathrm{M}_{\mathrm{e}} \text {. }
$$

Using the same procedure used above to determine the rotation requirements for piers adjacent to interior spans for homogeneous girders, the rotation requirements at piers adjacent to interior spans in hybrid girders are determined to be:

- For hybrid pier sections between an end span and an internal span

$$
\theta_{\mathrm{pR}}=90 \mathrm{M}_{\mathrm{rd} 1} / \mathrm{M}_{\mathrm{e} 1}+22.5 \mathrm{M}_{\mathrm{rdA} 1} / \mathrm{M}_{\mathrm{eA} 1},
$$

- And, for hybrid pier sections between two internal spans

$$
\theta_{\mathrm{pR}}=90 \mathrm{M}_{\mathrm{rd}} / \mathrm{M}_{\mathrm{e}}+22.5 \mathrm{M}_{\mathrm{rdA} 1} / \mathrm{M}_{\mathrm{eA} 1}+22.5 \mathrm{M}_{\mathrm{rdA} 2} / \mathrm{M}_{\mathrm{eA} 2} \text {. }
$$

By assuming that similar levels of redistribution moment will occur at each pier, the above equations can be reduced to,

$$
\theta_{\mathrm{pR}}=\mathrm{C}\left(\mathrm{M}_{\mathrm{rd}} / \mathrm{M}_{\mathrm{e}}\right)
$$

where:

- For homogeneous pier sections:

$$
\mathrm{C}=80+20 \mathrm{n} \text {, and }
$$

- For hybrid pier sections between two end spans:

$$
C=90+22.5 n
$$

and $\mathrm{n}$ represents the number of adjacent piers where moment redistribution occurs.

Thus the ultimate result of the rotation requirement study conducted herein is Eqs. 6.14 through 6.16. These relationships will be used in the following chapter to develop the rotation compatibility design and rating specifications by comparing these rotation requirements to the available rotations of steel I-girders. 


\section{Chapter 7: ROTATION COMPATABILITY - DESIGN AND RATING SPECIFICATIONS}

The primary focus of this chapter will be to present the development of the rotation compatibility approach to moment redistribution and its applications for steel bridge design and rating. This is carried out through synthesis of the available rotation/moment rotation characteristics presented in Chapter 5 and the rotation requirements presented in Chapter 6. It will be demonstrated herein that the suggested procedure provides a rational basis for conveniently determining the two key parameters required for design or rating: the maximum permissible level of redistribution and the minimum required girder capacity.

As previously discussed the rotation compatibility procedure is based on explicitly assuring that the available rotation of a girder is greater than the rotation required for the intended level of moment redistribution to occur. The first section of this chapter (Section 7.1) will summarize the expressions for required and available rotations developed in previous chapters and then proceed with evaluation of the conditions under which it can be assured that the available rotation is greater than the required rotation.

Section 7.2 presents rotation compatibility design specifications for steel bridges. Here the range of applicability of the specifications, an overview of the design process, and a series of equations in a format suitable for incorporation into design specifications are presented. Similarities and differences between the rotation compatibility design procedure and the AASHTO (2004) moment redistribution procedure are also discussed.

As discussed in Chapter 2, rating procedures consistent with the Load and Resistance Factor Design (LRFD) procedures were published in 2003 (AASHTO 2003). These rating specifications utilize the moment capacity equations from LRFD for load rating. Therefore,

it should by extension also be possible to utilize the optional moment redistribution specifications contained in the LRFD specifications for load rating. However, the limited applicability of the AASHTO (2004) moment redistribution specifications excludes many 
typical bridges from use with these procedures. The scope of the moment redistribution provisions (AASHTO 2004) is particularly restrictive in terms of lateral bracing distance, with many existing bridges violating the current lateral bracing requirements. The AASHTO (2004) moment redistribution provisions also are only applicable for compact compression flanges, which further restricts the use of these procedures. These limitations have been overcome in the present rotation compatibility procedure. The development of these procedures into a rating format is presented in Section 7.3.

Examples illustrating the use of the rotation compatibility procedure in both design and rating situations are then presented in Section 7.4. In the case of the design example, the design is also performed using the AASHTO (2004) moment redistribution procedures and comparisons are made between the two designs. These examples show that the use of the rotation compatibility procedures is both convenient and economically advantageous.

\subsection{Comparison of Required and Available Rotations}

\subsubsection{Summary of Available Rotations}

The available rotation of steel I-girder pier sections was previously discussed in Chapter 5, where it was shown that the moment-rotation behavior of any girder may be represented by one of two models. For girders satisfying the lateral bracing requirement of

$$
\mathrm{L}_{\mathrm{b}} \leq \frac{\mathrm{r}_{\mathrm{t}} \mathrm{E}}{\mathrm{F}_{\mathrm{yc}}}\left[0.1-0.06\left(\frac{\mathrm{M}_{1}}{\mathrm{M}_{2}}\right)\right],
$$

the moment-rotation behavior is represented by the model shown below in Fig. 7-1(a), while the moment-rotation behavior of girders that do not satisfy Eq. 7.1 is represented by Fig. 71(b). In both models, $\theta_{\mathrm{RL}}$ (in mrads) may be expressed by the equation

$$
\theta_{\mathrm{RL}}=128-143 \frac{\mathrm{b}_{\mathrm{fc}}}{\mathrm{t}_{\mathrm{fc}}} \sqrt{\frac{\mathrm{F}_{\mathrm{yc}}}{\mathrm{E}}}-43.2 \frac{\mathrm{D}_{\mathrm{cp}}}{\mathrm{b}_{\mathrm{fc}}}+48.2 \frac{\mathrm{b}_{\mathrm{fc}}}{\mathrm{t}_{\mathrm{fc}}} \frac{\mathrm{D}_{\mathrm{cp}}}{\mathrm{b}_{\mathrm{fc}}} \sqrt{\frac{\mathrm{F}_{\mathrm{yc}}}{\mathrm{E}}}-\max \left[0,0.5\left(\frac{\mathrm{L}_{\mathrm{b}}}{\mathrm{r}_{\mathrm{t}}}-30\right)\right] \geq 5 .
$$

Equation 7.2 gives nearly identical results to the current (AASHTO 2004) $\theta_{\mathrm{RL}}$ equation for girders that satisfy Eq. 7.1; linear decreasing values of $\theta_{\mathrm{RL}}$ then result as the lateral bracing distance is increased beyond the unbraced length given by Eq. 7.1. 


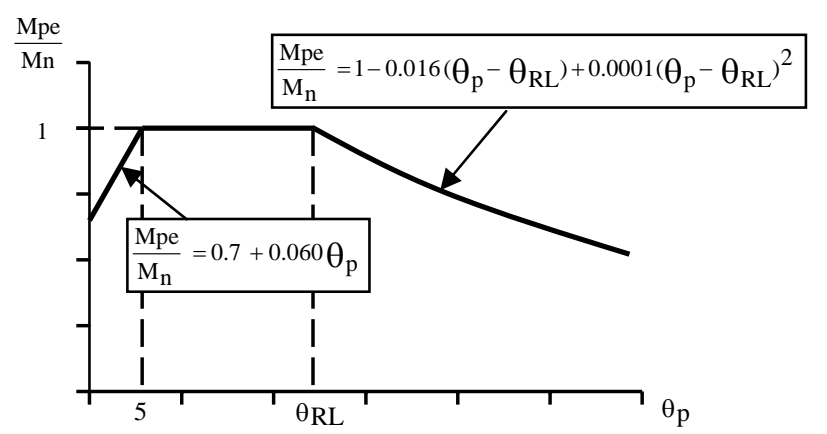

(a) For girders satisfying Eq. 7.1

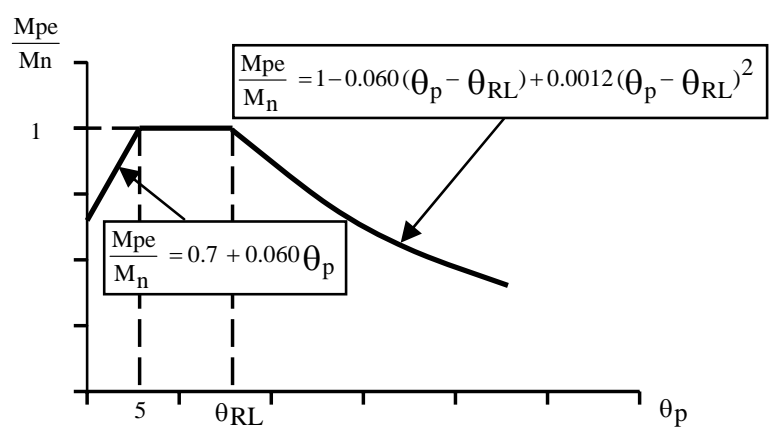

(b) For girders violating Eq. 7.1

Figure 7-1. Moment-Rotation Models

\subsubsection{Summary of Required Rotations}

As discussed in Chapter 6, the required rotation is expressed as a function of the percentage of moment to be redistributed and the pier location, with alternative expressions being required based on the grade of material used in the pier section. In summary, rotation requirements were formulated for homogeneous Grade 50 girders as well as hybrid girders comprised of Grade 70 flanges in negative bending and Grade 50 steel for positive bending flanges and webs; higher rotations are required for hybrid girders due to the smaller moment of inertia of these girders. Additionally, higher rotation requirements are required for piers between internal spans of the girder compared to piers adjacent to end spans due to the fact that the redistribution moments applied at adjacent piers also influence the rotation requirements. The alternative expressions for rotation requirements previously presented in Chapter 6 are reproduced below in Eqs. 7.3 through 7.5 where $\theta_{\mathrm{pR}}$ represents the required plastic rotation, $C$ is the rotation coefficient given by Eq. 7.4 or $7.5, \mathrm{M}_{\mathrm{rd}} / \mathrm{M}_{\mathrm{e}}$ represents the normalized redistribution moment, and $\mathrm{n}$ represents the number of adjacent piers where redistribution moments are applied. The reader is referred to Chapter 6 for a more detailed presentation of these equations.

$$
\theta_{\mathrm{pR}}=\mathrm{C}\left(\mathrm{M}_{\mathrm{rd}} / \mathrm{M}_{\mathrm{e}}\right)
$$

- For homogeneous pier sections:

$$
C=80+20 n
$$

- For hybrid pier sections:

$$
C=90+22.5 n
$$




\subsubsection{Comparison of Available and Required Rotations}

The rotation compatibility procedure is based on an explicit comparison of available and required rotations. Moment redistribution is valid for all cases where the available rotation is greater than or equal to that which is required, and conversely, moment redistribution may not be used when the available rotation is less than the required rotation. The comparison of available and required rotation is facilitated through the relationships for available and required rotations summarized in the previous two sections in conjunction with the general equation for moment redistribution,

$$
\mathrm{M}_{\mathrm{e}}=\mathrm{M}_{\mathrm{pe}}+\mathrm{M}_{\mathrm{rd}} \text {. }
$$

It may be observed that Eq. 7.3 expresses the rotation requirements in terms of $\mathrm{M}_{\mathrm{rd}} / \mathrm{M}_{\mathrm{e}}$ and that Fig. 7-1 presents the available rotations in terms of $\mathrm{M}_{\mathrm{pe}}$. Thus, through algebraic manipulation of Eq. 7.6, available and required rotations may be directly compared.

Equation 7.6 is used to express the required rotation in terms of $M_{p e} / M_{n}$. Because the available rotation is already expressed in this format, a similar expression for the required rotations will allow for direct comparison of the two rotations. This is accomplished by first dividing Eq. 7.6 by $\mathrm{M}_{\mathrm{e}}$.

$$
\frac{M_{p e}}{M_{e}}+\frac{M_{r d}}{M_{e}}=1
$$

This produces the term $\mathrm{M}_{\mathrm{rd}} / \mathrm{M}_{\mathrm{e}}$, which is also used in the rotation requirement expression given in Eq. 7.3. This term is then subtracted from both sides of the equation, giving

$$
\frac{M_{p e}}{M_{e}}=1-\frac{M_{r d}}{M_{e}} .
$$

Multiplication by $\mathrm{M}_{\mathrm{e}} / \mathrm{M}_{\mathrm{n}}$ then results in the desired format of the equation,

$$
\frac{M_{p e}}{M_{n}}=\left(1-\frac{M_{r d}}{M_{e}}\right) \cdot \frac{M_{e}}{M_{n}} .
$$

Examination of Eq. 7.9 reveals that the quantity $\mathrm{M}_{\mathrm{rd}} / \mathrm{M}_{\mathrm{e}}$ can be related to the required rotation while the quantity $\mathrm{M}_{\mathrm{pe}} / \mathrm{M}_{\mathrm{e}}$ can be related to the available rotation. However, further consideration must be given to the term $\mathrm{M}_{\mathrm{e}} / \mathrm{M}_{\mathrm{n}}$ and, as will be revealed in the following discussion, there are two alternative cases to consider when determining the value of this 
parameter, which are related to the relative slopes of the required rotation and available rotation curves. These two alternative cases will be illustrated by considering the Grade 50, two-span (90' - 90') continuous girder shown below in Fig. 7-2. First, Eq. 7.3 may be used to determine the rotation requirements. Because $\mathrm{n}$ is equal to zero for a two-span girder (see Section 6.4.6), the equation reduces to

$$
\theta_{\mathrm{pR}}=80 \mathrm{M}_{\mathrm{rd}} / \mathrm{M}_{\mathrm{e}},
$$

such that $\mathrm{M}_{\mathrm{rd}} / \mathrm{M}_{\mathrm{e}}$ may be expressed as

$$
\mathrm{M}_{\mathrm{rd}} / \mathrm{M}_{\mathrm{e}}=0.0125 \theta_{\mathrm{pR}} .
$$

Substitution of Eq. 7.11 into Eq. 7.9 gives

$$
\frac{M_{p e}}{M_{n}}=\left(1-0.0125 \theta_{p R}\right) \cdot \frac{M_{e}}{M_{n}}
$$

for the present design example. In general terms, Eq. 7.12 is expressed as

$$
\mathrm{M}_{\mathrm{pe}} / \mathrm{M}_{\mathrm{n}}=\left(1-\theta_{\mathrm{pR}} / \mathrm{C}\right)\left(\mathrm{M}_{\mathrm{e}} / \mathrm{M}_{\mathrm{n}}\right) \text {. }
$$

It is initially assumed that the lateral bracing requirements of Eq. 7.1 are satisfied such that $\theta_{\mathrm{RL}}$ is computed with the last term in Eq. 7.2 equal to zero. Furthermore, it is assumed that this girder is composite with the concrete deck resulting in $\mathrm{D}_{\mathrm{cp}}=25.91$ in. and $\mathrm{r}_{\mathrm{t}}=4.234 \mathrm{in}$, giving

$$
\theta_{\mathrm{RL}}=18 \text { mrads. }
$$

Equation 7.14 is then substituted for $\theta_{\mathrm{RL}}$ into the unloading equation in Fig. 7-1(a),

$$
\mathrm{M}_{\mathrm{pe}} / \mathrm{M}_{\mathrm{n}}=1-0.016\left(\theta_{\mathrm{p}}-18\right)+0.0001\left(\theta_{\mathrm{p}}-18\right)^{2} \text {, }
$$

which may also be expressed as

$$
\mathrm{M}_{\mathrm{pe}} / \mathrm{M}_{\mathrm{n}}=0.0001 \theta_{\mathrm{p}}{ }^{2}-0.0196 \theta_{\mathrm{p}}+1.3204 \text {. }
$$

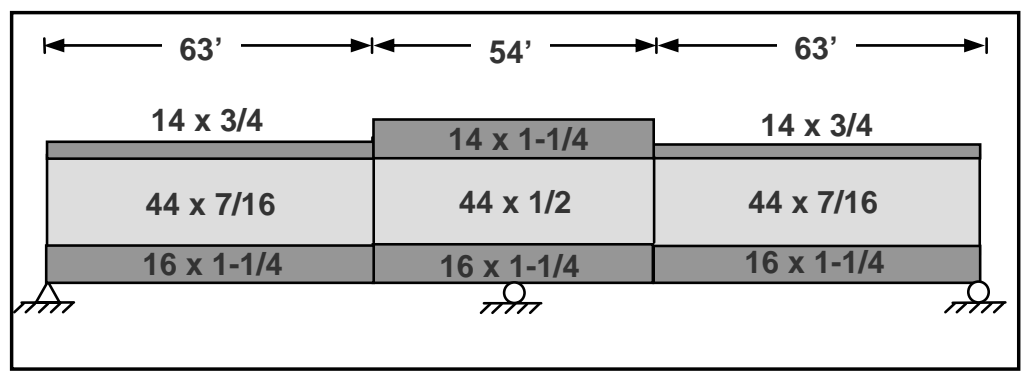

Figure 7-2. Example Girder 
Setting Eqs. 7.12 and 7.16 equal then gives

$$
0.0001 \theta_{p}{ }^{2}-0.0196 \theta_{p}+1.3204=\left(1-0.0125 \theta_{p R}\right) \bullet \frac{M_{e}}{M_{n}} .
$$

Thus, for the present example, moment redistribution is valid for any set of values of $M_{e} / M_{n}$, $\theta_{\mathrm{p}}$, and $\theta_{\mathrm{pR}}$ that satisfy Eq. 7.17 and where $\theta_{\mathrm{p}}$ is greater than or equal to $\theta_{\mathrm{pR}}$. The requirement that the available rotation must be greater than the required rotation can thus be represented by Eq. 7.18, which is valid for $\theta_{\mathrm{p}} \geq \theta_{\mathrm{RL}}$.

$$
0.0001 \theta_{\mathrm{p}}^{2}-0.0196 \theta_{\mathrm{p}}+1.3204 \geq\left(1-0.0125 \theta_{\mathrm{pR}}\right) \bullet \frac{\mathrm{M}_{\mathrm{e}}}{\mathrm{M}_{\mathrm{n}}}
$$

A graphical representation Eq. 7.18 is given below in Fig. 7-3 where the right side of the equation is graphed for various values of $M_{e} / M_{n}$. Here it is shown that because the slopes of the required rotation curves are less than the minimum slope of the available rotation curve (which occurs at $\theta_{R L}$ ), for $M_{e} / M_{n}$ ratios less than 1.29 the required rotations are less than the

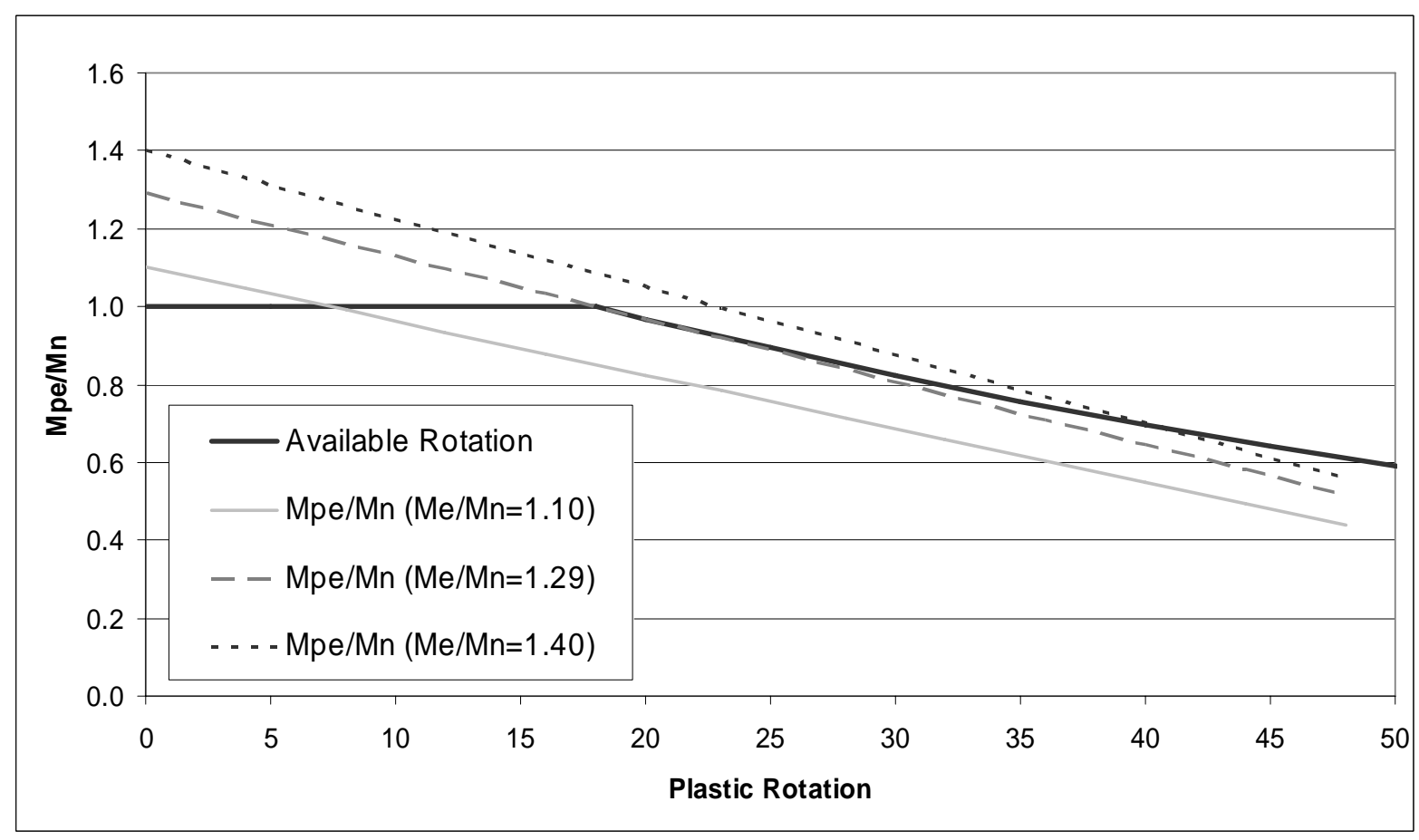

Figure 7-3. Comparison of Available and Required Rotations for Example Girder with $L_{h}$ satisfying Eq. 7.1 
available rotation throughout the unloading region of the moment versus rotation response and the curves do not intersect. Thus, the effective plastic moment capacity is equal to $M_{n}$ for $M_{e} / M_{n}$ ratios less than 1.29. Alternatively, for $M_{e} / M_{n}$ ratios greater than 1.29 the required rotations are initially greater than the available rotation. Consequently, the effective plastic moment in these cases is determined by the intersections of the two curves, which is shown to be $0.7 \mathrm{M}_{\mathrm{n}}$ for a $\mathrm{M}_{\mathrm{e}} / \mathrm{M}_{\mathrm{n}}$ ratio of 1.40 in Fig. 7-3. Lastly, it is shown by Fig. 7-3 that, for the example girder considered, 1.29 is the maximum $M_{e} / M_{n}$ ratio, where $M_{p e}$ may be taken as the maximum allowable value $\left(\mathrm{M}_{\mathrm{n}}\right)$.

It should be apparent from Fig. 7-3 that, above a certain limit (1.29 for this example), increasing ratios of $\mathrm{M}_{\mathrm{e}} / \mathrm{M}_{\mathrm{n}}$ reduce the effective plastic moment capacity of the girder. In terms of the physical girder, this represents a point where the girder can no longer sustain its nominal moment capacity due to excessive stresses in the girder. It should also be apparent that some maximum limit should be placed on the amount of moment that is permitted to be redistributed. For example, in the current AASHTO (2004) Specifications this limit is 20\% of the elastic moment. However, it is suggested that a more appropriate limit would be to limit the maximum allowable level of redistribution in accordance with the moment level at which the moment capacity decreases below $M_{n}$. Such an approach not only establishes a rational criterion for the maximum level of moment redistribution, but will also decrease girder distortions compared to allowing the girder to unload to moment levels below $M_{n}$. Thus, the objectives of the current 20\% limit (to control girder deformations) are also better satisfied. For these reasons, the maximum allowable elastic moment is limited to the magnitude of moment at which the effective plastic moment is equal to $M_{n}$ in the present work. This maximum value of elastic moment may also be thought of as the moment level where the available and required rotation curves intersect at a rotation value less than or equal to $\theta_{\mathrm{RL}}$. Mathematical expressions for determining the maximum allowable elastic moment are now derived based on this requirement; the ramifications of this requirement in comparison to the values of $\mathrm{M}_{\mathrm{pe}}$ less than $\mathrm{M}_{\mathrm{n}}$ permissible in the current AASHTO (2004) Specifications are subsequently discussed in Section 7.2.4. 
The maximum allowable value of $\mathrm{M}_{\mathrm{e}} / \mathrm{M}_{\mathrm{n}}$ may be obtained mathematically by setting $\theta_{\mathrm{pR}}$ $=\theta_{\mathrm{RL}}$ in Eq. 7.13. Realizing that $\mathbf{M}_{\mathrm{pe}} / \mathbf{M}_{\mathrm{n}}$ will equal one at $\theta_{\mathrm{RL}}$ and substituting this value into Eq. 7.13, the maximum $\mathrm{M}_{\mathrm{e}} / \mathrm{M}_{\mathrm{n}}$ ratio can be obtained for any girder where the slope of the required rotation is less than the slope of the available rotation.

$$
\mathrm{M}_{\mathrm{e}} / \mathrm{M}_{\mathrm{n}} \leq 1 /\left(1-\theta_{\mathrm{RL}} / \mathrm{C}\right)
$$

In order to develop the needed expressions to determine the applicable range of Eq. 7.19, (i.e., when the slope of the required rotation equation is less than the slope of the available rotation equation) we now consider the respective slopes of the required rotation and available rotation curves. From examination of Eq. 7.14 it is revealed that the slope of the required rotation equation is

$$
\mathrm{m}_{\mathrm{r}}=-\mathrm{M}_{\mathrm{e}} / \mathrm{CM}_{\mathrm{n}} .
$$

The slope of the available rotation curves is given by taking the first derivative of the unloading curves given in Figs. 7-1(a) and (b) with respect to $\theta_{\mathrm{p}}$. Expressing these equations in the standard quadratic format of

$$
y=a x^{2}+b x+c,
$$

the slope of the unloading curve is then expressed as

$$
\mathrm{m}_{\mathrm{a}}=-\mathrm{b}+2 \mathrm{a}\left(\theta_{\mathrm{p}}-\theta_{\mathrm{RL}}\right) .
$$

Because $b$ is negative and a is positive, the minimum slope of the available rotation curve occurs when $\theta_{\mathrm{p}}$ equals $\theta_{\mathrm{RL}}$. Thus,

$$
\mathrm{m}_{\mathrm{a}-(\min )}=-\mathrm{b} \text {. }
$$

By then requiring that $\mathrm{m}_{\mathrm{r}}<\mathrm{m}_{\mathrm{a}}$, from Eqs. 7.20 and 7.23, we obtain

$$
-\mathrm{M}_{\mathrm{e}} / \mathrm{CM}_{\mathrm{n}} \leq-\mathrm{b},
$$

and thus,

$$
\mathrm{M}_{\mathrm{e}} / \mathrm{M}_{\mathrm{n}} \leq \mathrm{Cb} .
$$

Because $b$ is a negative quantity, Eq. 7.26 can also be expressed as

$$
\mathrm{M}_{\mathrm{e}} / \mathrm{M}_{\mathrm{n}} \geq \mathrm{C}|\mathrm{b}| .
$$

Equation 7.26 must be satisfied to ensure that the slope of the required rotation expression is less than the slope of the available rotation, such that Eq. 7.19 may be used to compute the maximum allowable ratio of $\mathrm{M}_{\mathrm{e}} / \mathrm{M}_{\mathrm{n}}$. However, this condition alone is not sufficient to limit 
the required rotations to be less than the available rotations; in order for Eq. 7.19 to be valid, it must also be guaranteed that the minimum value of $\mathrm{M}_{\mathrm{e}} / \mathrm{M}_{\mathrm{n}}$ from Eq. 7.26 does not produce a required rotation greater than the available rotation at $\theta_{R L}$, i.e., at a moment level of $M_{p e} / M_{n}$ $=1$. If this requirement is satisfied, then it will be assured that the required rotation will be less than the available rotation throughout the unloading range of the girder. This requirement can be mathematically expressed by first using the basic equation of moment redistribution, Eq. 7.6, normalized by $\mathrm{M}_{\mathrm{n}}$, which gives

$$
\frac{M_{e}}{M_{n}}=\frac{M_{p e}}{M_{n}}+\frac{M_{r d}}{M_{n}} .
$$

$\mathrm{M}_{\mathrm{p}} / \mathrm{M}_{\mathrm{n}}=1$ may then be substituted into Eq. 7.27 giving

$$
\frac{M_{e}}{M_{n}}=1+\frac{M_{r d}}{M_{n}} .
$$

Multiplication of Eq. 7.28 by the ratio of $\mathrm{M}_{\mathrm{n}} / \mathrm{M}_{\mathrm{e}}$ and rearranging the resulting terms gives

$$
\frac{M_{n}}{M_{e}}=1-\frac{M_{r d}}{M_{e}} .
$$

Substituting $\theta_{\mathrm{pR}} / \mathrm{C}$ for $\mathrm{M}_{\mathrm{rd}} / \mathrm{M}_{\mathrm{e}}$ (which may be obtained from rearranging Eq. 7.3) subsequently results in

$$
\frac{\mathrm{M}_{\mathrm{n}}}{\mathrm{M}_{\mathrm{e}}}=1-\frac{\theta_{\mathrm{pR}}}{\mathrm{C}}
$$

Solving Eq. 7.30 for $\theta_{\mathrm{pR}}$ gives

$$
\theta_{\mathrm{pR}}=\mathrm{C}\left(1-\frac{\mathrm{M}_{\mathrm{n}}}{\mathrm{M}_{\mathrm{e}}}\right) .
$$

Then in order to determine if a value of $\theta_{p R}$ is less than $\theta_{R L}$ for $M_{p e} / M_{n}=1$, the maximum valid value of $\mathrm{M}_{\mathrm{n}} / \mathrm{M}_{\mathrm{e}}$, which may be computed from Eq. 7.26 as

$$
\frac{M_{n}}{M_{e}}(\max )=\frac{1}{C|b|},
$$

is substituted into Eq. 7.31 giving

$$
\theta_{\mathrm{pR}}(\min )=\mathrm{C}\left(1-\frac{1}{\mathrm{C}|\mathrm{b}|}\right),
$$

or, 


$$
\theta_{\mathrm{pR}}(\min )=\mathrm{C}-\frac{1}{|\mathrm{~b}|}
$$

Finally, it is required that the minimum value for $\theta_{p R}$ at $M_{p e} / M_{n}=1$ must be less than the available rotation at the same level of moment, i.e., $\theta_{\mathrm{RL}}$,

$$
C-\frac{1}{|b|} \leq \theta_{\mathrm{RL}}
$$

So, in conclusion, when both Eqs. 7.26 and 7.35 are satisfied, the maximum allowable value of $\mathrm{M}_{\mathrm{e}} / \mathrm{M}_{\mathrm{n}}$ is computed using Eq. 7.19 and any value of $\mathrm{M}_{\mathrm{e}} / \mathrm{M}_{\mathrm{n}}$ satisfying both Eqs. 7.19 and 7.26 is valid.

The allowable value of $\mathrm{M}_{\mathrm{e}} / \mathrm{M}_{\mathrm{n}}$ is now examined for other situations, beginning with the case when Eq. 7.35 is satisfied but Eq. 7.26 is violated. For this situation the available rotation will initially be greater than the required rotation, but the two curves may eventually intersect causing a situation where there is insufficient available rotation. This case is illustrated by using a value of $\mathrm{M}_{\mathrm{e}} / \mathrm{M}_{\mathrm{n}}$ equal to 1.10 with the previous example girder and is shown graphically in Fig. 7-3. The potential intersection of the two curves is found by setting the available and required rotation curves equal to one another as follows

$$
1-b\left(\theta-\theta_{R L}\right)+a\left(\theta-\theta_{R L}\right)^{2}=(1-\theta / R) M_{e} / M_{n} .
$$

Solution of Eq. 7.36 for $\theta$ gives the intersection point, which is expressed as

$$
\theta=\theta_{R L}+\frac{b}{2 a}-\frac{M_{e}}{2 a R M_{n}} \pm \frac{\sqrt{\left(\frac{M_{e}}{M_{n}}\right)^{2}-4 \frac{M_{e}}{M_{n}} a R \theta_{R L}-2 \frac{M_{e}}{M_{n}} b R+b^{2} R^{2}+4 a R^{2}\left(\frac{M_{e}}{M_{n}}-1\right)}}{2 a R}
$$

Examination of the square root term in Eq. 7.37 reveals that for all cases where $M_{e} / M_{n}<b C$ (i.e., when Eq. 7.26 is violated), this term is negative. This indicates that there is no solution for Eq. 7.37, which signifies that the available and required rotation curves do not intersect for any case where Eq. 7.35 is satisfied. Furthermore, we may state that the maximum allowable ratio of $\mathrm{M}_{\mathrm{e}} / \mathrm{M}_{\mathrm{n}}$ is given by Eq. 7.19 whenever Eq. 7.35 is satisfied. 
We now turn to the case when Eq. 7.35 is not satisfied. This situation is illustrated by considering the previous example girder although it is now assumed that the lateral bracing is placed at 4 equal segments per span equal to $22.5 \mathrm{ft}$, such that Eq. 7.1 is violated. Thus, $\theta_{\mathrm{RL}}$ is computed using Eq. 7.2 to equal 5 mrad. Substituting this value of $\theta_{\mathrm{RL}}$, b equal to -0.06 , and the previously computed value of $\mathrm{C}$ equal to 80 into Eq. 7.33 shows that the requirement to assure that the available and required rotation curves do not interest is not satisfied. This is shown graphically for three different $\mathrm{M}_{\mathrm{e}} / \mathrm{M}_{\mathrm{n}}$ ratios in Fig. 7-4. Here it is obvious that a $\mathrm{M}_{\mathrm{e}} / \mathrm{M}_{\mathrm{n}}$ ratio of 1.10 (or greater) is not valid since the required rotation is greater than the available rotation throughout the unloading region. It is also shown that for a $M_{e} / M_{n}$ ratio of 1.067 the available and required rotation curves will intersect at $\theta_{R L}$, while for lower $M_{e} / M_{n}$ ratios the two curves will intersect at higher rotations and lower levels of moment. Because the most economical design situation will result when $M_{e} / M_{n}$ is maximized, it will now be proven that a $\mathrm{M}_{\mathrm{e}} / \mathrm{M}_{\mathrm{n}}$ ratio of 1.067 will yield sufficient rotation for moment redistribution if the rotations are limited to $\theta_{\mathrm{RL}}$.

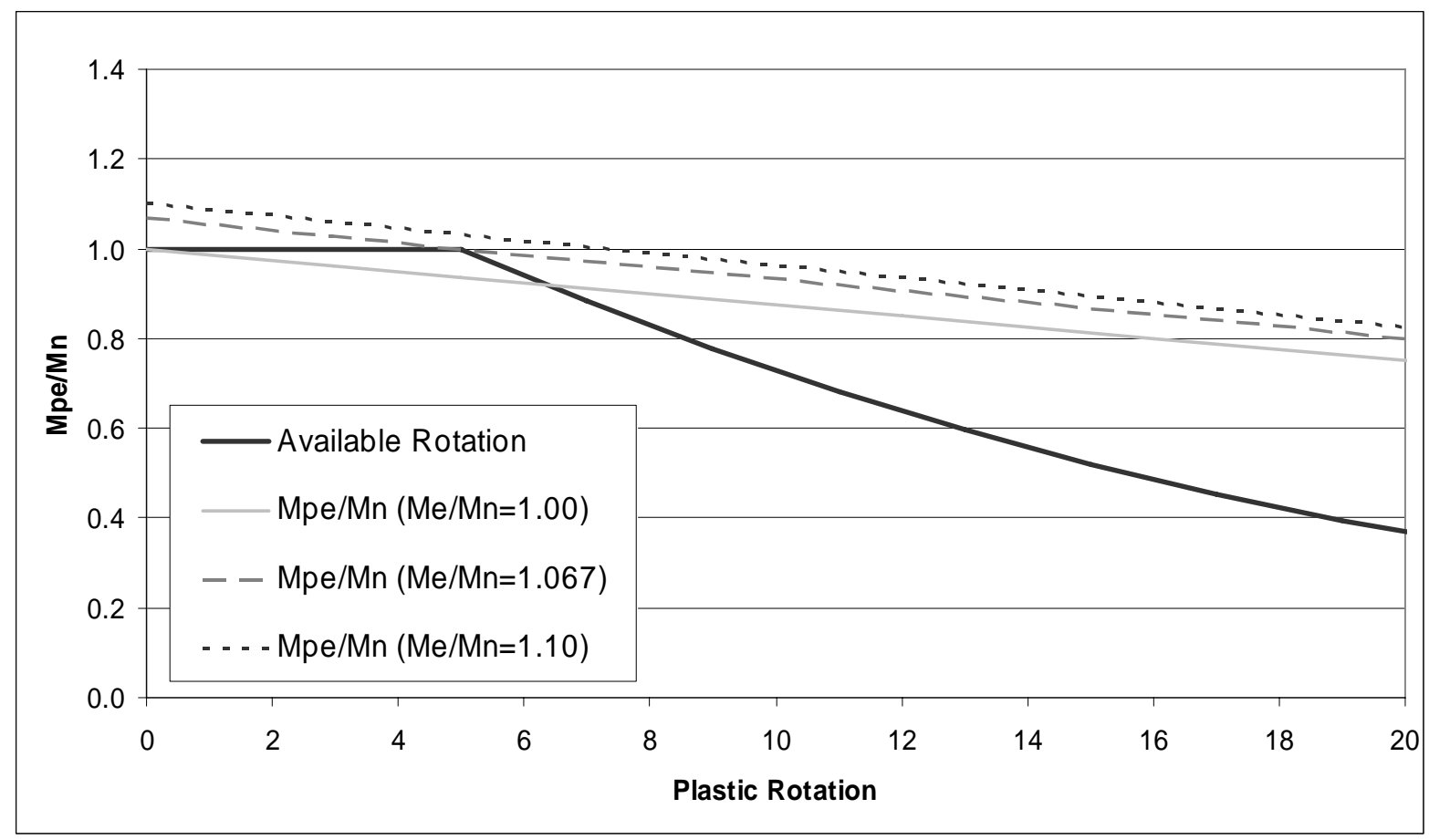

Figure 7-4. Comparison of Available and Required Rotations for Example Girder with $L_{h}$ violating Eq. 7.1 
In order to assure that there is sufficient rotation available to redistribute moment when a $\mathrm{M}_{\mathrm{e}} / \mathrm{M}_{\mathrm{n}}$ ratio of 1.067 is used, the sum of the effective plastic moment and the maximum redistribution moment that may be developed at $\theta_{\mathrm{RL}}$ must equal a minimum of the elastic moment at the pier. The effective plastic moment, as determined from the available rotation curve at $\theta_{R L}$, is shown to be equal to $M_{n}$, i.e., $M_{p e} / M_{n}=1$ and

$$
\frac{\mathrm{M}_{\mathrm{pe}}}{\mathrm{M}_{\mathrm{e}}}=\frac{\mathrm{M}_{\mathrm{pe}}}{\mathrm{M}_{\mathrm{n}}} \cdot \frac{\mathrm{M}_{\mathrm{n}}}{\mathrm{M}_{\mathrm{e}}}=1 \cdot \frac{1}{1.067}=0.9375 .
$$

The maximum redistribution moment (occurring at $\theta_{\mathrm{RL}}$ ), computed by rearranging Eq. 7.3, is equal to

$$
\frac{\mathrm{M}_{\mathrm{rd}}}{\mathrm{M}_{\mathrm{e}}}=\frac{\theta_{\mathrm{pR}}}{\mathrm{C}}=\frac{\theta_{\mathrm{RL}}}{\mathrm{C}}=\frac{5}{80}=0.0625 .
$$

Summing Eqs. 7.38 and 7.39 gives

$$
\frac{\mathrm{M}_{\mathrm{pe}}}{\mathrm{M}_{\mathrm{e}}}+\frac{\mathrm{M}_{\mathrm{rd}}}{\mathrm{M}_{\mathrm{e}}}=0.9375+0.0625=1 .
$$

Thus the requirement that the effective plastic moment and redistribution moment sum to the elastic moment is exactly satisfied and it is shown that moment redistribution is valid for the girder under consideration for a $\mathrm{M}_{\mathrm{e}} / \mathrm{M}_{\mathrm{n}}$ ratio up to 1.067.

The maximum allowable $\mathrm{M}_{\mathrm{e}} / \mathrm{M}_{\mathrm{n}}$ ratio for the case when Eq. 7.35 is violated may then be computed in the same manner as before, using Eq. 7.20. This is true because the intersection point of the available and required rotation curves occurs at $\theta_{\mathrm{RL}}$ in all cases.

\subsubsection{Summary}

The above discussion has compared the available and required rotations and examined the conditions necessary to assure that the available rotation is greater than the required rotations. All possible scenarios were investigated with regard to the various relationships that may exist between the required and available rotation functions and the results of this analysis show that it can be assured that the available rotation is greater than that which is required if the intersection of the available and required rotation curves occurs at a rotation less than or equal to $\theta_{\mathrm{RL}}$. Furthermore, by setting the rotation at which the two curves intersect to $\theta_{\mathrm{RL}}$, the maximum allowable ratio of $\mathrm{M}_{\mathrm{e}} / \mathrm{M}_{\mathrm{n}}$ may then by computed using Eq. 7.19. 


\subsection{Rotation Compatibility Design Specifications}

\subsubsection{Scope}

As with the AASHTO 2004 moment redistribution specifications, the moment redistribution specifications suggested herein are limited to straight girders with skews less than 10 degrees having neither staggered cross-frames nor holes in the top/tension flange over a distance of two times the web depth from either side of interior-pier sections where moments are redistributed. Also consistent with the AASHTO 2004 procedures, the current specifications restrict the length over which the girder may be analyzed for reduced negative moments due to moment redistribution to a distance equal to one unbraced length on each side of the pier. However, there are no cross-section limits outside of the cross-section proportion limits for general I-girders contained in AASHTO 2004 Section 6.10.2, which allows the rotation compatibility specifications to be applicable for a considerably larger class of girders. The procedures are applicable for girders having yield strengths up to $70 \mathrm{ksi}$.

\subsubsection{Overview of Design Procedure}

While there are no explicit requirements on lateral bracing distance in the rotation compatibility specifications, the beneficial effects of shorter bracing spacing are incorporated through the last term of the $\theta_{\mathrm{RL}}$ equation (Eq. 7.2). For designs satisfying Eq. 7.1, this last term is equal to zero. Thus, for preliminary design, the designer may wish to initially assume that the last term of Eq. 7.2 is equal to zero when determining cross-section geometry requirements, and then subsequently selecting the lateral bracing distance.

The rotation coefficient, $C$, is then used in combination with $\theta_{\mathrm{RL}}$ to determine the maximum permissible level of elastic moment. It was shown by Eq. 7.19 that the maximum allowable ratio of $\mathrm{M}_{\mathrm{e}} / \mathrm{M}_{\mathrm{n}}$ is computed as

$$
\mathrm{M}_{\mathrm{e}} / \mathrm{M}_{\mathrm{n}} \leq 1 /\left(1-\theta_{\mathrm{RL}} / \mathrm{C}\right) .
$$

By rearranging this equation, we arrive at the moment requirements at the pier section using rotation compatibility moment redistribution design,

$$
\mathrm{M}_{\mathrm{e}}\left(1-\theta_{\mathrm{RL}} / \mathrm{C}\right) \leq \mathrm{M}_{\mathrm{n}}
$$


Positive bending sections must also be analyzed for the effects of moment redistribution. This is performed by computing the redistribution moment. At the piers the redistribution moment is equal to the difference between the applied moments and the moment capacity,

$$
\mathrm{M}_{\mathrm{rd}}=\mathrm{M}_{\mathrm{e}}-\mathrm{M}_{\mathrm{n}} \text {. }
$$

The redistribution moment at other locations is then determined by linear interpolation between the redistribution moments at the ends of each span, where redistribution moments are equal to zero at abutments as is done in the AASHTO (2004) procedures.

The following section will present the equations given above in a format suitable for incorporation into design specifications.

\subsubsection{Rotation Compatibility Design Specification Equations}

- Negative bending capacity at the strength limit state may be evaluated using

$$
M_{e}\left(1-\theta_{R L} / C\right) \leq M_{n},
$$

where:

$\mathrm{M}_{\mathrm{n}}=$ nominal moment capacity of the girder computed using applicable AASHTO Specifications,

$\theta_{\mathrm{RL}}=128-143 \frac{\mathrm{b}_{\mathrm{fc}}}{\mathrm{t}_{\mathrm{fc}}} \sqrt{\frac{\mathrm{F}_{\mathrm{yc}}}{\mathrm{E}}}-21.6 \frac{2 \mathrm{D}_{\mathrm{cp}}}{\mathrm{b}_{\mathrm{fc}}}+24.1 \frac{\mathrm{b}_{\mathrm{fc}}}{\mathrm{t}_{\mathrm{fc}}} \frac{2 \mathrm{D}_{\mathrm{cp}}}{\mathrm{b}_{\mathrm{fc}}} \sqrt{\frac{F_{\mathrm{yc}}}{\mathrm{E}}}-\max \left[0,0.5\left(\frac{\mathrm{L}_{\mathrm{b}}}{\mathrm{r}_{\mathrm{t}}}-30\right)\right] \geq 5$

$\mathrm{C}=80+20 \mathrm{n}$ for homogeneous girders with $\mathrm{F}_{\mathrm{y}} \leq 50 \mathrm{ksi}$,

$\mathrm{C}=90+22.5 \mathrm{n}$ for hybrid girders with $\mathrm{F}_{\mathrm{yf}} \leq 70 \mathrm{ksi}$, and

$\mathrm{n} \quad=$ number of piers where moment redistribution occurs adjacent to the pier under consideration.

- Positive bending capacity must then be evaluated using

$$
M_{n} \geq M_{e}+M_{r d L}+(x / L)\left(M_{r d R}-M_{r d L}\right),
$$

where:

$\mathrm{M}_{\mathrm{n}}=$ nominal moment capacity of the girder computed using applicable AASHTO Specifications,

$\mathrm{M}_{\mathrm{rdL}}=$ redistribution moment at left end of the span under consideration, computed using Eq. 7.42, 
$\mathrm{M}_{\mathrm{rdR}}=$ redistribution moment at right end of the span under consideration, computed using Eq. 7.42,

$\mathrm{x}=$ location where moment is being evaluated expressed as distance from left end of span

$\mathrm{L} \quad=$ span length

\subsubsection{Comparison to AASHTO 2004 Moment Redistribution Procedures}

The AASHTO 2004 moment redistribution procedures were previously summarized in Chapter 2. Although the underlying theory of these procedures and the rotation compatibility design procedures described above is the same, the resulting design equations are markedly different. The AASHTO 2004 moment redistribution procedures are primarily based on the computation of two quantities, the effective plastic moment $\left(\mathrm{M}_{\mathrm{pe}}\right)$ and the redistribution moment $\left(\mathrm{M}_{\mathrm{rd}}\right)$. Examination of the derivation of the rotation compatibility procedures presented in Section 7.1.3, reveals that the basis of these procedures is also the quantities $\mathrm{M}_{\mathrm{pe}}$ and $\mathrm{M}_{\mathrm{rd}}$, although the primary quantities used in the design computations in the rotation compatibility procedure are $\theta_{\mathrm{RL}}$ and $\mathrm{C}$.

In the AASHTO 2004 procedures, $\mathrm{M}_{\mathrm{pe}}$ for a given limit state (strength or service) is expressed by one of two equations depending on the web slenderness and transverse stiffener configuration. These equations express $\mathrm{M}_{\mathrm{pe}}$ as a function of $\mathrm{M}_{\mathrm{n}}$ and are limited to a maximum value of $\mathrm{M}_{\mathrm{n}}$. This is based on the premise that compact beams will have sufficient rotation available to redistribute moment at $\mathrm{M}_{\mathrm{n}}$, but that more slender beams will have reduced rotation available at $\mathrm{M}_{\mathrm{n}}$ and a lower level of moment must be used to achieve the needed rotation. Furthermore, the AASHTO (2004) specifications limit the maximum allowable level of moment redistribution to $20 \%$ of the elastic moment. Conversely, in the present rotation compatibility procedures, the $M_{p e}$ value is equal to $M_{n}$ in all cases and the maximum allowable level of redistribution varies based on the rotation requirements and girder ductility.

To compare the consequences of these two alternative approaches to limiting maximum levels of redistribution, a girder that has an $\mathrm{M}_{\mathrm{pe}}$ value equal to $\mathrm{M}_{\mathrm{n}}$ according to the AASHTO 
(2004) specifications is first considered. Substituting $M_{n}$ for $M_{p e}$ in Eq. 7.6 and setting the magnitude of redistribution moment equal to the maximum value permissible using the AASHTO (2004) specifications, $\mathrm{M}_{\mathrm{rd}}=0.2 \mathrm{M}_{\mathrm{e}}$, gives

$$
\mathrm{M}_{\mathrm{e}}=\mathrm{M}_{\mathrm{n}}+0.2 \mathrm{M}_{\mathrm{e}}
$$

which can be solved to determine the maximum allowable value of the elastic moment in terms of $\mathrm{M}_{\mathrm{n}}$, giving

$$
\mathrm{M}_{\mathrm{e}} \leq 1.25 \mathrm{M}_{\mathrm{n}} .
$$

In the rotation compatibility procedure, the maximum value of $\mathrm{M}_{\mathrm{rd}}$ varies based on the available and required rotations of the girder under consideration and can be expressed as

$$
\mathrm{M}_{\mathrm{rd}} / \mathrm{M}_{\mathrm{e}}=\theta_{\mathrm{RL}} / \mathrm{C} \text {. }
$$

For illustrative purposes, a representative girder for which $M_{p e}$ is equal to $M_{n}$ (Girder 90-2-E50-20-B from Chapters 5 and 6 and also described in Appendix E) is selected and the maximum value of normalized redistribution moment is computed using Eq. 7.39. First, Eq. 7.2 is used to compute a $\theta_{\mathrm{RL}}$ value equal to 24.1 mrads. Then, from Eqs. 7.4 and 7.5, it is shown that C may vary between a minimum value of 80 and a maximum value of 135 . Substituting the minimum value of C into Eq. 7.39 and rearranging the equation gives that the maximum allowable value of redistribution moment is equal to

$$
\mathrm{M}_{\mathrm{rd}}=\left(\theta_{\mathrm{RL}} / \mathrm{C}\right) \mathrm{M}_{\mathrm{e}}=24 \cdot 1 / 80 \mathrm{M}_{\mathrm{e}}=0.30 \mathrm{M}_{\mathrm{e}} .
$$

Substituting Eq. 7.46 for $\mathrm{M}_{\mathrm{rd}}$ and $\mathrm{M}_{\mathrm{n}}$ for $\mathrm{M}_{\mathrm{pe}}$ into Eq. 7.6 gives

$$
\mathrm{M}_{\mathrm{e}}=\mathrm{M}_{\mathrm{n}}+0.30 \mathrm{M}_{\mathrm{e}} \text {. }
$$

The maximum allowable value for the elastic moment is then equal to

$$
\mathrm{M}_{\mathrm{e}} \leq 1.43 \mathrm{M}_{\mathrm{n}} \text {, }
$$

which represents a $15 \%$ increase in the allowable value of the elastic moment compared to the AASHTO (2004) specifications. Alternatively, substituting the maximum value of $\mathrm{C}$ into Eq. 7.39 gives

$$
\mathrm{M}_{\mathrm{rd}}=\left(\theta_{\mathrm{RL}} / \mathrm{C}\right) \mathrm{M}_{\mathrm{e}}=24.1 / 135 \mathrm{M}_{\mathrm{e}}=0.18 \mathrm{M}_{\mathrm{e}} \text {, }
$$

which subsequently results in a maximum allowable elastic moment of

$$
\mathrm{M}_{\mathrm{e}} \leq 1.22 \mathrm{M}_{\mathrm{n}} .
$$

The above example illustrates that for girders that have a $M_{p e}$ value equal to $M_{n}$ in the AASHTO (2004) procedures, the rotation compatibility procedure results in maximum 
allowable elastic moment values that are appreciably higher (15\% in this example) or slightly lower (2\% in this example) than those permitted by the AASHTO (2004) specifications. The consequences of the alternative methodologies in the AASHTO (2004) and rotation compatibility procedures are also illustrated for a girder that has a $M_{p e}$ value less than $M_{n}$ in the AASHTO (2004) specifications. Specifically, for this consideration Girder CF-1-65-4-50 from the parametric study presented in Section 5.2 is employed. The AASHTO (2004) $\mathrm{M}_{\mathrm{pe}}$ equations give a value of $0.65 \mathrm{M}_{\mathrm{n}}$ for this girder. Thus, the maximum value of the elastic moment using the AASHTO (2004) moment redistribution procedures is

$$
\mathrm{M}_{\mathrm{e}}=0.65 \mathrm{M}_{\mathrm{n}} / 0.8=0.81 \mathrm{M}_{\mathrm{n}} .
$$

Alternatively, using the rotation compatibility procedures and the maximum possible value of $\mathrm{C}$ (which results in the minimum allowable value of $\mathrm{M}_{\mathrm{e}}$ ) gives a maximum redistribution moment of

$$
\mathrm{M}_{\mathrm{rd}}=\left(\theta_{\mathrm{RL}} / \mathrm{C}\right) \mathrm{M}_{\mathrm{e}}=5 / 135 \mathrm{M}_{\mathrm{e}}=0.04 \mathrm{M}_{\mathrm{e}} .
$$

Substituting this value of $\mathrm{M}_{\mathrm{rd}}$ into Eq. 7.6 gives

$$
\mathrm{M}_{\mathrm{e}}=\mathrm{M}_{\mathrm{n}}+0.04 \mathrm{M}_{\mathrm{e}},
$$

and an allowable elastic moment of

$$
\mathrm{M}_{\mathrm{e}} \leq 1.04 \mathrm{M}_{\mathrm{n}},
$$

which is $28 \%$ higher than the allowable moment permitted by the AASHTO (2004) specifications. Thus, it is concluded that limiting the value of $M_{p e}$ to $M_{n}$ in the rotation compatibility procedures does not adversely affect the economy that may result from the use of moment redistribution provisions.

The AASHTO 2004 moment redistribution provisions also give alternative design equations for the strength and service limit states, where the differing equations are based on the premise that girders will need to undergo less rotation to achieve moment redistribution at service limit states due to the lower magnitudes of load at this limit state. Conversely, the rotation compatibility procedures suggested above can be applied for either limit state as these procedures directly evaluate the moment redistribution characteristics of the girder for a given magnitude of applied loading. 


\subsection{Rotation Compatibility Rating Specifications}

\subsubsection{Scope}

The rotation compatibility specifications are applicable to both design and rating of continuous-span steel bridges and the scope of the rating and design procedures are identical. The reader is referred to section 7.2.1 for a description of the scope of these specifications.

\subsubsection{Overview of Rating Procedure}

As discussed in Section 2.2, the LRFR procedures use the member capacities calculated from LRFD procedures. Thus, because the effective plastic moment capacity is equal to $M_{n}$ in the rotation compatibility procedures, $\mathrm{M}_{\mathrm{n}}$ is the girder capacity used for both inelastic and elastic rating. However, inelastic rating procedures offer the engineer the ability to account for the reduction in moment levels that occurs in the negative bending regions due to moment redistribution. This effect is incorporated into the rotation compatibility rating specifications through the use of moment redistribution load factors that decrease the applied moment in negative bending regions and increase the applied moments in positive bending regions. These load factors are represented by the variables $\gamma_{\theta-}$ and $\gamma_{\theta^{+}}$, respectively. The rating procedure then proceeds in the same manner as would be used for an elastic design.

The load factor $\gamma_{\theta-}$ is easily computed as

$$
\gamma_{\theta-}=1-\theta_{\mathrm{RL}} / \mathrm{C} \text {. }
$$

If this optional load factor is used in the rating analysis of negative bending regions, the inelastic action that this assumes must be accounted for in the rating of the positive bending sections. This is done by using Eq. 7.40 to compute the redistribution moment as a ratio of the elastic moment at each end of the span under consideration,

$$
\mathrm{M}_{\mathrm{rd}} / \mathrm{M}_{\mathrm{e}}=\theta_{\mathrm{RL}} / \mathrm{C} .
$$

Linear interpolation of the $\theta_{\mathrm{RL}} / \mathrm{C}$ or $\mathrm{M}_{\mathrm{rd}} / \mathrm{M}_{\mathrm{e}}$ ratio at each end of the span is used to determine the factor by which the elastic positive bending moments should be increased in the rating analysis, represented as $\gamma_{\theta+}$. Using the subscripts " $L$ " and " $R$ " to refer to the left and right ends of the span, $x$ to represent the distance from left end of the span to the point where the

rating factor is to be computed, and L to represent the span length, Eq. 7.46 gives the equation to compute $\gamma_{\theta+}$, 


$$
\gamma_{\theta+}=1+\left(\frac{M_{r d}}{M_{e}}\right)_{L}+\frac{x}{L}\left[\left(\frac{M_{r d}}{M_{e}}\right)_{R}-\left(\frac{M_{r d}}{M_{e}}\right)_{L}\right]
$$

\subsubsection{Rotation Compatibility Rating Specification Equations}

The rotation compatibility procedure is easily incorporated into LRFR specifications through the addition of the load factor $\gamma_{\theta}$. Specifically, the general rating equation of LRFR is given by

$$
R F=\frac{C-\gamma_{D C}(D C)-\gamma_{D W}(D W) \pm \gamma_{P}(P)}{\gamma_{L}(L L+I M)},
$$

which for rating using the rotation compatibility procedures may be modified as

$$
\mathrm{RF}=\frac{\mathrm{C}-\gamma_{\theta}\left[\gamma_{\mathrm{DC}}(\mathrm{DC})+\gamma_{\mathrm{DW}}(\mathrm{DW}) \pm \gamma_{\mathrm{P}}(\mathrm{P})\right]}{\gamma_{\theta} \gamma_{\mathrm{L}}(\mathrm{LL}+\mathrm{IM})} .
$$

As discussed in the previous section, alternative formulas $\gamma_{\theta}$ are given for the negative and positive bending regions, where

$$
\gamma_{\theta-}=1-\theta_{\mathrm{RL}} / \mathrm{C}
$$

and

$$
\gamma_{\theta+}=1+\left(\frac{M_{r d}}{M_{e}}\right)_{L}+\frac{x}{L}\left[\left(\frac{M_{r d}}{M_{e}}\right)_{R}-\left(\frac{M_{r d}}{M_{e}}\right)_{L}\right] .
$$

\subsection{Examples}

This section will illustrate the rotation compatibility procedure using both design and rating examples. First an example bridge will be designed using the procedures outlined above; this same bridge is then redesigned using both the standard and refined procedures given in the AASHTO 2004 specifications and the resulting designs from the various methods are compared. Second, a rating example is presented. This example illustrates one potential area of application for the rotation compatibility rating procedures: a bridge initially designed using elastic procedures experiencing section loss due to corrosion. 


\subsubsection{Design Example}

This section will present the design of a two-span continuous bridge having equal 90' spans and the bridge cross-section shown in Fig. 7-5. The bridge is designed for all applicable limit states, i.e., strength, service, fatigue, and constructibility. It is assumed that the girders are comprised of Grade 50 steel and the concrete deck of the bridge is assumed to have a minimum compressive strength of $4 \mathrm{ksi}$ and standard Grade 60 steel reinforcement. In addition to the self weight of the girders and concrete deck, the bridge is designed to support stay-in-place forms (15 psf), cross-frames and miscellaneous steel details (with an assumed weight equal to $5 \%$ of the girder weight), parapets weighing $520 \mathrm{lb} / \mathrm{ft}$ that are applied to the composite sections, a future wearing surface (25 psf), and all relevant live loads (HL-93 live loading) as prescribed by AASHTO (2004) specifications. It is also assumed that dead load effects are equally resisted by each girder, while the AASHTO (2004) distribution factors are used to determine the live load effects on each girder.

At the constructibility limit state, the vertical bending moments are determined by considering the deck casting sequence illustrated in Fig. 7-6 and the variable stiffness of the girder as sections become composite. Lateral bending forces induced by the deck form brackets are also considered at the constructibility limit state. These form brackets are assumed support: deck overhang weight (255 plf), overhang forms (40 plf), screed rail (85 plf), railing (25 plf), walkway (125 plf), and fininshing machine (3000 lbs).

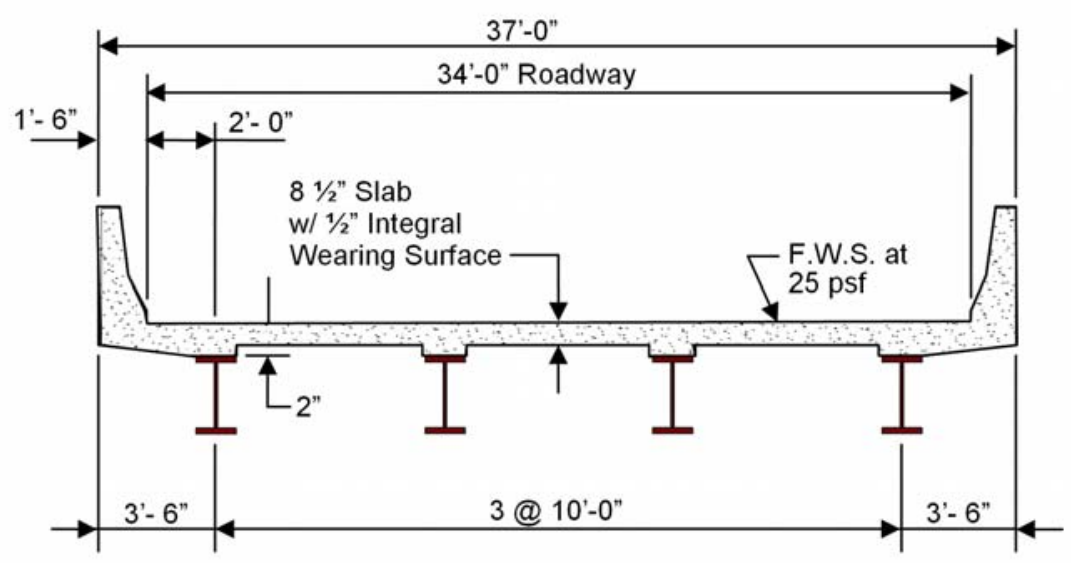

Figure 7-5. Design Example Bridge Cross-Section 
Given these design assumptions, the girder design $\mathrm{u}$ is presented in the following sections sing the rotation compatibility and AASHTO 2004 moment redistribution (standard and refined) procedures. For all designs, section transitions are assumed to occur at 27' from the pier requiring separate design of the positive and negative bending sections. Preliminary analyses were conducted to determine the optimum L/d ratio (ratio of span length to total super-structure depth) resulting in the most economical cross-section. This showed that the optimum L/d ratio was equal to 20, which corresponds to a web depth of 42 in. Using a web depth of 42 in., the web thickness was then selected to satisfy AASHTO (2004) shear requirements without the need for intermediate transverse stiffeners. This resulted in a web thickness of $1 / 2$ in. at the pier and $7 / 16$ in. at the abutments.

Preliminary analysis also showed that the design of positive bending section was highly controlled by fatigue requirements. In fact, the performance ratio (applied force divided by allowable force) for the fatigue limit state is $40 \%$ to $50 \%$ higher than the performance ratios for the other limit states, where fatigue is governed by the welded connection between the stiffener and bottom flange at the lateral bracing locations $50 \mathrm{ft}$. from each abutment. Thus, in order to reduce the cross-section requirements for the positive bending section to satisfy fatigue requirements, it is assumed that this connection is bolted, which is a Category B fatigue detail with an allowable stress range of $8 \mathrm{ksi}$, as opposed to welded, which results in a allowable stress range of $6 \mathrm{ksi}$.

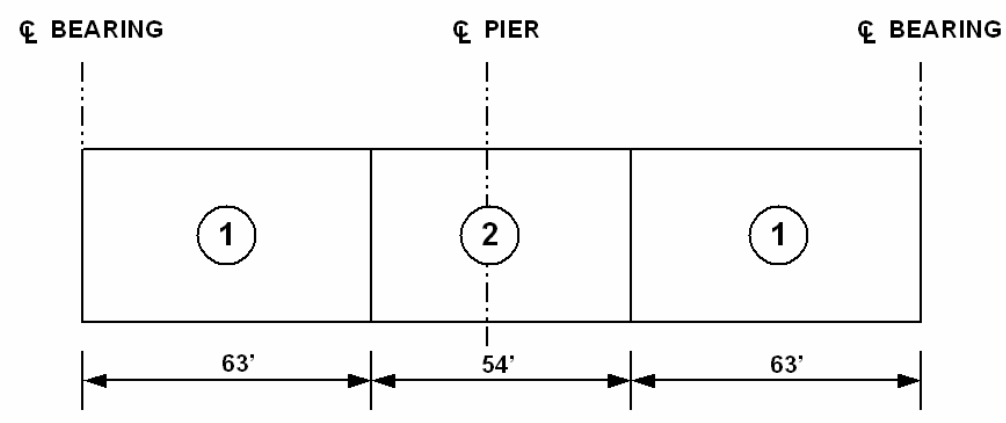

Figure 7-6. Casting Sequence for Design Example 


\subsubsection{Rotation Compatibility}

The girder design using the rotation compatibility procedure begins with selection of a trial lateral bracing layout, where three lateral bracing segments of $25 \mathrm{ft}$. followed by a $15 \mathrm{ft}$. lateral bracing segment at the pier are initially assumed. The girder geometry in the positive bending section is then determined. Constructibility requirements dictate the geometry of the top flange; based on a lateral bracing distance of $25 \mathrm{ft}$., a 16 in. by 3/4 in. top flange is selected. As previously stated this positive bending section design is controlled by fatigue requirements; by utilization of a bolted stiffener to bottom flange connection, fatigue is then governed by the stress at the termination of the web to stiffener weld, which is assumed to terminate at a distance equal to 5 times the web thickness above the bottom flange. Given the previously stated web and top flange dimensions, a 16 in. by $1-1 / 4 \mathrm{in}$. bottom flange is required to satisfy the fatigue limit state.

For the design of the negative bending section, it is initially assumed that the geometry of the negative bending cross-section is the same as the positive bending section. Evaluation of the pier section at the strength limit state, which typically controls the negative bending section design, is carried out using Eq. 7.43,

$$
\mathrm{M}_{\mathrm{e}}\left(1-\theta_{\mathrm{RL}} / \mathrm{C}\right) \leq \mathrm{M}_{\mathrm{n}}
$$

where

$$
\begin{aligned}
& \mathrm{M}_{\mathrm{n}}=4035 \mathrm{ft}-\mathrm{kips}, \\
& \theta_{\mathrm{RL}}=27.65 \text { mrads using Eq. 7.2, } \\
& \mathrm{C}=80, \text { and } \\
& \mathrm{M}_{\mathrm{e}}=5353 \mathrm{ft}-\mathrm{kips} .
\end{aligned}
$$

Substituting the above quantities into Eq. 7.41 gives,

$$
5353(1-27.65 / 80)=3502<4035,
$$

and thus the strength requirements at the pier are satisfied with a performance ratio of 3502/ $4035=0.86$.

The negative bending section is then evaluated for strength 15' from the pier (moment

redistribution is only permitted within one lateral bracing segment), service, fatigue, and constructibility requirements. These design checks are performed using the same processes 
and design equations that would be utilized in an elastic analysis and result in performance ratios equal to $0.50,0.92,0.66$, and 0.77 , respectively. Thus, the assumed geometry of the negative bending section results in an acceptable design using a uniform flange dimension.

It must then be verified that the positive bending section has adequate capacity to resist the elastic positive bending moments in addition to the redistribution moments at the strength limit state. Using Eq. 7.43 to evaluate this requirement at $36 \mathrm{ft}$ from the abutment (the critical positive bending location) gives

$$
\mathrm{M}_{\mathrm{n}}=6198 \mathrm{ft}-\mathrm{kips}>4169 \mathrm{ft}-\mathrm{kips}+\mathrm{M}_{\mathrm{rdL}}+(36 / 90)\left(\mathrm{M}_{\mathrm{rdR}}-\mathrm{M}_{\mathrm{rdL}}\right) \text {, }
$$

where:

$$
\begin{aligned}
& \mathrm{M}_{\mathrm{rdL}}=0 \text { (abutment) and } \\
& \mathrm{M}_{\mathrm{rdR}}=\mathrm{M}_{\mathrm{e}}-\mathrm{M}_{\mathrm{n}}=5353-4035=1318 \mathrm{ft} \text {-kips. }
\end{aligned}
$$

Substitition of these values into Eq. 7.43 then gives

$$
\mathrm{M}_{\mathrm{n}}=6198>4169+0+(36 / 90)(1318-0)=4696,
$$

resulting in a performance ratio of 0.76 (4696/6198) at the strength limit state and indicating that the positive bending section has sufficient reserve capacity to resist redistribution moments. The performance ratios at the service (0.70), fatigue (1.00), and constructibility (0.78) limit states also indicate that the positive bending section design is adequate.

The high performance ratio (1.00) of the positive bending section and the desire to have a negative bending section that is not smaller than the positive bending section indicates that the girder geometry has been optimized for the assumed lateral bracing configuration. However, the use of a longer lateral bracing distance at the piers will translate into a shorter lateral bracing distance in positive bending sections and may allow for the use of a smaller top flange. The maximum allowable bracing distance at the pier for the present girder crosssection may be computed by first solving for the value of $\theta_{\mathrm{RL}}$ that will result in the left and right sides of Eq. 7.41 being equal

$$
\mathrm{M}_{\mathrm{e}}\left(1-\theta_{\mathrm{RL}} / \mathrm{C}\right) \leq \mathrm{M}_{\mathrm{n}}
$$

Rearranging Eq. 7.41 gives

$$
\theta_{\mathrm{RL}} \geq \mathrm{C}\left(1-\mathrm{M}_{\mathrm{n}} / \mathrm{M}_{\mathrm{e}}\right)
$$


and substituting the applicable values for the present design situation results in

$$
\theta_{\mathrm{RL}} \geq 80(1-4035 / 5353)=19.7 \text { mrads. }
$$

Equation 7.2 can then be used to solve for the maximum allowable brace spacing that will give $\theta_{\mathrm{RL}}$ equal to 19.7 mrads,

$$
\begin{aligned}
& 19.7 \geq 0.128-0.143 \frac{16}{1.25} \sqrt{\frac{50}{29000}}-0.0432 \frac{22.16}{16} \\
& +0.0482 \frac{16}{1.25} \frac{22.16}{16} \sqrt{\frac{50}{29000}}-0.0005\left(\frac{\mathrm{L}_{\mathrm{b}}}{4.253}-30\right)^{\prime}
\end{aligned}
$$

resulting in $\mathrm{L}_{\mathrm{b}} \leq 16.3 \mathrm{ft}$. Thus the lateral bracing distance in the negative bending region cannot be appreciably altered and it is concluded that the optimal design has been obtained.

\subsubsection{AASHTO 2004 - Standard Method}

The same girder design is now evaluated using the AASHTO (2004) moment redistribution procedures, beginning with the evaluation of the negative bending region. This begins with computing $\mathrm{M}_{\mathrm{pe}}$ using the AASHTO (2004) procedures, where

$$
\mathrm{M}_{\mathrm{pe}}=\left[2.63-2.3 \frac{\mathrm{b}_{\mathrm{fc}}}{\mathrm{t}_{\mathrm{fc}}} \sqrt{\frac{F_{\mathrm{yc}}}{\mathrm{E}}}-0.35 \frac{\mathrm{D}}{\mathrm{b}_{\mathrm{fc}}}+0.39 \frac{\mathrm{b}_{\mathrm{fc}}}{\mathrm{t}_{\mathrm{fc}}} \frac{\mathrm{D}}{\mathrm{b}_{\mathrm{fc}}} \sqrt{\frac{\mathrm{F}_{\mathrm{yc}}}{\mathrm{E}}}\right] \mathrm{M}_{\mathrm{n}} \leq \mathrm{M}_{\mathrm{n}} .
$$

Substitution of the girder properties gives

$$
M_{p e}=[1.033] M_{n} \leq M_{n},
$$

or,

$$
\mathrm{M}_{\mathrm{pe}}=\mathrm{M}_{\mathrm{n}}=4035 \mathrm{ft}-\text { kips } .
$$

AASHTO (2004) then requires that the redistribution moment must not be greater than $20 \%$ of the elastic moment at the pier. The redistribution moment is equal to

$$
\mathrm{M}_{\mathrm{rd}}=\mathrm{M}_{\mathrm{e}}-\mathrm{M}_{\mathrm{pe}}=5353-4035=1318 \mathrm{ft} \text {-kips, }
$$

which represents $25 \%$ of the elastic moment. Thus, the negative bending section design obtained using the rotation compatibility procedures violates AASHTO (2004) requirements.

The thickness of the top flange of the negative bending section is consequently increased to 1 in. This gives 


$$
\mathrm{M}_{\mathrm{pe}}=[1.033] \mathrm{M}_{\mathrm{n}} \leq \mathrm{M}_{\mathrm{n}}
$$

or,

$$
\mathrm{M}_{\mathrm{pe}}=\mathrm{M}_{\mathrm{n}}=4424 \mathrm{ft}-\text { kips . }
$$

The redistribution moment is then equal to

$$
\mathrm{M}_{\mathrm{rd}}=\mathrm{M}_{\mathrm{e}}-\mathrm{M}_{\mathrm{pe}}=5362-4424=938 \mathrm{ft} \text {-kips, }
$$

This value of $\mathrm{M}_{\mathrm{rd}}$ represents $17 \%$ of the elastic moment, and in AASHTO 2004 this is the only strength requirement at the pier section. The negative bending section is also satisfactory for strength 15 ' from the pier, service, fatigue, and constructibility requirements, resulting in performance ratios equal to $0.46,0.90,0.66$, and 0.66 , respectively. The positive bending section performance ratios also indicate an acceptable design with 0.73 for strength, 0.70 for service, 1.00 for fatigue, and 0.77 for constructibility.

\subsubsection{AASHTO 2004 - Refined Method}

Because the maximum value of $M_{p e}$ is equal to $M_{n}$ and the maximum magnitude of the redistribution moment is limited to $0.2 \mathrm{M}_{\mathrm{e}}$ in the AASHTO (2004) procedures, the smallest cross-section that may satisfy the moment redistribution procedures is that presented above for the standard method of moment redistribution design. This design is also evaluated using the refined methods given in the AASHTO (2004) procedures, which indicates that the design is also acceptable using these procedures. Furthermore, identical performance ratios result whether the refined or standard methods of analysis are used as the effective plastic moment capacity is the same using both procedures.

\subsubsection{Comparision of Rotation Compatibility and AASHTO 2004 Designs}

Figure 7-7 illustrates the alternative designs resulting from the rotation compatibility procedures and the AASHTO (2004) moment redistribution specifications. Here it is shown that the only difference between the two designs is that the rotation compatibility procedures result in a top flange that is $1 / 4$ in. thinner than that required by the moment redistribution procedures indicating that the use of the rotation compatibility procedure results in a reasonable girder design with increased economy compared to the AASHTO (2004) procedures. 

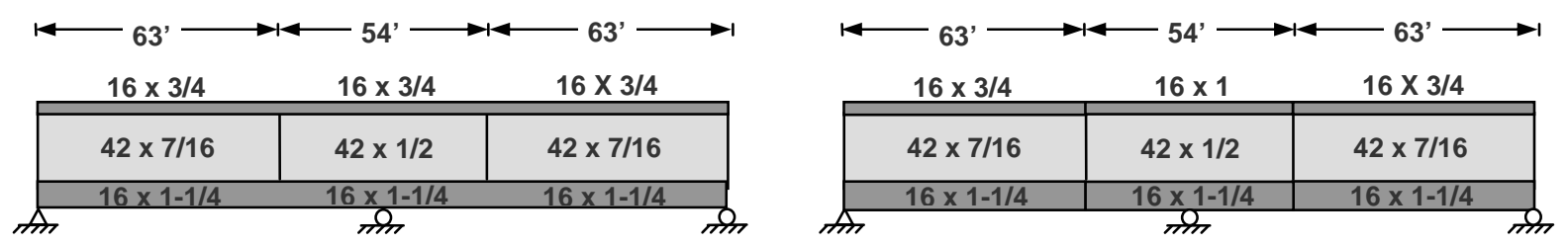

Figure 7-7. Rotation Compatibility (left) and Moment Redistribution (right) Designs

The change to a thinner top flange allows for the use of uniform flange dimensions. This results in the ability to eliminate either costly full-penetration groove welds of field splices that would otherwise be required at the flange transition locations at one or more locations in the girder. Furthermore, not only does the smaller flange result in reduced steel weight and steel cost, but a fewer number of plate thicknesses are required for the girder fabrication, resulting in the need for stocking less steel inventory and less scrap steel.

Table 7-1 compares the performance ratios resulting from the two designs. This shows that the two designs result in nearly equal performance ratios. The most significant difference between the performance ratios for the two designs results for the strength limit state in the positive bending section. This is due to the larger redistribution moment applied in the rotation compatibility design, which is made possible by explicitly computing the maximum allowable level of redistribution as opposed to uniformly limiting the redistribution moment to $20 \%$ of the elastic moment as specified in AASHTO (2004). However, even with the

Table 7-1. Comparison of Performance Ratios from Rotation Compatibility and Moment Redistribution Designs

\begin{tabular}{|c|l|c|c|}
\cline { 3 - 4 } \multicolumn{2}{c|}{} & Rotation Compatibility & $\begin{array}{c}\text { AAHSTO (2004) } \\
\text { Moment Redistribution }\end{array}$ \\
\hline \multirow{4}{*}{ Negative Bending } & Strength (@ 15' from pier) & 0.50 & 0.46 \\
\cline { 2 - 4 } & Service & 0.92 & 0.90 \\
\cline { 2 - 4 } & Fatigue & 0.66 & 0.66 \\
\cline { 2 - 4 } & Constructibility & 0.77 & 0.66 \\
\hline \multirow{4}{*}{ Positive Bending } & Strength & 0.76 & 0.73 \\
\cline { 2 - 4 } & Service & 0.70 & 0.70 \\
\cline { 2 - 4 } & Fatigue & 1.00 & 1.00 \\
\cline { 2 - 4 } & Constructibility & 0.78 & 0.77 \\
\hline
\end{tabular}


higher redistribution moments applied in the rotation compatibility design, the reserve strength of the positive bending section at the strength limit state is significant.

The other primary difference between the rotation compatibility and AASHTO (2004) procedures is that longer lateral bracing distances are permitted at interior piers in the rotation compatibility procedures. While this only allowed for a small increase in the present example, it is recommended that the procedure shown above to determine the maximum allowable bracing distance be implemented in design practices.

\subsubsection{Rating Example}

This section illustrates the use of the rotation compatibility procedures for rating applications. In this example, it is assumed that the subject bridge was initially designed using elastic provisions and has since undergone corrosion at the pier location. Specifically, it is assumed that the bottom flange of the pier section has $3 / 8$ in. of section loss. The subject bridge for this example is comprised of the same bridge cross-section used in the previous example (illustrated in Fig. 7-5) and two continuous spans $120 \mathrm{ft}$ in length. An elevation of the original girder (prior to corrosion) is shown below in Fig. 7-8. Lateral bracing is provided at four intervals of $25 \mathrm{ft}$. and one interval of $20 \mathrm{ft}$. at the pier. This girder satisfies all applicable limit states (prior to corrosion) when designed using the same loads and design assumptions used in the design of the examples presented in the previous sections.

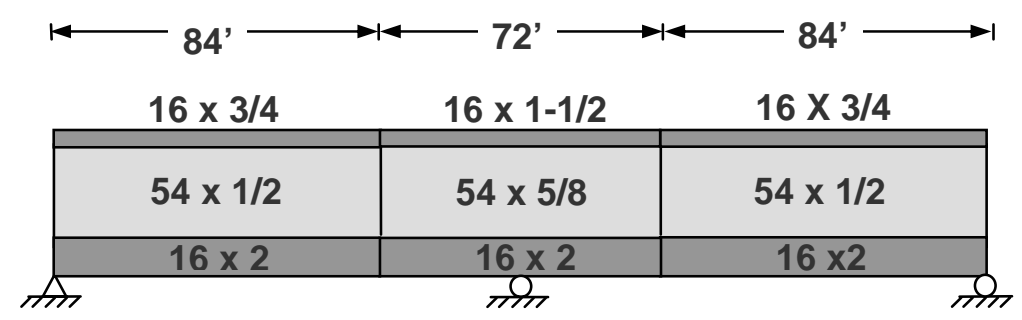

Figure 7-8. Girder Elevation Rating Example 
Equation 7.57 is first used to perform an elastic rating for moment capacity of the pier section,

$$
\mathrm{RF}=\frac{\mathrm{C}-\gamma_{\mathrm{DC}}(\mathrm{DC})-\gamma_{\mathrm{DW}}(\mathrm{DW}) \pm \gamma_{\mathrm{P}}(\mathrm{P})}{\gamma_{\mathrm{L}}(\mathrm{LL}+\mathrm{IM})} .
$$

In order to determine if posting or strengthening of the bridge is required, the rating procedure is performed at the legal load level. Comparing the moments induced at the pier by the various legal live loads, it is determined that the legal lane loading controls and the distributed live load moment (including impact on axle loads only) is equal to $1571 \mathrm{ft}$-kips. Similarly, the moments at the pier from dead loads and wearing surface loads are $3033 \mathrm{ft}$ kips and $396 \mathrm{ft}$-kips, respectively. Assuming an ADTT greater than 5000, the applicable load factors are $\gamma_{\mathrm{DC}}=1.25, \gamma_{\mathrm{DW}}=1.50$, and $\gamma_{\mathrm{L}}=1.80$. The girder capacity is computed using Eq. 7.72,

$$
\mathrm{C}=\varphi_{\mathrm{c}} \varphi_{\mathrm{s}} \varphi \mathrm{R}_{\mathrm{n}}
$$

where,

$$
\begin{aligned}
\varphi_{\mathrm{c}}= & 0.85 \text { (for members in poor condition, which is appropriate for the given level } \\
& \text { of section loss), } \\
\varphi_{\mathrm{s}}= & 1.00 \\
\varphi= & 1.00 \\
\mathrm{R}_{\mathrm{n}}= & M_{\mathrm{n}}=8441
\end{aligned}
$$

resulting in

$$
\mathrm{C}=(0.85)(1.00)(1.00)(8441)=7175 \mathrm{ft}-\mathrm{kips} .
$$

Substituting the above values into Eq. 7.57 gives

$$
\mathrm{RF}=\frac{7175-1.25(3033)-1.5(396) \pm \gamma_{\mathrm{P}}(0)}{1.8(1571)}=0.99<1,
$$

indicating that the pier section is not adequate to resist the applied moments.

To perform the same rating using the rotation compatibility procedures, the procedure is nearly identical. The only change is the use of the moment redistribution load factors

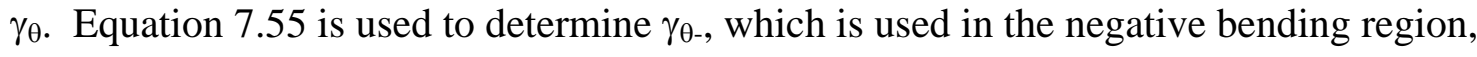

$$
\gamma_{\theta-}=1-\theta_{\mathrm{RL}} / \mathrm{C} \text {. }
$$


Using $\theta_{\mathrm{RL}}$ equal to 9.73 and $\mathrm{C}$ equal to 80 gives

$$
\gamma_{\theta-}=1-9.73 / 80=0.878 \text {. }
$$

Equation 7.58 is then used to compute the inelastic rating factor at the pier

$$
\mathrm{RF}=\frac{\mathrm{C}-\gamma_{\theta}\left[\gamma_{\mathrm{DC}}(\mathrm{DC})+\gamma_{\mathrm{DW}}(\mathrm{DW}) \pm \gamma_{\mathrm{P}}(\mathrm{P})\right]}{\gamma_{\theta} \gamma_{\mathrm{L}}(\mathrm{LL}+\mathrm{IM})},
$$

which gives a rating factor of

$$
\mathrm{RF}=\frac{7175-0.878\left[1.25(3033)+1.5(396) \pm \gamma_{\mathrm{P}}(0)\right]}{0.878(1.8)(1571)}=1.34>1 .
$$

indicating that the pier section is adequate to resist the legal loads and that posting or strengthening of the bridge is not required.

Equations 7.39, 7.56, and 7.58 are then used to rate the positive bending section for moment capacity. First Eq. 7.39 is used to determine the redistribution moment ratio,

$$
\mathrm{M}_{\mathrm{rd}} / \mathrm{M}_{\mathrm{e}}=\theta_{\mathrm{RL}} / \mathrm{C}=9.73 / 80=0.1216 .
$$

Equation 7.56 is next used to determine the value of $\gamma_{\theta}$,

$$
\gamma_{\theta}=1+\left(\frac{M_{r d}}{M_{e}}\right)_{L}+\frac{x}{L}\left[\left(\frac{M_{r d}}{M_{e}}\right)_{R}-\left(\frac{M_{r d}}{M_{e}}\right)_{L}\right],
$$

where,

$$
\begin{array}{ll}
\left(\mathrm{M}_{\mathrm{rd}} / \mathrm{M}_{\mathrm{e}}\right)_{\mathrm{L}} & =0, \\
\left(\mathrm{M}_{\mathrm{rd}} / \mathrm{M}_{\mathrm{e}}\right)_{\mathrm{R}} & =0.1216 \text { (from Eq. 7.78), } \\
\mathrm{x} & =48 \mathrm{ft} . \text { (the critical positive bending location), and } \\
\mathrm{L} & =120 \mathrm{ft},
\end{array}
$$

which results in

$$
\gamma_{\theta}=1+0+\frac{48}{120}[0.1216-0]=1.049
$$

Lastly, using Eq. 7.58 the rating factor moment in the positive bending section is determined to be

$$
\mathrm{RF}=\frac{0.85(9473)-1.25(1.049)(1611)-1.5(1.049)(210) \pm(0)}{1.8(1.049)(1558)}=1.91>1 \text {. }
$$

Thus it is shown that this bridge rates successfully for legal loads. 
In summary, this rating example has illustrated a rating situation where the current rating procedures result in a rating factor less than one at the legal load level, suggesting that the bridge should be posted or strengthened. However, by simply using the moment redistribution load factors, $\gamma_{\theta_{-}}$and $\gamma_{\theta_{+}}$, the bridge rates successfully.

\subsection{Summary}

This chapter has demonstrated the development of the rotation compatibility procedure, which was formulated through comparison of the relationships for required and available rotations developed in previous chapters. These procedures were then further developed into design and rating specifications and illustrative examples were presented demonstrating the use of these specifications. The design and rating examples demonstrate that use of the rotation compatibility procedures is convenient and results in rational economic savings. 


\section{Chapter 8: SUMMARY, CONCLUSIONS, \& RECOMMENDATIONS}

\subsection{Summary}

This work has resulted in the development of moment redistribution specifications that represent a significant improvement over previous inelastic specifications in that they are valid for an increased range of cross-sections and are based on an explicit evaluation of girder ductility. Additionally, the procedures developed herein are convenient for use with either design or rating of continuous-span I-girders and may be conveniently incorporated into AASHTO specifications.

Introductory material, including general information on moment redistribution procedures, was discussed in Chapter 1. Chapter 2 presented a detailed literature review of topics related to this research. Components of this work included a description of previous moment redistribution specifications, a summary of the current rating specifications and previous work on inelastic rating of steel bridges, a review of previous studies on the available rotation of steel I-girders, and details of previous studies on rotation requirements.

The experimental testing conducted in this work was presented in Chapter 3. Here detailed information was given on the test girder properties, testing configurations, and experimental procedures. Results of the experimental testing were also detailed. The most significant conclusion resulting from this work was the decrease in girder ductility that occurs with increasing lateral bracing distance.

Chapter 4 presented the FEA techniques used in this work. Element selection, mesh density considerations, material modeling issues, incorporation of residual stresses, modeling of geometric imperfections, and the solution algorithm implemented were detailed.

Results of the FEA of experimental and hypothetical girders performed in this research were described in Chapter 5. This chapter first presented FEA of the experimental girders discussed in Chapter 3, which illustrated that the modeling procedures described in Chapter 4 
were accurate for predicting the behavior of steel I-girders of the type used in this work. Chapter 5 also presented the FEA of three series of hypothetical girders: a series of parametric designs having large slenderness ratios, a series of girders obtained from a design study of typical moment redistribution designs, and an extended series of design study girders with large flange slenderness values and unbraced lengths. This work along with the experimental results was used to develop moment-rotation models to predict the available rotations of any (straight, non-skewed) steel I-girder.

A study on the rotation requirements was presented in Chapter 6. This included a description of the analysis method used in this work and a discussion of the relationship of this method to those used in previous studies. In order to determine rotation requirements, it was necessary to obtain representative section properties of moment redistribution girders. Thus, a series of representative girders were designed. This chapter concluded with a detailed discussion of rotation requirements for moment redistribution, including analysis of factors influencing these requirements and the development of rotation requirement prediction equations.

The available rotation relationships presented in Chapter 5 are then used with the required rotation relationships introduced in Chapter 6 to develop the rotation compatibility approach to moment redistribution. The derivation of the rotation compatibility procedures were based on examining the conditions necessary to assure that the available rotation of a member is greater than that required for moment redistribution and were presented in Chapter 7 . This chapter also presented methods for conveniently incorporating these procedures into design and rating specifications and presented examples of use of the suggested rotation compatibility specifications. These examples illustrated that use of these procedures is convenient and results in a rational degree of economic savings.

\subsection{Conclusions}

The current and previous AASHTO moment redistribution specifications have aimed to assure that adequate ductility is provided by limiting the applicability of these specifications to a restricted class of cross-sections and requiring that rather stringent lateral bracing limits 
be satisfied. Alternatively, by using the explicit evaluation of girder ductility inherent to the rotation compatibility procedures suggested herein, it is no longer necessary to limit moment redistribution procedures to the relatively compact members previous specifications have required.

In order to extend the moment redistribution procedures to a wider class of steel I-girders, a primary component of this research was the development of a moment-rotation model that accurately represents the behavior of more slender members. Specifically, of interest was to investigate increased lateral bracing distances and compression flange slenderness ratios compared to those permitted in previous moment redistribution specifications as these are the two areas where past methods are most restrictive. For this purpose, the experimental testing described in Chapter 3 and the FEA modeling presented in Chapters 4 and 5 were completed. The result of these efforts was the moment rotation model shown below in Fig. 8-1. The key parameter in this model is the value of $\theta_{\mathrm{RL}}$, which represents the moment at which the moment capacity drops below $\mathrm{M}_{\mathrm{n}}$ and is equal to

$$
\theta_{\mathrm{RL}}=128-143 \frac{\mathrm{b}_{\mathrm{fc}}}{\mathrm{t}_{\mathrm{fc}}} \sqrt{\frac{\mathrm{F}_{\mathrm{yc}}}{\mathrm{E}}}-21.6 \frac{2 \mathrm{D}_{\mathrm{cp}}}{\mathrm{b}_{\mathrm{fc}}}+24.1 \frac{\mathrm{b}_{\mathrm{fc}}}{\mathrm{t}_{\mathrm{fc}}} \frac{2 \mathrm{D}_{\mathrm{cp}}}{\mathrm{b}_{\mathrm{fc}}} \sqrt{\frac{\mathrm{F}_{\mathrm{yc}}}{\mathrm{E}}}-\max \left[0,0.5\left(\frac{\mathrm{L}_{\mathrm{b}}}{\mathrm{r}_{\mathrm{t}}}-30\right)\right] \geq 5 .
$$

Equation 8.1 is similar to the $\theta_{\mathrm{RL}}$ equation given in the AASHTO (2004) specifications, but differs in two respects. First Eq. 8.1 contains the quantity $2 \mathrm{D}_{\mathrm{cp}}$, which is used in place of D

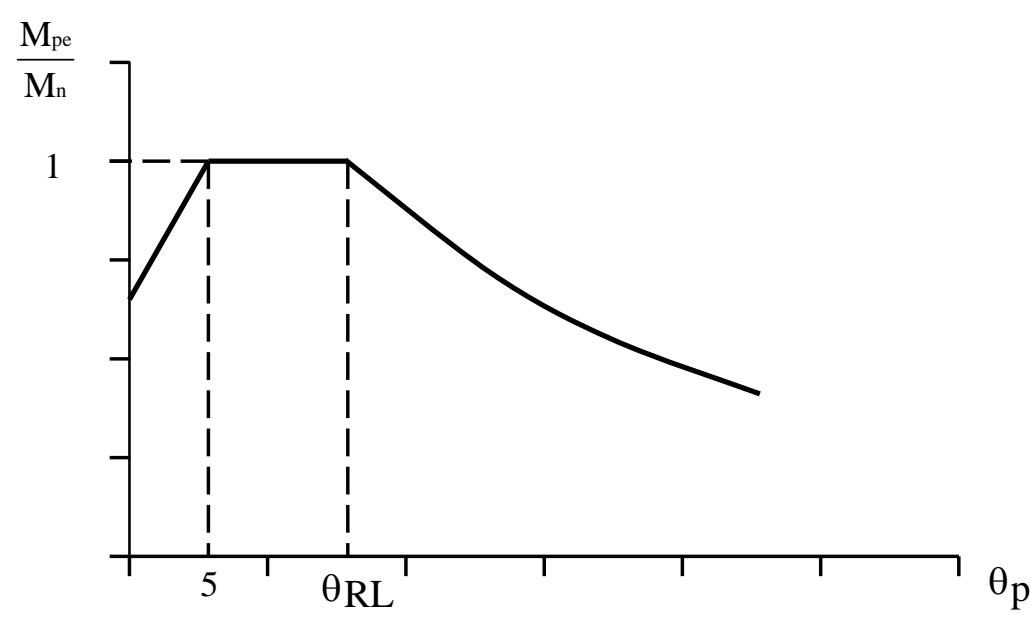

Figure 8-1. Moment Rotation Model 
in the AASHTO $\theta_{\mathrm{RL}}$ equation as it was found herein that improved accuracy results with this modification. Second, the last term in Eq. 8-1 is added to incorporate the decreased ductility that results with increasing lateral bracing distances. For lateral bracing distances that are relatively compact, this last term equals zero, thus giving $\theta_{\mathrm{RL}}$ values that are similar to those resulting from the AASHTO (2004) $\theta_{\mathrm{RL}}$ equation. However, as the compact bracing distance is exceeded, linearly decreasing $\theta_{\mathrm{RL}}$ values result.

The rotation compatibility approach was then founded on comparison of the ductility of steel girders, as represented by the model shown in Fig. 8-1, to the rotations required for moment redistribution. Thus, a second primary component of this work was to determine the rotation requirements for moment redistribution to occur, which was presented in Chapter 6. Here it was concluded that three parameters are needed to accurately predict the plastic rotations required for moment redistribution. First, it is decided that the rotation requirements are accurately represented as a linear function of the ratio between the redistribution moment and the elastic moment of the girder. Additionally, it was found that the material properties of the girder are also influential to the rotation requirements. Lastly, due to the increased rotation requirements that result when redistribution moments are applied to multiple piers, consideration was also given to the number of piers where moment redistribution occurs. The resulting required plastic rotation, $\theta_{\mathrm{pR}}$, is given by Eq. 8.2.

$$
\theta_{\mathrm{pR}}=\mathrm{C}\left(\mathrm{M}_{\mathrm{rd}} / \mathrm{M}_{\mathrm{e}}\right)
$$

where:

- For homogeneous pier sections:

$$
C=80+20 n \text {, and }
$$

- For hybrid pier sections between two end spans:

$$
\mathrm{C}=90+22.5 n
$$

and $\mathrm{n}$ represents the number of adjacent piers where moment redistribution occurs.

Chapter 7 then compared the rotation requirements given by Eq. 8.2 and the available rotations given by the model shown in Fig. 8.1. By requiring that the available rotations must be greater than the required rotations, equations for inelastic design and rating were 
developed. In summary, the rotation compatibility design equations reduce the elastic moment of the girder at the locations of peak negative moment according to Eq. 8.5

$$
\mathrm{M}_{\mathrm{e}}\left(1-\theta_{\mathrm{RL}} / \mathrm{C}\right) \leq \mathrm{M}_{\mathrm{n}},
$$

where $\theta_{\mathrm{RL}}$ is given by Eq. 8.1 and $\mathrm{C}$ is given by Eq. 8.3 or 8.4 . The rotation compatibility procedures are incorporated in the rating specifications through the use of the moment redistribution load factor $\gamma_{\theta}$, as shown by Eq. 8.6,

$$
\mathrm{RF}=\frac{\mathrm{C}-\gamma_{\theta}\left[\gamma_{\mathrm{DC}}(\mathrm{DC})+\gamma_{\mathrm{DW}}(\mathrm{DW}) \pm \gamma_{\mathrm{P}}(\mathrm{P})\right]}{\gamma_{\theta} \gamma_{\mathrm{L}}(\mathrm{LL}+\mathrm{IM})} .
$$

The moment redistribution load factor acts to decrease the applied negative moments and is given by the equation

$$
\gamma_{\theta-}=1-\theta_{\mathrm{RL}} / \mathrm{C} .
$$

Conversely, $\gamma_{\theta}$ increases the applied positive moments and is expressed as

$$
\gamma_{\theta+}=1+\left(\frac{M_{r d}}{M_{e}}\right)_{L}+\frac{x}{L}\left[\left(\frac{M_{r d}}{M_{e}}\right)_{R}-\left(\frac{M_{r d}}{M_{e}}\right)_{L}\right]
$$

where $M_{r d} / M_{e}=\theta_{R L} / C$, the subscripts "L" and "R" refer to the piers at the left and right ends of the span under consideration, $\mathrm{x}$ represents the distance from the left pier where the rating analysis is being performed, and $\mathrm{L}$ represents the span length of the girder.

Through a direct evaluation of girder ductility, the rotation compatibility procedures developed herein provide the needed evidence that moment redistribution is permissible for girders more slender than those incorporated in previous moment redistribution provisions. Specifically, the rotation compatibility procedures can be used with any steel I-girder that satisfies the AASHTO requirements for general I-girders. Additionally, the rotation compatibility procedures directly compute the maximum allowable levels of moment redistribution (equal to $\theta_{\mathrm{RL}} / \mathrm{C}$ ) based on the girder characteristics. This is also a notable improvement over past moment redistribution specifications where either: (1) there were no limitations on the maximum allowable level of redistribution (e.g., ALFD 1986) or (2) the maximum amount of redistribution was limited to an assumed quantity (e.g., AASHTO 2004). 


\subsection{Recommendations for Future Research}

The rotation requirements developed in this work are based on conservative assumptions regarding the extent of concrete cracking in negative bending regions. It is possible that this conservativism could be reduced if experimental data were available to better quantify the concrete behavior as moment redistribution occurs. Thus, obtaining such data would be a beneficial supplement to the existing work.

The rotation compatibility procedure presented herein is applicable to either the strength or service limit state. However, service related issues of moment redistribution, such as the resulting deflections under repeated occurrences of moment redistribution, investigation of levels of concrete cracking, and quantifying appropriate limits to control this cracking, should be investigated. Presently there is no maximum level of redistribution suggested for the rotation compatibility procedures, but future research may suggest such a limit is rational. With respect to the study of deflections due to the use of the suggested moment redistribution procedures, it is suggested that the concepts used in conjunction with the residual deformation analysis method discussed in Chapter 2 is appropriate for this purpose.

The present work is focused on moment redistribution for steel I-girders, which are currently the most popular configuration of steel bridges. Because of the ductility offered by all steel members, it is suggested that these procedures could be extended to other girder configurations. Specifically, as the number of steel box girder bridges that are constructed continues to increase, future efforts focusing on extending moment redistribution specifications to these member types may be of benefit.

Also, the current work is focused on a girder approach to moment redistribution, i.e, only the redistribution of moments along the length of the girder is considered. Future work incorporating the additional transverse redistribution of moments that occurs as one overstressed girder redistributes moment to lesser stressed girders is also likely to have positive economic impacts. Such a system approach to inelastic design has been studied by Galambos et al (1993) and one suggested method of formulating this system approach to moment redistribution would be through the use of inelastic distribution factors, which would 
account for transverse moment redistribution through lower distribution factors. Cheung et al (1986) provide guidelines for such distribution factors that may be of benefit to future researchers investigating this topic.

The experimental testing conducted in this work suggests that improvements to the current AASHTO moment capacity equations are possible. It is suggested that these specifications may be unconservative for girders where failure is due to a combination of buckling modes and future research is recommended to better evaluate the influences of such combined modes of buckling. Furthermore, the moment capacities predicted for the experimental girders in this work that had nominal yield strengths higher of $70 \mathrm{ksi}$ were typically unconservative and this data suggests that there is the potential for improving upon the moment capacity prediction equations for these types of girders.

AASHTO Specifications do not currently include requirements for lateral bracing members. Instead designers often adopt AISC Specifications for guidance in determining the required stiffness of these members. Future research determining lateral bracing requirements for bridges, with a specific focus on the additional lateral bracing demands that may be required for moment redistribution designs, is suggested. 


\section{REFERENCES}

AASHTO (1973). Standard Specifications for Highway Bridges, $11^{\text {th }}$ ed., AASHTO, Washington, D. C.

AASHTO (1986). Guide Specifications for Alternate Load Factor Design Procedures for Steel Beam Bridges Using Braced Compact Sections, AASHTO, Washington, D. C.

AASHTO (1994). LRFD Bridge Design Specifications, $1^{\text {st }}$ ed., Washington, D. C.

AASHTO (1998). LRFD Bridge Design Specifications, $2^{\text {nd }}$ ed., Washington, D. C.

AASHTO (2003). Guide Manual for Condition Evaluation and Load and Resistance Factor Rating (LRFR) of Highway Bridges, $1^{\text {st }}$ ed., AASHTO, Washington, D. C., 2003.

AASHTO (2004). LRFD Bridge Design Specifications, $3^{\text {rd }}$ ed., Washington, D. C.

ABAQUS (2002). ABAQUS/Standard User's Manual, version 6.3, Hibbitt, Karlsson \& Sorensen, Inc.

AISC (1973). Specification for the Design, Fabrication and Erection of Structural Steel for Buildings, AISC.

ASCE (1971). Plastic Design in Steel, ASCE, New York, NY.

AWS (1995). Bridge Welding Code, ANSI/AASHTO/AWS D1.5-95.

Adams, P. F., M. G. Lay, T. V. Galambos. (1964). “Experiments on High Strength Steel Members”, Fritz Engineering Laboratory Report No. 297.8, Fritz Engineering Laboratory, Lehigh University, Bethlehem, PA. 
Alpsten, G. A. (1972). "Variation in Mechanical and Cross-Sectional Properties of Steel”, International Conference of Planning and Design of Tall Buildings, Lehigh University, ASCE-IABSE International Conference Preprints: Report 1b-9, 1-51.

Barker, M. G. and J. A. Zacher. (1997). "Reliability of Inelastic Capacity Rating Limits for Steel Bridges”, Journal of Bridge Engineering, 2(2), 45-52.

Barker, M. G., B. A. Hartnagel, C. G. Schilling, and B. E. Dishongh. (2000). "Simplified Inelastic Design of Steel Girder Bridges”, ASCE Journal of Bridge Engineering, 5(1), 58-66.

Barth, K. E. (1996). "Moment-rotation characteristics for inelastic design of steel bridge beams and girders”, Dissertation, Purdue University, West Lafayette, Ind.

Barth, K. E., B. A. Hartnagel, D. W. White, and M. G. Barker. (2004). "Recommended Procedures for Simplified Inelastic Design of Steel I Girder Bridges”, ASCE Journal of Bridge Engineering, 9, 230.

Barth, K. E. and D. W. White. (1998). "Finite Element Evaluation of Moment-Rotation Characteristics”, Engineering Structures, 20(8), 761-778.

Barth, K. E. and D. W. White. (2000). "Inelastic Design of Steel I-Girder Bridges”, Journal of Bridge Engineering, 5(3), 179-190.

Basler, K. (1959). “Strength of Plate Girders”, Dissertation, Lehigh University, Bethlehem, PA.

Bazant, Z. P. and L. Cedolin (1980). "Effect of Finite Element Choice in Blunt Crack Band Analysis”, Computer Methods in Applied Mechanics and Engineering, 24(3), 305316. 
Boeraeve, P. H., B. Lognard, J. Janss, J. C. Gerardy, and J. B. Schleich. (1993). "Elastoplastic Behaviour of Steel Frameworks”, Journal of Constructional Steel Research, 27, 3-21.

Carskaddan, P. S. (1980). "Autostress design of highway bridges, Phase 3: Initial moment rotation tests”, Research Laboratory Report, United States Steel Corporation, Monroeville, PA.

Cheung, M. S. Jategaonkar, and Jaeger, L. G. (1986). "Effects of Intermediate Diaphragms in Distributing Live Loads in Beam-and-Slab Bridges”, Canadian Journal of Civil Engineering, 13, 278-292.

Climenhaga, J. J. and Johnson, R. P. (1972). "Local buckling in continuous composite beams”, The Structural Engineer_50(9), 367-374.

Crisfield, M. A. (1983). "An Arc-Length Method Including Line Searches and Accelerations”, International Journal for Numerical Methods in Engineering, 19, 1269-1289.

Dinno, D. K. and P. C. Birkemoe (2001). “The Rotational Capacity of Beams Exceeding Compact Section Slenderness Requirements”, Annual Technical Session, Structural Stability Research Council, Proceedings, p 291-312.

Dishongh, B. E. and T. V. Galambos (1992). "Residual Deformation Analysis for Inelastic Bridge Rating”, Journal of Structural Engineering, 118(6), 1494-1508.

Earls, C. J. and B. J. Shah (2000). "Compactness and Bracing Requirements for Use in the Analysis and Design of High Performance Steel Highway Bridges”, Report No. CE/ST17, Department of Civil and Environmental Engineering, University of Pittsburgh. 
FHWA (1992). FHWA Model Bridge Study, Volume II: Component Test Report, Publication No. FHWA-RD-90-066, , Turner-Fairbank Highway Research Center, McLean, VA.

Galambos, T. V. (1968). "Deformation and Energy Absorption Capacity of Steel Structures in the Inelastic Range”, American Iron and Steel Institute, Bulletin No. 8.

Galambos, T. V., R. T. Leon, C. W. French, M. Barker, B. Dishongh (1993). "Inelastic Rating Procedures for Steel Beam and Girder Bridges”, National Cooperative Highway Research Program, Report 352, Transportation Research Board, National Academy Press, Washington, D. C.

Gioncu, V. and D. Petcu. (1997a). “Available Rotation Capacity of Wide-Flange Beams and Beam-Columns, Part 1: Theoretical Approaches”, Journal of Constructional Steel Research, 45, 161-217.

Gioncu, V. and D. Petcu. (1997b). “Available Rotation Capacity of Wide-Flange Beams and Beam-Columns, Part 2: Experimental and Numerical Tests”, Journal of Constructional Steel Research, 45, 219-244.

Green, P. S., Ricles, J. M., and Sause, R. (2001). "The Inelastic Behavior of High Performance Steel Flexural Members”, Structural Stability Research Council Proceedings, Ft. Lauderdale, FL.

Green, P. S., Sause, R., and Ricles, J.M. (2002). "Strength and Ductility of HPS Flexural Members”, Journal of Constructional Steel Research, 58, 907-941.

Greschik, G., D. W. White, and W. McGuire (1989). "Evaluation of the rotation capacity of wide-flange beams using shell finite elements”, Proceedings Structures Congress, San Francisco, 590-599. 
Grubb, M. A. and P. S. Carskaddan. (1979). “Autostress design of highway bridges, Phase 3: Initial Moment Rotation Tests”, Research Laboratory Report, United States Steel Corporation, Monroeville, PA.

Grubb, M. A., and P. S. Carskaddan (1981). "Autostress design of highway bridges, Phase 3: Initial Moment Rotation Tests”, Research Laboratory Report, United States Steel Corporation, Monroeville, PA.

Haaijer, G., P. S. Carskaddan, and M. A. Grubb. (1980). "Plastic Design with Noncompact Sections Including Composite Bridge Members”, Structural Stability Research Council Annual Meeting.

Hartnagel, B. A. (1997) "Inelastic design and experimental testing of compact and noncompact steel girder bridges," $\mathrm{PhD}$ dissertation, University of MissouriColumbia, Columbia, Mo.

Hartnagel, B. A. and A. V. Tran. (2003). "Pier Moment-Rotation of Compact and Noncompact HPS70W I- Girders”. Published by Department of Transportation, University Centers Program.

Holtz, N. M., and Kulak, G. L. (1973) "Web slenderness limits for compact beams”, Struct. Engr. Rep. No. 43, Dept. of Civ. Engr., University of Alberta, Edmonton, Alberta, Canada.

Holtz, N. M., and Kulak, G. L. (1975) "Web slenderness limits for non-compact beams", Struct. Engr. Rep. No. 51, Dept. of Civ. Engr., University of Alberta, Edmonton, Alberta, Canada.

Hope-Gill, M. C. (1979). "Redistribution in Composite Beams", Structural Engineer, 57B(1), 7-10. 
Huang, P. C. (1994). "Finite Element Analysis of Inelastic Behavior of Structural Steel Beams and Bridge Girders”, Ph.D. Dissertation, Cornell University, Ithaca, N. Y.

Kato, B. (1989) "Rotation Capacity of H-Section Members as Determined by Local Buckling”, Journal of Constructional Steel Research, 13, 95-109.

Kato, B. (1990) “Deformation Capacity of Steel Structures”, Journal of Constructional Steel Research, 17, 33-94.

Kemp, A. R. (1986). "Factors Affecting the Rotation Capacity of Plastically Designed Members”, The Structural Engineer, 64B(2), 28-35.

Kemp, A. R. (1987). “Quantifying Ductility in Continuous Composite Beams”, Engineering Foundation Conference on Composite Construction, Proceedings, New England College, Henniker, NH, 107-121.

Kemp, A. R. (1990). "Quantifying Limit-States of Rotation Capacity in Flexural Members”, Proceedings - Institution of Civil Engineers, Part 2: Research and Theory, Sept. 1990, 387-406.

Kemp, A. R. and N. W. Dekker (1991). “Available rotation capacity in steel and composite beams”, Structural Engineer, 69(5), 88-97.

Lääne, A. and J. Lebet. (2005) "Available Rotation Capacity of Composite Bridge Plate Girders Under Negative Moment and Shear", Journal of Constructional Steel Research, 61, 305-327.

Li, T. Q., B. S. Choo, and D. A. Nethercot. (1995). "Determination of Ratation Capacity Requirements for Steel and Composite Beams”, Journal of Constructional Steel Research, Vol. 32, p. 303-332. 
Lukey, A. F., and P. F. Adams. (1969). "Rotation Capacity of Beams Under MomentGradient”, Journal of the Structural Division, ASCE, 95(ST6), 1173-1188.

Lukey, A. F., R. J. Smith, M. U. Hosain, P. F. Adams. (1969). "Experiments on WideFlange Beams Under Moment Gradient”, WRC Bulletin No. 142.

Neal, B. G. (1963). “The Plastic Methods of Structural Analysis”, $2^{\text {nd }}$ Edition, John Wiley and Sons, New York.

Nethercot, D. A., T. Q. Li, and B. S. Choo (1995). "Required Rotations and Moment Redistribution for composite Frames and Continuous Beams”, Journal of Constructional Steel Research, 35, 121-163.

Prasad, J. and T. V. Galambos (1963). "The Influence of the Adjacent Spans on the Rotation Capacity of Beams”, Fritz Engineering Laboratory Report No. 205H.12, Lehigh University, Bethlehem, PA.

Powell, G. and J. Simons (1981). “Improved Iteration Strategy for Nonlinear Structures”, International Journal for Numerical Methods in Engineering, 17(10), 1455-1467.

Riks, E. (1979). "An Incremental Approach to the Solution of Snapping and Buckling Problems”, International Journal of Solids and Structures, 15, 529-551.

Schilling, C. G. (1985). "Moment-rotation tests of steel bridge girders," Report on Project 188, American Iron and Steel Institute, Washington, D. C.

Schilling, C. G. (1986). "Exploratory Autostress Girder Designs” Report on Project 188, American Iron and Steel Institute, Washington, D. C. 
Schilling, C. G. and S. S. Morcos. (1988). "Moment-rotation tests of steel girders with ultracompact flanges”, Report on Project 188, American Iron and Steel Institute, Washington, D. C.

Schilling, C. G. (1989). “A Unified Autostress Method” Report on Project 51, American Iron and Steel Institute, Washington, D. C.

Schilling, C. G., M. G. Barker, B. E. Dishong, and B. A. Hartnagel. (1997). "Inelastic Design Procedures and Specifications”, Final Report submitted to American Iron and Steel Institute.

Southward, R. E. (1969). “Local Buckling in Universal Sections”, Internal Report, University of Cambridge Engineering Department.

Spangemacher, R. (1991). “Zum Rotationsnachweisvon Stahlkonstruktion, die nach dem traglastverfahren berechnet warden”, Dissertation, Technischen Hochshule Aachen.

Stowell, E. Z. (1950). “Compressive Strength of Flanges”, Technical Note No. 2020, National Advisory Committee for Aeronautics.

Suzuki, T., T. Ogawa, and K. Ikarashi (1994). "A study on Local Buckling Behaviour of Hybrid Beams”, Thin-Walled Structures, 19, 337-351.

Tansil, T. C. (1991). "Behavior of a composite plate girder in negative bending,” M. S. Thesis, University of Texas, Austin, TX.

Thomas, S. J. and C. J. Earls. (2003). "Cross-Sectional Compactness and Bracing Requirements for HPS483W Girders”, ASCE Journal of Structural Engineering, 129(12), 1569-1583.

White, D. W. and K. E. Barth. (1998). “Strength and Ductility of Compact-Flange I-Girders in Negative Bending”, Journal of Constructional Steel Research, 45(3), 241-280. 
Wright, W. J. (1997). Personal communication.

Yakel, A. J., Mans, P. and Azizinamini, A. (1999). "Flexural capacity of HPS-70W bridge girders.” NaBRO Rep. University of Nebraska-Lincoln, Lincoln, NE.

Yang, L. (2003). "Evaulation of Moment Redistribution for Hybrid HPS 70W Bridge Girders”, M. S. Thesis, West Virginia University, Morgantown, WV. 


\section{Appendix A: NOMENCLATURE}

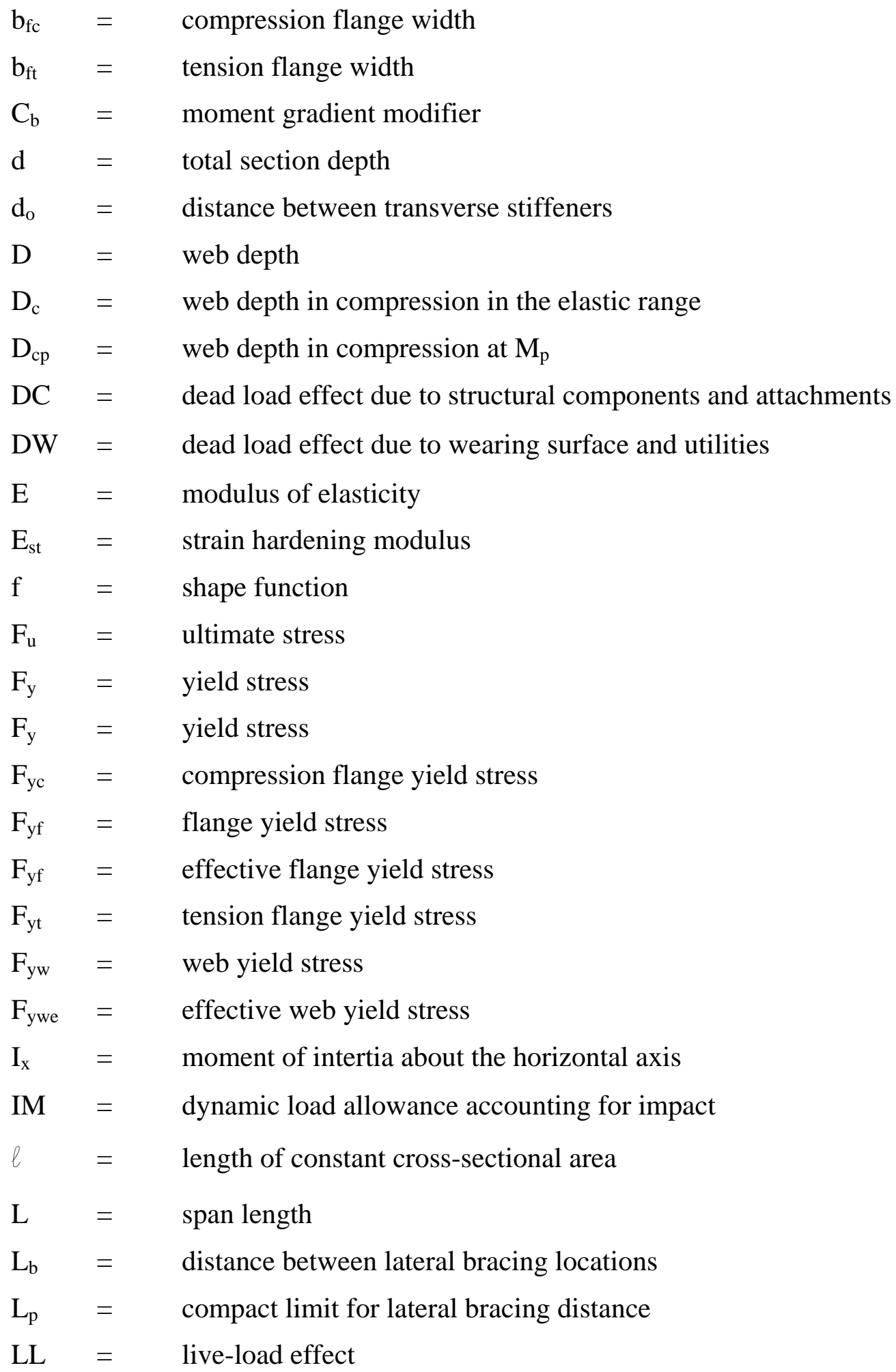




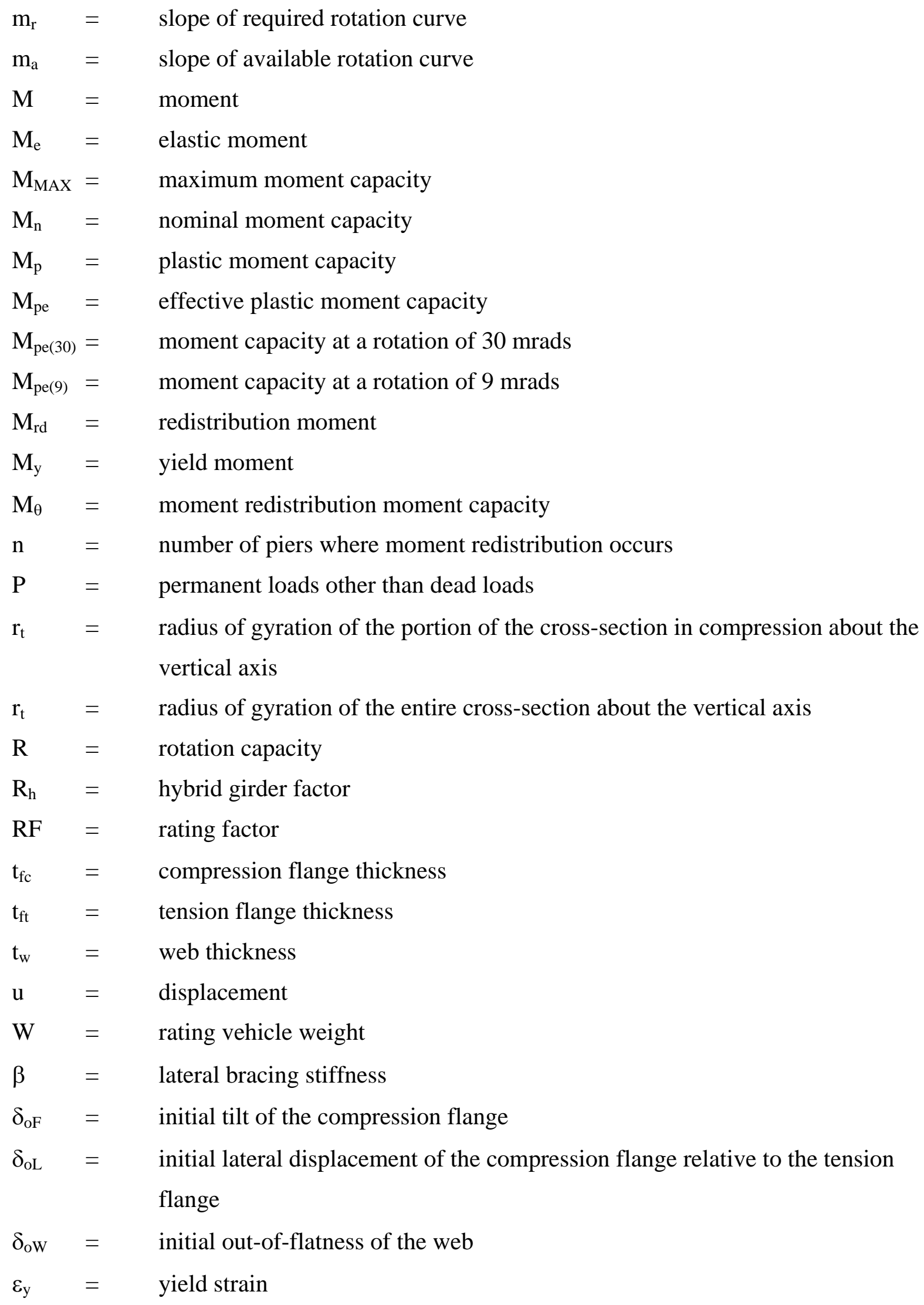




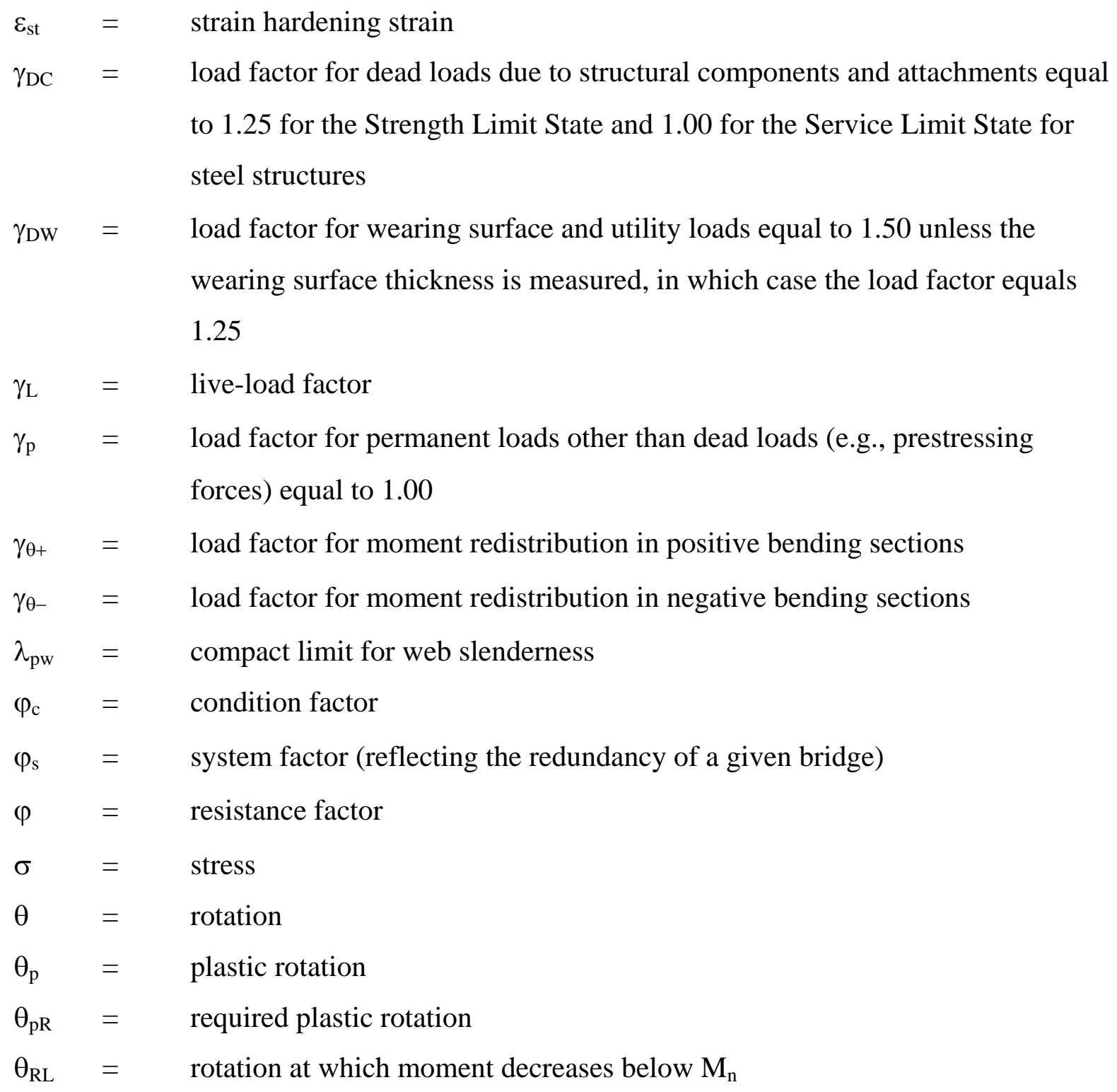




\section{Appendix B: EXISTING TEST DATA}

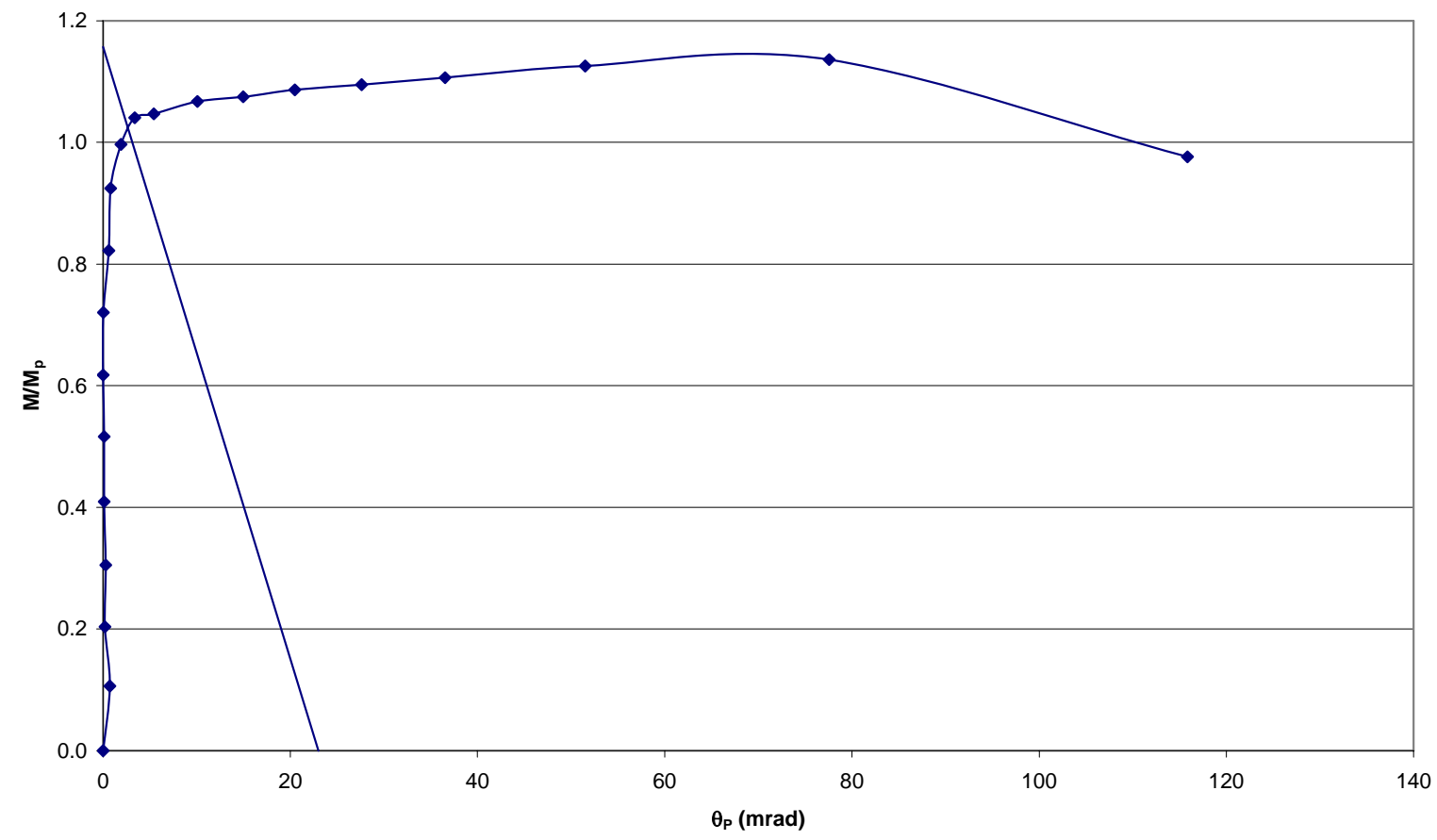

Figure B-1. Moment versus Rotation for Girder HT-28 (Adams et al. 1964)

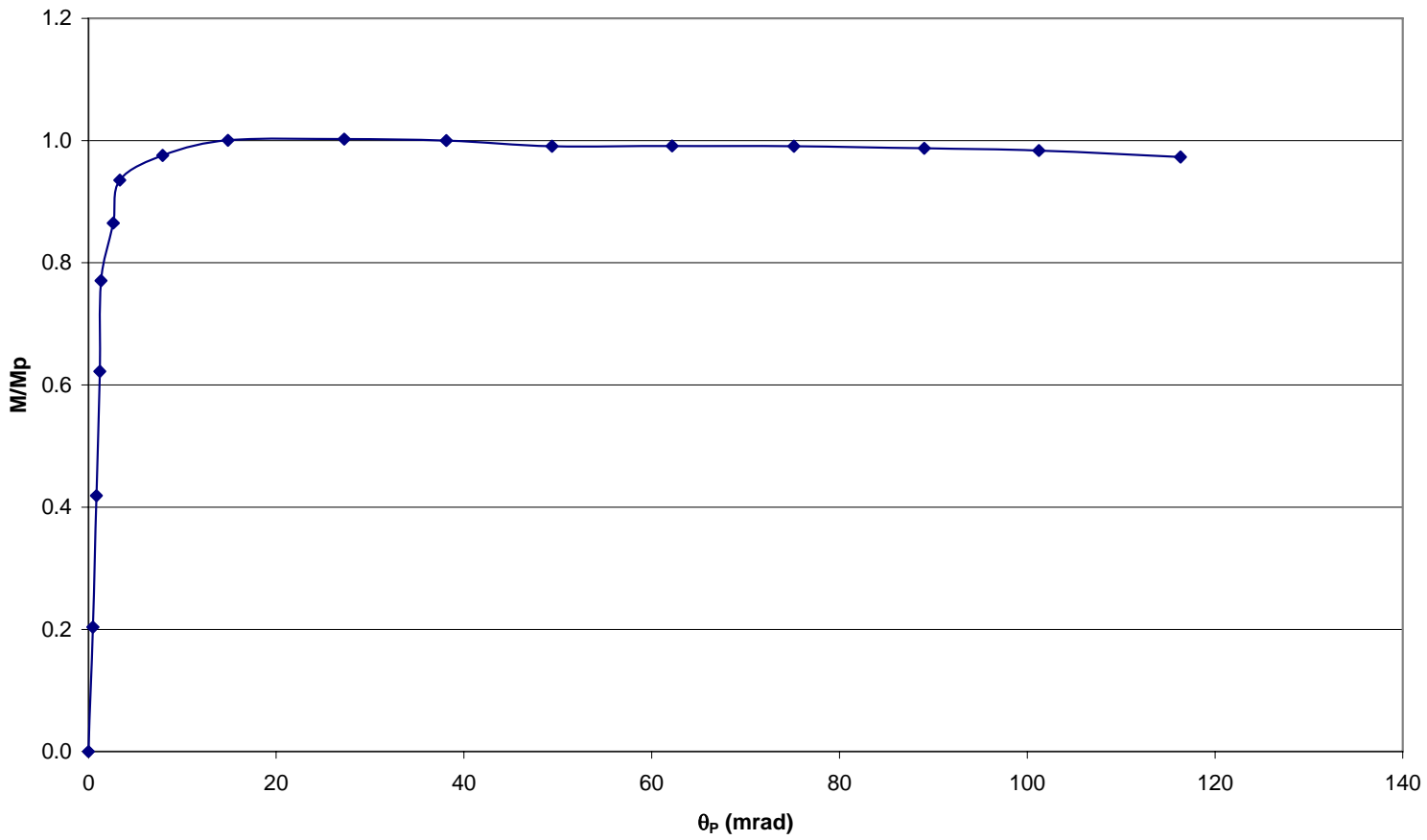

Figure B-2. Moment versus Rotation for Girder HT-29 (Adams et al. 1964) 


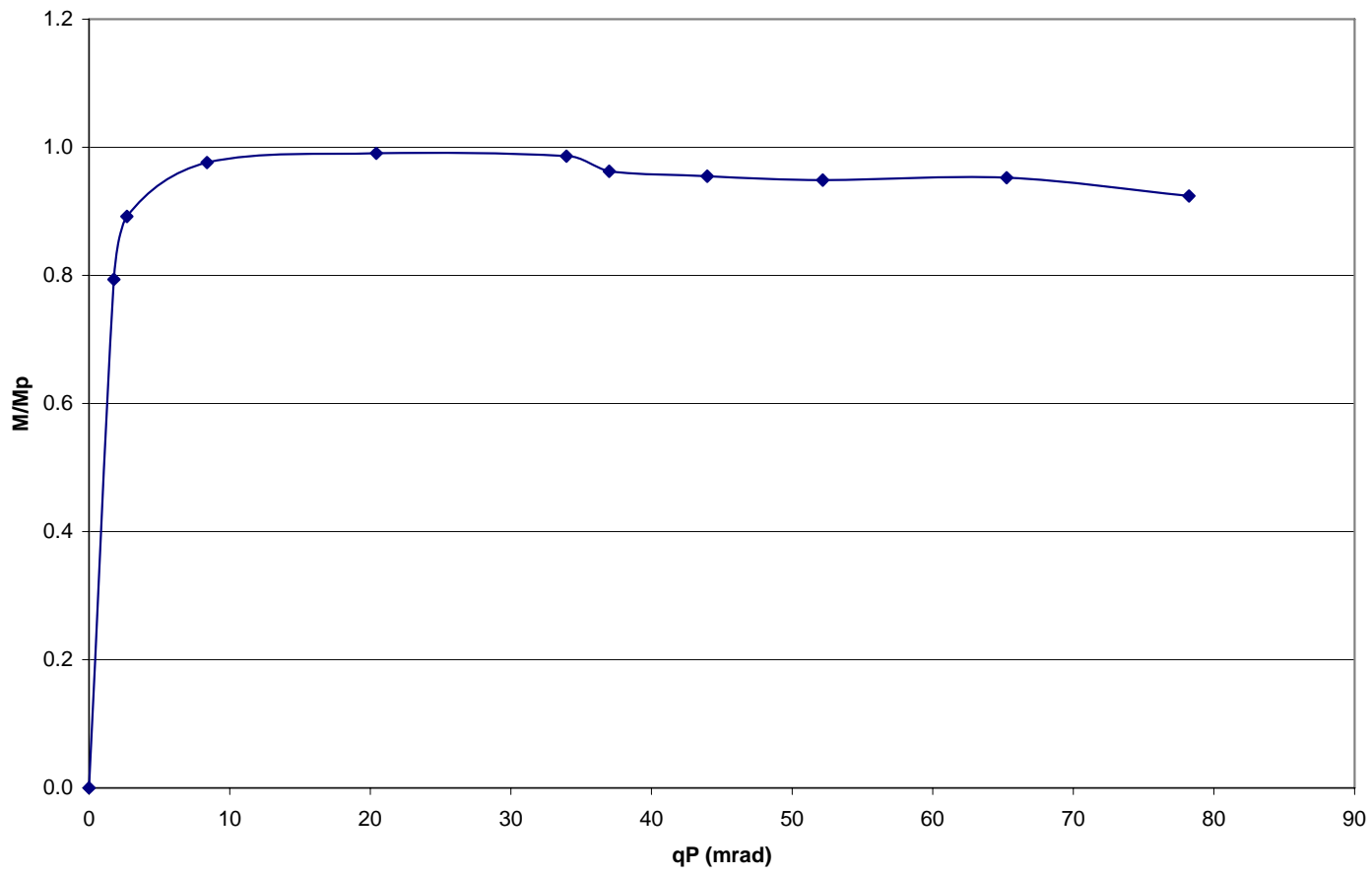

Figure B-3. Moment versus Rotation for Girder HT-30 (Adams et al. 1964)

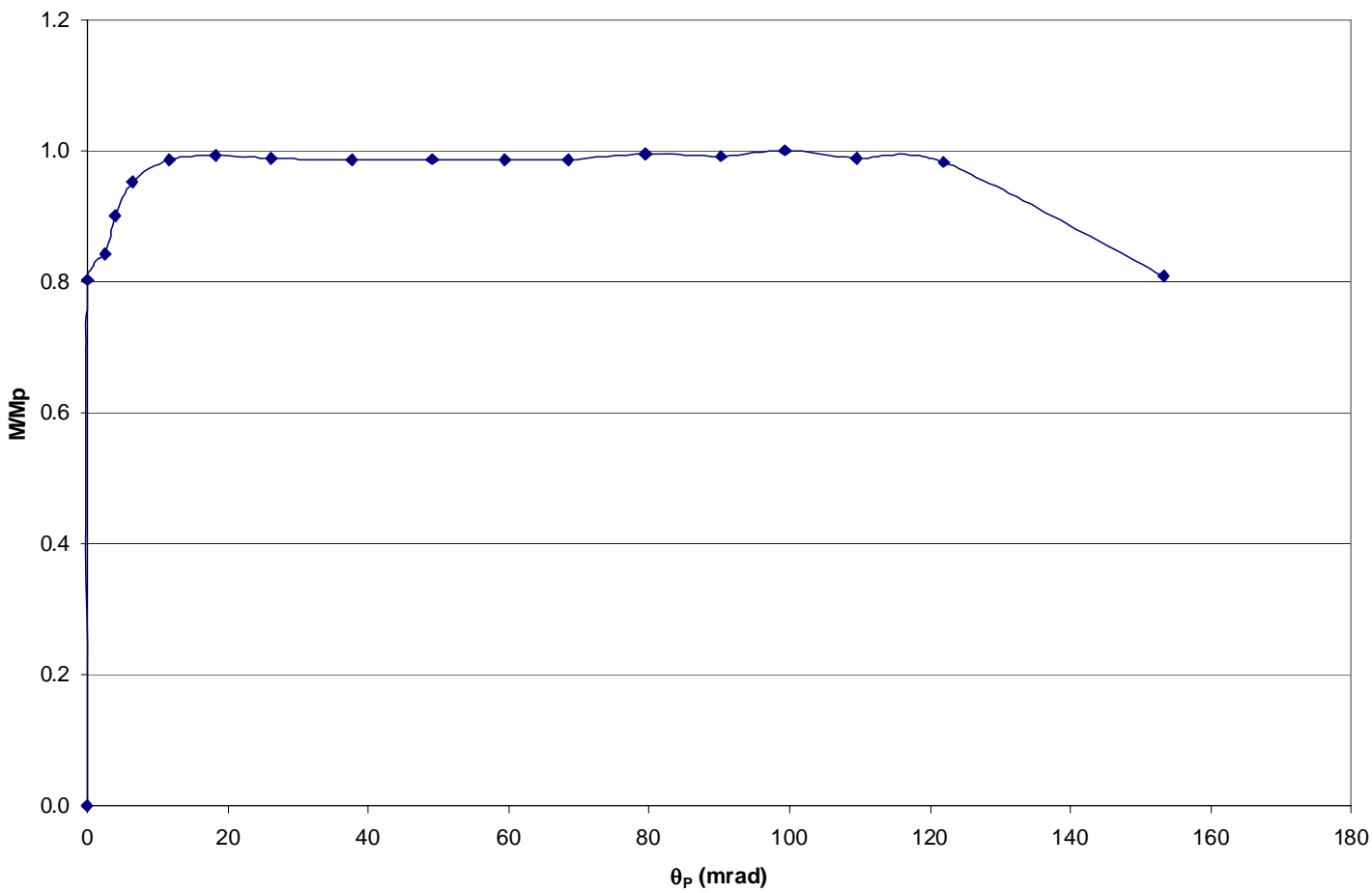

Figure B-4. Moment versus Rotation for Girder HT-31 (Adams et al. 1964) 


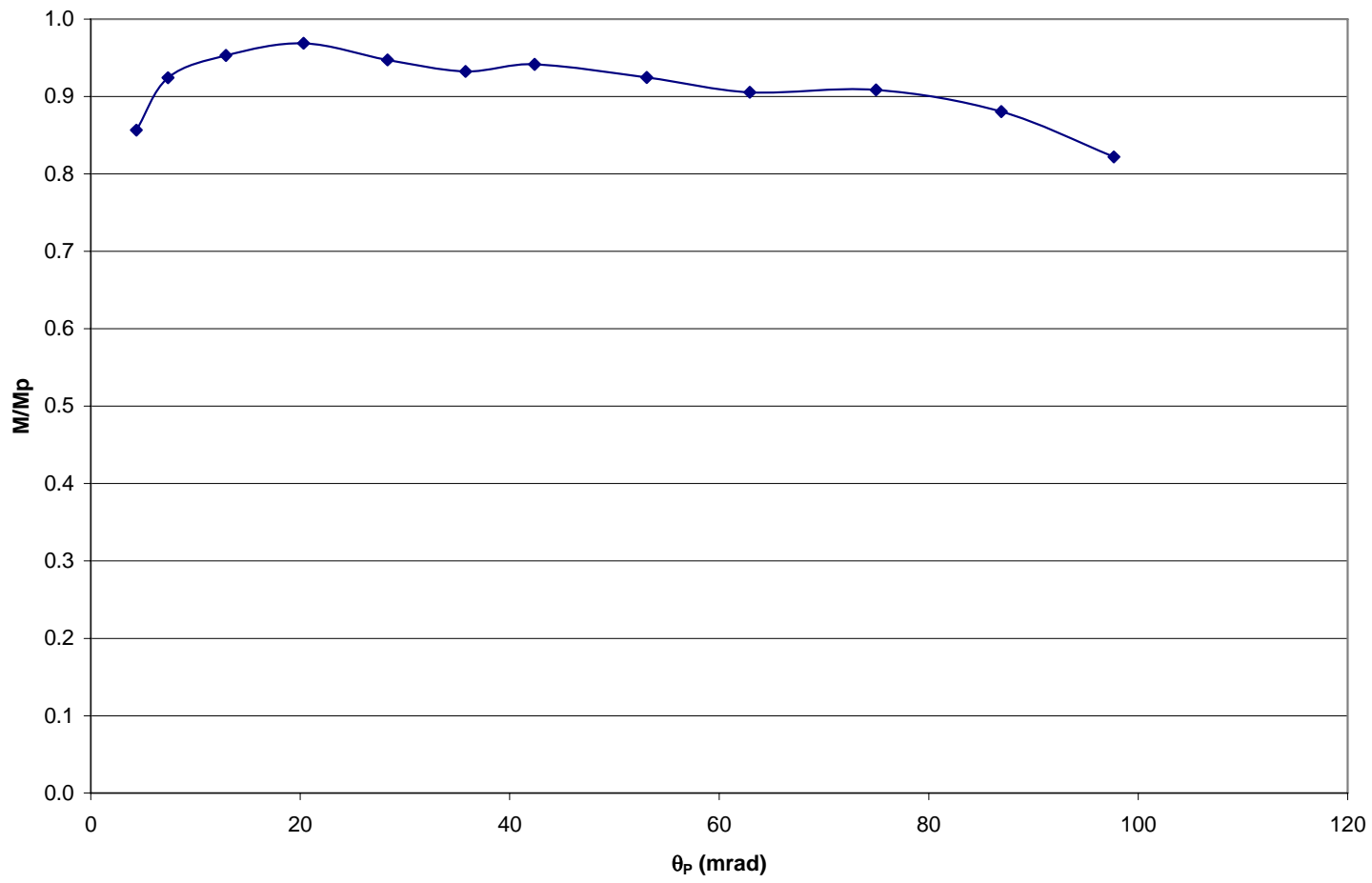

Figure B-5. Moment versus Rotation for Girder HT-36 (Adams et al. 1964)

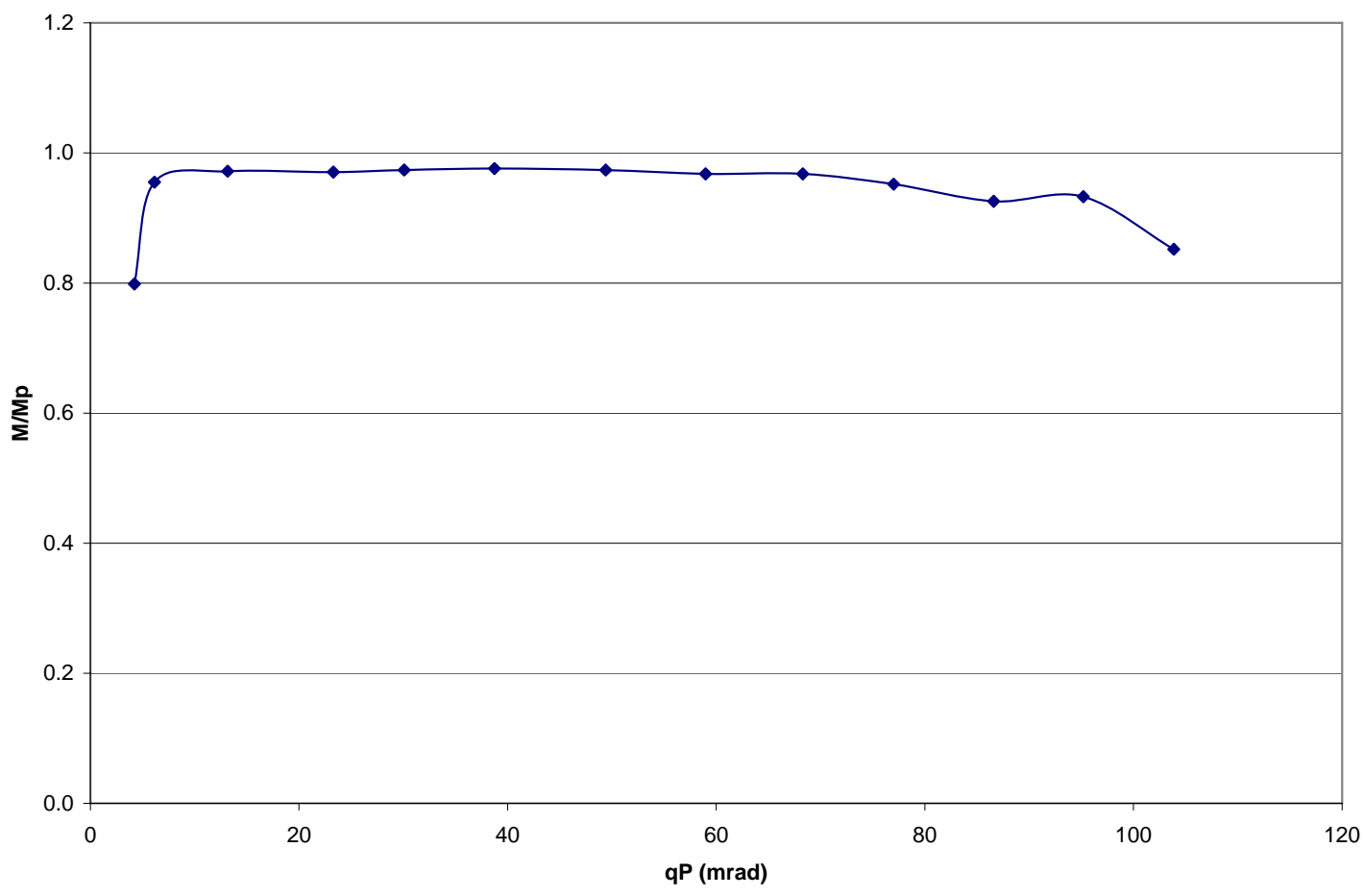

Figure B-6. Moment versus Rotation for Girder HT-37 (Adams et al. 1964) 


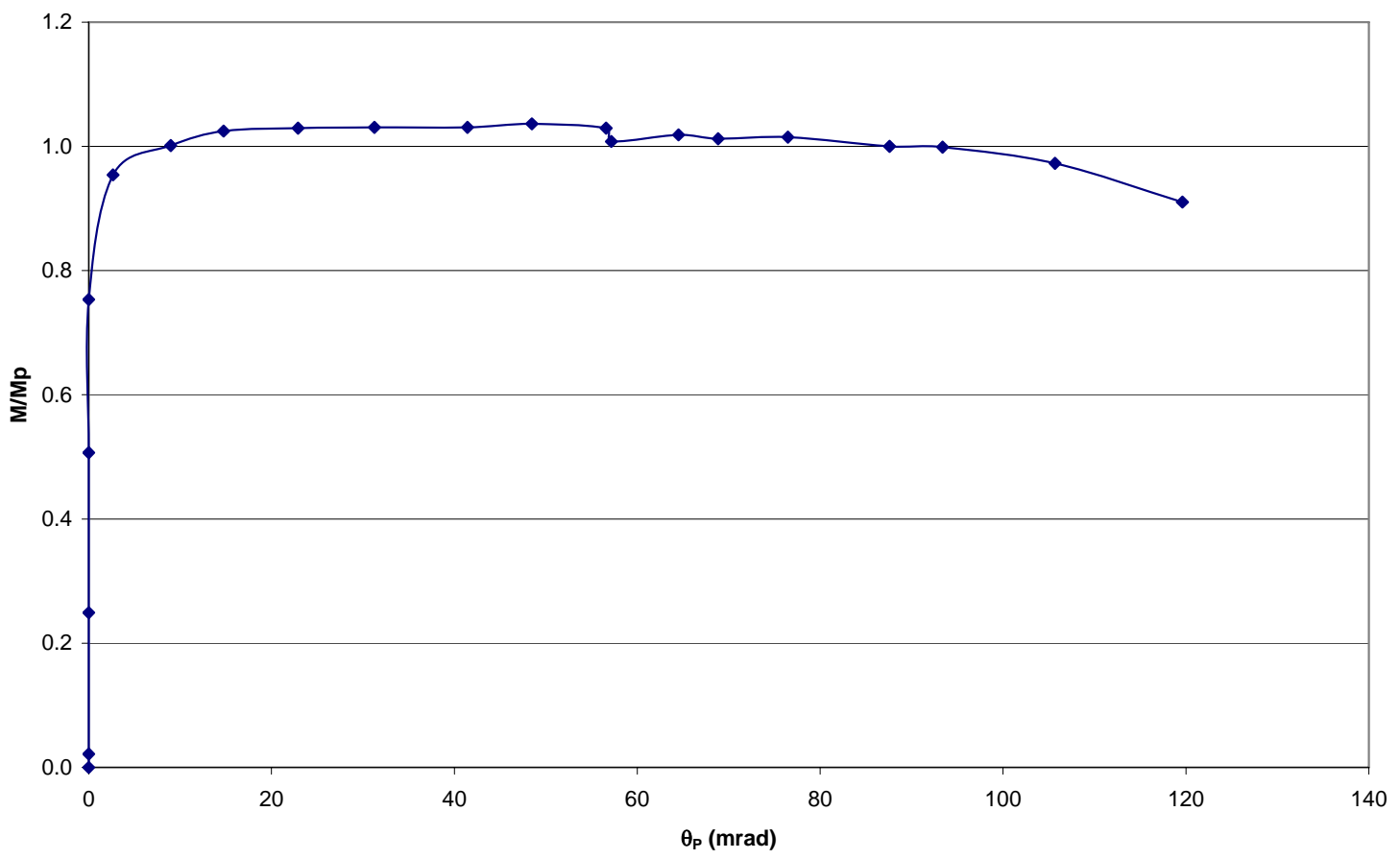

Figure B-7. Moment versus Rotation for Girder HT-38 (Adams et al. 1964)

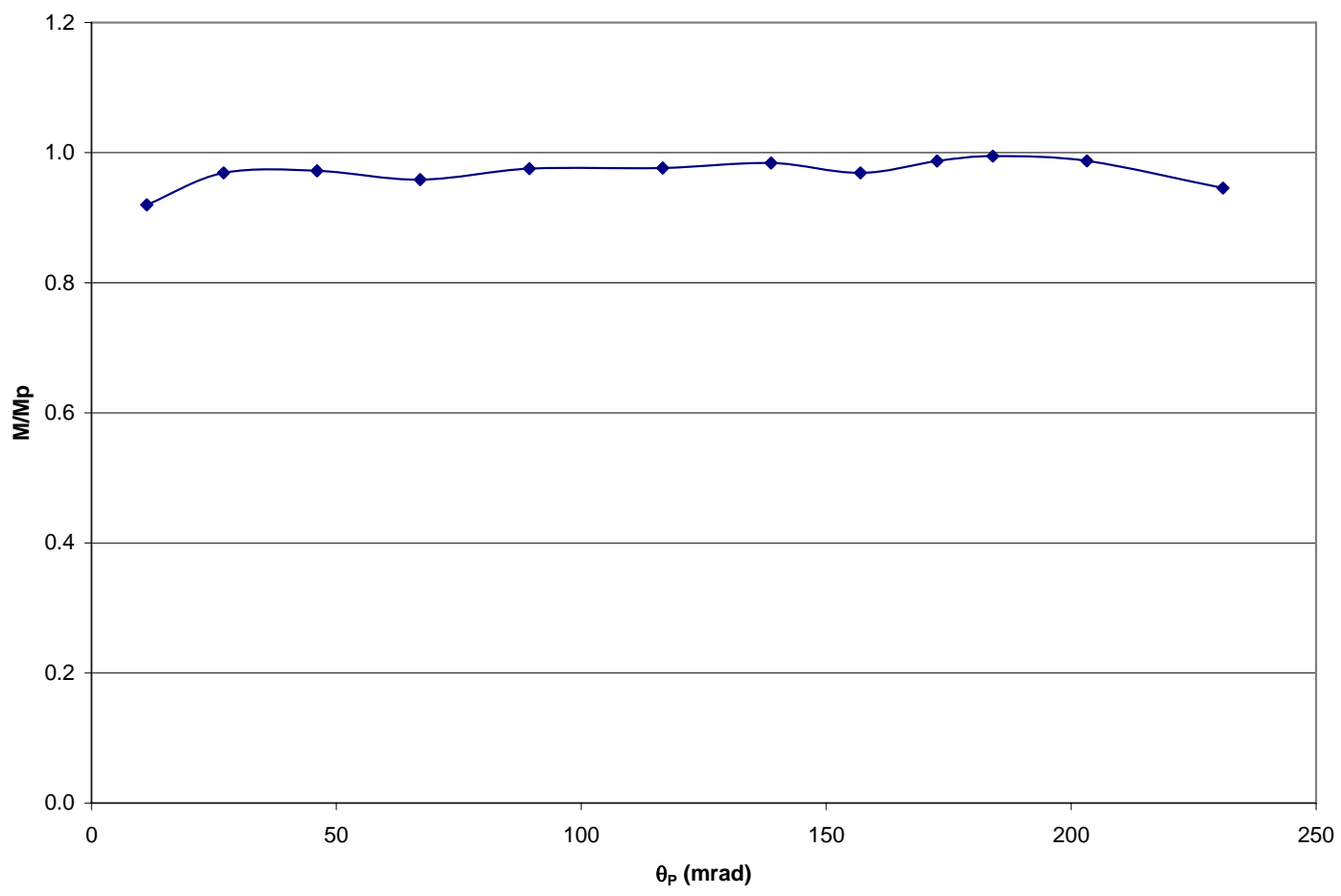

Figure B-8. Moment versus Rotation for Girder HT-41 (Adams et al. 1964) 


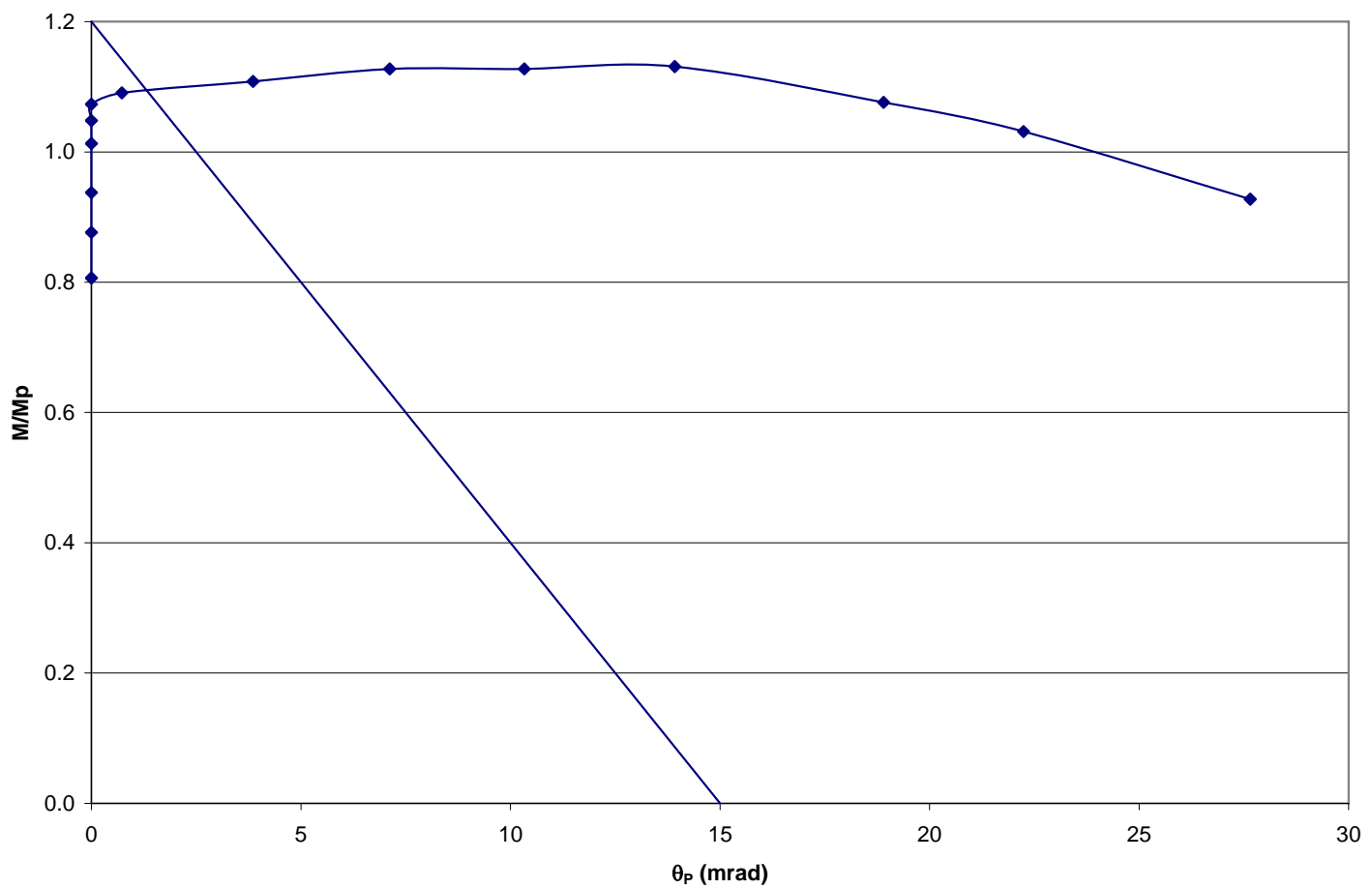

Figure B-9. Moment versus Rotation for Girder HT-43 (Adams et al. 1964)

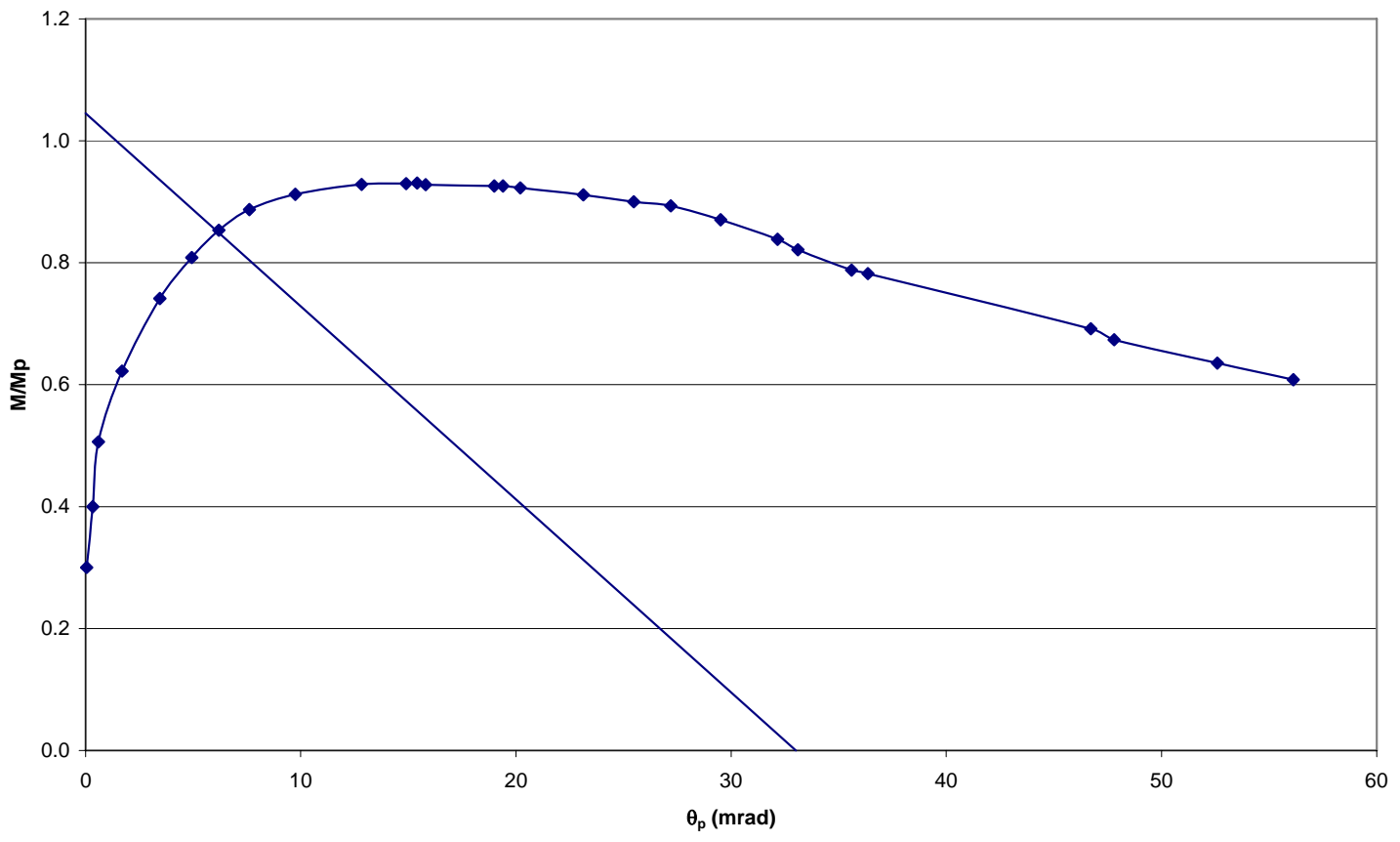

Figure B-10. Moment versus Rotation for Girder P2 (Barth 1996) 


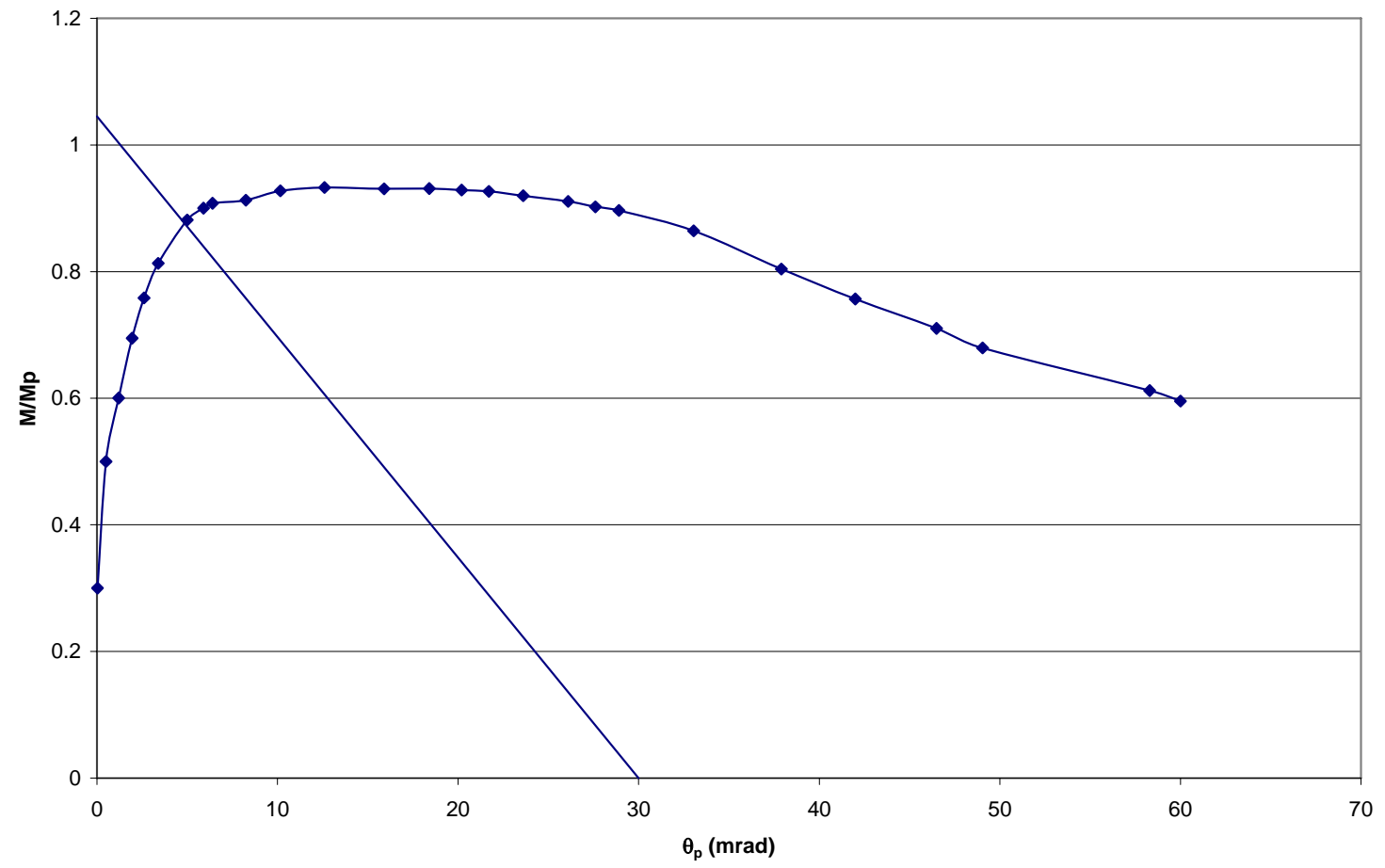

Figure B-11. Moment versus Rotation for Girder P3 (Barth 1996)

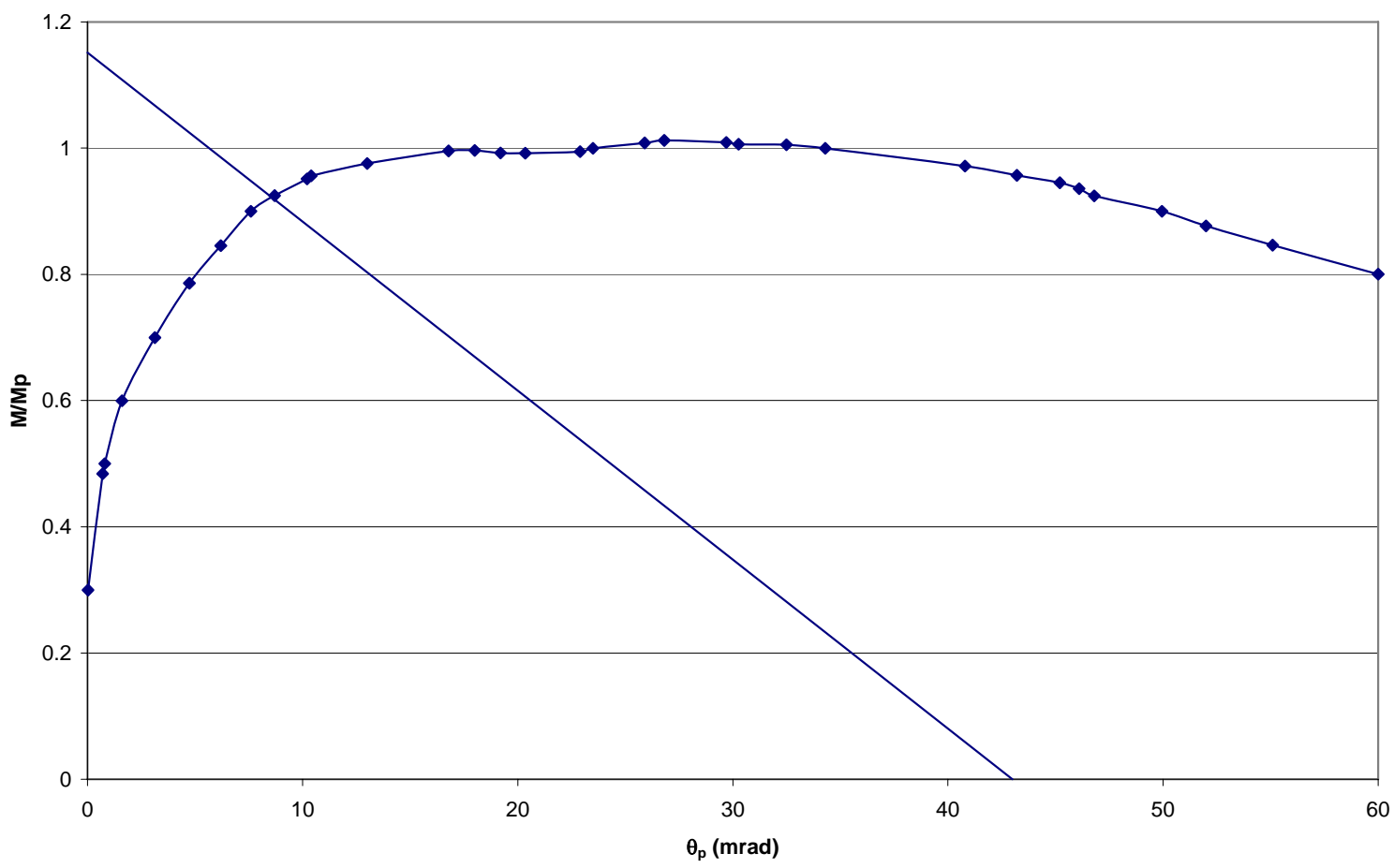

Figure B-12. Moment versus Rotation for Girder P6 (Barth 1996) 


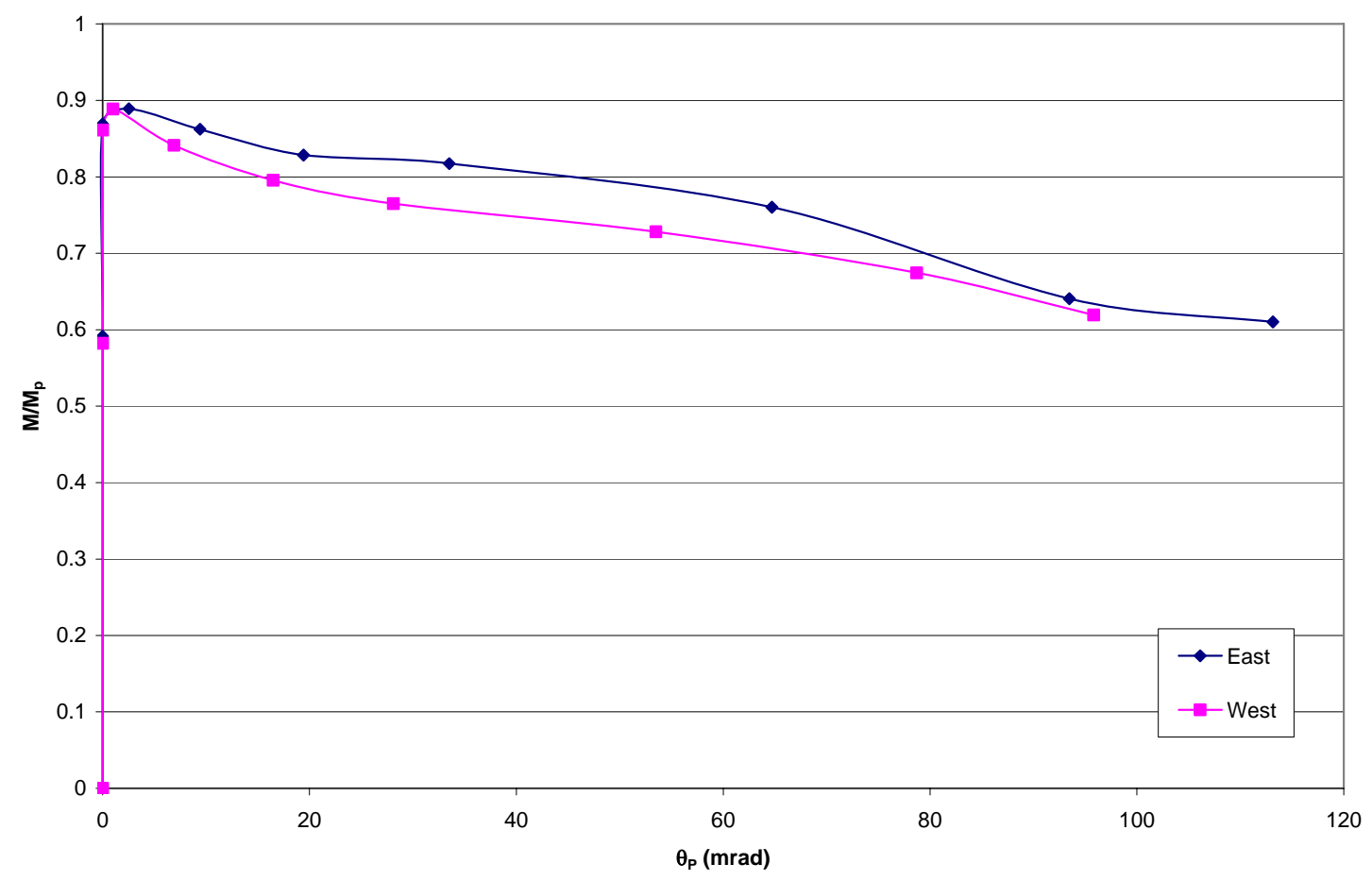

Figure B-13. Moment versus Rotation for Girder 188-3-4 Carskaddan (1980)

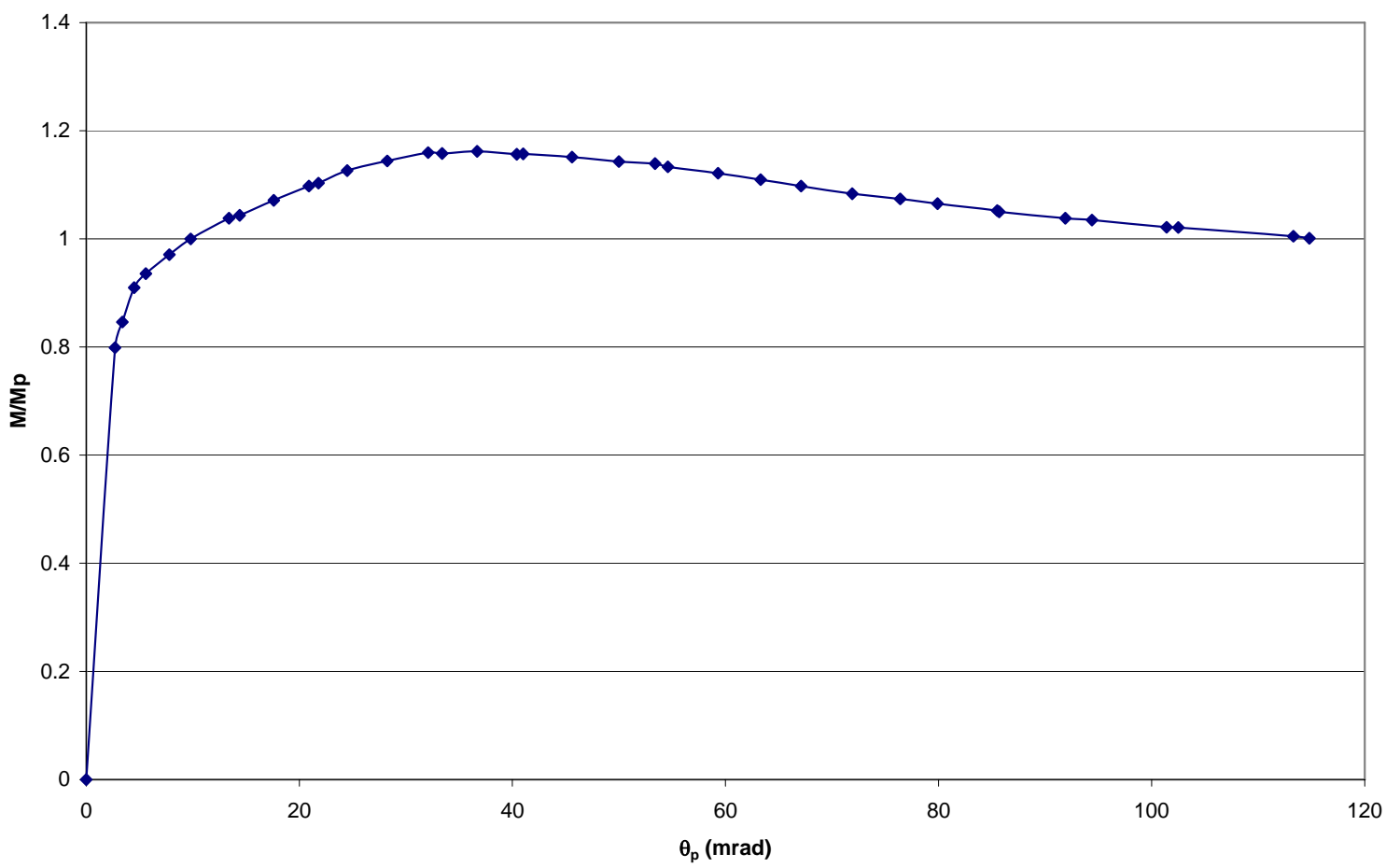

Figure B-14. Moment versus Rotation for Girder HB-40

(Climenhaga and Johnson 1972) 


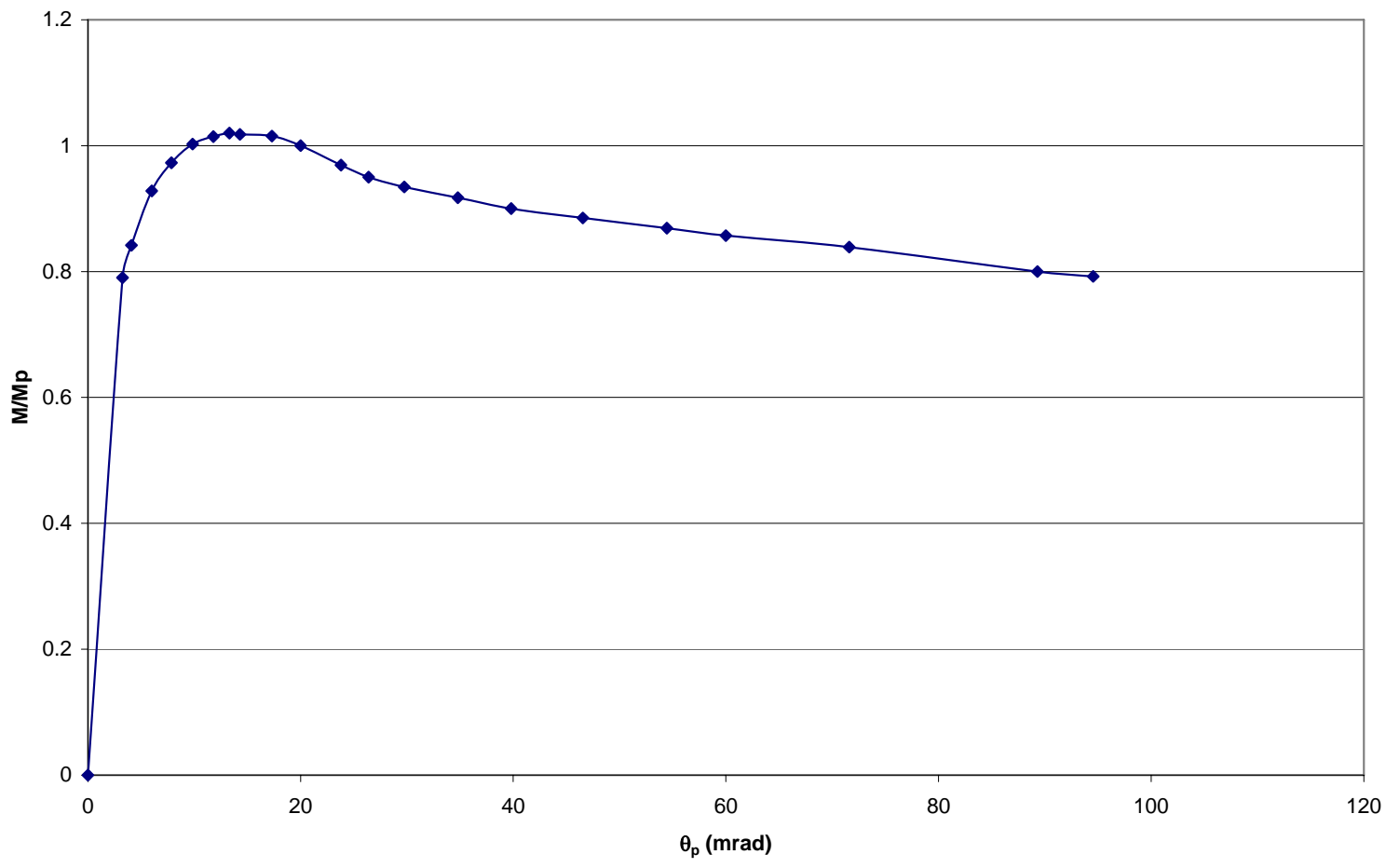

Figure B-15. Moment versus Rotation for Girder HB-41

(Climenhaga and Johnson 1972)

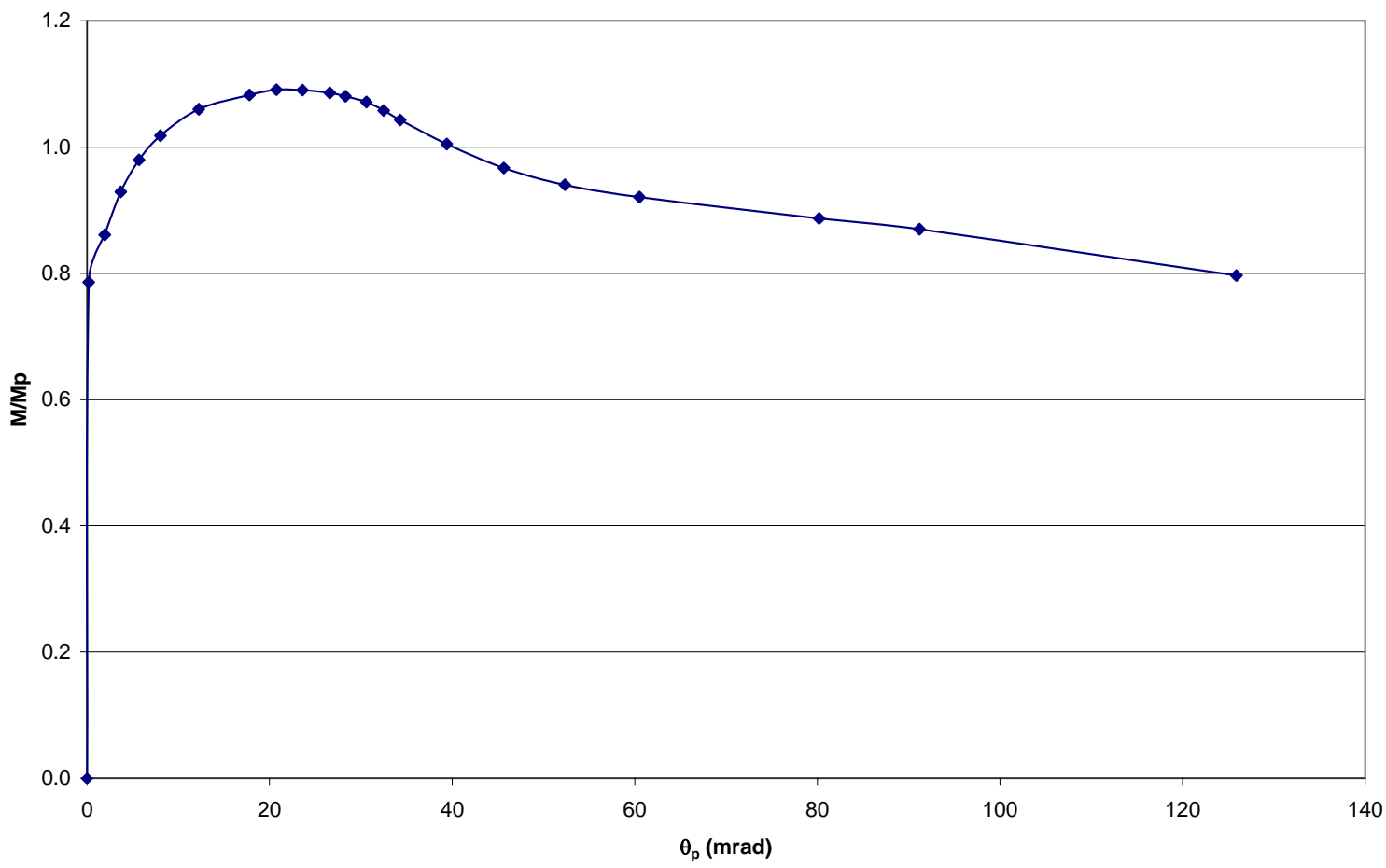

Figure B-16. Moment versus Rotation for Girder SB-2 (Climenhaga and Johnson 1972) 


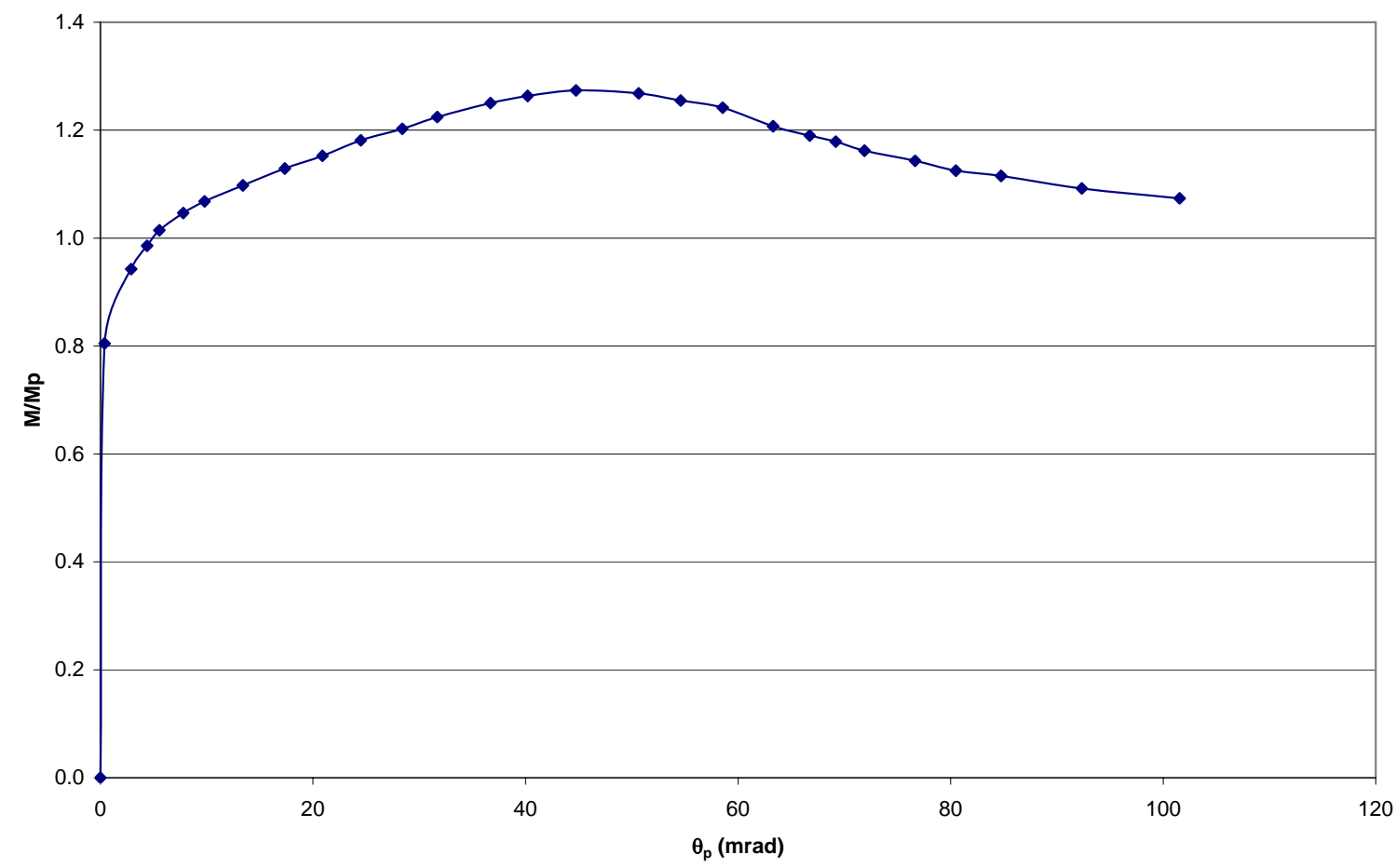

Figure B-17. Moment versus Rotation for Girder SB-3 (Climenhaga and Johnson 1972)

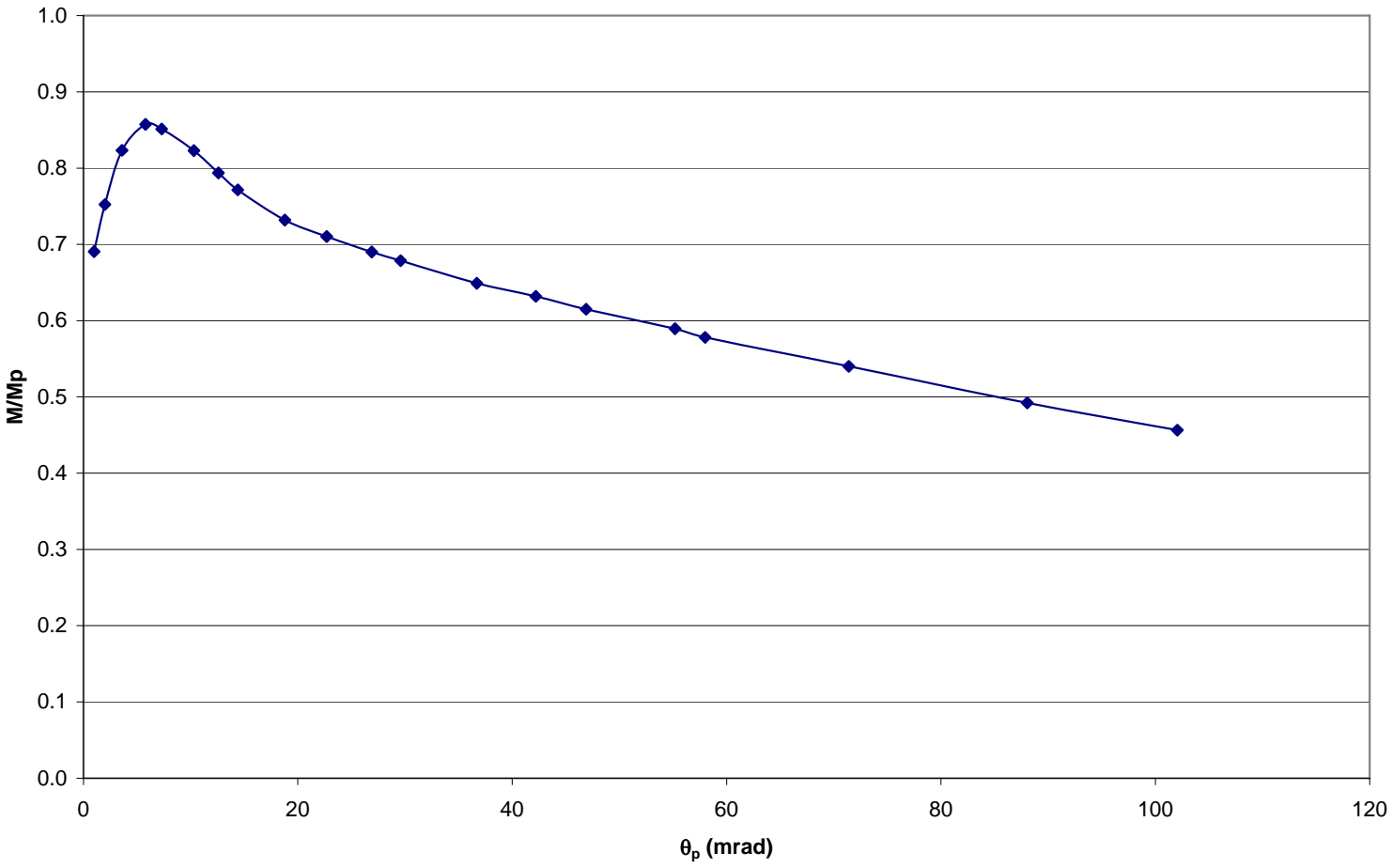

Figure B-18. Moment versus Rotation for Girder SB-5 (Climenhaga and Johnson 1972) 


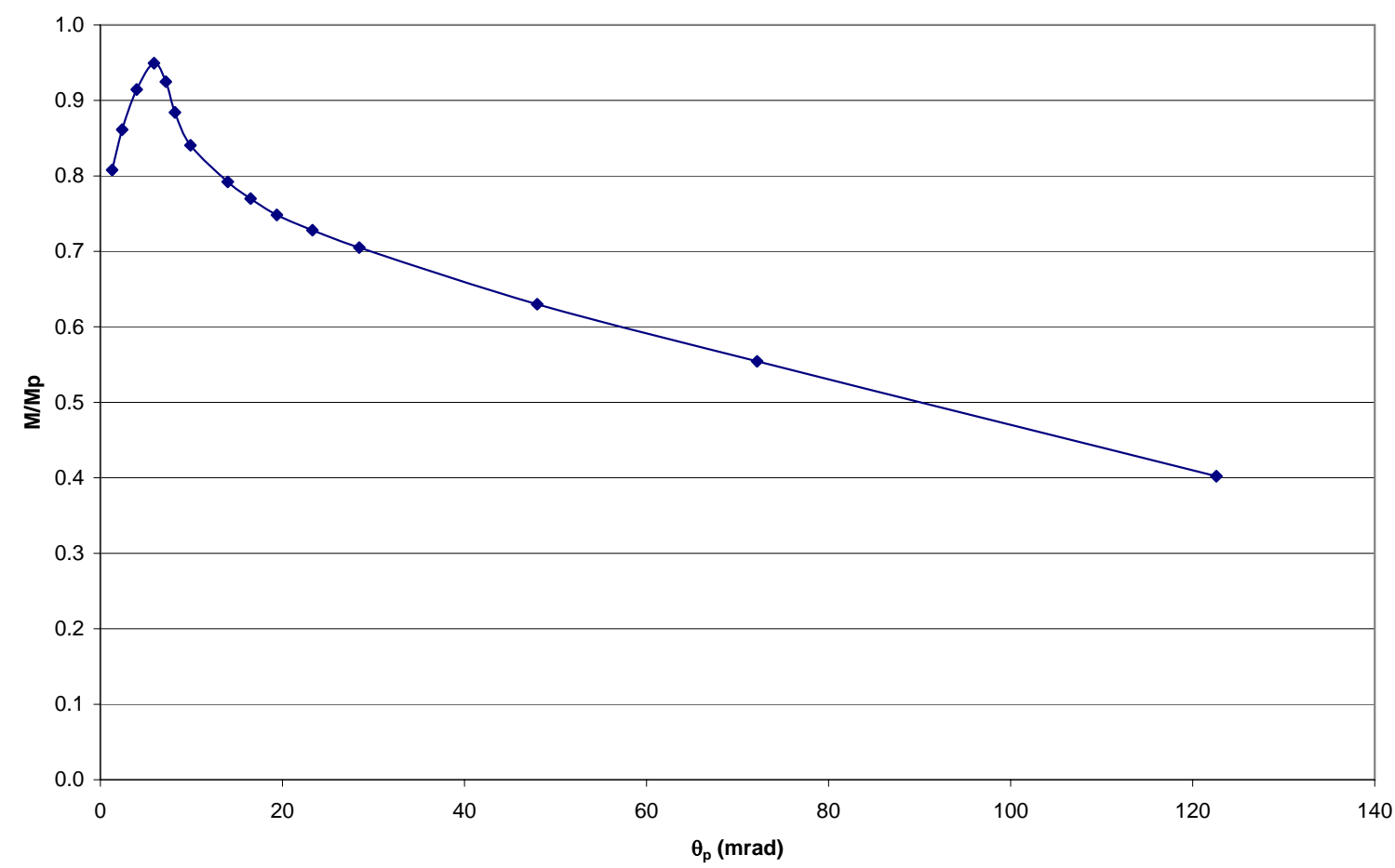

Figure B-19. Moment versus Rotation for Girder SB-6 (Climenhaga and Johnson 1972)

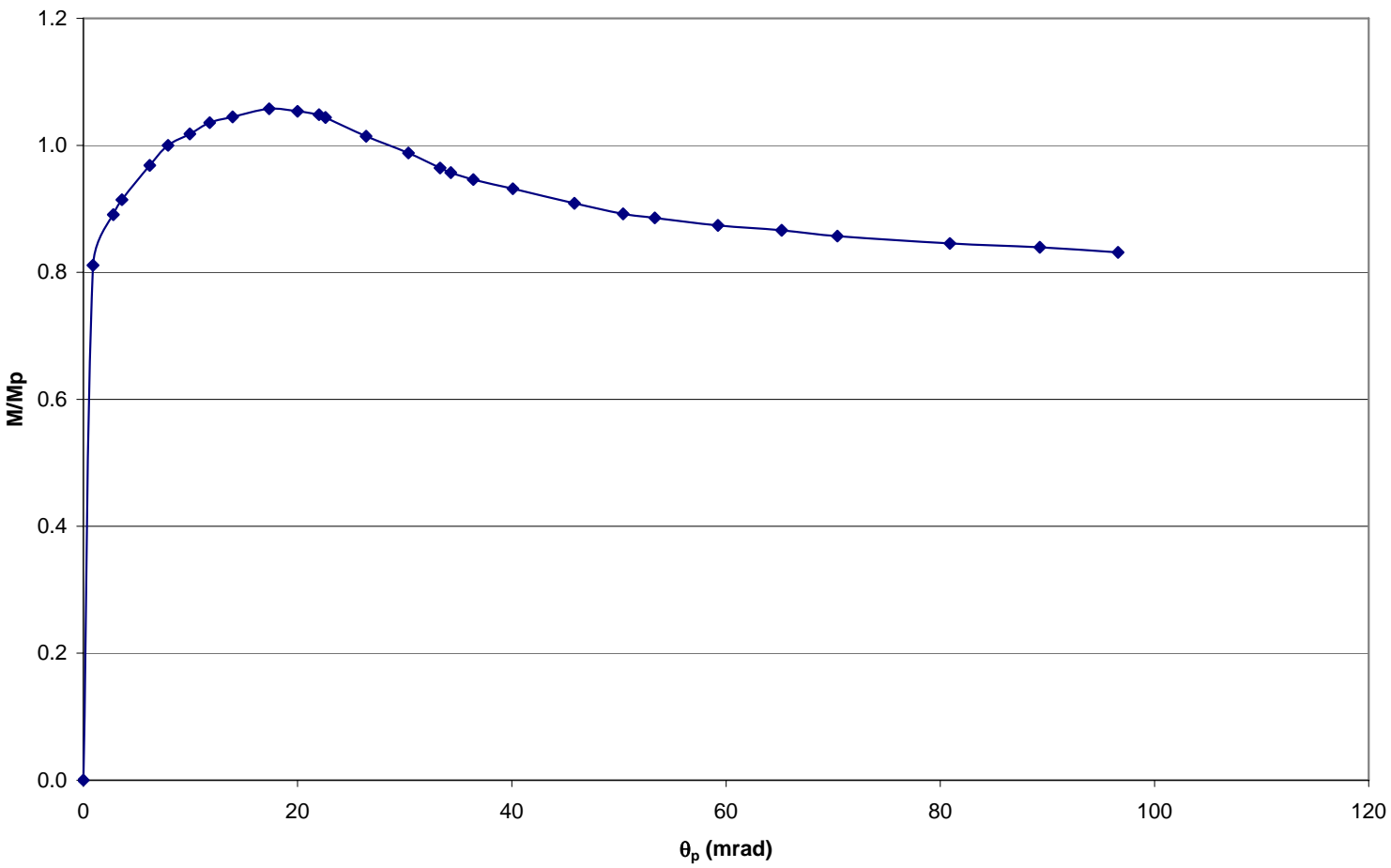

Figure B-20. Moment versus Rotation for Girder SB-8 (Climenhaga and Johnson 1972) 


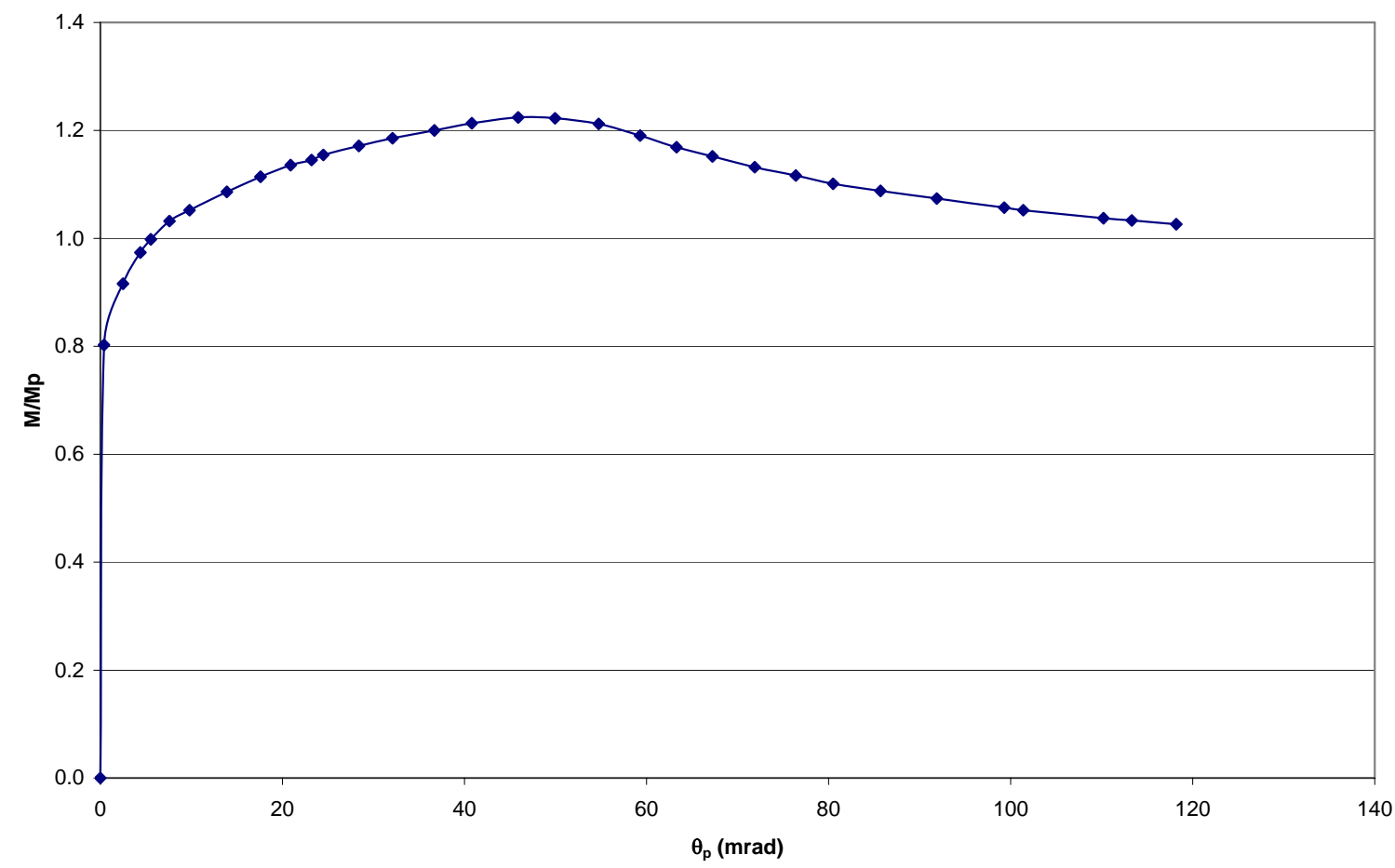

Figure B-21. Moment versus Rotation for Girder SB-9 (Climenhaga and Johnson 1972)

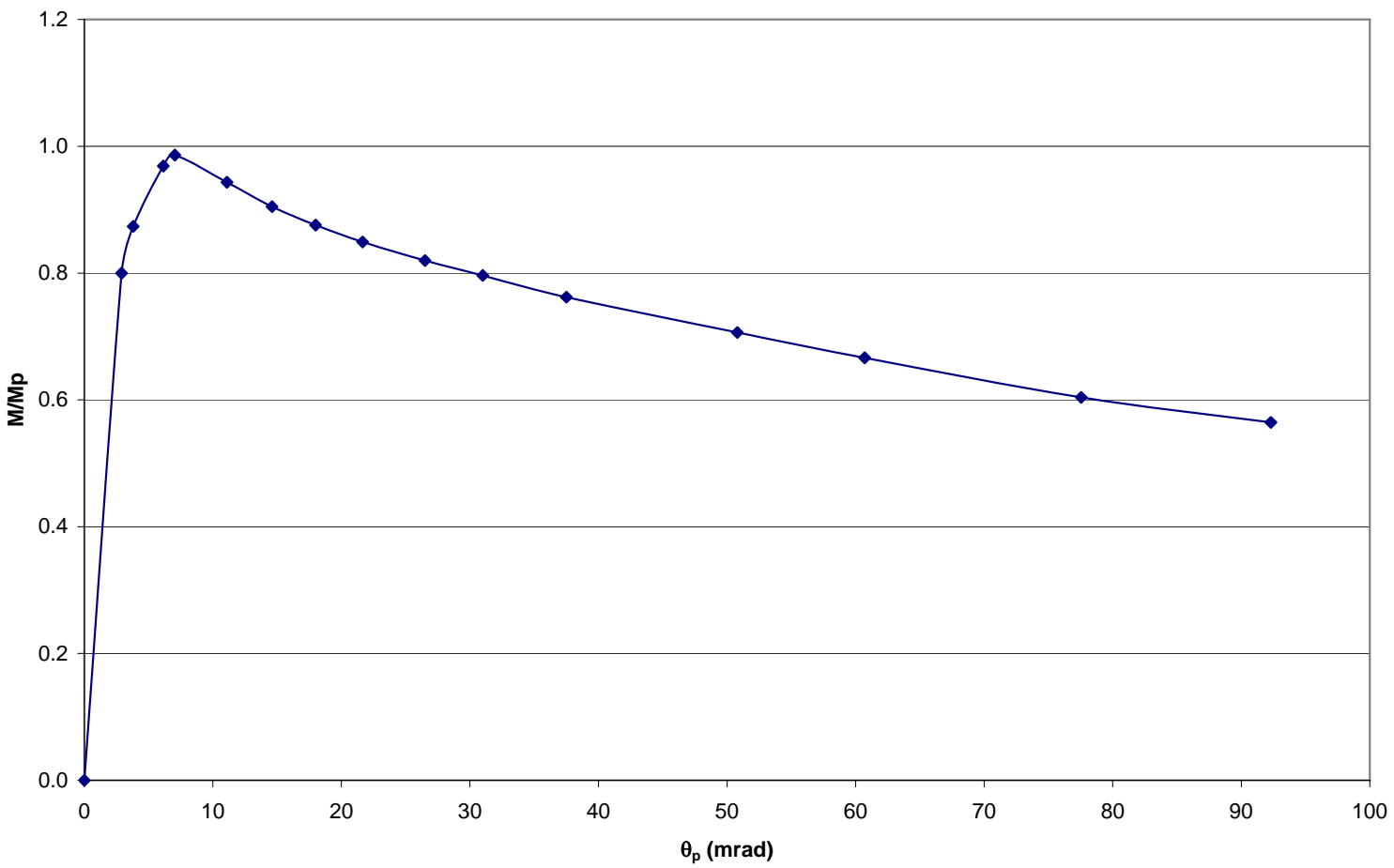

Figure B-22. Moment versus Rotation for Girder SB-10

(Climenhaga and Johnson 1972) 


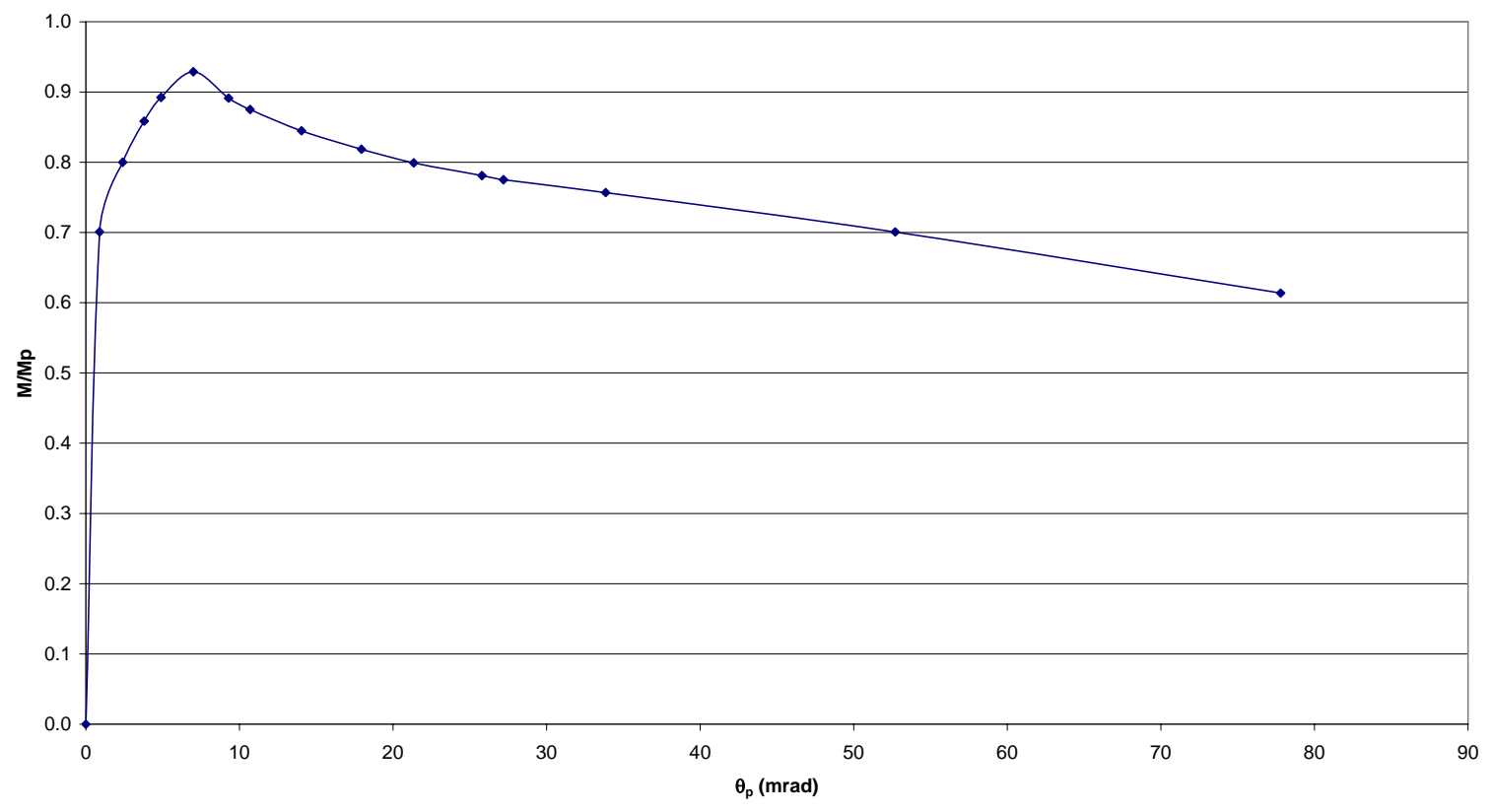

Figure B-23. Moment versus Rotation for Girder SB-11

(Climenhaga and Johnson 1972)

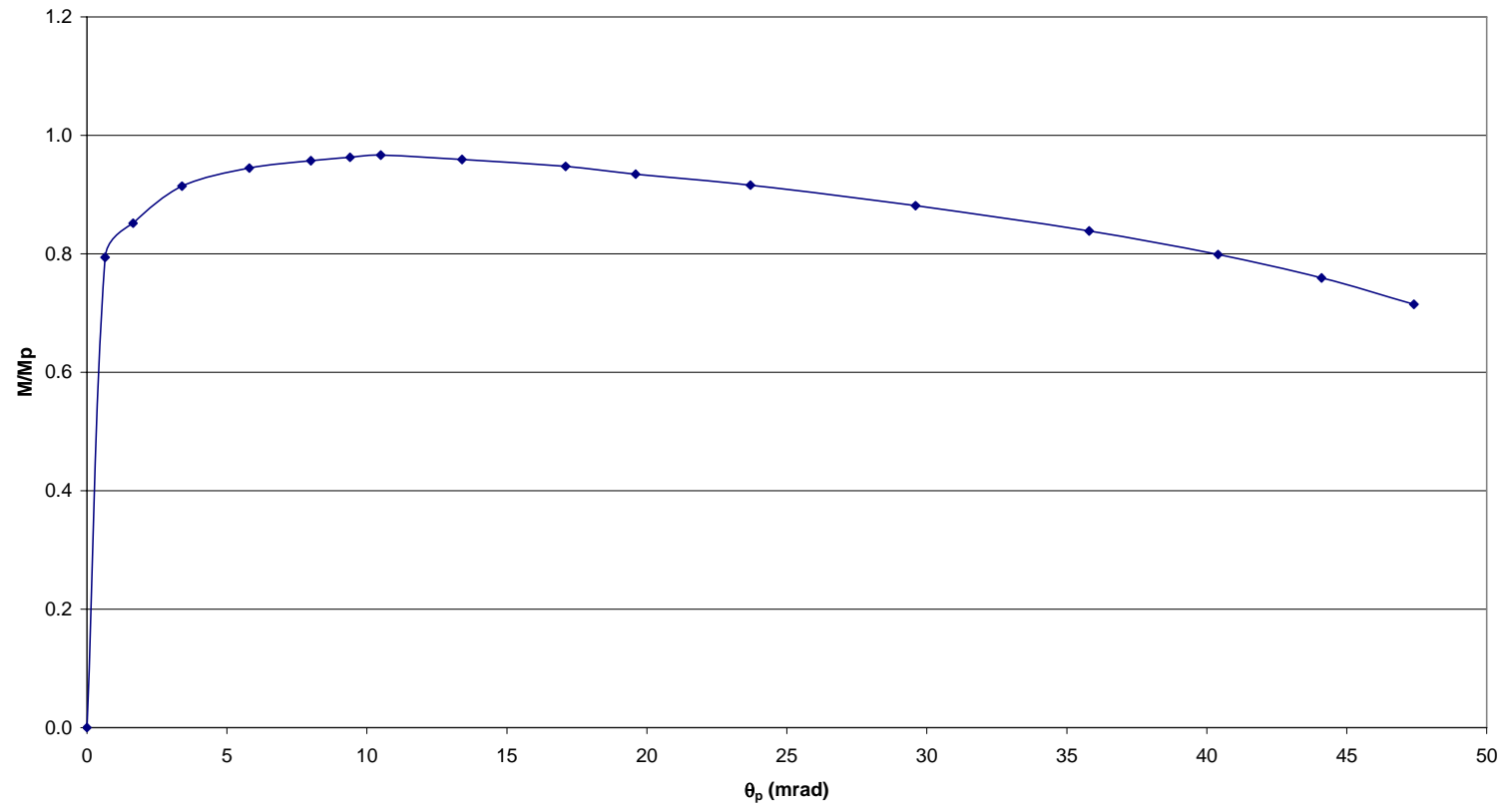

Figure B-24. Moment versus Rotation for Girder SB-14

(Climenhaga and Johnson 1972) 


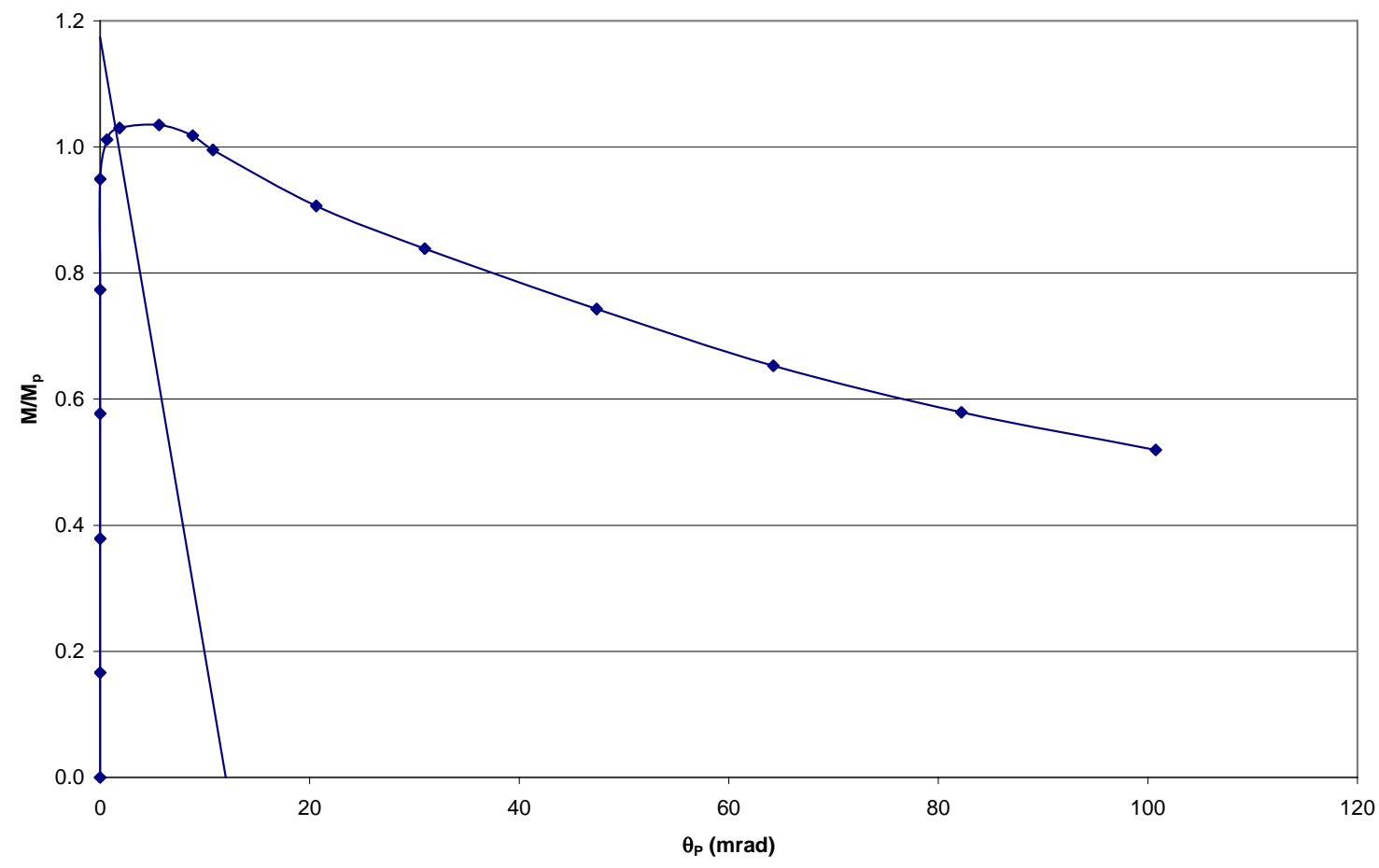

Figure B-25. Moment versus Rotation for Girder 188-3-1

(Grubb and Carskaddan 1979)

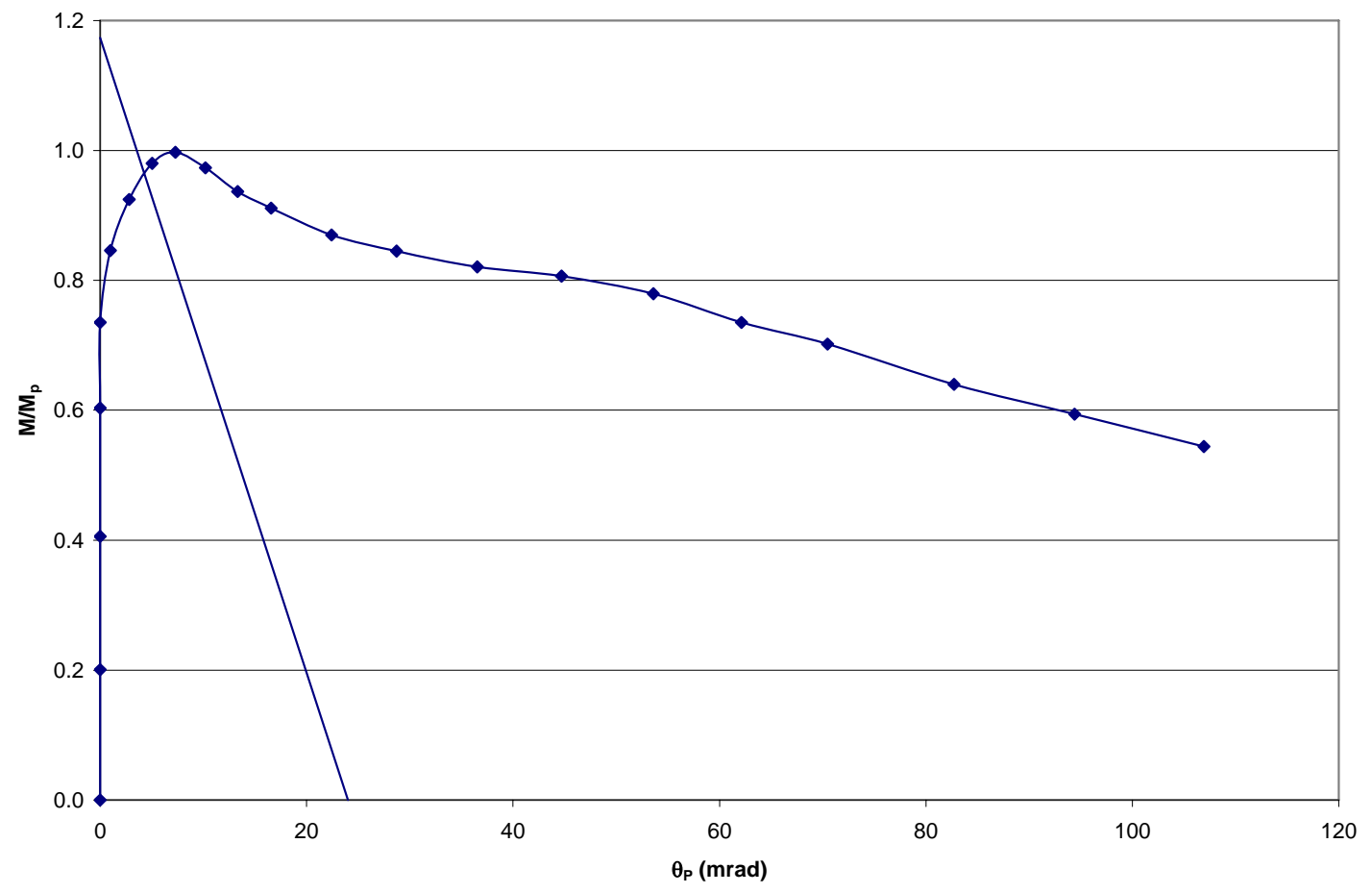

Figure B-26. Moment versus Rotation for Girder 188-3-2

(Grubb and Carskaddan 1979) 


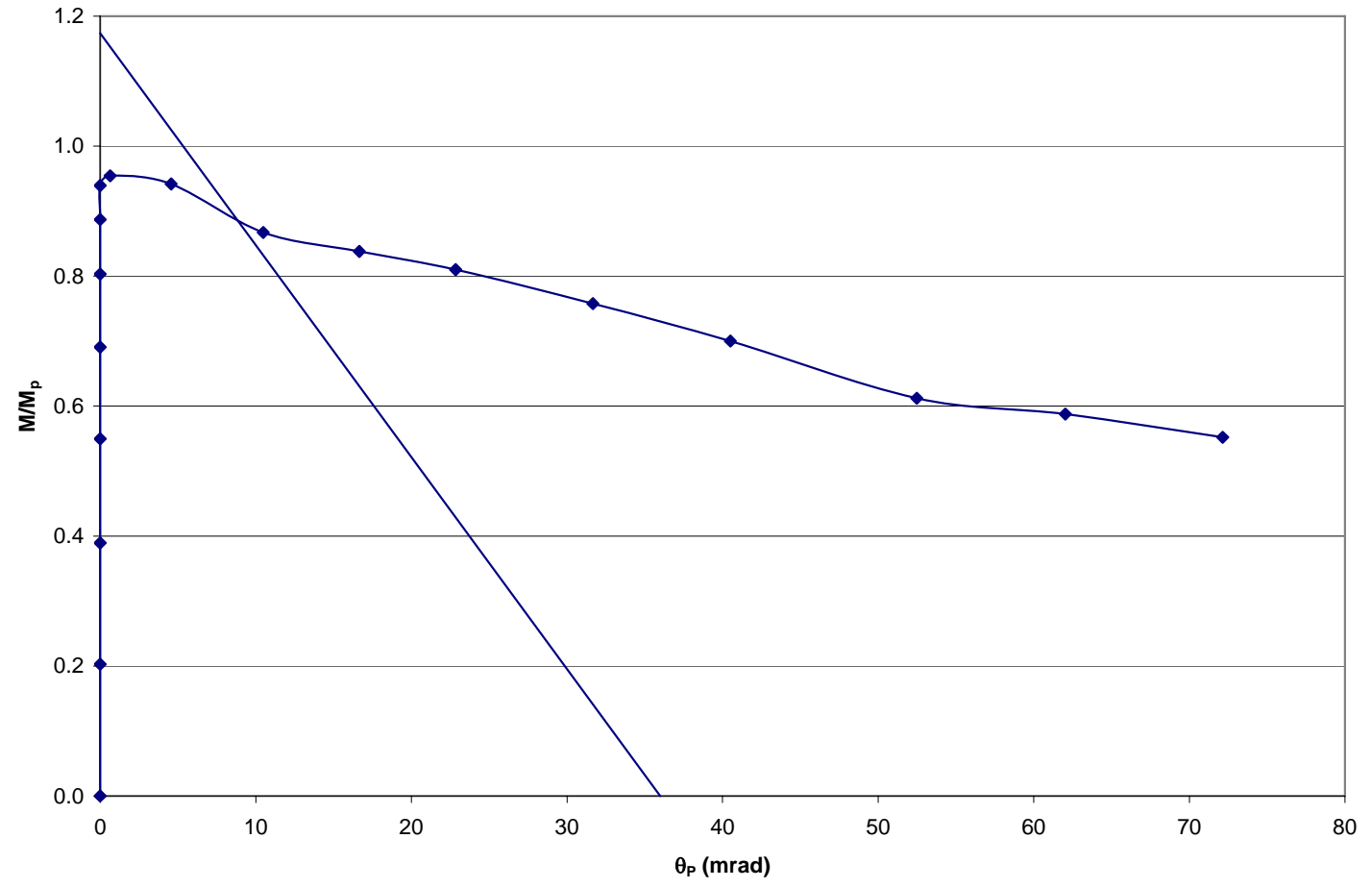

Figure B-27. Moment versus Rotation for Girder 188-3-3 (Grubb and Carskaddan 1979)

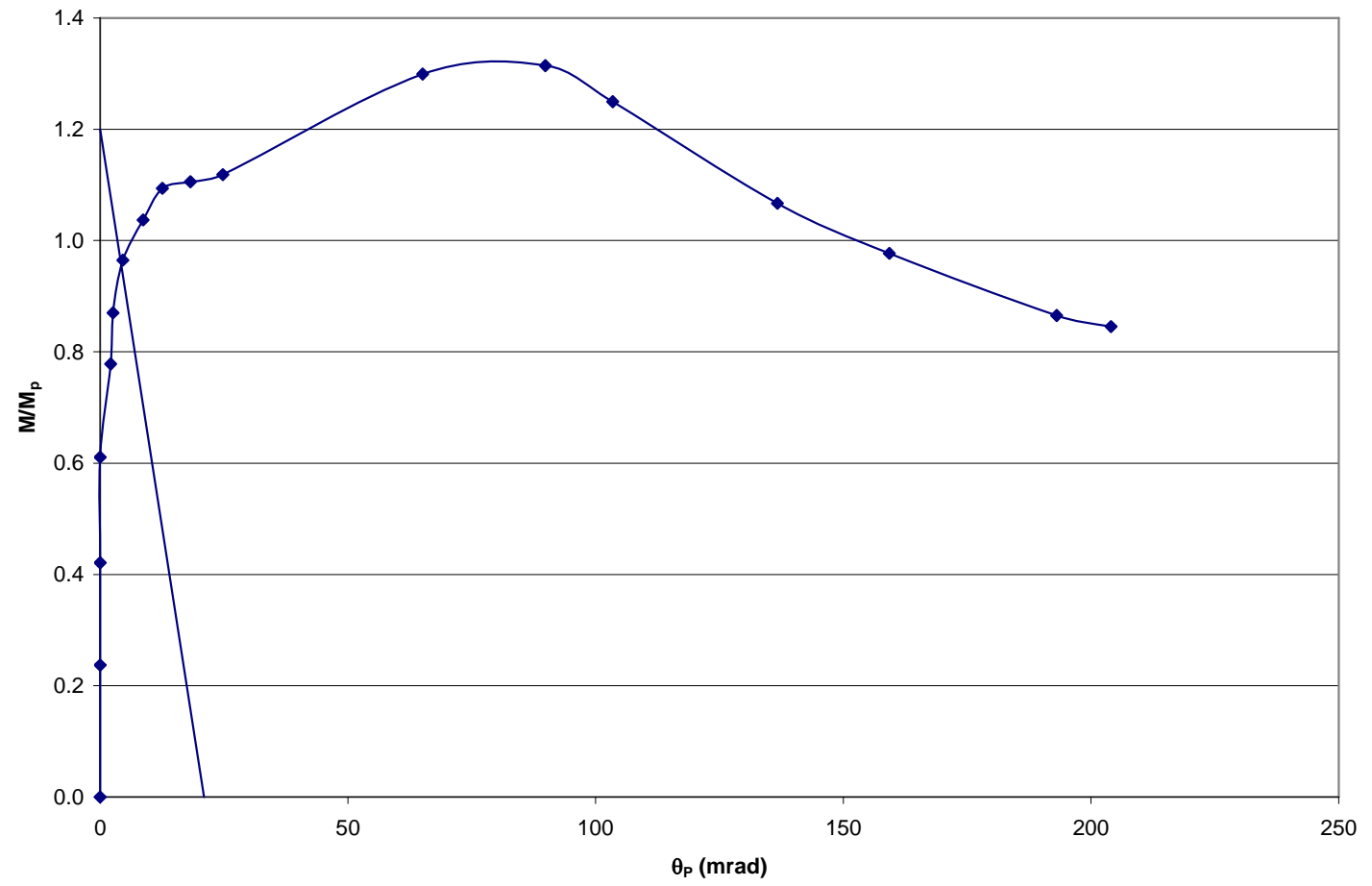

Figure B-28. Moment versus Rotation for Girder 188-3-5 (Grubb and Carskaddan 1981) 


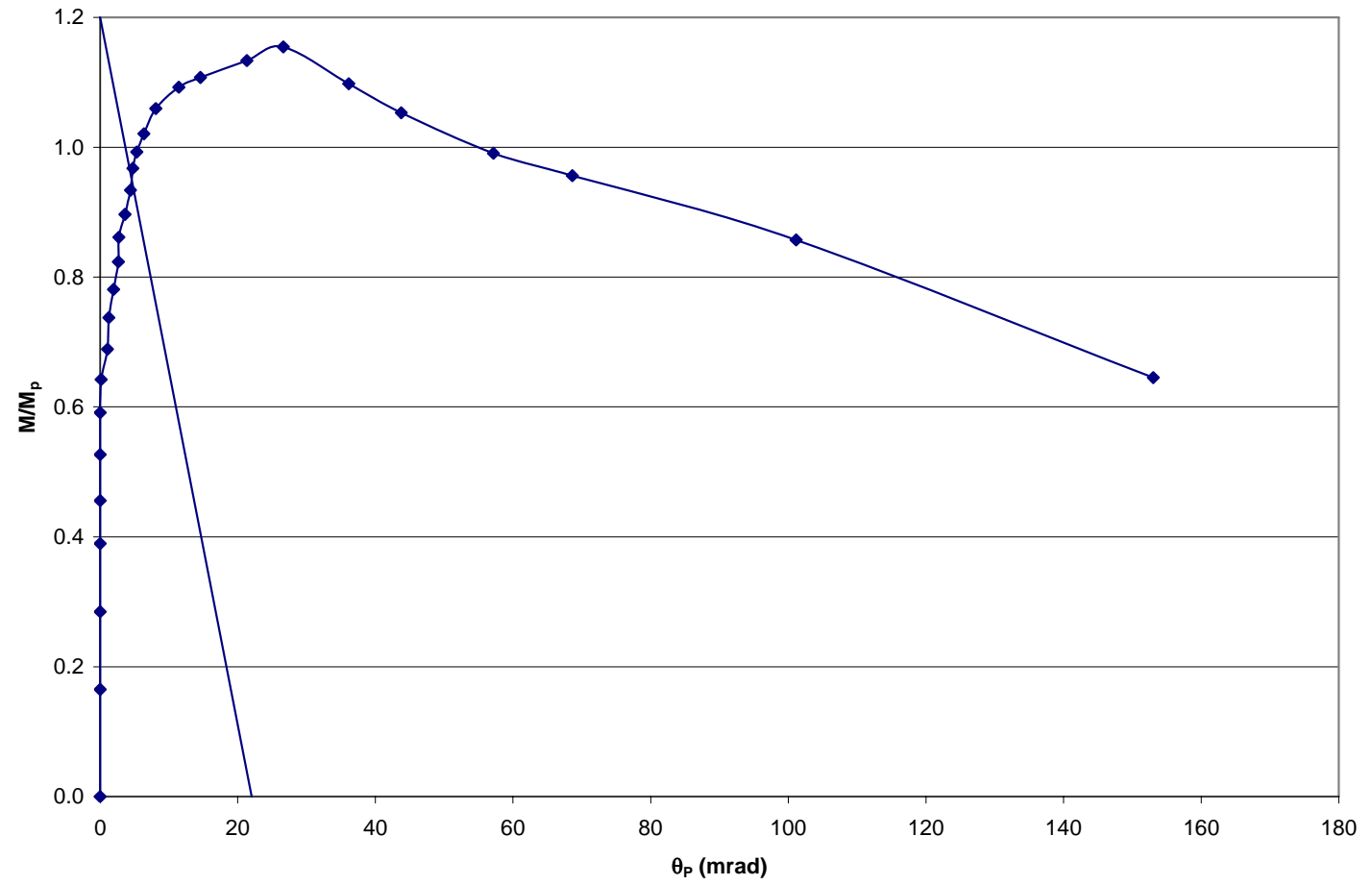

Figure B-29. Moment versus Rotation for Girder 188-3-6 (Grubb and Carskaddan 1981)

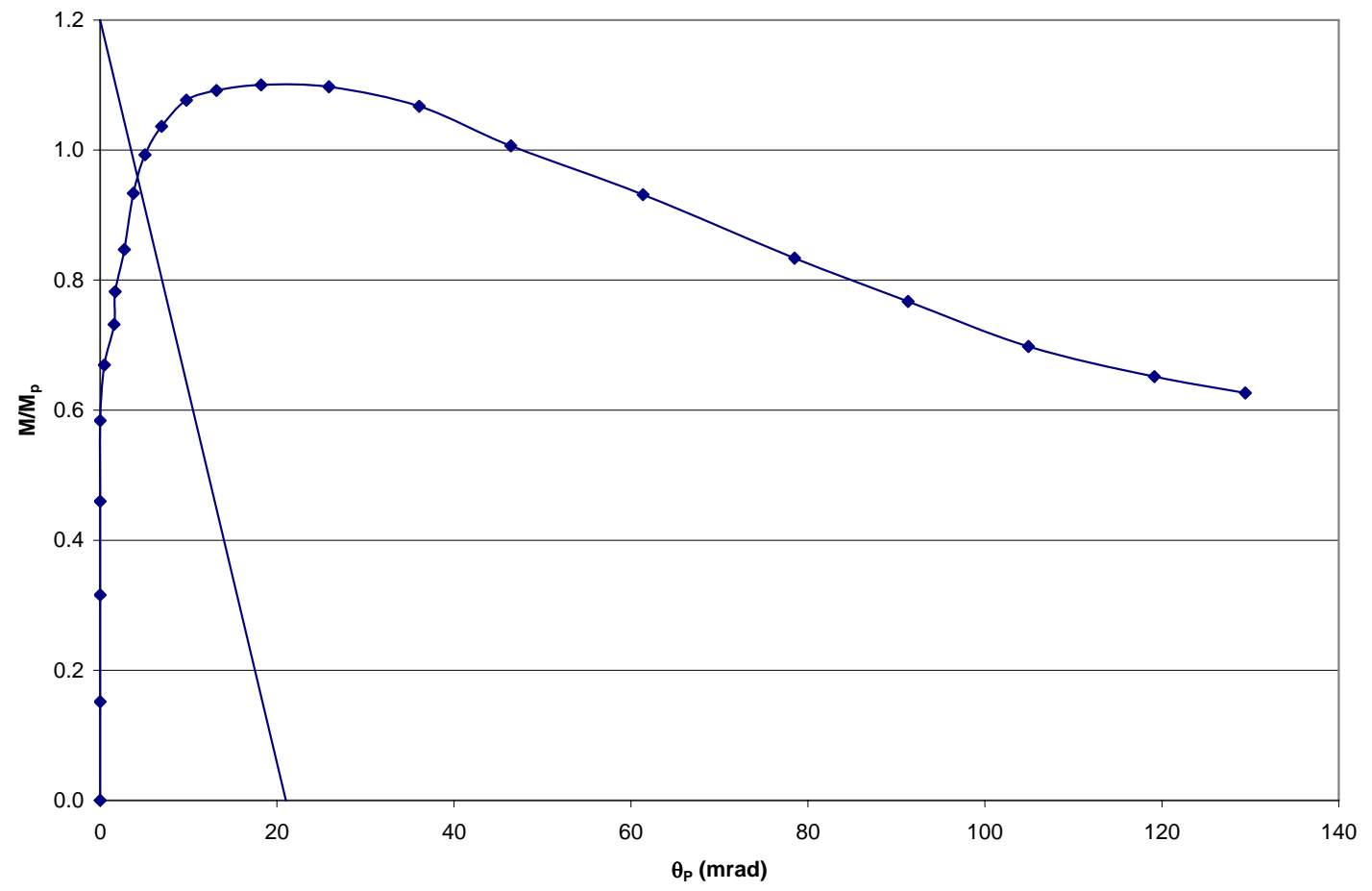

Figure B-30. Moment versus Rotation for Girder 188-3-7

(Grubb and Carskaddan 1981) 


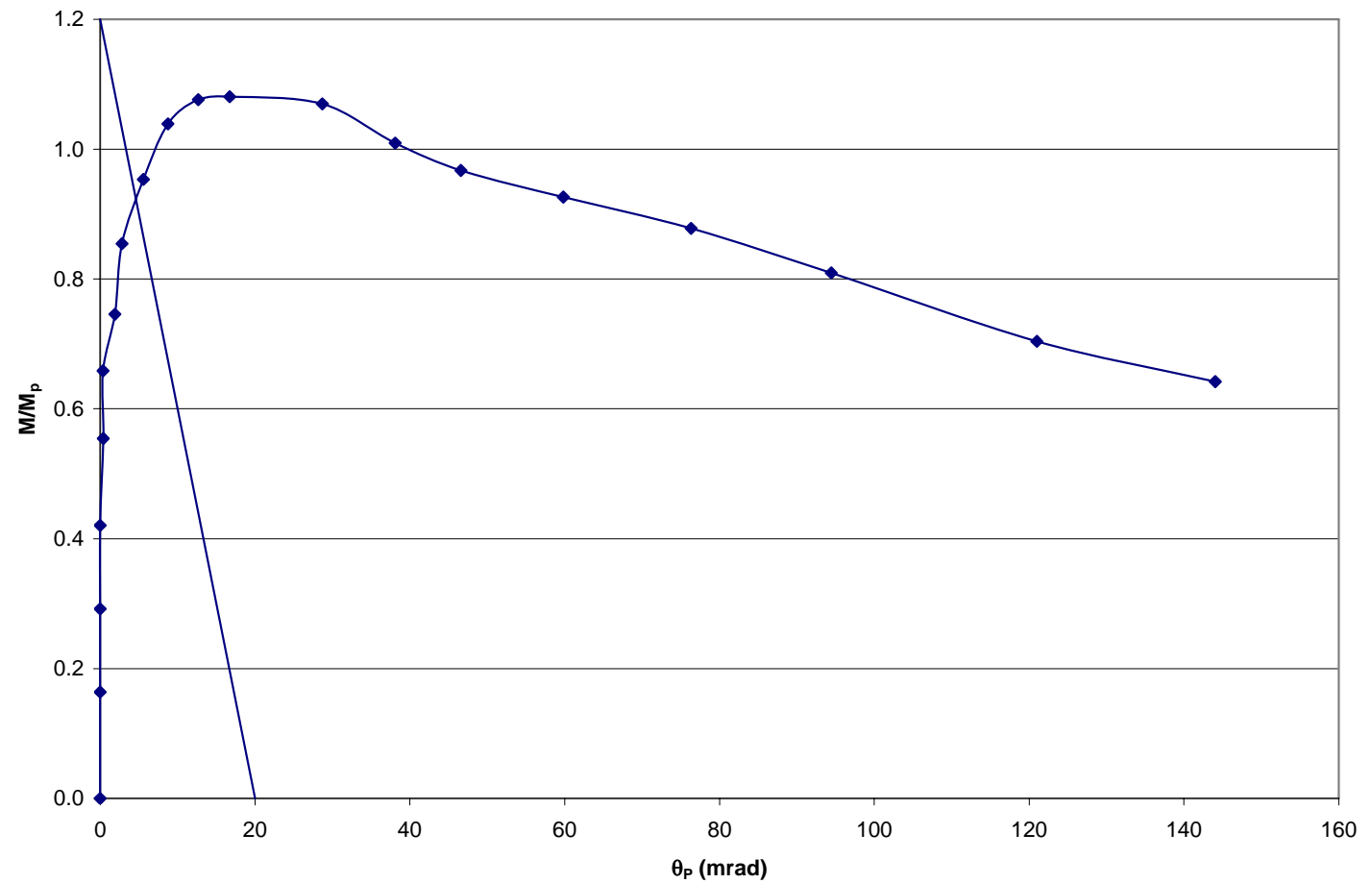

Figure B-31. Moment versus Rotation for Girder 188-3-8 (Grubb and Carskaddan 1981)

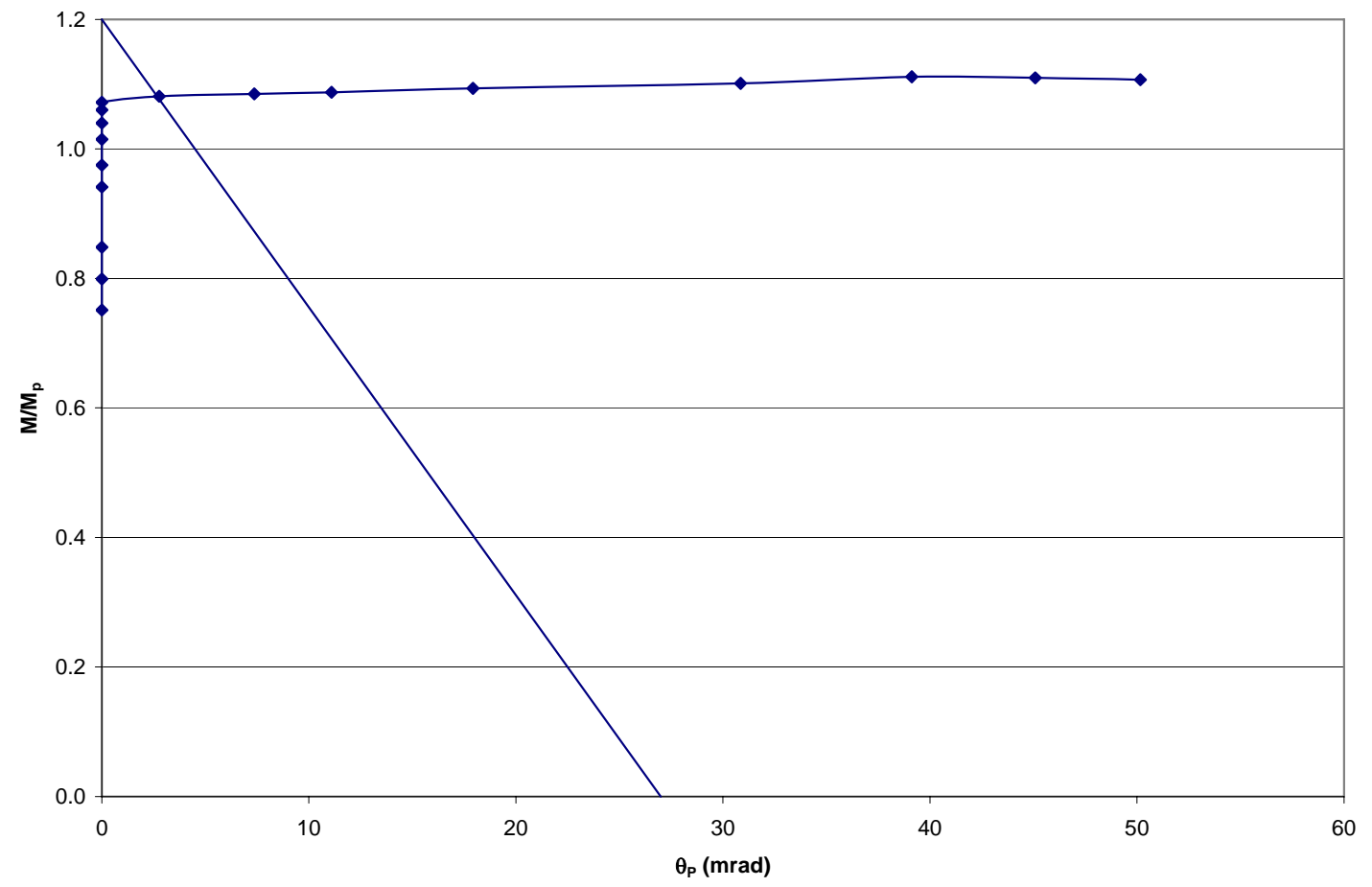

Figure B-32. Moment versus Rotation for Girder W14x26 (Hartnagel 1997) 


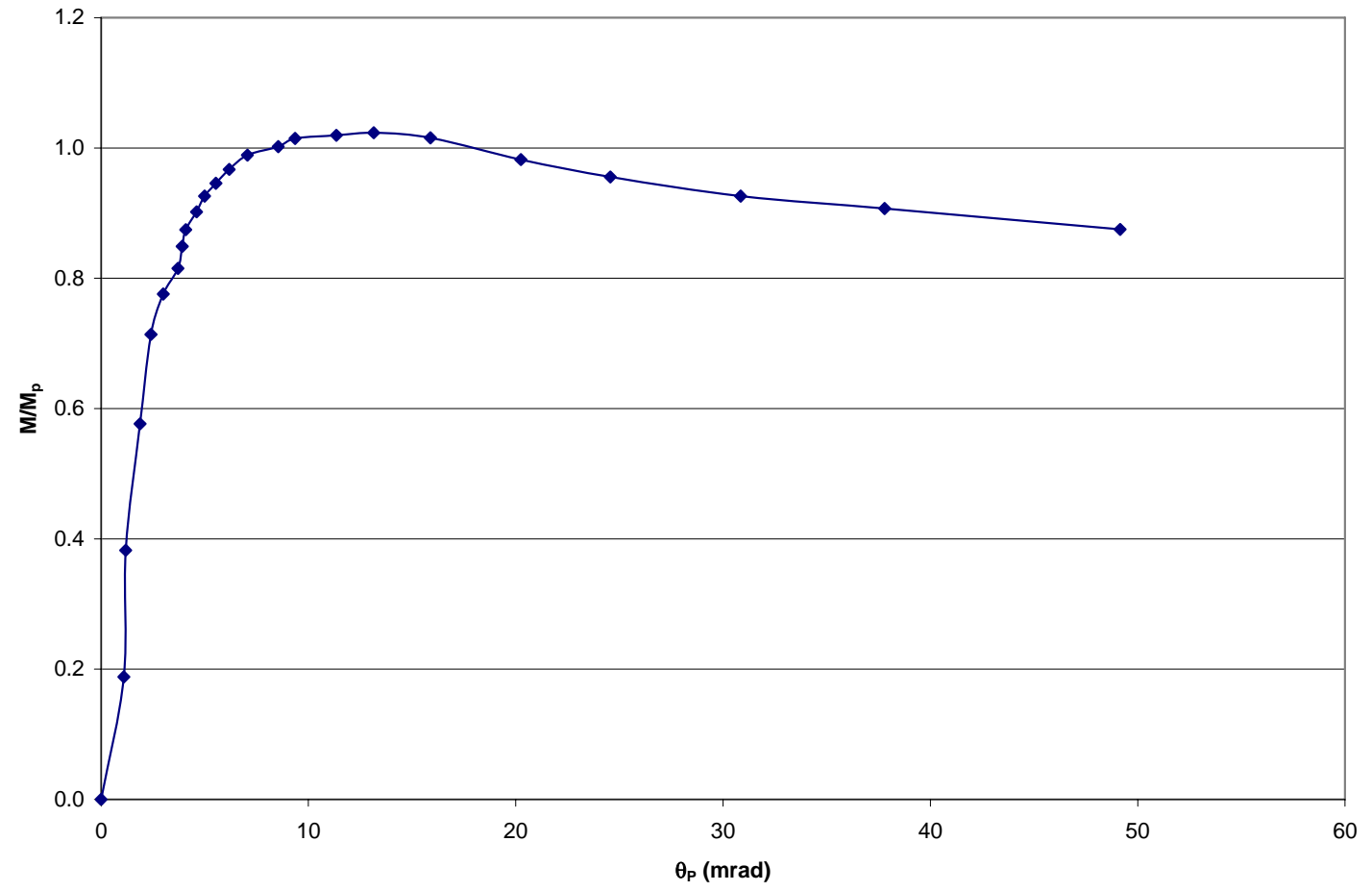

Figure B-33. Moment versus Rotation for Girder WS-1 (Holtz and Kulak 1973)

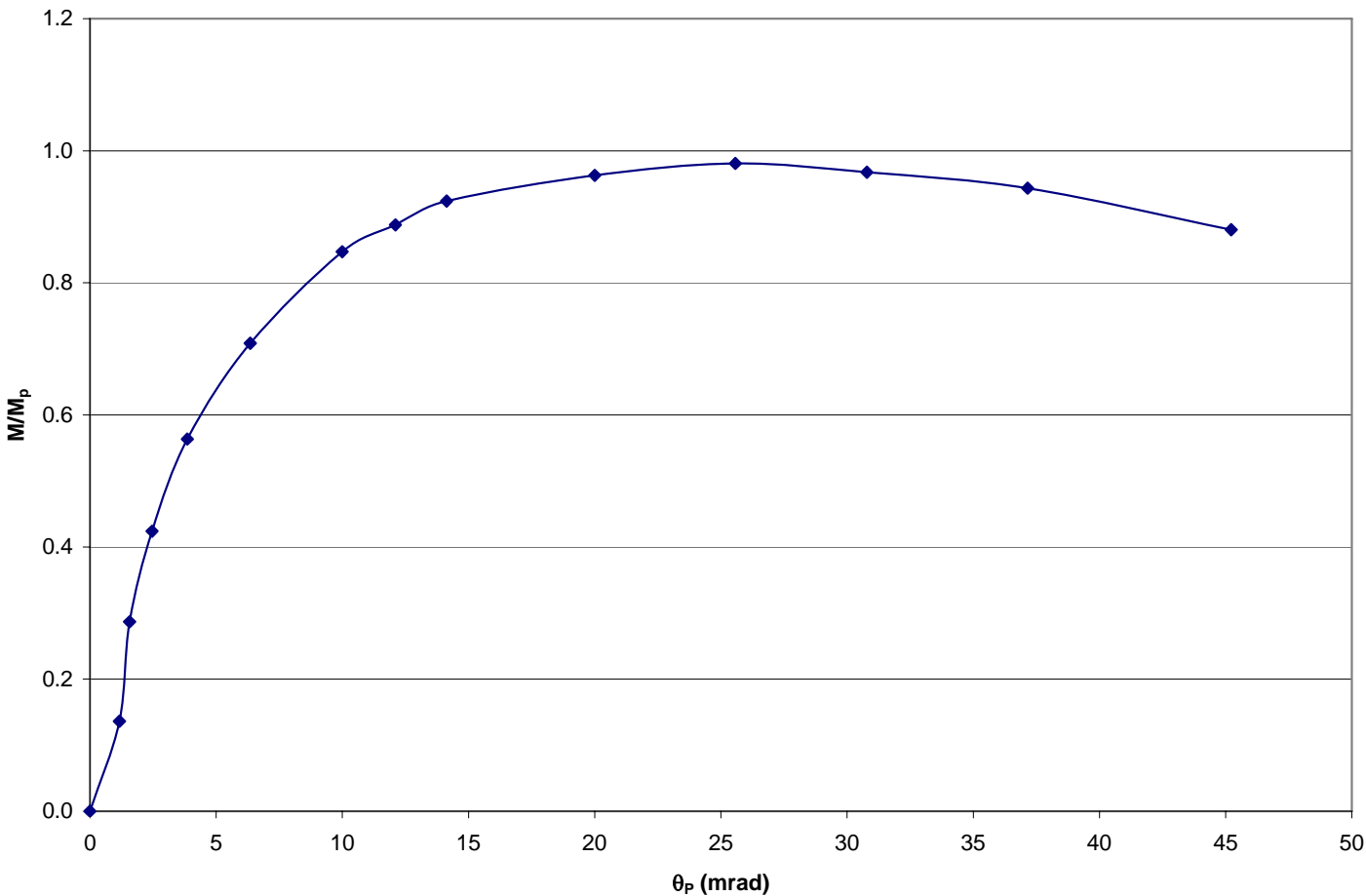

Figure B-34. Moment versus Rotation for Girder WS-2 (Holtz and Kulak 1973) 


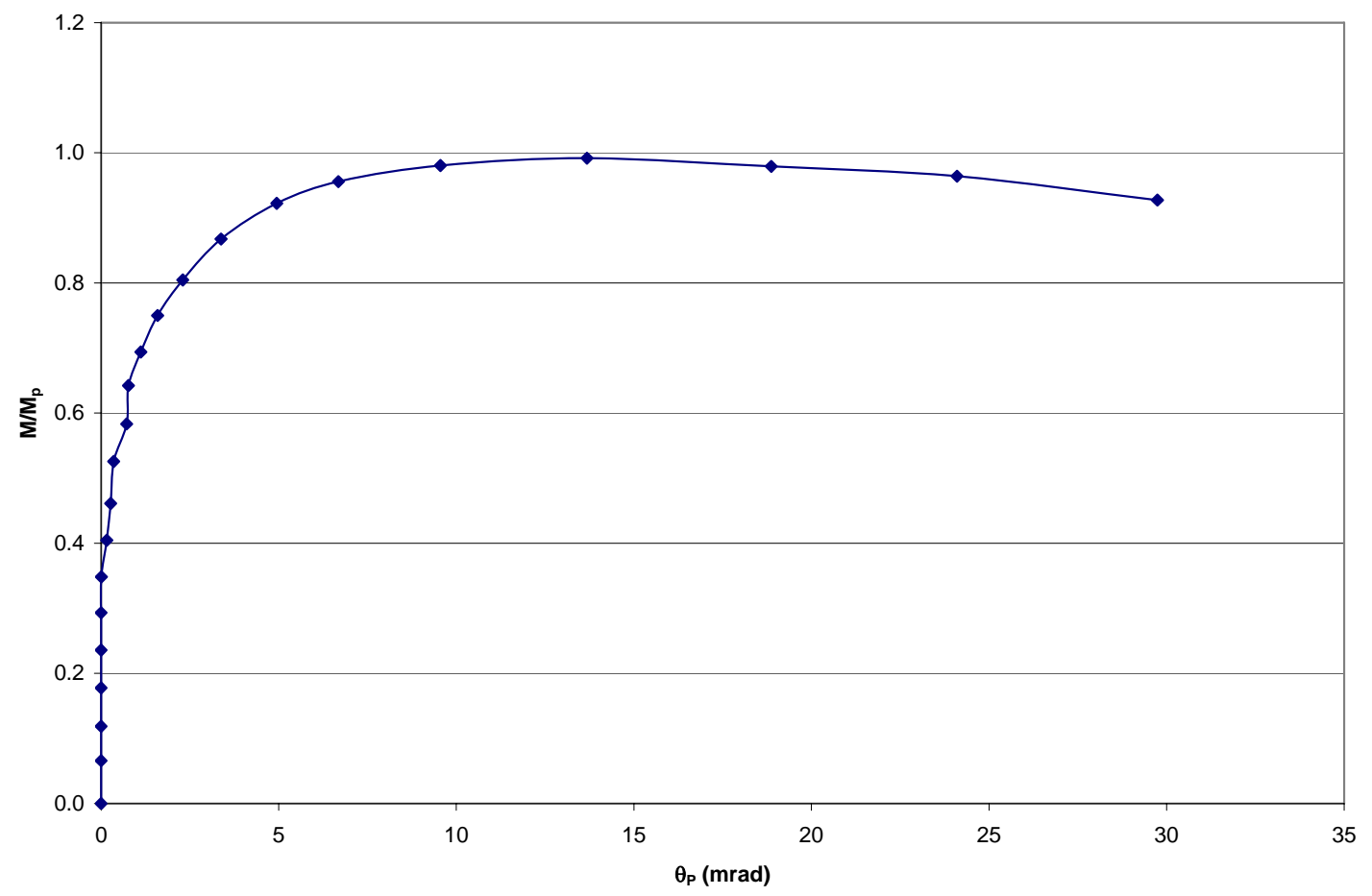

Figure B-35. Moment versus Rotation for Girder WS-7-P (Holtz and Kulak 1973)

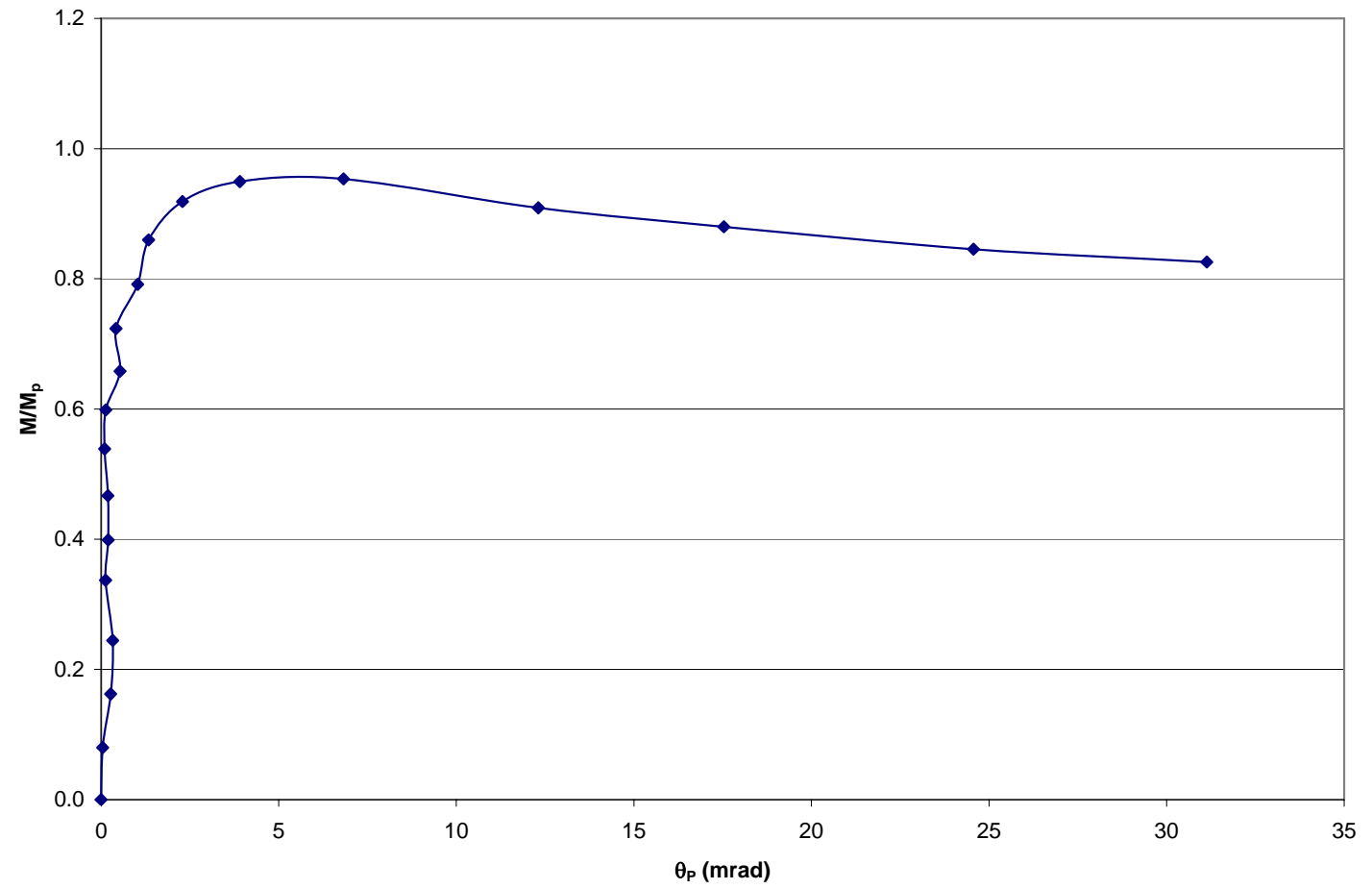

Figure B-36. Moment versus Rotation for Girder WS-8-P (Holtz and Kulak 1973) 


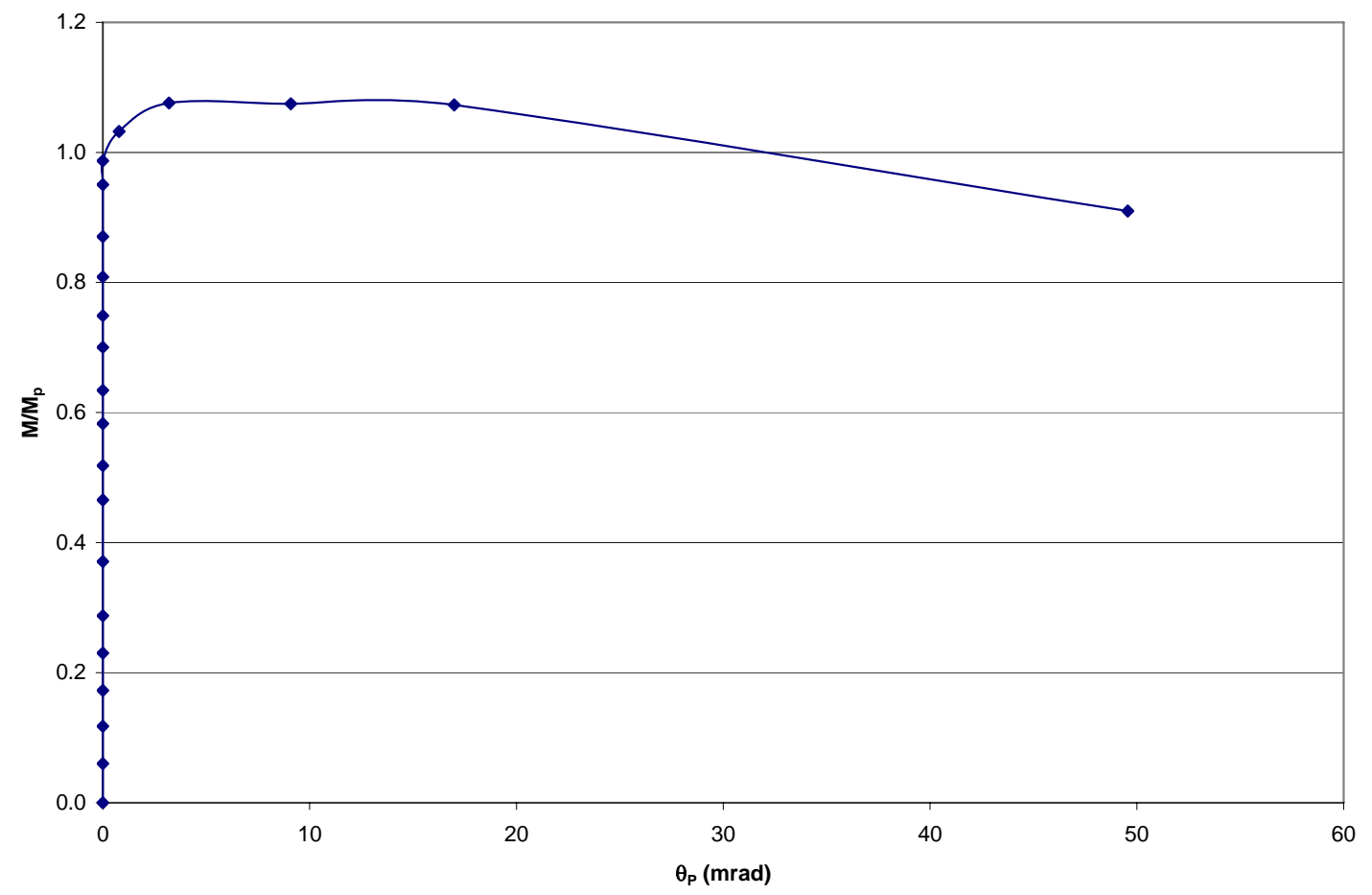

Figure B-37. Moment versus Rotation for Girder WS-9 (Holtz and Kulak 1973)

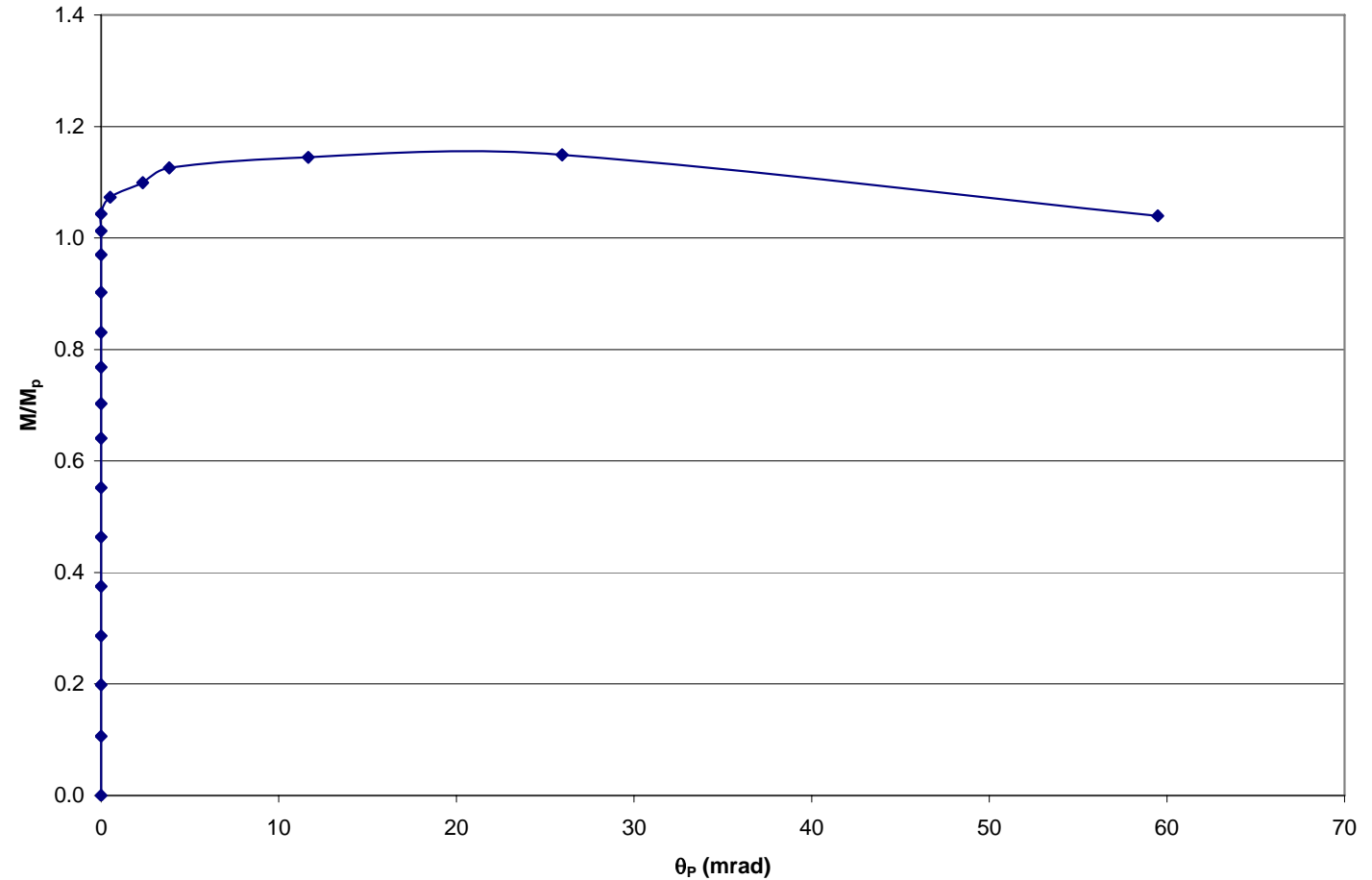

Figure B-38. Moment versus Rotation for Girder WS-10 (Holtz and Kulak 1973) 


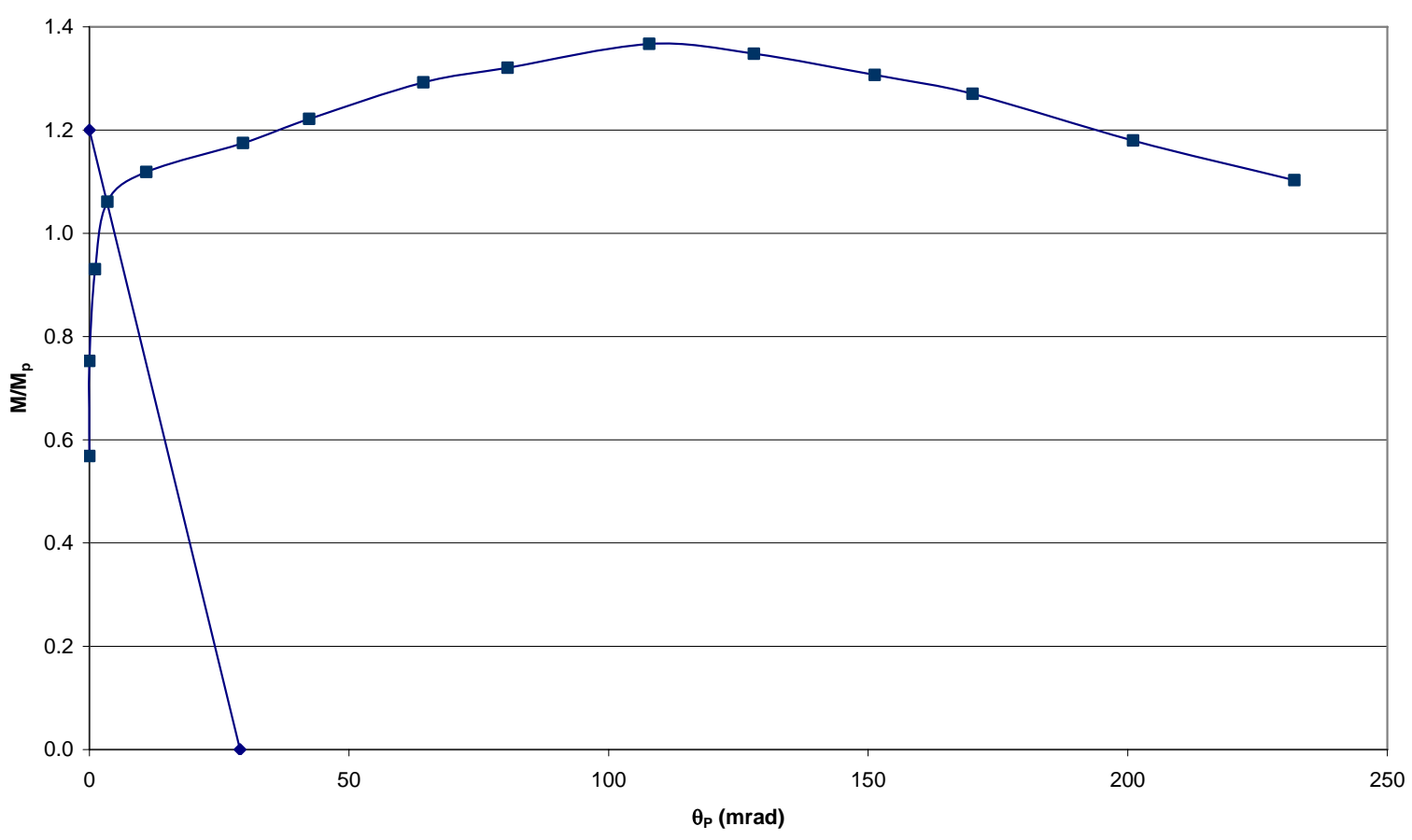

Figure B-39. Moment versus Rotation for Girder A-1 (Lukey et al. 1969)

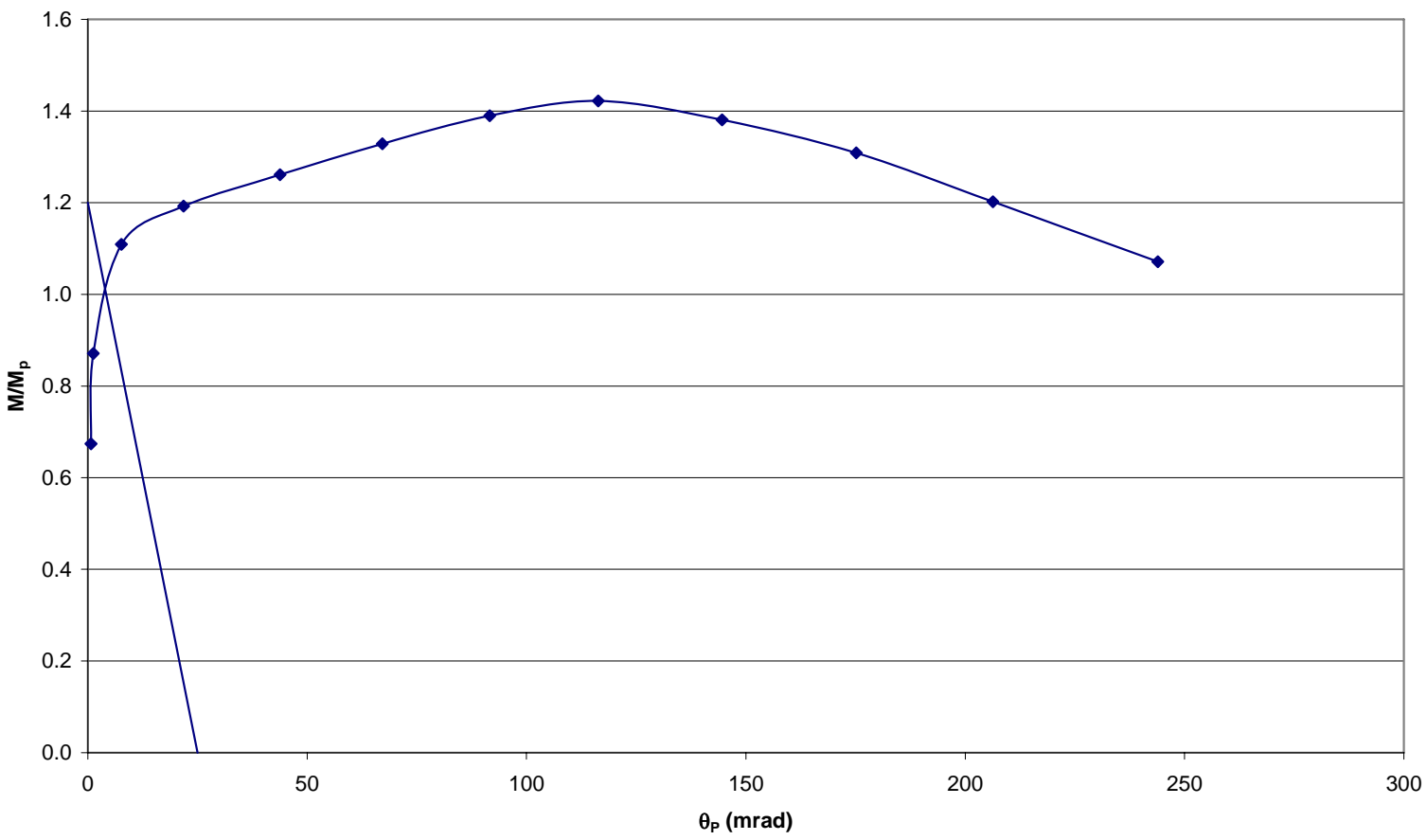

Figure B-40. Moment versus Rotation for Girder A-2 (Lukey et al. 1969) 


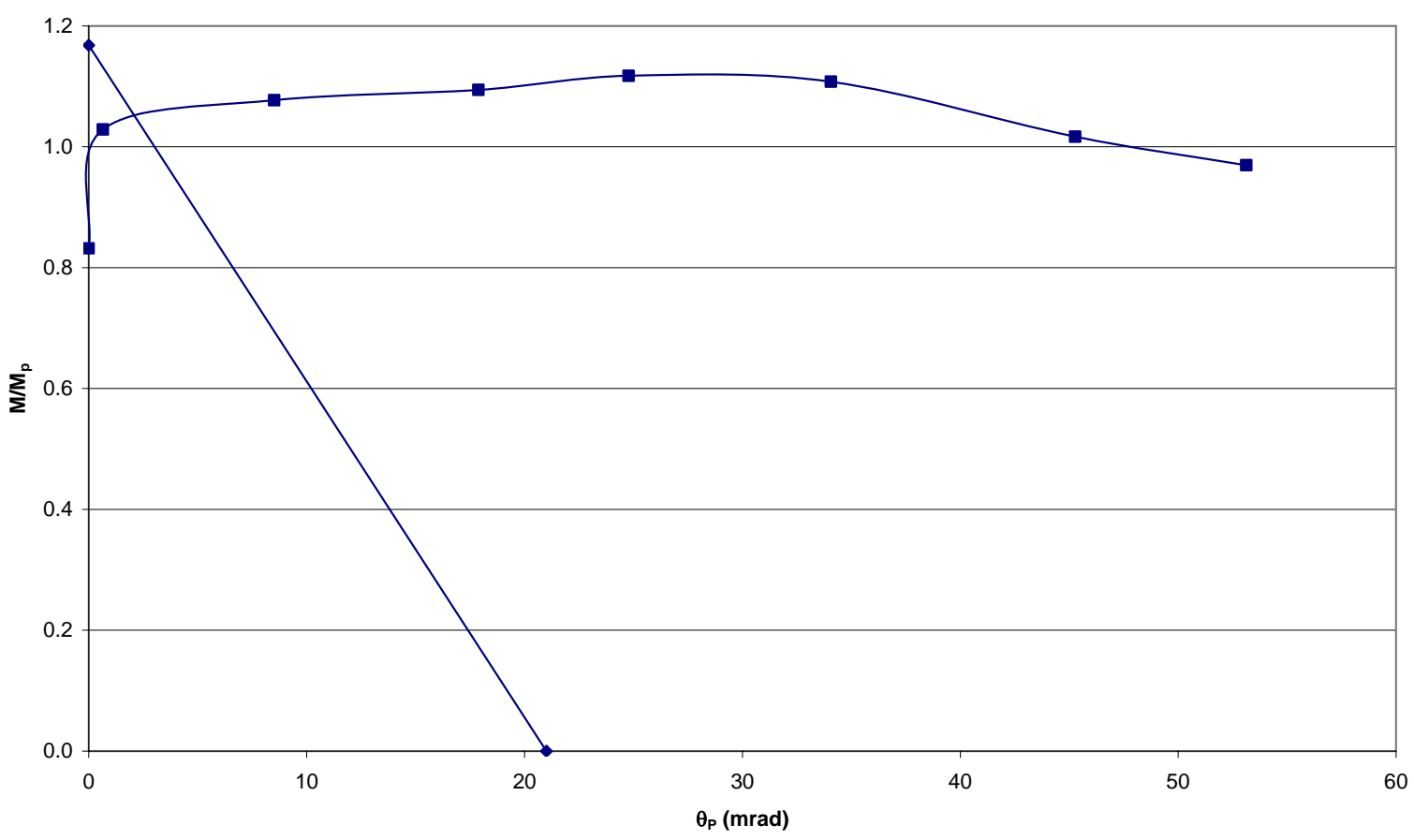

Figure B-41. Moment versus Rotation for Girder B-1 (Lukey et al. 1969)

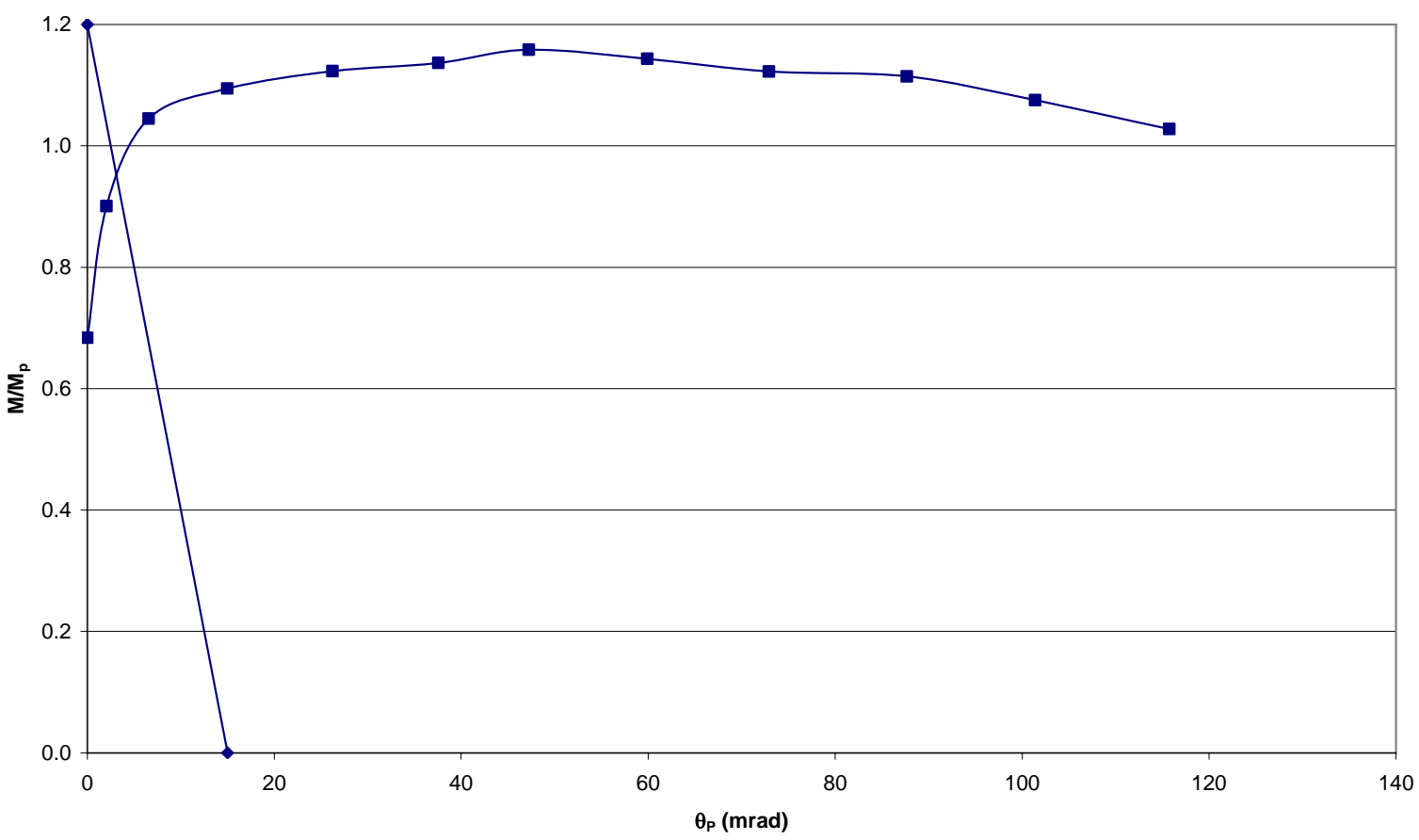

Figure B-42. Moment versus Rotation for Girder B-2 (Lukey et al. 1969) 


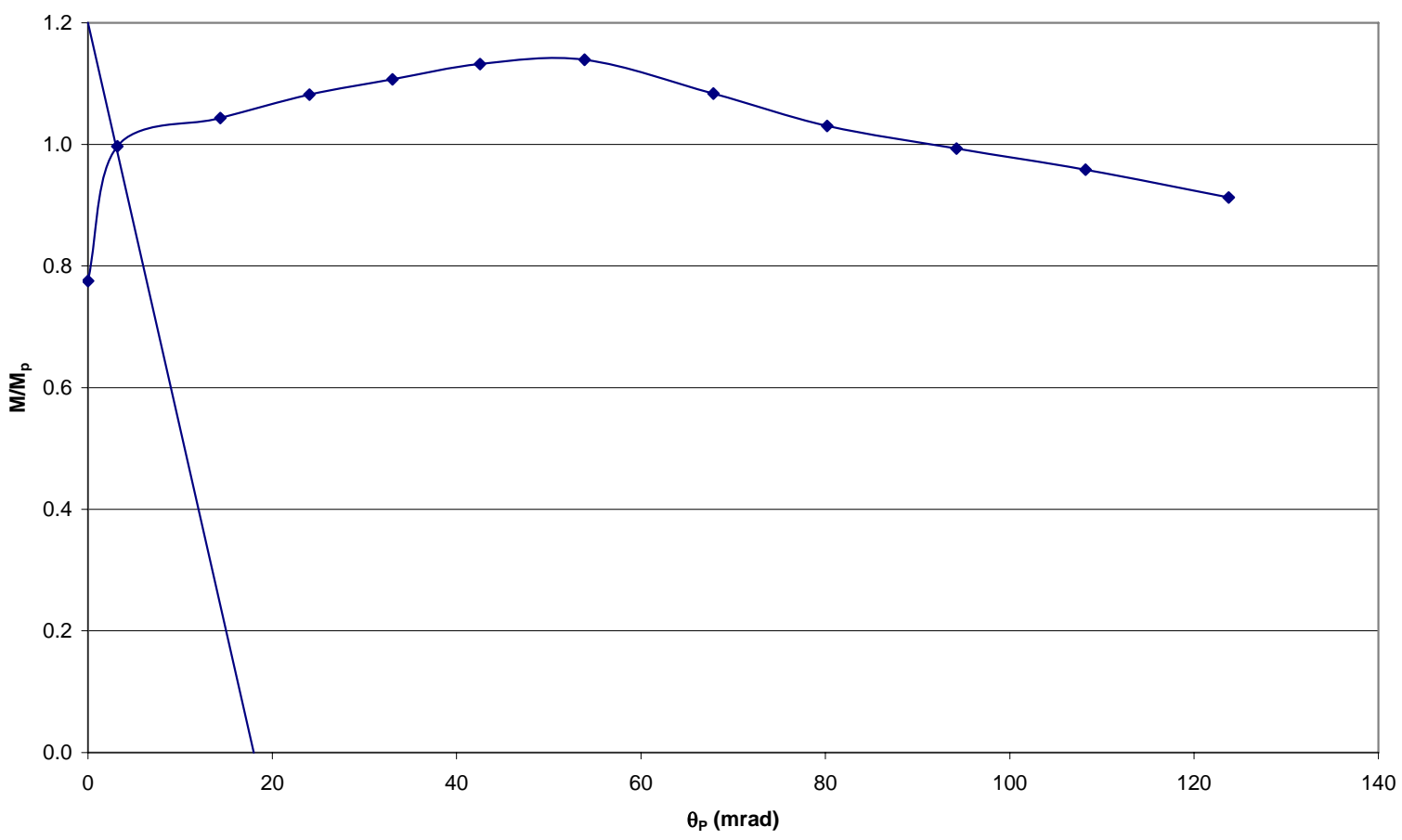

Figure B-43. Moment versus Rotation for Girder B-3 (Lukey et al. 1969)

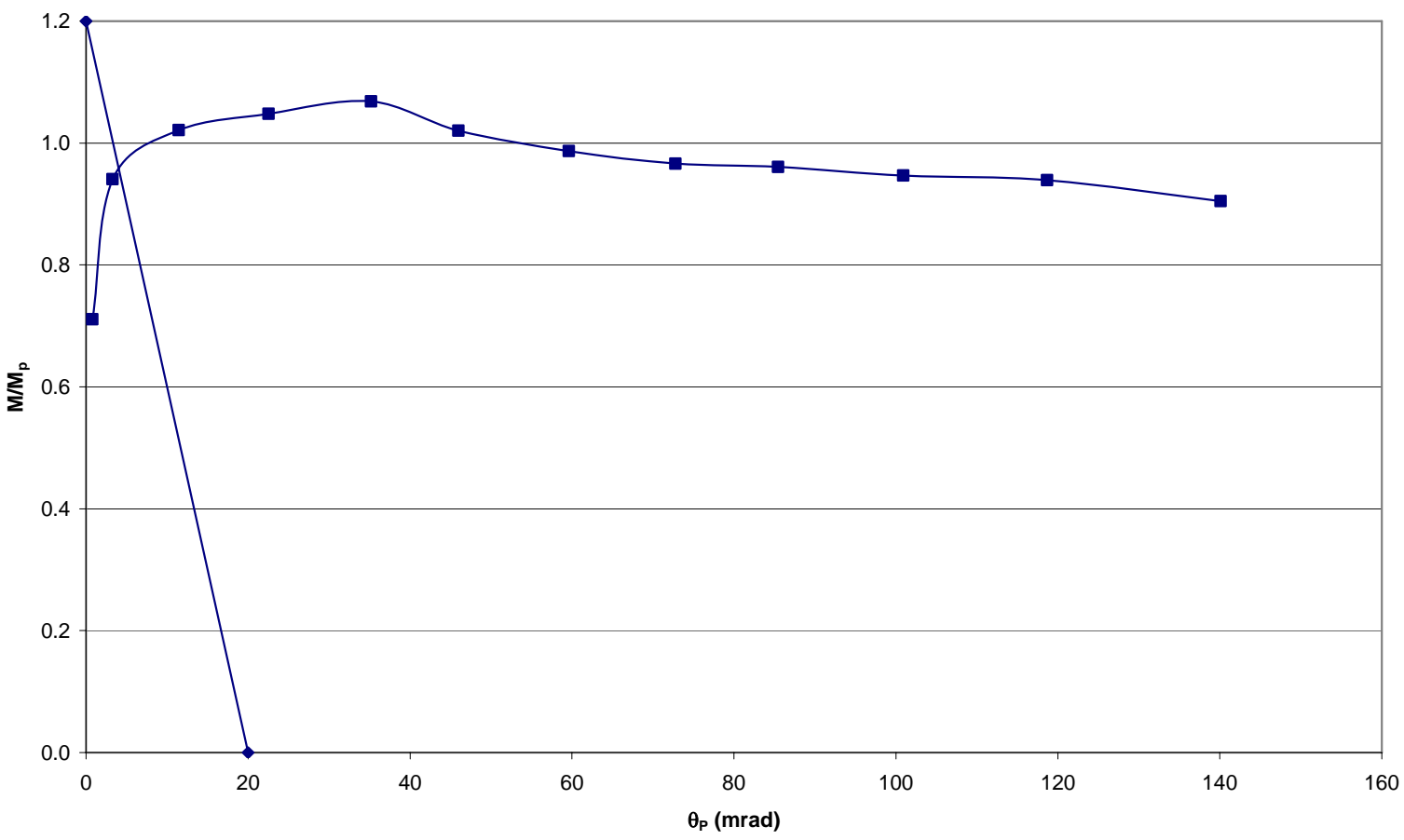

Figure B-44. Moment versus Rotation for Girder B-4 (Lukey et al. 1969) 


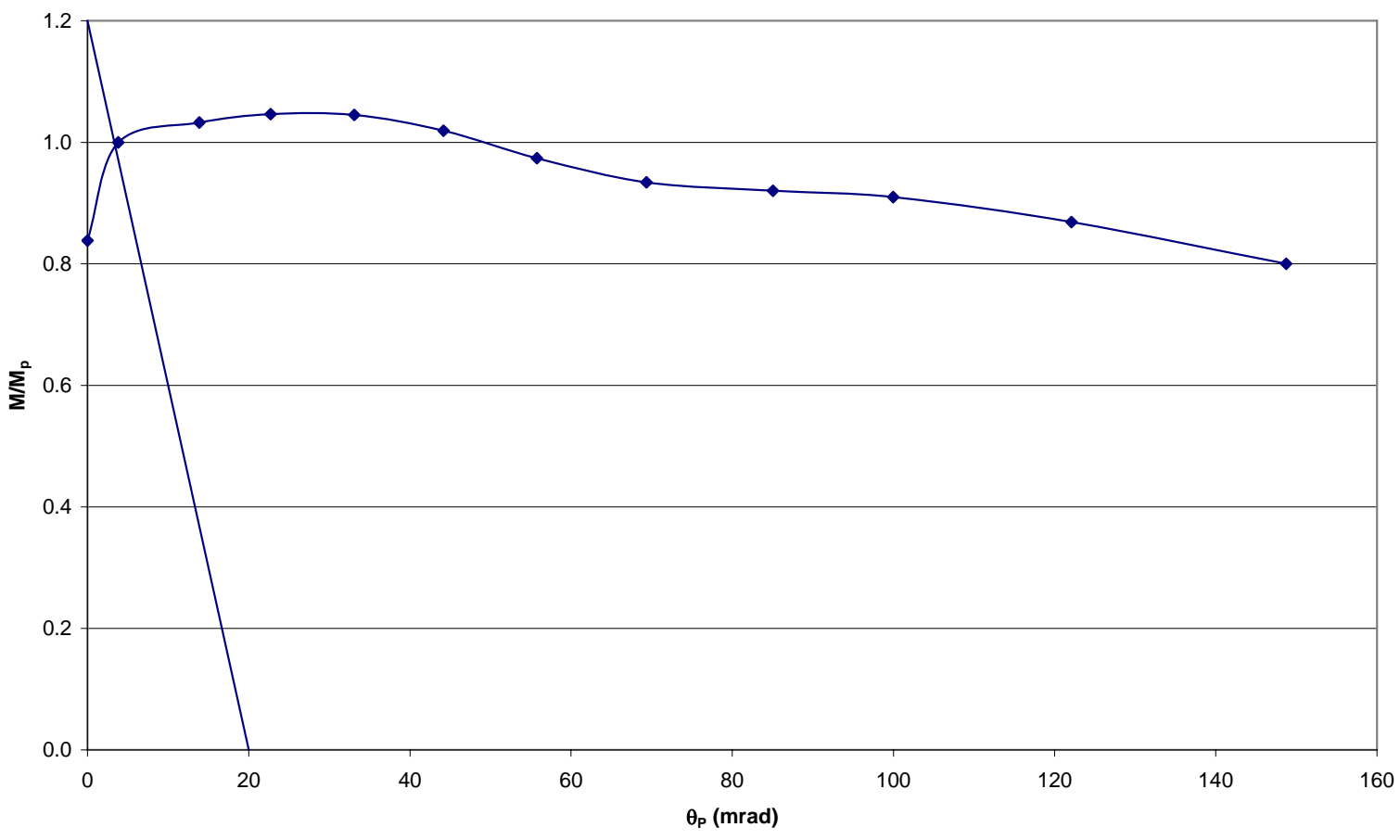

Figure B-45. Moment versus Rotation for Girder B-5 (Lukey et al. 1969)

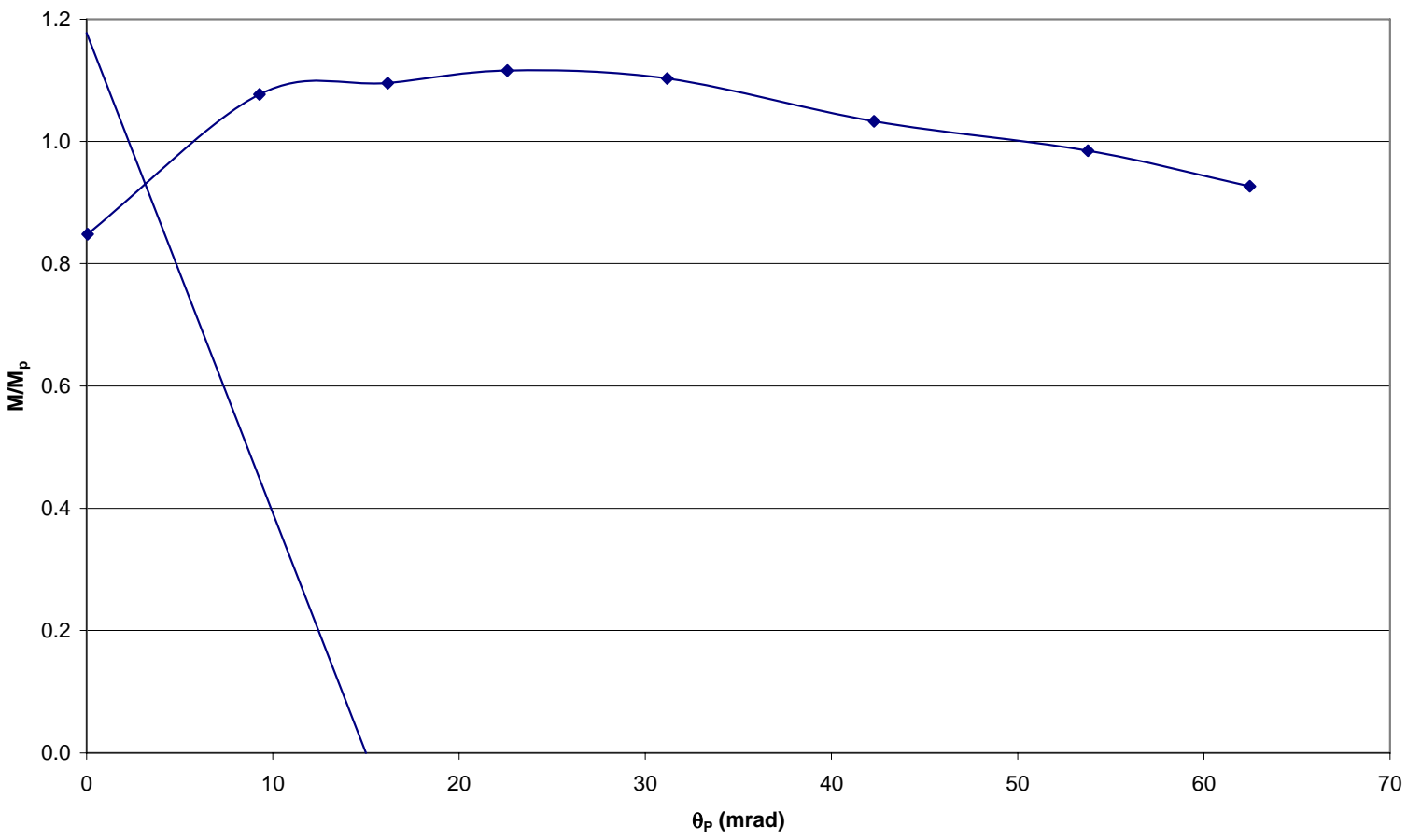

Figure B-46. Moment versus Rotation for Girder C-1 (Lukey et al. 1969) 


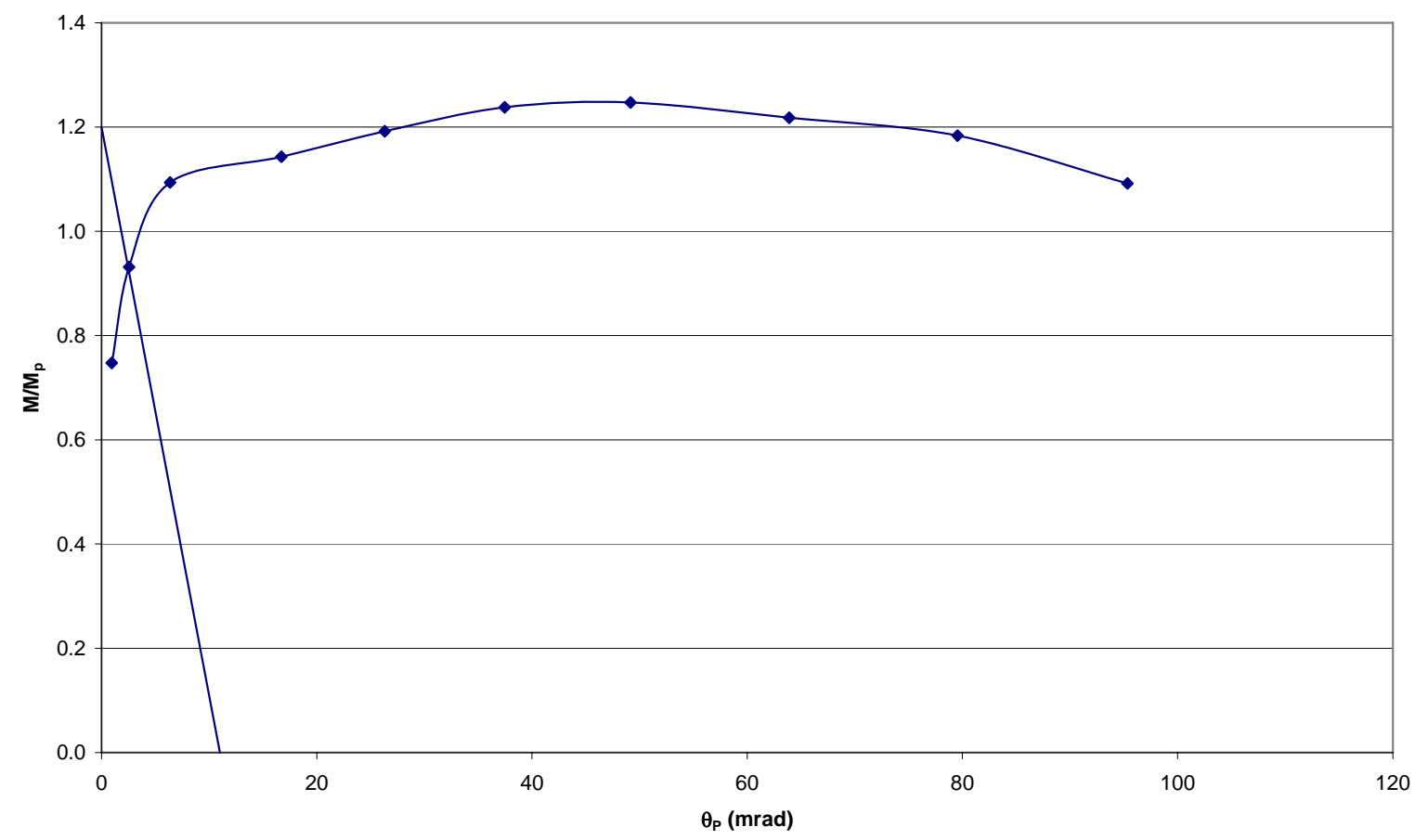

Figure B-47. Moment versus Rotation for Girder C-2 (Lukey et al. 1969)

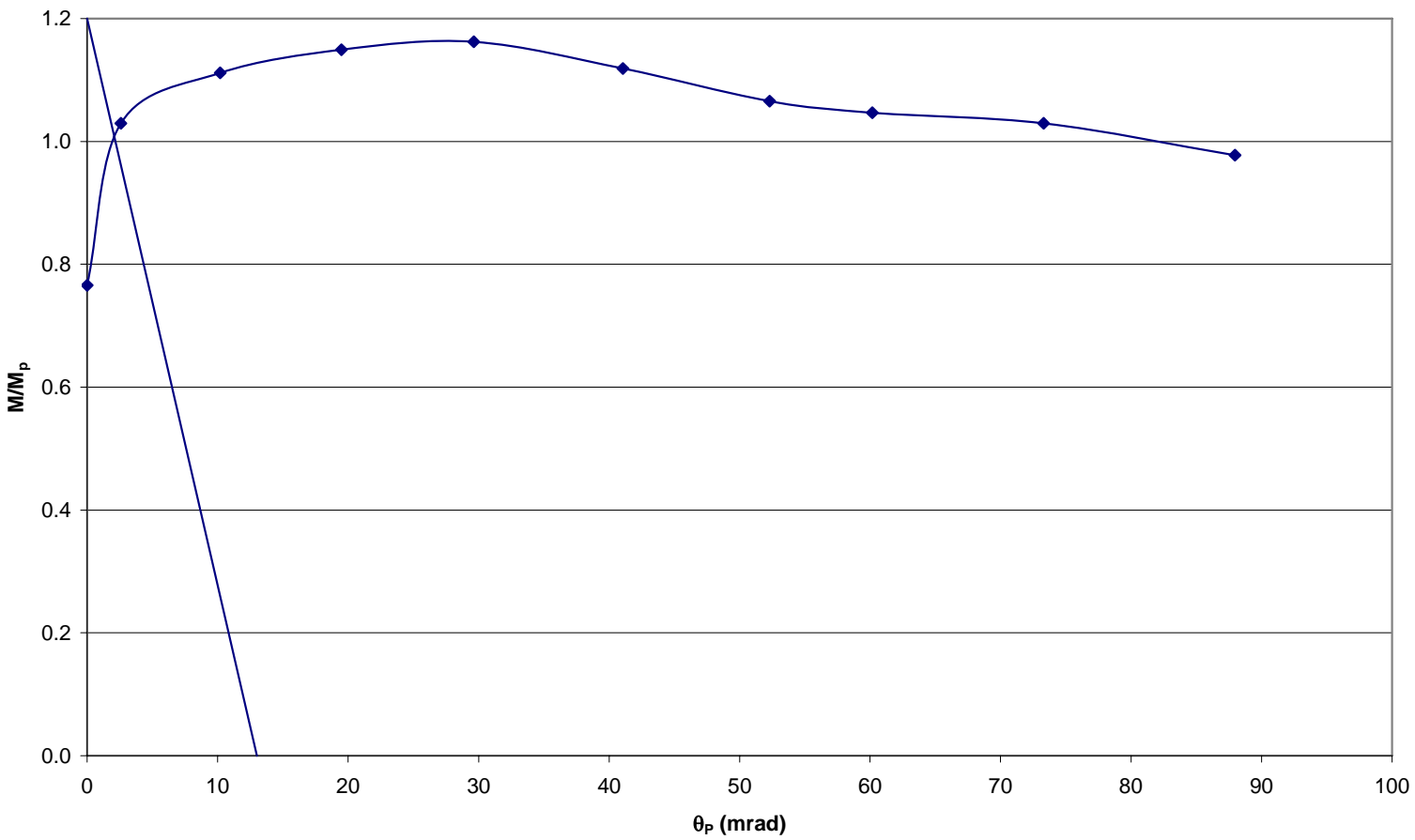

Figure B-48. Moment versus Rotation for Girder C-3 (Lukey et al. 1969) 


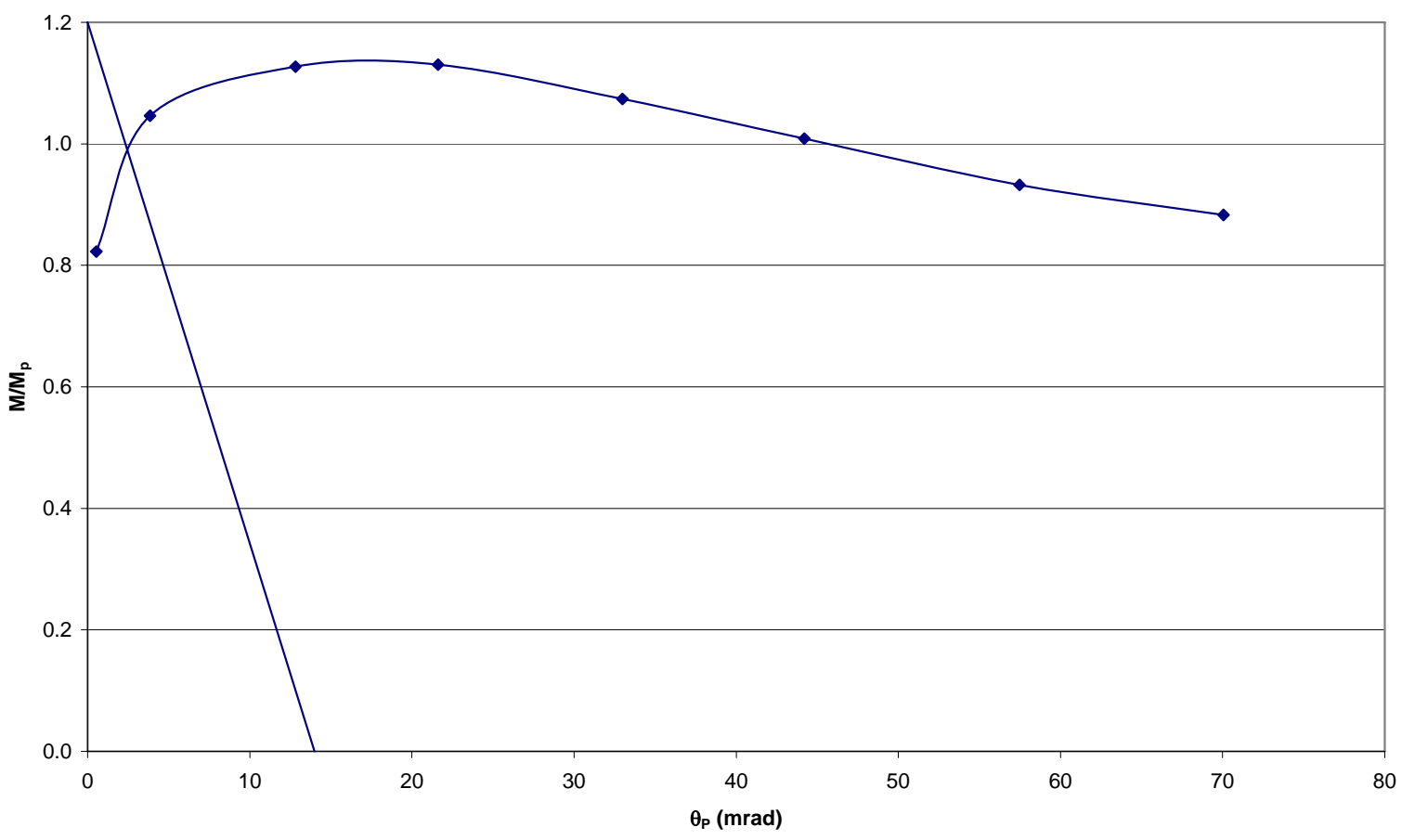

Figure B-49. Moment versus Rotation for Girder C-4 (Lukey et al. 1969)

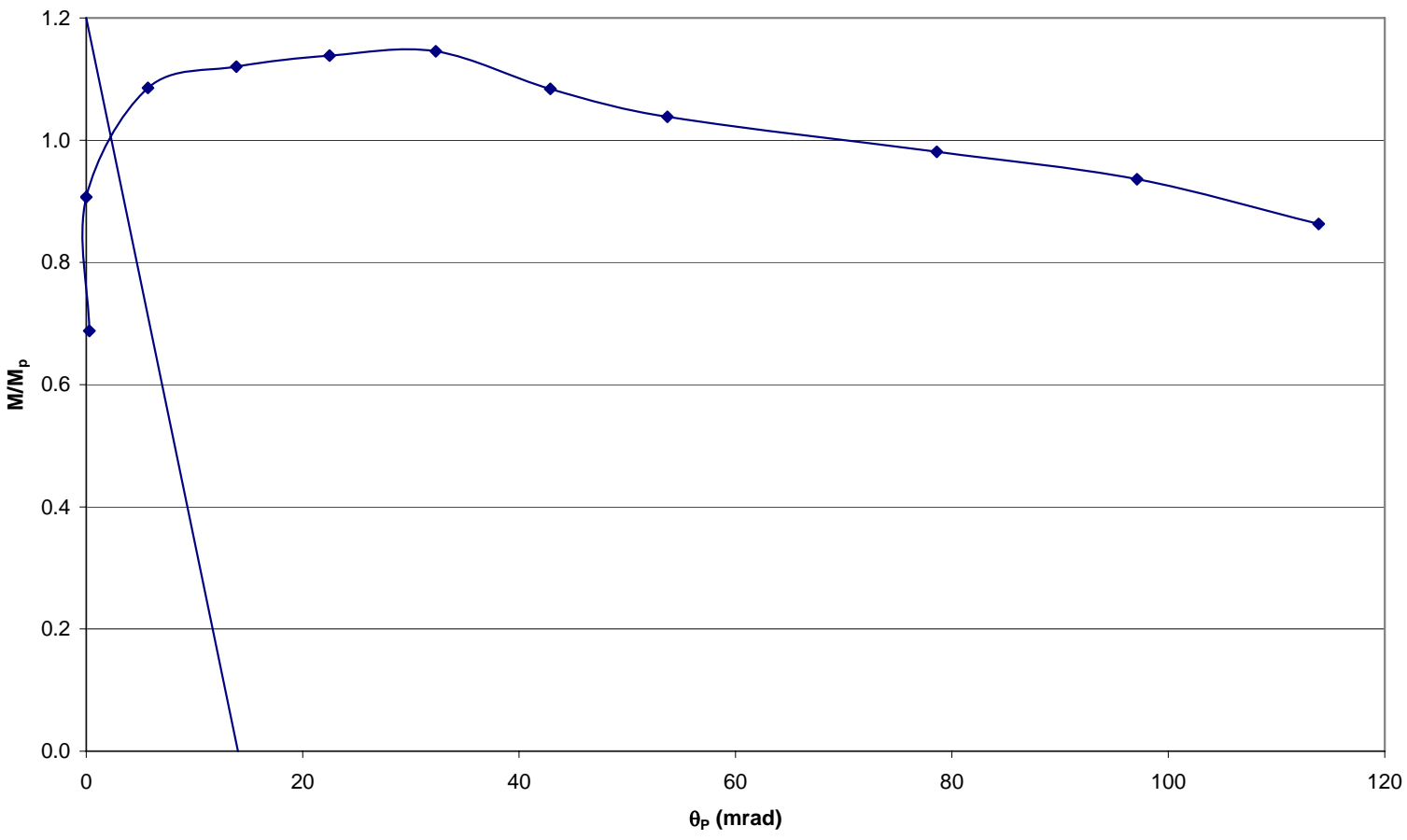

Figure B-50. Moment versus Rotation for Girder C-5 (Lukey et al. 1969) 


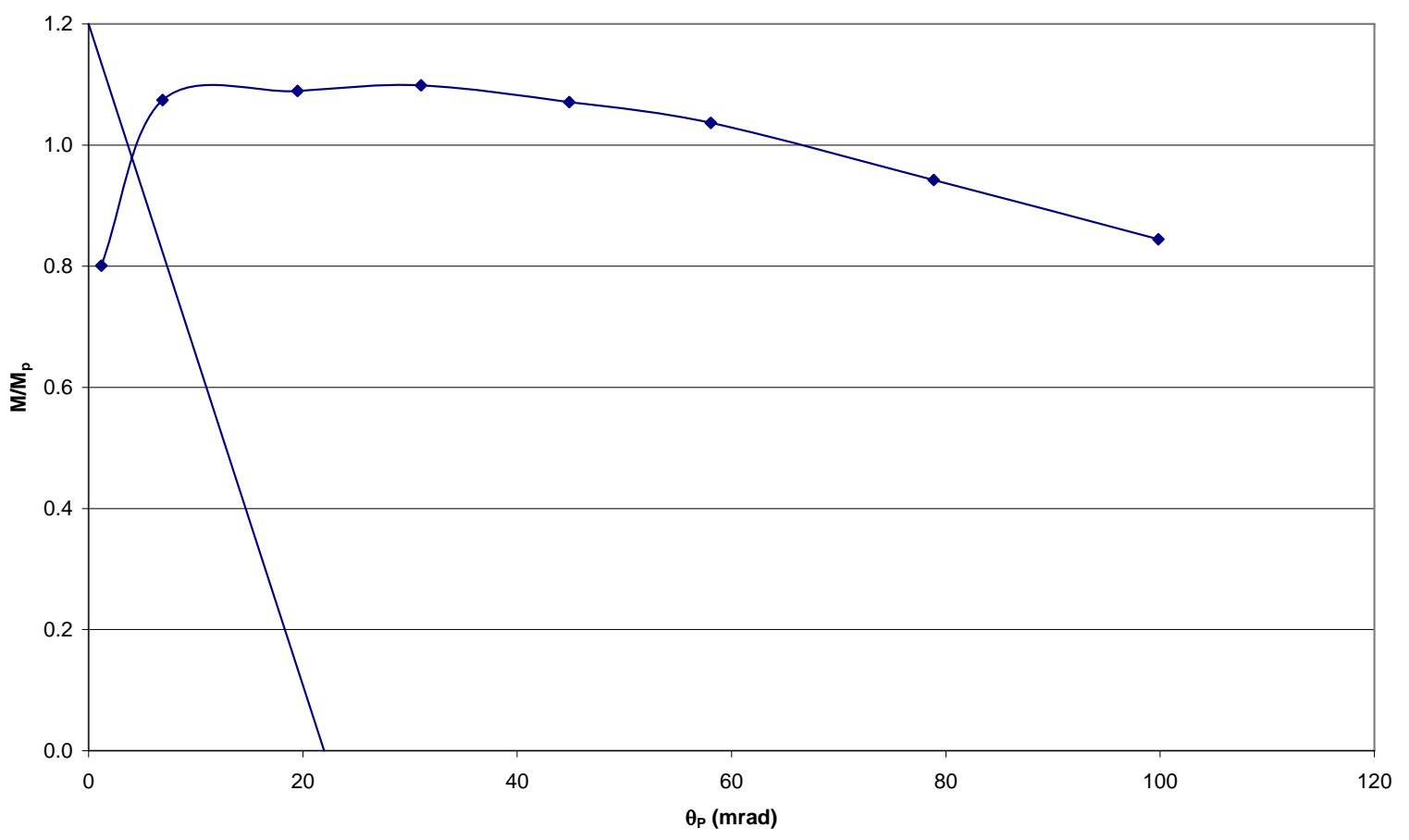

Figure B-51. Moment versus Rotation for Girder D-1 (Lukey et al. 1969)

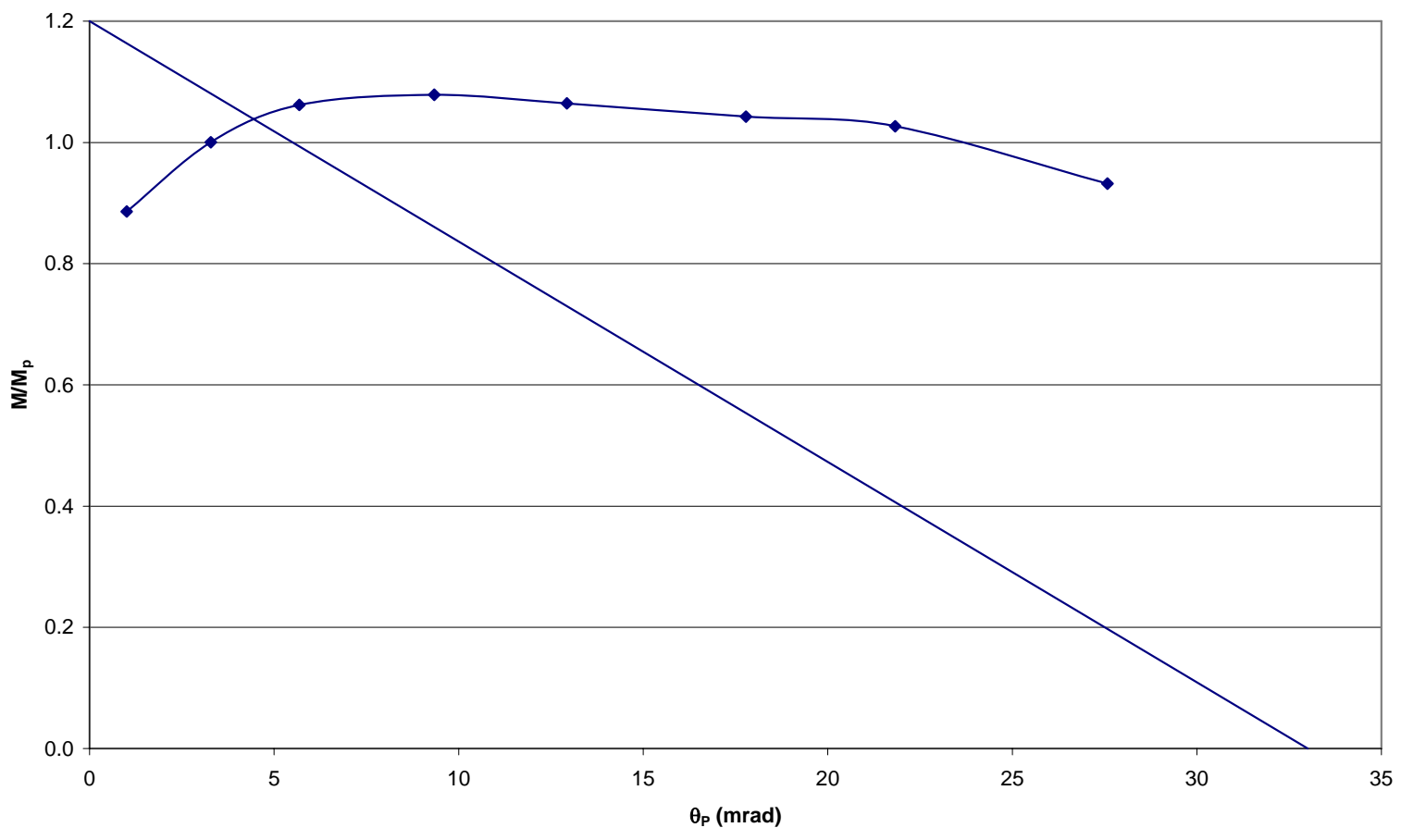

Figure B-52. Moment versus Rotation for Girder D-2 (Lukey et al. 1969) 


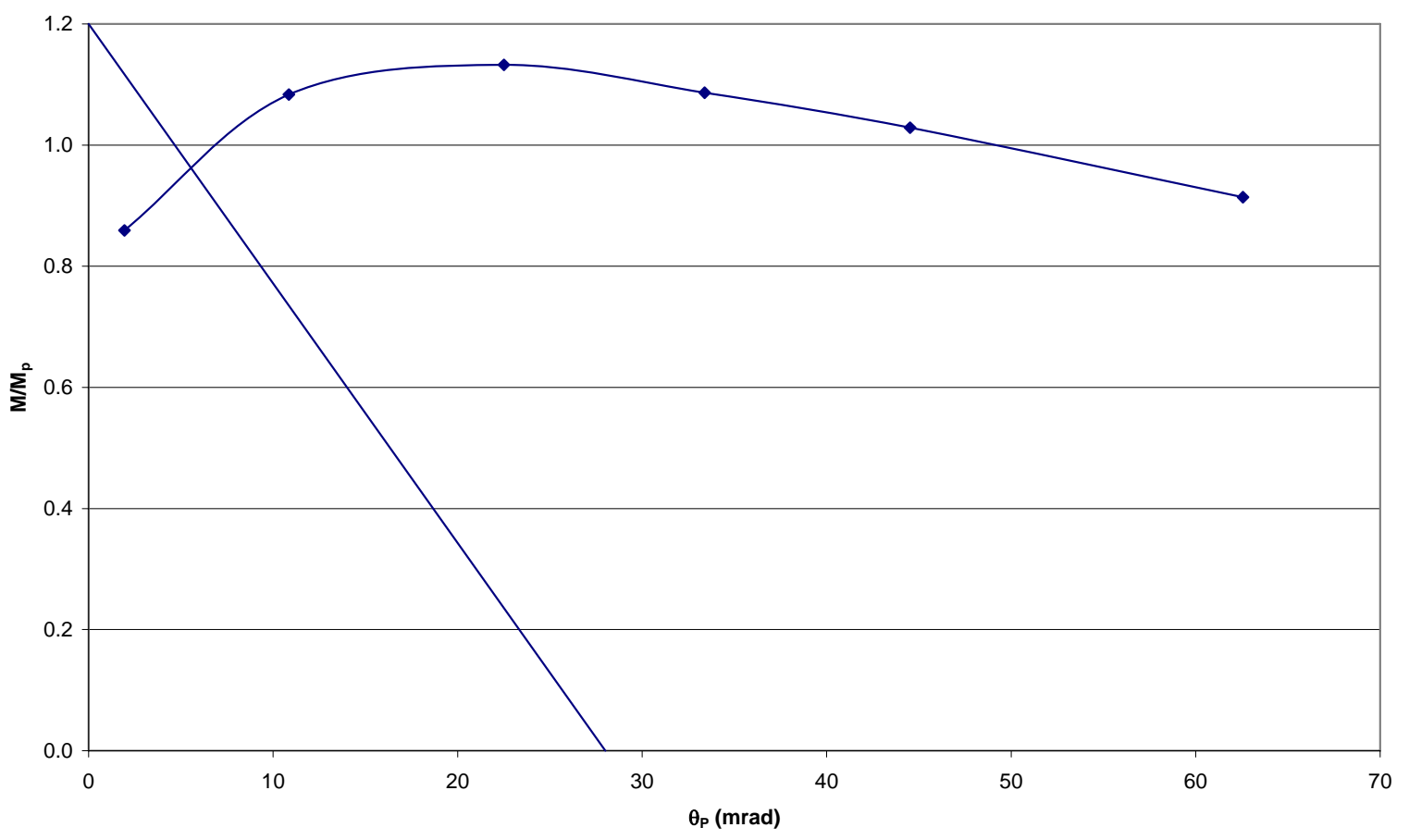

Figure B-53. Moment versus Rotation for Girder D-5 (Lukey et al. 1969)

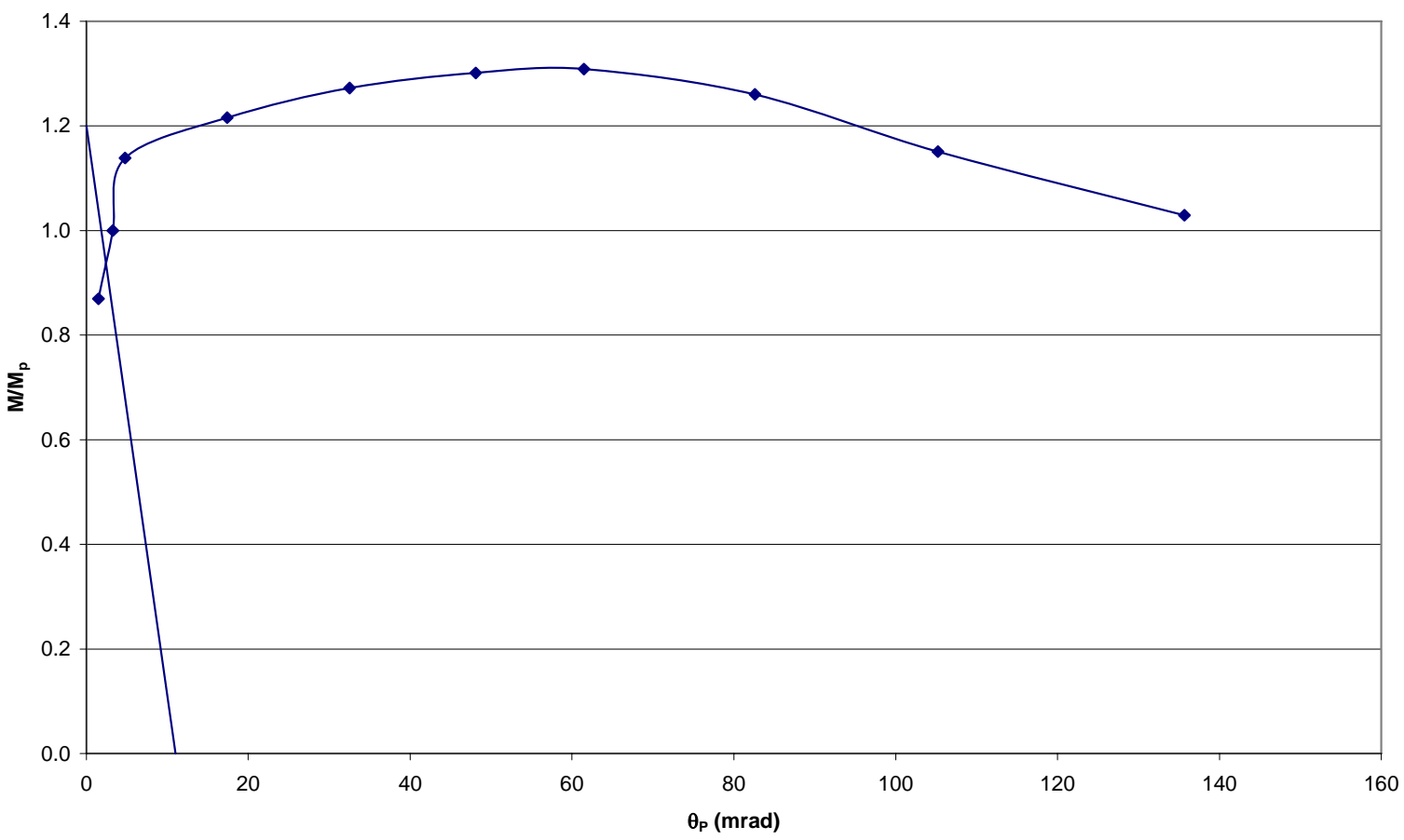

Figure B-54. Moment versus Rotation for Girder D-6 (Lukey et al. 1969) 


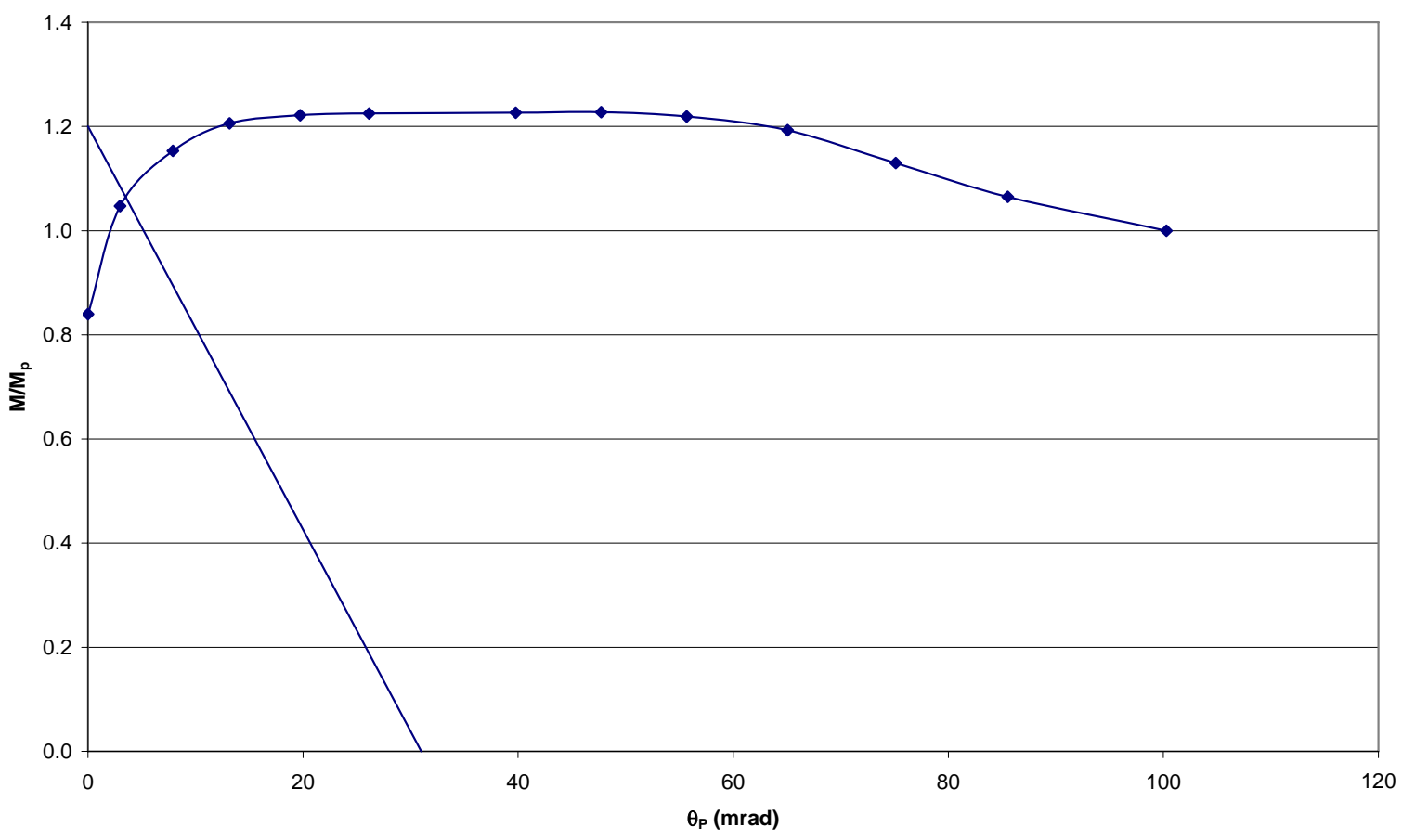

Figure B-55. Moment versus Rotation for Girder E-1 (Lukey et al. 1969)

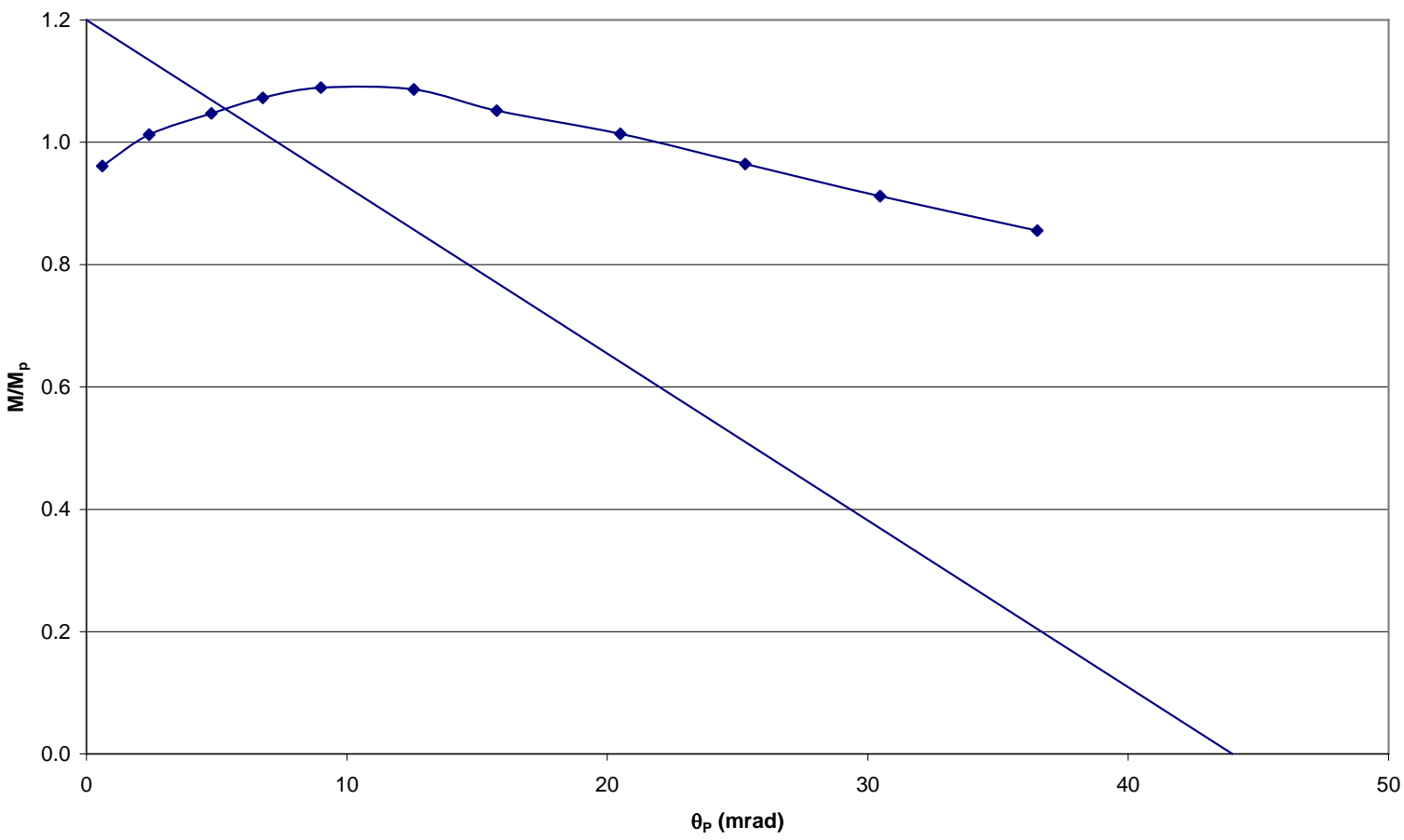

Figure B-56. Moment versus Rotation for Girder E-2 (Lukey et al. 1969) 


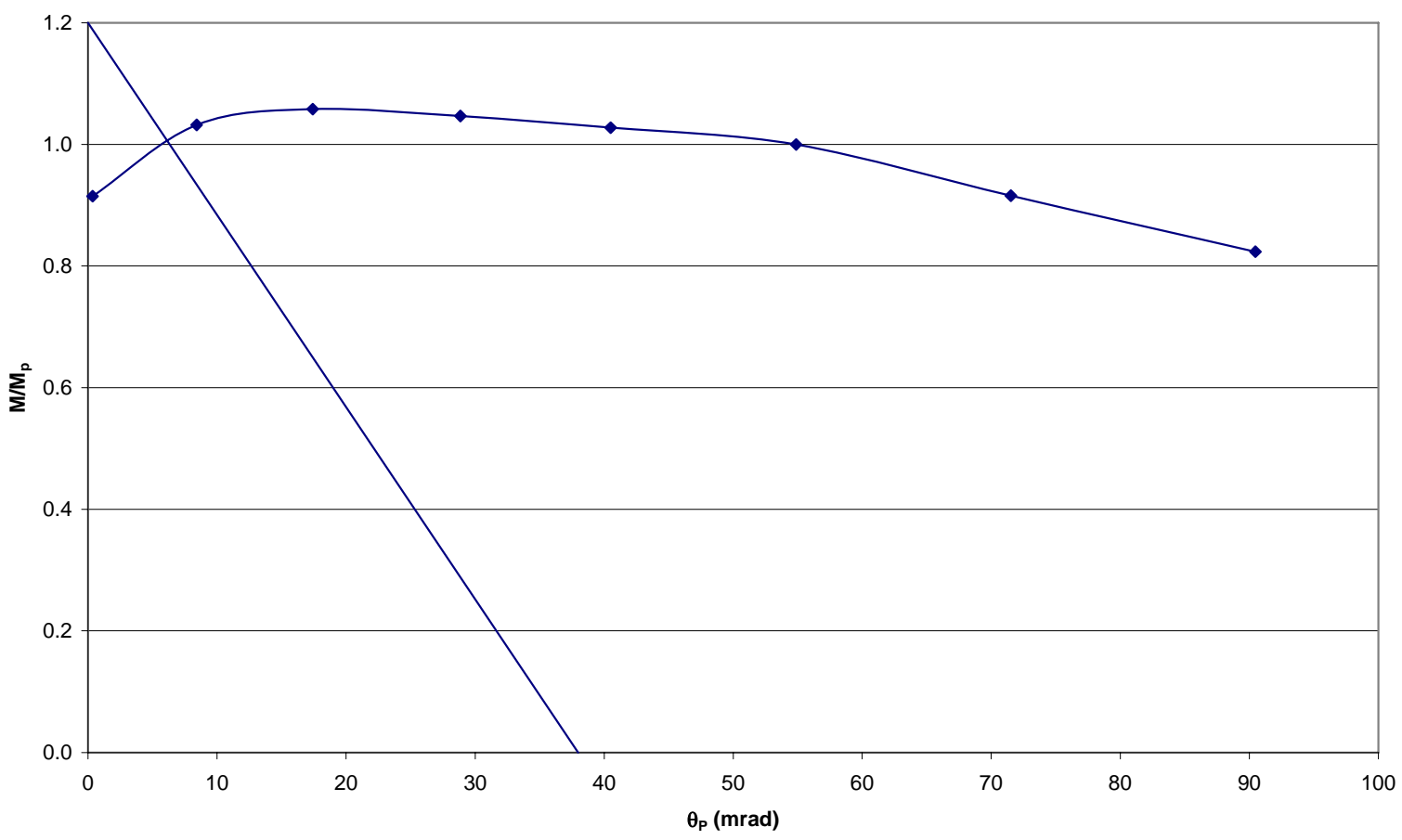

Figure B-57. Moment versus Rotation for Girder E-5 (Lukey et al. 1969)

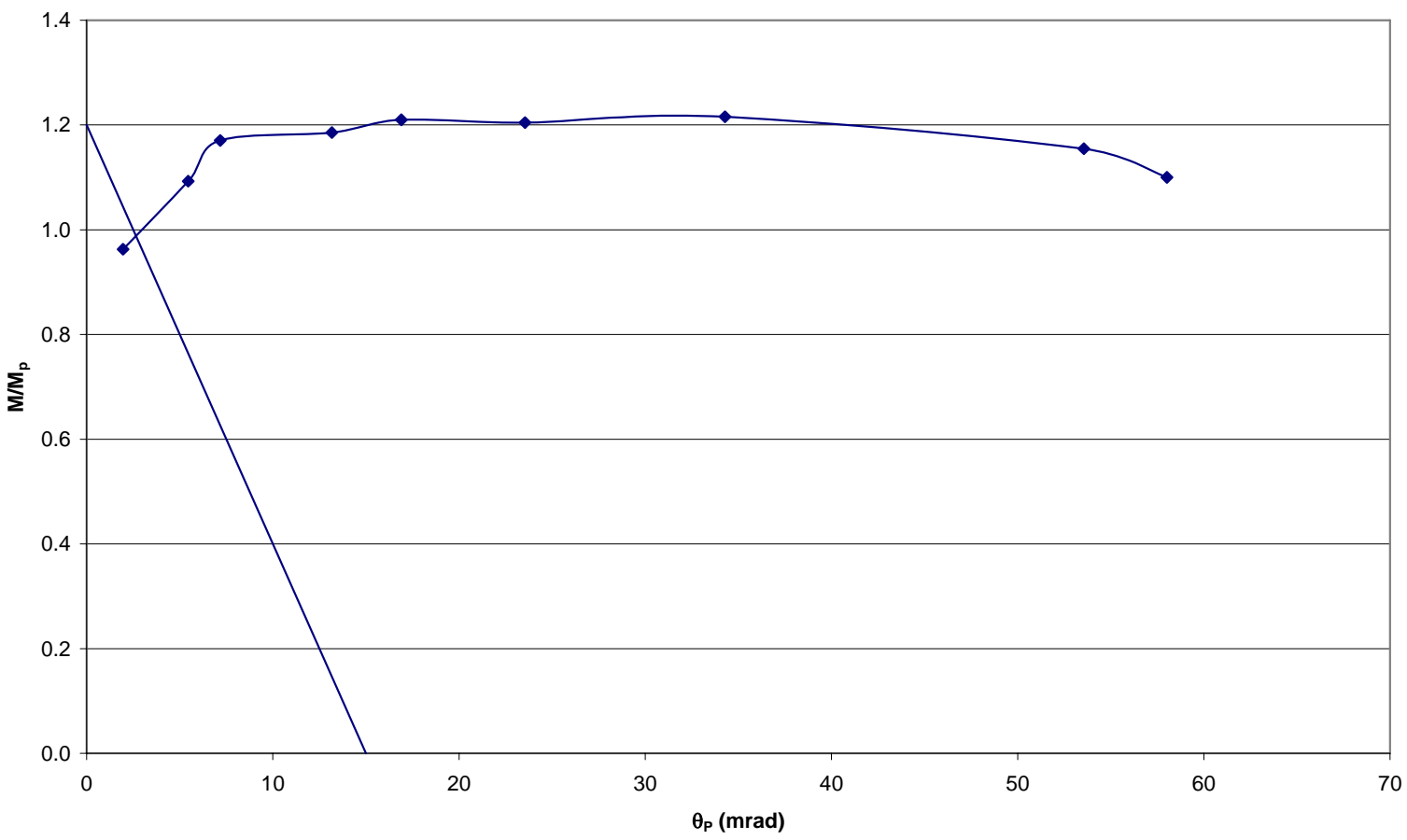

Figure B-58. Moment versus Rotation for Girder E-6 (Lukey et al. 1969) 


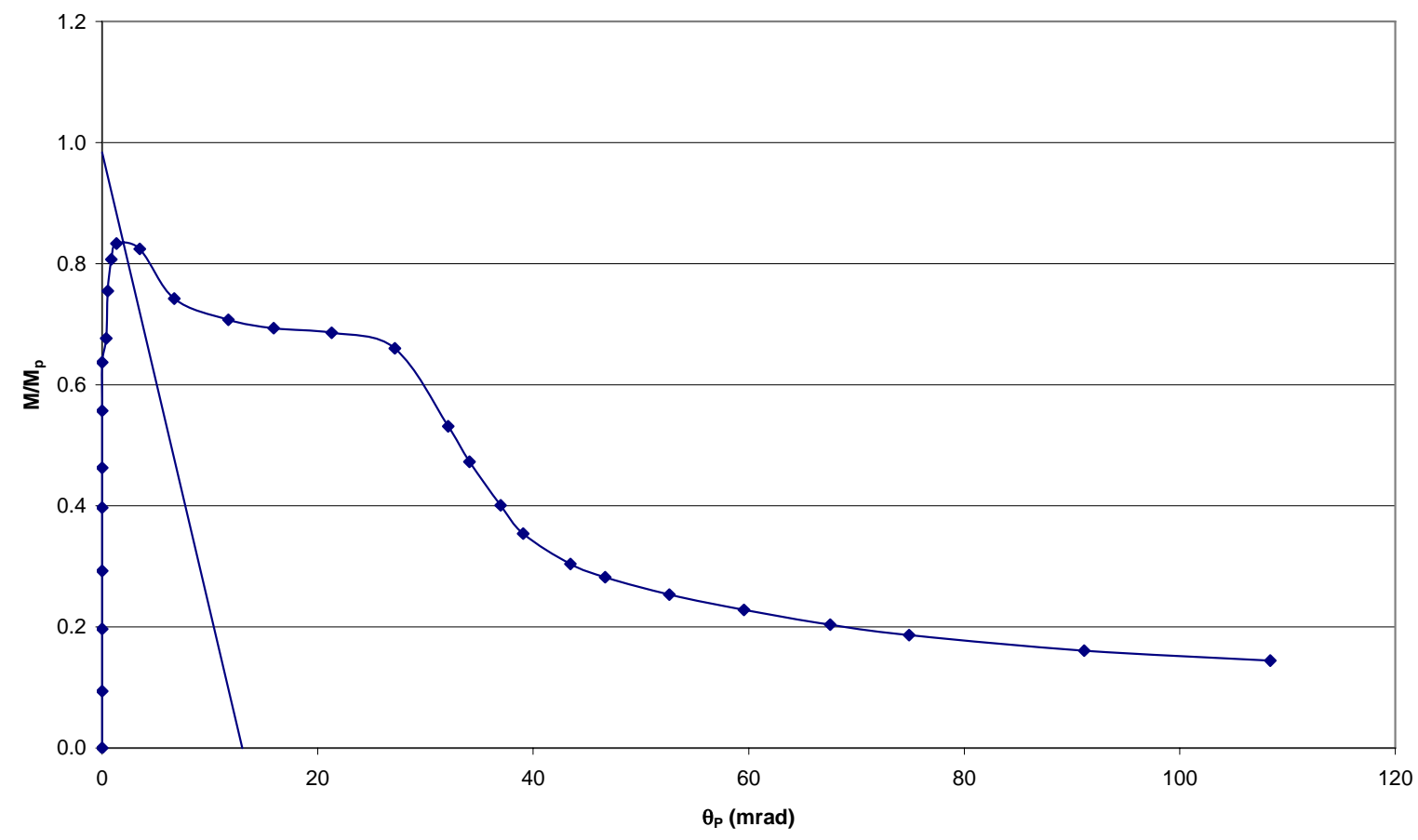

Figure B-59. Moment versus Rotation for Girder SL (Schilling 1985)

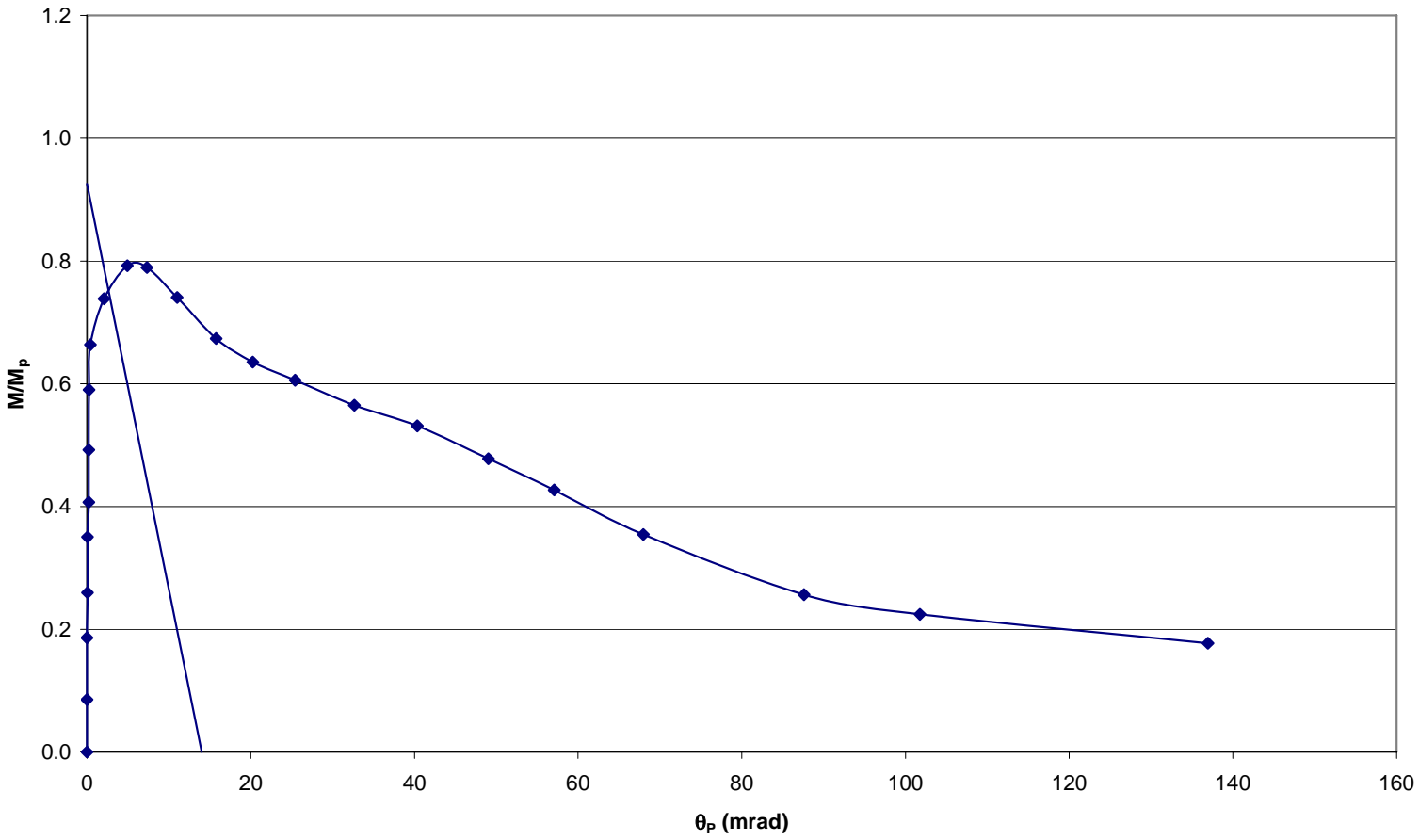

Figure B-60. Moment versus Rotation for Girder UL (Schilling 1985) 


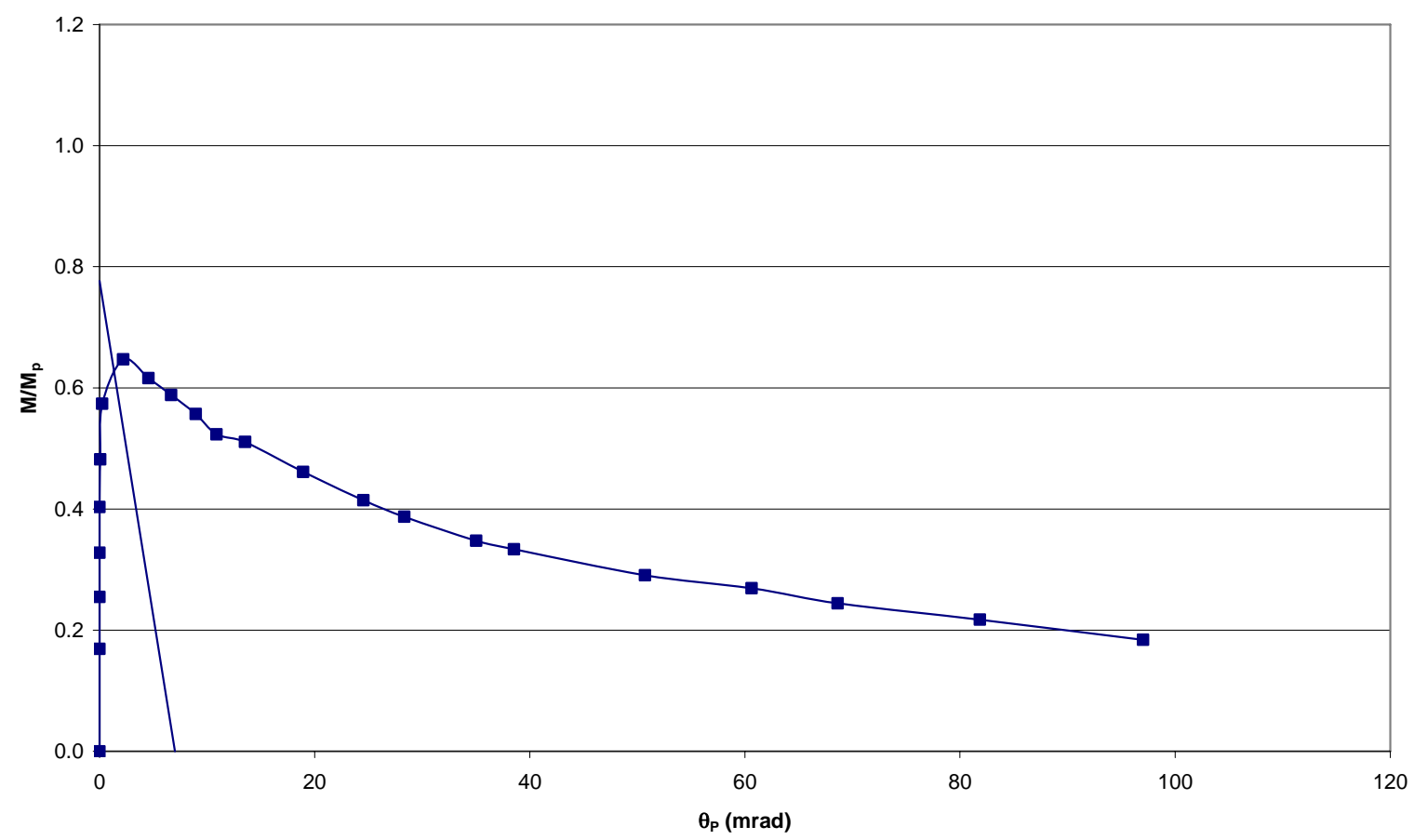

Figure B-61. Moment versus Rotation for Girder US (Schilling 1985)

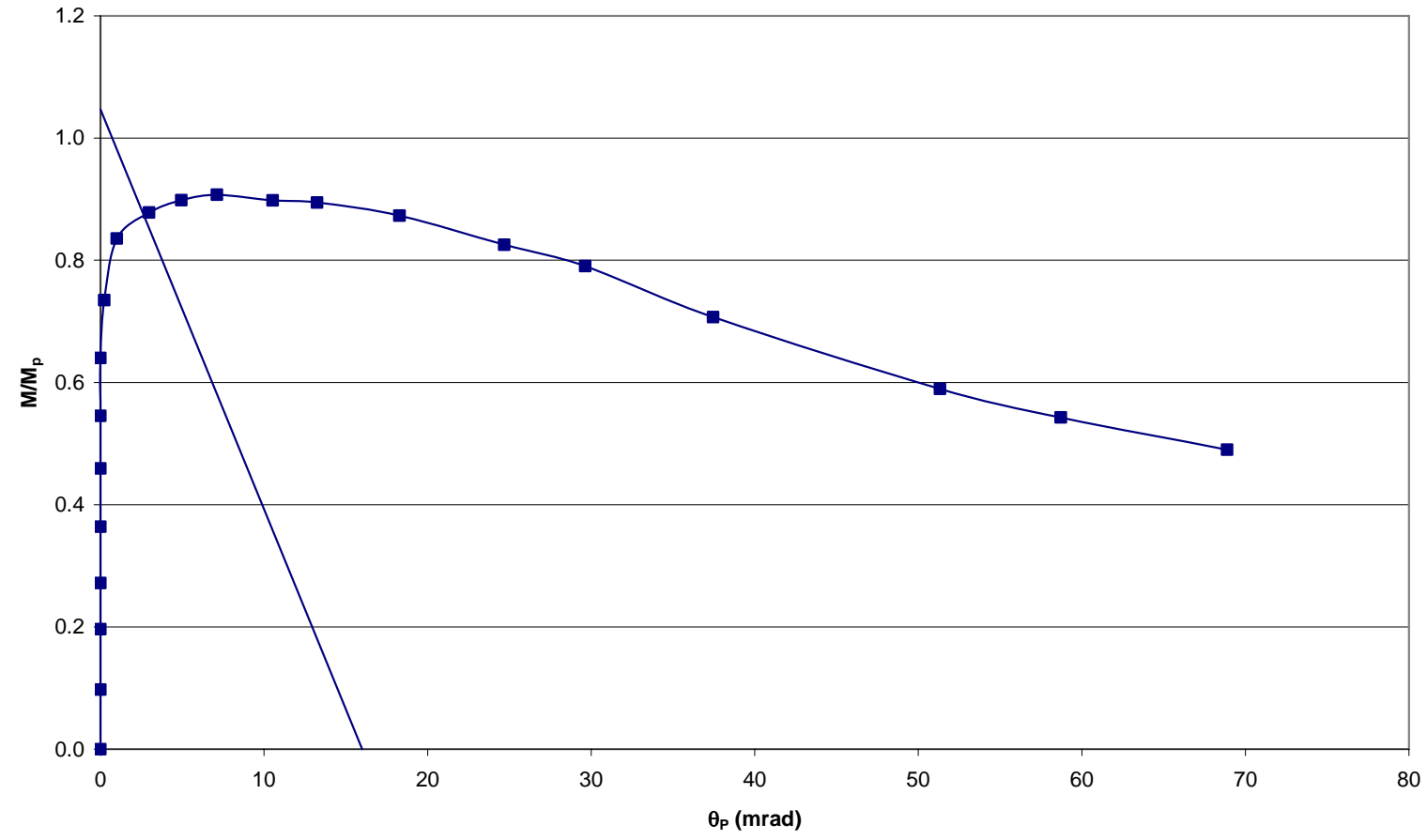

Figure B-62. Moment versus Rotation for Girder D (Schilling and Morcos 1988) 


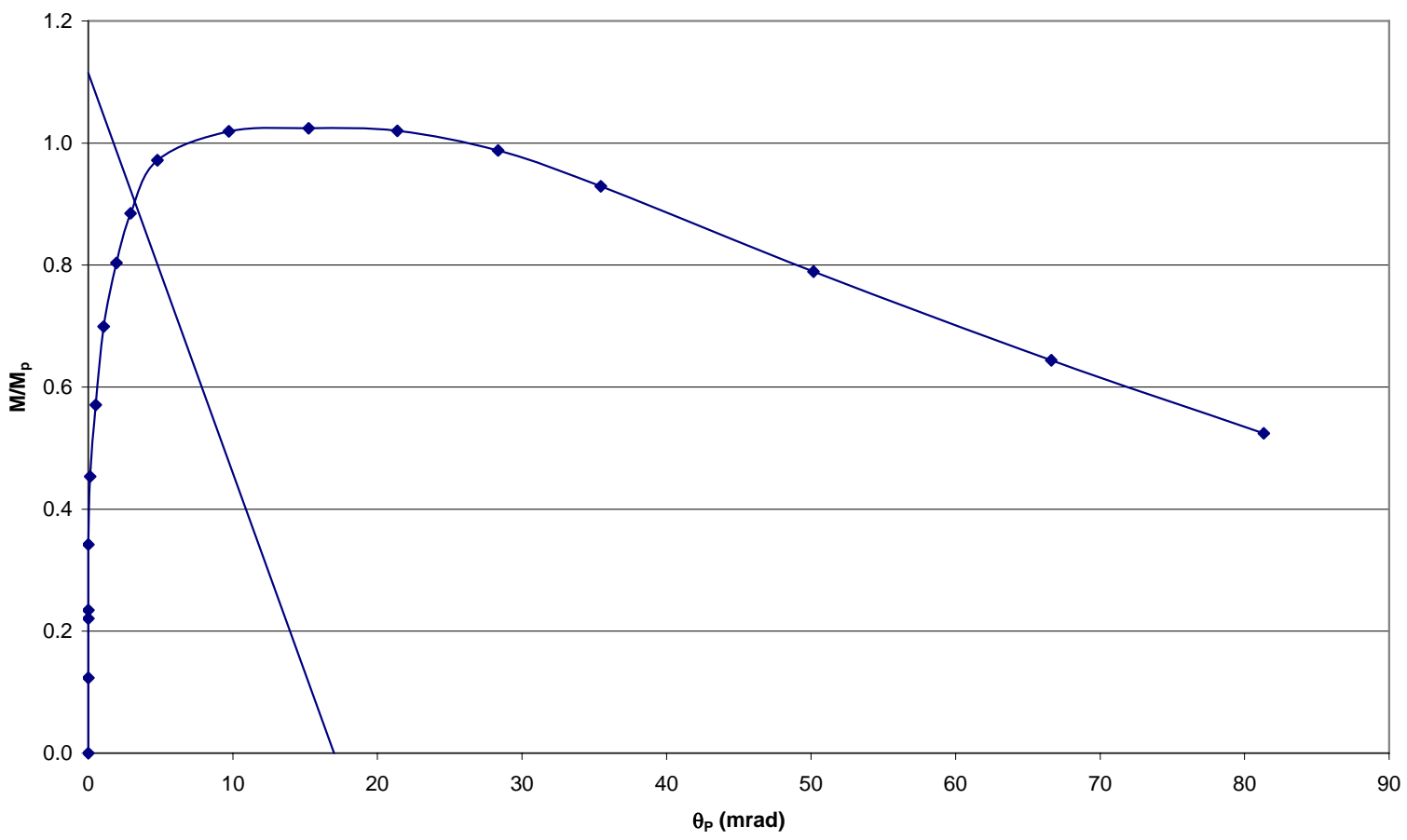

Figure B-63. Moment versus Rotation for Girder M (Schilling and Morcos 1988)

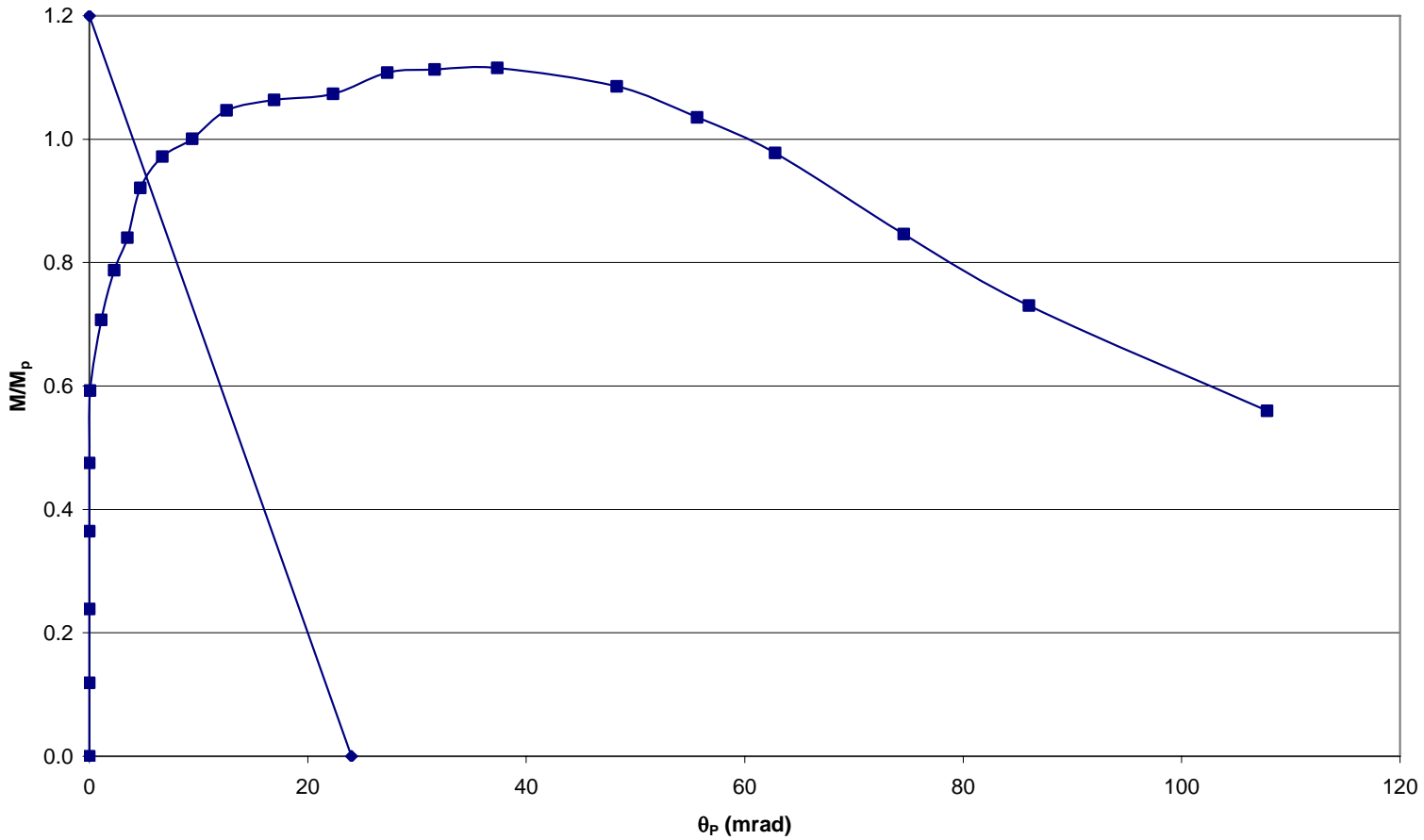

Figure B-64. Moment versus Rotation for Girder S (Schilling and Morcos 1988) 


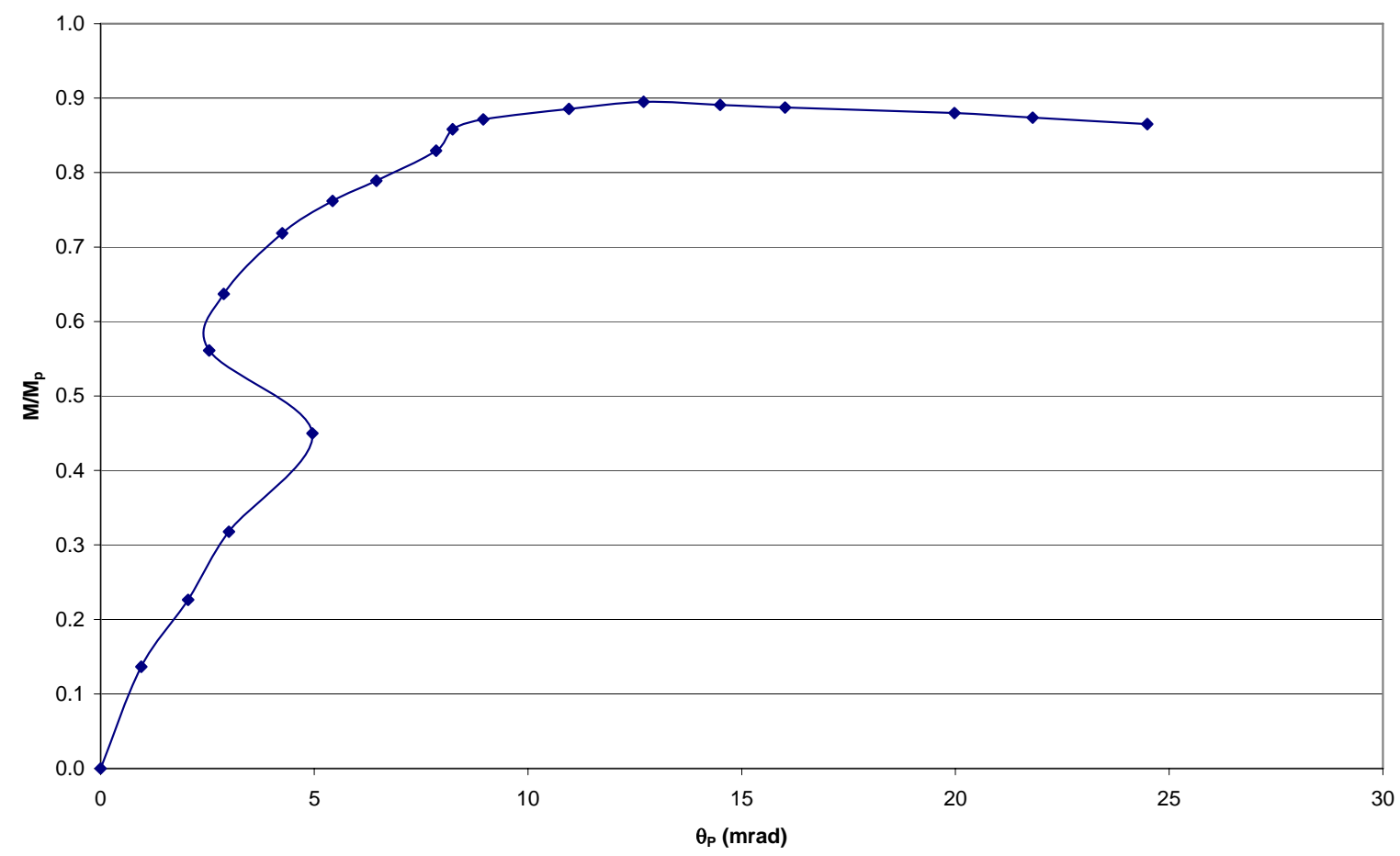

Figure B-65. Moment versus Rotation for Tansil Girder (1991)

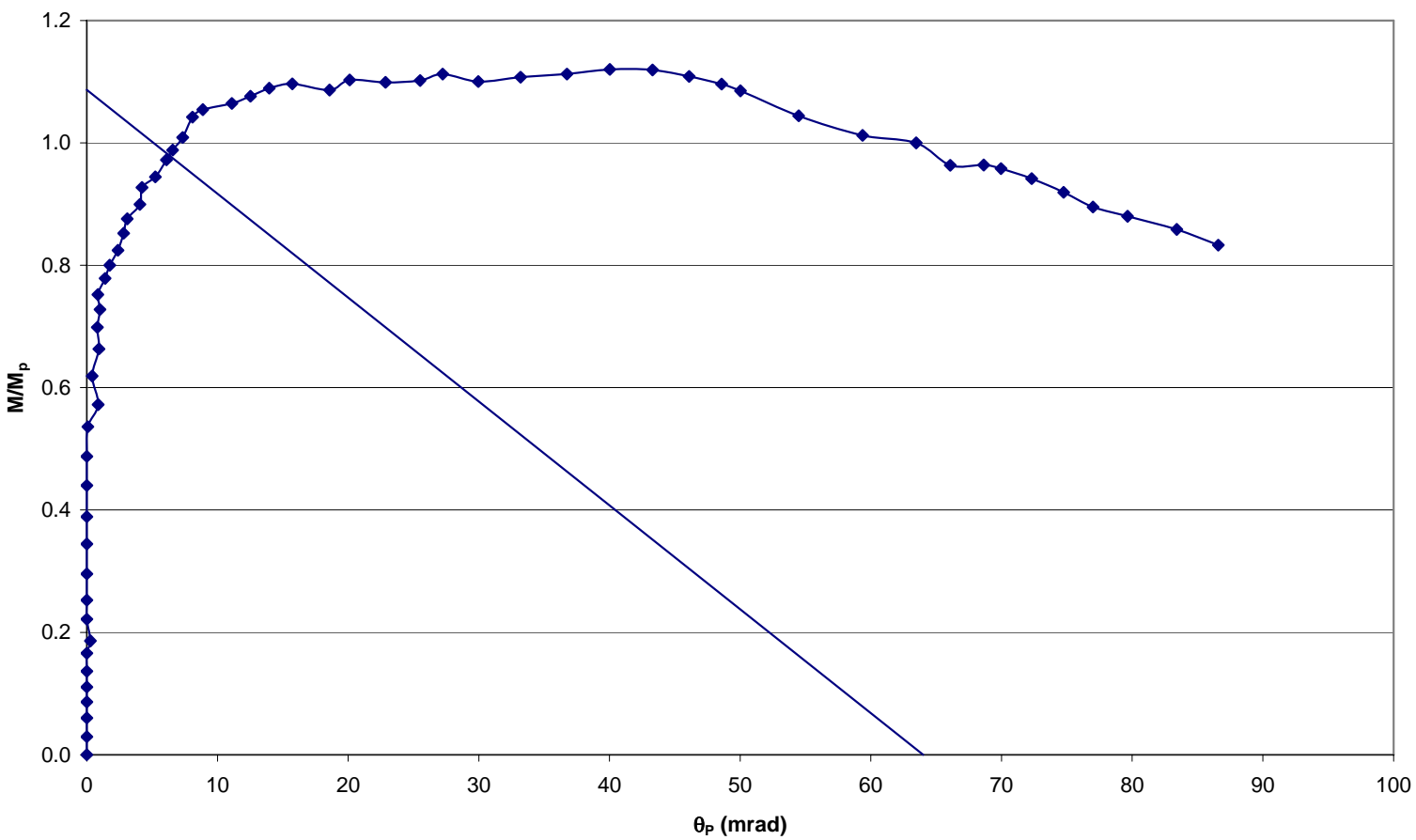

Figure B-66. Moment versus Rotation for Girder A (Yakel et al. 1999) 


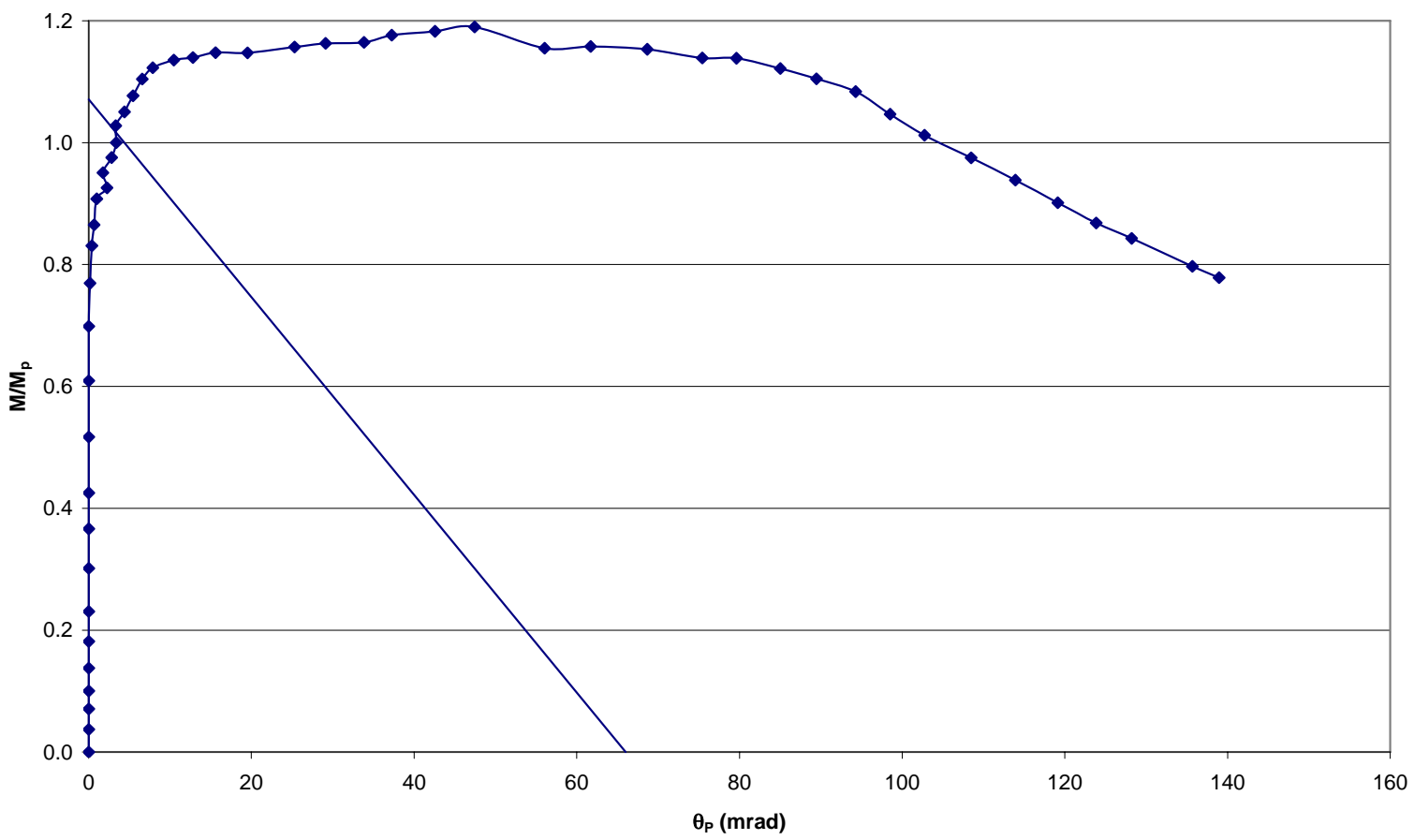

Figure B-67. Moment versus Rotation for Girder D (Yakel et al. 1999)

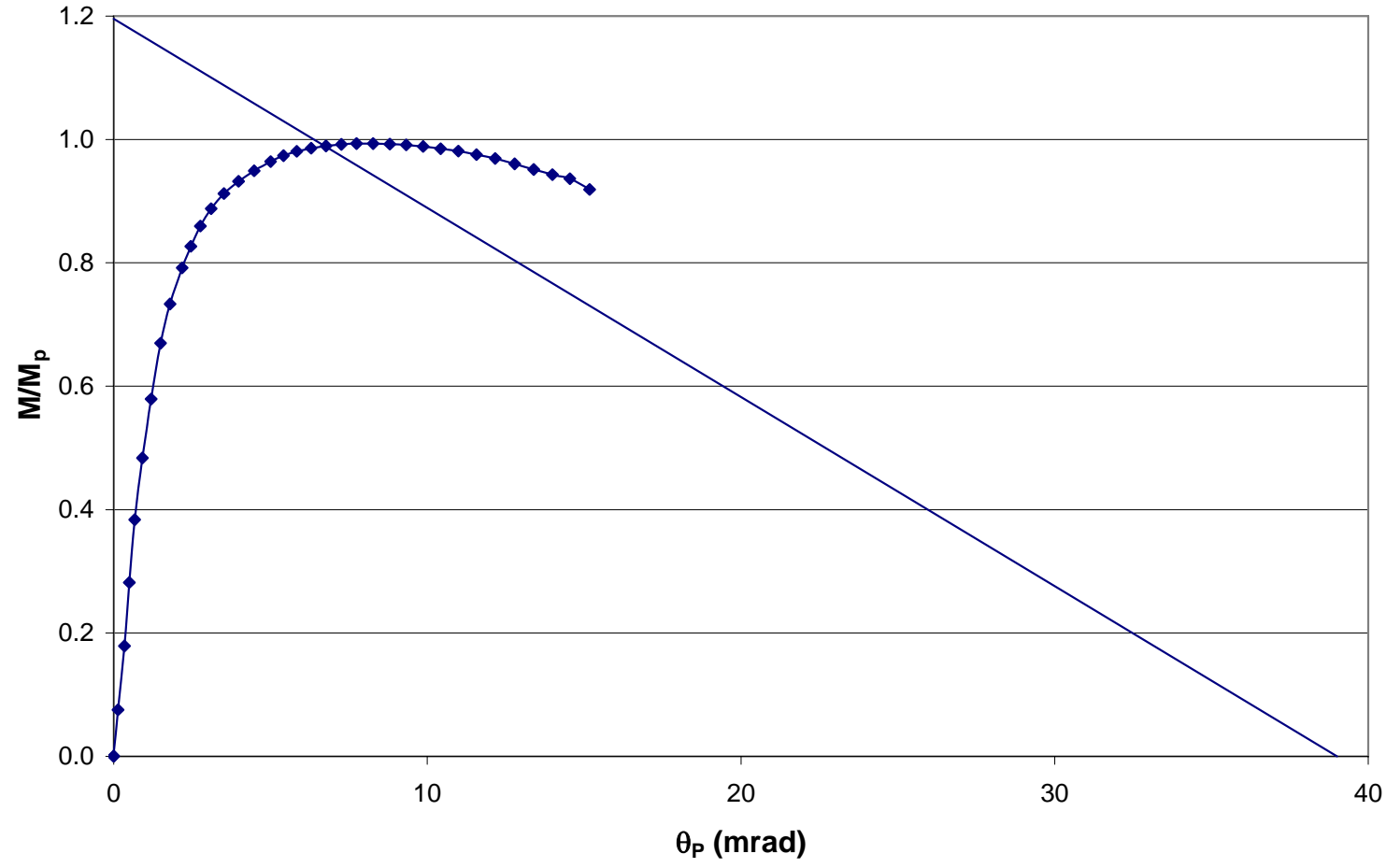

Figure B-68. Moment versus Rotation for Girder 1MG (Righman 2005) 


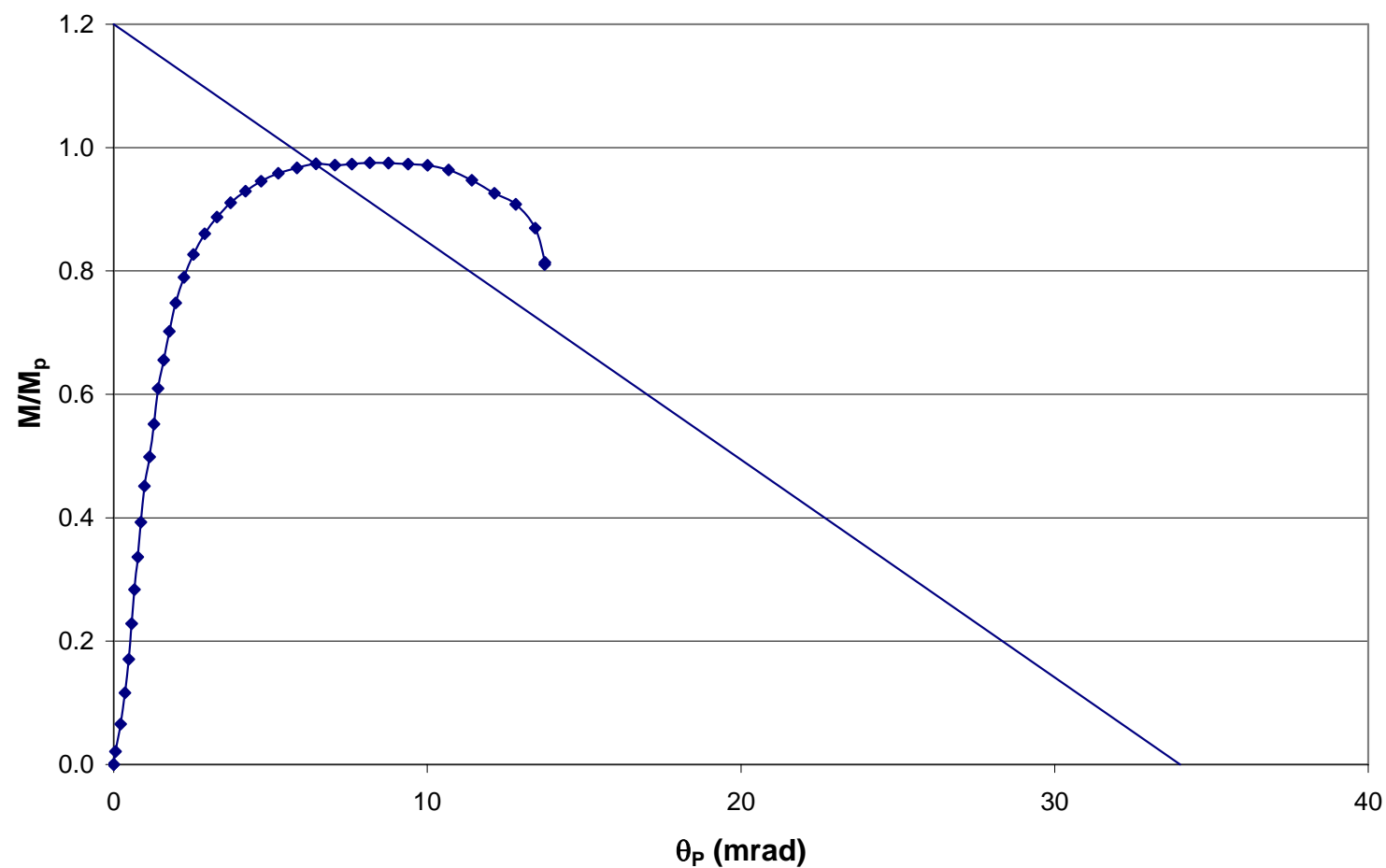

Figure B-69. Moment versus Rotation for Girder 2MG (Righman 2005)

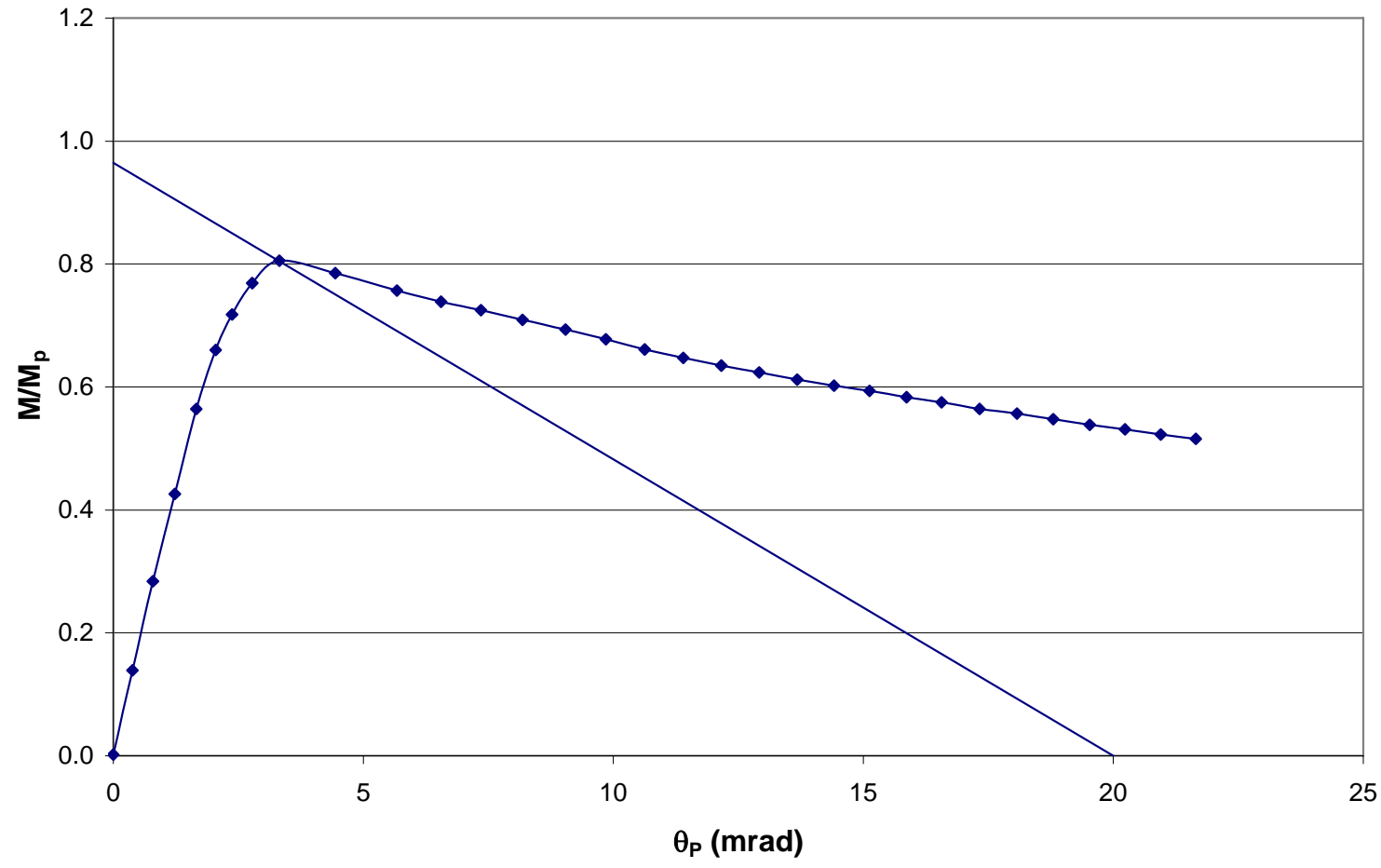

Figure B-70. Moment versus Rotation for Girder 3MG (Righman 2005) 


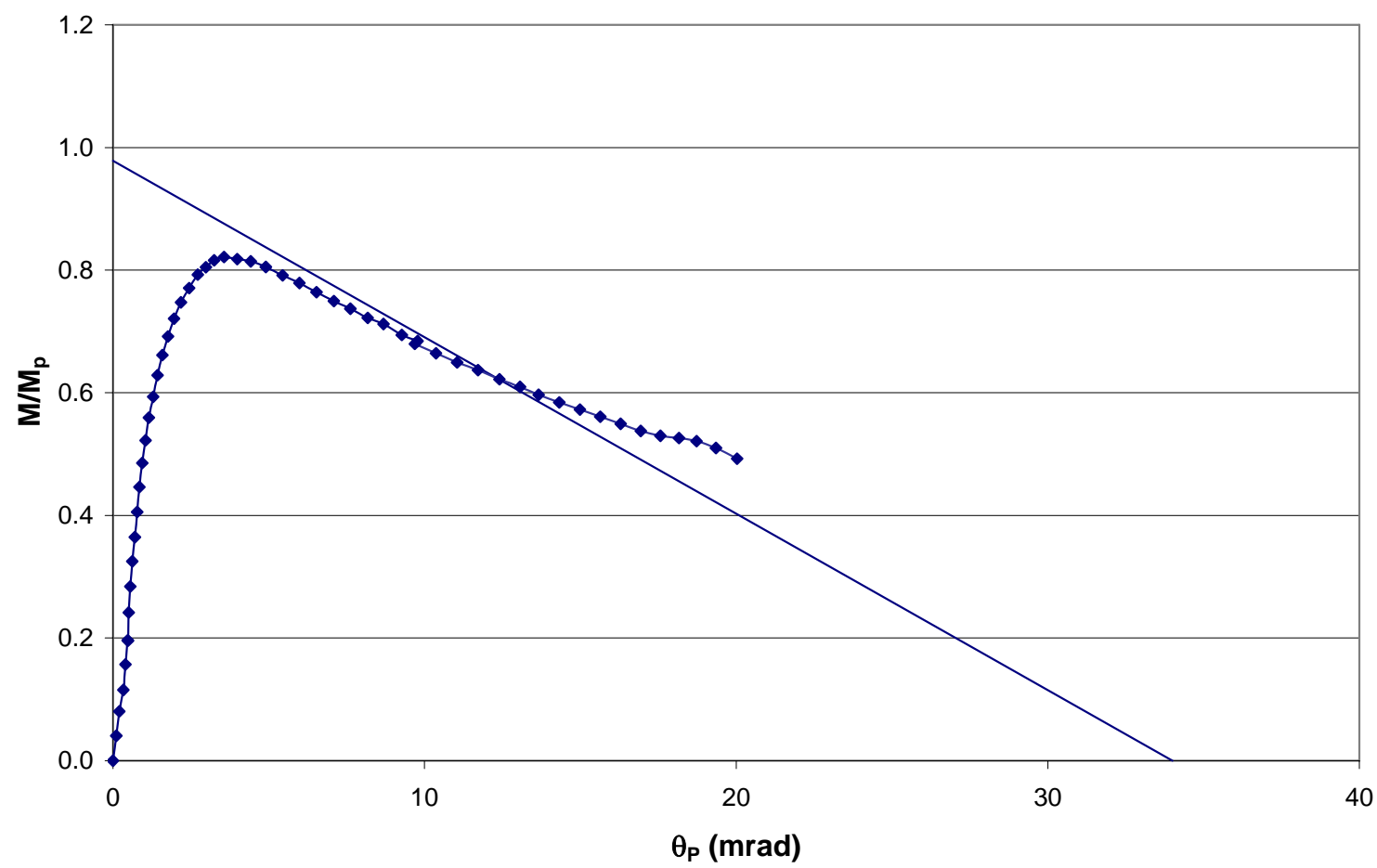

Figure B-71. Moment versus Rotation for Girder 4MG (Righman 2005)

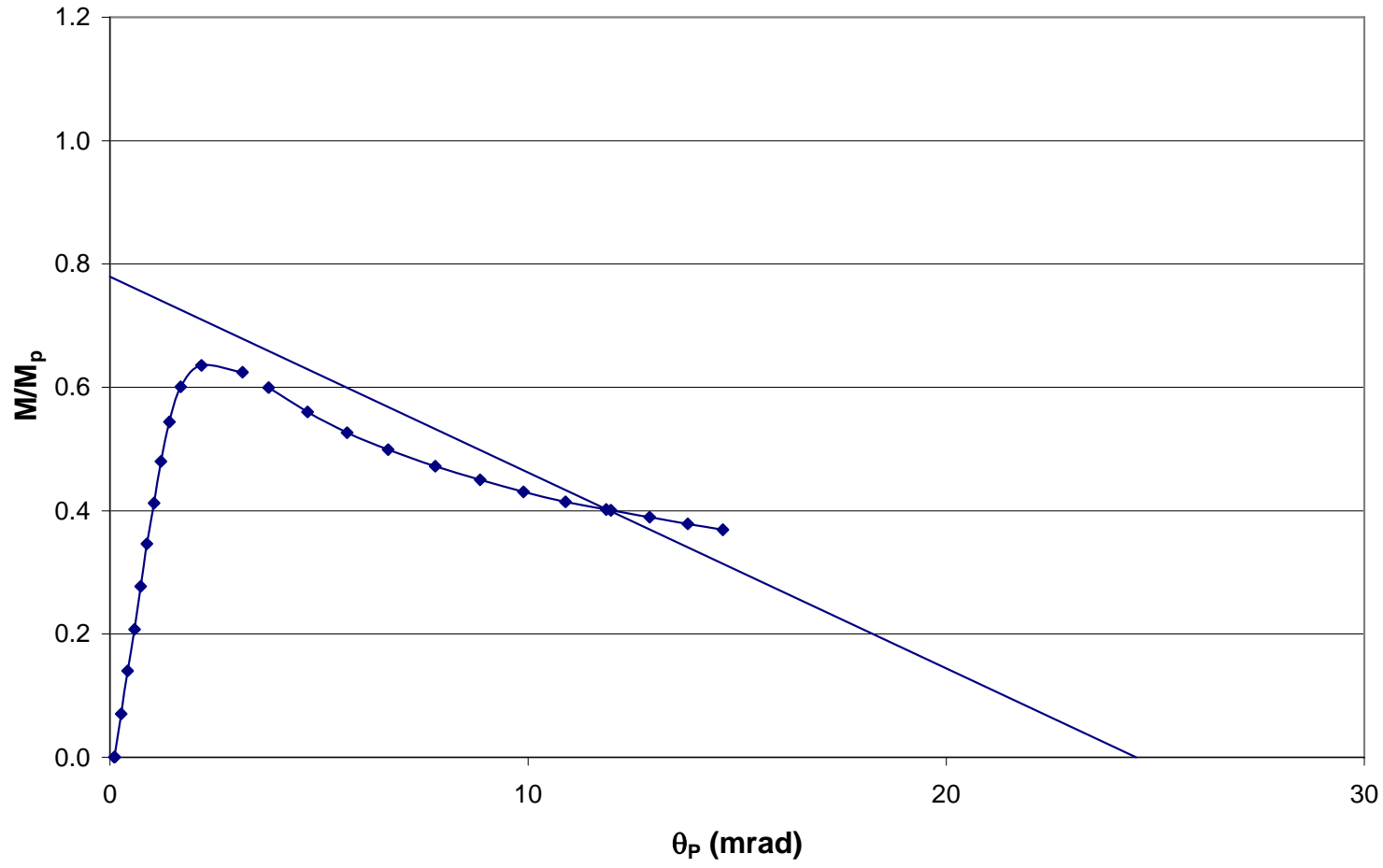

Figure B-72. Moment versus Rotation for Girder 5MG (Righman 2005) 


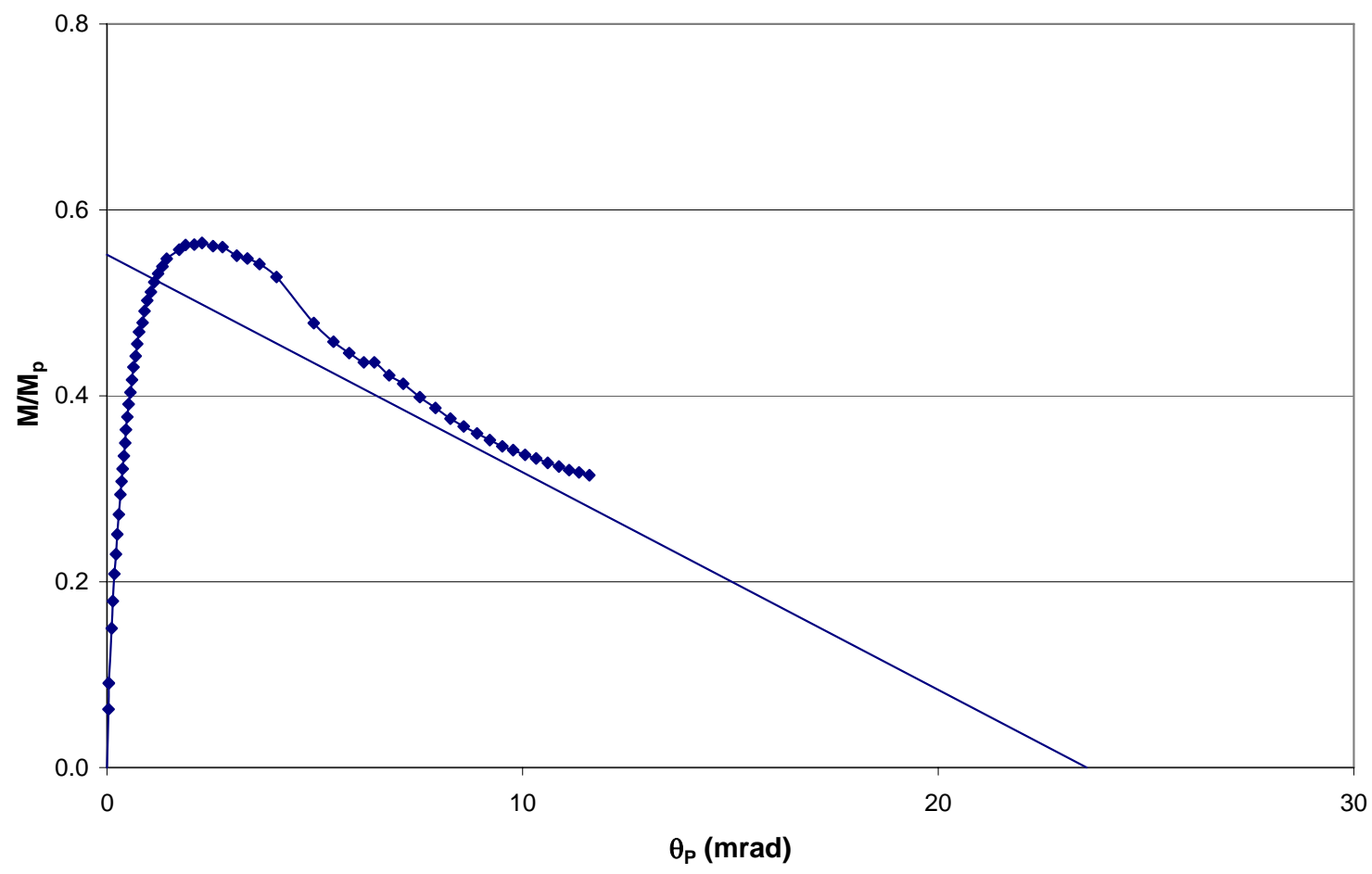

Figure B-73. Moment versus Rotation for Girder 6MG (Righman 2005)

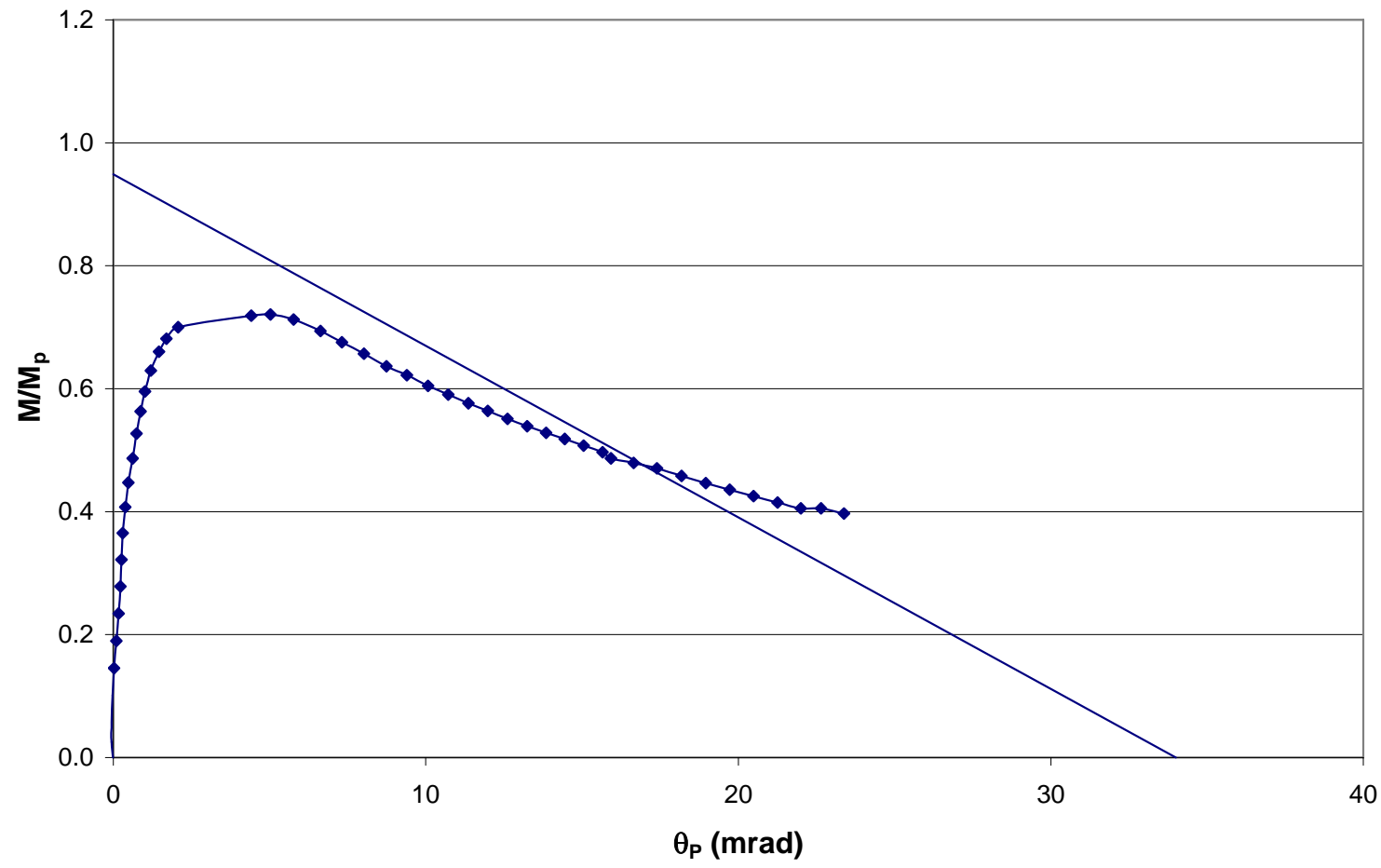

Figure B-74. Moment versus Rotation for Girder 7MG (Righman 2005) 


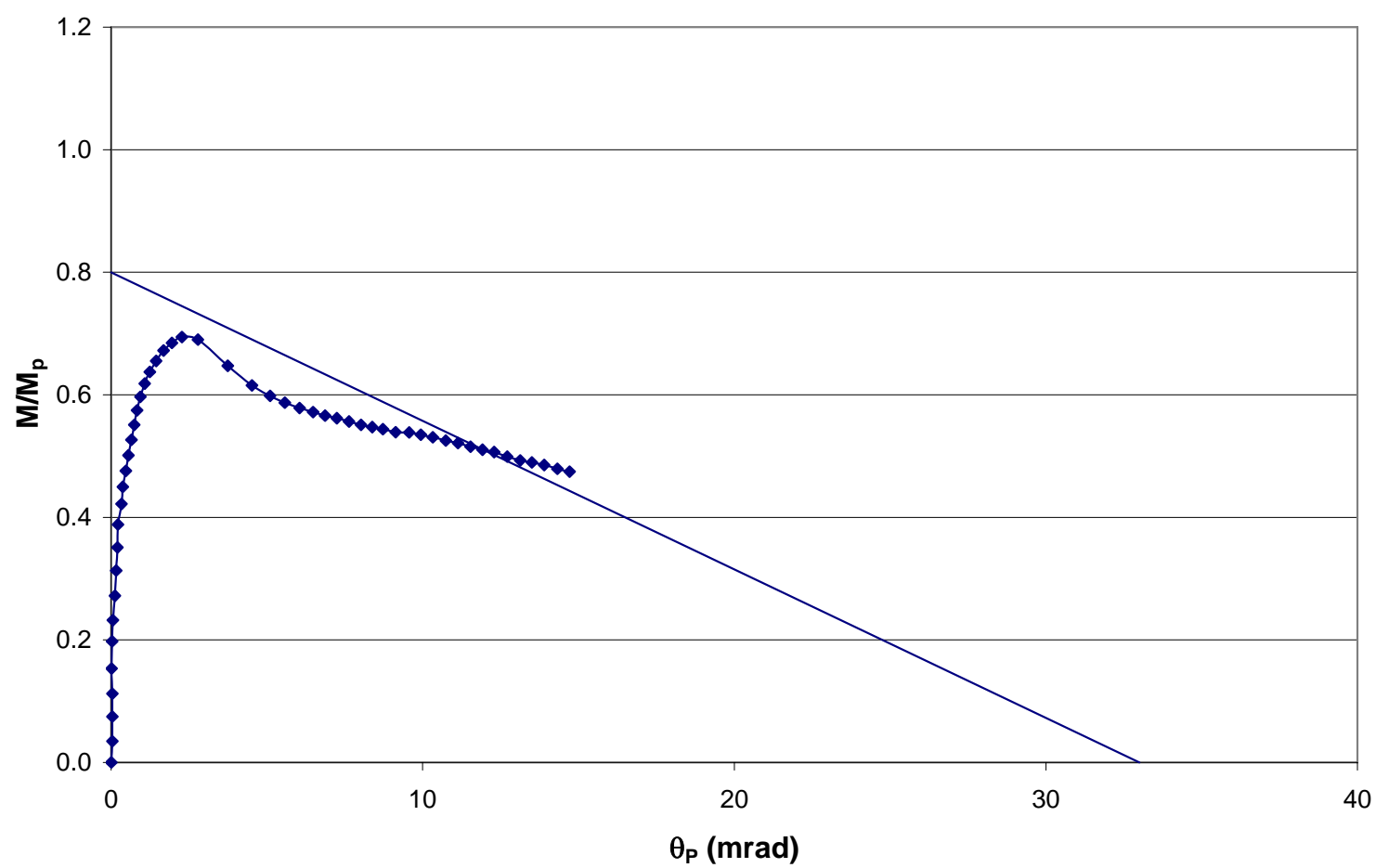

Figure B-75. Moment versus Rotation for Girder 8MG (Righman 2005)

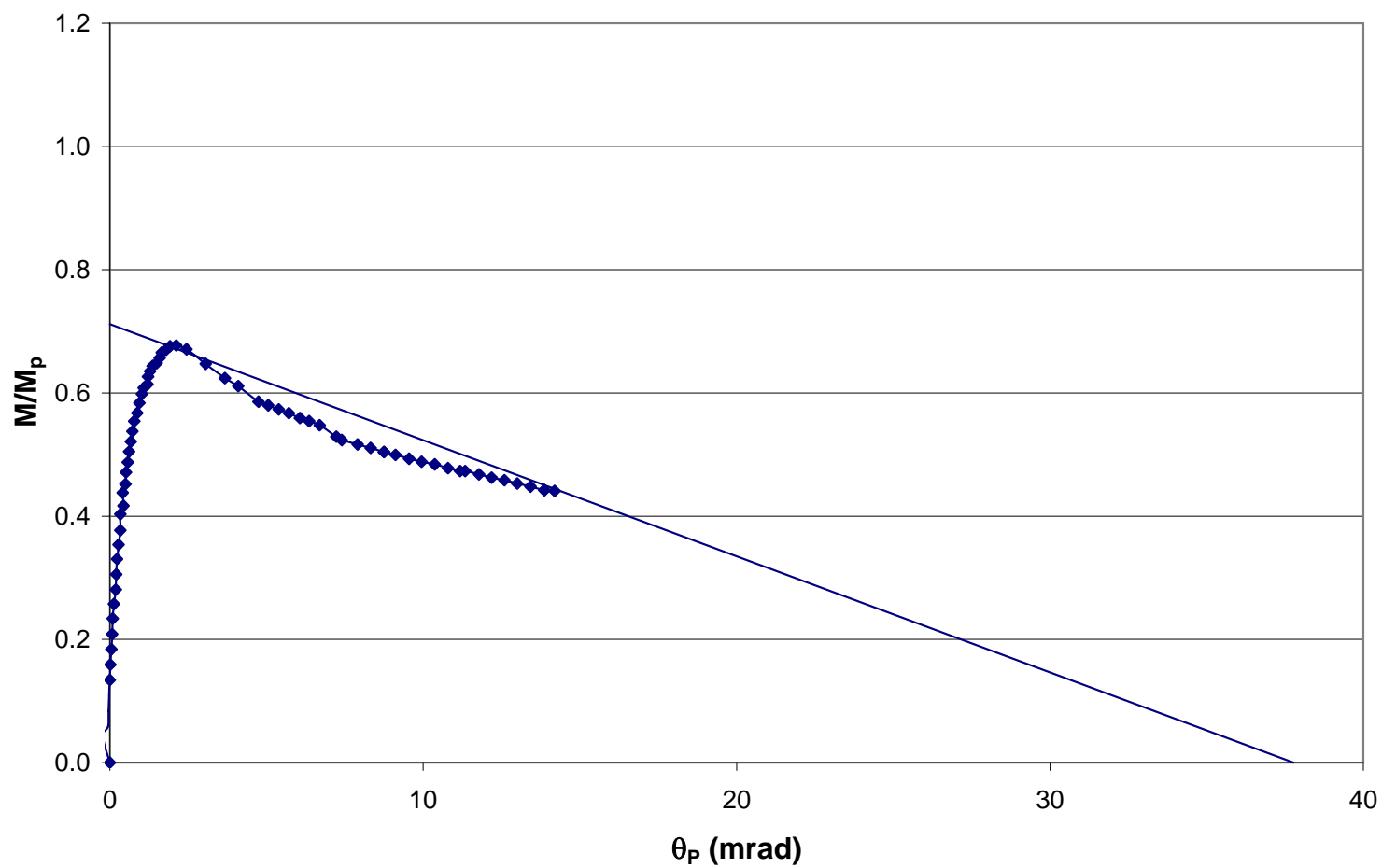

Figure B-76. Moment versus Rotation for Girder 9MG (Righman 2005) 


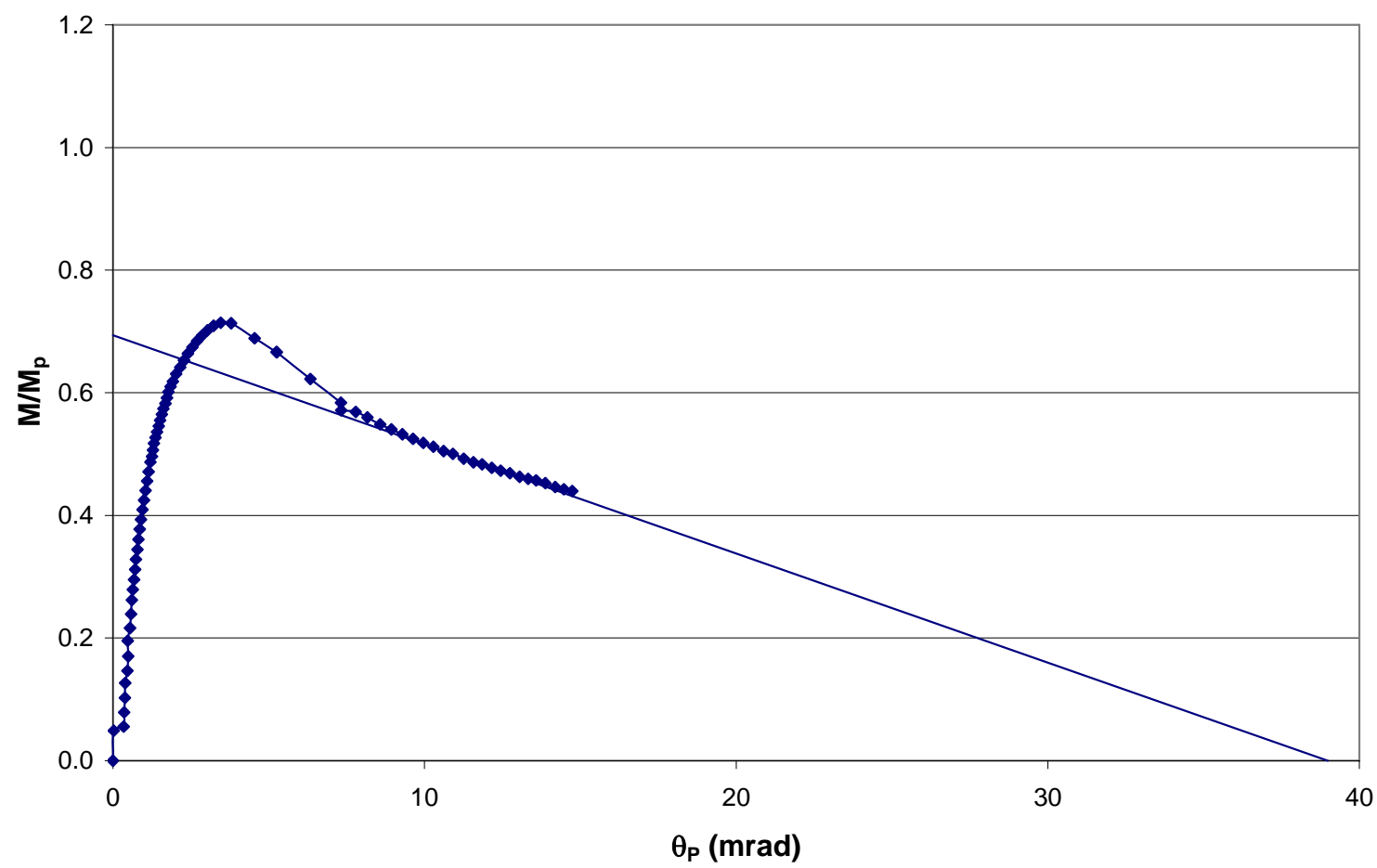

Figure B-77. Moment versus Rotation for Girder 10MG (Righman 2005)

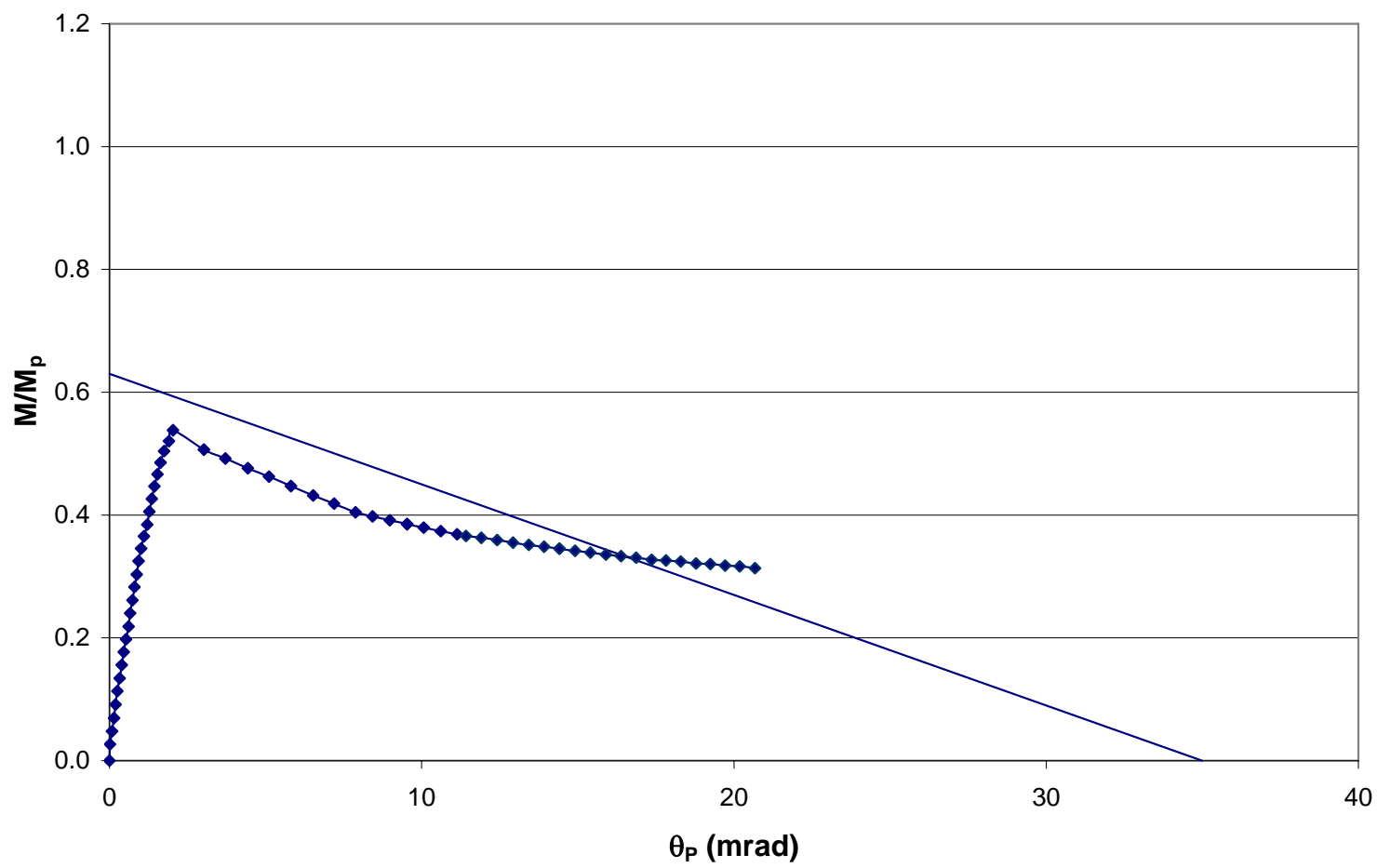

Figure B-78. Moment versus Rotation for Girder 11MG (Righman 2005) 


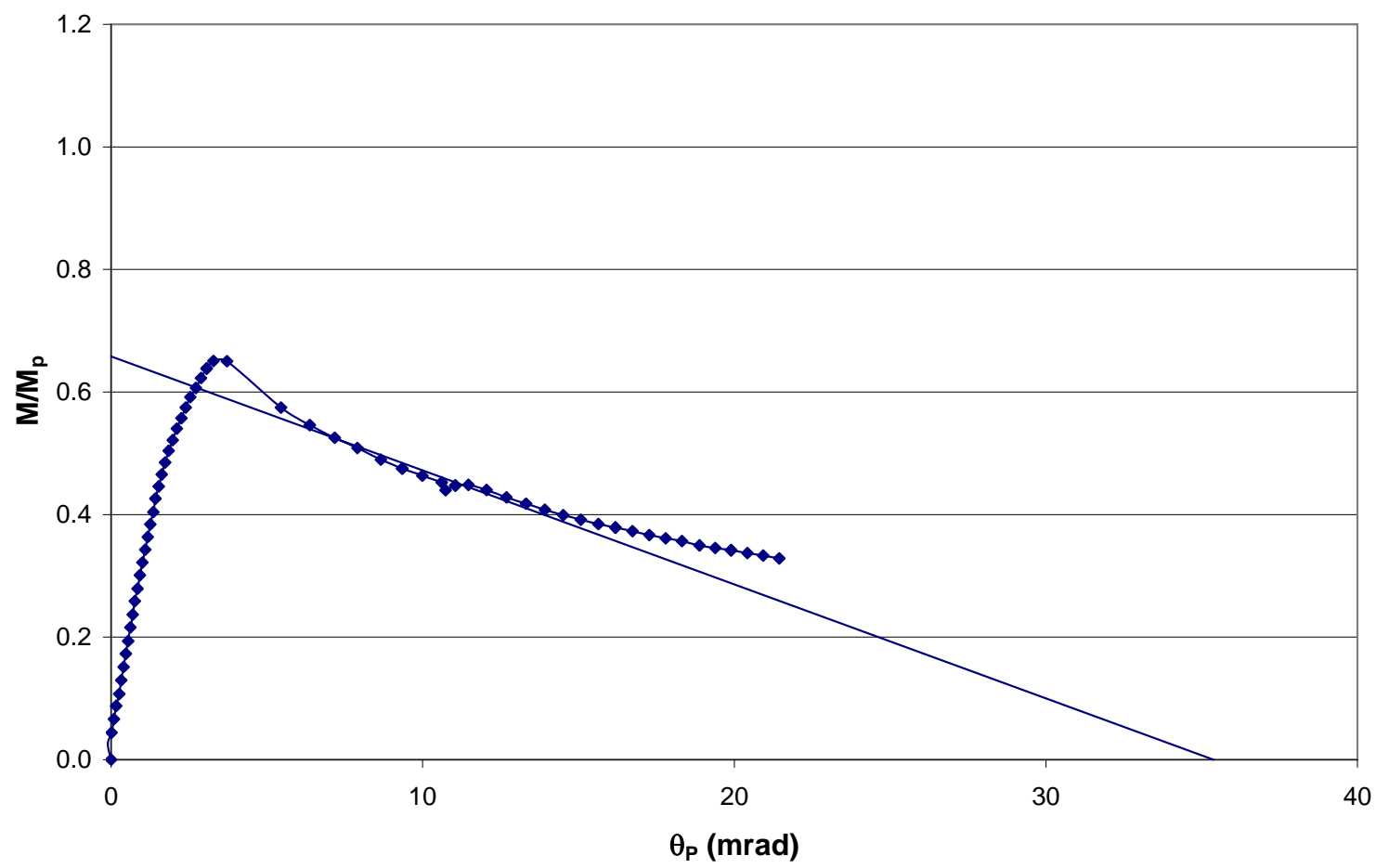

Figure B-79. Moment versus Rotation for Girder 12MG (Righman 2005) 


\section{Appendix C: MATERIAL TESTING RESULTS}

The experimental girders analyzed in this work were fabricated from nine different combinations of plate thicknesses, material properties, and steel heats. A minimum of three material coupons were taken from each plate of steel and tension tested to obtain material properties. Tension testing of heats JUN and AUG was performed at West Virginia University, while testing of heats A and B were commercially tested by Non-Destructive Testing Group of Aliquippa, PA.

Table C-1 details the specific plates used to fabricate each girder. Each plate is labeled according to the following naming convention: the first label indicates the plate thickness, the second label indicates the nominal yield strength of the material, and the last label references the heat of the material.

The stress versus strain diagrams obtained for each plate type are presented in Figs. C-1 through C-9. These diagrams depict the data resulting from the testing of each coupon with thin lines and the average of all specimens, which is used in the FEA of the experimental girders, by a heavy line. The inset in each graph tabulates the specific stress-strain data taken

Table C-1. Steel Designations for Experimental Girders

\begin{tabular}{|c|c|c|c|}
\hline Specimen & Top Flange & Bottom Flange & Web \\
\hline $1 \mathrm{MG}$ & $0.5 "-50 \mathrm{KSI}-\mathrm{JUN}$ & 0.875" - 50KSI - B & "0.75" - $50 \mathrm{KSI}-\mathrm{JUN}$ \\
\hline $2 M G$ & $0.5 "-50 \mathrm{KSI}-\mathrm{JUN}$ & $0.875 "-50 \mathrm{KSI}-\mathrm{A}$ & $0.75 "-50 \mathrm{KSI}-\mathrm{JUN}$ \\
\hline $3 M G$ & $0.5^{\prime \prime}-50 \mathrm{KSI}-\mathrm{JUN}$ & $0.5^{\prime \prime}-50 \mathrm{KSI}-\mathrm{JUN}$ & $0.3125 "-50 \mathrm{KSI}-\mathrm{AUG}$ \\
\hline $4 M G$ & $0.5^{\prime \prime}-50 \mathrm{KSI}$ - AUG & $0.5 "-50 \mathrm{KSI}$ - AUG & 0.3125" - $50 \mathrm{KSI}-\mathrm{JUN}$ \\
\hline $5 M G$ & $0.5 "-50 \mathrm{KSI}-\mathrm{JUN}$ & $0.75 "-50 \mathrm{KSI}-\mathrm{JUN}$ & $0.5^{\prime \prime}-50 \mathrm{KSI}-\mathrm{JUN}$ \\
\hline $6 M G$ & $0.3125 "$ - $50 \mathrm{KSI}$ - AUG & $0.5^{\prime \prime}-50 \mathrm{KSI}-\mathrm{JUN}$ & $0.3125 "-50 \mathrm{KSI}-\mathrm{JUN}$ \\
\hline $7 \mathrm{MG}$ & $0.5 "$ - $70 \mathrm{KSI}$ - JUN & $0.5 "$ - $70 \mathrm{KSI}$ - JUN & $0.3125 "-50 \mathrm{KSI}-\mathrm{JUN}$ \\
\hline $8 M G$ & 0.375" - $70 \mathrm{KSI}-\mathrm{JUN}$ & $0.5^{\prime \prime}-70 \mathrm{KSI}-\mathrm{JUN}$ & $0.3125 "-50 \mathrm{KSI}-\mathrm{JUN}$ \\
\hline 9MG & $0.5^{\prime \prime}-70 \mathrm{KSI}-\mathrm{JUN}$ & $0.5^{\prime \prime}-70 \mathrm{KSI}-\mathrm{JUN}$ & $0.3125^{\prime \prime}-50 \mathrm{KSI}-\mathrm{AUG}$ \\
\hline $10 M G$ & 0.375" - 70 KSI - JUN & $0.5 "$ - 70 KSI - JUN & $0.3125 "-50 \mathrm{KSI}-\mathrm{JUN}$ \\
\hline $11 \mathrm{MG}$ & $0.5 "$ - $70 \mathrm{KSI}$ - JUN & $0.5 "$ - 70 KSI - JUN & 0.375" - $70 \mathrm{KSI}$ - JUN \\
\hline $12 \mathrm{MG}$ & 0.375" - $70 \mathrm{KSI}-\mathrm{JUN}$ & $0.5^{\prime \prime}-70 \mathrm{KSI}-\mathrm{JUN}$ & $0.375 "-70 \mathrm{KSI}-\mathrm{JUN}$ \\
\hline
\end{tabular}


from the average results that is converted into true stress versus plastic strain and then input into the ABAQUS analysis.

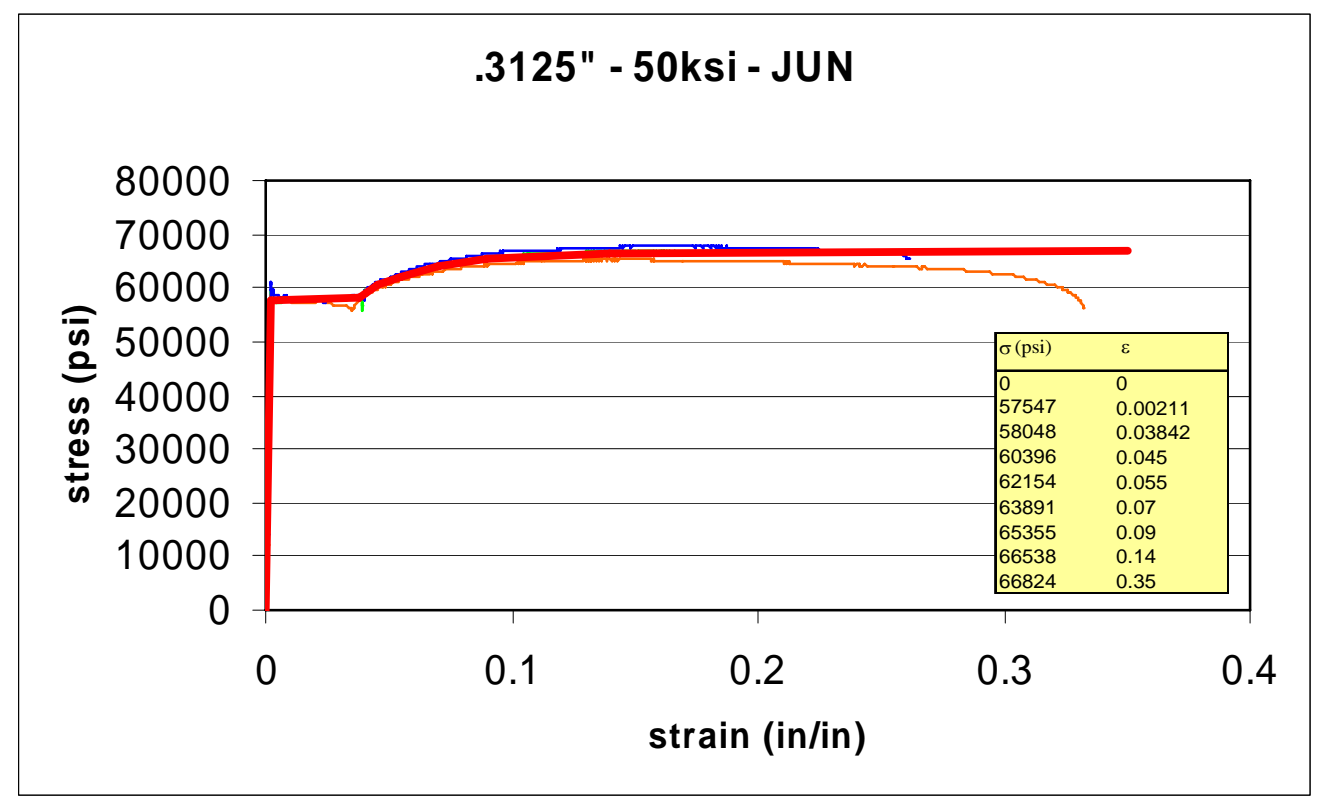

Figure C-1. Tension Test Data for 0.3125” - 50ksi - JUN

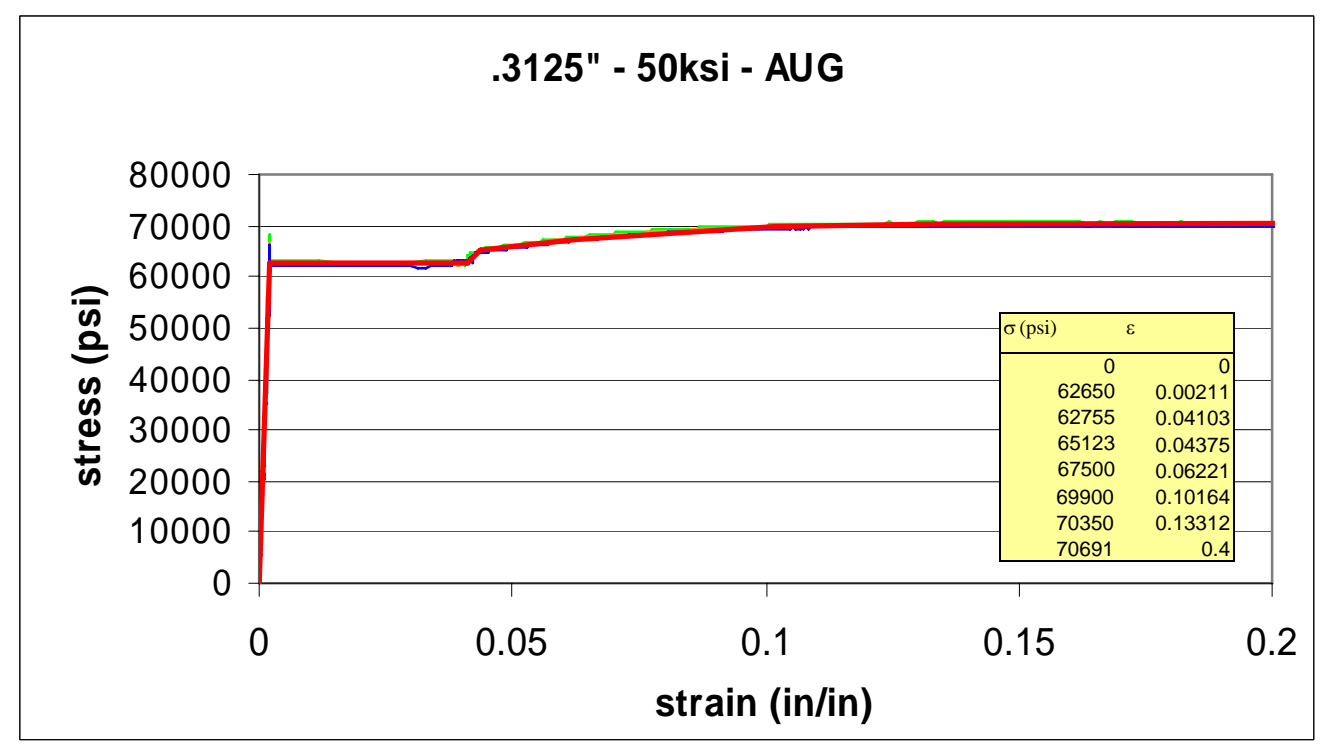

Figure C-2. Tension Test Data for 0.3125” - 50ksi - AUG 


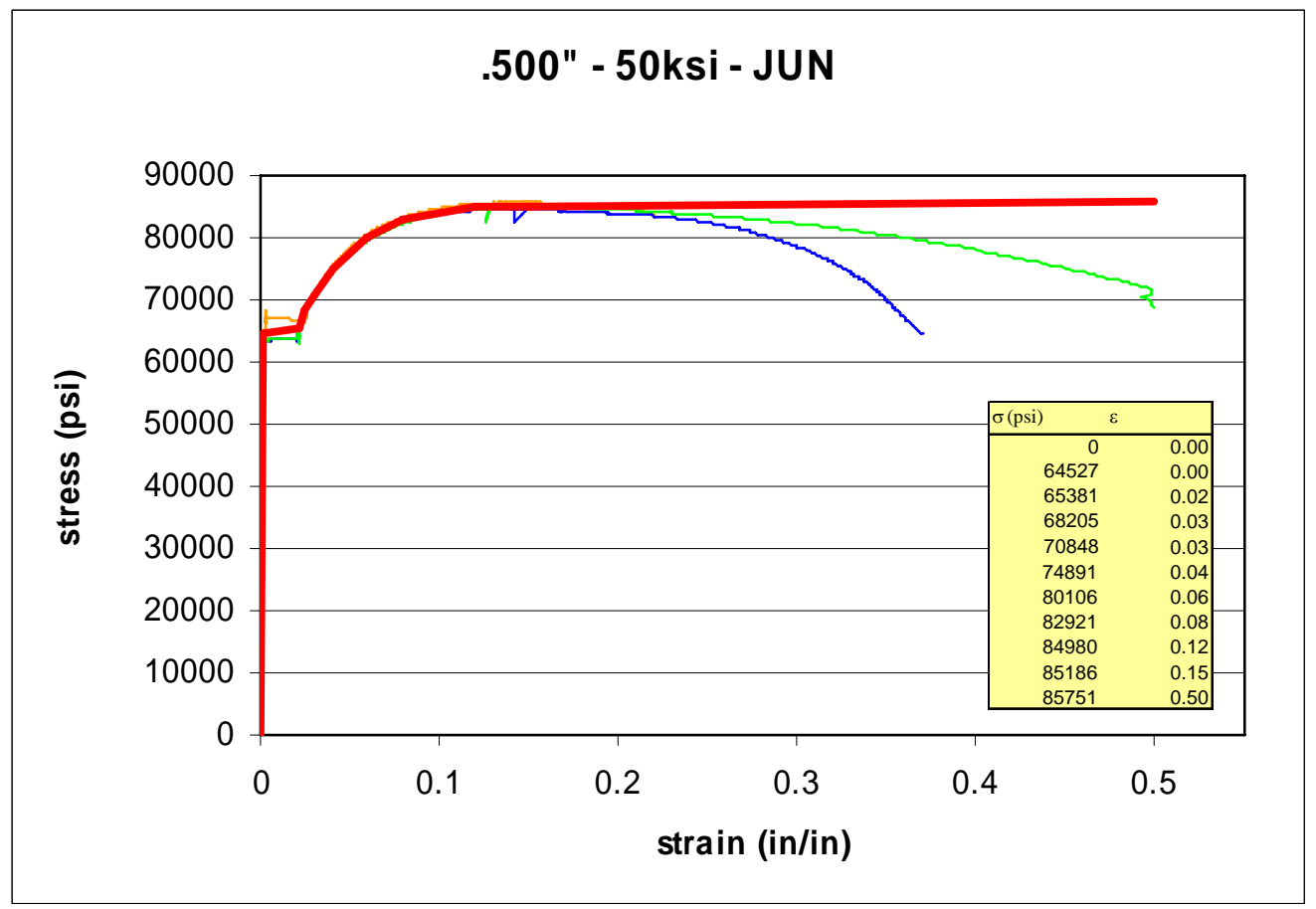

Figure C-3. Tension Test Data for 0. 5” - 50ksi - JUN

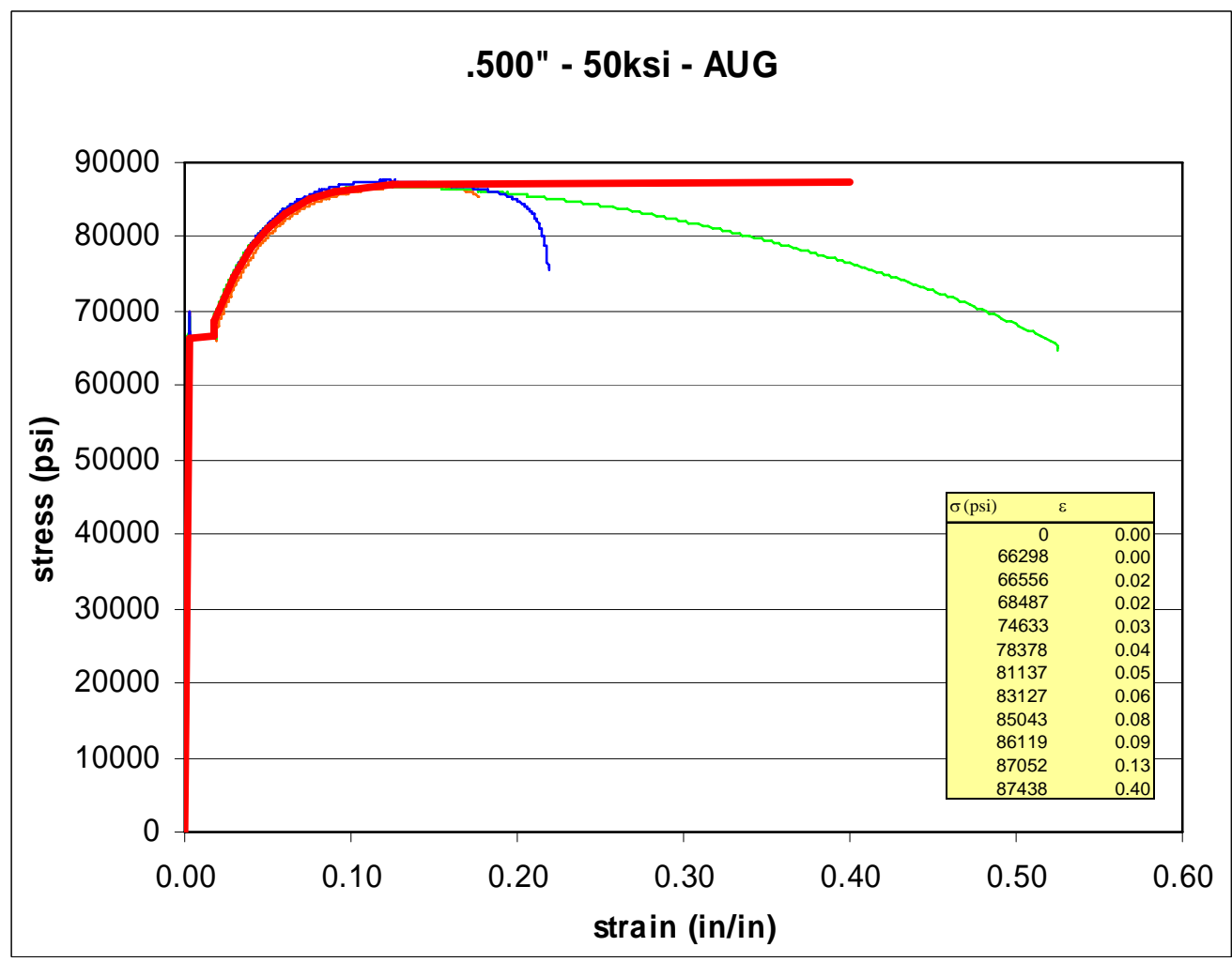

Figure C-4. Tension Test Data for 0.5” - 50ksi - AUG 


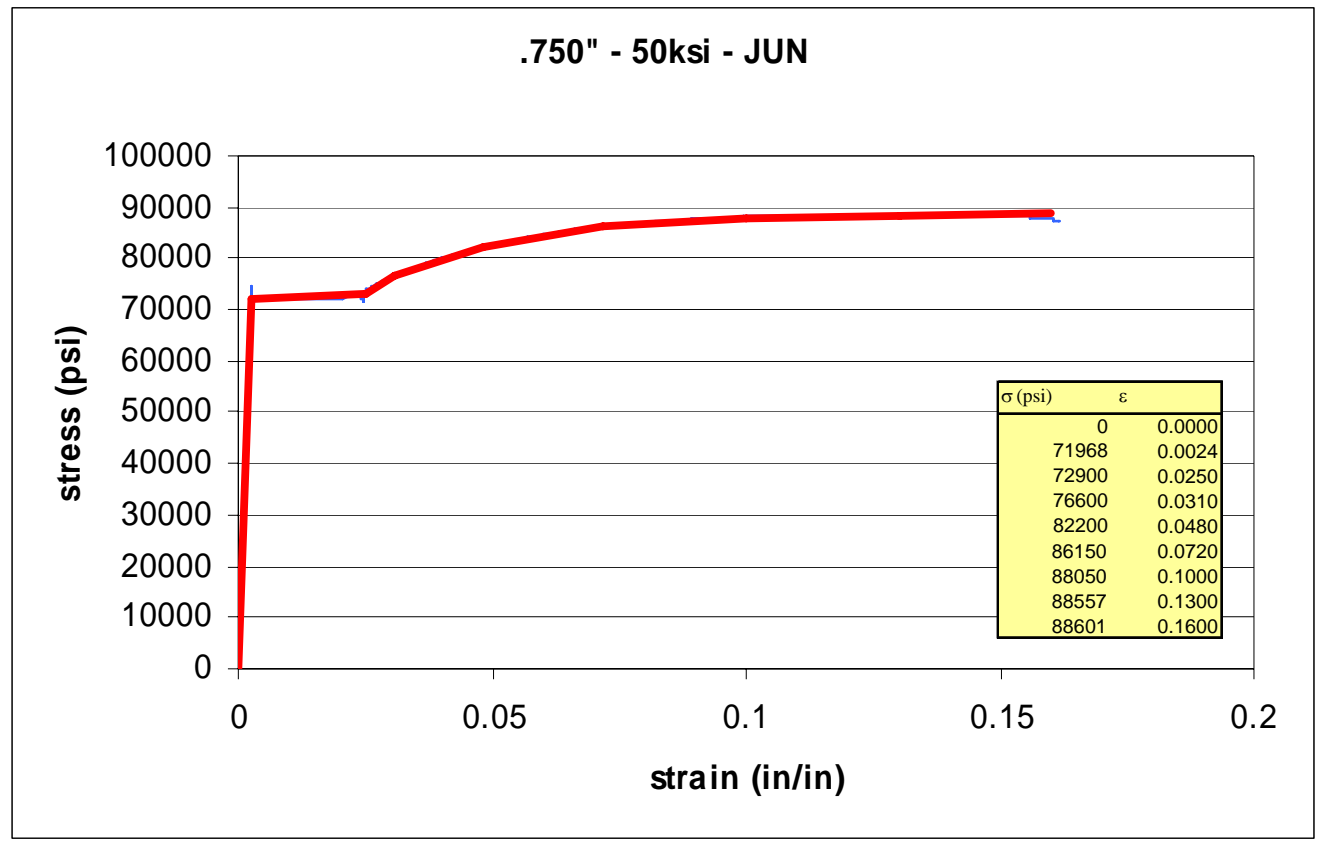

Figure C-5. Tension Test Data for 0. 75”-50ksi - JUN

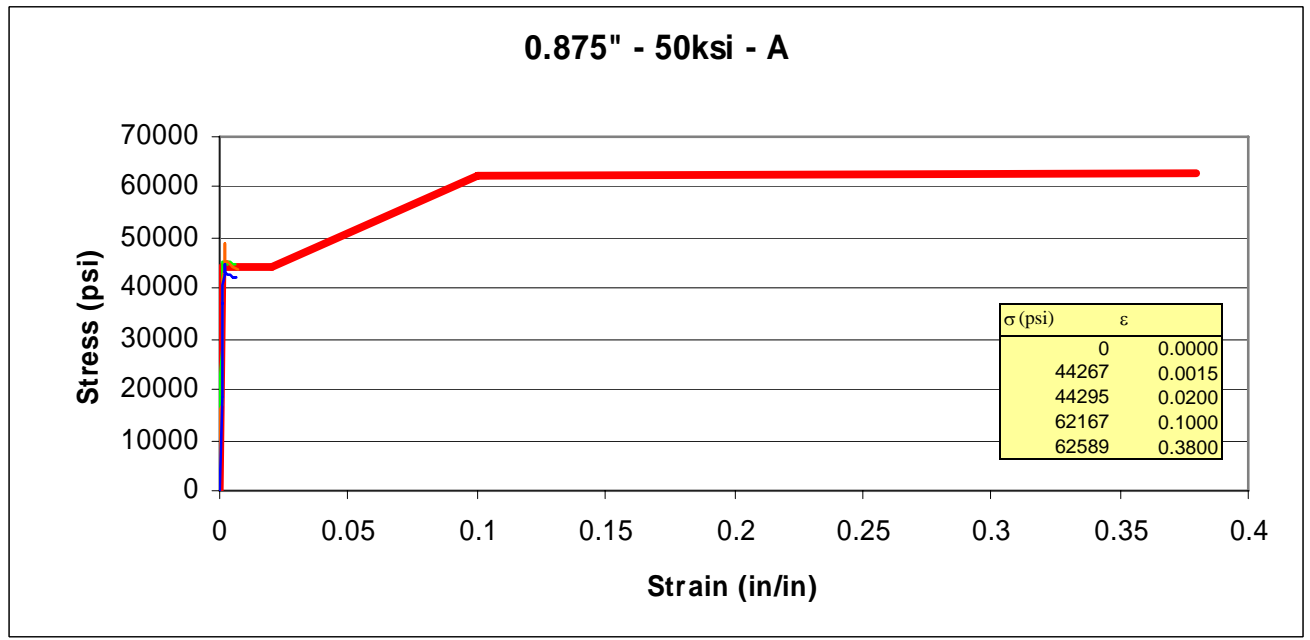

Figure C-6. Tension Test Data for 0. 875” - 50ksi - A 


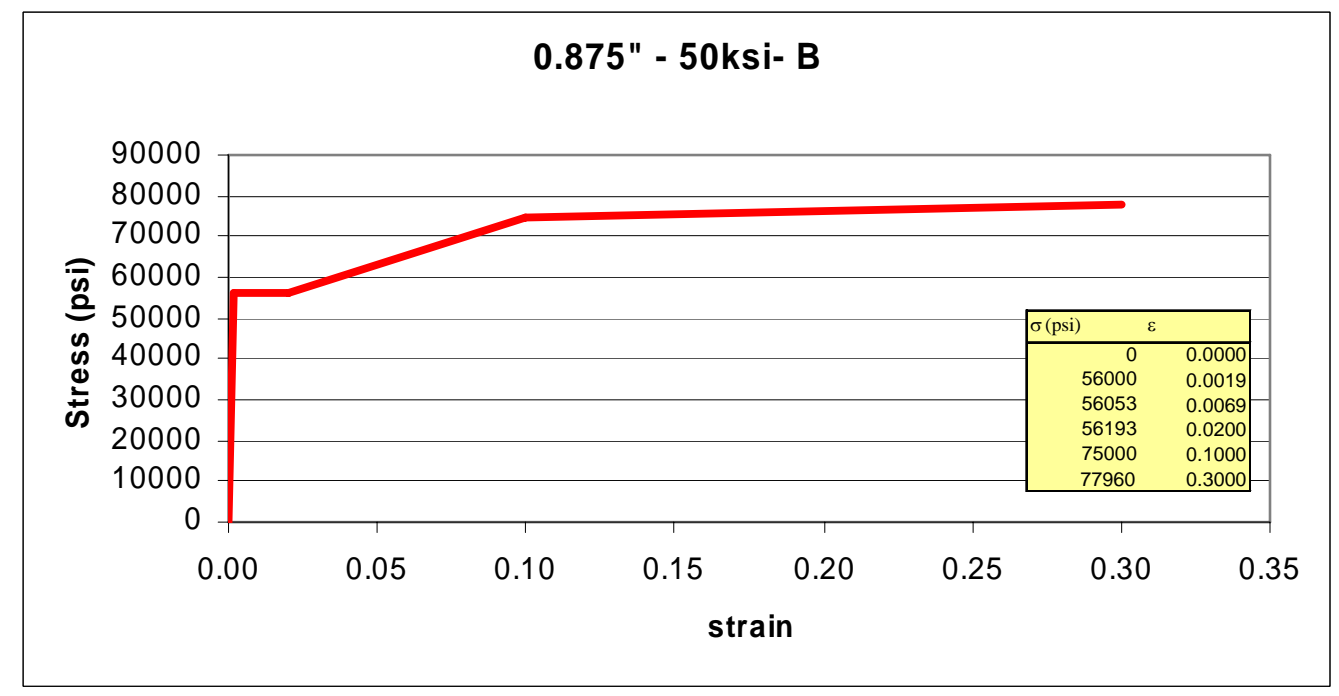

Figure C-7. Tension Test Data for 0. 875” - 50ksi - B

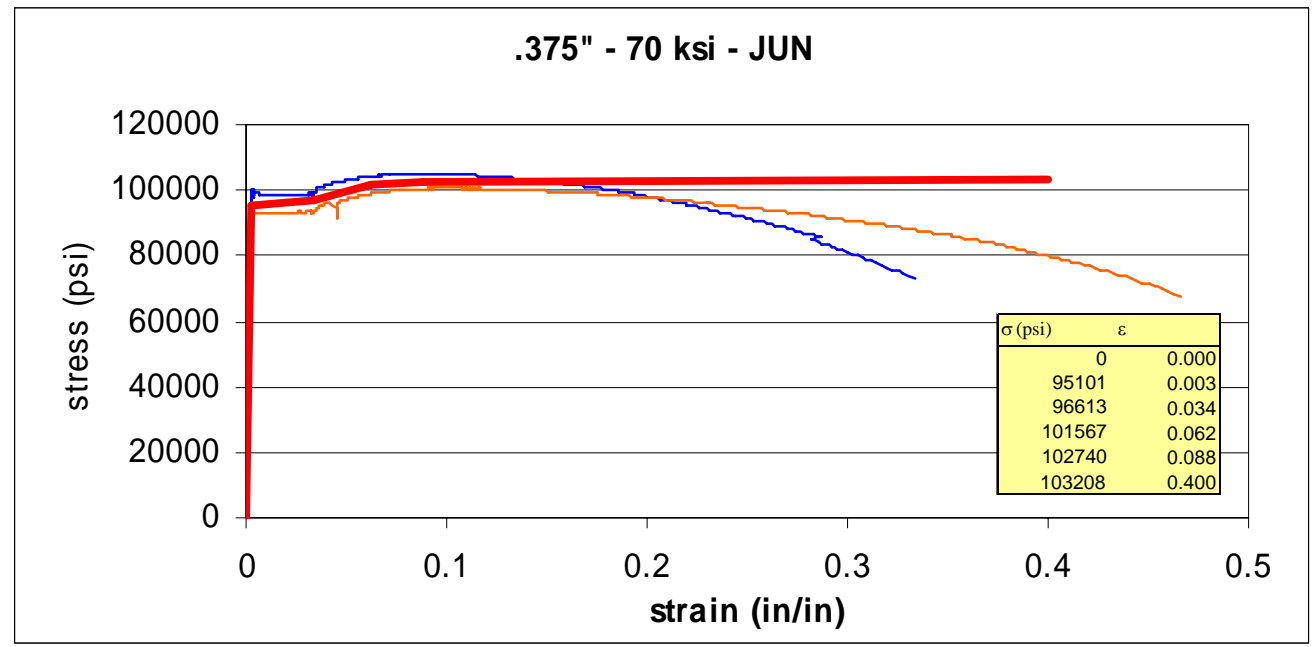

Figure C-8. Tension Test Data for 0. 375” - 70ksi - JUN 


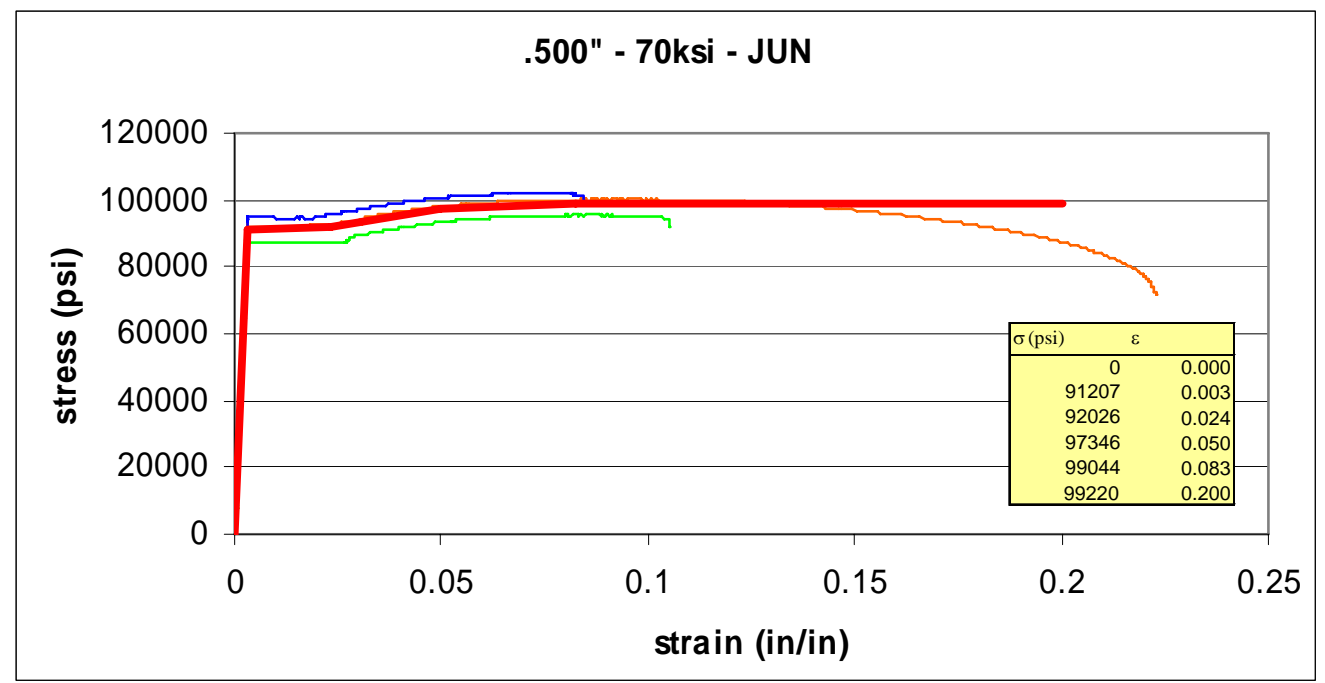

Figure C-9. Tension Test Data for 0. 5” - 70ksi - JUN 


\section{Appendix D: FEA MOMENT-ROTATION CURVES}

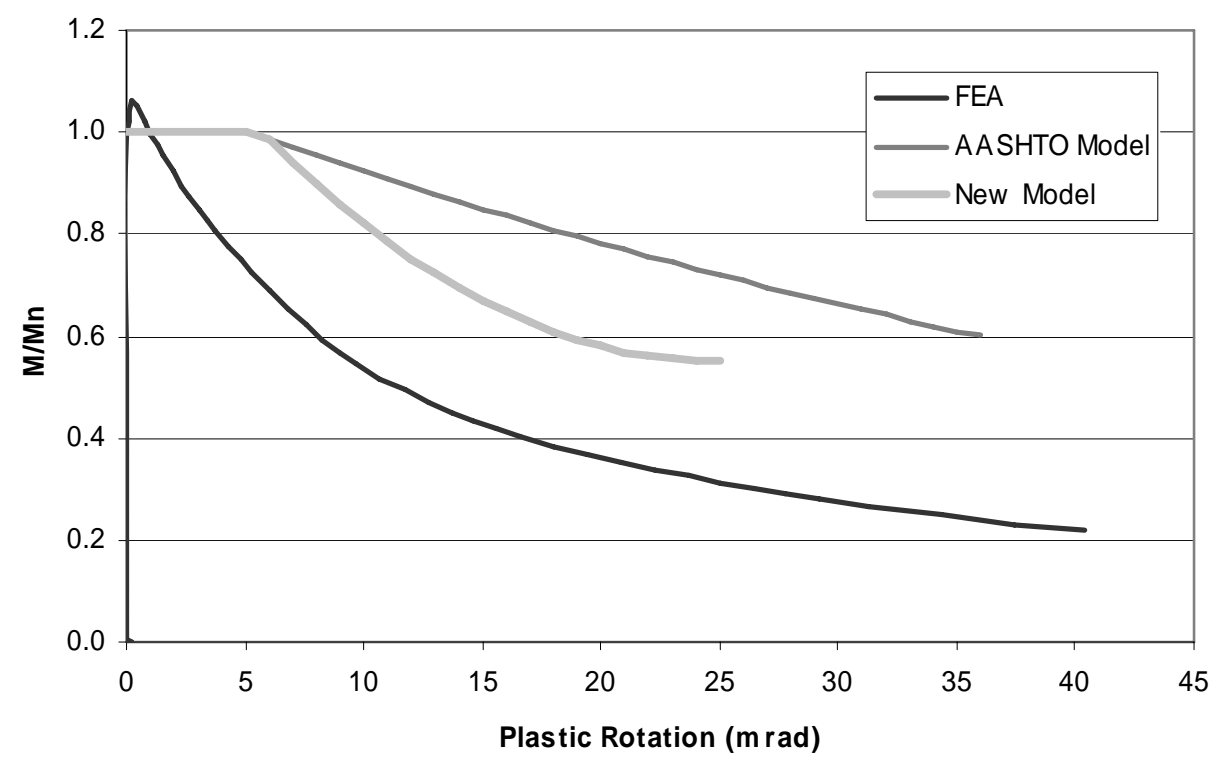

Figure D-1. Moment versus Rotation for Girder CF-1-65-4-50

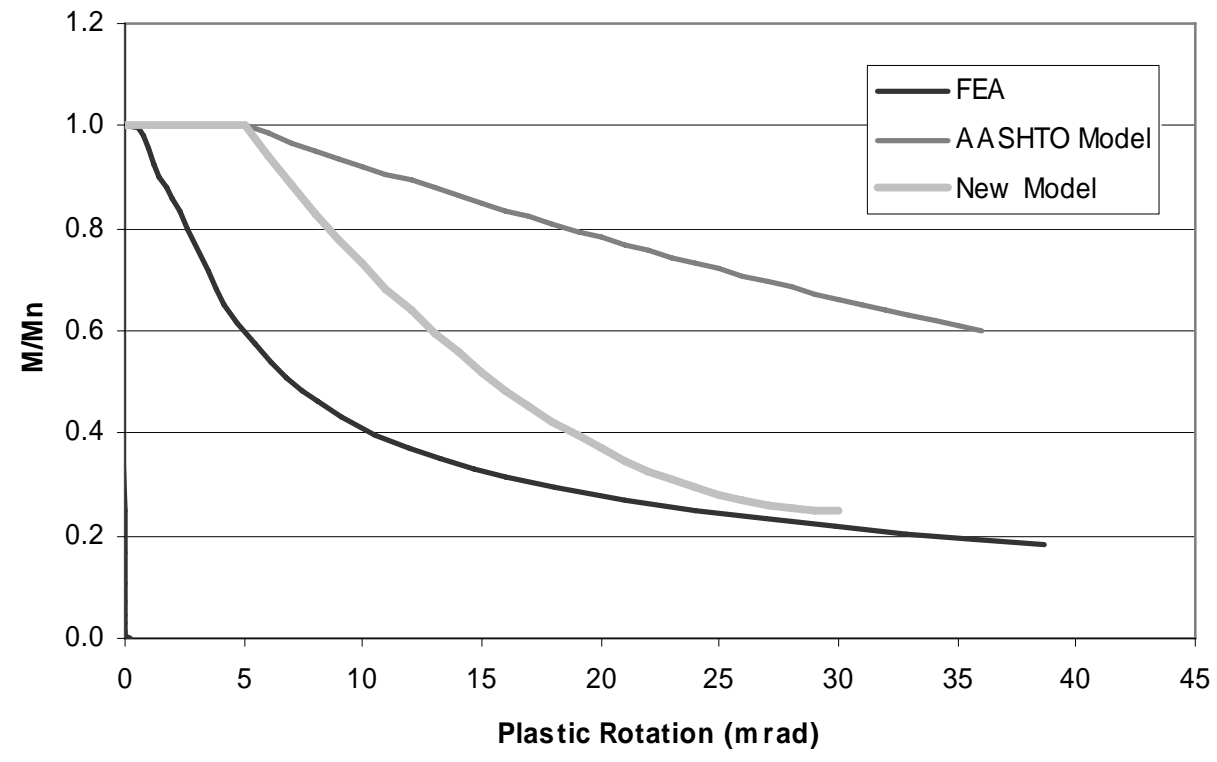

Figure D-2. Moment versus Rotation for Girder CF-2-65-4-50 


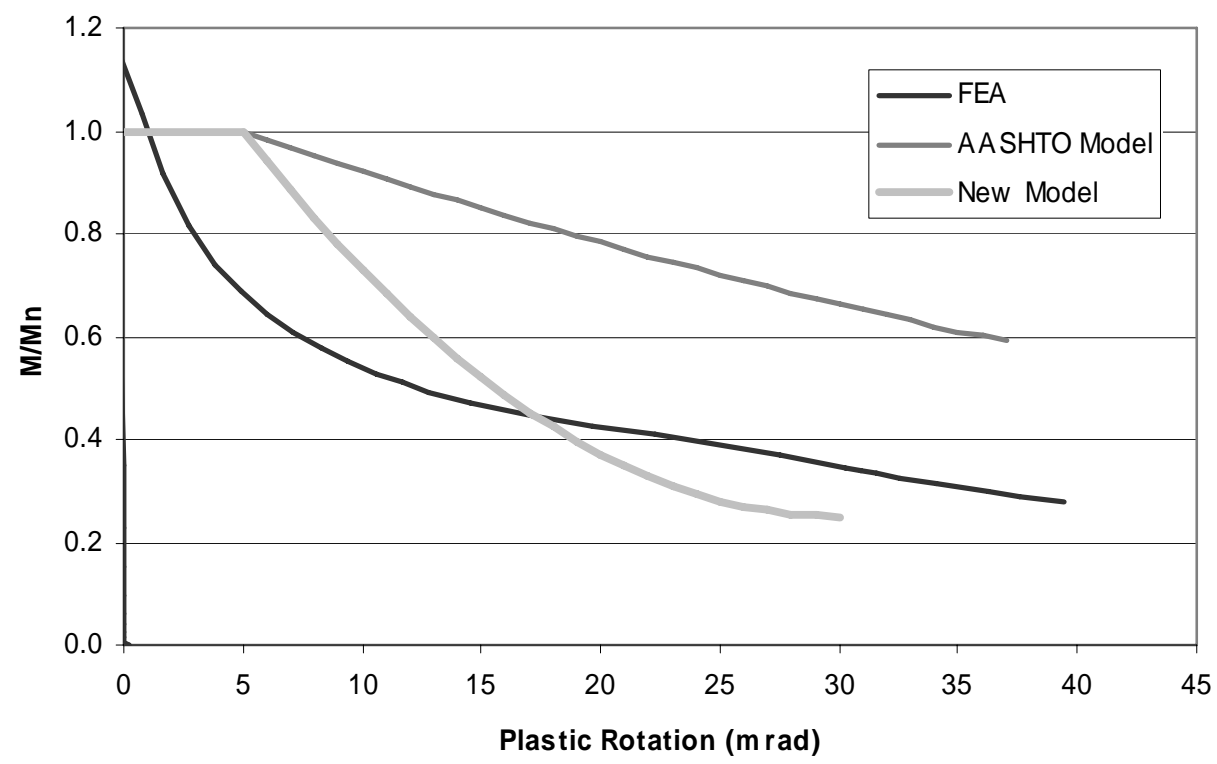

Figure D-3. Moment versus Rotation for Girder CF-30-65-4-50

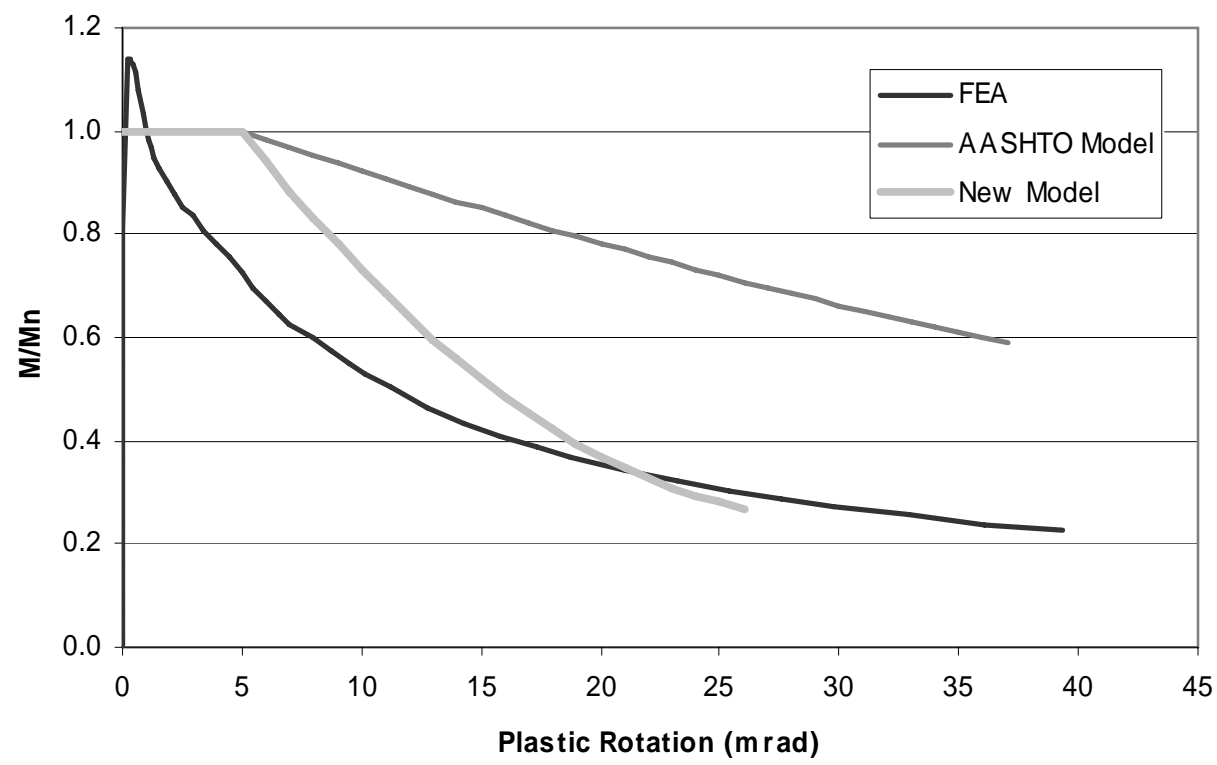

Figure D-4. Moment versus Rotation for Girder SF-1-65-4-50 


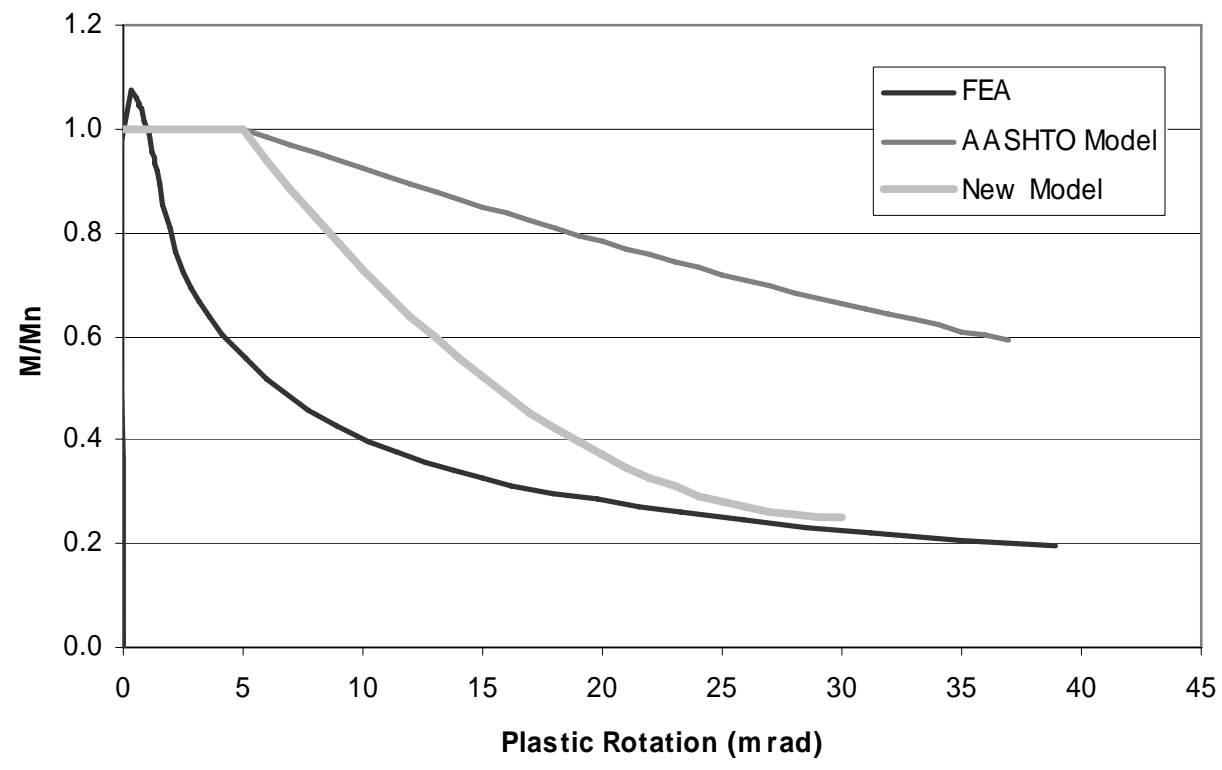

Figure D-5. Moment versus Rotation for Girder SF-2-65-4-50

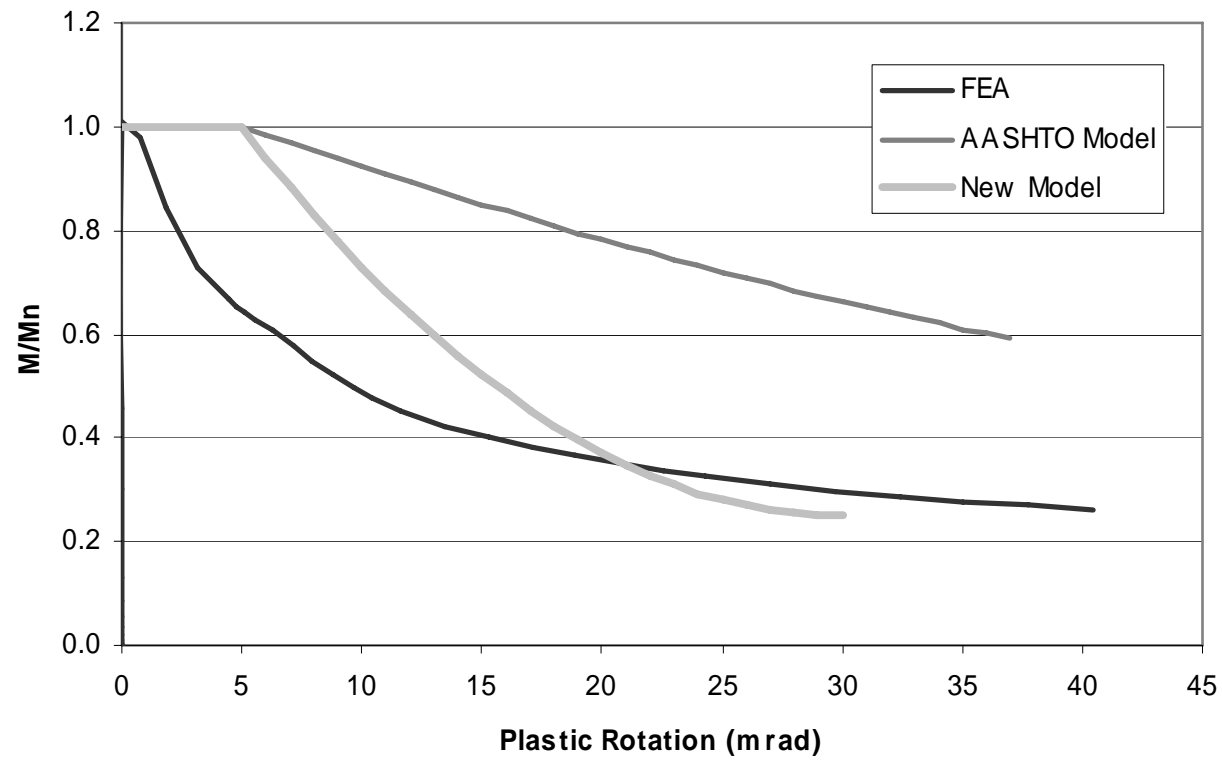

Figure D-6. Moment versus Rotation for Girder SF-30-65-4-50 


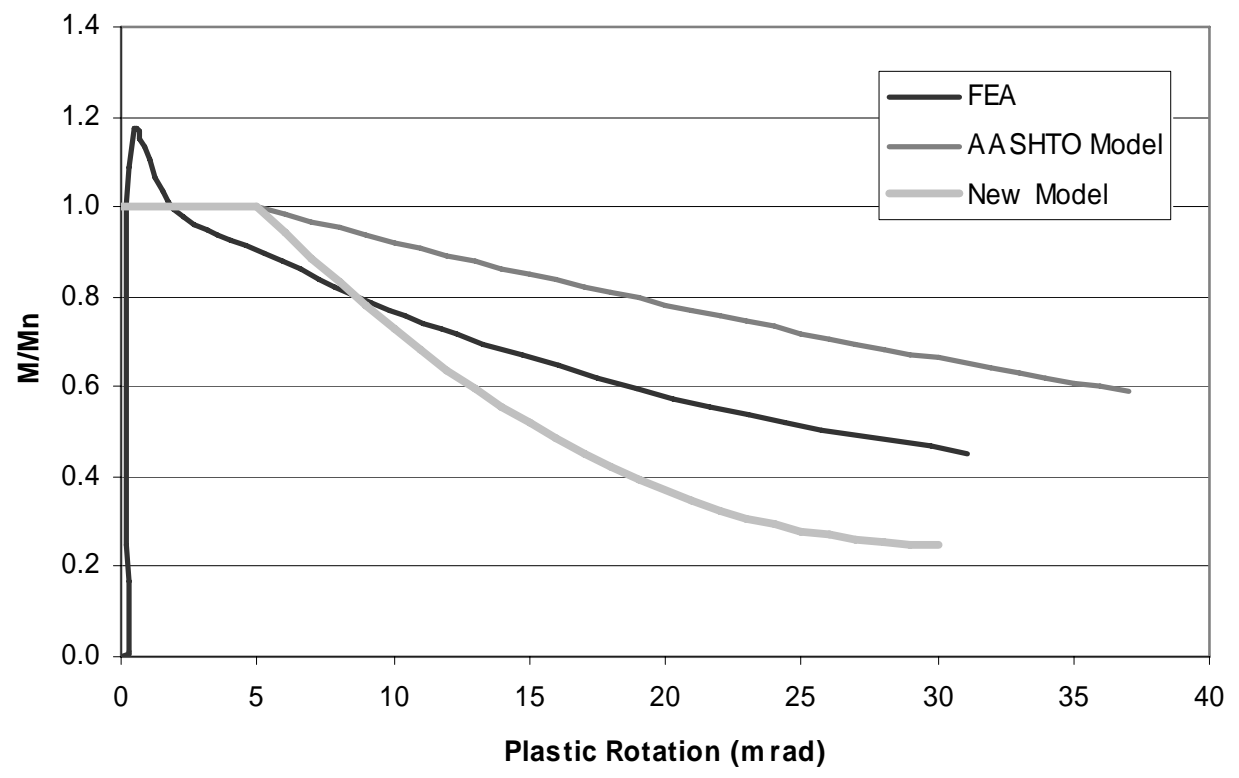

Figure D-7. Moment versus Rotation for Girder SF-1-65-3-50

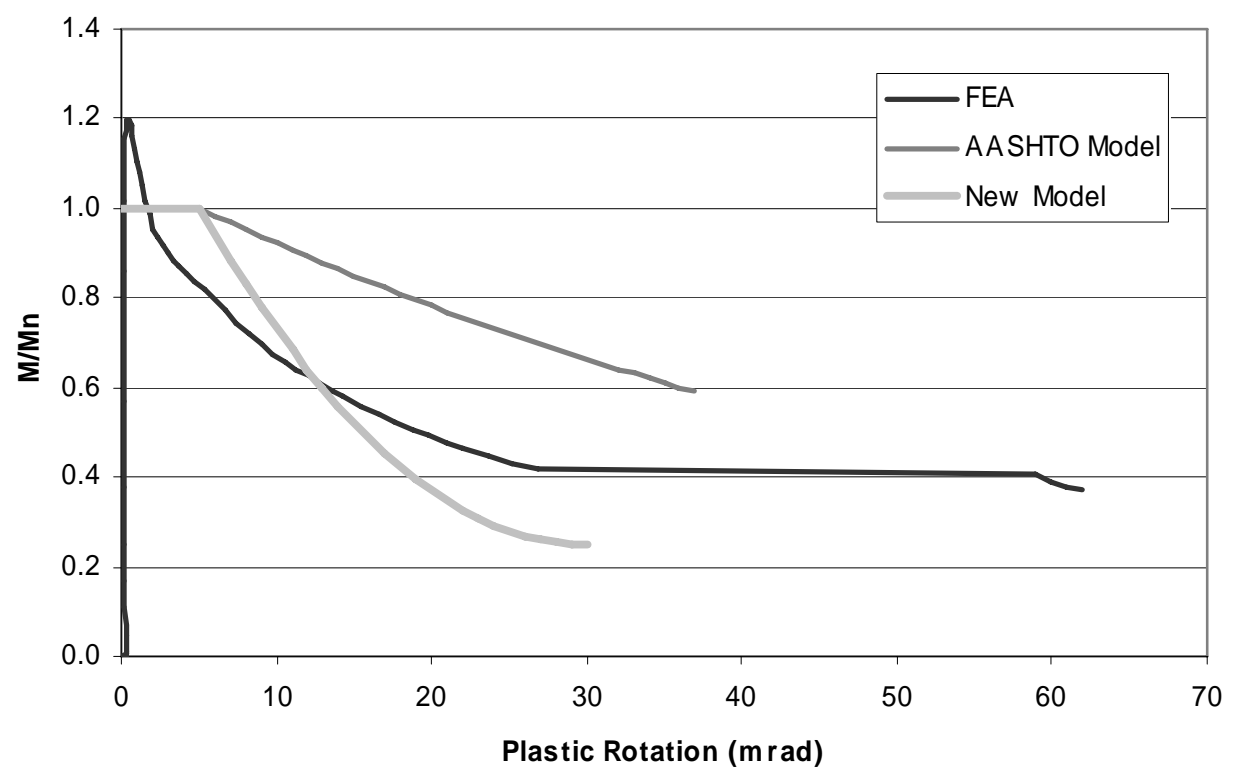

Figure D-8. Moment versus Rotation for Girder SF-2-65-3-50 


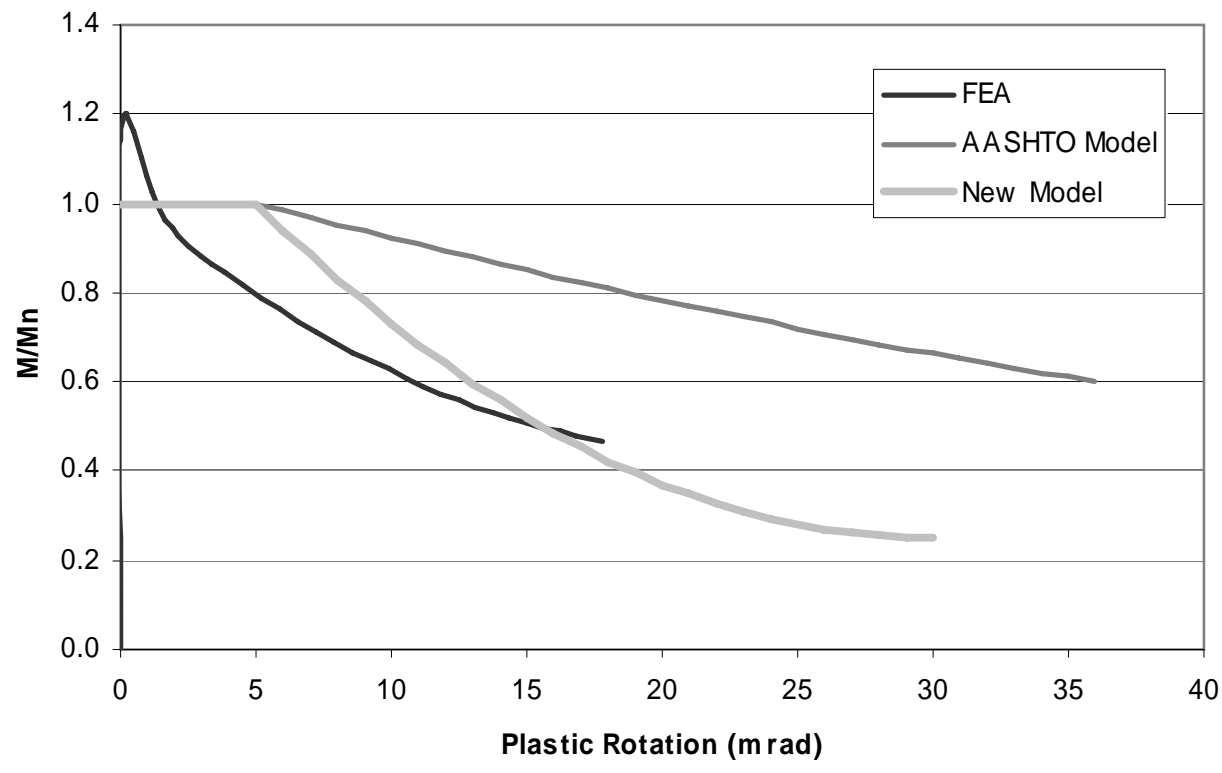

Figure D-9. Moment versus Rotation for Girder SF-30-65-3-50

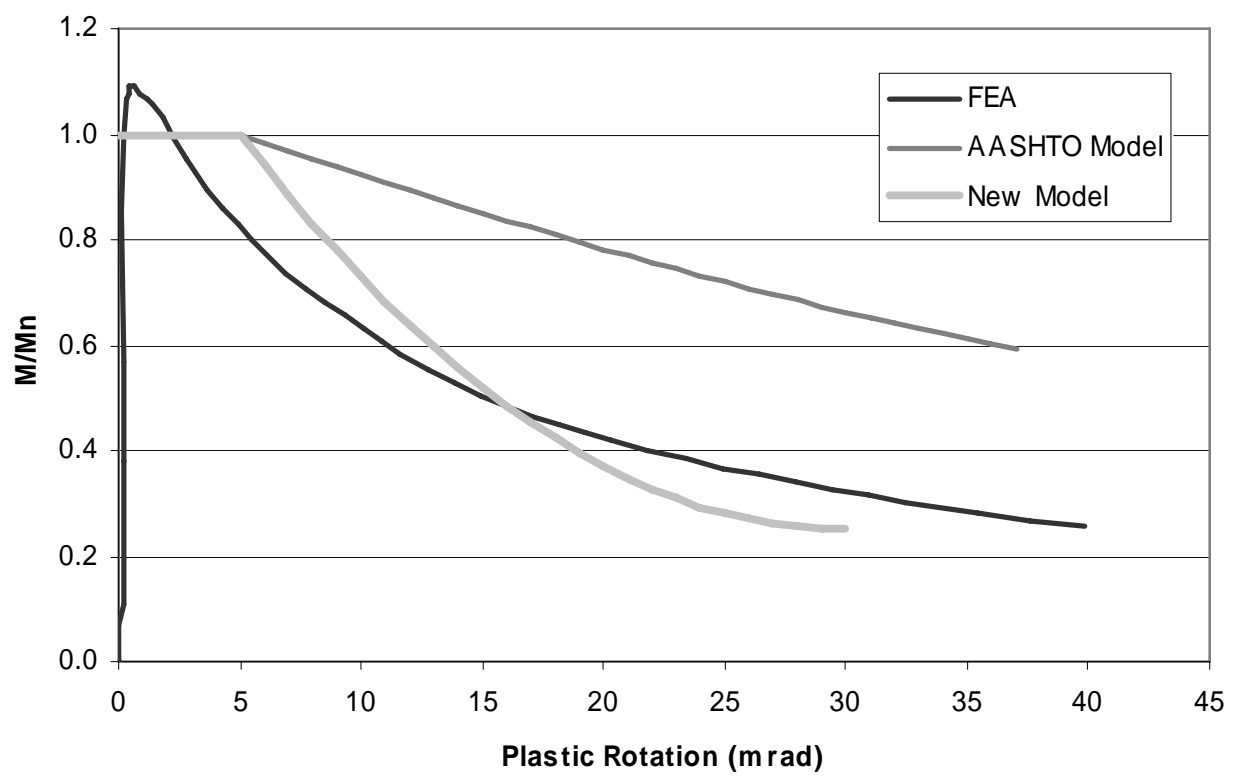

Figure D-10. Moment versus Rotation for Girder CF-1-65-4-H 


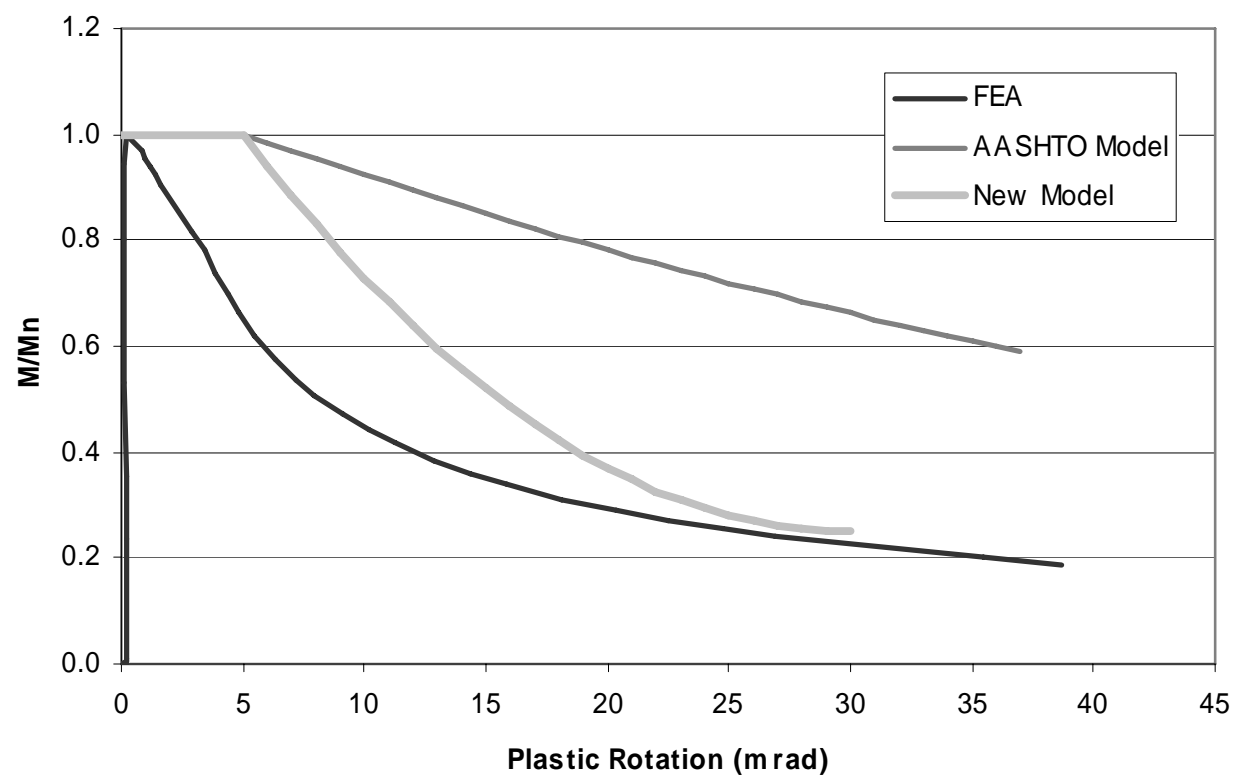

Figure D-11. Moment versus Rotation for Girder CF-2-65-4-H

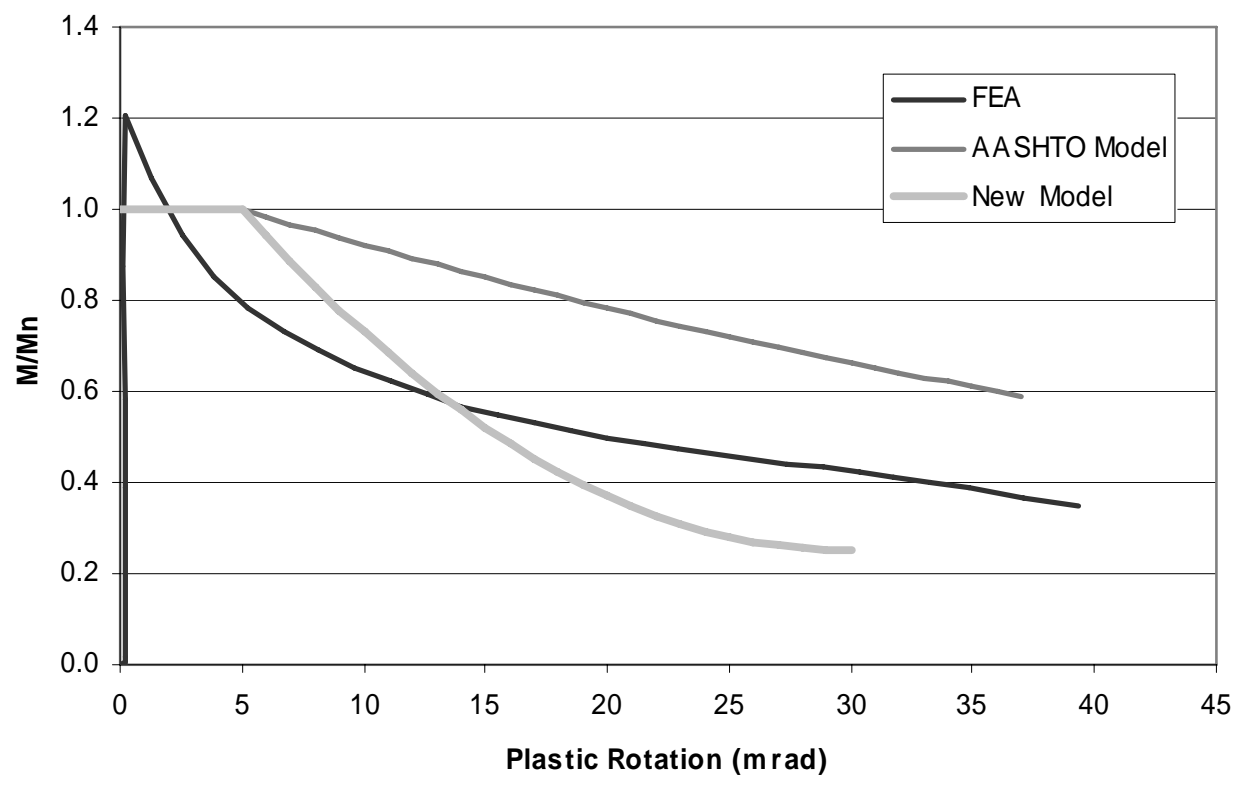

Figure D-12. Moment versus Rotation for Girder CF-30-65-4-H 


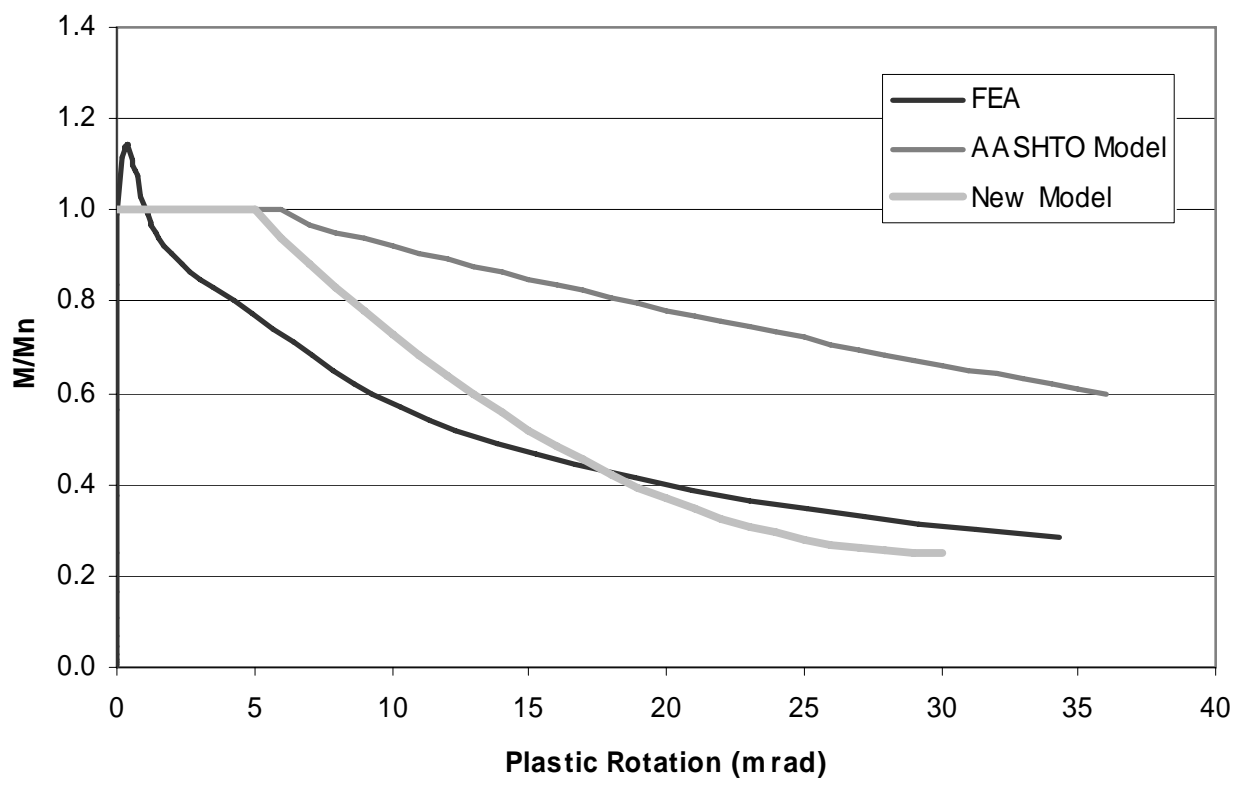

Figure D-13. Moment versus Rotation for Girder SF-1-65-4-H

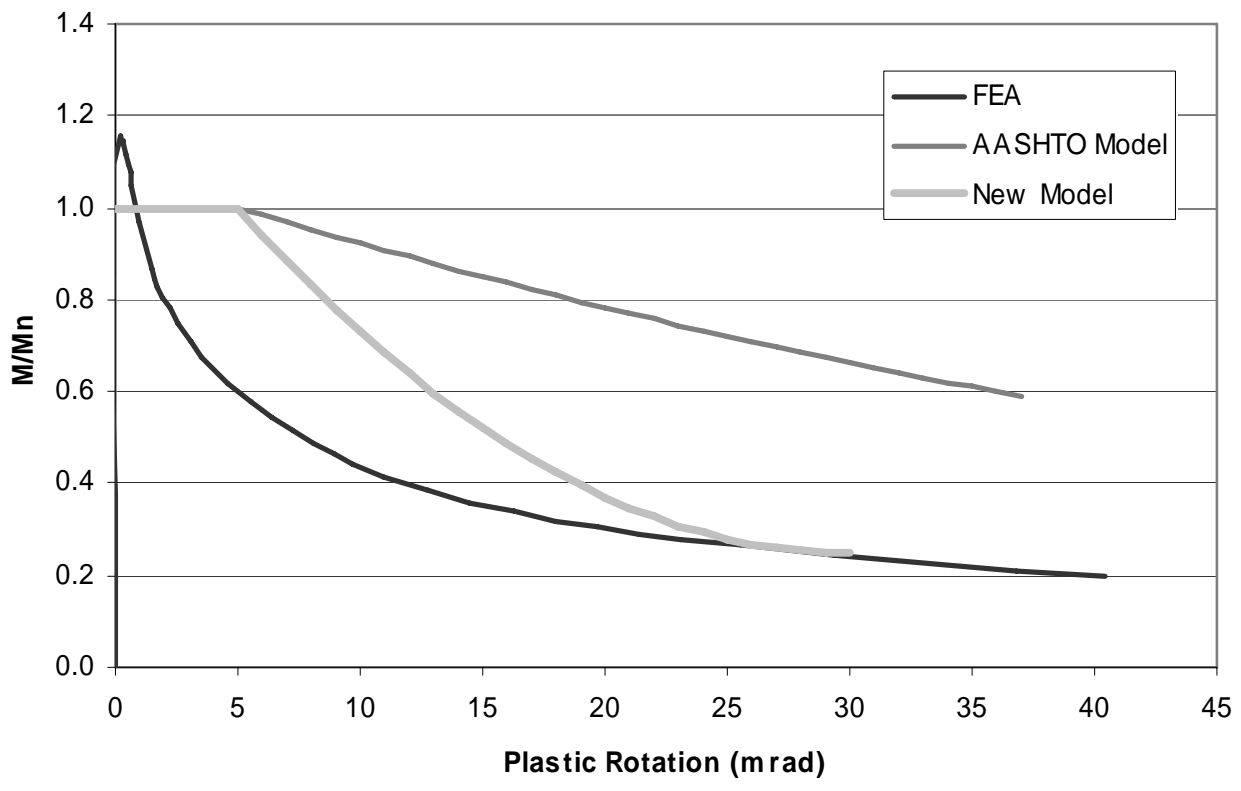

Figure D-14. Moment versus Rotation for Girder SF-2-65-4-H 


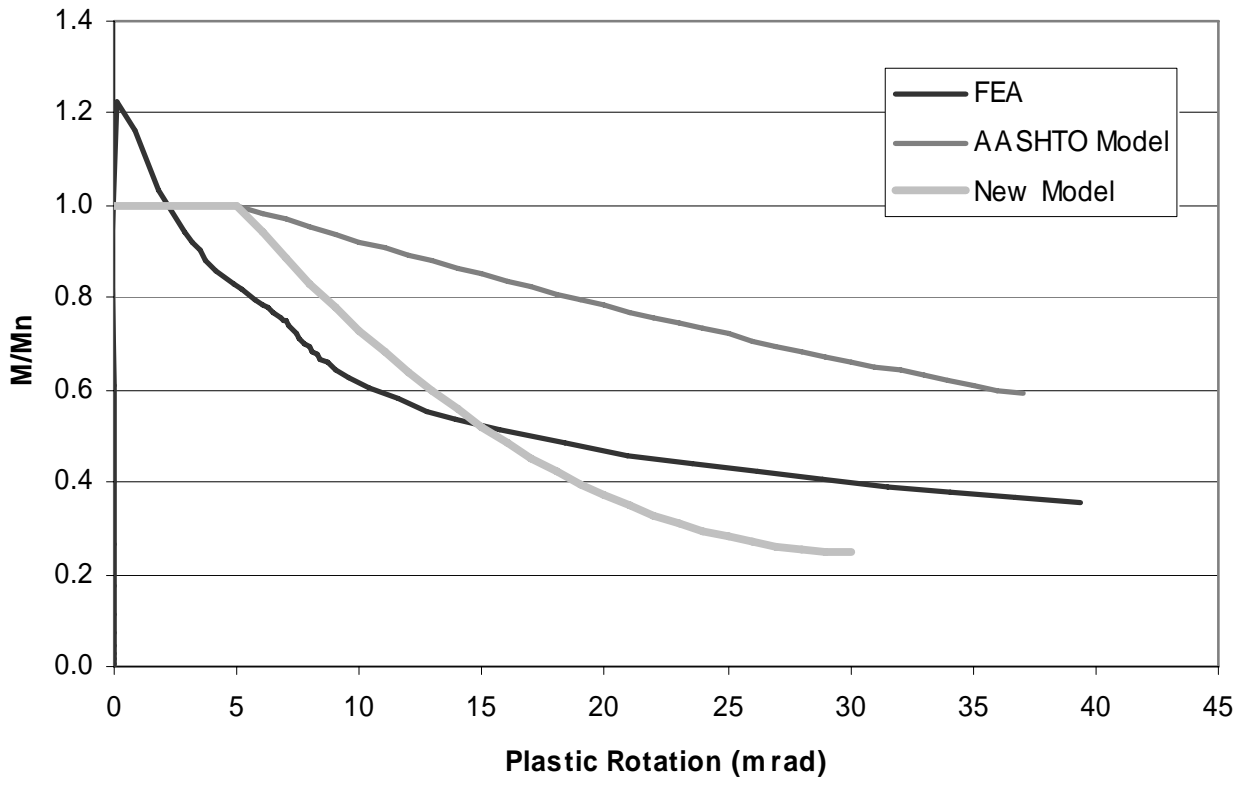

Figure D-15. Moment versus Rotation for Girder SF-30-65-4-H

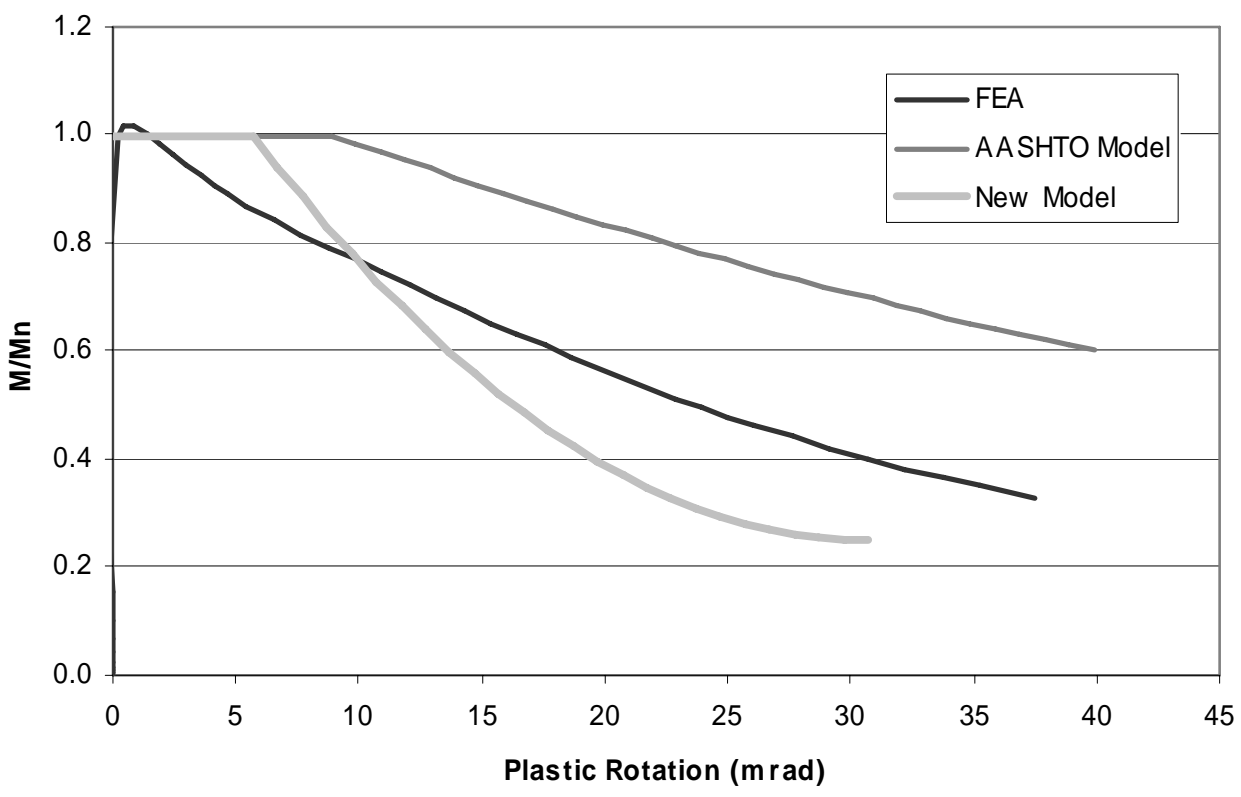

Figure D-16. Moment versus Rotation for Girder CF-0.5-65-3-H 


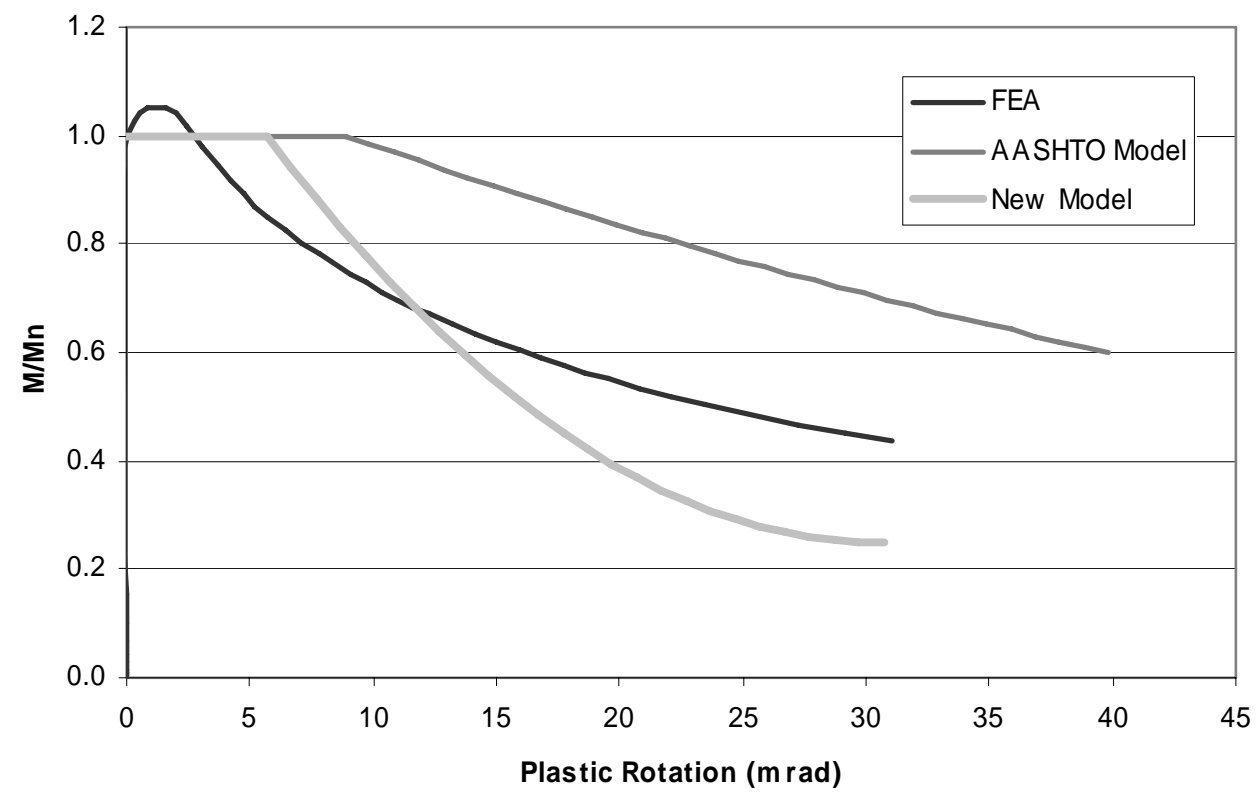

Figure D-17. Moment versus Rotation for Girder CF-1-65-3-H

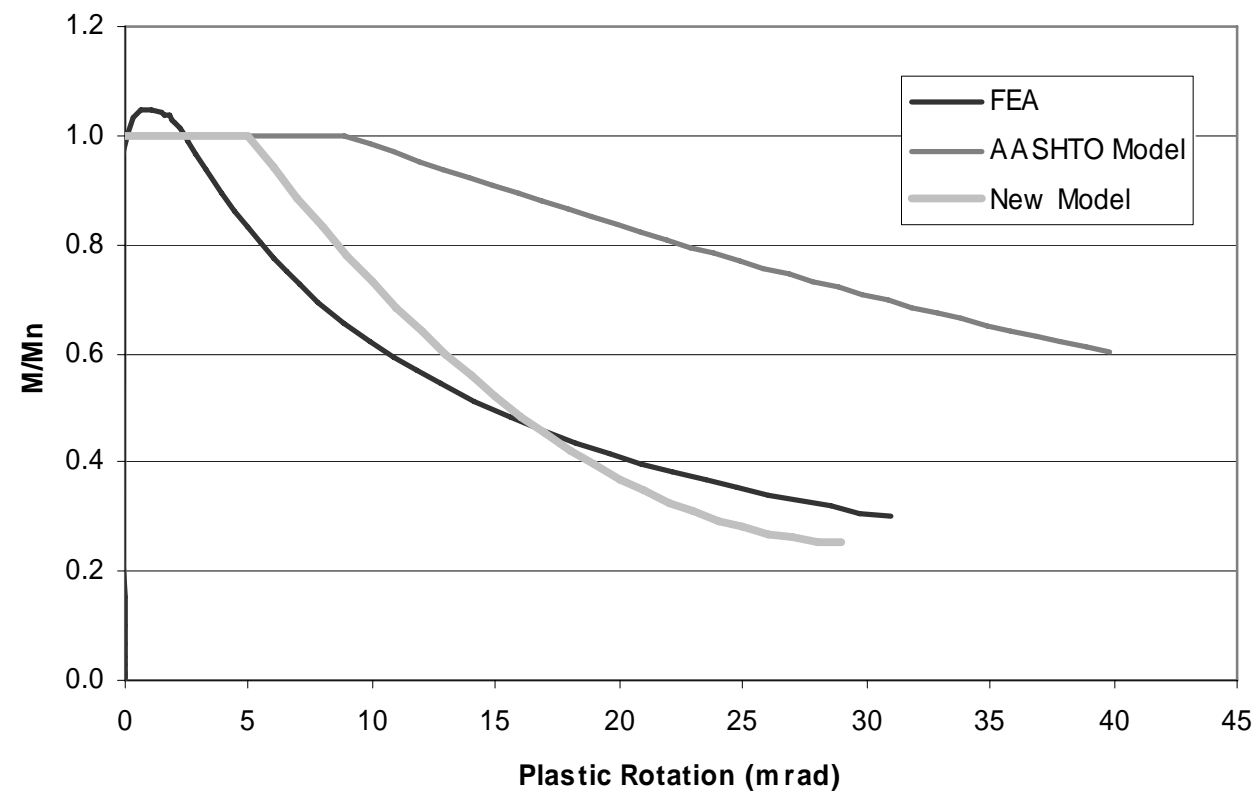

Figure D-18. Moment versus Rotation for Girder CF-1.65-65-3-H 


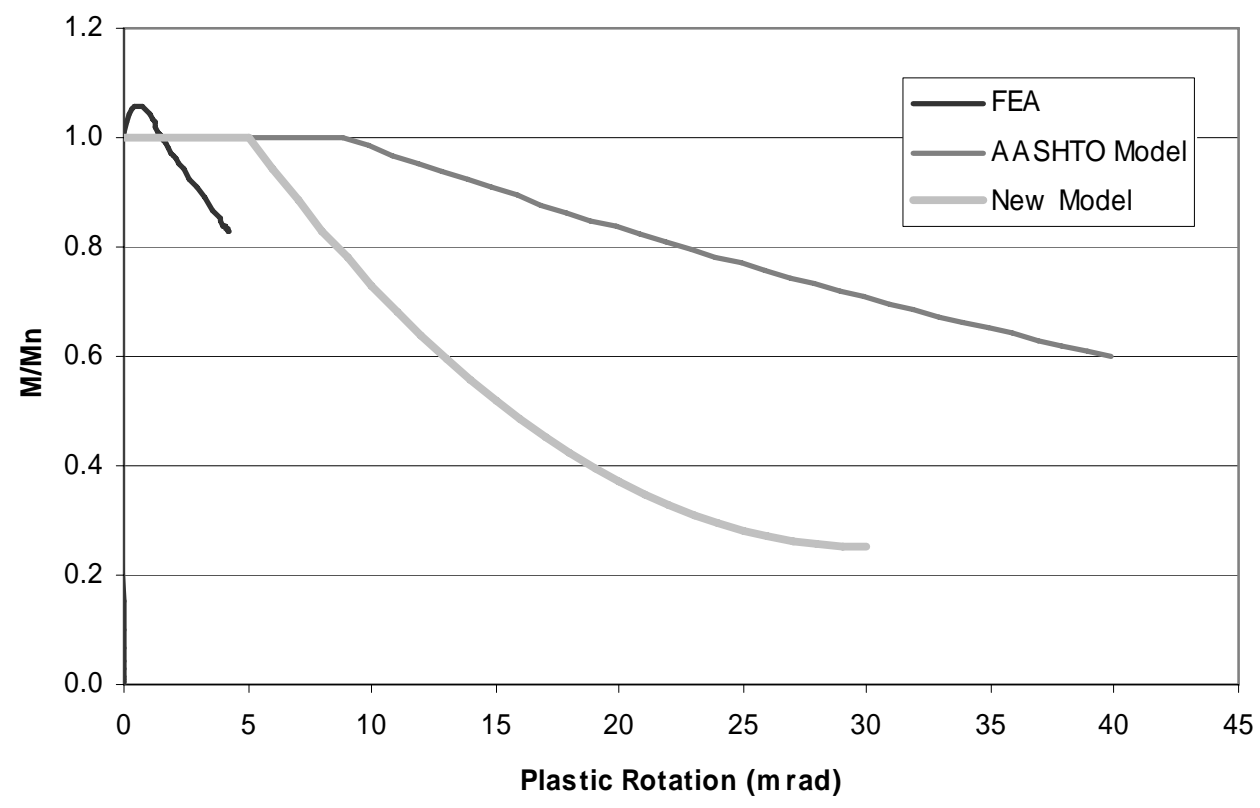

Figure D-19. Moment versus Rotation for Girder CF-30-65-3-H

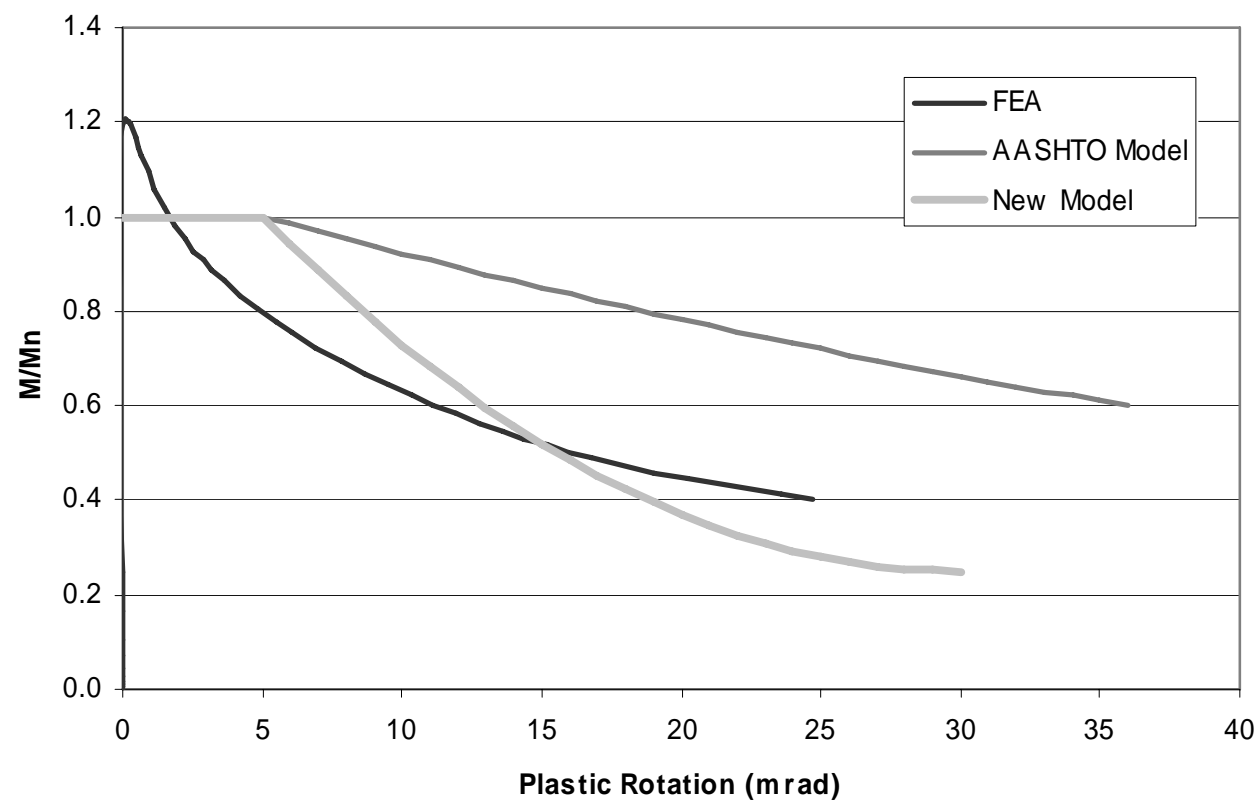

Figure D-20. Moment versus Rotation for Girder SF-1.75-65-3-H 


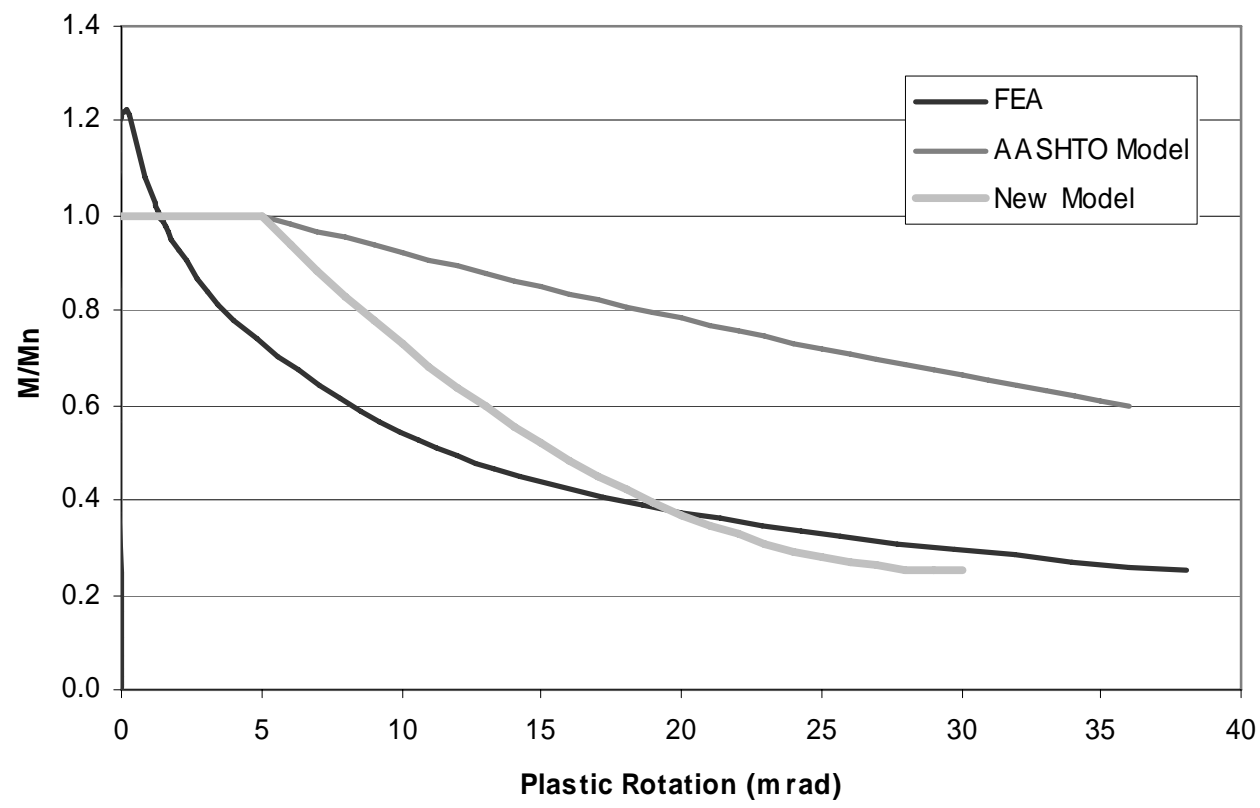

Figure D-21. Moment versus Rotation for Girder SF-30-65-3-H

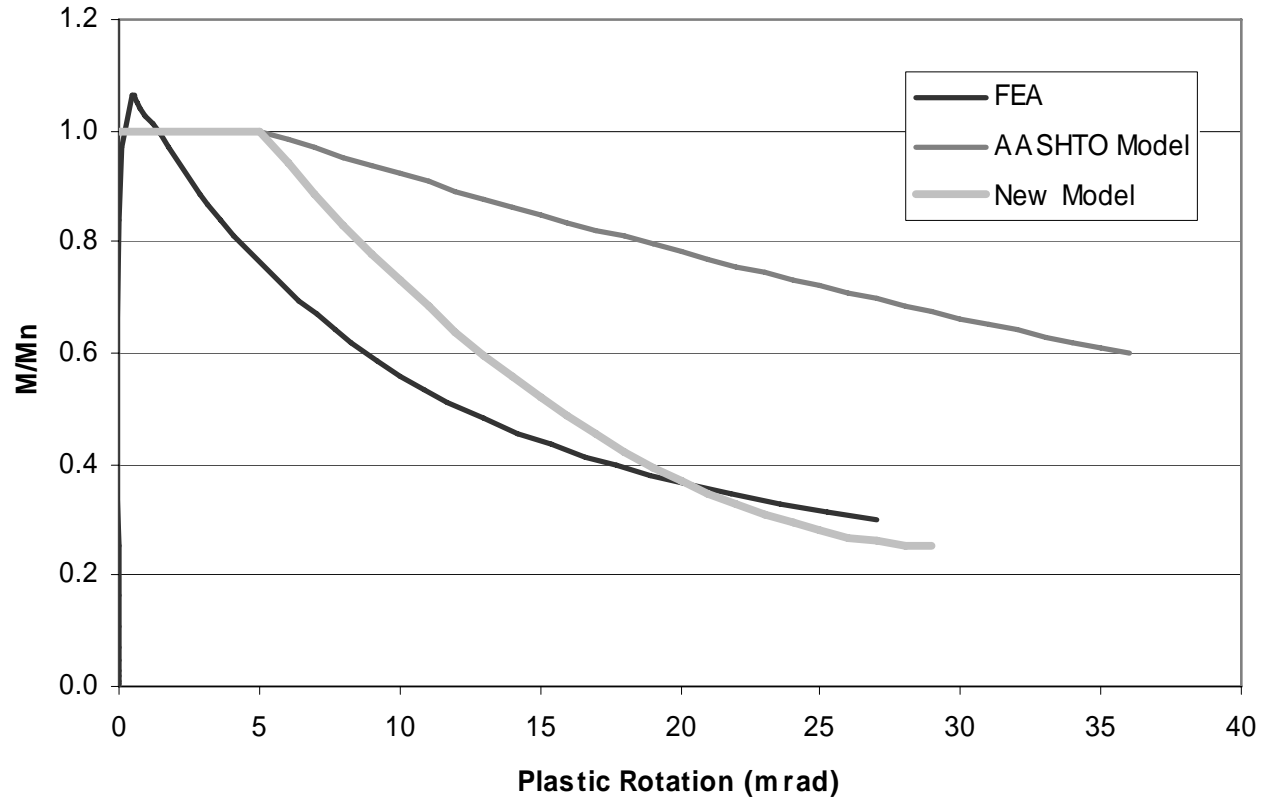

Figure D-22. Moment versus Rotation for Girder CF-1-50-4-50 


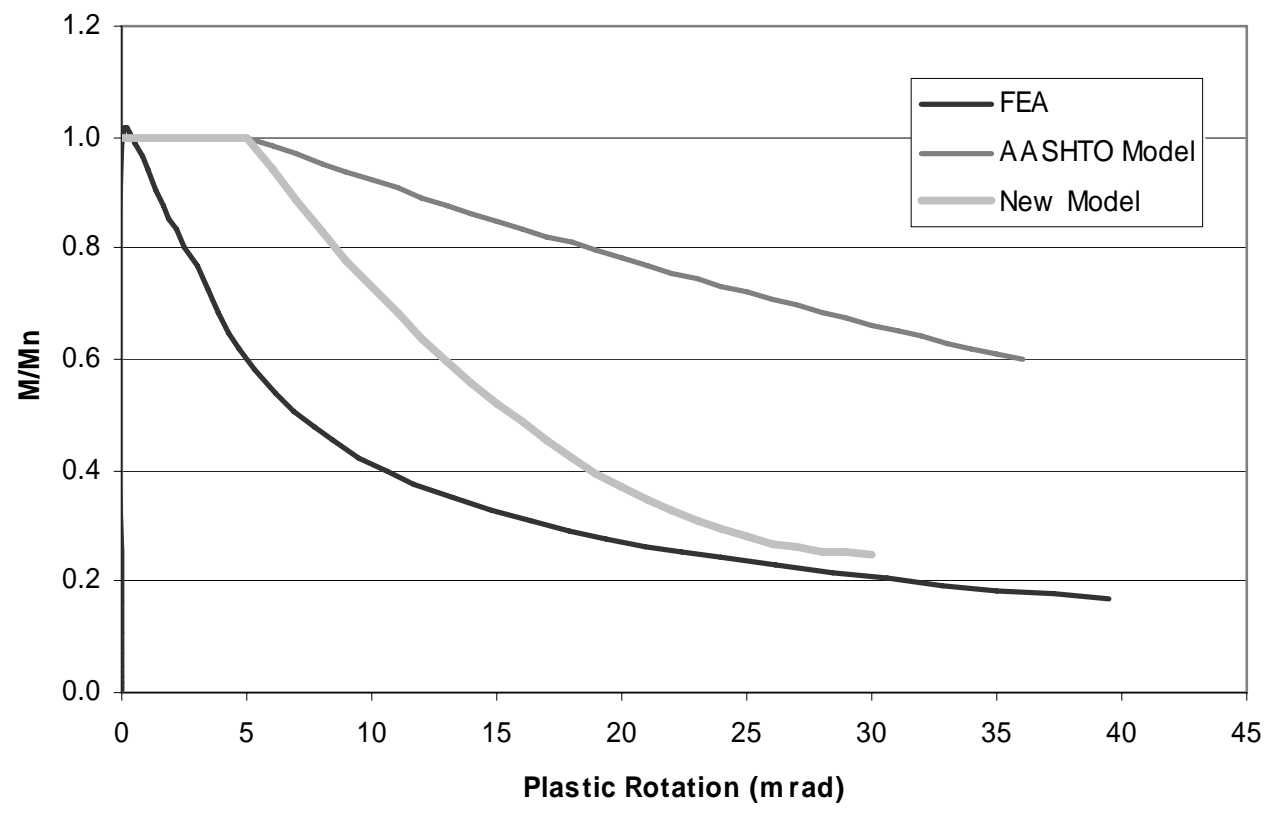

Figure D-23. Moment versus Rotation for Girder CF-2-50-4-50

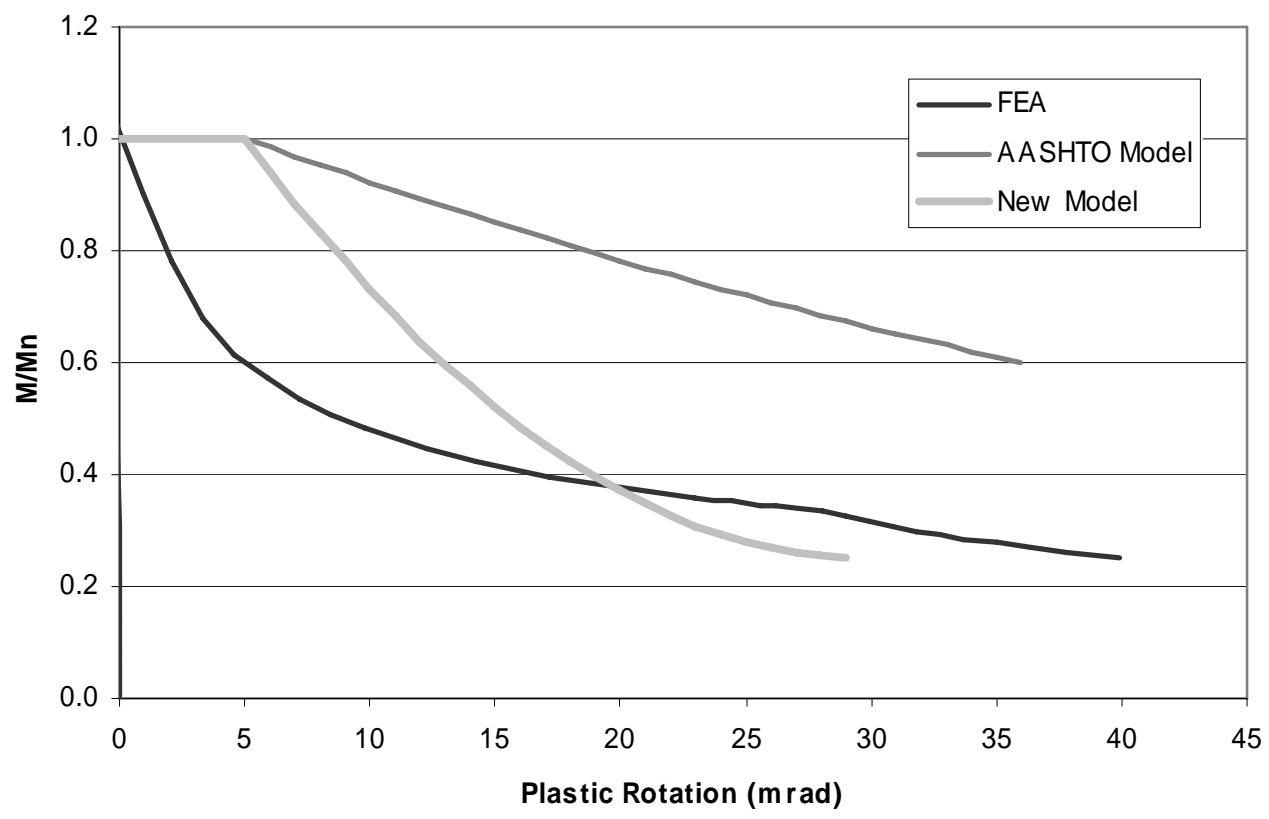

Figure D-24. Moment versus Rotation for Girder CF-30-50-4-50 


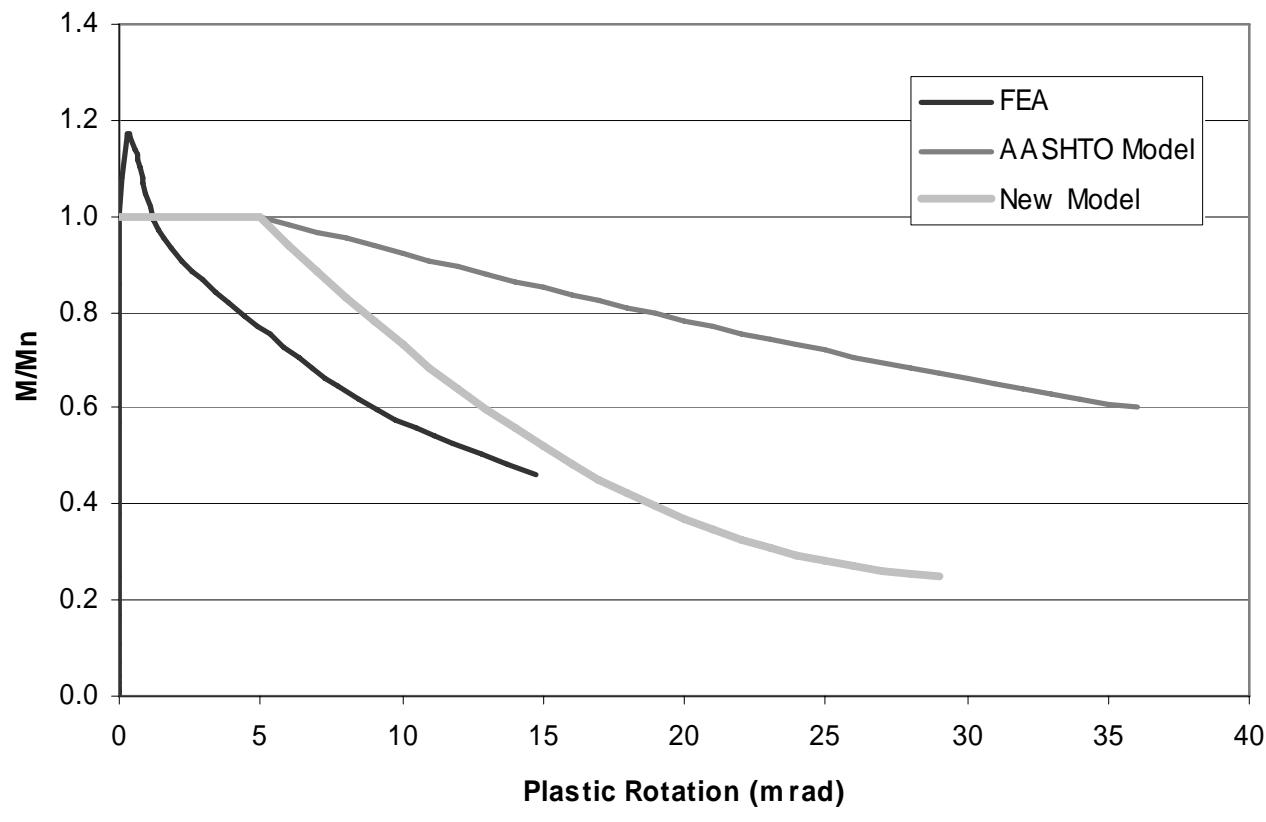

Figure D-25. Moment versus Rotation for Girder SF-1-50-4-50

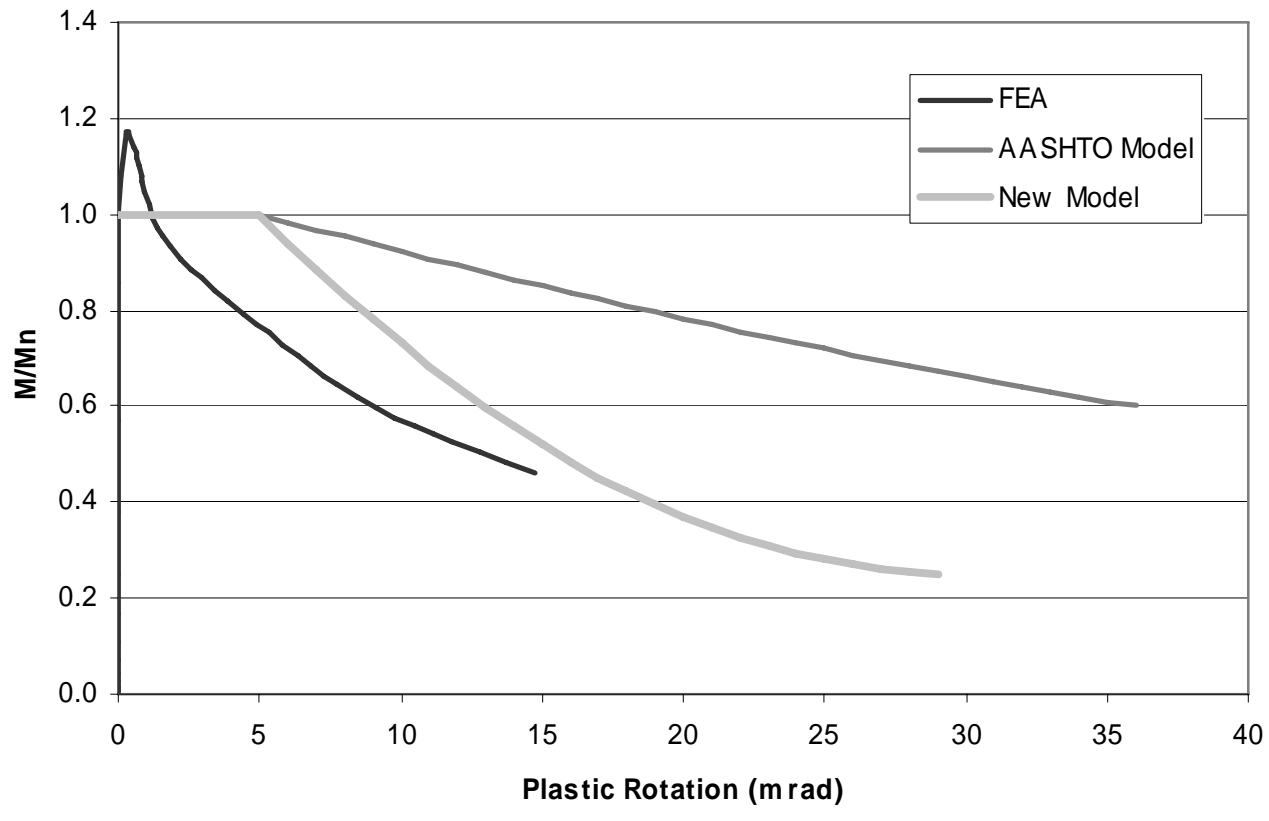

Figure D-26. Moment versus Rotation for Girder SF-2-50-4-50 


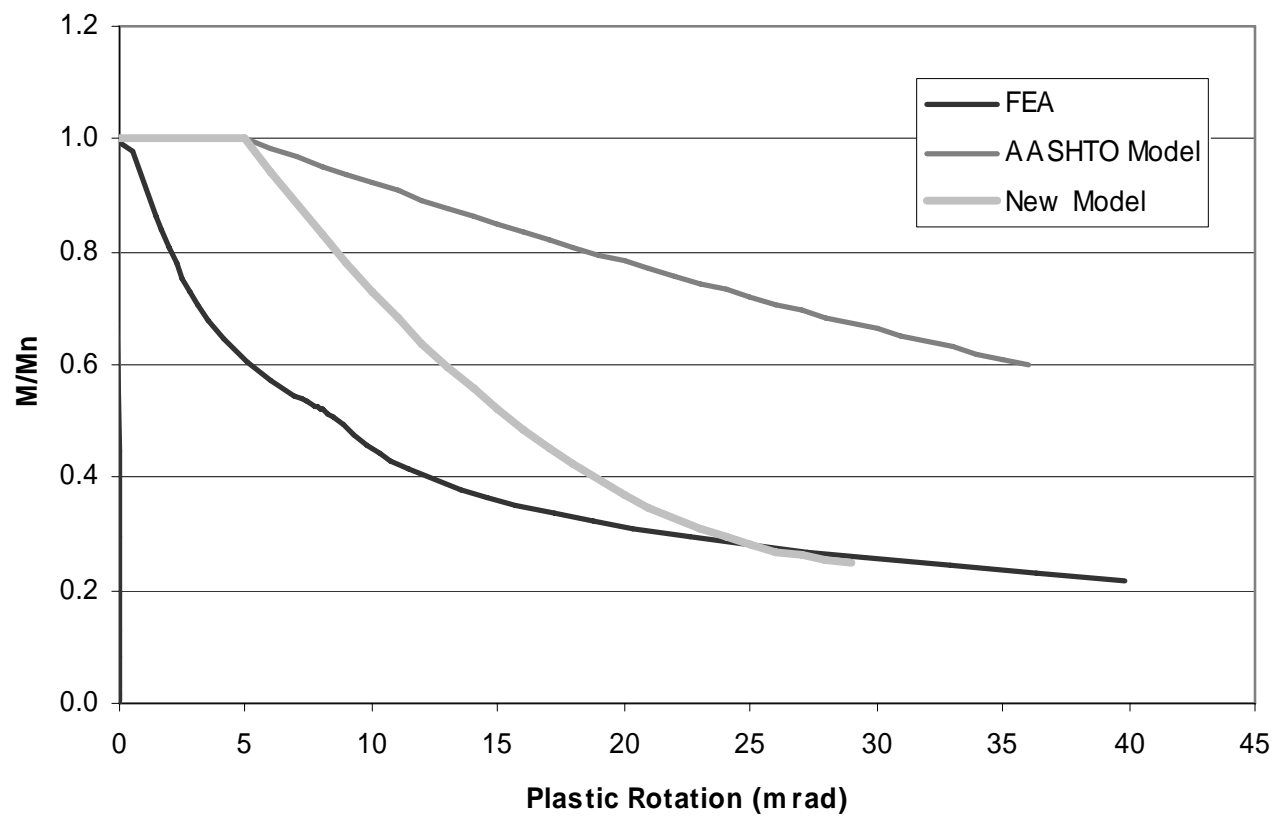

Figure D-27. Moment versus Rotation for Girder SF-30-50-4-50

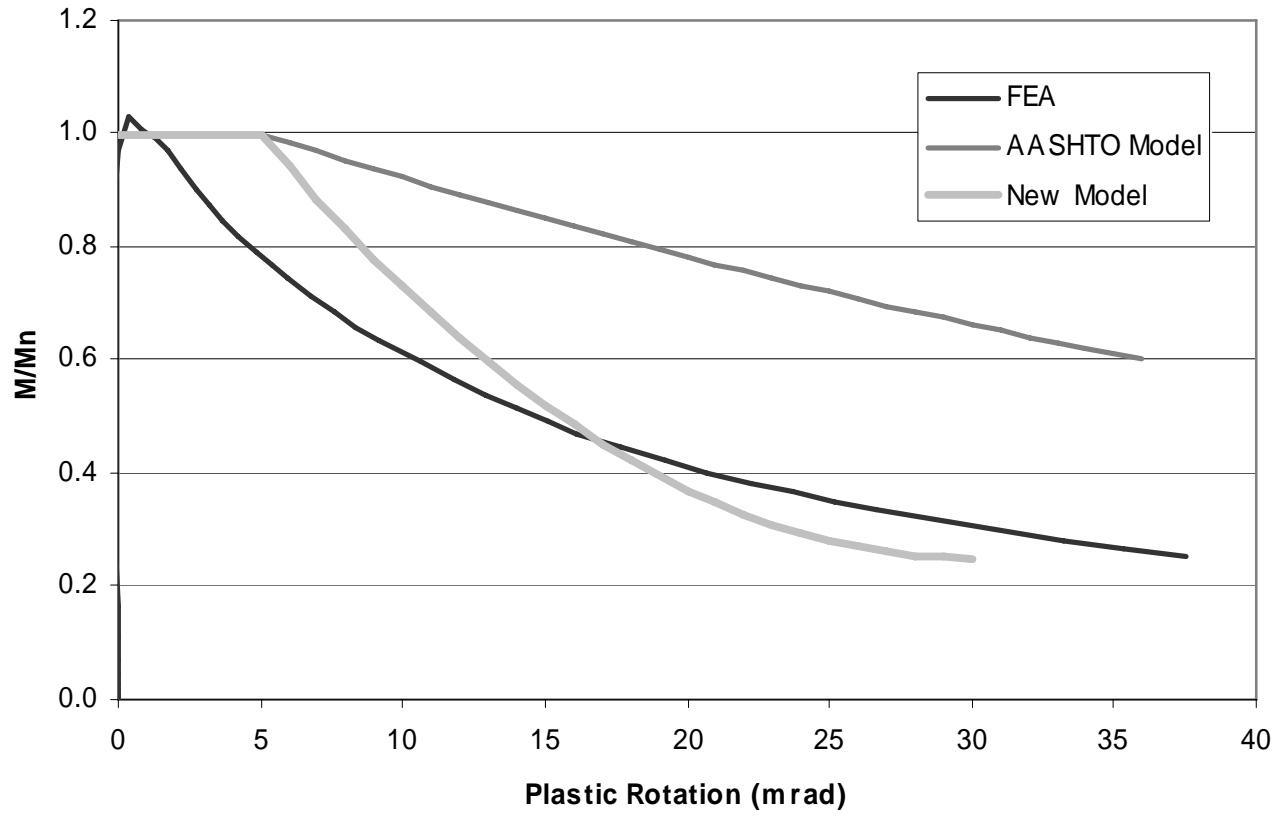

Figure D-28. Moment versus Rotation for Girder CF-1-50-4-H 


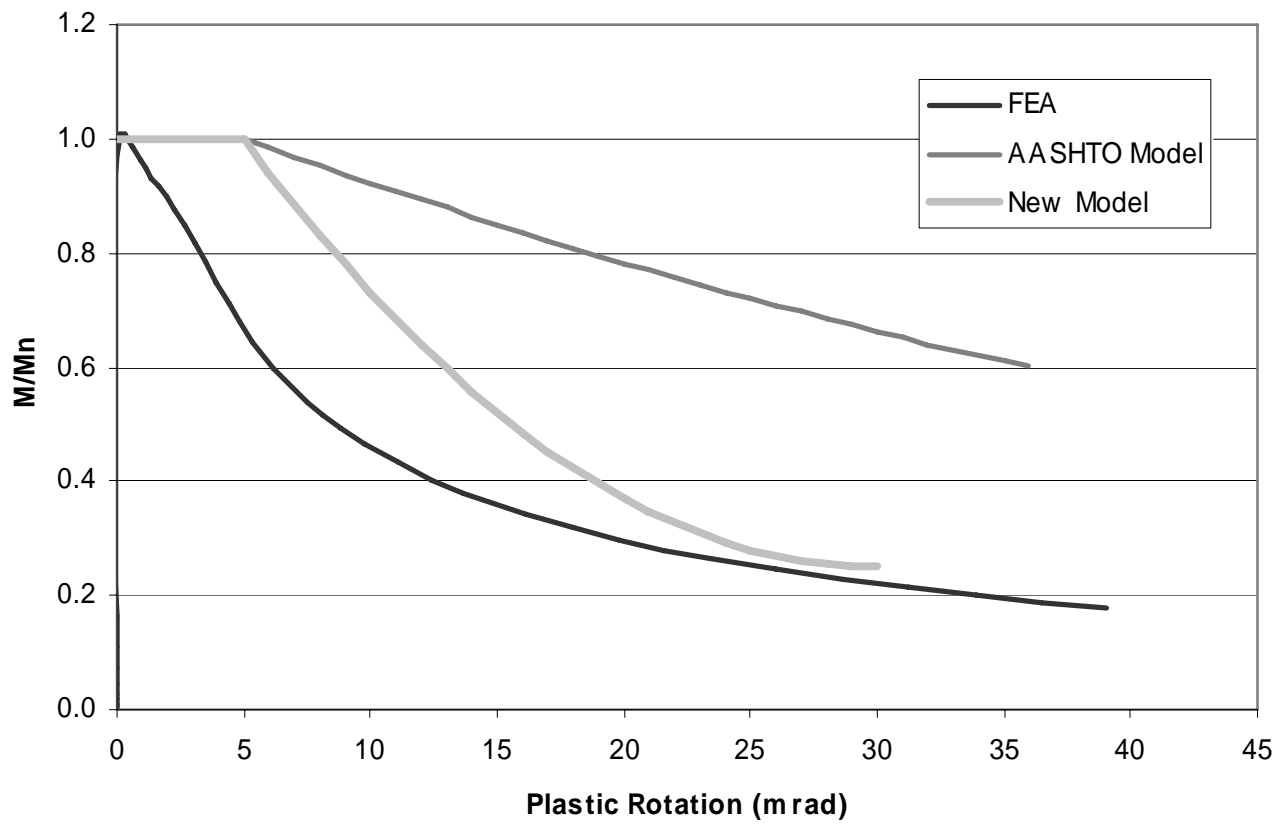

Figure D-29. Moment versus Rotation for Girder CF-2-50-4-H

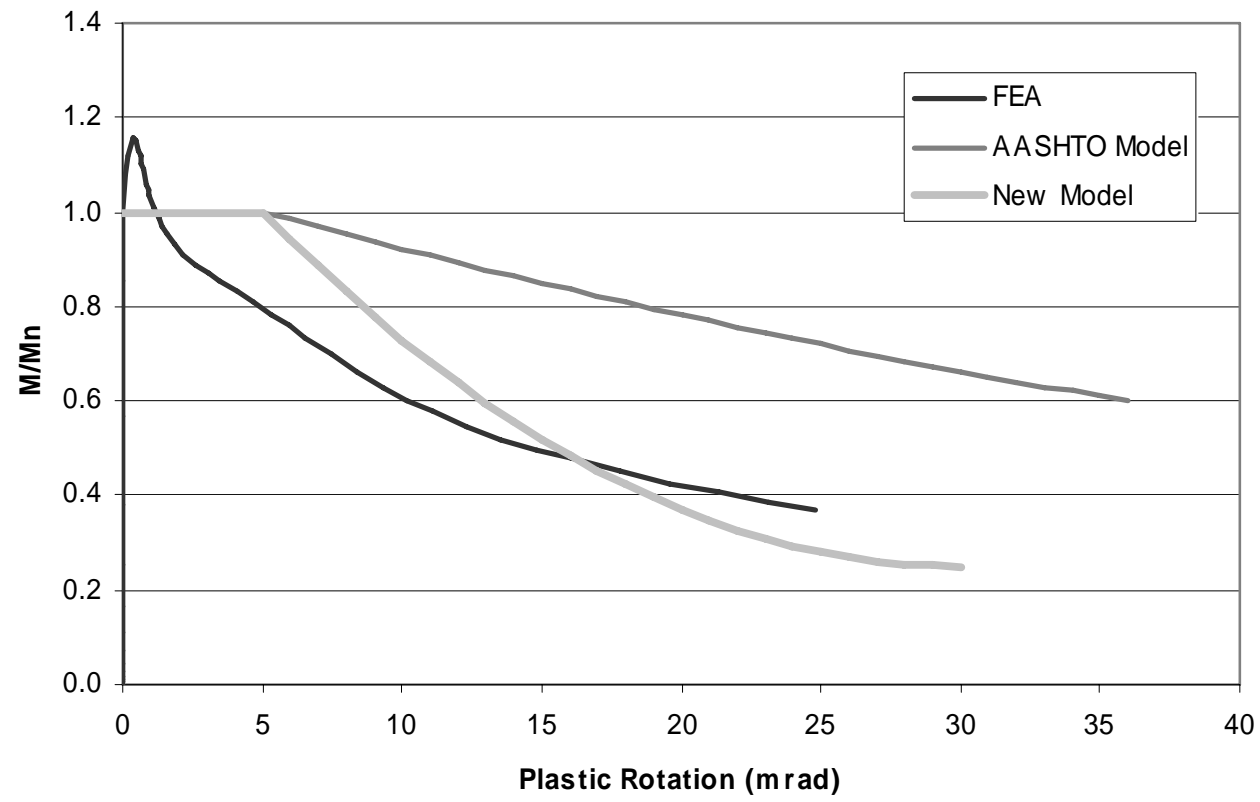

Figure D-30. Moment versus Rotation for Girder SF-1-50-4-H 


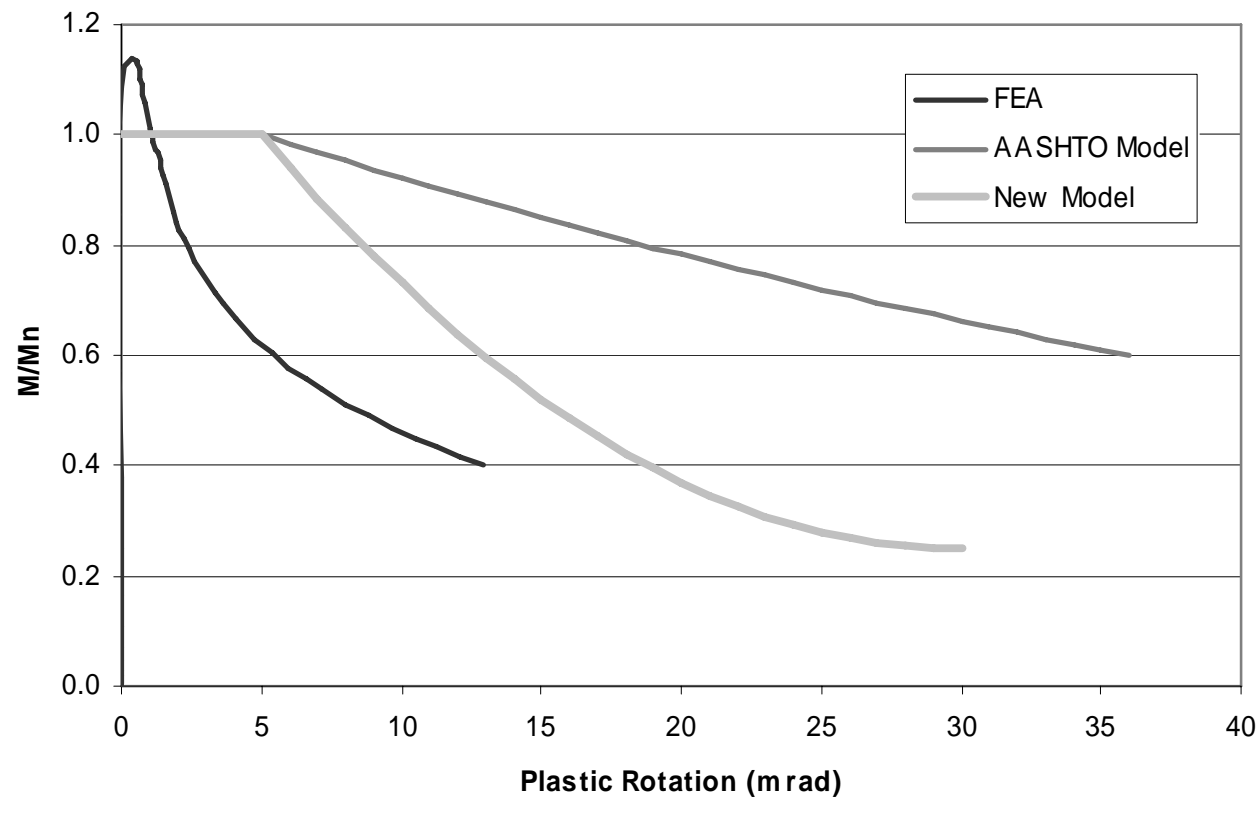

Figure D-31. Moment versus Rotation for Girder SF-2-50-4-H

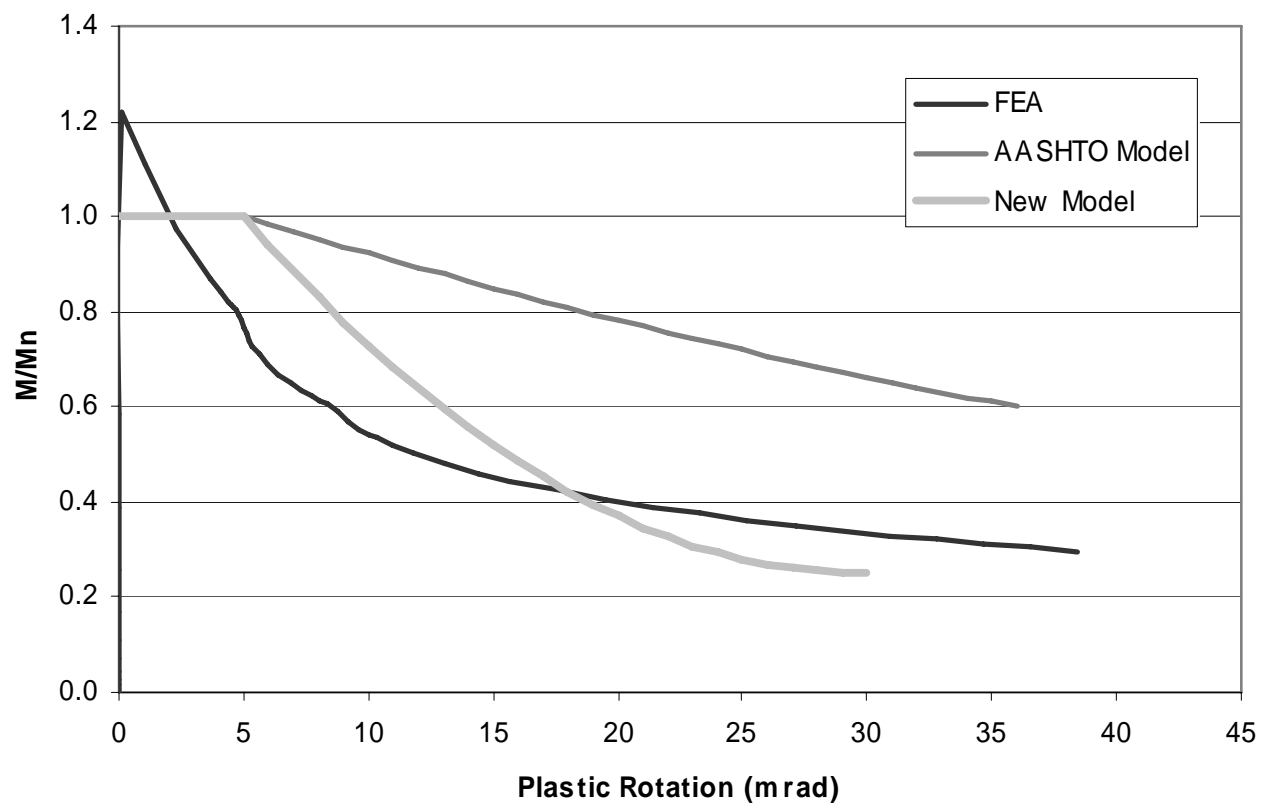

Figure D-32. Moment versus Rotation for Girder SF-30-50-4-H 


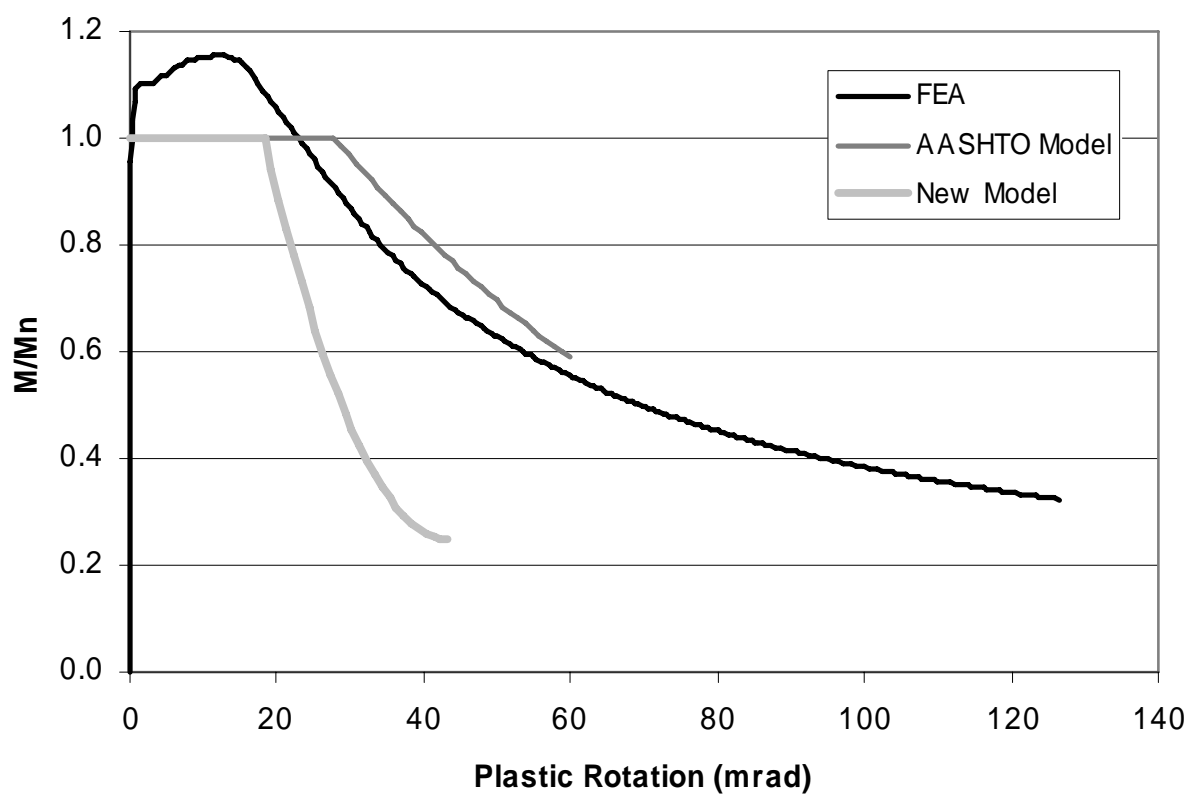

Figure D-33. Moment versus Rotation for Girder 90-2-50-10-A

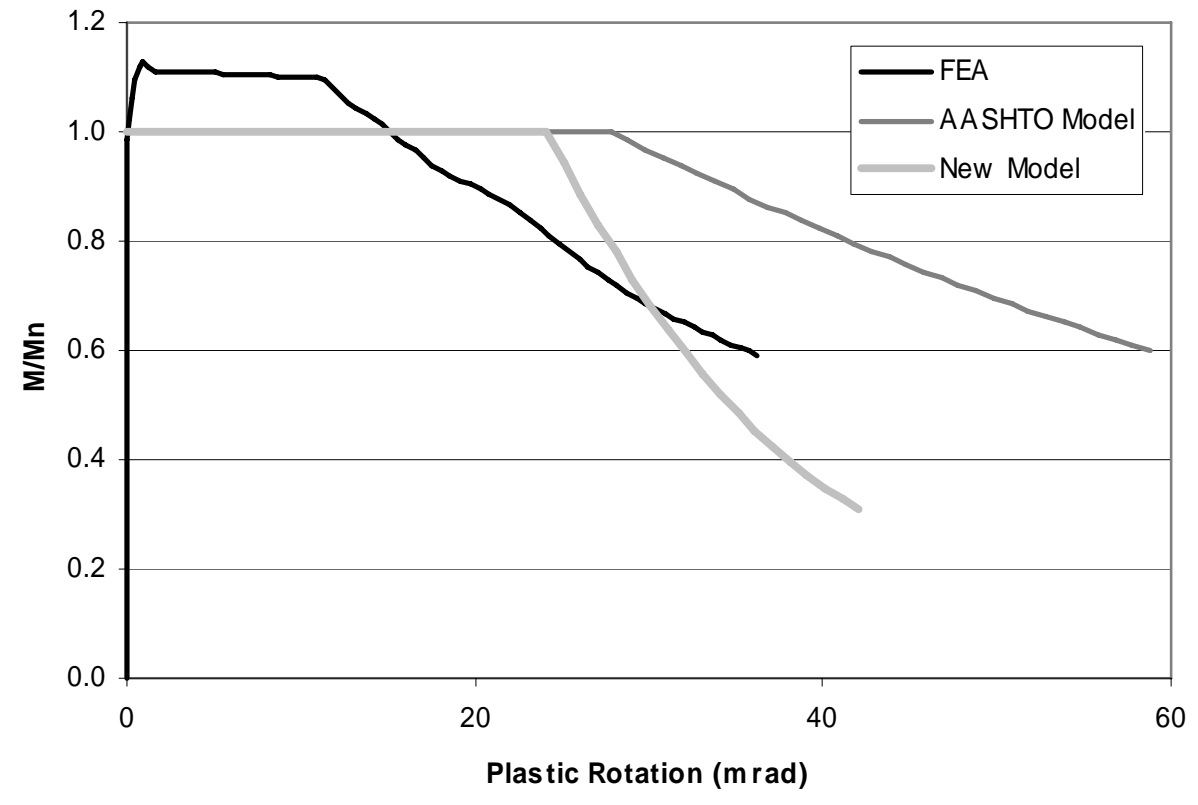

Figure D-34. Moment versus Rotation for Girder 90-2-E-50-20-B 


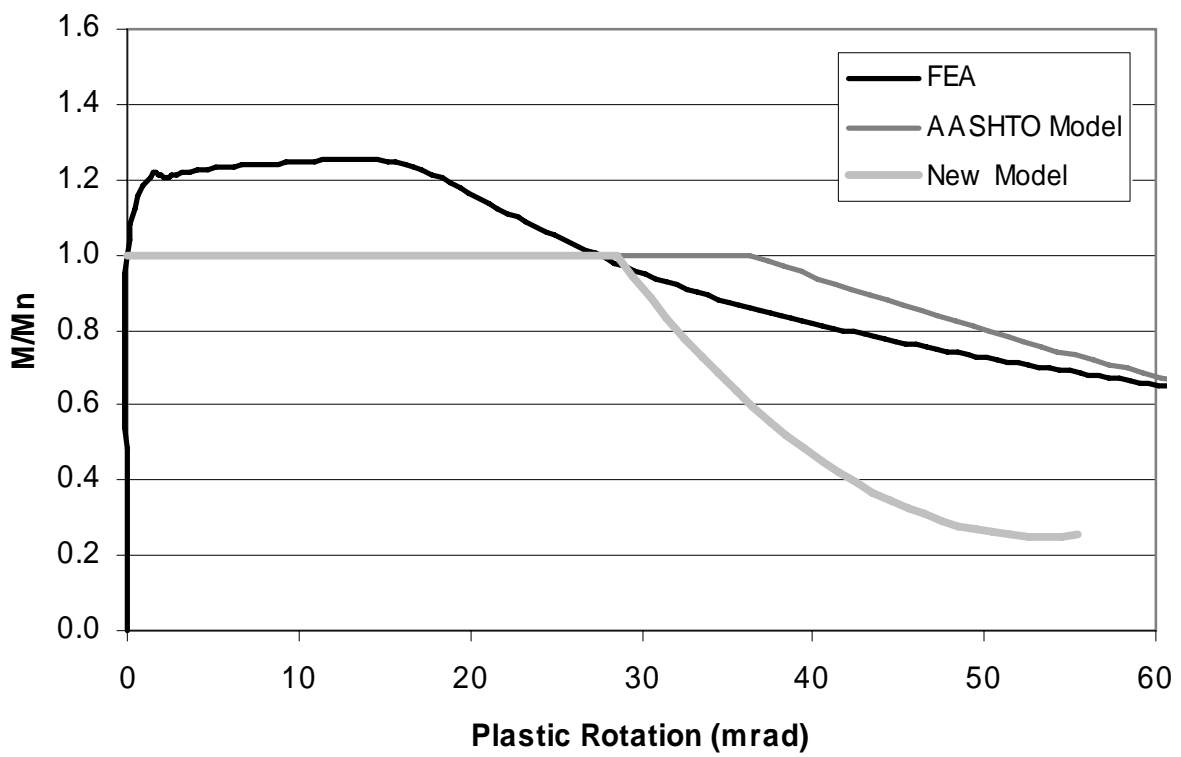

Figure D-35. Moment versus Rotation for Girder 120-2-E-50-10-A

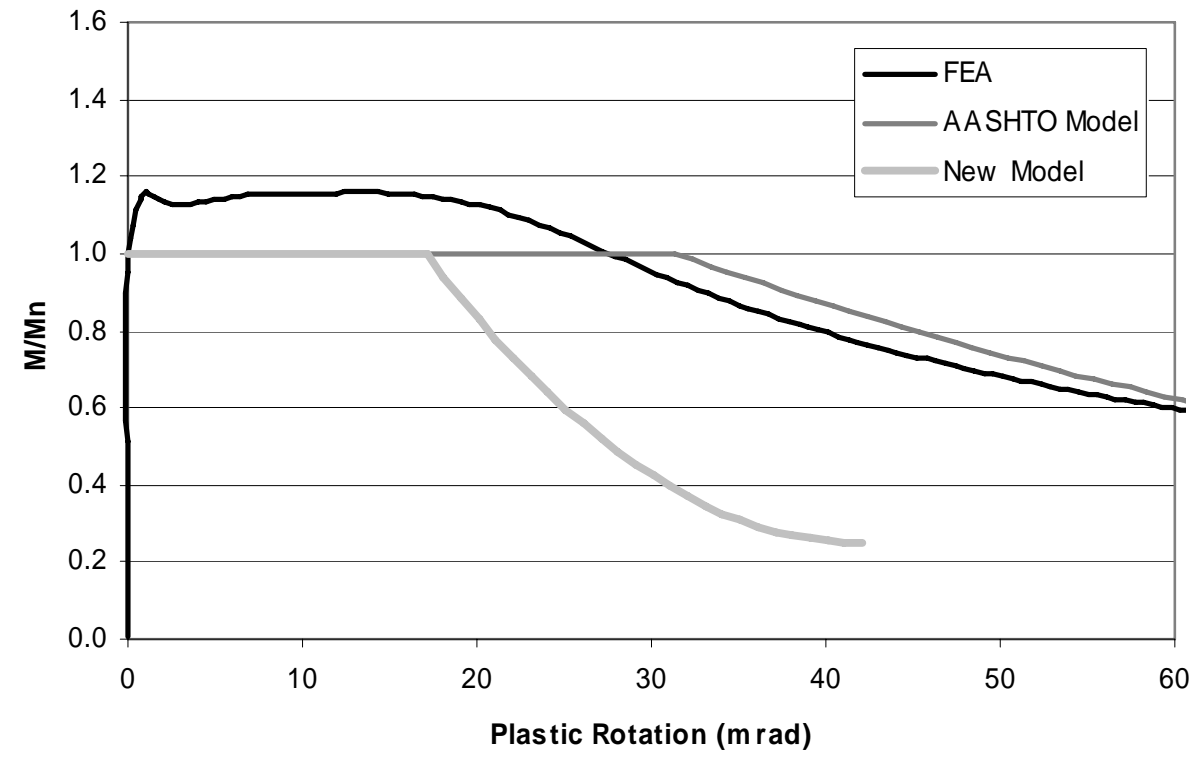

Figure D-36. Moment versus Rotation for Girder 120-2-E-50-20-A 


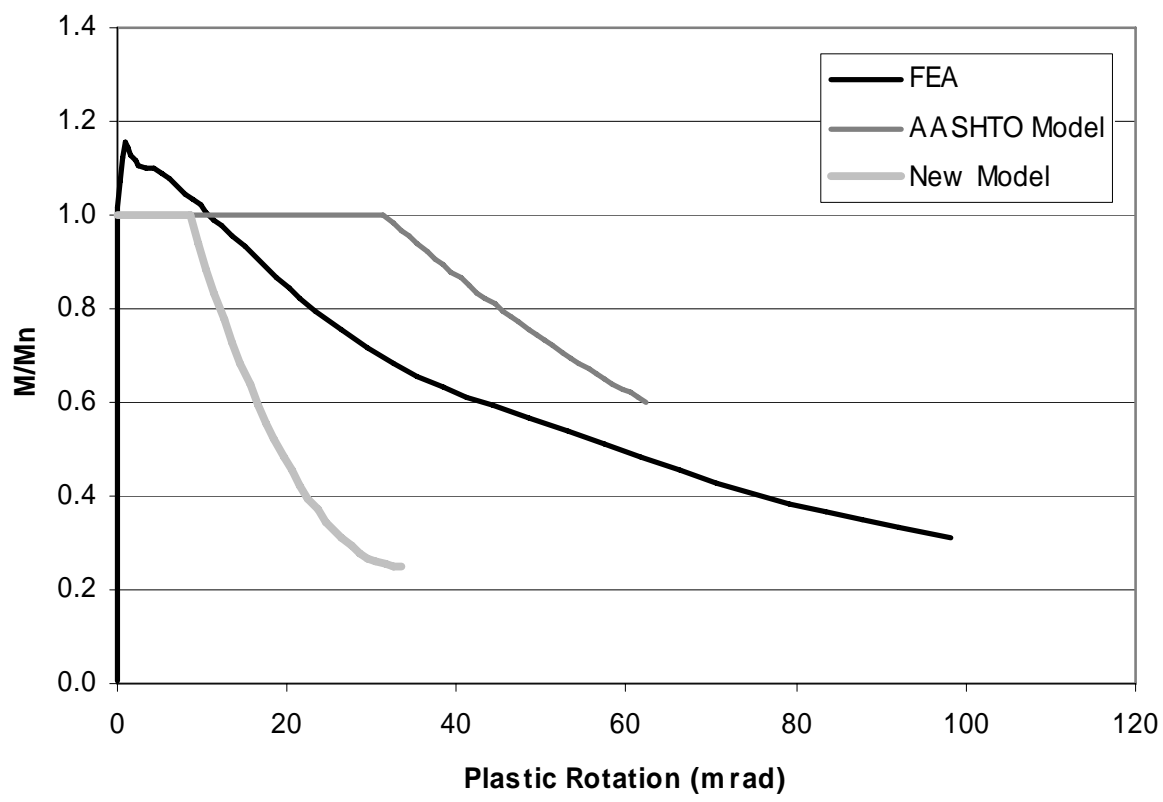

Figure D-37. Moment versus Rotation for Girder 120-2-E-50-20- $\mathrm{L}_{\mathbf{b}}=20$

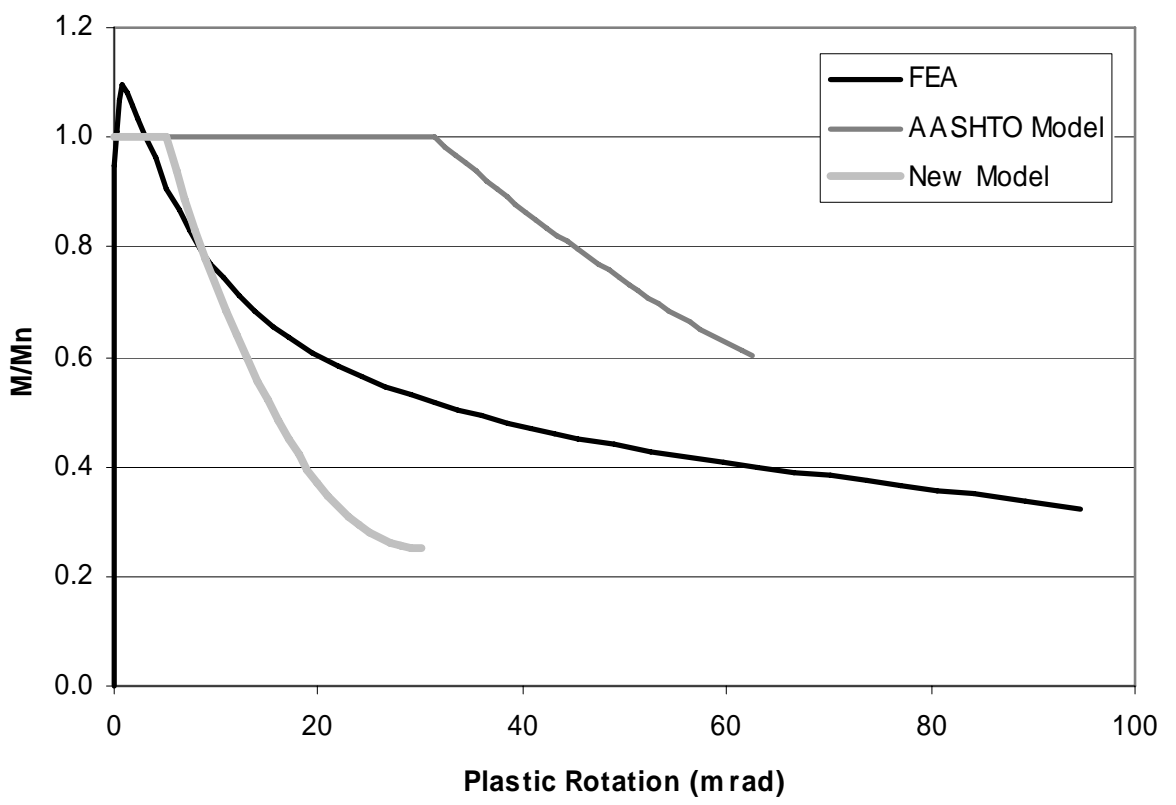

Figure D-38. Moment versus Rotation for Girder 120-2-E-50-20- $\mathrm{L}_{\mathbf{b}}=30$ 


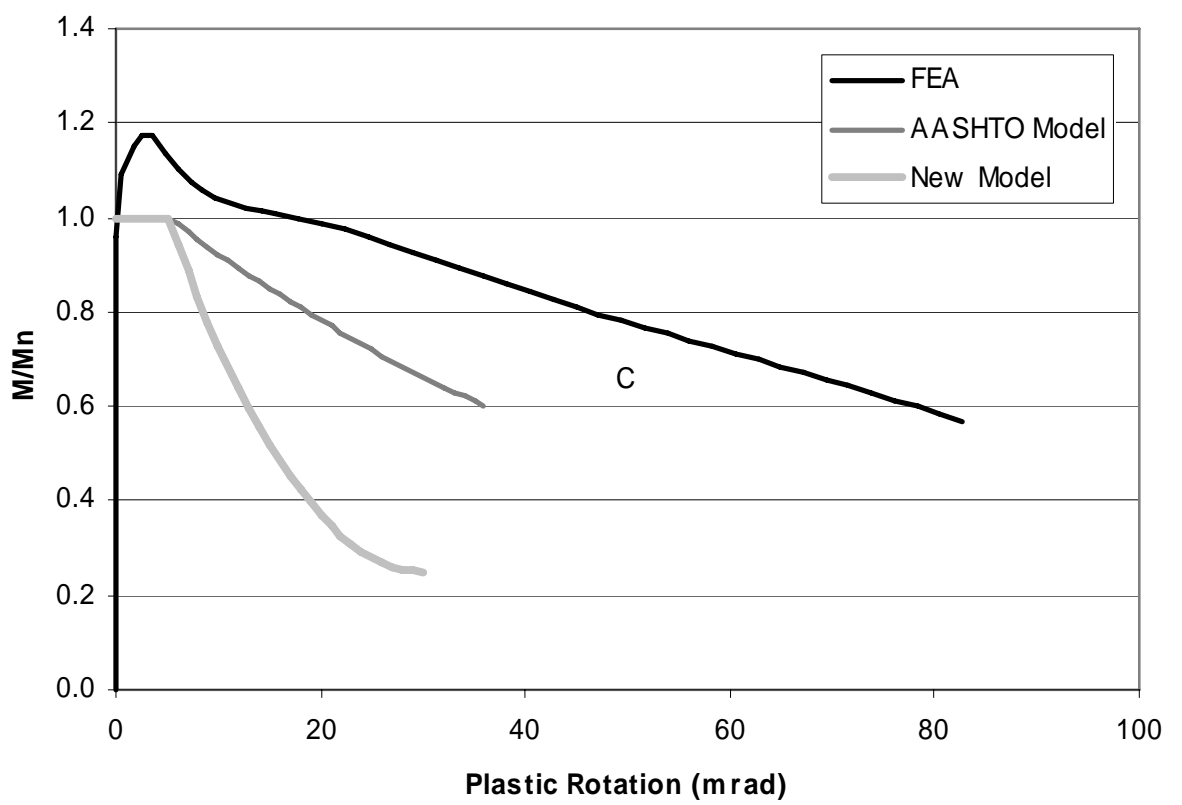

Figure D-39. Moment versus Rotation for Girder 120-2-E-50-20-NCF

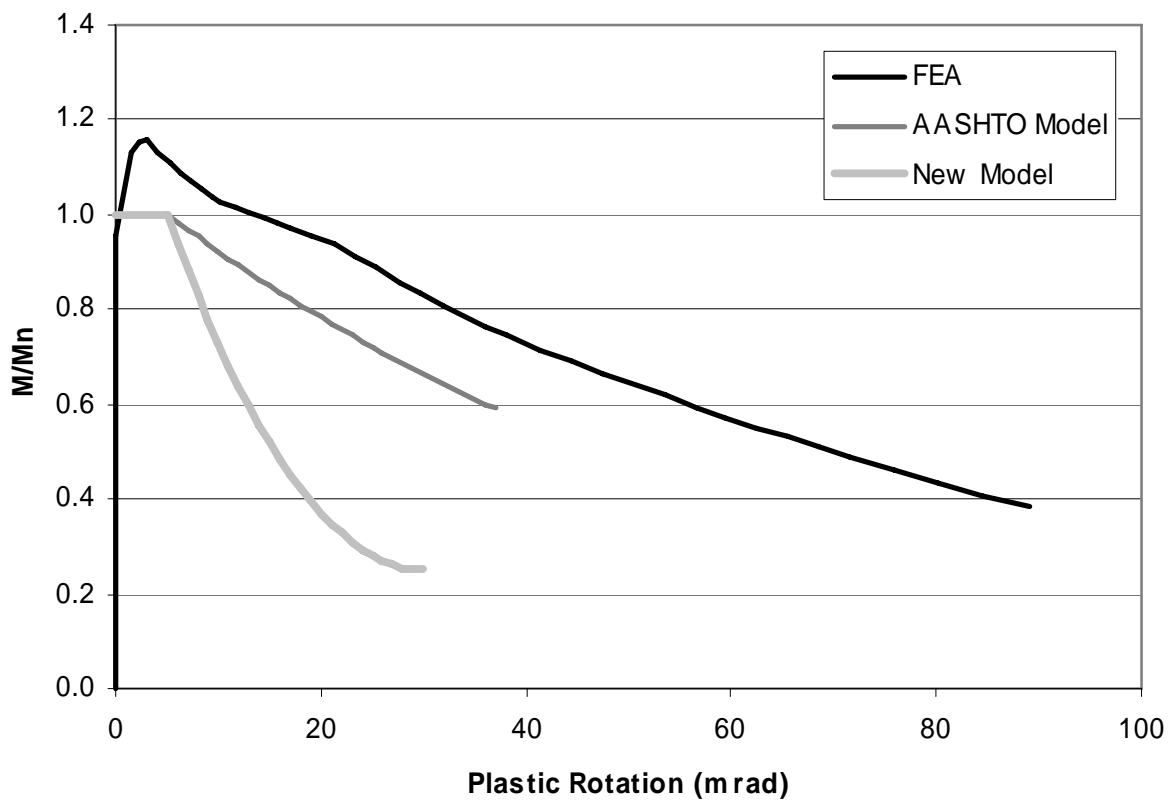

Figure D-40. Moment versus Rotation for Girder 120-2-E-50-20-NCF- $\mathrm{L}_{b}=30$ 


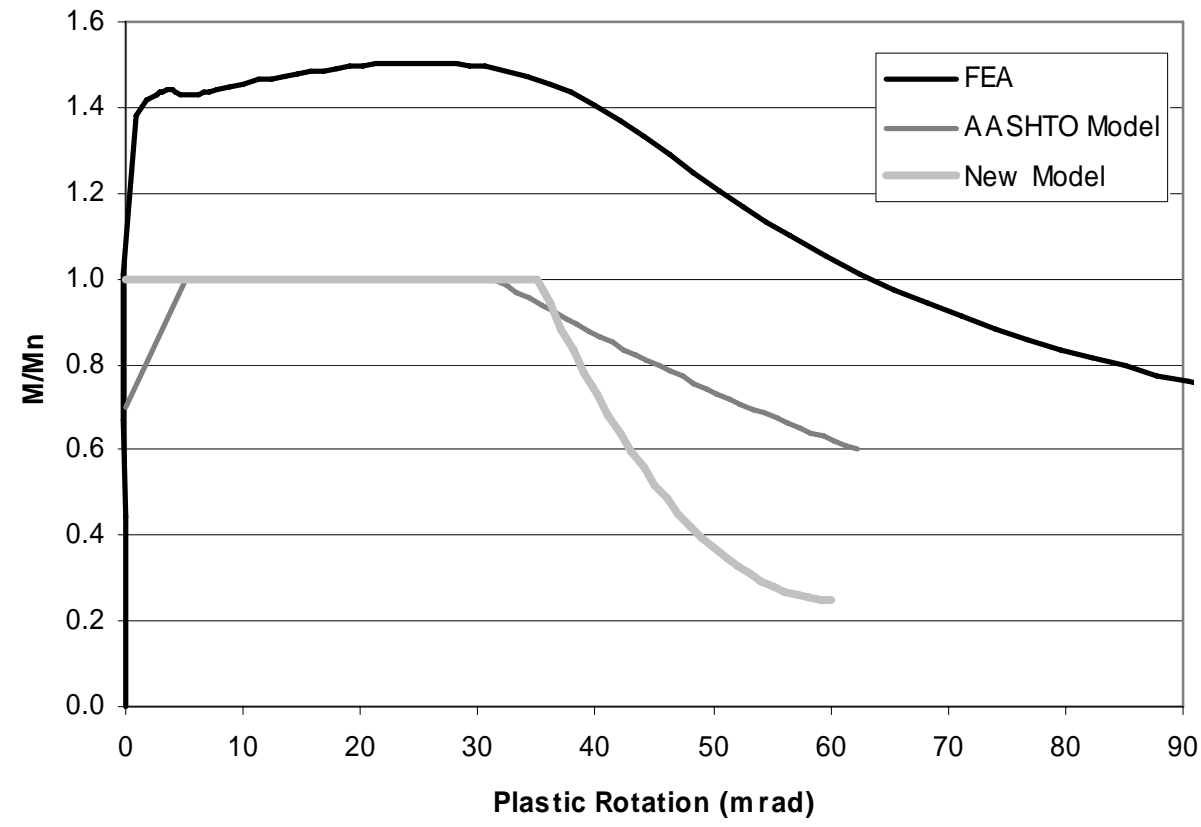

Figure D-41. Moment versus Rotation for Girder 120-2-E-50-UF(30)-E

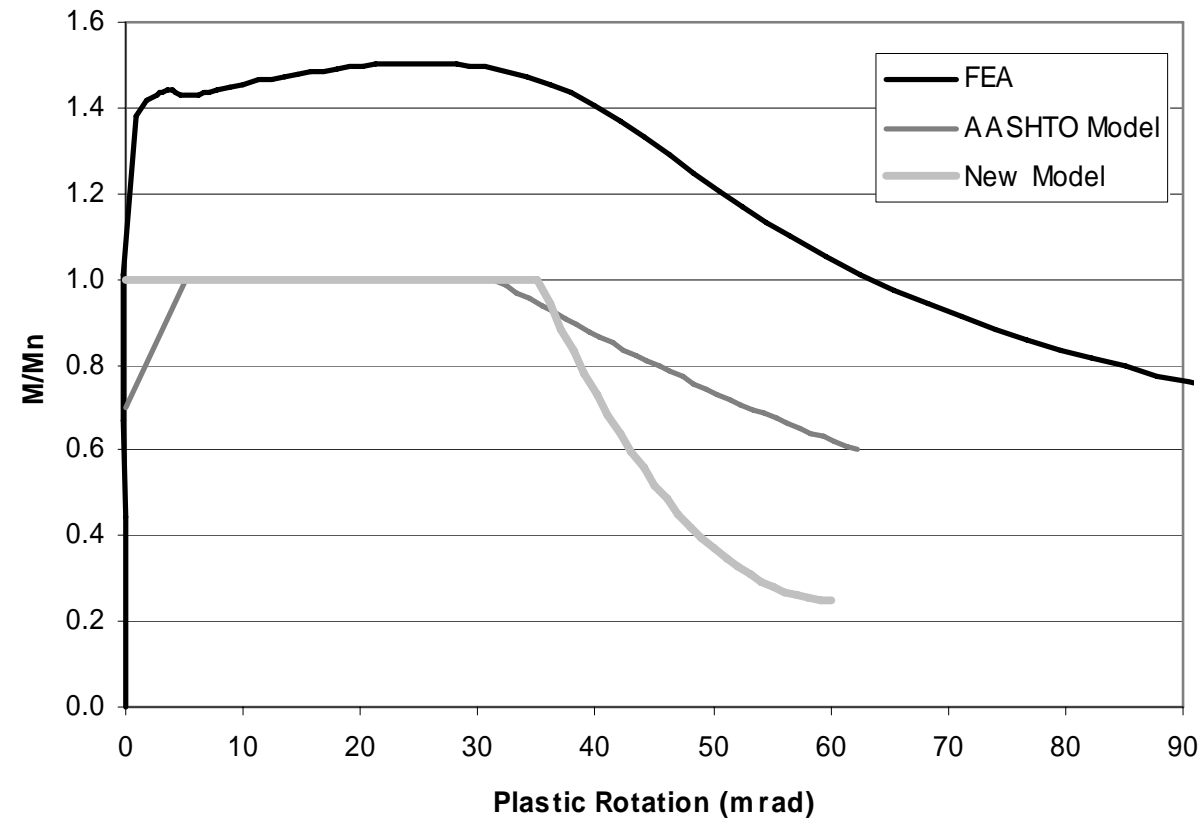

Figure D-42. Moment versus Rotation for Girder 120-2-E-50-U(30)-E 


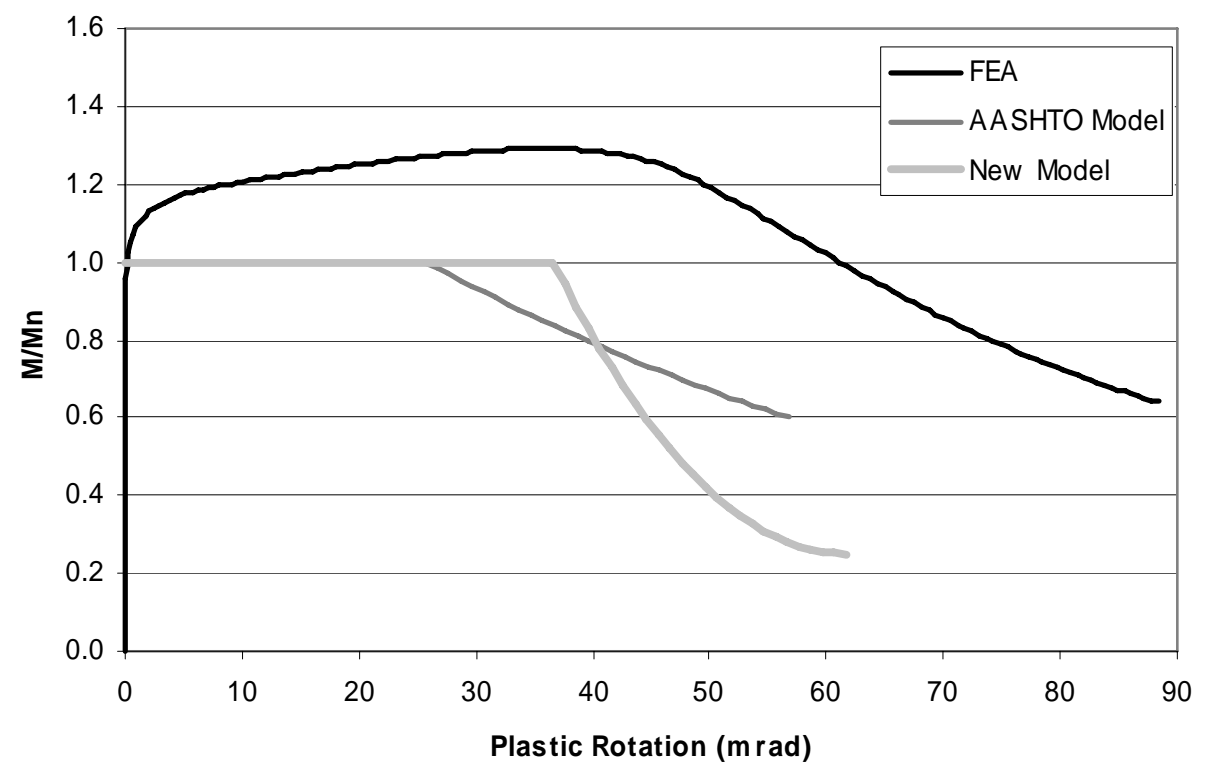

Figure D-43. Moment versus Rotation for Girder 120-2-H-10-A

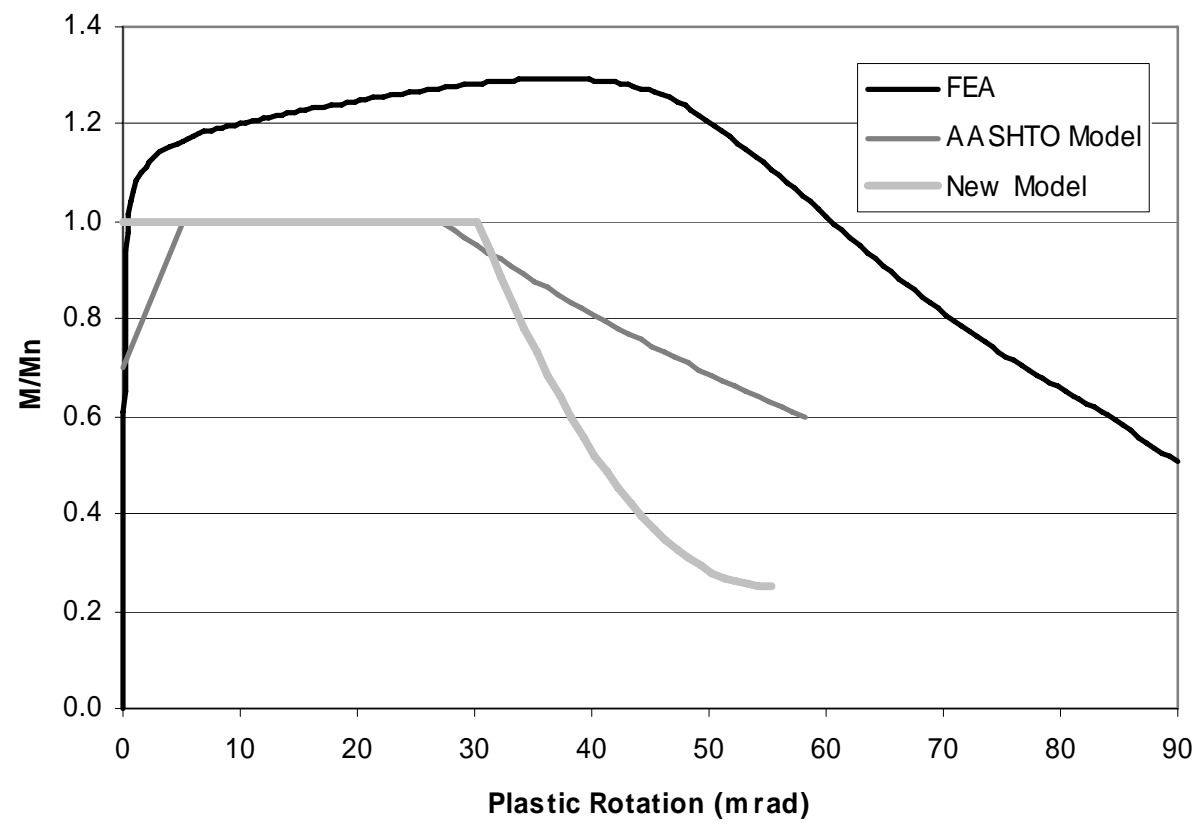

Figure D-44. Moment versus Rotation for Girder 120-2-H-10-B 


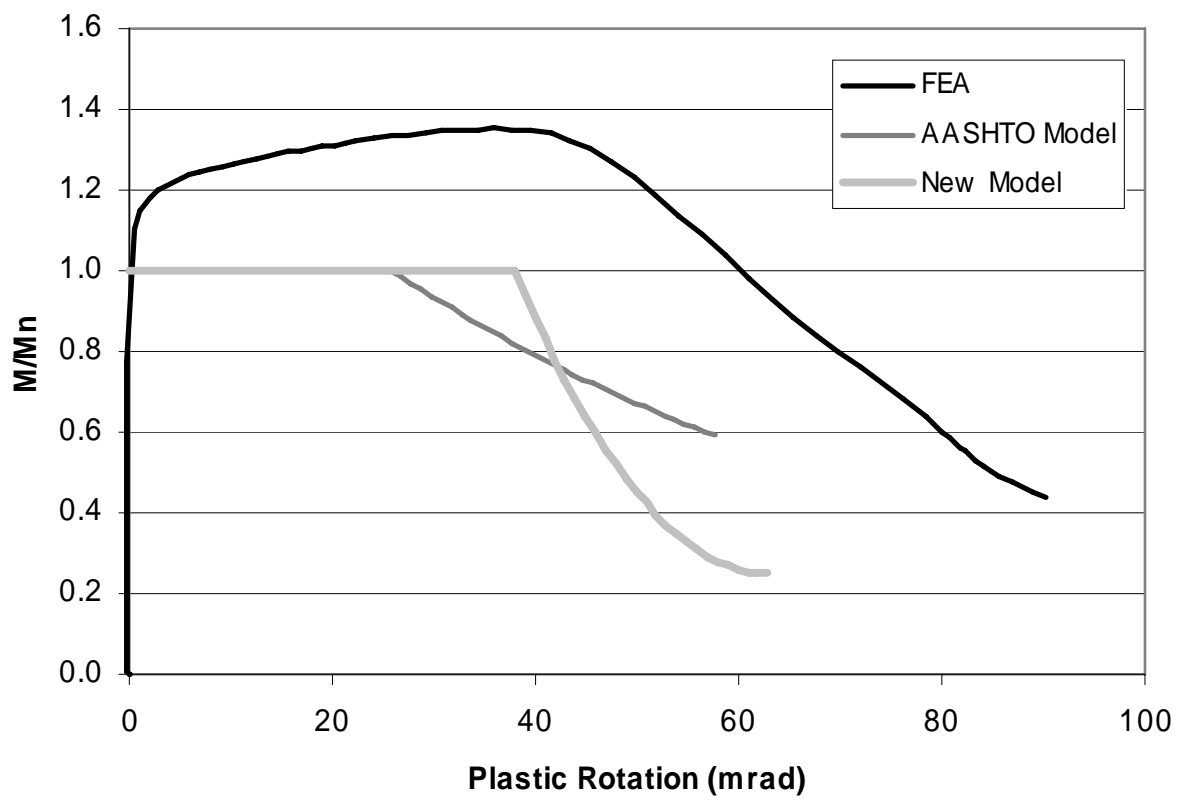

Figure D-45. Moment versus Rotation for Girder 120-2-E-H-10-B(S)

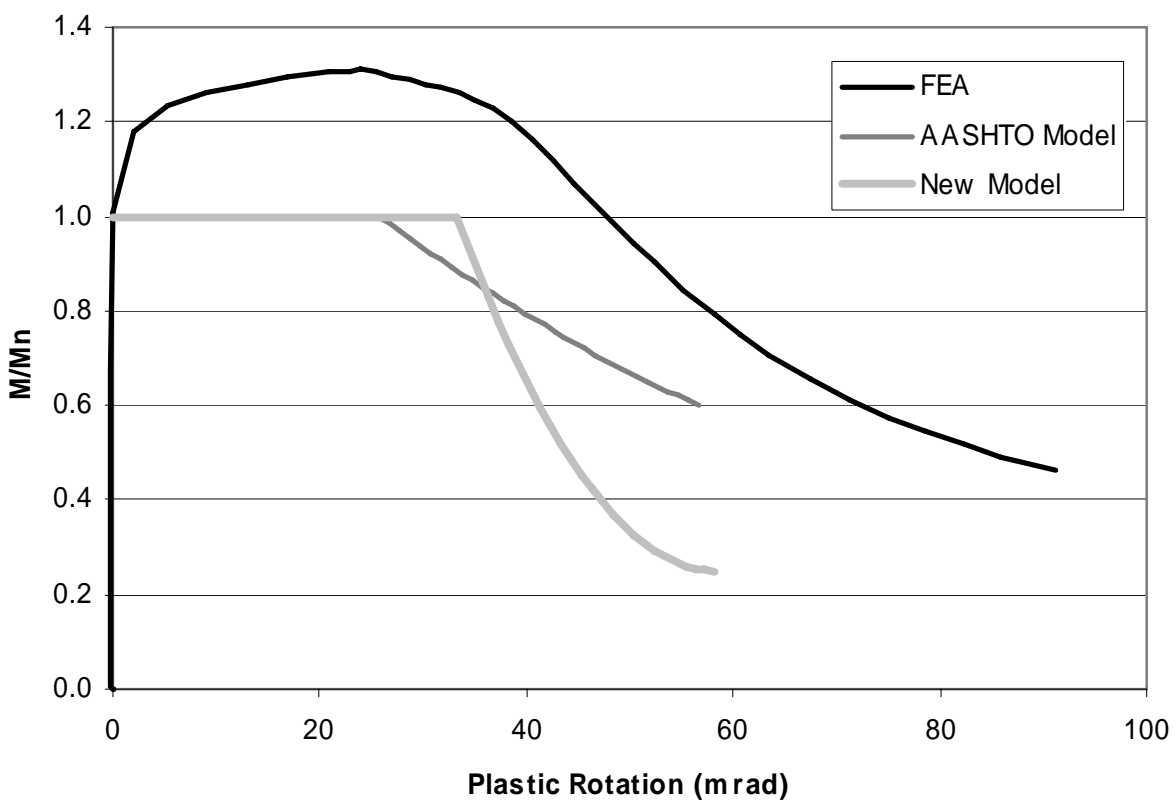

Figure D-46. Moment versus Rotation for Girder 120-2-H-10-B(S)-16 


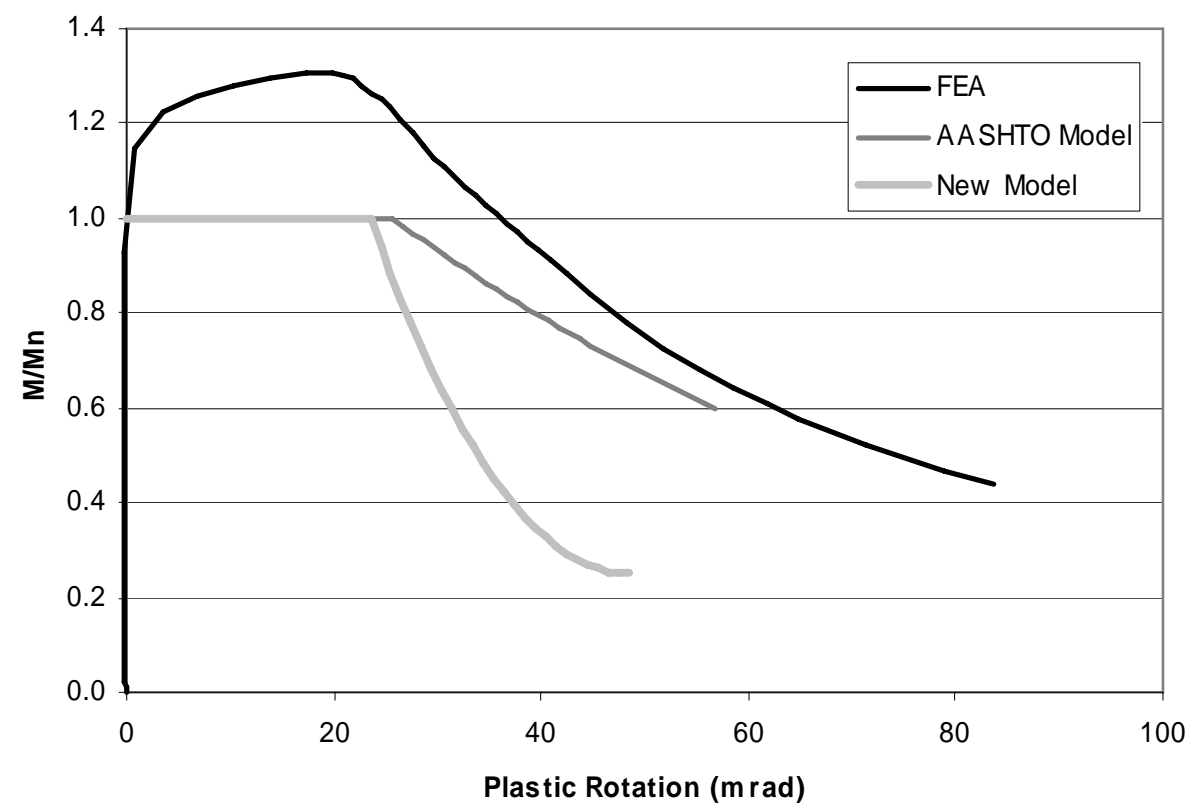

Figure D-47. Moment versus Rotation for Girder 120-2-H-10-B(S)-24

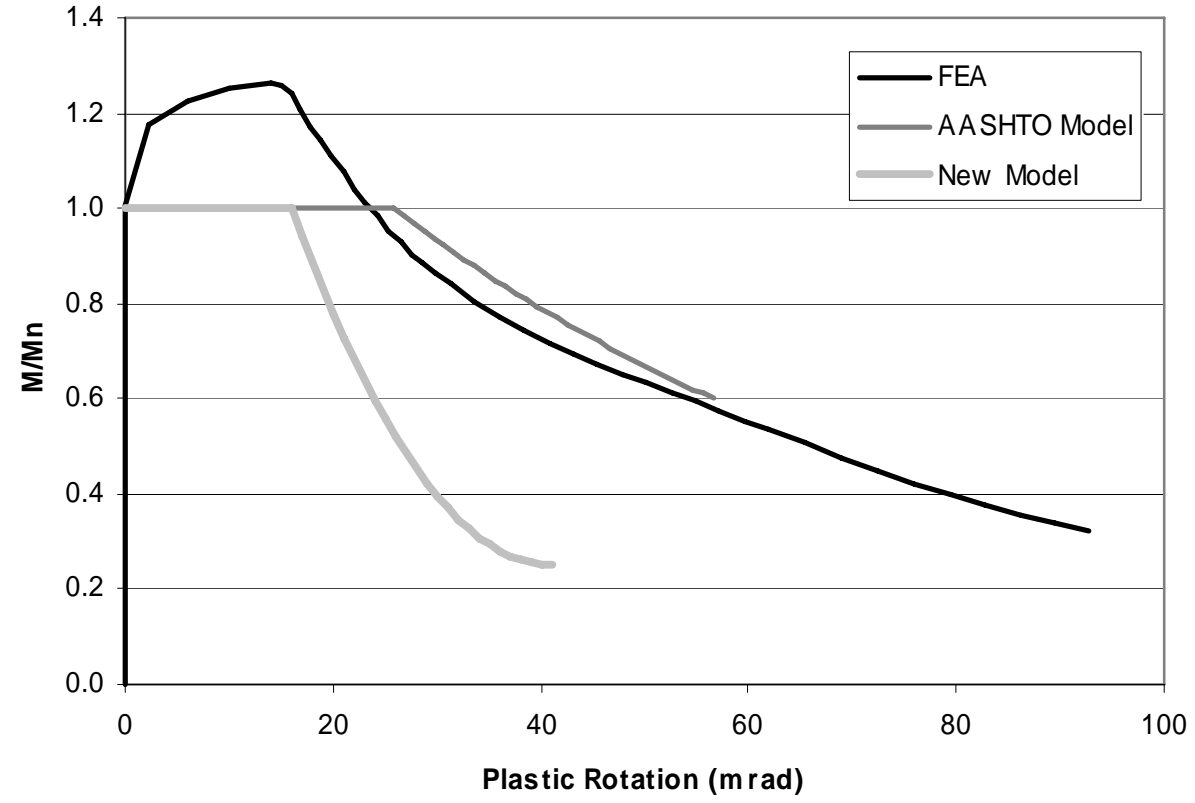

Figure D-48. Moment versus Rotation for Girder 120-2-H-10-B(S)-30 


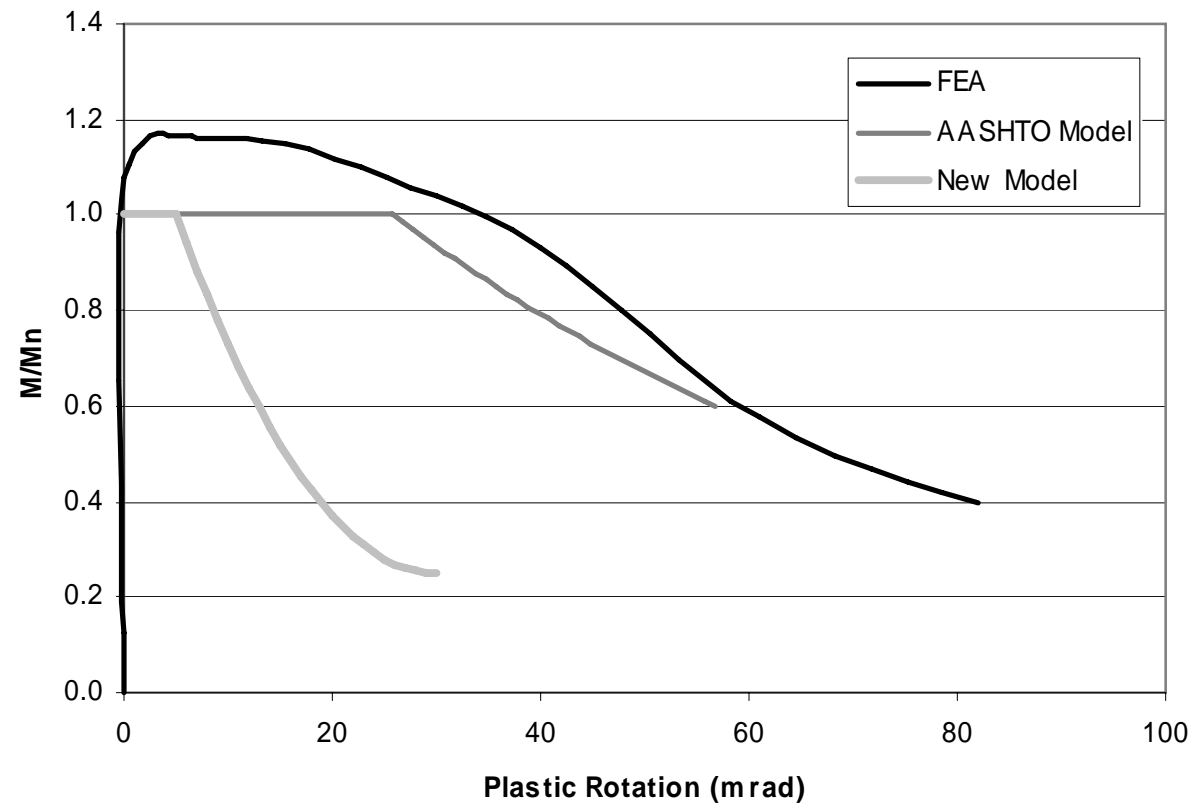

Figure D-49. Moment versus Rotation for Girder 120-2-H-10-B(S)-NCF

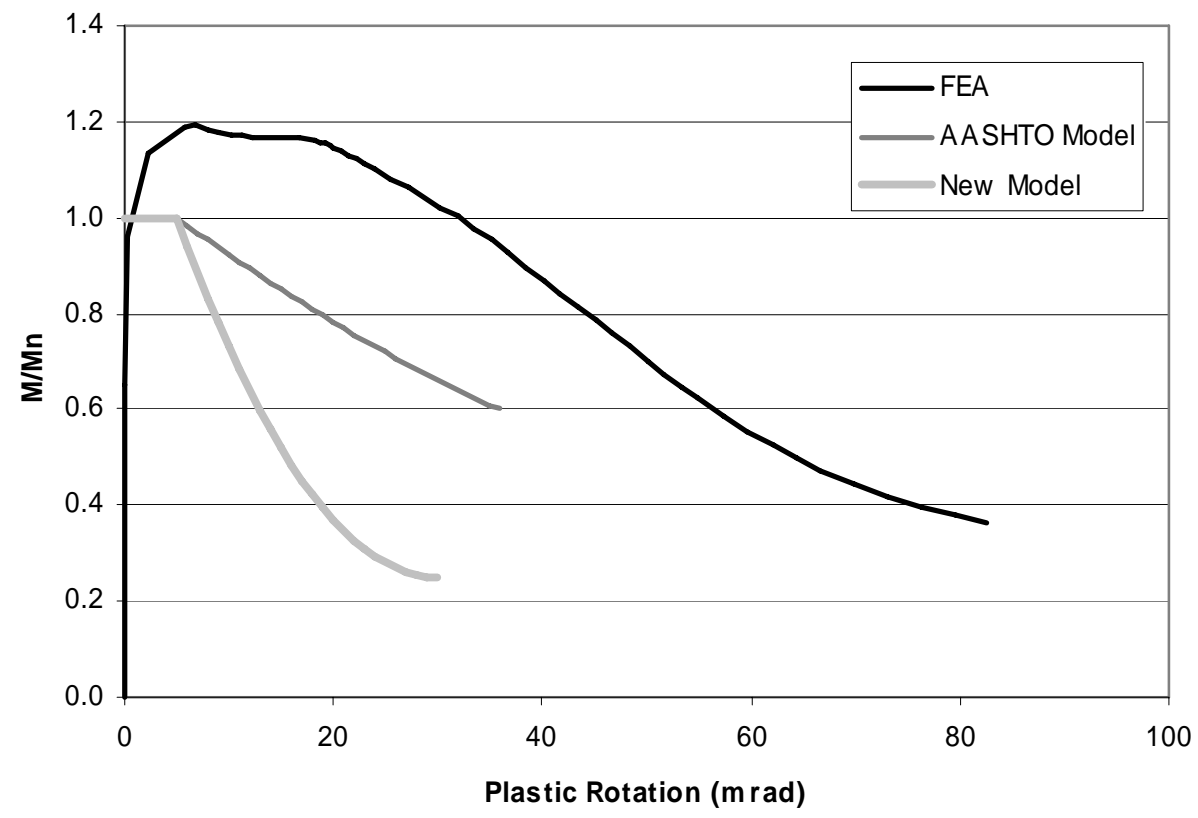

Figure D-50. Moment versus Rotation for Girder 120-2-H-10-B(S)-NCF-30 


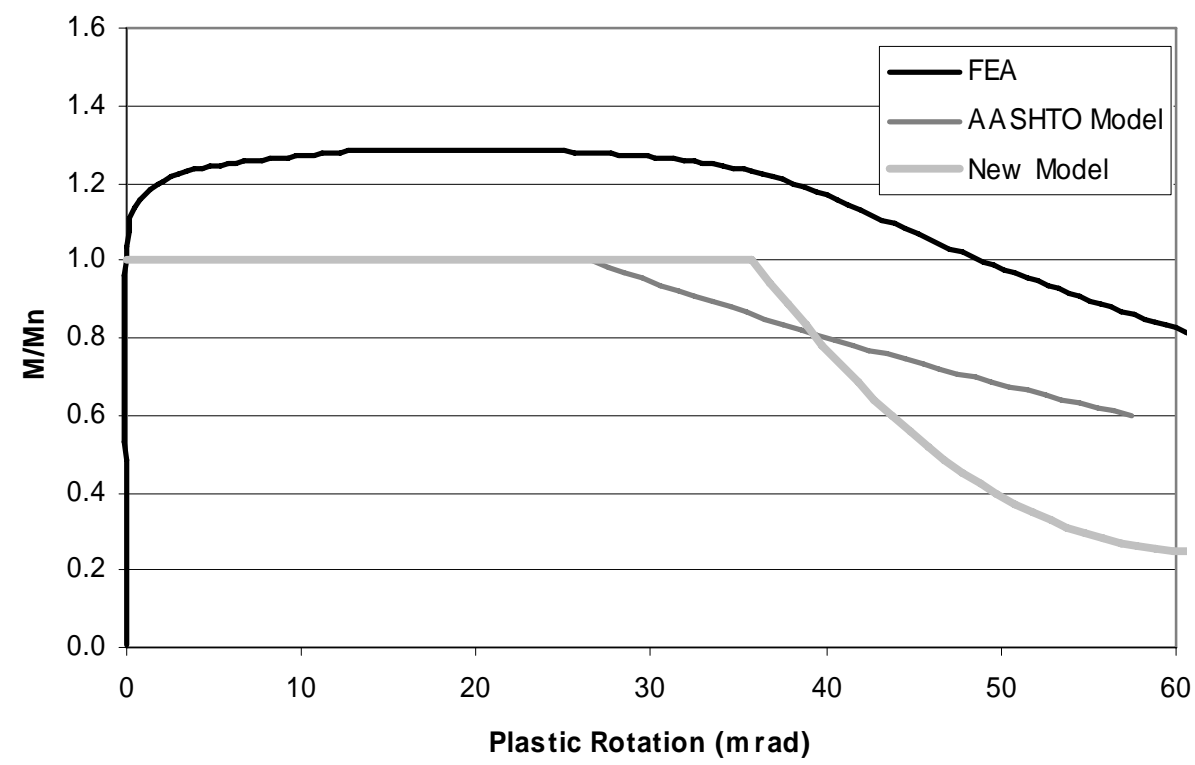

Figure D-51. Moment versus Rotation for Girder 120-2-H-20-A

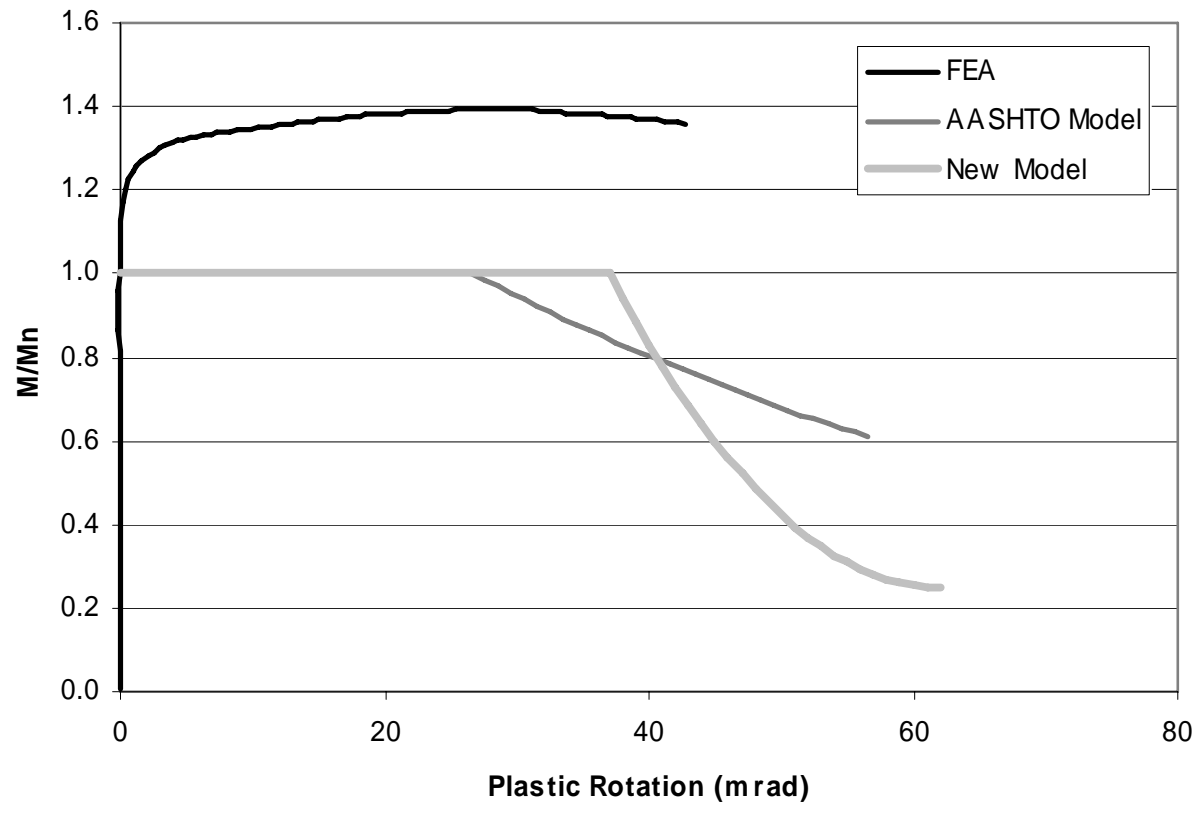

Figure D-52. Moment versus Rotation for Girder 120-2-H-20-A(S) 


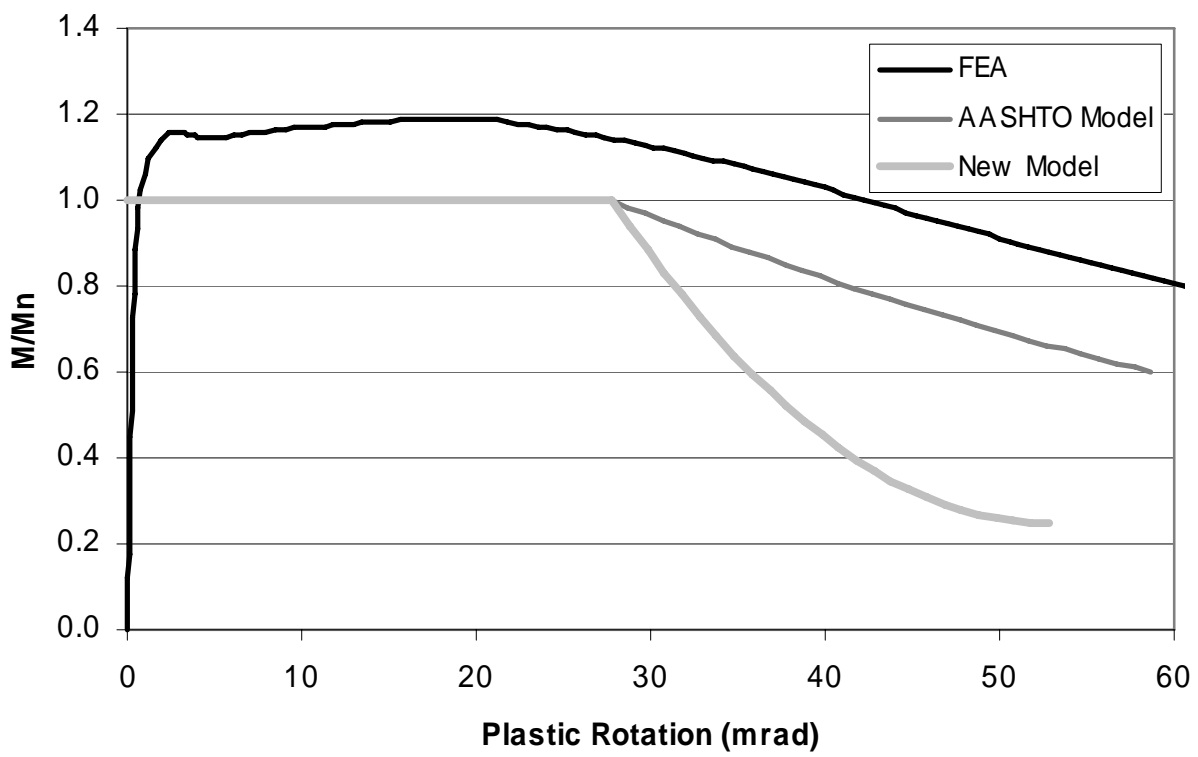

Figure D-53. Moment versus Rotation for Girder 150-2-50-10-A

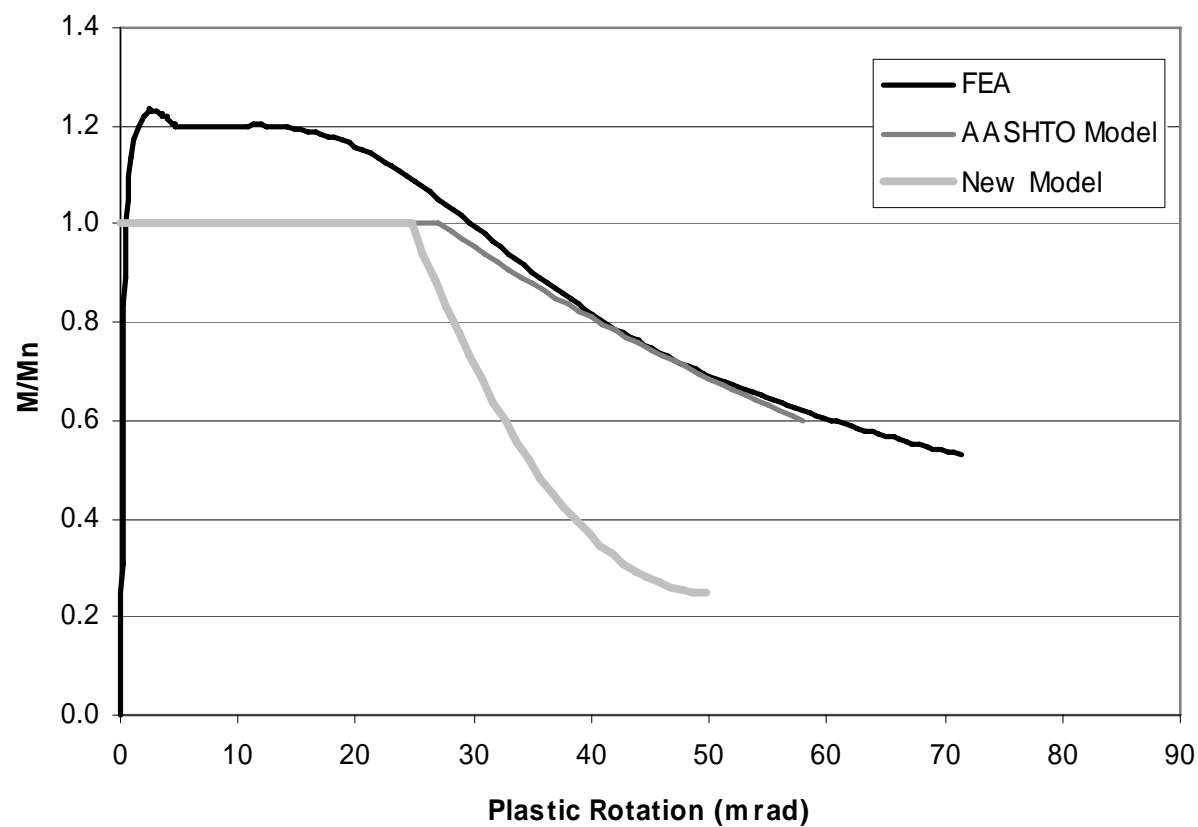

Figure D-54. Moment versus Rotation for Girder 150-2-50-20-C 


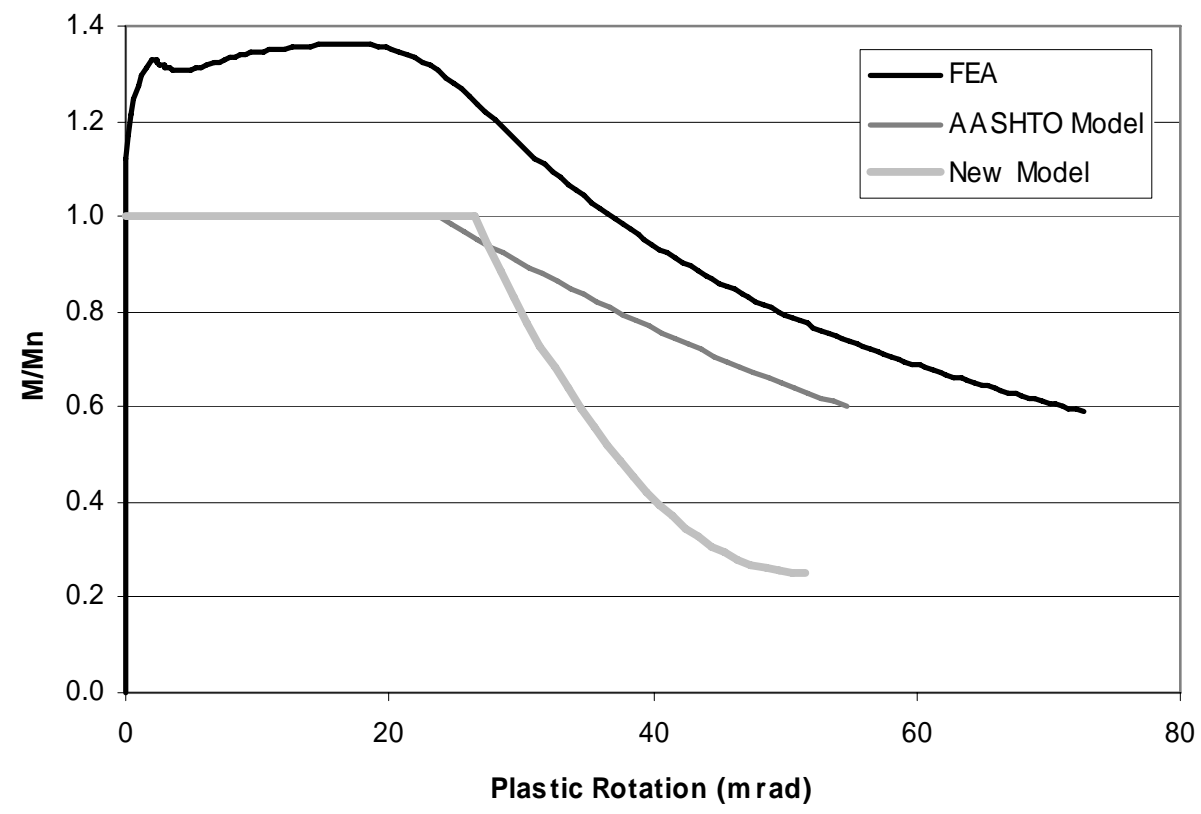

Figure D-55. Moment versus Rotation for Girder 150-2-50-40-A

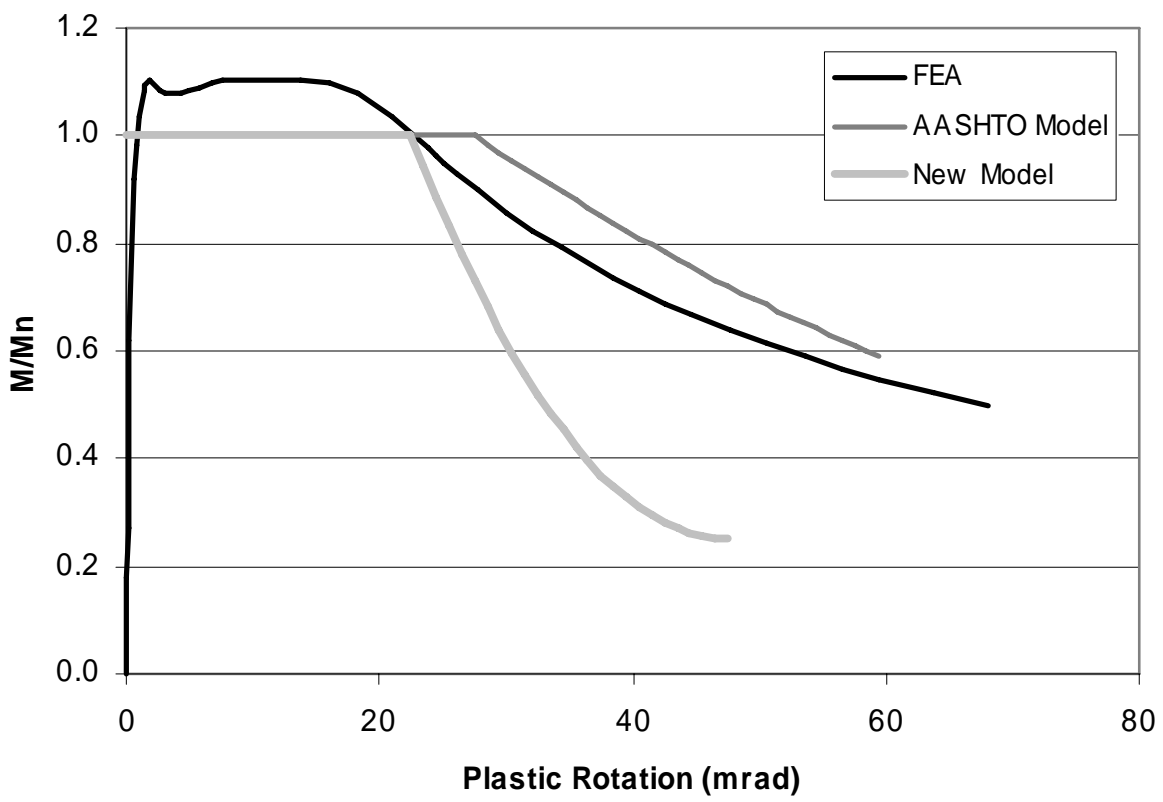

Figure D-56. Moment versus Rotation for Girder 180-2-E-50-10-A 


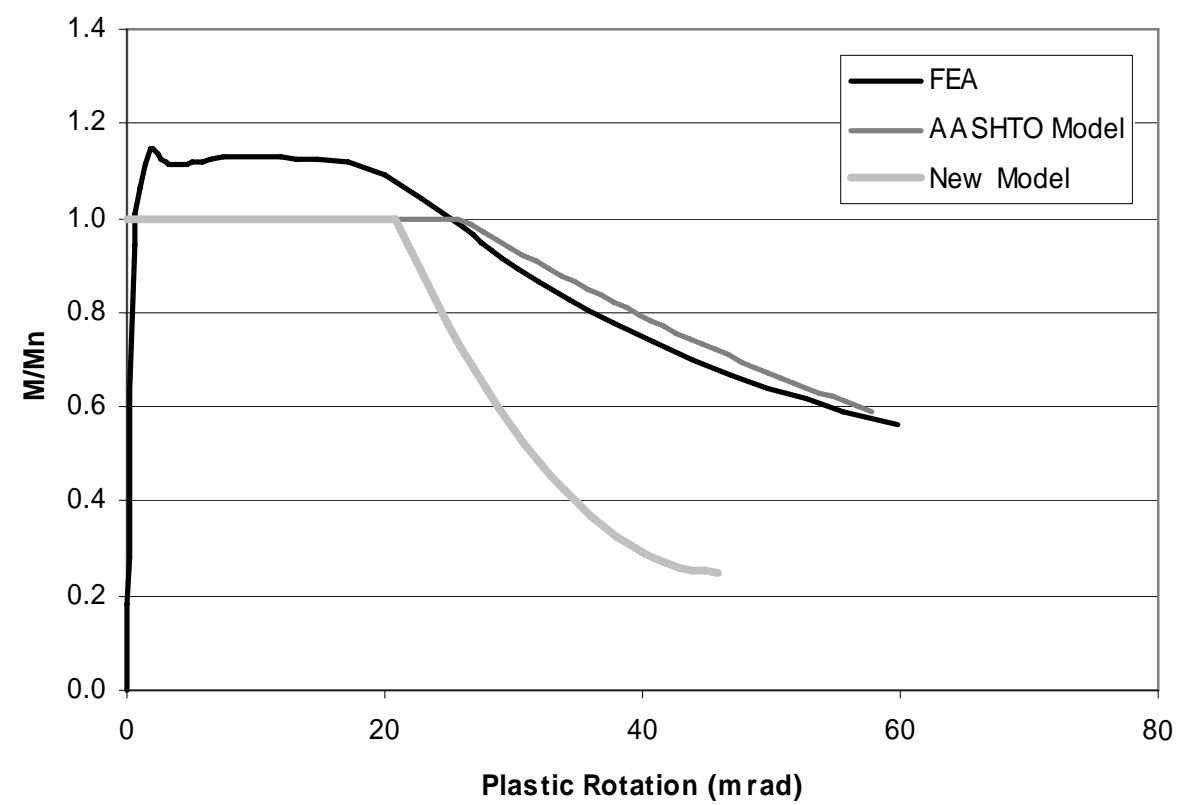

Figure D-57. Moment versus Rotation for Girder 180-2-E-50-20-B

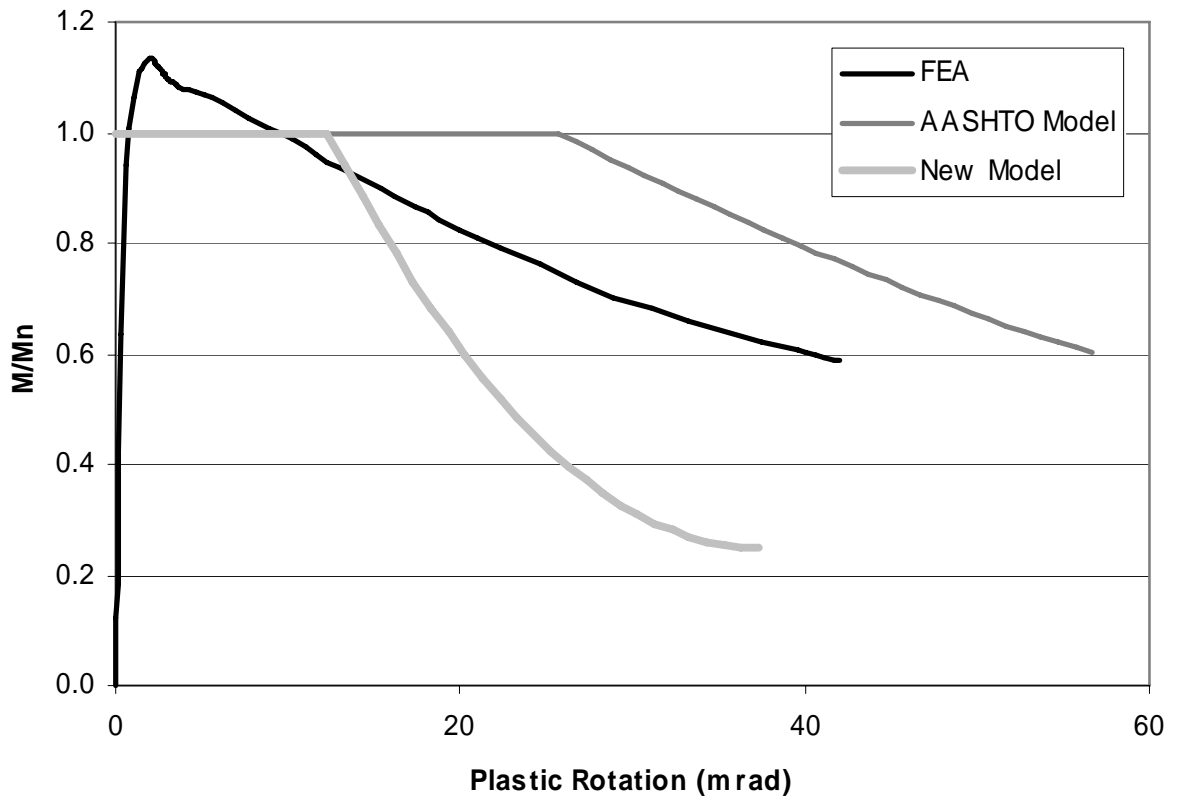

Figure D-58. Moment versus Rotation for Girder 180-2-E-50-20-22.5 


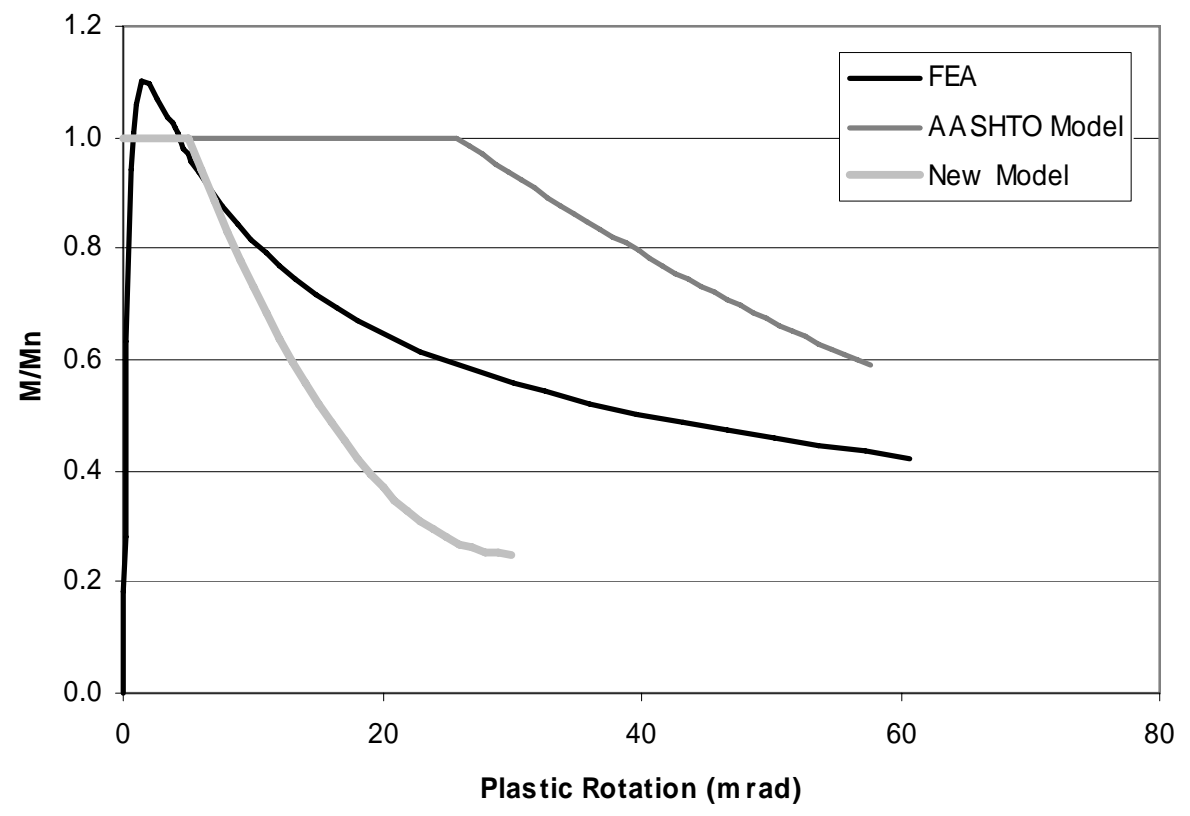

Figure D-59. Moment versus Rotation for Girder 180-2-E-50-20-30

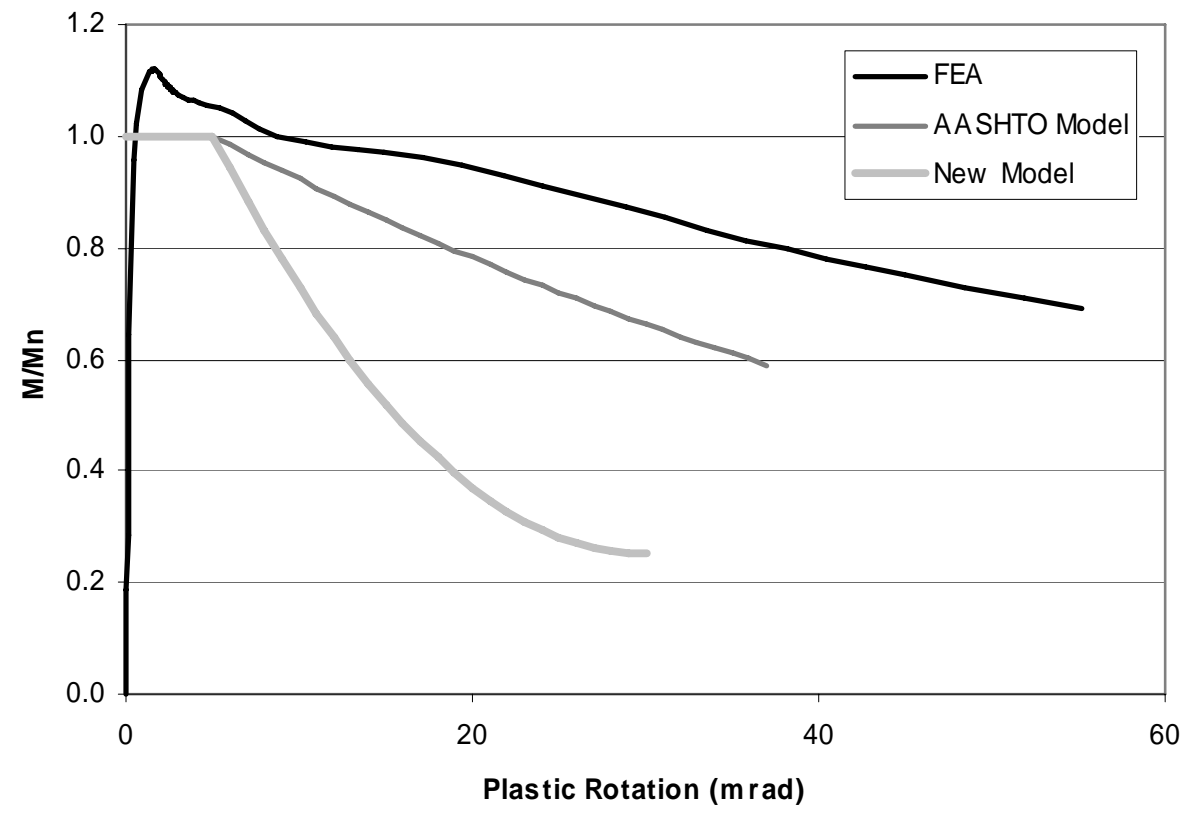

Figure D-60. Moment versus Rotation for Girder 180-2-E-50-20-NCF 


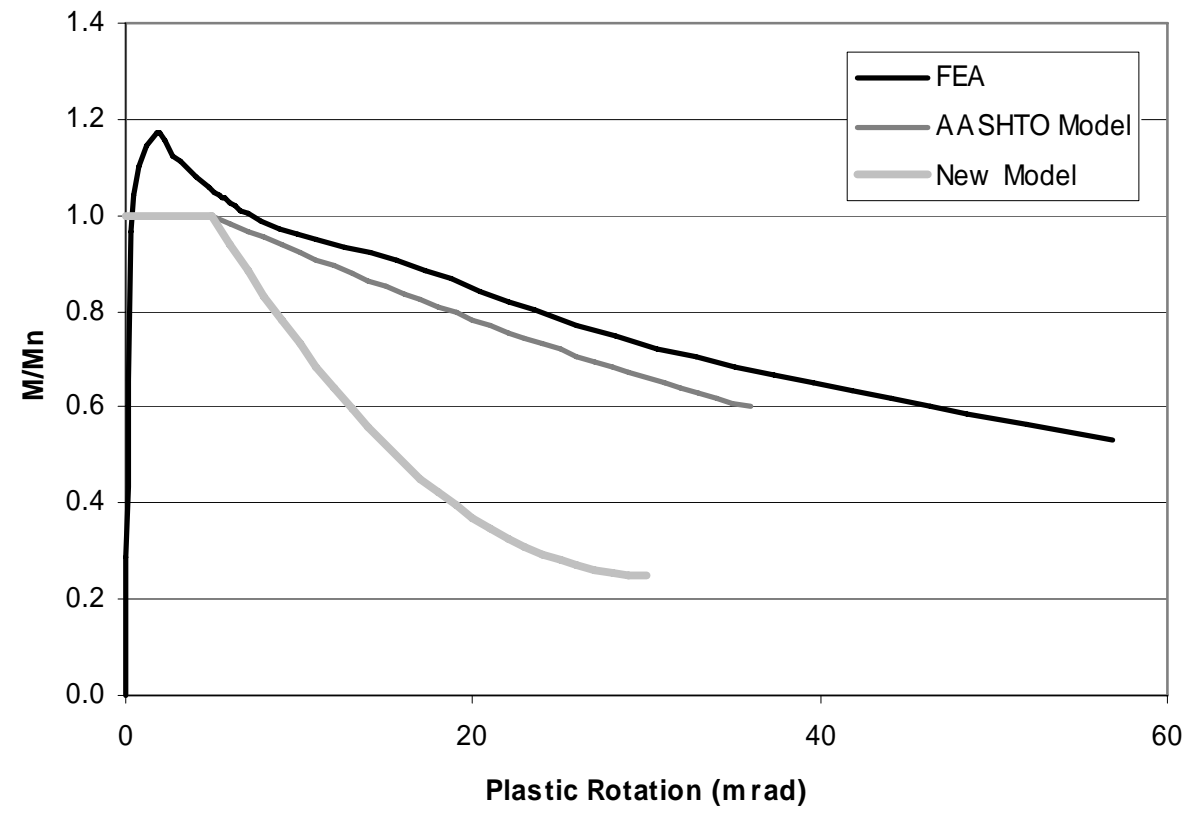

Figure D-61. Moment versus Rotation for Girder 180-2-E-50-20-NCF- $\mathrm{L}_{b}=30$

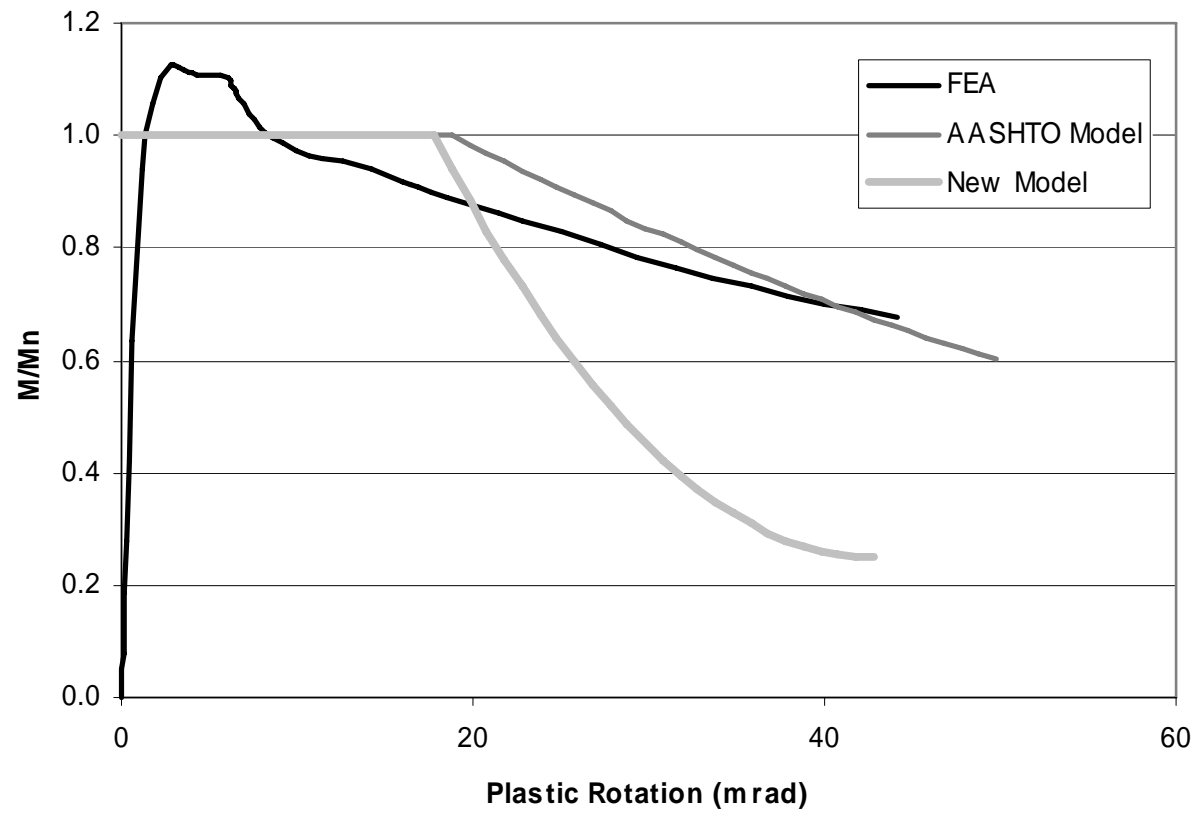

Figure D-62. Moment versus Rotation for Girder 210-2-E-50-10-E 


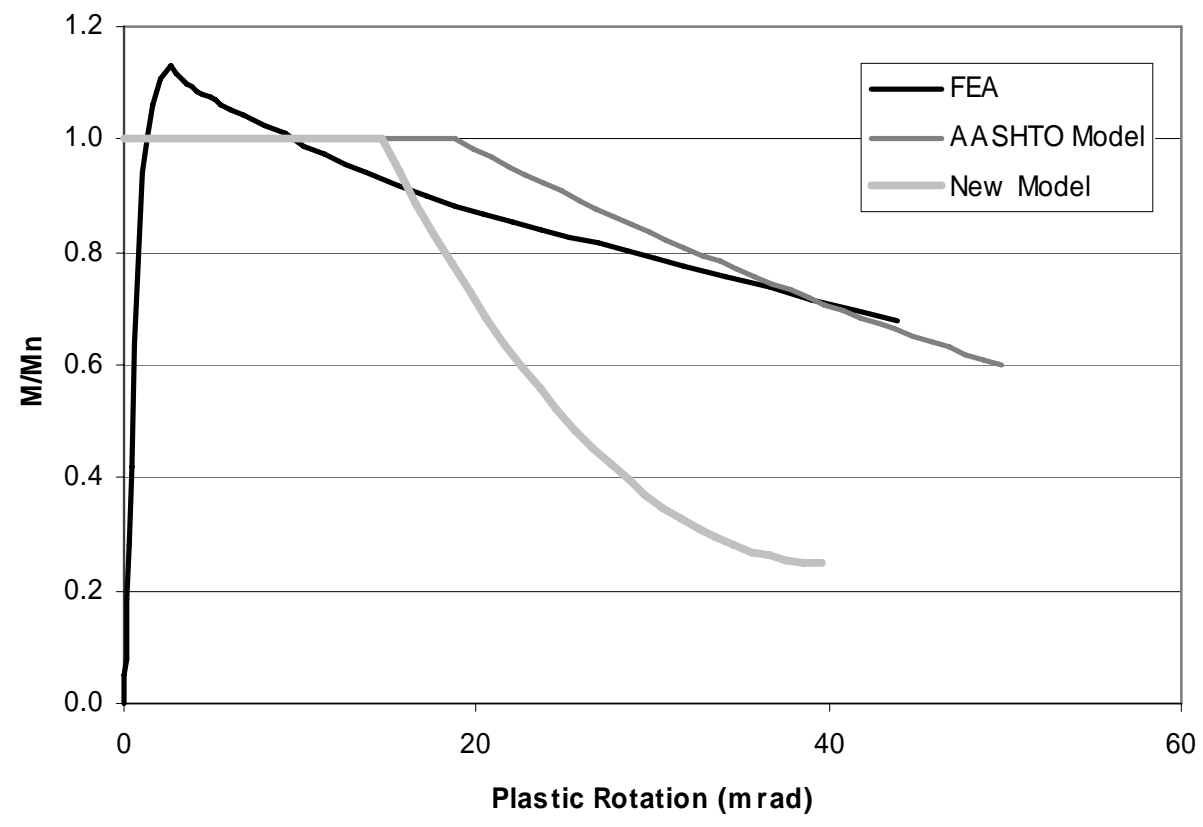

Figure D-63. Moment versus Rotation for Girder 210-2-E-50-10- $\mathbf{L}_{b}=18$

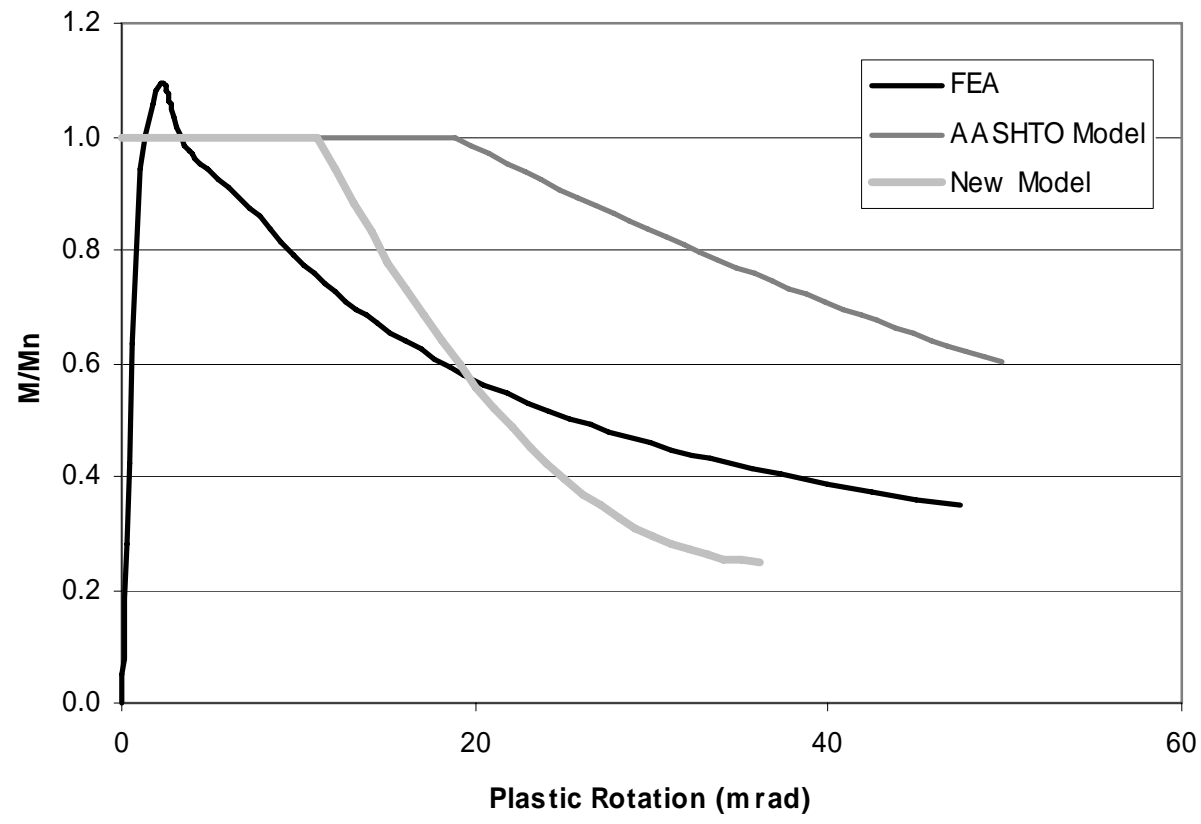

Figure D-64. Moment versus Rotation for Girder 210-2-E-50-10- $L_{b}=26.25$ 


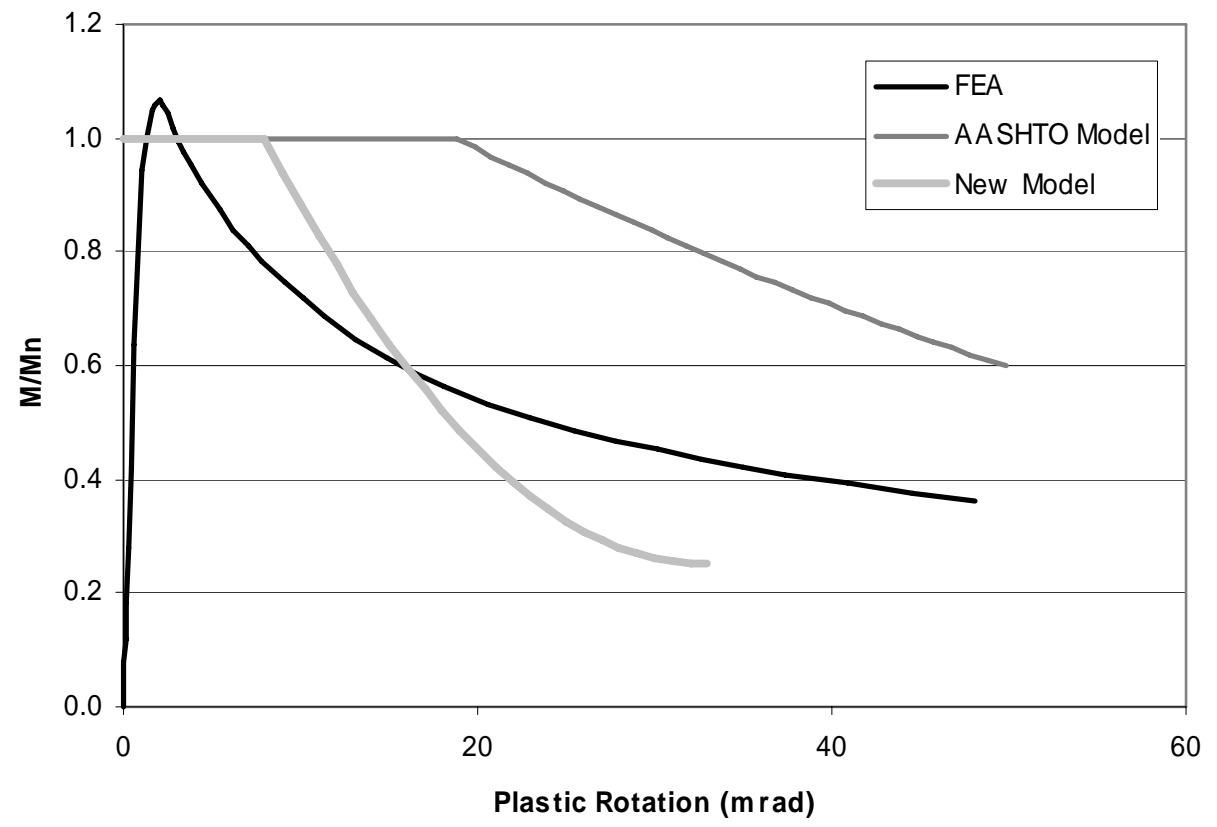

Figure D-65. Moment versus Rotation for Girder 210-2-E-50-10- $\mathbf{L}_{b}=30$

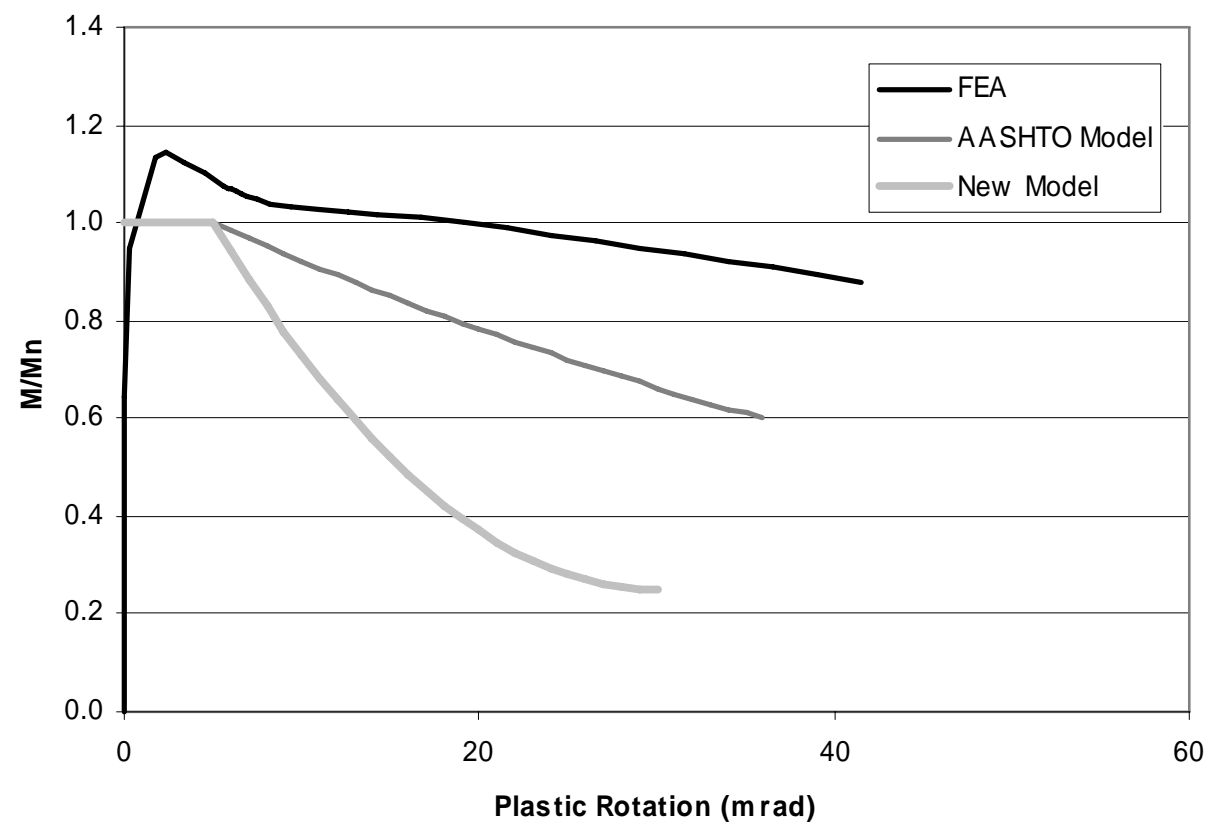

Figure D-66. Moment versus Rotation for Girder 210-2-E-50-10-NCF 


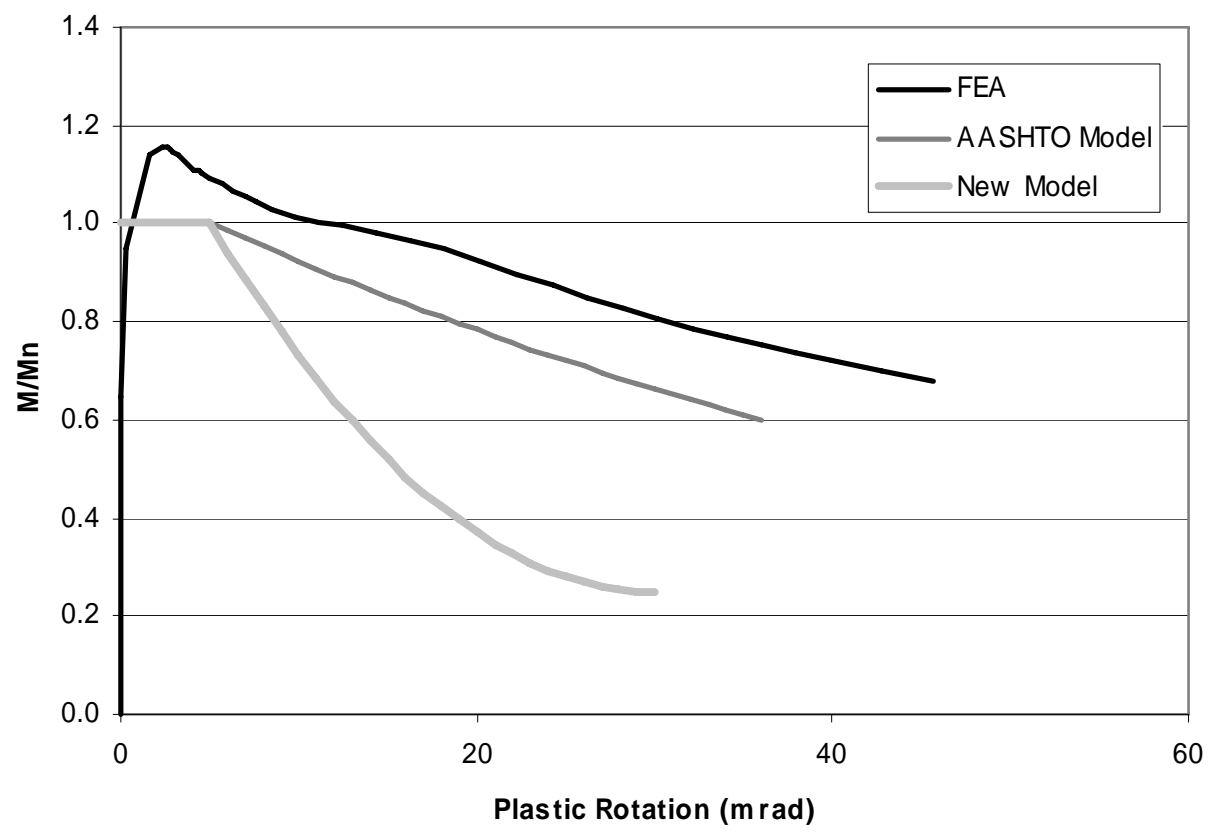

Figure D-67. Moment versus Rotation for Girder 210-2-E-50-10-NCF- $\mathrm{L}_{\mathbf{b}}=30$

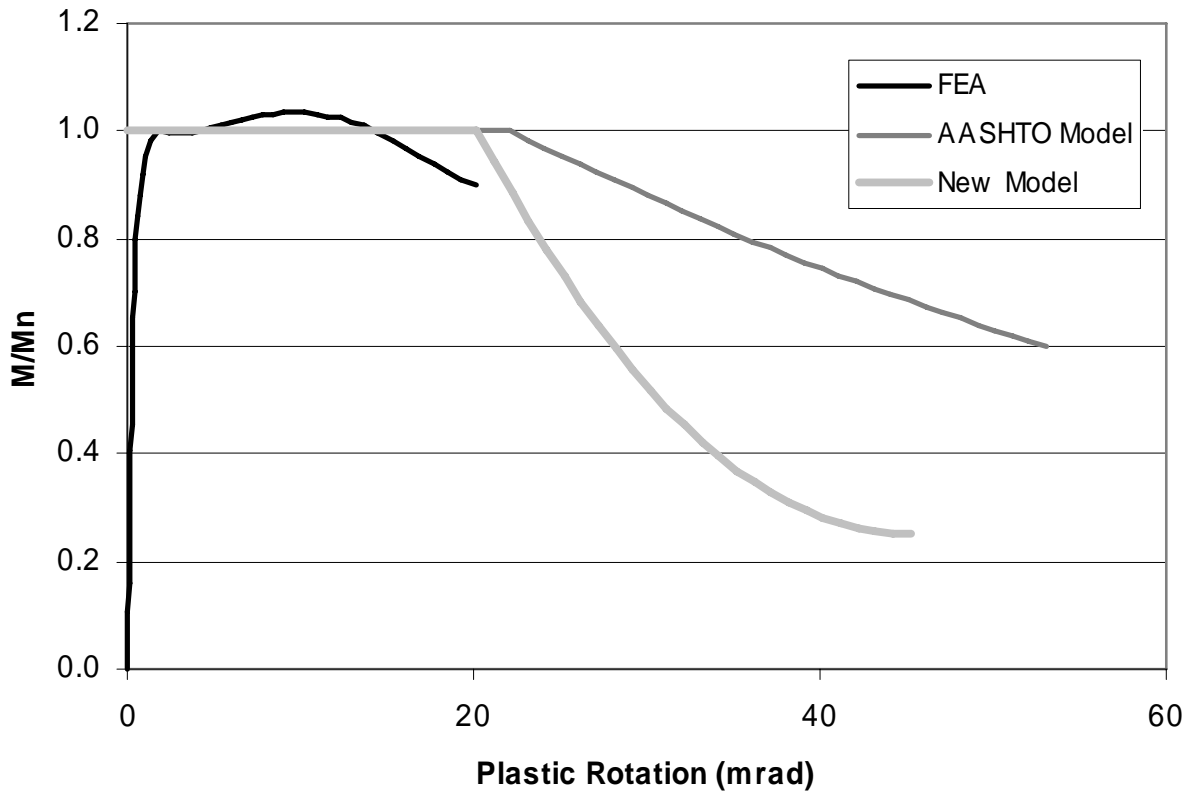

Figure D-68. Moment versus Rotation for Girder 210-2-E-50-20-F 


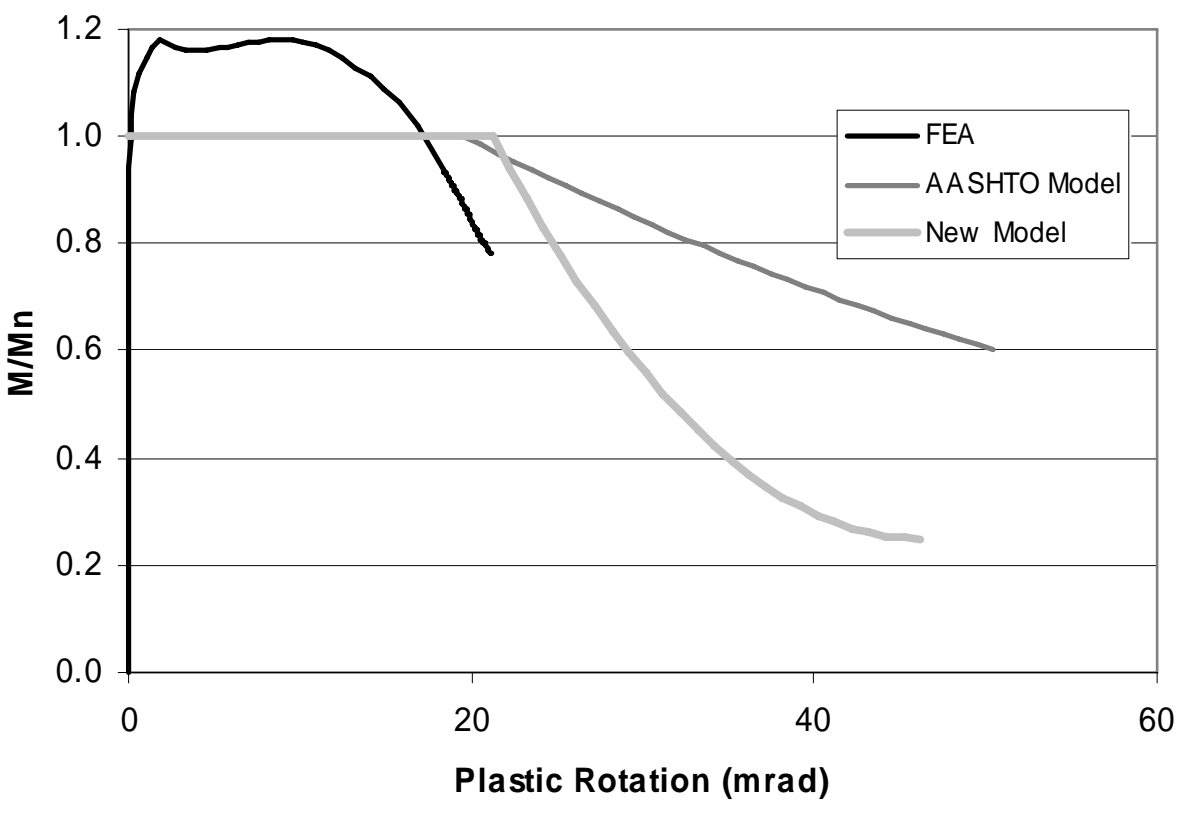

Figure D-69. Moment versus Rotation for Girder 210-2-E-50-30-B

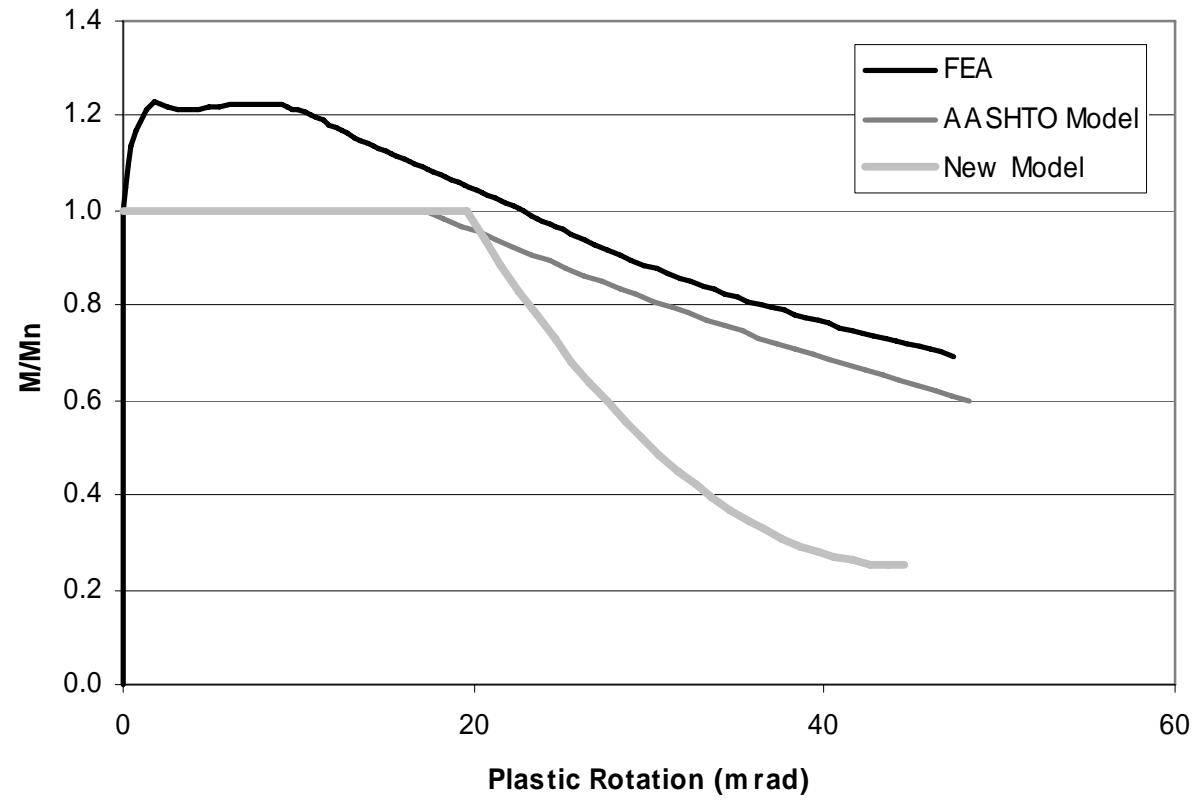

Figure D-70. Moment versus Rotation for Girder 210-2-E-50-40-C 


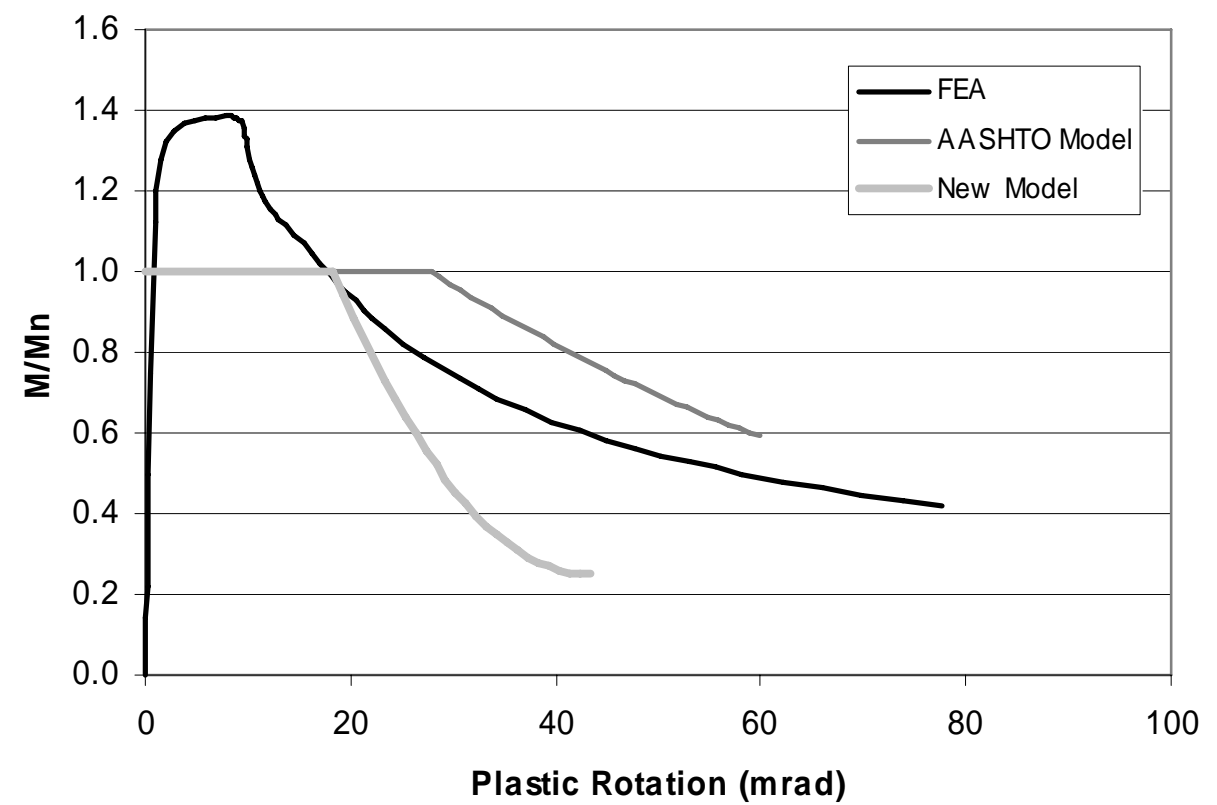

Figure D-71. Moment versus Rotation for Girder 140-175-140-H-20-D 


\section{Appendix E: EXAMPLE DESIGNS}
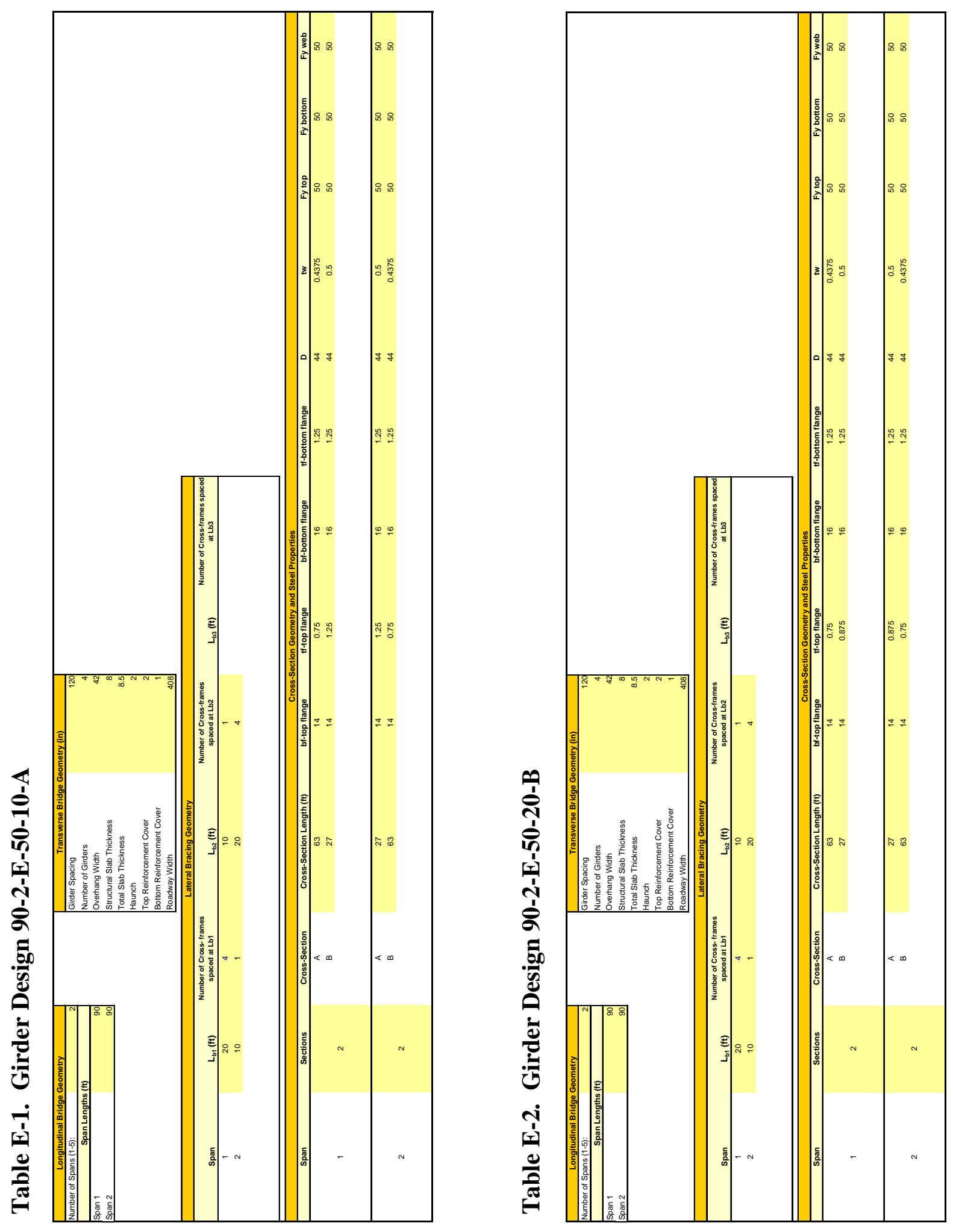

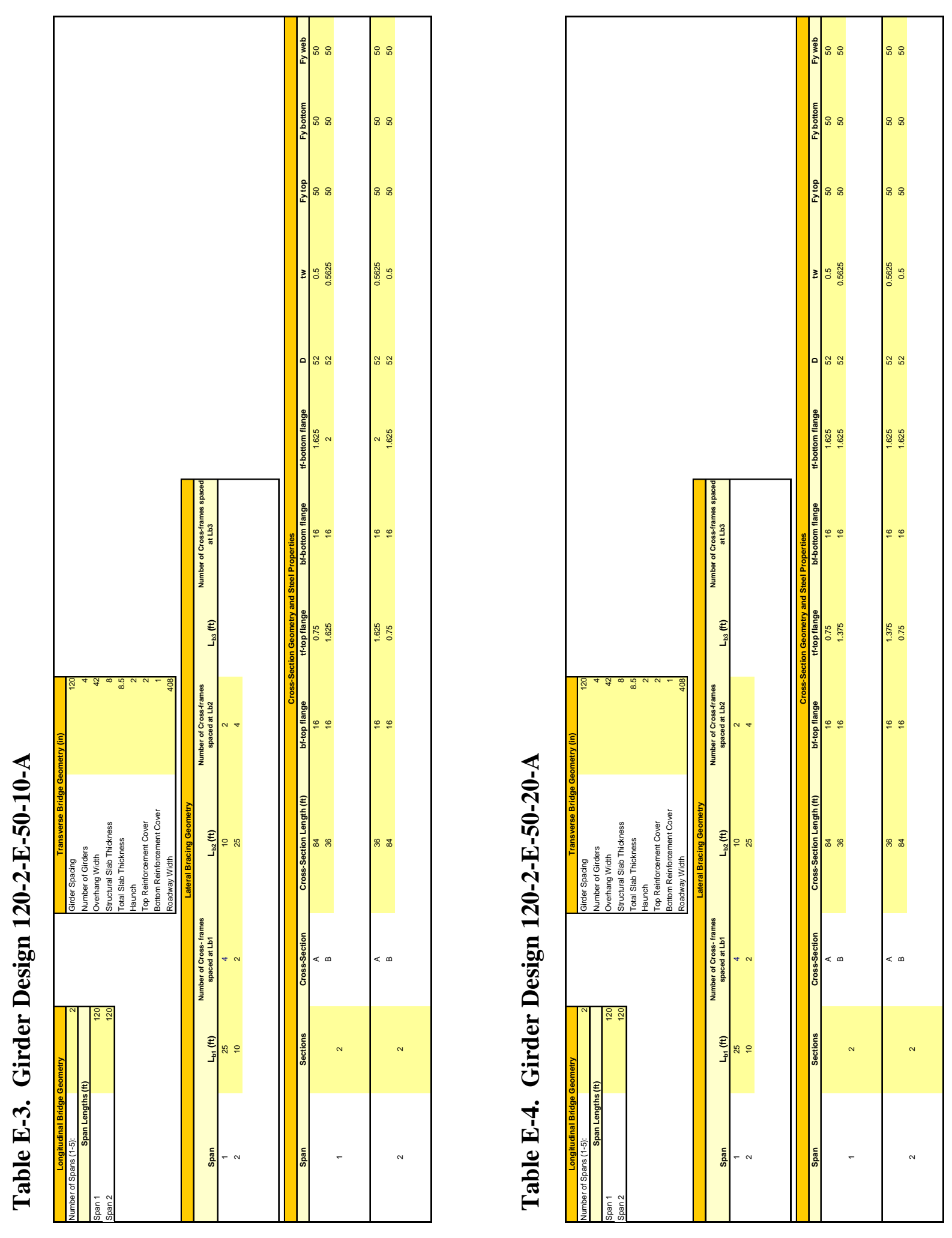

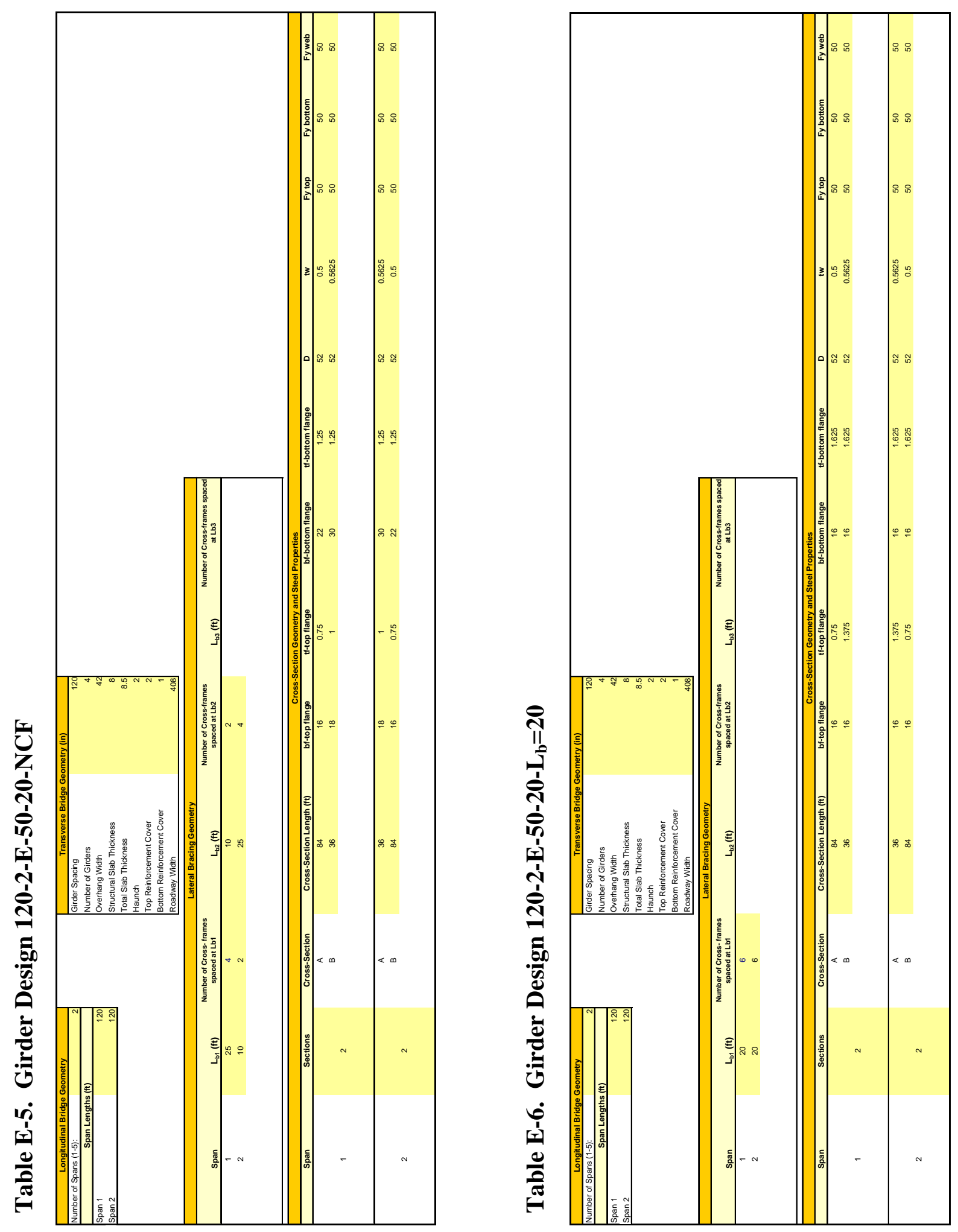

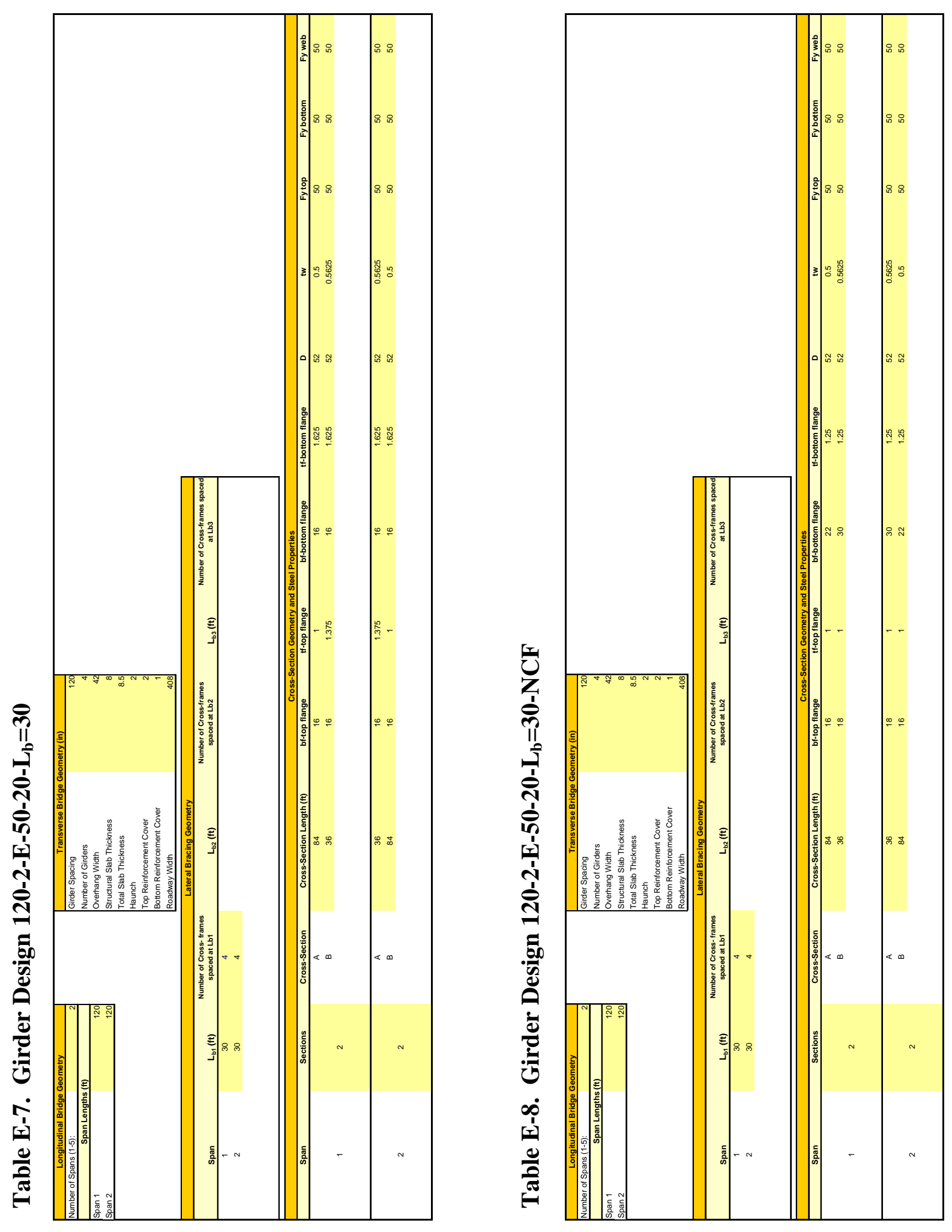

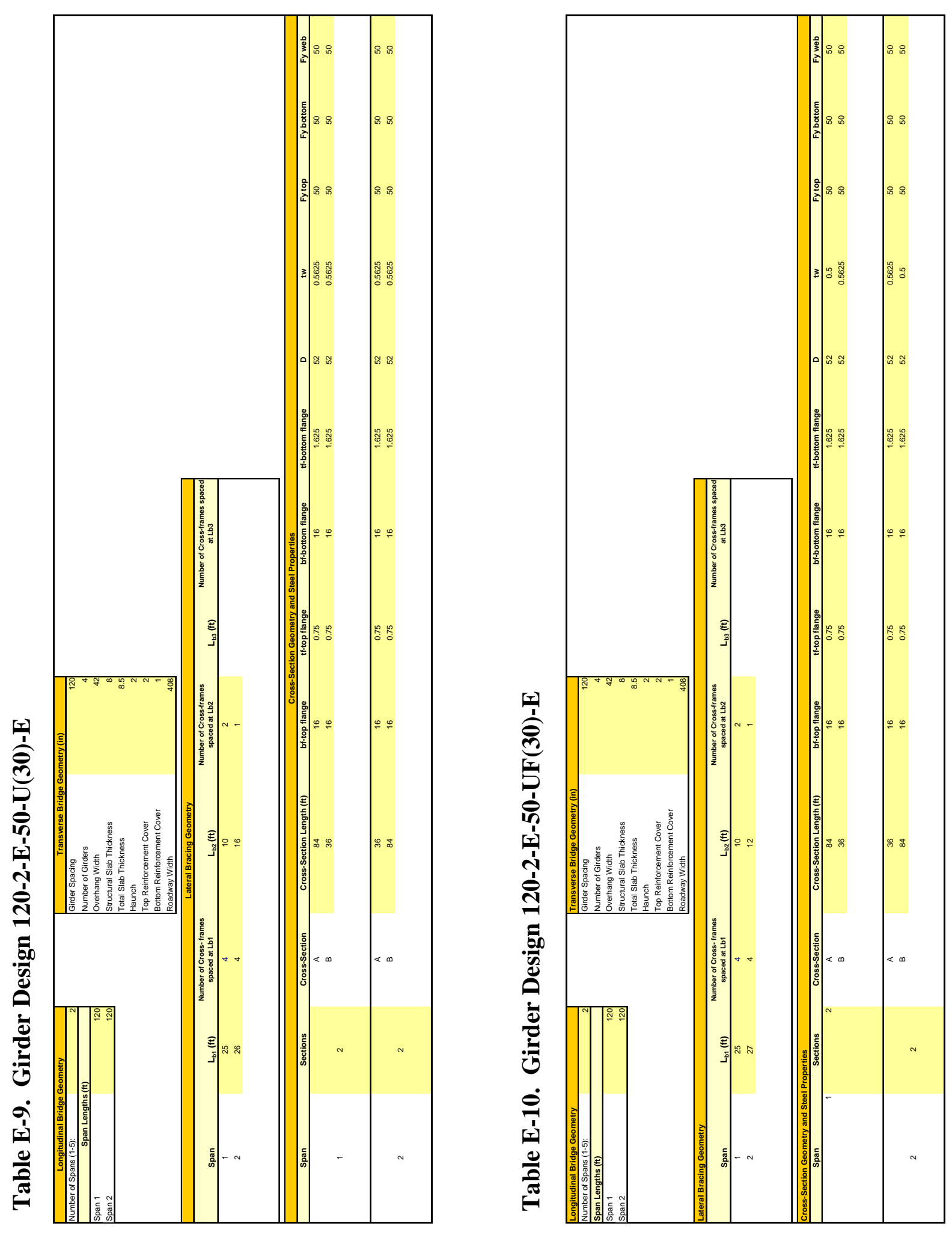

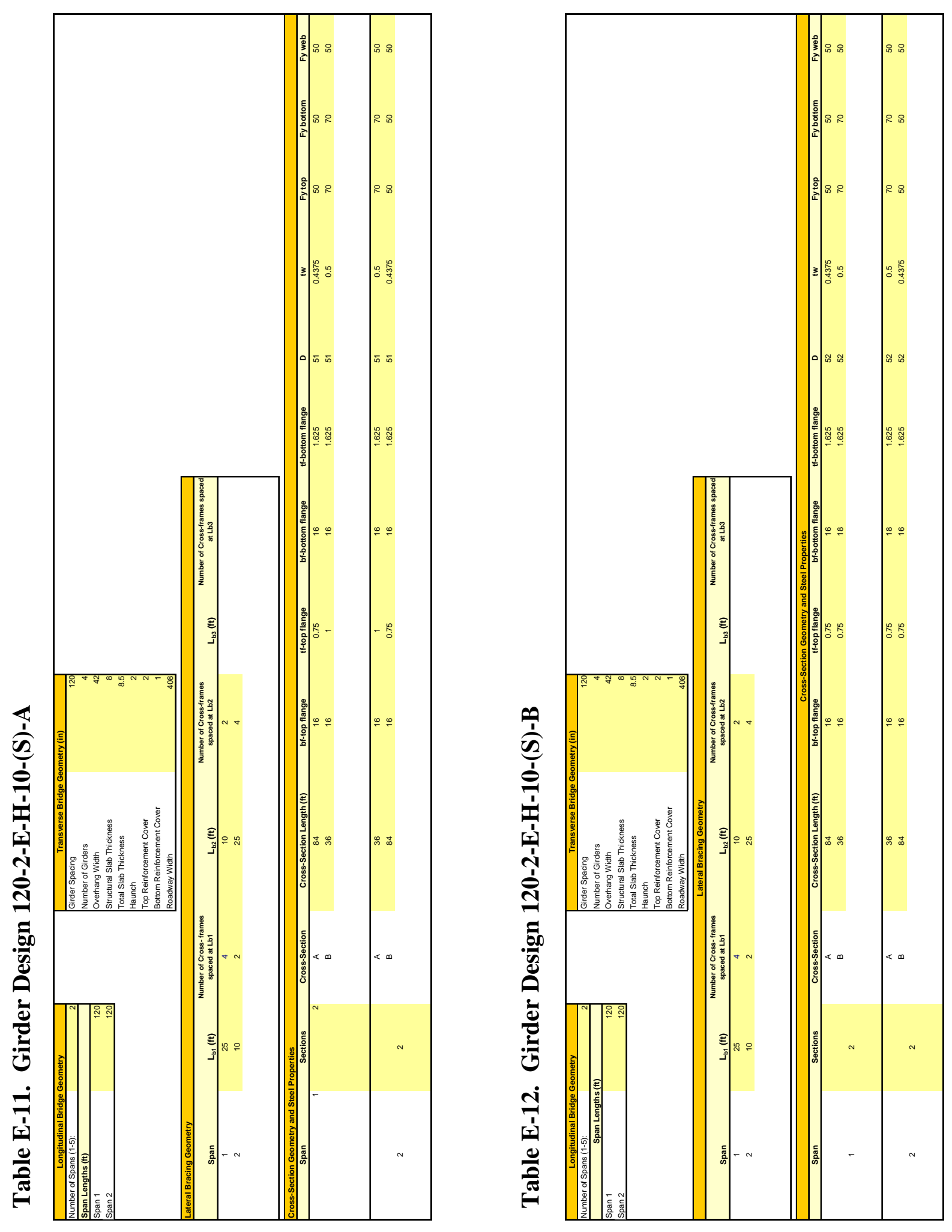

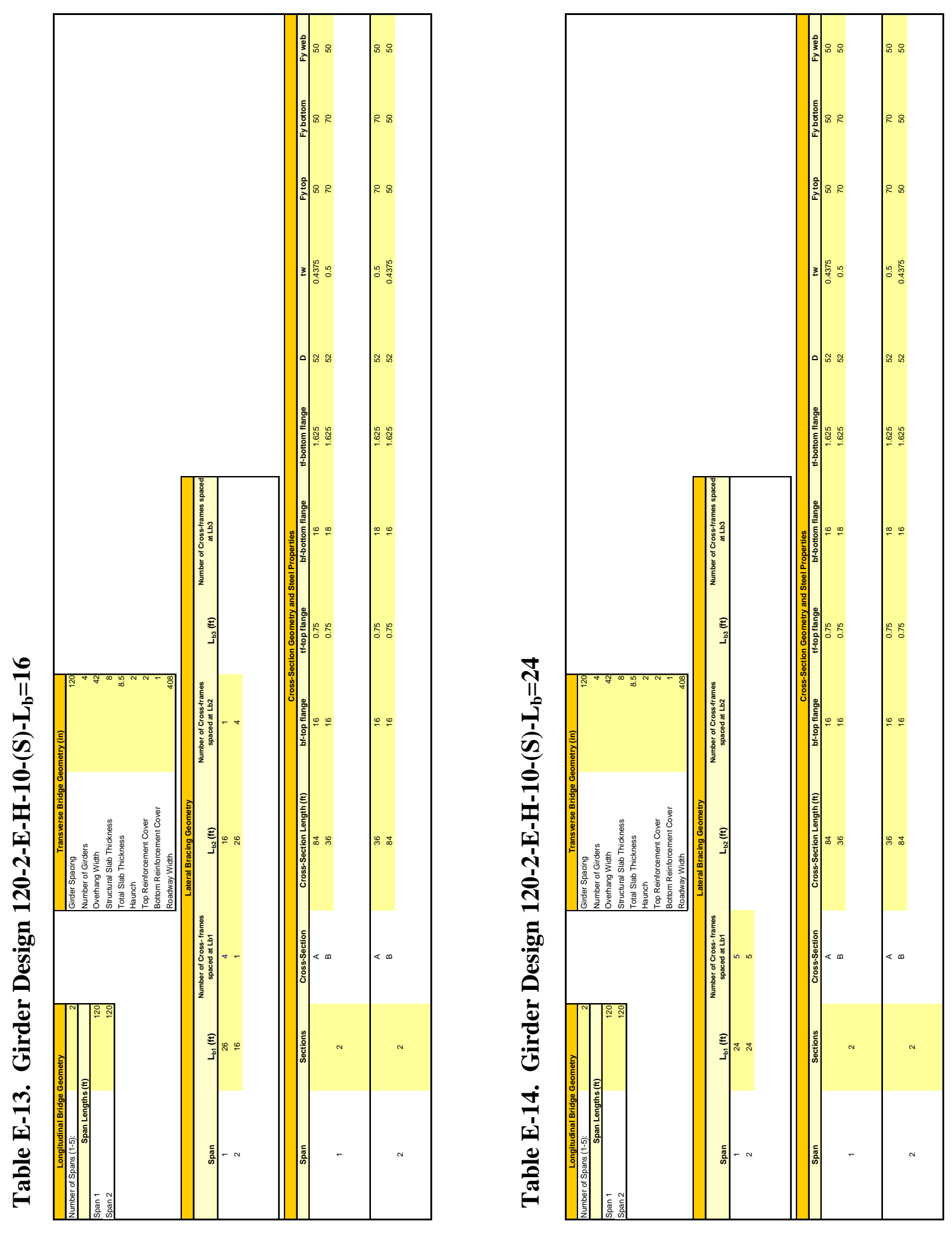

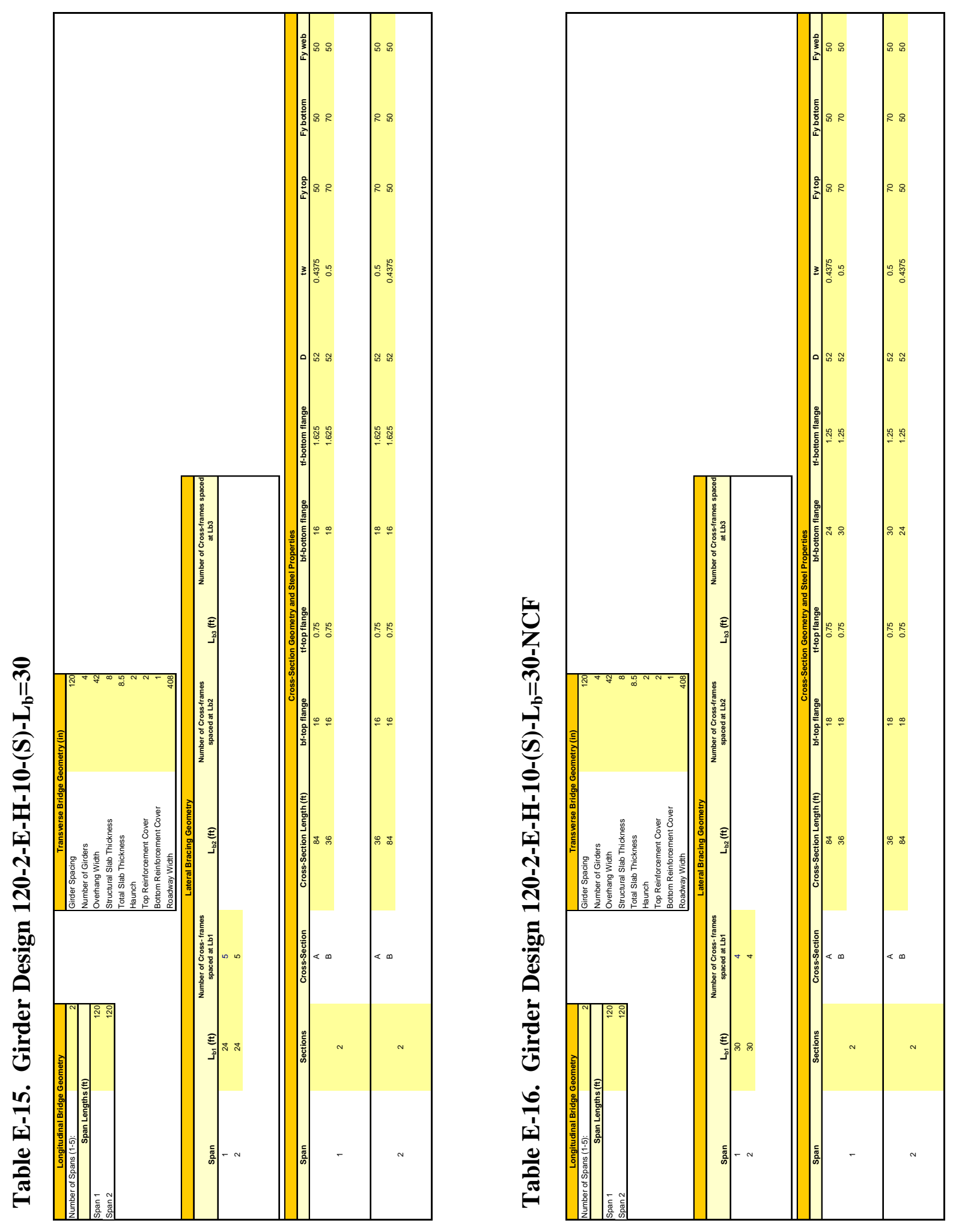

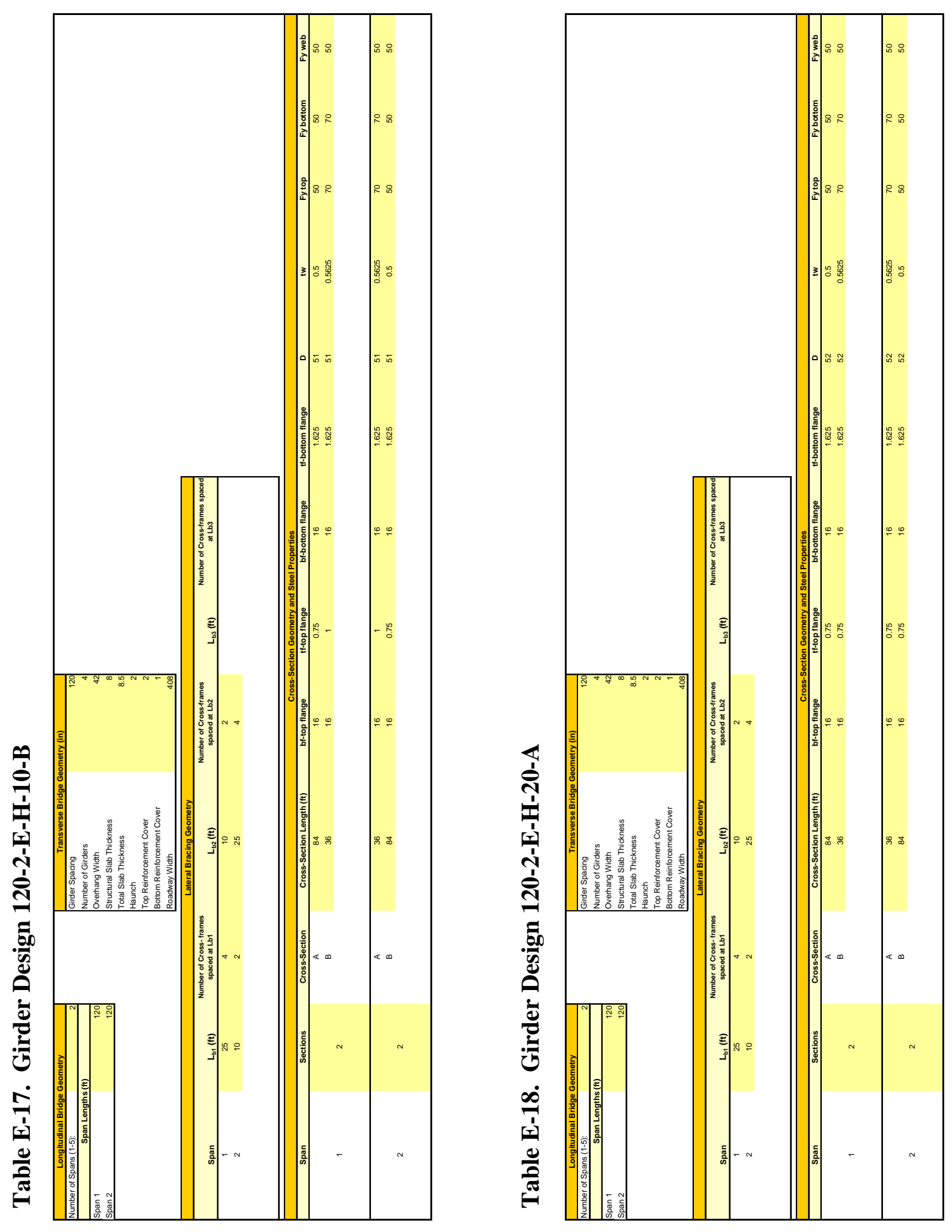

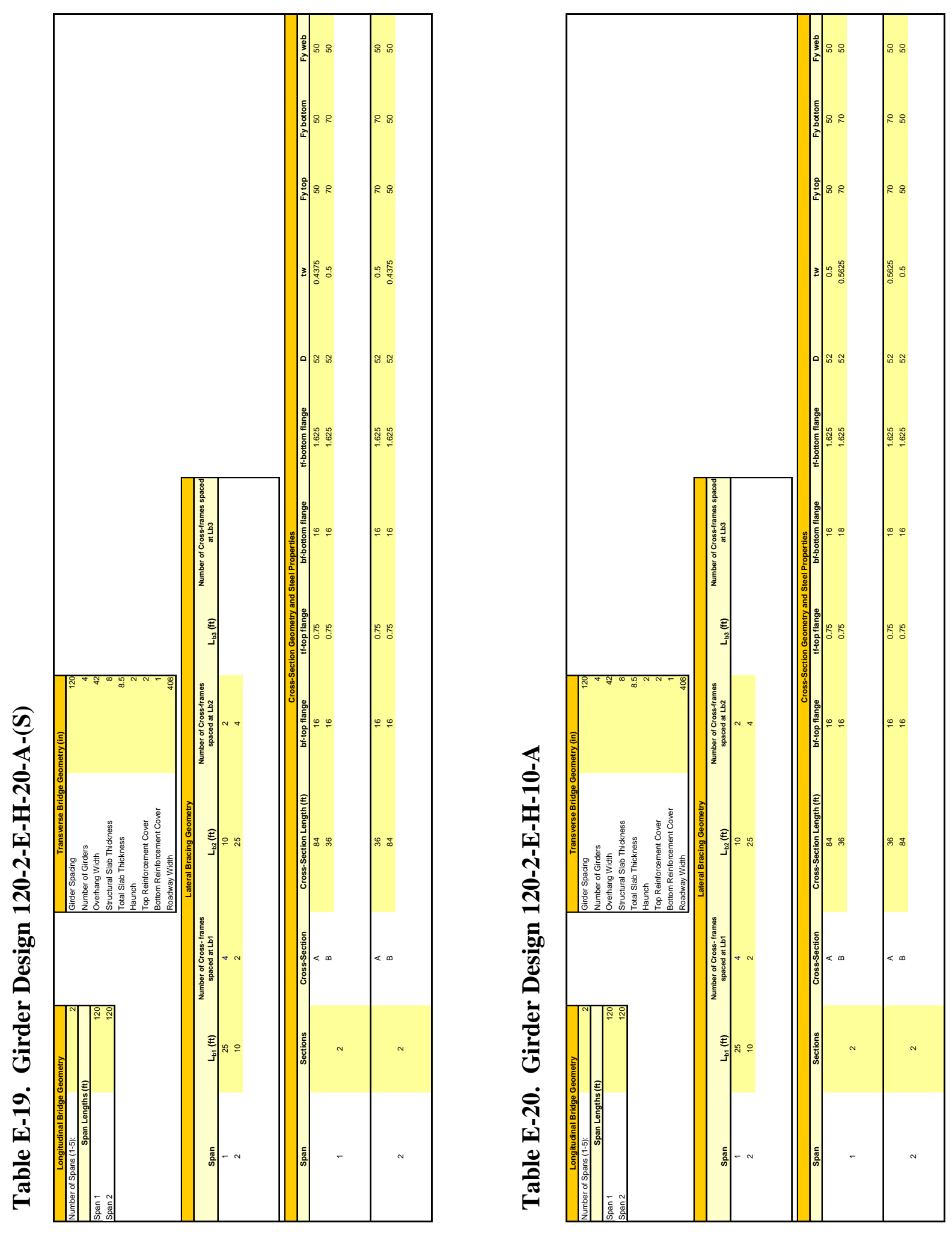

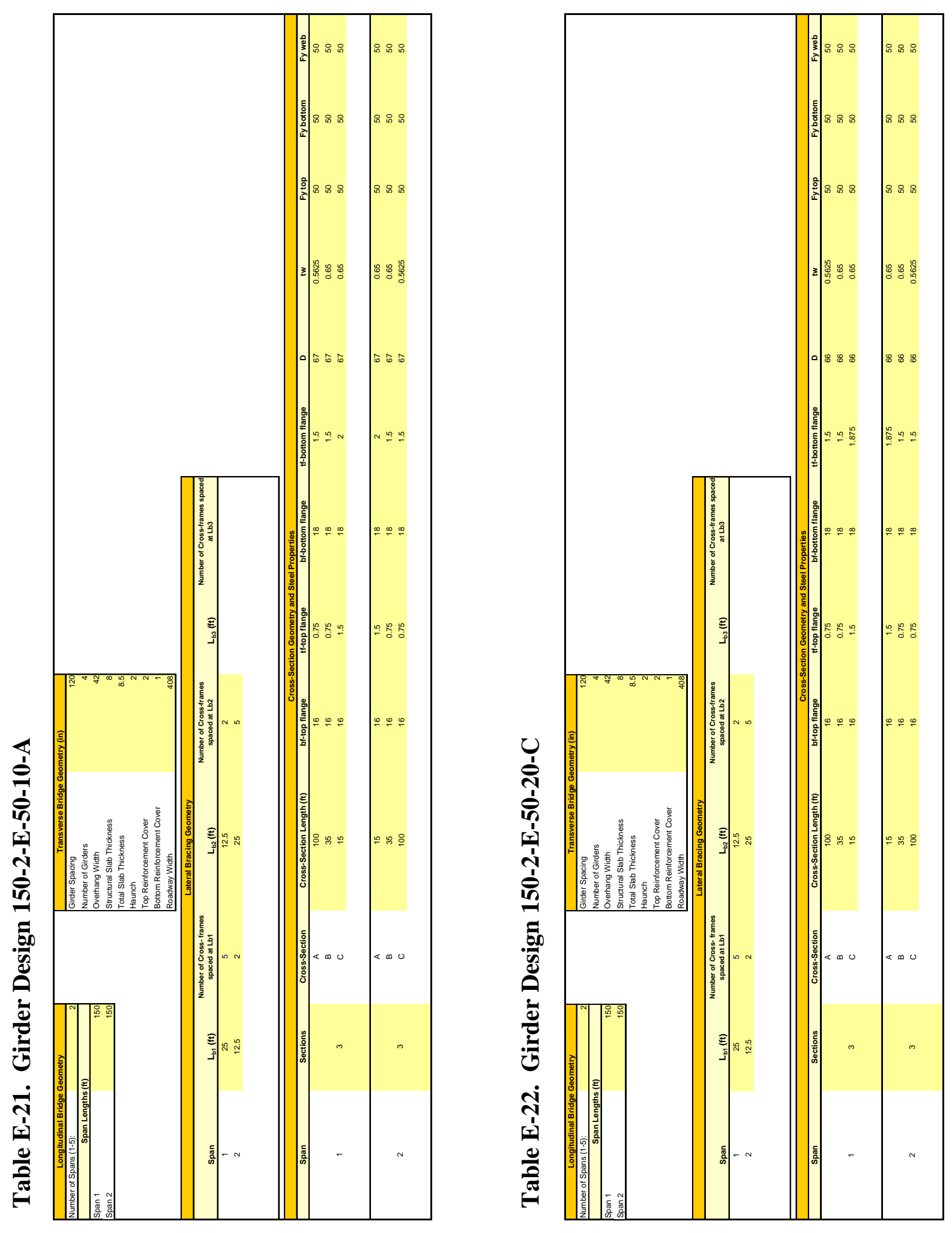

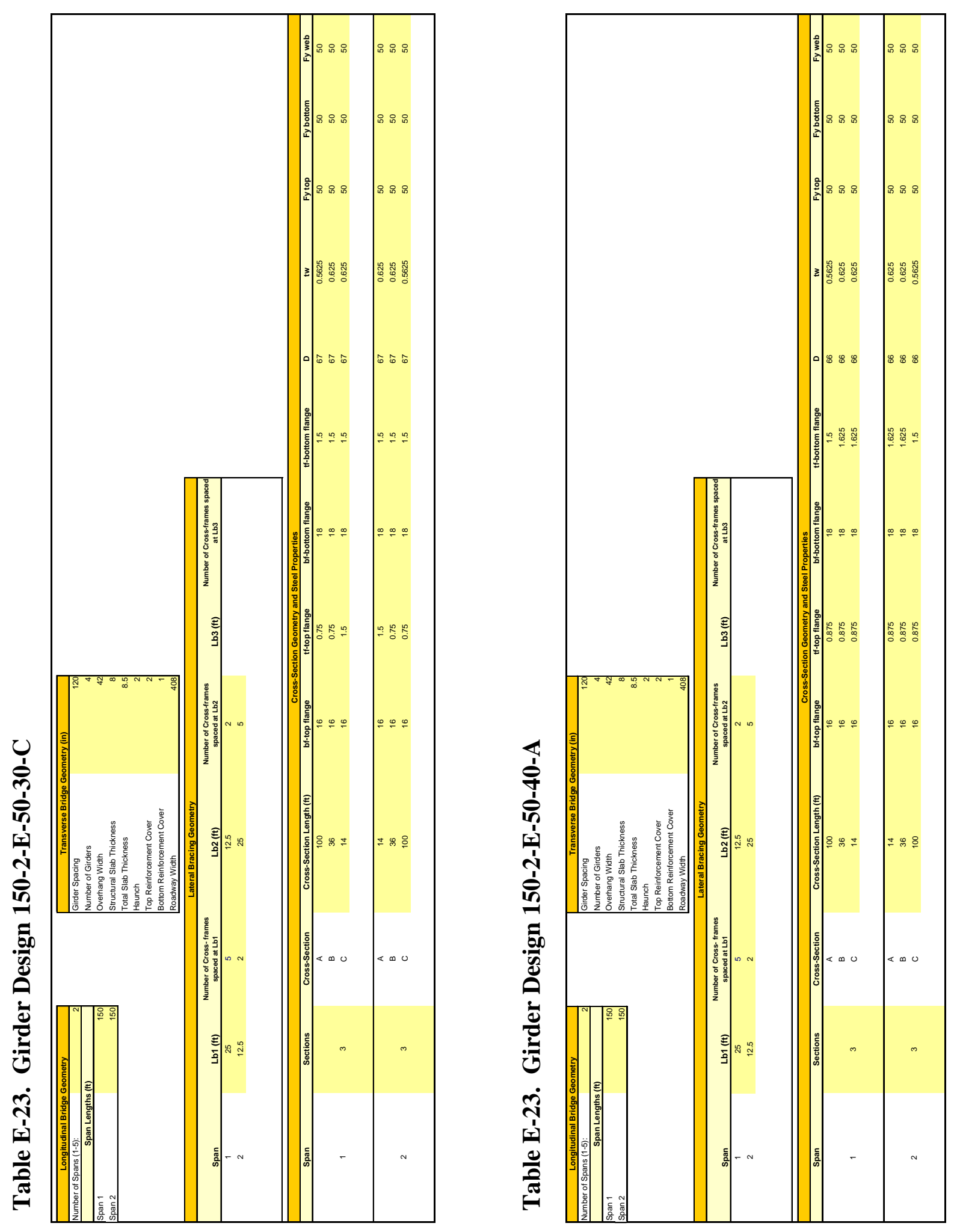

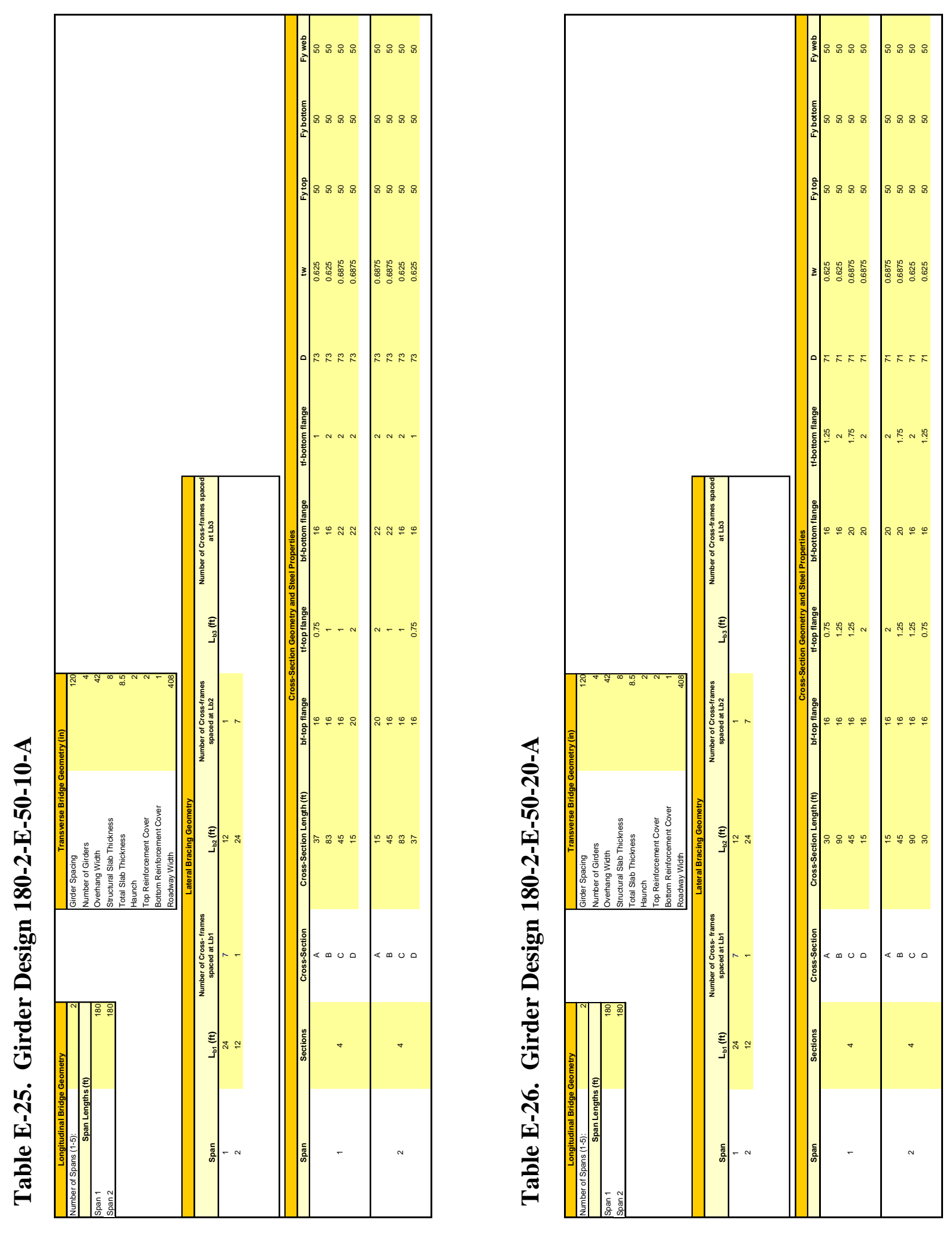

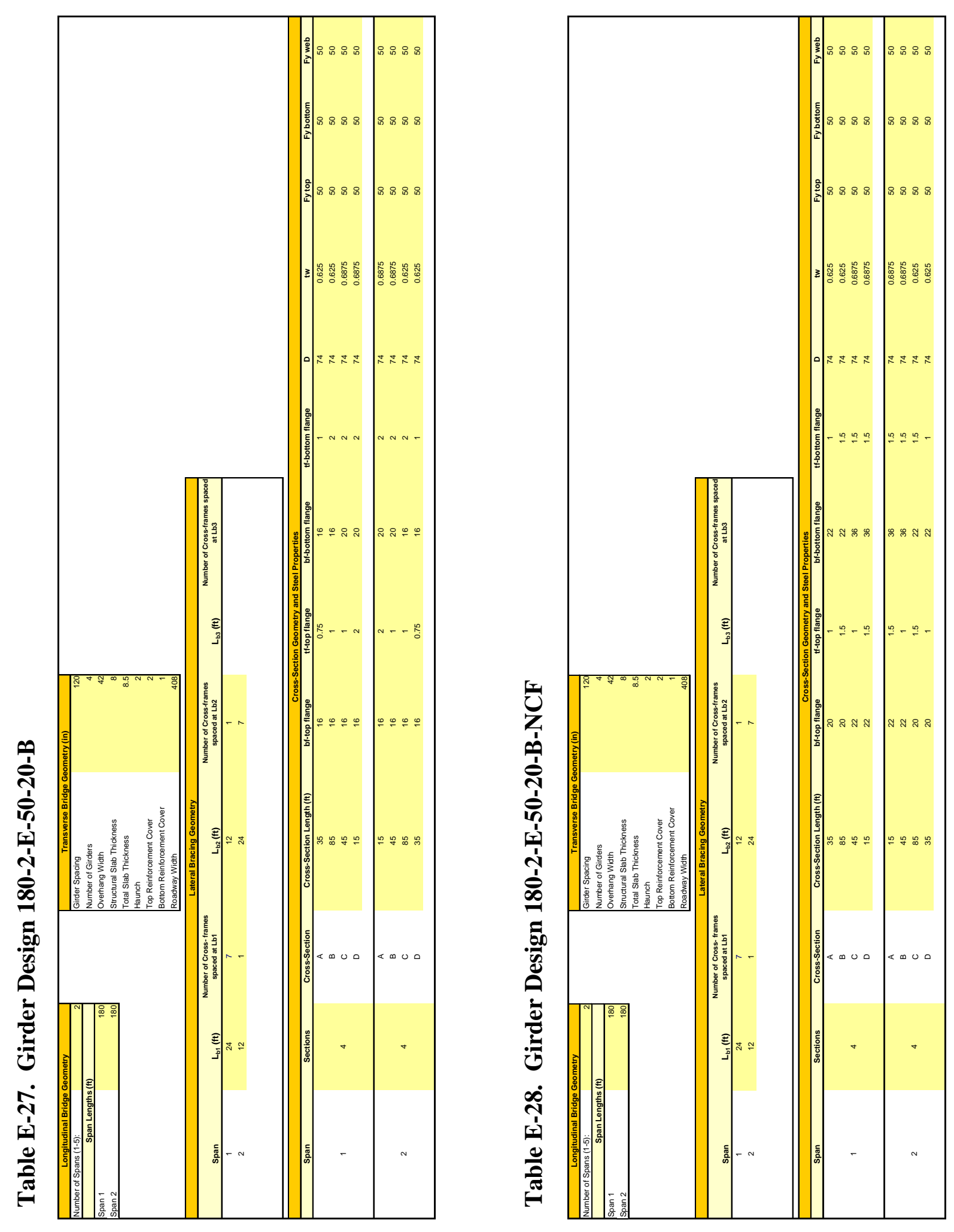

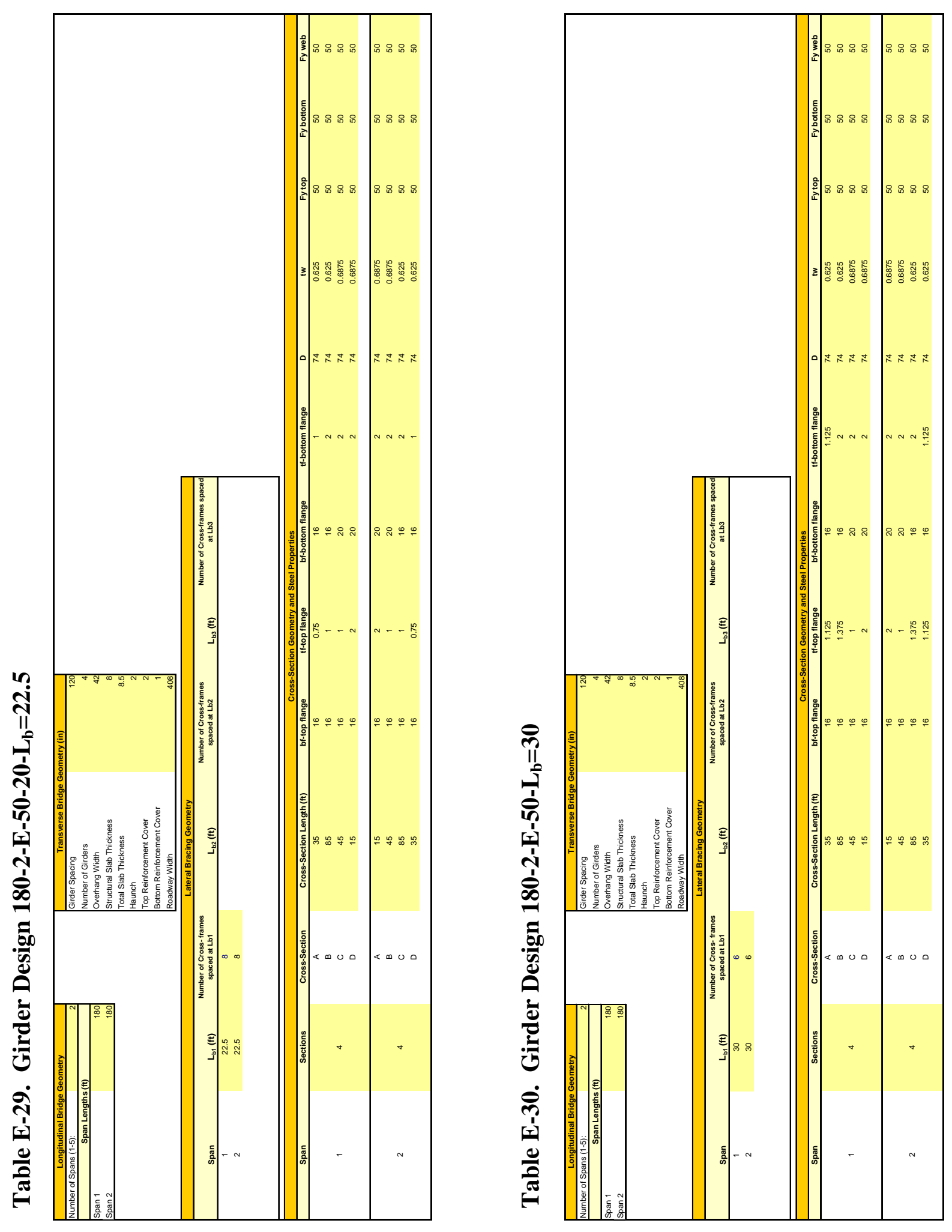

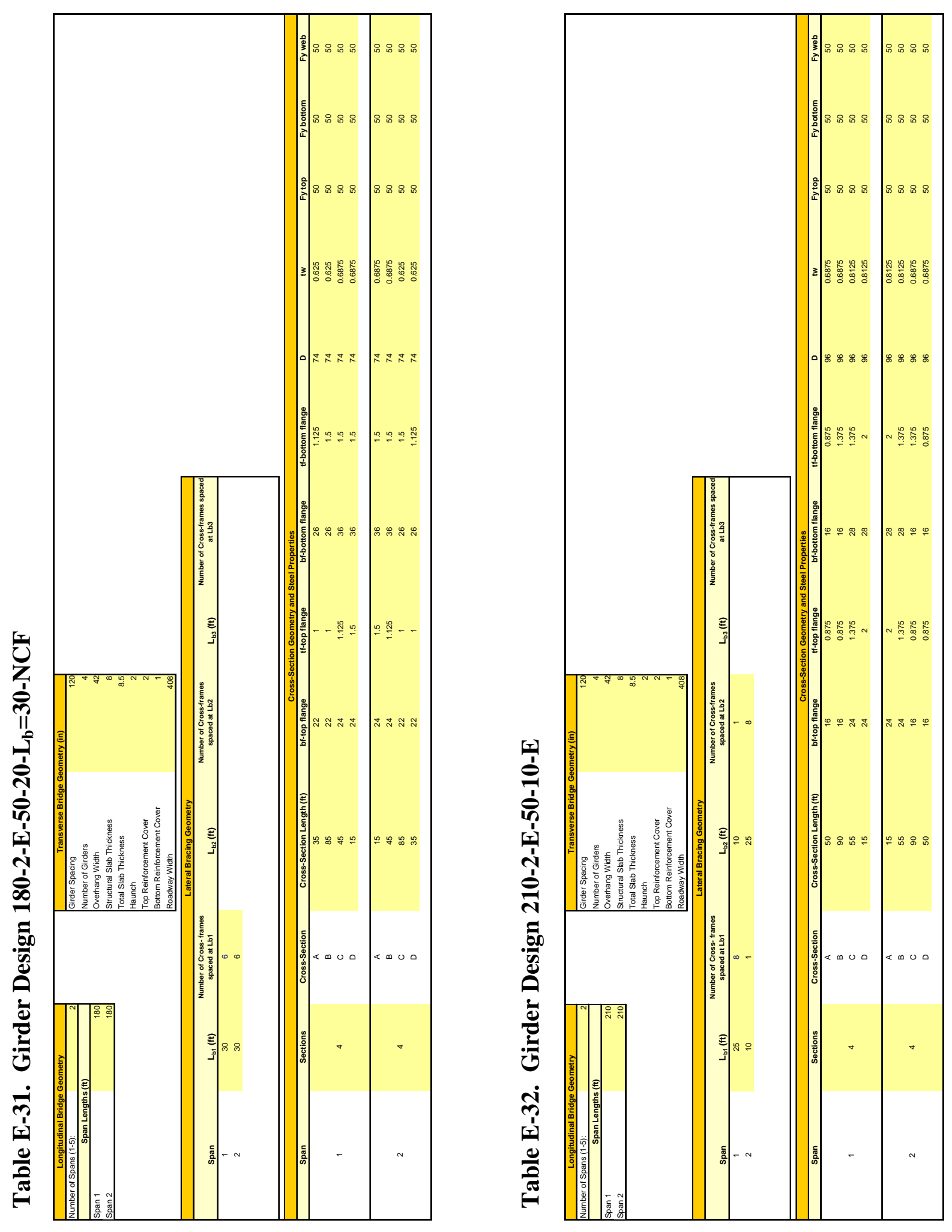

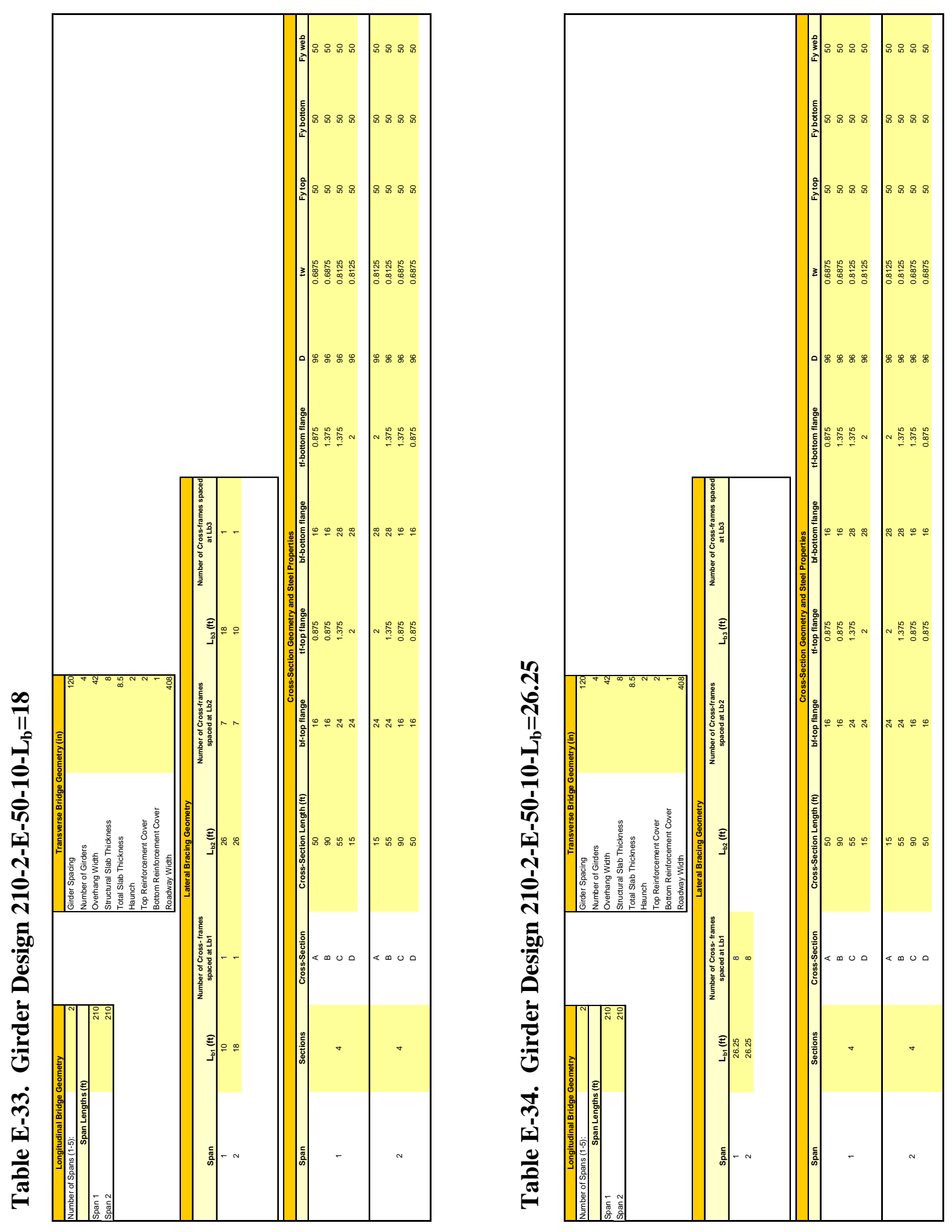

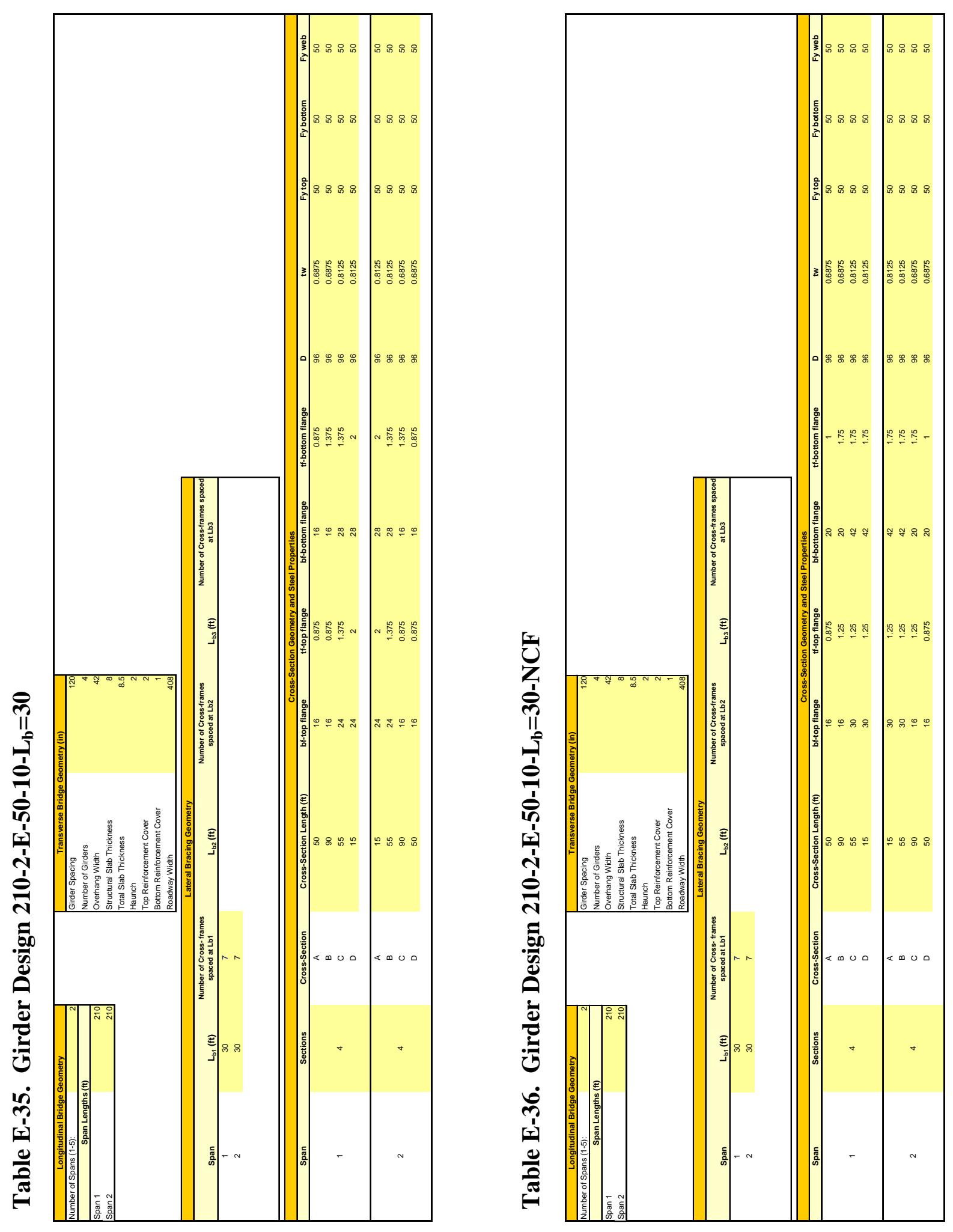

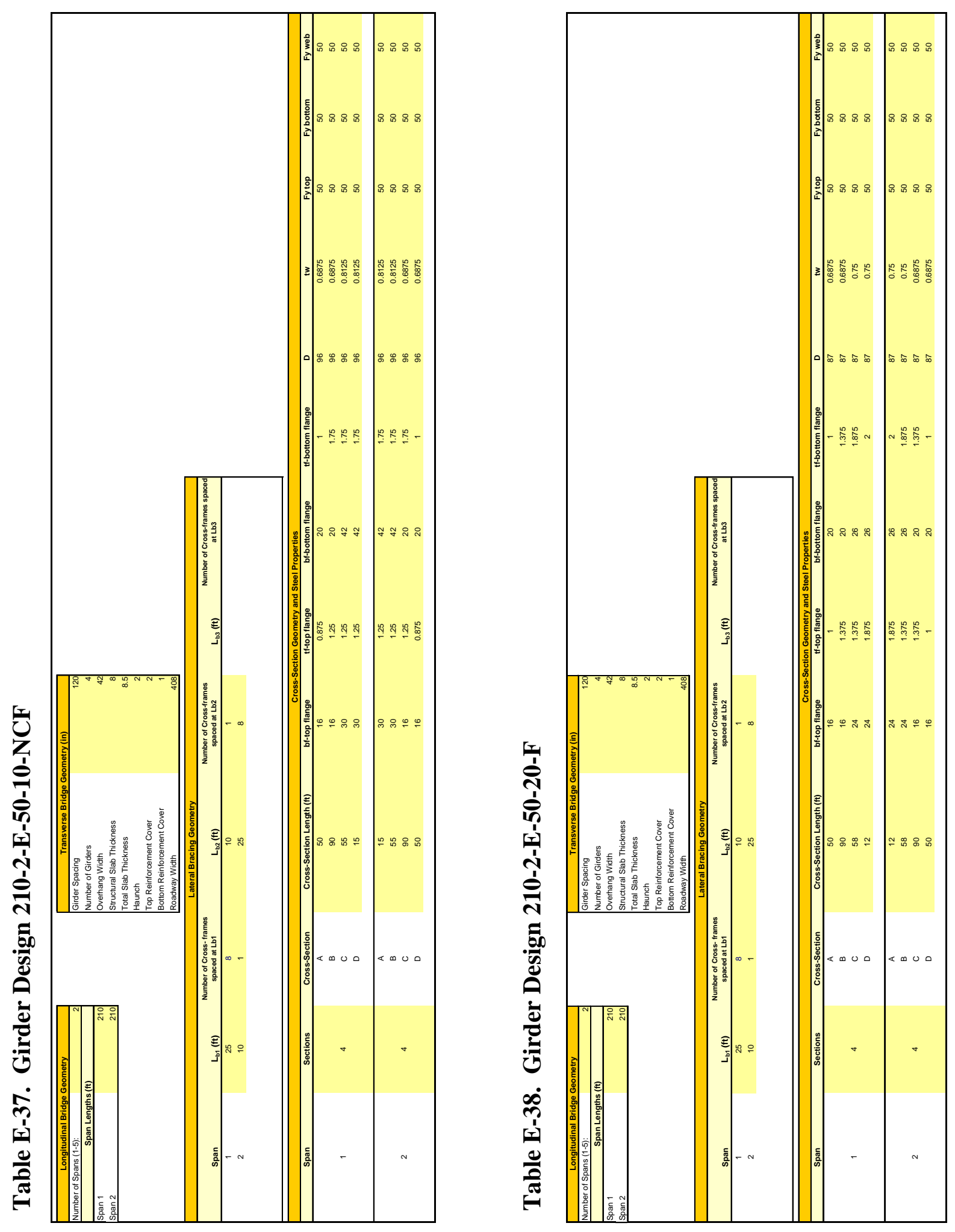

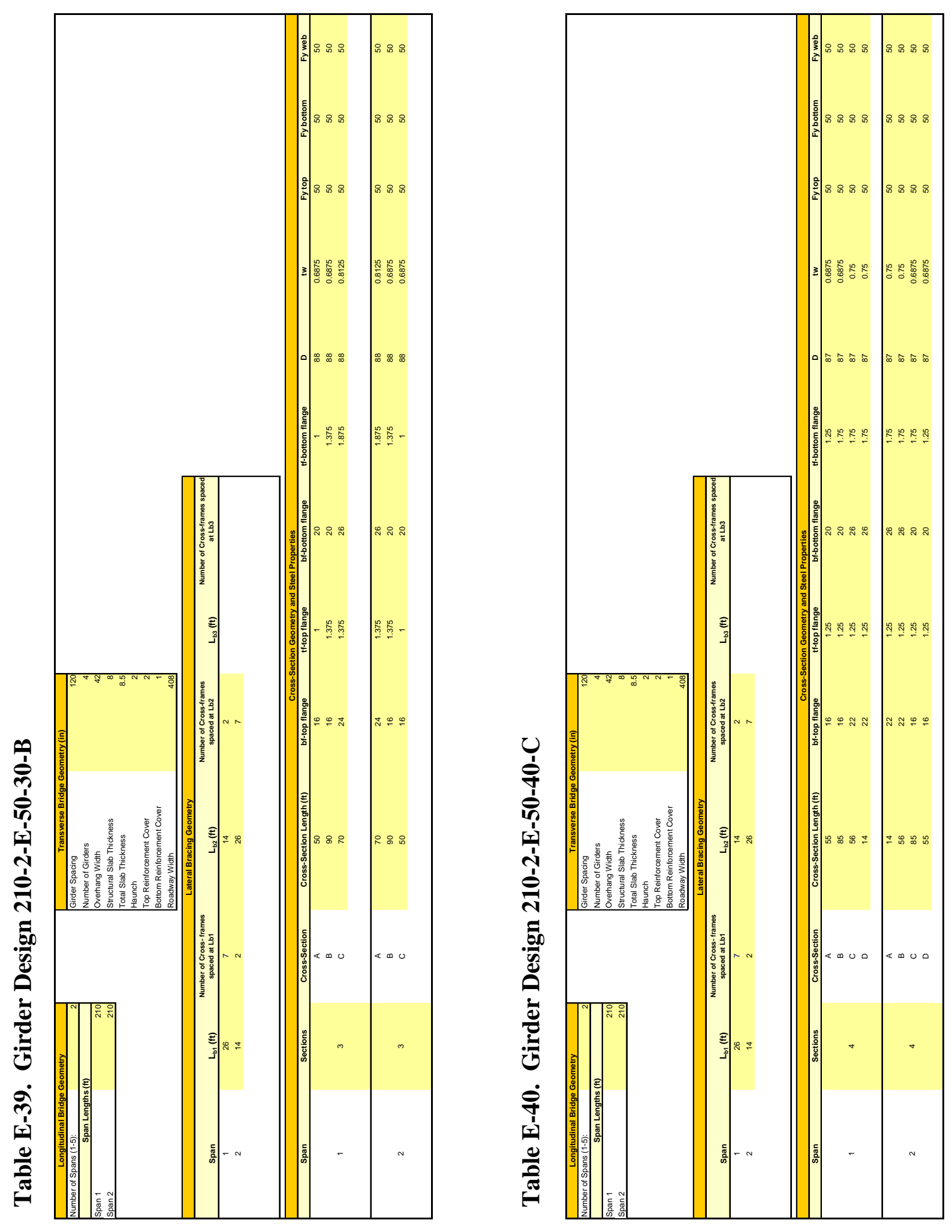

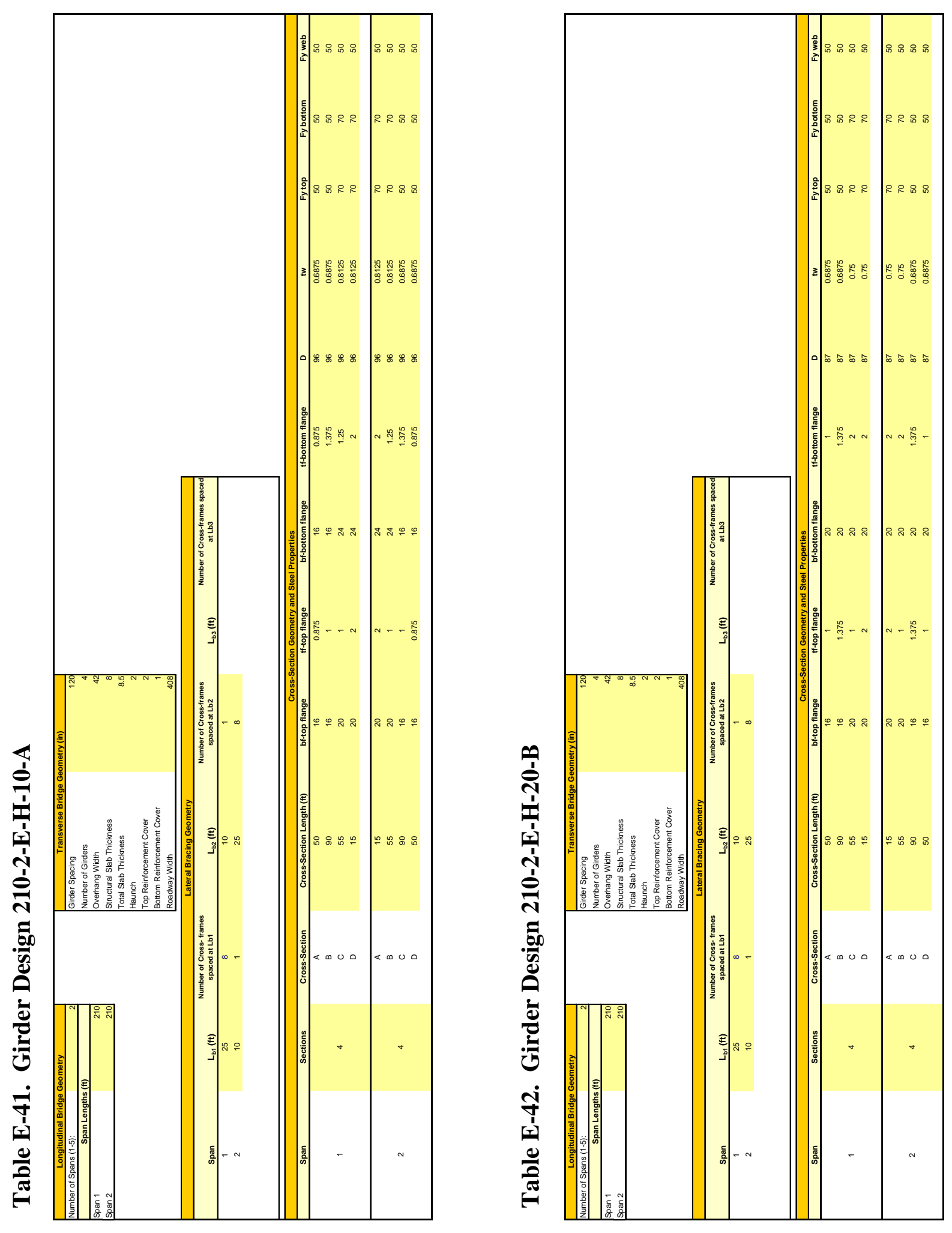

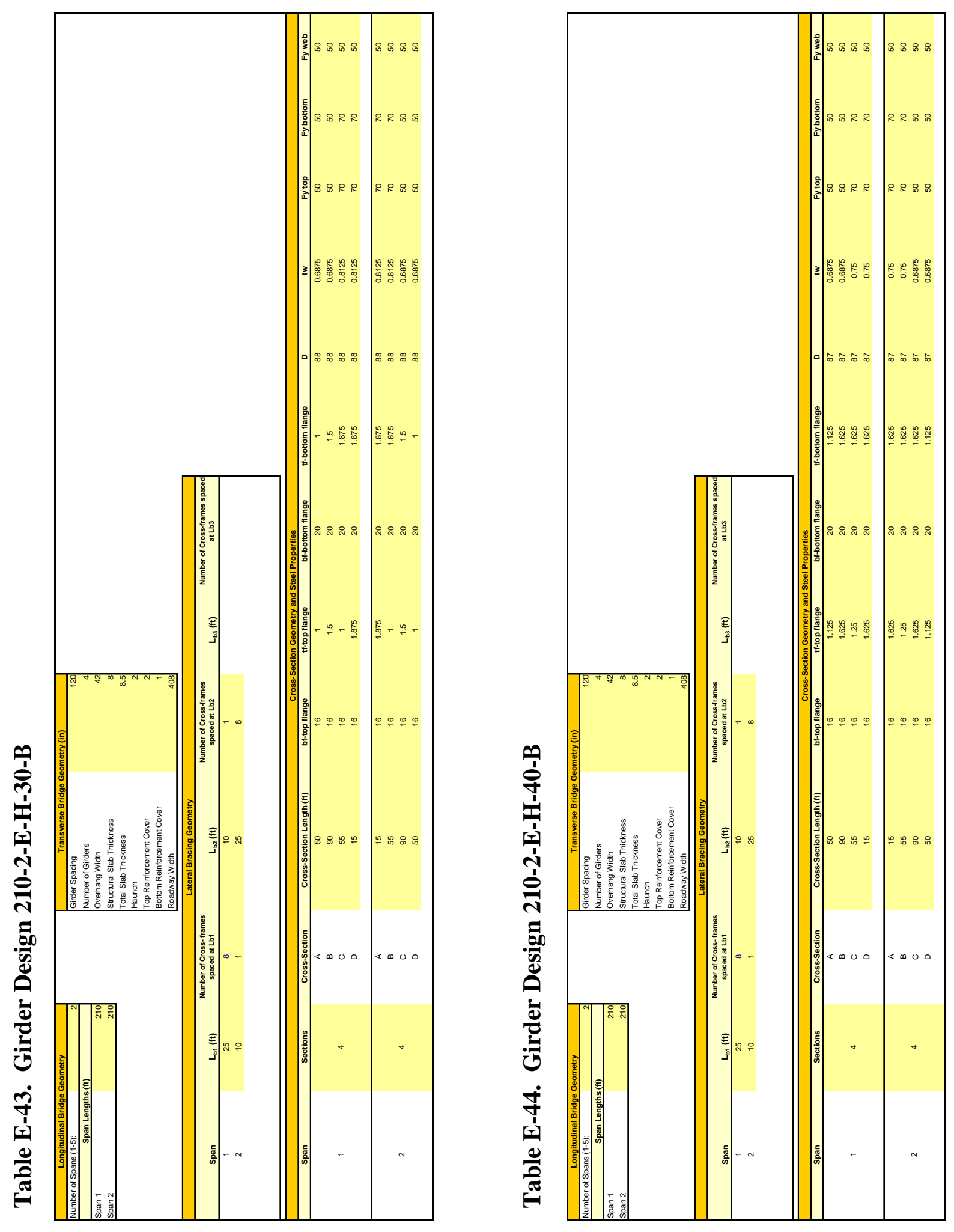


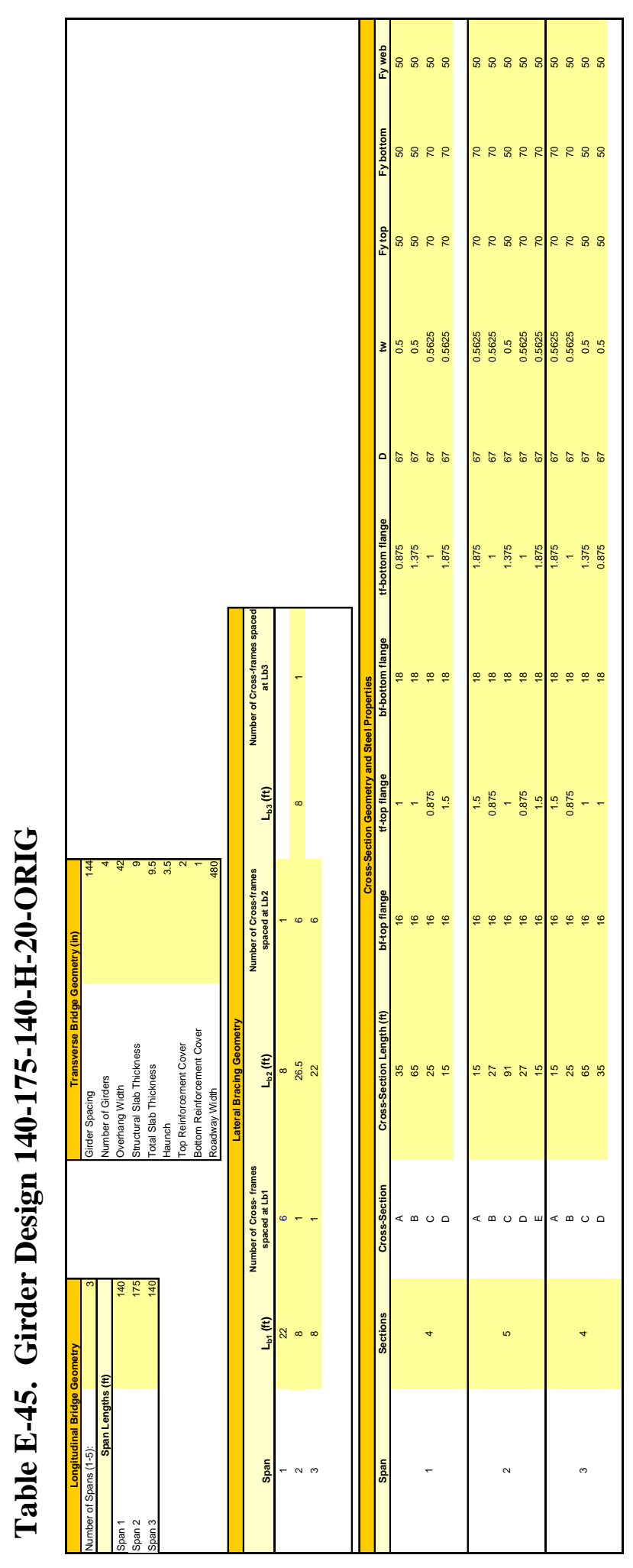




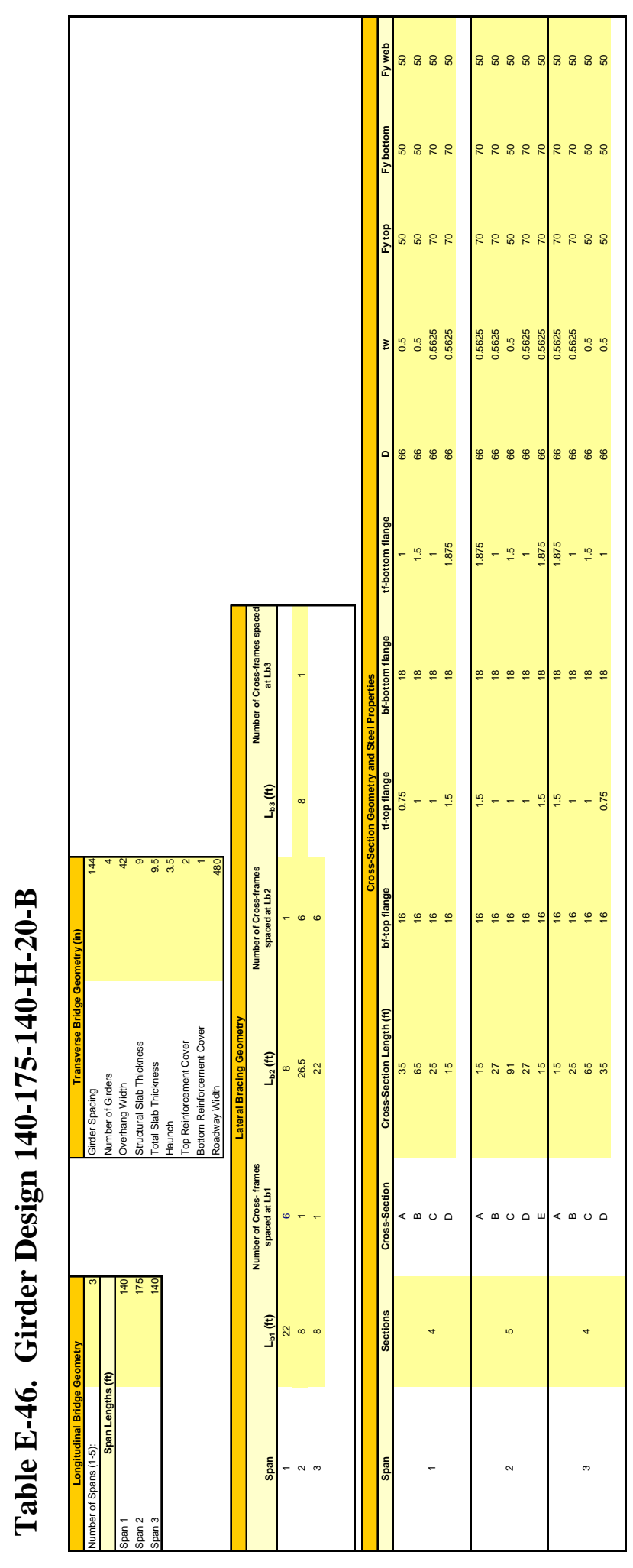




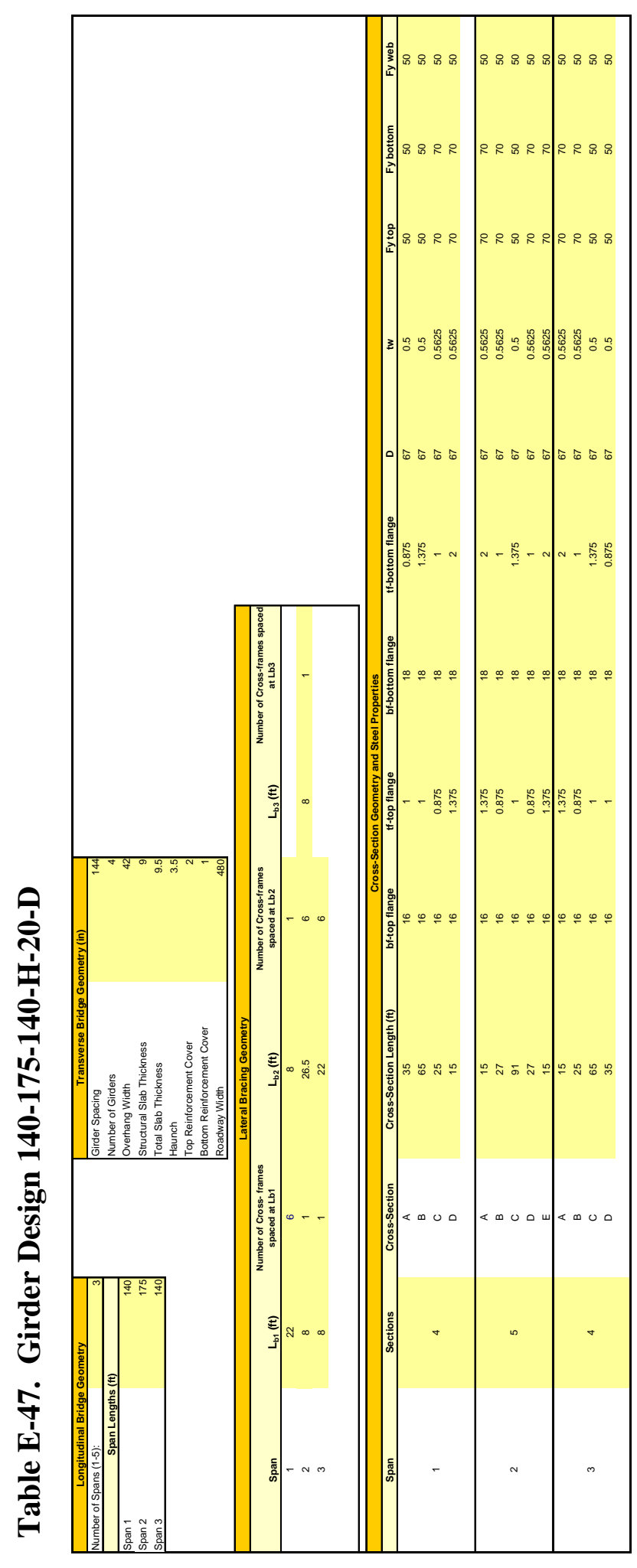




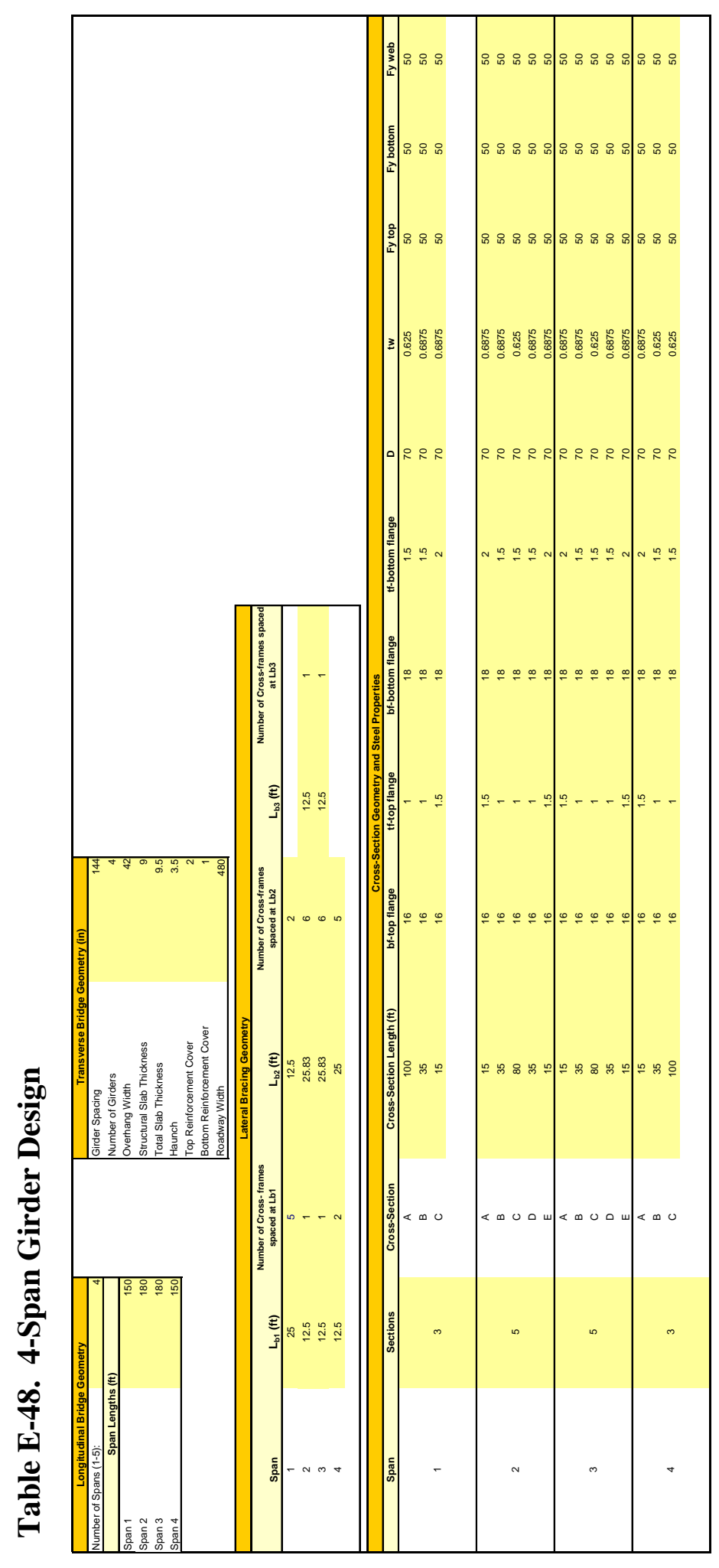

